\title{
Kapitalrationierung \\ am deutschen Aktiensekundärmarkt
}

\section{institutionenökonomisch untersucht}

\author{
Dissertation \\ zur Erlangung des wirtschaftswissenschaftlichen Doktorgrades der \\ Wirtschaftswissenschaftlichen Fakultät der Universität Göttingen
}

vorgelegt von Jörg Oelschläger aus Hagen i.Westf.

Göttingen, 2002 
Erstgutachter: Prof. Dr. G. Kucera

Zweitgutachter: Prof. Dr. W. König

Tag der mündlichen Prüfung: 05.02.2002 


\section{- Gliederung -}

Abkürzungsverzeichnis $\quad 5$

$\begin{array}{ll}\text { Abbildungsverzeichnis } & 7\end{array}$

$\begin{array}{lr}\text { Symbolverzeichnis } & 9\end{array}$

1 Problemskizze 11

2 Niveaubedingte Unterliquidität als Hemmnis wirtschaftlicher Entwicklung 18

2.1 Die Segmentierung des deutschen Aktiensekundärmarktes 19

$\underline{2.2}$ Unterliquidität am deutschen Aktiensekundärmarkt 37

$\underline{2.3}$ Arten der Unterliquidität $\quad 46$

2.4 Niveaubedingte Unterliquidität und wirtschaftliche Entwicklung 57

$\underline{3}$ Niveaubedingte Unterliquidität am Capital asset Pricing model 74

3.1 Der Funktionsmechanismus des sekundärmarktes 75

3.2 Niveaubedingte Unterliquidität und CAPM 90

$\underline{4}$ Niveaubedingte Unterliquidität als Ergebnis einer Kapitalrationierung $\begin{array}{ll}\text { am Sekundärmarkt } & 103\end{array}$

$\begin{array}{ll}4.1 & 104\end{array}$

4.2 Institutionenökonomischer Modellrahmen 113

4.2.1 Die Anteilseignerschaft als relationaler Vertrag 114

4.2.2 Informations- und Ertragsasymmetrien 121

4.2.3 Selektion des Sekundärmarktes? 136

4.3 Sekundärmarktrationierung 142

4.3.1 Poolbildung im Anlegerportefeuille 143

4.3.2 Adverse Selektion $\quad 150$

4.3.3 Adverse Anreize 163

4.3.4 Unterinvestition am Sekundärmarkt 165 
4.3.5 Modellerweiterungen

4.3.5.1 Bimodale Netto-Renditeerwartung

4.3.5.2 Ungewissheit

4.3.5.3 Mehrperiodigkeit

4.3.5.4 Favorable Selektion

$\underline{4.4}$ Geld- und Fiskalpolitik im „equity channel“ 196

$\underline{4.5}$ Unterliquiditätsfalle

$\underline{5}$ Wirtschaftspolitische Implikationen

$\underline{5.1}$ Risikoüberwachung: Externes Mittelstands-Rating

5.2 Risikotransfer: Garantieleistungen

221

5.3 Risikosignale: Eigeninvestitionen ins Downgrade-Risiko 228

$\underline{5.4}$ Risikogemeinschaften: Co-opetition 233

$\underline{5.5}$ Finanzielle Anreize staatlicher Förderung 


\section{Abkürzungsverzeichnis}

a.

AG

AktG

APT

BAKred

Bd.

BFuP

BIP

BIZ

BSP

BörsG

BMWi

CAPM

$\mathrm{CDO}$

CEO

c.p.

csV

d.A.

DAI

Dax

dcf

DIW

EASDAQ

EU

EZB

F.A.Z.

FRBNY

FWB

GG

HGB

auch

Aktiengesellschaft

Aktiengesetz

Arbitrage Pricing Theory

Bundesaufsichtsamt für das Kreditwesen

Band

Betriebswirtschaftliche Forschung und Praxis

Bruttoinlandsprodukt

Bank für internationalen Zahlungsausgleich

Bruttosozialprodukt

Börsengesetz

Bundesministerium für Wirtschaft und Technologie

Capital Asset Pricing Model

Credit Default Option

Chief Executive Officer

ceteris paribus

costly state verification

der Autor

Deutsches Aktieninstitut

Deutscher Aktienindex

discounted cash flow

Deutsches Institut für Wirtschaftsforschung

European Association of Securities Dealers Automated

Quotations

Europäische Union

Europäische Zentralbank

Frankfurter Allgemeine Zeitung

Federal Reserve Bank of New York

Frankfurter Wertpapierbörse

Grundgesetz

Handelsgesetzbuch 


\begin{tabular}{|c|c|}
\hline HWWA & Hamburger Weltwirtschaftsarchiv \\
\hline IAS & International Accounting Standards \\
\hline IPO & Initial Public Offering \\
\hline JEI & Journal of Economic Issues \\
\hline JITE & Journal of Institutional and Theoretical Economics \\
\hline $\mathrm{JuS}$ & Juristische Schriften \\
\hline KfW & Kreditanstalt für Wiederaufbau \\
\hline KGV & Kurs/Gewinn-Verhältnis \\
\hline $\mathrm{kmU}$ & kleine und mittlere Unternehmen \\
\hline LSE & London School of Economics \\
\hline $\mathrm{MBI} / \mathrm{MBO}$ & Management Buy In / Out \\
\hline Mdax & Mid Cap Deutscher Aktienindex \\
\hline MEW & Marx-Engels-Werke \\
\hline Mid Caps & Mid Capitalized Enterprises \\
\hline NBER & National Bureau of Economics Research \\
\hline Nemax & Neuer Markt Index \\
\hline NJ & New Jersey \\
\hline NY & New York \\
\hline p.a. & per annum / pro anno \\
\hline ROA & rate of return on total assets \\
\hline ROE & rate of return on equity \\
\hline ROI & rate of return on total investment \\
\hline SEC & The Securities and Exchange Commission \\
\hline Small Caps & Small Capitalized Enterprises \\
\hline Smax & Small Caps Index \\
\hline UBBG & Gesetz für Unternehmensbeteiligungsgesellschaften \\
\hline US-GAAP & US-Generally Accepted Accounting Principles \\
\hline WiSt & Wirtschaftswissenschaftliches Studium \\
\hline WISU & Das Wirtschaftsstudium \\
\hline ZEW & Zentrum für Europäische Wirtschaftsforschung GmbH \\
\hline Zfo & Zeitschrift für Organisation \\
\hline $\mathrm{ZgS}$ & Zeitschrift für die gesamte Staatswissenschaft \\
\hline $3 \mathrm{i}$ & 3i Deutschland Gesellschaft für Industriebeteiligungen $\mathrm{mbH}$ \\
\hline
\end{tabular}




\section{Abbildungsverzeichnis}

Abbildung 2.1 „Lorenzkurve“ zum deutschen Aktiensekundärmarkt

Abbildung 2.2 Konzentrationsmaße zum deutschen Aktiensekundärmarkt

Abbildung 2.3 Kurs-Gewinn-Verhältnisse einzelner Marktsegmente

Abbildung 2.4 Bid-Ask-Spreads nach Segmenten / Segmentausschnitten

Abbildung 2.5 Arten der Unterliquidität in zeitlicher Abfolge

Abbildung 3.1 Wertpapiermarktlinie im Up-Market

Abbildung 3.2 Die Wertpapiermarktlinie als Konjunkturindikator

Abbildung 3.3 Eine niveaubedingt unterliquide Aktie am CAPM

Abbildung 4.1 Asymmetrische Beteiligung an den unternehmerischen Investitionserträgen

Abbildung 4.2 Adverse Selektion im Up-Market

Abbildung 4.3 Adverse Selektion in Abhängigkeit vom Informationsgrad und der Verteilung der Einzelrisiken

Abbildung 4.4 Adverser Anreizmechanismus

Abbildung 4.5 Unterinvestition am Sekundärmarkt

Abbildung 4.6 Maximale Angleichung der Pool- an die Wertpapiermarktlinie

Abbildung 4.7 Bimodale Netto-Renditeerwartung

Abbildung 4.8 Sekundärmarktrationierung bei abnehmender Risikoaversion und zunehmender Ungewissheit 
Abbildung 5.1 Handel eines Downgrade-Risikos per CDO

Abbildung 5.2 Separationsgleichgewicht durch eigene Garantieverpflichtungen 


\section{Symbolverzeichnis}

\begin{tabular}{|c|c|}
\hline \multirow{3}{*}{$\begin{array}{l}\beta \mathrm{n} \\
\mathrm{dcf}_{\mathrm{i}}^{\text {prinz/agent }}\end{array}$} & Beta-Risiko von $n$ unsicheren Anlagen im CAPM \\
\hline & diskontierter Einzahlungsüberschuss des Unternehmens i aus \\
\hline & seinen Investitionen, die im Erfolgsfall den Prinzipalen / \\
\hline & Agenten zufallen. \\
\hline $\mathrm{dcf}_{\mathrm{i}}^{\text {free }}$ & frei verfügarer Cash Flow des Unternehmens $\mathrm{i}\left(=\mathrm{dcf}_{\mathrm{i}}{ }^{\text {agent }} \times \mathrm{p}_{\mathrm{i}}\right)$ \\
\hline \multirow[t]{2}{*}{$\mathrm{E}$} & erwarteter Netto-Ertrag für die Eigenkapitalgeber des \\
\hline & Unternehmens $\mathrm{i}\left(=\mathrm{dcf}_{\mathrm{i}}^{\mathrm{prinz}} \times \mathrm{p}_{\mathrm{i}}\right)$ \\
\hline EK & eingesetztes Eigenkapital \\
\hline$\mu(r)$ & Erwartungswert der Renditeverteilung $r$ \\
\hline$\mu_{\mathrm{i}}^{\mathrm{erw}}$ & erwartete Rendite der Aktie i \\
\hline$\mu_{\mathrm{i}}^{\text {gef }}$ & geforderte Rendite der Aktie i \\
\hline \multirow[t]{3}{*}{$\mu_{\mathrm{n}}^{\text {gef* }}$} & Wertpapiermarktlinie: geforderte und erwartete \\
\hline & Gleichgewichtsrendite von n Einzelaktien im CAPM bei \\
\hline & vollkommenen Informationen \\
\hline \multirow[t]{3}{*}{$\mu_{\mathrm{n}}^{\text {gefø }}$} & Poollinie: geforderte und (brutto) erwartete Rendite der \\
\hline & Durchschnittsrisiken von n Pools eines Anlegerportefeuilles \\
\hline & bei asymmetrischer Informationsverteilung \\
\hline \multirow[t]{2}{*}{$\mathrm{p}_{\mathrm{i}}$} & Wahrscheinlichkeit der Investitionsprojekte des Unternehmens \\
\hline & $\mathrm{i}$ \\
\hline \multirow[t]{2}{*}{$\rho_{i}$} & erwartete Netto-Rendite für die Eigenkapitalgeber des \\
\hline & Unternehmens i auf das eingesetzte Eigenkapital (= E/EK) \\
\hline \multirow[t]{2}{*}{$\mathrm{R}_{\mathrm{i}}$} & Investitionsertrag des Unternehmens i zur Bedienung der \\
\hline & Aktionärsforderungen $\left[=\left(1+\mu_{\mathrm{i}}^{\mathrm{gef}}\right) \mathrm{EK}\right]$ \\
\hline \multirow[t]{2}{*}{$r_{i}^{\text {int }}$} & Interne Verzinsung des eingesetzten Eigenkapitals im \\
\hline & Unternehmen i \\
\hline$r_{i}^{\text {tat }}$ & tatsächliche Rendite der Aktie i \\
\hline \multirow[t]{2}{*}{$\mathrm{r}_{\mathrm{i} / \mathrm{M}}$} & Renditeverteilung einer einzelnen Aktie i / des \\
\hline & Marktportefeuilles M \\
\hline $\mathrm{r}_{\mathrm{f}}$ & risikoloser Zinssatz \\
\hline$\sigma(\mathrm{r}) / \sigma^{2}(\mathrm{r})$ & Standardabweichung / Varianz der Renditeverteilung $\mathrm{r}$ \\
\hline
\end{tabular}


$\tau_{\mathrm{i}}^{\mathrm{adv}}$ alle bewertungsrelevanten Risiken der n Aktien

durchschnittliches bewertungsrelevantes Risiko des Pools i

gemäß der Poollinie

durchschnittlich bewertungsrelevantes Risiko des Pools i nach

Anhebung der Renditeforderung 
„Der Marktmechanismus hat es seit der Industriellen Revolution nicht fertiggestellt, wenn immer die Wirtschaft in den Griff der Stagnation in den jeweiligen Leitindustrien geriet und ins technologische Patt hineintrieb, beizeiten Kapital und Arbeit aus den stagnierenden Industriebereichen abzuziehen und in neue Technologien umzulenken." (Mensch, 1975, 166.)

\section{Problemskizze}

Die deutsche Wirtschaft verfügt über ein ausgeprägt mittelständisches Rückgrat. Innovationen, Neu- und Ausgründungen kleiner und mittlerer Unternehmen bilden den „Humus“ für gesamtwirtschaftliches Wachstum und Beschäftigung. Seit einigen Jahren ist allerdings die gesamtwirtschaftliche Entwicklung zu schwach, um die Unterbeschäftigung in Deutschland entscheidend abzubauen. Zur Behebung wachstumsdefizitärer Arbeitslosigkeit kommt insbesondere Produktinnovationen eine hohe Bedeutung $\mathrm{zu}$, tragen sie doch wesentlich zur Überwindung allgemeiner Sättigungstendenzen bei. Produktinnovationen gehen in den Unternehmen zumeist mit riskanten Investitionen einher, die auf lange Sicht hohe Finanzvolumina binden. Um Investitionen dieser Art aus eigener Kraft tätigen zu können, ist das Korsett der Eigenkapitalausstattung kleiner und mittlerer Unternehmen oft $\mathrm{zu}$ eng geschnürt. ${ }^{1}$ Auch sind ihre Möglichkeiten zur Innenfinanzierung allzu begrenzt. Langfristiges Haftungskapital von außen zuzuführen, wird deshalb von vielen Ökonomen für notwendig erachtet, um das Innovationspotenzial des deutschen Mittelstandes fruchtbar zu machen.

Eine Möglichkeit, den Finanzierungsengpass kleiner und mittlerer Unternehmen zu weiten, besteht in der Bereitstellung von Eigenkapital über den Aktienmarkt. ${ }^{2}$ Aktienmärkte stellen hohe Summen langfristig haftenden Eigenkapitals für unsichere unternehmerische Investitionsprojekte über den Emissions- beziehungsweise den Primärmarkt bereit. Ähnlich der Finanziellen im Vorfeld der Industriellen Revolution

Der Anteil mittelständischer Betriebe mit einer Eigenkapitalquote unter 10\% - gemessen an der Bilanzsumme - liegt bei 38,8\%. Vgl. Jahresbericht der Creditreform AG (2000). Zu den durchschnittlich höheren Fremdfinanzierungsquoten der $\mathrm{kmU}$ im Verhältnis $\mathrm{zu}$ Großunternehmen vgl. Tholen (2000). Vgl. a. Rasch 1996, 32-37. 
des vorletzten Jahrhunderts nimmt seit einigen Jahren die kapitalmarktorientierte Unternehmensfinanzierung stark zu. Verstärkt durch Umbrüche an den deutschen Finanzmärkten zeichnet sich auch in der „Old Economy ${ }^{63}$ des deutschen Mittelstands eine Tendenz hin zur Finanzierung über Aktienmärkte $a b .{ }^{4}$ Doch läuft mit den entsprechend negativen Folgen für Wachstum und Beschäftigung nicht eine ,stärkere Kapitalmarktorientierung prinzipiell auf eine relative Benachteiligung kleinerer Unternehmen hinaus?“5

Der deutsche Aktienzirkulations- beziehungsweise -sekundärmarkt ist trotz seiner allgemeinen Tendenz zur Vervollkommnung stark segmentiert (Kapitel 2). Es besteht eine Anlegerpräferenz für die wenigen großen Unternehmen bei gleichzeitiger Vernachlässigung einer breiten Schicht kleiner und mittlerer Gesellschaften. Das Kapitalaufkommen zwischen ihren Segmenten ist in hohem Maße ungleich verteilt. Während sich der Aktienhandel auf die Standardwerte des Dax 30 konzentriert, nimmt sich bereits das Anlegerinteresse an Werten aus der zweiten Reihe bescheiden aus. Die Nachfrage nach kleineren börsennotierten Aktientiteln der Old Economy stellt gar eine quantité négligable dar. Und die jüngeren Entwicklungen am Neuen Markt erinnern daran, dass „,[n]amentlich aber die Spekulation, die besonders die Aktien neuer Unternehmungen oft ganz allein auf dem Markt hält, fast nur mit solchen kurzfristigen, die heute gewährt und morgen versagt werden können. “6 Es scheint, als sei der zu erwirtschaftende Cash Flow eines Unternehmens im Verhältnis zu seinem Risiko nicht immer die treibende Kraft für die Allokation von Finanzkapital am Sekundärmarkt. So sind die Aktien zahlreicher kleiner und mittlerer Gesellschaften abseits des Medieninteresses im Vergleich zu denen der Standardwerte dauerhaft unterbewertet, weil anlegerseits unbeachtet. Auch liegen ihre Aktienkurse oft unterhalb ihrer fundamentalen Unternehmenswerte (Fair Value). ${ }^{7}$ Folgeemissionen am Primärmarkt zu plazieren, ist dank eines geringen Anlegerinteresses für kleine und mittlere Gesellschaften außerdem stark eingeschränkt, zumindest jedoch mit höheren Eigenkapitalkosten verbunden. Sowohl

\footnotetext{
2 „Aus Untersuchungen des Deutschen Aktieninstituts geht hervor, dass wichtige Motive für den Börsengang die Wachstumsfinanzierung, die Erhöhung der Eigenkapitalquote ... sind." Scholz 2000, 520.

Zur Abgrenzung der Old von der New Economy vgl. Funk (2000); EZB 2000, 48f.

Vgl. Reden 2000, 533f.

Kotz 2000, 638.

Schumpeter 1926, 204.
} 
die Unterbewertung ihrer emittierten Aktien als auch die Einschränkungen im Emissionsvolumen führen $\mathrm{zu}$ einer im Verhältnis $\mathrm{zu}$ Standardwerten geringeren Marktkapitalisierung kleiner und mittlerer Aktiengesellschaften. ${ }^{8}$ Der Zugang zum Aktienmarkt als dauerhafte Quelle der Eigenkapitalbeschaffung - er scheint für viele kleine und mittlere Unternehmen faktisch versperrt. „Es sollte daher nicht wundern, wenn 2001 ein weiterer Schritt auf dem Weg zur Renaissance der Privatunternehmen getan würde - mit Deutschland in führender Position. ${ }^{\text {(9) }}$ Mit erwarteten 5-10\% aller deutschen börsennotierten Aktiengesellschaften werden nicht wenige von ihnen vom Aktienmarkt verschwinden. Auch jene unter den kleinen und mittleren Gesellschaften, deren Investitionstätigkeit den Rendite/Risiko-Anforderungen der Anleger genügen, stoßen am deutschen Aktiensekundärmarkt großenteils auf ein geringes Anlegerinteresse. Die Aktien mitunter auch dieser für die Eigenkapitalgeber eigentlich „guten“ Einzelrisiken werden nicht in hinreichendem Umfang zu ihren fundamentalen Aktienkursen nachgefragt. Die Nachfrage auch ihrer Aktien reagiert kursunelastisch; ihre Unterbewertung ist ein bleibendes Phänomen. Anders gewendet: Zum fundamental richtig bewerteten Aktienkurs wird am Sekundärmarkt von den Anlegern weniger Eigenkapital angeboten, als von den Unternehmen an Aktienkapital über den Primärmarkt emittiert wurde. Ein insofern „unterschießendes“ Eigenkapitalangebot geht für die Anteilseigner vernachlässigter Gesellschaften mit Unterliquidität einher. Ein geplanter Aktienverkauf sucht zum Fair Value in Zeitpunkt und Volumen oft vergeblich ein entsprechendes Kaufinteresse. Nicht nur zu einzelnen Zeitpunkten liegen Profildiskrepanzen zwischen dem geplanten Aktienangebot und der geplanten Aktiennachfrage am Sekundärmarkt vor. Vielmehr ist das Niveau der geplanten Aktiennachfrage grundsätzlich geringer als das des Aktienangebots. Eine insofern niveaubedingte Unterliquidität geht für die Eigner vernachlässigter Gesellschaften mit teils empfindlichen Kosten einher.

Um sich niveaubedingte Unterliquidität als Gleichgewichtsphänomen eines unterschießenden Angebots an Eigenkapital am Aktiensekundärmarkt zu erschließen, erweist sich das in Theorie und Praxis vorherrschende statische Referenzmodell, das

Allein „3i hat mindestens 150 börsennotierte Firmen in Deutschland identifiziert, die durch die Börse unterbewertet sind." Cullom 2001, 18.

Die Kapitalisierung einer Gesellschaft entspricht dem Produkt aus der Anzahl emittierter Aktien und dem Aktienkurs.

Marsh 2001, 25. 
„Capital Asset Pricing Model“, als ungeeignet (Kapitel 3). Das CAPM beschreibt das (zum Fair Value) markträumende Gleichgewicht von Eigenkapitalangebot durch die Anleger und von „Eigenkapitalnachfrage“ am Sekundärmarkt durch die Unternehmen in Höhe der ausgeschütteten Aktien. Im CAPM halten rationale Anleger unter Unsicherheit geplantermaßen alle umlaufenden Aktien im Verhältnis ihrer fundamental richtig bewerteten Marktwerte. ${ }^{10}$ Niveaubedingte Unterliquidität der Aktien kleiner und mittlerer Gesellschaften als das Ergebnis eines insgesamt unterschüssigen Eigenkapitalangebots ist unter den neoklassischen Annahmen des CAPM mithin nicht zu erklären. Auch in seiner oft bemühten „dynamisierten“ Form impliziert das CAPM ein langfristig markträumendes Eigenkapitalangebot in Höhe des fundamental richtig bewerteten emittierten Aktienkapitals. Denn auch hierin wird der Preis- respektive Renditemechanismus als gänzlich flexibel unterstellt.

Um das CAPM für die Erklärung einer Rationierung der unternehmerischen Eigenkapitalnachfrage am Sekundärmarkt durch ein „unterschießendes“ Eigenkapitalangebot nutzbar $\mathrm{zu}$ machen, bedarf es gewissermaßen seiner Institutionalisierung (Kapitel 4). Insbesondere die Verteilung von Informationen zwischen den Eigenkapitalgebern (Prinzipalen) und den Eigenkapitalnehmern (Agenten) sowie die Verteilung der Cash Flows aus den Unternehmensinvestitionen zwischen ihnen ist den realen Verhältnissen anzupassen. ${ }^{11}$ An einem nur mehr als unvollkommen unterstellten Aktienmarkt mit asymmetrisch verteilten Informationen zu Lasten der Eigenkapitalgeber zeigt der Preis für die Übernahme einer bewertungsrelevanten Einheit Risikos nicht nur die relative Knappheit von Eigenkapital an; die Renditeforderung der Anleger übernimmt zusätzlich eine Indikatorfunktion für die Höhe der $\mathrm{zu}$ finanzierenden, ihm unbekannten Einzelrisiken. Außerdem ist vor allem für eine Stakeholder-Ökonomie „rheinischer“ Prägung eine abnehmende Beteiligung der Eigenkapitalgeber an den Investitionserträgen der Unternehmen kennzeichnend. Unter beiden AsymmetrieBedingungen stellt ein markträumendes Eigenkapitalangebot am Sekundärmarkt -

10 Liegt die fundamental richtig bewertete Marktkapitalisierung der annahmegemäß einzigen beiden Gesellschaften bei 400.000 EUR (Aktie A) und 600.000 EUR (Aktie B), besteht das eine Million EUR teure Portefeuille des rationalen Anlegers im CAPM zu 40\% aus A- und zu $60 \%$ aus B-Aktien.

11 Mit der Einführung von Informations- und Ertragsasymmetrien zwischen den Eigenkapitalgebern und -nehmern wird der neoklassische Kern des CAPM in modifizierter Form beibehalten. Damit folgt die Argumentation der Aufforderung Leijonhufvuds 1989, 18: „Try to beat them at their own game“... ,Refuse to play their rules“. 
im CAPM als Regel unterstellt - nur eine theoretische Möglichkeit, jedoch keine Notwendigkeit dar. Asymmetrischen Informations- und Ertragsverteilungen zu Lasten der Prinzipale ist es geschuldet, dass adverse Selektions- und Anreizrisiken, die vom Markt nicht entgolten werden, die Funktionstüchtigkeit des Preis- respektive Renditemechanismus am Aktiensekundärmarkt einschränken. Um eine Negativauslese in seinem Portefeuille zu verhindern, fordert der rationale Anleger an einem (wohlgemerkt!) polypolistischen Sekundärmarkt maximale Renditen, die möglicherweise unterhalb des markträumendem Niveaus liegen. Eine nach oben starre Netto-Renditeerwartung der Eigenkapitalgeber führt dabei regelmäßig zu einem Angebotsmangel an Eigenkapital im dauerhaften Gleichgewicht. Die Wahrscheinlichkeit des Auftretens adverser Selektions- und Anreizrisiken nimmt c.p. in so genannten Up-Markets, das heißt bei zunehmenden Renditeforderungen für die Übernahme einer weiteren bewertungsrelevanten Risikoeinheit, zu. Wie im Boom eines Konjunkturverlaufs bedeutet dies bei einem hohen Zufluss von Finanzkapital nicht zwangsläufig, dass die Bewertung der vernachlässigten, das heißt rationierten Gesellschaften absolut, so doch zumindest relativ zu den Standardwerten sinkt. Die Berücksichtigung weiterer relevanter, im Rationierung-Grundmodell nicht vorkommender Aspekte wie etwa ein mehrperiodiger Planungshorizont der Eigenkapitalgeber oder ihre Ungewissheit entwickelt eine zusätzliche Annäherung des Rationierungsansatzes an jüngere Entwicklungen am Kapitalmarkt. Ihre Auswirkungen lassen dabei auf ein künftig eher ver- als entschärfendes Ausmaß an Sekundärmarktrationierung schließen.

Das insgesamt eingeschränkte und damit rationierende Eigenkapitalangebot erstreckt sich im Zuge der Kapitalrationierung am Sekundärmarkt nicht auf alle am Sekundärmarkt notierenden Gesellschaften in gleichem Maße. Dem Rendite/RisikoProfil nach identisch erscheinende Gesellschaften werden je nach „sekundär“ökonomischen Attributen wie der Größe einer Gesellschaft, ihrem Alter, ihrer Branchenzugehörigkeit, ihrem Renommé sowie ihrer allgemeinen Medienbeachtung teils be-, teils übervorteilt und damit vollrationiert. Das „primär“-ökonomische Kriterium des erwarteten Rendite/Risiko-Profils einer einzelnen Aktie spielt für die Aufteilung der Kapitalströme auf die einzelnen Segmente unter den genannten Bedingungen eine nur mehr nachgeordnete Rolle. Bei einer Vollrationierung bestimmter Gesellschaftstypen weisen die Teilmärkte eines Aktiengesamtmarktes 
trotz geringer (formaler) Substitutionslücken zum Teil deutlich unterschiedliche Liquiditätsgrade auf. ${ }^{12}$ Es sind insbesondere kleine und mittlere Aktiengesellschaften der Old Economy von geringer Medienbeachtung, die in die „Unterliquiditätsfalle“ einer nach oben starren Renditeforderung tappen. Eigendynamiken sowie soziologische und (massen-)psychologische Aspekte tragen zur Konzentration des unterschießenden Eigenkapitalangebots auf die Aktien nur weniger Gesellschaften zusätzlich bei. Den vernachlässigten Unternehmen hingegen widerfährt zunächst am Sekundär-, im Gefolge auch am Primärmarkt eine Eigenkapitalrationierung.

Anders als zu Zeiten der Finanziellen Revolution ist für kleine und mittlere Unternehmen dieser Tage der Zugang zum Aktienmarkt formal geöffnet. Heute sind es eher vom Markt unentgoltene adverse Selektions- und Anreizrisiken, die die Finanzierung von Pionierideen über den Aktienmarkt erschweren. Es rücken damit die faktischen Zutrittsbarrieren zur Finanzquelle „Aktienmarkt“" in den Mittelpunkt wirtschaftspolitischer Betrachtungen. Denn infolge einer Rationierung am Sekundärmarkt unterbleiben solche Unternehmensinvestitionen, die für die gesamtwirtschaftliche Entwicklung möglicherweise notwendig sind. Dabei beeinträchtigt eine Kapitalrationierung am Sekundärmarkt insbesondere den Beginn eines Lange-Wellen-Aufschwungs. ${ }^{13}$ Sie mindert die Informations-, Bewertungs- und Transaktionskosteneffizienz des Sekundärmarktmechanismus und verhindert auf diese Weise, dass das ohnehin eingeschränkte Eigenkapital in seine volkswirtschaftlich beste Verwendung über den Primärmarkt gelenkt wird. Negative Wachstumswirkungen, aber auch katalysierende Effekte auf die Geld- und Fiskalpolitik im „equity channel“ verlangen nach wirtschaftspolitischen Gegenmaßnahmen.

Sollen sich geeignete Maßnahmen unmittelbar aus der institutionenökonomischen Ursachenforschung ableiten, müssen sie sich grundsätzlich an der Informationsausstattung der Eigenkapitalgeber, an der Beseitigung und Transformation adverser Selektions- und Anreizrisiken oder direkt an der Anhebung

12 Während sich die Keynes'sche Liquiditätstheorie mit der optimalen Aufteilung eines Vermögens auf Anlagen unterschiedlicher Liquiditätsgrade befasst, geht es hier um die Erklärung unterschiedlicher Liquiditätsgrade identisch erscheinender Anlagen. Vgl. Keynes 1936, chap. 17.

13 Es „wird z.B. in Europa zu wenig Venture Kapital angeboten und kleine Firmen haben es sehr schwer, sich am Markt zu finanzieren.“ Nefiodow 1996, 88. 
der zu erwartenden Netto-Rendite ansetzen (Kapitel 5). Im Einzelnen wird einem wirtschaftspolitischen Maßnahmen-Mix aus einer verbesserten Erkennung guter Einzelrisiken durch Aktienratings, aus der Verbriefung adverser Anreiz- und Selektionsrisiken mit Hilfe so genannter Credit Default Options, aus einer RisikoSelbstanzeige guter Einzelrisiken durch eigene Garantieleistungen, aus verschiedenen Arten von Kooperationen vernachlässigter Gesellschaften sowie aus einer negativen „Tobinsteuer“ das Wort geredet. Mit der Implementierung entsprechender Institutionen ist einerseits die Notwendigkeit einer wirtschaftspolitischen Mikrosteuerung gegeben. ${ }^{14}$ Mehr noch als die Schaffung verlässlicher Rahmenbedingungen ist das aktive Mitwirken verschiedener Träger der Wirtschaftspolitik im Bereich der Risikoreduzierung und -steuerung wesentliche Voraussetzung für die Eindämmung einer Kapitalrationierung am Sekundärmarkt. ${ }^{15}$ „Wenn die Wirtschaftspolitik effizienter werden soll, dann muß sie wieder näher an den Markt herangeführt werden."16 Andererseits sind die genannten staatlichen Eingriffe das Ergebnis eines zielgerichteten wirtschaftspolitischen Entwurfs. Denn adäquate Institutionen bilden sich nicht „ohne alle Uebereinkunft, ohne legislativen Zwang, ja selbst ohne Berücksichtigung des öffentlichen Interesses ${ }^{\text {(117 }}$ quasi aus sich heraus; die wirtschaftspolitischen Vorschläge der vorliegenden Arbeit folgen somit eher einem konstruktivistischen, denn einem evolutionären Rationalismus. ${ }^{18}$ Doch kann man in einer traditionell bankorientierten deutschen Förderlandschaft künftig mit dem Ergreifen entsprechender Maßnahmen rechnen (Kapitel 6)?

Vgl. Reuter 1994a, 363.

Vgl. Landeszentralbank Hessen (2001).

Nefiodow 1996, 84.

Menger 1969, 176.

Vgl. Hayek 1973, 33 u. 38f. 
2 Niveaubedingte Unterliquidität

als

Hemmnis wirtschaftlicher Entwicklung 


\subsection{Segmentierung des deutschen Aktiensekundärmarktes}

Im internationalen Vergleich lange Zeit als unterentwickelt geltend, ${ }^{19}$ mauserte sich in den letzten Jahren der deutsche Aktiensekundärmarkt zu einem hoch organisierten Finanzmarkt. ${ }^{20}$ Sein Wachstum ist erheblich. ${ }^{21}$ Bereits 67\% betrug der Anteil der Börsenkapitalisierung aller inländischen Aktiengesellschaften am BIP im Jahr 2000. ${ }^{22}$ Mit seinem Wachstum erfüllt der deutsche Aktiensekundärmarkt immer mehr die Bedingungen eines vollkommenen Marktes: ${ }^{23}$ Erstens fordern Anleger eine immer größere Markttransparenz ein. Publizitätsvorschriften für die Emittenten sowie die Veröffentlichungen der Analysten, der Presse und der Börsenbetreiber tragen $\mathrm{zu}$ mehr Klarheit über die Unternehmen bei. ${ }^{24}$ Strenge Börsenregeln bewerkstelligen eine transparente Preisermittlung. Für Anleger wie Emittenten sinken durch diverse institutionelle Maßnahmen seitens der Intermediäre, der Emittenten, der Börsenorganisationen und des Gesetzgebers zweitens die Zutrittsbarrieren zum Aktienmarkt. ${ }^{25}$ Die Folge: die Anzahl der an die „Tränke“ des

19 Vgl. The World Development Report 1989, Tab. 7.1. In: Rasch 1996, 51. Im Zuge der Privatisierung von Preussag (1959) und VW (1961) wurde zwar Anfang der 60er Jahre fast jede vierte Mark des Geldvermögens der Bundesbürger in Aktien investiert. Im Durchschnitt der Jahre 1962 und 1982 stieg der Index der Börsenzeitung - der Vorgänger des Dax - jedoch nur um ein knappes Prozent im Verhältnis zum Rentenmarkt mit fast 8\% p.a.; Häuser (1985) bezeichnete diese Entwicklung als „Renditenparadoxie“. Der Aktienanteil am Geldvermögen der Bundesbürger schrumpfte in dieser Zeit von $12 \%$ auf $4 \%$.

$\mathrm{Zu}$ den Kriterien eines hoch organisierten Finanzmarktes vgl. Franke, Hax 1990, 368. Nach Ansicht der Bundesbank verdienten bereits die 80er Jahre einschließlich des Jahres 1990 das Prädikat „Jahrzehnt der Aktie“. Vgl. Deutsche Bundesbank 1991, 24. „,Der Anteil der im Wege von Aktienemissionen beschafften Finanzierungsmittel an den langfristigen Mittelaufnahmen aller im Produktionssektor tätigen Unternehmen (ohne Wohnungswirtschaft) an den Finanzmärkten hat 1990 mit gut einem Viertel wieder Größenordnungen erreicht, wie sie zuletzt verschiedentlich in den fünfziger und sechziger Jahren zu beobachten gewesen waren.“ Deutsche Bundesbank 1991, 22.

21 Vgl. Deutsche Bundesbank 1998, 57f. Bereits „[d]ie Wachstumsrate des Umsatzes zwischen 1980 und 1992 betrug jahresdurchschnittlich für Aktien ... zehn Prozent und lag damit weit über der von Exporten und Produktion." The Economist v. 07.10.95, 6. 1993 betrug die Börsenkapitalisierung am BIP 25,4\%, vgl. Ramb 2000, 2; 1997: 31,4\%, vgl. Jürgens, Naumann, Rupp 2000, 56; 1998: 48,1\%, 1999: 70,2\%, eigene Berechnungen laut Angaben des Statistischen Bundesamtes und der Deutsche Börse AG. Vgl. http://www.statistisches bundesamt.de und http://deutsche-boerse.com. $\mathrm{Zu}$ den Kriterien eines vollkommenen Marktes vgl. Ahrns, Feser 1991, 41-43; Felderer, Homburg 1987, 210.

24 Ein Mehr an Transparenzvorschriften spiegelt sich etwa in der Rechnungslegung nach IAS, US-GAAP, in mehrsprachigen Quartalsberichten mit Aussagen über den Geschäftsverlauf und die Ertragsentwicklung, in der ad hoc-Publizität und in der Offenlegung des Anteilsbesitzes von Aufsichtsrat und Vorstand wider.

25 Hierbei ist insbesondere an Folgendes $\mathrm{zu}$ denken: Regelungen zum Insiderwissen; Finanzmarktförderungsgesetze II (Neuordnung von Wertpapieraufsicht und Handelsüberwachung, Errichtung des Bundesaufsichtsamt für Wertpapierwesen) und III (Erweiterung des Anlagespektrums für Investmentfonds); Schaffung diverser Handelssegmente; Maßnahmen technischer Natur wie die Einführung des Xetra-Handels; Kooperationen der Börsen; Einschränkung der Prospekthaftung; Emissionsbündelung; 
Aktienmarktes geführten Unternehmen steigt; ${ }^{26}$ eine im Übrigen beachtliche Entwicklung nach den fehlgeschlagenen Implementierungsversuchen von Börsenmittelsegmenten auf breiter europäischer Front zu Anfang der 90er Jahre. ${ }^{27}$ Auch eine ansteigende Zahl von Unternehmen in der Rechtsform der Aktiengesellschaft sorgt für weiteren Nachschub potenzieller Börsengänge. ${ }^{28}$ Aber nicht nur als Finanzierungs-, sondern insbesondere als Anlageform ist seit Mitte der 90er Jahre - nicht zuletzt infolge einer weitgehenden Liberalisierung des Kapitalverkehrs sowie sinkender Realzinsen seit Anfang der 90er $-{ }^{29}$ das allgemeine Interesse an Aktien gestiegen. ${ }^{30}$ Mit circa einem Zehntel der Geldvermögensbildung floss seit 1991 in Deutschland direkt oder indirekt deutlich mehr Kapital in die Aktie als in den 80er Jahren. ${ }^{31}$ Mit zunehmender Zahl atomisieren private und institutionelle Anleger somit angebotsseitig ${ }^{32}$ und börsennotierte Gesellschaften nachfrageseitig den deutschen Aktiensekundärmarkt. Dank sinkender Transaktionskosten bei immer ausgereifteren Kommunikationstechnologien, dank einer Professionalisierung und Institutionalisierung des Anlagemanagements können

Etablierung von Benchmark-Produkten; laufende Stellung von Kursen; Einstellung von Mehrfachnotierungen; freier Wettbewerb der Preisbildungsmodelle; Verbesserungen bei der Aktienemission (Bookbuilding-Verfahren, Greenshoe); Derivatisierung von Mid Caps. Zur Zeit notieren die Anteilsscheine von ca. 1.000 Gesellschaften am Aktienmarkt. 1990 waren 649, 1995 nur 681 inländische Aktiengesellschaften börsennotiert. Von den anstehenden Regelungen des Generationenwechsels in mittelständischen Unternehmen, der zunehmenden Anzahl von Spin-offs im Zuge von Konzernverschlankungen, von grenzüberschreitenden Investitionen, von Expansionen und Bridge-Finanzierungen geht auch künftig eine Nachfrage nach Aktienfinanzierungen aus.

27 Vgl. Rasch 1994b, 512-515.

28 Tiefststand in 1982: 2.122 deutsche Aktiengesellschaften; Ende September 1998: 5.007 Gesellschaften. Vgl. Die Aktiengesellschaft 12/1998, R500f. Zum Gesetz für Kleine Aktiengesellschaften (1994) sowie zu seiner kontroversen Diskussion vgl. Frick (1994); Vollmer (1993). Vgl. Deutsche Bundesbank 2001, 43; Obstfeld 1998, 10-13; Weber 1999, $304 f$.

Vgl. Deutsche Bundesbank 1998, 58; vgl. Deutsche Bundesbank (1997, 1999a). Nach einer Infratest-Umfrage im Auftrag des DAI stieg die Zahl der Aktien- oder Aktienfonds-Besitzer auf 12,3 Millionen. Damit halten 19,3\% der bundesdeutschen Bevölkerung Wertpapiere. Im Jahr 1999 besaßen 8,2 Millionen Deutsche direkt oder über Fonds Aktien. Vgl. F.A.Z. v. 10.01.01, Nr. 8, 25. Populär wurde die Aktie hierzulande erst wieder mit dem Börsengang der Telekom im November 1996. Gegen Ende 1999 übersprang die Zahl der Aktionäre in Deutschland erstmals die 5-Millionen-Grenze. Der Anteil der Aktie am Geldvermögen ist mit ca. $10 \%$ im Steigen begriffen, allerdings deutlich unterhalb der 26,5\% im Jahre 1950. Allerdings nimmt sich Deutschland trotz des jüngsten Aktienbooms im internationalen Vergleich bescheiden aus. In den USA sind rund 25\% der Bevölkerung direkte Aktienbesitzer, in Großbritannien 23\% und in Schweden sogar 35\%. Vgl. F.A.Z. v. 02.08.00, Nr.177, 27. Vgl. Deutsche Bundesbank 1999a, 40.

Im Vergleich zu angelsächsischen Ländern ist die Anteilseignerstruktur der deutschen Wirtschaft allerdings vielfach noch durch Großaktionäre gekennzeichnet. Vgl. Shleifer, Vishny 1997, 754-772. Mit Beginn des Veranlagungszeitraums 2002 müssen Kapitalgesellschaften die Gewinne aus dem Verkauf von Beteiligungen an anderen Kapitalgesellschaften nicht versteuern, sofern die Beteiligung länger als ein Jahr dauerte. Damit erhalten die Entflechtungsbestrebungen der deutschen Wirtschaft einen weiteren, steuerlichen Anschub. Vgl. Jürgens, Naumann, Rupp 2000, 58. 
Investoren drittens mit höherer Anpassungsgeschwindigkeit reagieren und global diversifizierte Portfeuilles bilden. ${ }^{33}$ Moderne Finanzinstrumente zur Bewertung bedingter Ansprüche helfen, nicht diversifizierbare Risiken abzusichern. ${ }^{34}$ Vor dem Hintergrund erweiterter Transaktionsmöglichkeiten wirken Präferenzen sachlicher und räumlicher Art - wider das Kalkül der Gewinnmaximierung - viertens als Anachronismus. Das Hauptaugenmerk zunehmend rationaler Anleger fällt im Allgemeinen auf das erwartete Rendite/Risiko-Profil als Schlüsseleigenschaft einer Aktie. Mehr denn je ist die Renditeorientierung der Eigenkapitalgeber bewegendes Element einer Anlage. Die Geschwindigkeit, mit der auf gute wie auch vor allem auf schlechte Performance in großem Transaktionsvolumen reagiert wird, ist in den letzten Jahren deutlich angestiegen. Nur mehr auf das Rendite/Risiko-Profil reduziert, wird der Zirkulationsmarkt für Unternehmensanteile somit insgesamt homogener. Der damit entstehende Zwang für die Unternehmen, geforderte Aktienrenditen zu erwirtschaften, wird auf einzelne Unternehmensbereiche, die jeweils auf den Prüfstand gestellt werden, weitergereicht. Kurzum: Die homogenisierten Unternehmensprofile werden bei immer mehr Markttransparenz an einem zunehmend polypolistischen deutschen Aktiensekundärmarkt mit höherer Geschwindigkeit zu sinkenden Transaktionskosten gehandelt. „Der Aktienmarkt erfüllt die Kriterien des vollkommenen Marktes so gut wie kaum ein anderer Markt". ${ }^{35}$

Mit zunehmender Vollkommenheit des Aktienmarktes ist $\mathrm{zu}$ erwarten, dass die Grenzen zwischen seinen Teilmärkten fallen. ${ }^{36}$ Präferenzen für bestimmte Aktienwerte müssten erwartungsgemäß in den Hintergrund treten und ein „grenzüberschreitendes“ Anlegen erleichtern. Entgegen dem allgemeinen Prozess fortschreitender Vervollkommnung des deutschen Aktiensekundärmarktes bilden sich jedoch realiter immer neue Segmente und Teilmärkte heraus. Während

33 Wegweisend für die Strukturierung von Portefeuilles auf der Grundlage von Gleichgewichtsmodellen sind Markowitz (1952), Tobin (1957/58) und Sharpe (1970). Vgl. a. Abschnitt 3.1.

34 Die Bewertung derartiger Instrumente geht auf Modelle von Merton (1973a) und Black, Scholes (1972) zurück.

$35 \quad$ Soros 1998a, 57.

36 Nach Stackelberg (1934) ist ein Teil- bzw. Elementarmarkt als größter vollkommener Markt eines unvollkommenen Gesamtmarktes zu verstehen. Vgl. Stackelberg 1934, 29ff. Innerhalb eines Elementarmarktes beträgt die Kreuzpreiselastizität Eins. Eine weniger strikte Definition liefert Robinson (1969). Danach bilden alle Produkte eine Kette von Substituten, die durch so genannte Substitutionslücken unterbrochen wird. Diese Substitutionslücken kennzeichnen die Grenzen der Teilmärkte. Vgl. Robinson 1969, 4. 
Segmente durch formelle Kriterien sichtbar voneinander abgegrenzt werden, können die Grenzen der Teilmärkte auch innerhalb eines Segmentes verlaufen, ohne institutionell kenntlich gemacht zu sein. Das Börsensegment des Neuen Marktes grenzt sich beispielsweise durch formelle Kriterien vom Dax $30 \mathrm{ab}$. Innerhalb des Neuen Marktes lassen sich mit so genannten Schwergewichten, Neben- und Spezialwerten einerseits und unterschiedlichen Branchen (Mikroelektronik und Internet, Medien, Biotechnologie, Logistik etc.) andererseits bestimmte Teilmärkte ausmachen.

Der börsliche Aktiensekundärmarkt ist nach gesetzlichen Kriterien mit dem Amtlichen Handel, ${ }^{37}$ dem Geregelten Markt ${ }^{38}$ und dem Freiverkehr ${ }^{39}$ dreigeteilt. $^{40}$ Die drei Segmente unterscheiden sich in den Zulassungsbedingungen der Unternehmen, in den Publizitätsvorschriften sowie in der Art der Kursermittlung. ${ }^{41}$ An den acht inländischen Aktienbörsen notierten etwa Mitte März 2000 960 inländische Unternehmen. ${ }^{43}$ Mit 464 Unternehmen waren börsenrechtlich zwar nur knapp die Hälfte dem Amtlichen Handel zugeteilt; der Geregelte Markt umfasste 157, der Freiverkehr 339 Unternehmen. Doch fällt mit über neunzig Prozent der Börsenumsätze aller inländischen Aktiengesellschaften der Löwenanteil auf den Amtlichen Handel. Die gesetzliche Dreiteilung des deutschen Aktiensekundärmarktes teils überlagernd, haben die Betreiber der Börsen in den letzten Jahren mehrere Handelsplattformen mit einem je eigenen institutionellen Profil geschaffen. Neben der paneuropäisch ausgerichteten EASDAQ handelt es sich für den spezifisch deutschen Aktiensekundärmarkt hierbei um Segmente, die sich in der Größe der notierten Unternehmen - gemessen an der Marktkapitalisierung und dem Börsenumsatz ${ }^{44}$, in ihrer Branchenzugehörigkeit sowie in ihrer räumlichen

Vgl. §§ 36ff BörsG.

Vgl. §§ 71ff BörsG; Schrader (1993).

Vgl. § 78 BörsG.

Im Rahmen des geplanten Vierten Finanzmarktförderungsgesetzes wird eine Zusammenführung des Geregelten Marktes mit dem Freiverkehr erwogen. Auf diese Weise sollen im Zuge einer Deregulierung des Börsenrechts die Börsen einen größeren Spielraum für die Ausgestaltung des Nicht-Amtlichen Handels erhalten. Zum außerbörslichen Handel vgl. Stenzel (1995).

$41 \quad$ Vgl. Grill, Perczynski 1988, 319-321; Gerke, Rapp 1993, 292ff.; www.ip.exchange.de.

42 Stichtag: 13.03 .00$.

43 Darunter ist die Frankfurter Wertpapierbörse (FWB) die bedeutendste. Entfielen 1990 auf sie 62\% des Gesamtumsatzes, erhöhte sich der Anteil in 1999 auf 84\%. Vgl. Deutsche Börse AG: Historical Facts - Cash Market vom 05.01.00. In: www.exchange.de, $10 \mathrm{f}$.

44 „Es existiert kein einheitliches, objektiv „richtiges“ Kriterium für die Beurteilung der Unternehmensgröße. Als dynamischer, börsentäglich am Kapitalmarkt ermittelter, theoretischer Gesamtwert einer Aktiengesellschaft reflektiert der Marktwert des Eigenkapitals, 
Tragweite unterscheiden. Um nur die Wesentlichen zu nennen: Der Composite Deutsche Aktienindex (Cdax), in dem alle Aktienwerte des Amtlichen Handels notieren, umfasst mit dem Dax 100 die größten deutschen börsennotierten Aktiengesellschaften. Der Dax 100 unterteilt sich wiederum in den seine Blue Chips beinhaltenden Dax $30^{45}$ sowie in den siebzig Titel umfassenden Mdax der so genannten zweiten Reihe. ${ }^{46}$ Die größten Werte des Dax 30 notieren zudem im Aktienindex der europäischen Schwergewichte Euro Stoxx 50. Ein Segment für kleine und mittlere Aktiengesellschaften ist der Smax, einem 1999 gegründeten Börsensegment vornehmlich für Werte der Old Economy. Mitte März 2000 notierten in ihm 117 Titel aus dem Amtlichen Handel und dem Geregelten Markt. ${ }^{47}$ Die Schwergewichte des Smax werden in einem besonderen Index, dem so genannten Sdax hervorgehoben. Als bedeutendstes Mitglied im Verbund des Euro.NM stellt der 1997 aus der Taufe gehobene Neue Markt (Mitte März 2000: 188 Unternehmen) innerhalb des Freiverkehrs ein eigenständiges Handelssegment für Unternehmen dar, die vornehmlich auf Wachstumsmärkten agieren. ${ }^{48}$ Die große Mehrheit der am Neuen Markt gelisteten Gesellschaften sind High-Tech-Unternehmen aus der New Economy, ${ }^{49}$ deren Unternehmenswerte regelmäßig hohe Einkünfte erst in weiter Zukunft aufweisen. Auch die Schwergewichte des Nemax all shares Index werden im Nemax 50 hervorgehoben. Weitere Wachstums- und sonstige Themensegmente sind an den deutschen Regionalbörsen etabliert. ${ }^{50}$

bei Unterstellung eines effizienten Kapitalmarktes sowohl die zukünftige als auch die augenblickliche Bewertung der relevanten Einflußfaktoren und Kennzahlen eines Unternehmens. Er kann damit im Vergleich $\mathrm{zu}$ allen anderen Kriterien, trotz seiner Abhängigkeit von den auf die Börse einwirkenden psychologischen und gesamtwirtschaftlichen Einflußfaktoren, als die umfassendste Kennzahl zur Messung der Unternehmensgröße und damit als geeigneter Größenmaßstab qualifiziert werden." Beiker 1993, 263.

Der Dax 30 ist ein gewichteter Durchschnitt. Es handelt sich um einen so genannten Performanceindex. Bei seiner Berechnung wird unterstellt, dass die ausgeschütteten Dividenden reinvestiert werden. Damit sollen die Kursabschläge am Tag der Ausschüttung vermieden werden. Zur Konstruktion des Dax vgl. Janßen, Rudolph 1992, 8-33; Richard 1992, 106-141. Als Gewichte fungiert zur Zeit noch das zum Börsenhandel angemeldete Grundkapital. Von Juni 2002 an legt die Deutsche Börse AG bei der Berechnung der DaxGewichte nur noch den Streubesitz der liquidesten Aktiengattung zugrunde.

46 Vgl. Köhler (2000).

47 Zu den Teilnahmevoraussetzungen und Folgepflichten des Smax vgl. ww.ip.exchange.de.

$48 \quad$ Zu den Zulassungskriterien des Neuen Marktes vgl. Gerke 1999, 204-206; Gruppe Deutsche Börse - Infoline, FWB09-up3, Stand 01.04.99.

49 Flach, Schwarz 1999, 277f. Zur jungen Vergangenheit des Neuen Marktes vgl. F.A.Z. v. 07.03.01, Nr.56, 35.

50 Hierunter fallen etwa der Münchener Prädikatsmarkt, der Berliner Freiverkehr und der Hamburger Start-Up-Market. 
Das Interesse der Anleger an Aktien großer Gesellschaften ist ungleich größer als das an Aktien kleiner und mittlerer Gesellschaften. Dies wird deutlich, stellt man das Kapitalaufkommen an den einzelnen Segmenten vergleichend gegenüber. Eine Anlegerpräferenz für bestimmte Segmente und Teilmärkte schlägt sich zum einen in vergleichsweise hohen Börsenumsätzen nieder. Zum anderen ist das Bewertungsniveau der bevorzugten Aktienwerte infolge einer höheren Nachfrage ein grundsätzlich höheres, was auf zumindest lange Sicht zu einem höheren Emissionsvolumen führt. ${ }^{51}$ Beides - Kursniveau und Emissionsvolumen - zusammen genommen, schlägt sich eine Anlegerpräferenz für bestimmte Aktien in einer höheren Marktkapitalisierung der präferierten Gesellschaften nieder. Wie in keinem anderen Land steht der weltweit viertgrößte, deutsche Aktienmarkt auf einer verhältnismäßig schmalen Basis. ${ }^{52}$ Die Verteilung des Börsenumsatzes und der Kapitalisierung bietet ein Bild der Konzentration, das ausgeprägter kaum sein könnte. War der deutsche Aktienmarkt bis in die 80er Jahre hinein aus formalen Gründen im Wesentlichen ein Vorbehaltsgut für Großunternehmen, hat sich seit dem Börsenboom der letzten Jahre und den institutionell verbesserten Rahmenbedingungen daran faktisch wenig verändert. ${ }^{53}$ Während 1999 allein der Dax 30 gut vier Fünftel des gesamten inländischen Börsenumsatzes auf sich vereinte, nehmen sich bereits die Unternehmen des Mdax insbesondere für die zunehmend wichtiger werdenden institutionellen Anleger bescheiden aus. ${ }^{54}$ Durch zahlreiche IPO's nahm die Zahl kleiner und mittlerer Aktienunternehmen in den letzten Jahren zwar zu, doch ist ihr Auftreten am Sekundärmarkt weniger Ausfluss eines relativen Bedeutungsgewinns gegenüber den Standardwerten. Vielmehr ist die steigende Anzahl kleiner und mittlerer Gesellschaften am deutschen Aktienmarkt einem Trickle Down-Effekt im Zuge einer allgemeinen Liquiditätshausse geschuldet. ${ }^{55}$ So

51 Das AktG unterscheidet neben der Ausgabe von Aktien bei Gründung ( $\S 23 \mathrm{ff}$. AktG) vier Arten der Eigenkapitalbeschaffungen: a) Kapitalerhöhungen gegen Einlagen ( $\S 182 \mathrm{ff}$. AktG), b) Bedingte Kapitalerhöhung ( $\S 192 \mathrm{ff} . \mathrm{AktG}), \mathrm{c})$ Genehmigtes Kapital ( $\S 202 \mathrm{ff} . \mathrm{AktG}), \mathrm{d})$ Kapitalerhöhung aus Gesellschaftsmitteln (\$§207ff. AktG). Vgl. Grill, Perczynski 1988, 350358; Brakmann 1993, 12-24.

52 Vgl. Deutsche Börse AG, Geschäftsbericht 1995, 22; Hansen 1996a, 26; Rasch 1996, 53-55.

53 Einige Zahlen zur Verdeutlichung: Allein die drei Spitzengesellschaften des Börsenumsatzes Deutsche Bank, Daimler-Benz und Siemens - erreichten 1997 20\%, die drei höchst kapitalisierten Unternehmen - Allianz, Deutsche Bank und Siemens - 19\% am Gesamtergebnis der 155 größten inländischen Gesellschaften. Vgl. Die Aktiengesellschaft 3/1998, R79ff. Mehr als die Hälfte der kleinsten Titel (367 von insgesamt 678) machten 1995 zusammen 2,8\% der Börsenkapitalisierung und nur 0,2\% des Börsenumsatzes aus. Vgl. Hansen 1996b, R88. In Deutschland falle - so Rasch - das Augenmerk institutioneller Anleger auf etwa 50 bis 100 Titel. Vgl. Rasch 1994a, 25.

55 Vgl. Die Zeit v. 22.04.99, Nr. 17, 30. 
führte ein insgesamt gestiegenes Angebot an Eigenkapital am Sekundärmarkt zwar zu einer (Erst-)Emissionswelle unbefristet haftenden Eigenkapitals am Primärmarkt. Am „unteren Ende“ des deutschen Sekundärmarktes ging der Börsenboom der letzten Jahre jedoch weitgehend vorbei. „Das Phänomen, dass Unternehmen mit einer großen Börsenkapitalisierung von den Anlegern im allgemeinen bevorzugt werden, hat die Börsenentwicklung im [letzten] Jahrzehnt entscheidend geprägt. ${ }^{\text {" } 56}$

Abbildung 2.1 „Lorenzkurve“ zum deutschen Aktiensekundärmarkt (Periode: 03/99 - 02/00; Kapitalisierungsstichtag: 29.02.00)

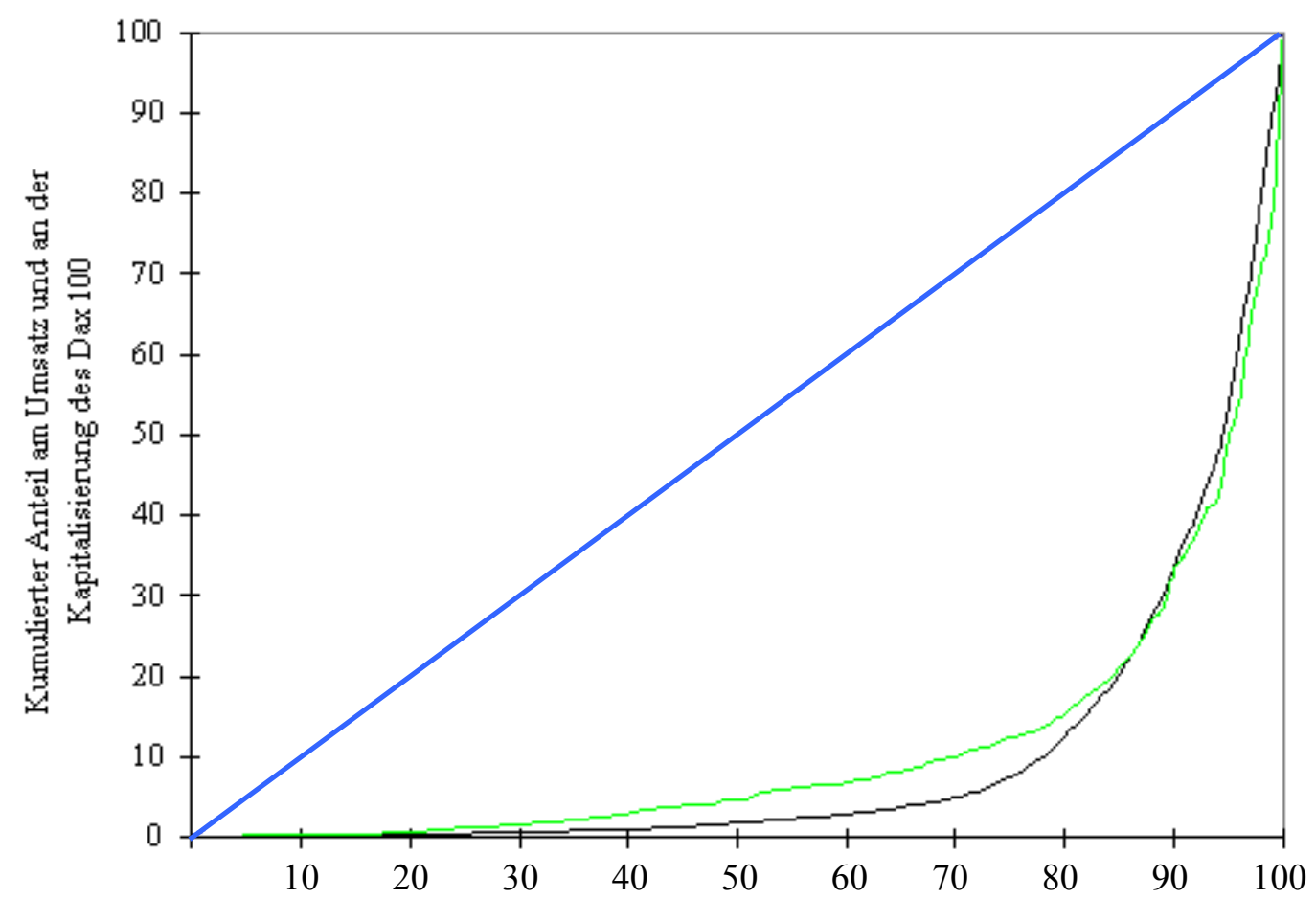

Kumulierter Anteil der Dax 100-Titel nach Börsenumsätzen

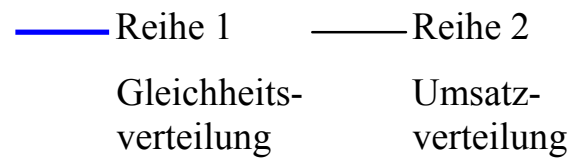

Reihe 3

Kapitalisierungsverteilung

Dass der meiste Börsenumsatz innerhalb des Dax 30 erfolgt, verwundert allein aus formalen Gründen zunächst nicht. Schließlich haben Titel des Dax 30 das so genannte 25/25 - Kriterium zu erfüllen. Um aufgenommen zu werden, müssen sie sich unter den 25 größten oder den 25 umsatzstärksten Aktiengesellschaften 
befinden. ${ }^{57}$ Allerdings ist das Ausmaß der „Kopflastigkeit“ des deutschen Aktiensekundärmarktes sehr wohl frappierend. In Bezug auf Börsenumsatz und Marktkapitalisierung besteht zwischen den Blue Chips des Dax 30 einerseits und den Small und Mid Cap andererseits eine große Bandbreite. Die ungleiche Verteilung von Börsenumsatz und -kapitalisierung veranschaulichen in Abbildung 2.1 exemplarische „Lorenzkurven“ des Dax 100 zu einem für den Börsenalltag repräsentativen Zeitpunkt beziehungsweise Zeitraum. ${ }^{58}$ Aus ihr ergibt sich ein GiniKoeffizient von 0,81 für die Konzentration der Börsenumsätze im Dax 100 sowie von 0,79 für jene der Marktkapitalisierung. Bereits die Mdax-Titel fristen demnach ein „Mauerblümchendasein“;59 ein noch krasseres Bild der Ungleichverteilung ergäbe sich, würde man die Lorenzkurven nicht nur des Dax 100, sondern des zum Zeitpunkt knapp tausend Titel umfassenden Gesamtmarktes entwerfen. Der Dax 100 kam in der betrachteten Periode auf einen Gesamtumsatz in Höhe von 636.589 Mio. EUR. 95\% entfielen auf den Dax 30, allein 66\% auf die zehn umsatzstärksten Titel und nur 5\% auf die gesamten siebzig Titel des Mdax. Hinsichtlich der Kapitalisierung zeichnet der deutsche Aktiensekundärmarkt ein ähnliches Bild der Konzentration auf die Titel des Dax 30. Die Börsenkapitalisierung aller Dax 100Titel betrug zum Kapitalisierungsstichtag 1.142.600 Mio. EUR. Der Dax 30 macht $90 \%$, allein die zehn höchst kapitalisierten Titel 67\% und der Mdax nur 10\% aus. ${ }^{60}$ Der Anteil des Mdax am Börsenumsatz des Dax 100 beträgt somit nur die Hälfte seines Anteils an der Börsenkapitalisierung.

Dass hoch kapitalisierte Aktiengesellschaften einen hohen, Small und Mid Caps einen entsprechend geringeren Umsatz in absoluten Zahlen auf sich vereinen, ist bereits aus stochastischen Gründen evident. Insofern verwundert die Fast-Kongruenz obiger Lorenzkurven und die Ähnlichkeit ihrer Gini-Koeffizienten kaum. Errechnet man allerdings den durchschnittlichen Umschlag der Kapitalisierung der Unternehmen, gelangt man je nach Unternehmensgröße zu unterschiedlichen

57 Weitere Bedingungen für eine Aufnahme in den Dax 30 bestehen für ein Unternehmen etwa darin, über einen Mindeststreubesitz in Höhe von 15\% zu verfügen und nicht zu mehr als $75 \%$ einer anderen Dax-Gesellschaft anzugehören. Eigene Berechnungen auf Grundlage von Umsatz- und Kapitalisierungsstatistiken der Deutsche Börse AG. Vgl. Deutsche Börse AG v. 29.02.00. In: www.exchange.de. Andere Zeitpunkt- respektive Zeitreihenbetrachtungen bestätigen die Aussage im exemplarisch angeführten Beispiel.

60 Die Aktien der Mannesmann AG bleiben in der Berechnung aufgrund ihrer Übernahme durch Vodafone unberücksichtigt. 
Werten. Die Aktien hoch kapitalisierter Unternehmen zielen nicht nur in absoluten Zahlen einen höheren Umsatz auf sich. Auch wird die Aktie eines hoch kapitalisierten Unternehmens im Durchschnitt wesentlich häufiger umgeschlagen, wie Abbildung 2.2 verdeutlicht.

Abbildung 2.2 Konzentrationsmaße zum deutschen Aktiensekundärmarkt

\begin{tabular}{|c|c|c|c|c|}
\hline Segmente / Segmentausschnitte & $\begin{array}{l}\begin{array}{l}\text { Börsen- } \\
\text { umsatz }^{\text {a }}\end{array} \\
\sum_{i=1}^{n} U i\end{array}$ & $\begin{array}{l}\text { Marktkapitali } \\
\text { sierung }{ }^{\mathrm{a}, \mathrm{b}} \\
\sum_{i=1}^{n} K i\end{array}$ & $\begin{array}{l}\varnothing \text {-lich } \\
\text { umgeschlagene } \\
\text { Kapitalisierung } \\
\qquad \frac{1}{n} \sum_{i=1}^{n} \frac{U i}{K i}\end{array}$ & $\begin{array}{l}\varnothing \text {-lich } \\
\text { umgeschlagener } \\
\text { Streubesitz } \\
\frac{1}{n} \sum_{i=1}^{n} \frac{U i}{K i} \times \text { freefloat } i\end{array}$ \\
\hline & Spalte 1 & Spalte 2 & Spalte 3 & Spalte 4 \\
\hline $\operatorname{Dax} 30, \mathrm{n}=30$ & 605.336 & 1.026 .759 & $80,0 \%$ & $125,7 \%$ \\
\hline Mdax, $n=70$ & 31.252 & 115.840 & $42,5 \% ; 35,6 \% *$ & $79,1 \% ; 109,9 \% *$ \\
\hline $\begin{array}{l}\text { umsatzschwächste Mdax-Titel, } \\
n=30\end{array}$ & 3.727 & 18.502 & $31,4 \%$ & $71,8 \%$ \\
\hline $\begin{array}{l}\text { umsatzstärkste Neue Markt- } \\
\text { Titel, } n=30\end{array}$ & 42.247 & 107.674 & $70,6 \%$ & $188,1 \%$ \\
\hline $\begin{array}{l}\text { umsatzschwächste Neue Markt- } \\
\text { Titel, } n=30\end{array}$ & 1.450 & 4.428 & $61,8 \%$ & $171,1 \%$ \\
\hline umsatzstärkste Sdax-Titel, n=30 & 3.620 & 9.583 & $50,0 \%$ & $102,3 \%$ \\
\hline $\begin{array}{l}\text { umsatzschwächste Sdax-Titel, } \\
n=30\end{array}$ & 255 & 1.885 & $21,5 \%$ & $44,6 \%$ \\
\hline $\begin{array}{l}\text { besonders umsatzschwache Titel } \\
\text { des Geregelten Marktes und } \\
\text { Amtlichen Handels, } n=30\end{array}$ & 95 & 1.432 & $12,2 \%$ & $41,2 \%$ \\
\hline
\end{tabular}

${ }^{\mathrm{a}}$ Alle Werte in Mio. Euro; betrachteter Zeitraum: 03/99 - 02/00; ${ }^{\mathrm{b}}$ Kapitalisierungsstichtag: 29.02.00;

${ }^{\mathrm{c}}$ Stammaktien in Xetra am 14.03.00; * ohne Philipp Holzmann AG)

Wurden im betrachteten Zeitraum (03/99 bis 02/00) vier Fünftel der Kapitalisierung eines durchschnittlichen Dax 30-Titels umgeschlagen, so sind es bei den kleinen - in absoluten Zahlen - besonders umsatzschwachen Unternehmen des Geregelten

61 Wenn der gewichtete Aktienkurs eines Unternehmens i über den betrachteten Transaktionszeitraum dem Kurswert des Kapitalisierungsstichtages vom 29.02.00 in $\mathrm{K}_{\mathrm{i}}$ entspricht, würde sich beim Quotienten aus Börsenumsatz und Kapitalisierung der Kurswert aus Zähler und Nenner kürzen. Wir erhielten dann das Verhältnis zwischen der Anzahl gehandelter und ausgegebener Aktien, ihre Umschlaghäufigkeit also. 
Marktes und des Amtlichen Handels nur knapp ein Achtel (Spalte 3). ${ }^{62}$ Allein umsatzstarke Neue Markt-Werte aus dem Nemax 50 reichen mit gut $70 \%$ umgeschlagener Marktkapitalisierung an die Dax 30-Titel heran - wohlgemerkt für den betrachteten Zeitraum. ${ }^{63}$ Die ausgewählten Mdax-Werte verzeichnen einen nur halb so großen Umschlag ihrer Kapitalisierung wie die umsatzschwächsten Neue Markt-Titel. Und die durchschnittlich umgeschlagene Kapitalisierung für das „untere“ Ende des Sdax liegt um ein Weiteres darunter. „Der Versuch der Börse, für mittelständische Unternehmen ein eigenes attraktives Marktsegment - Smax - zu schaffen, hat das Interesse der Investment Community noch nicht hervorgerufen." ${ }^{664}$ In der aufgezeigten Hierarchie der durchschnittlich umgeschlagenen Kapitalisierung kommt eine Anlegerpräferenz für hochkapitalisierte Dax 30- und für die (zumindest im betrachteten Zeitraum) medienwirksamen Nemax 50-Titel deutlich zum Ausdruck. Sie besteht fort, legt man statt des Umschlags der gesamten Kapitalisierung nur den des Streubesitzes zugrunde, das heißt den Anteil der frei zirkulierenden Kapitalisierung (Spalte 4). ${ }^{65}$

Die genannten Indikatoren bringen das allgemeine Interesse der Anleger für größere Unternehmen zum Ausdruck. Fraglich ist, ob ihre Größen- und im Falle des Neuen Marktes auch ihre Branchenpräferenz mit fundamentalen Kennzahlen zu erklären ist. In der geschilderten Anlegerpräferenz würde sich dann lediglich das rationale Anlegerinteresse für attraktive Rendite/Risiko-Profile äußern. Wäre dem so, müsste das Bewertungsniveau der Dax 30- und der Neue Markt-Titel, dokumentiert etwa am

62 Zu ähnlich differierenden Ergebnissen gelangt Rasch (1996, 103).

63 Jüngeren Entwicklungen zufolge hat besonders der Neue Markt an Attraktivität für Anleger verloren. Vgl. Deutsche Bundesbank 2001, 31; F.A.Z. v. 04.09.01, Nr. 205, 31: „Der Neue Markt verdient eine zweite Chance“. Gegenüber seinem Höchststand am 10. März 2000 hat der Neue Markt-Index über 85\% eingebüßt. Im Juli/August 2001 lagen die Notierungen im Durchschnitt nur noch knapp über dem Niveau, zu dem im März 1997 das gesamte Börsensegment eingeführt wurde. Die Marktkapitalisierung ist von $240 \mathrm{Mrd}$. Euro bei 226 Gesellschaften auf 46 Mrd. Euro von 319 Emittenten gegen Ende Februar 2002 eingebrochen. 14 Unternehmen haben inzwischen einen Insolvenzantrag gestellt. Die hohen Renditeerwartungen vieler Anleger an die oftmals sehr jungen Unternehmen mit zum Teil schwer vorhersagbaren Gewinnpotenzialen sind enttäuscht worden.

64 Cullom 2001, 17.

65 Die Daten der Streubesitzanteile entstammen den Angaben der Unternehmen aus ihren Geschäftsberichten. Der Streubesitz bzw. Free Float umfasst definitionsgemäß alle Aktien, die sich nicht in Blockbesitz befinden. Als Blockbesitz gelten Aktien in den Händen des Staates, Überkreuzbeteiligungen von Unternehmen und Anteile von Privatpersonen oder Familien ab einem gewissen Anteil. Die definitorische Grenze zwischen Streu- und Festbesitz ist fließend. Vgl. F.A.Z. v. 05.07.00, Nr. 153, 31. Im Zuge einer Indexneugewichtung zählen Stoxx und die Deutsche Börse AG (ab Juni 2002) zum Festbesitz Staatsanteile und Überkreuzbeteiligungen über 5\%, größere Aktienpakete von Fondsgesellschaften hingegen nicht. Vgl. F.A.Z. v. 
Kurs-Gewinn-Verhältnis (kurz: $\quad \mathrm{KGV}){ }^{66} \quad$ vergleichsweise gering ausfallen. Umgekehrt müssten die Aktien kleiner und mittlerer Gesellschaften im Verhältnis zu den Standardwerten teurer sein. Wie jedoch Abbildung 2.3 verdeutlicht, lassen sich die genannten Anlegerpräferenzen an Hand des KGV nicht erklären.

Abbildung 2.3 Kurs-Gewinn-Verhältnisse einzelner Marktsegmente (Mittelwerte)

\begin{tabular}{|l|l|l|l|l|}
\hline Marktsegmente & KGV 1999 & KGV 2000 & KGV* 2001 $^{*} \mathrm{KGV}^{*} 2002$ \\
\hline Euro Stoxx 50 & 33,2 & 26,3 & 23,1 & 18,5 \\
\hline Dax 30 & 35 & 28,2 & 23,3 & 20,1 \\
\hline Mdax & 27,2 & 20,6 & 16,6 & 16,7 \\
\hline Sdax & 20,6 & 15,42 & 12,1 & 15,5 \\
\hline$($ Nemax 50 & $127)$ & 115,6 & 41,2 & 44,3 \\
\hline
\end{tabular}

Quelle: Eigene Berechnungen auf Grundlage der Angaben der Börsenzeitung v. 20.01.01, Nr. 14, 15f. sowie der Financial Times Deutschland v. 12.10.01 für 2002, Nr. 198/41, 27-29; Gesellschaften mit einem (geschätzten) Verlust nach Steuern blieben unberücksichtigt, was insbesondere die durchschnittlichen KGV-Werte für den Nemax 50-Werte - nach oben beeinträchtigt.

Ganz im Gegenteil: Das durchschnittliche KGV der Sdax-Titel ist für den betrachteten Zeitraum deutlich geringer als das der Standardwerte. Für die Jahre 1999 bis 2001 beträgt es nur etwas mehr als die Hälfte desjenigen der Dax 30Werte, die mit den Euro Stoxx 50-Werten in etwa gleich aufliegen. Das Sdax-KGV des Jahres 1999 ist mit fast 21 erstaunlich hoch, was aber sicherlich mit dem Aufsehen des im selben Jahr gegründeten Smax bei einem (noch) boomenden Neuen Markt zu erklären ist. Bemerkenswert - und für die nachfolgende Analyse im Hinterkopf zu behalten - ist die Beobachtung, dass das Ausmaß der relativen Unterbewertung kleiner und mittlerer Aktienwerte im Vergleich $\mathrm{zu}$ den

10.08.00, Nr. 184, 29. Der von der Deutsche Börse AG empfohlene Streubesitz für Smax- und Neue Markt-Werte liegt bei $25 \%$, mindestens jedoch bei $20 \%$.

Das KGV gilt als Preis einer Aktie. Es gibt das Verhältnis zwischen der jeweils aktuellen Börsenbewertung eines Unternehmens und dem (geschätzten) realwirtschaftlichen Gewinn pro Aktie in einer Periode wieder. Bei einem hohen KGV gilt eine Aktie als teuer, bei einem niedrigen als günstig. Die Aussagekraft des KGV ist insofern eingeschränkt, als mit dem InsVerhältnis-Setzen des Unternehmensgewinns nicht der mehrere Jahre in die Zukunft reichende Cash Flow zugrunde gelegt wird.

67 Ein im Verhältnis zur Aktiennachfrage geringes Aktienangebot nach Wachstumswerten ließ die Kurse wegen ihrer erwarteten Erträge deutlich ansteigen und induzierte weitere IPO's. 
Standardwerten scheinbar danach variiert, ob es sich in den einzelnen Jahren beim Aktienmarkt allgemein um einen so genannten Up- oder um einen so genannten Down-Market handelt. Setzt man etwa das KGV des Sdax aus dem Up-Market 1999, einem Jahr des konjunkturellen Aufschwungs mit einem ansteigenden und vergleichsweise hohen Kursniveau, ins Verhältnis zum KGV des Dax 30 und vergleicht diesen Wert (55\%) mit dem entsprechenden (geschätzten) Wert für den Down-Market des (Rezessions-)Jahres 2002 auf einem derzeit eher geringen und weniger ansteigenden Bewertungsniveau (77\%), so scheint die Unterbewertung des Sdax in solchen Phasen relativ hoch zu sein, in denen die Übernahme von Risiko am Aktienmarkt allgemein mit einer hohen Rendite entgolten wird. Umgekehrt nimmt die relative Unterbewertung kleiner und mittlerer Gesellschaften bei einer (wenn überhaupt) geringen Entlohnung übernommenen Risikos, in einem Down-Market also, ab. Gleiches ist für den Mdax zu beobachten, wenn auch bei einer insgesamt geringeren Unterbewertung; wie bei den Konzentrationsmaßen nimmt der Mdax auch bei der Bewertung durch das KGV eine Mittelstellung zwischen Sdax und Dax 30 ein. Neben dem KGV lassen auch andere Kennzahlen wie etwa das Kurs-CashFlow-Verhältnis ${ }^{68}$ oder die Umsatzbewertung (Median Marktkapitalisierung zu Umsatz) eine „fundamental oft nur schwer nachvollziehbare Unterbewertung einiger Unternehmen ${ }^{669}$ erkennen.

Aus verschiedenen Bewertungsindikatoren ergibt sich, dass Standardwerte von Anlegern nicht deshalb präferiert werden, weil sie relativ günstig sind. Es gilt vielmehr umgekehrt: Weil ihnen eine allgemeine Anlegerpräferenz zuteil wird, sind sie teurer und relativ hoch bewertet. Aufgrund der Größenpräferenz der Anleger, die wie verdeutlicht insbesondere in Hochrendite-Phasen offen zu Tage tritt, kommt es nicht $\mathrm{zu}$ wesentlichen Wanderungsbewegungen des Finanzkapitals hin zu den Segmenten der günstigeren Small und Mid Caps. Arbitrage zwischen diesen Segmenten findet allenfalls eingeschränkt statt. Die Bewertungsdifferenzen zwischen den Segmenten der Standardwerte einerseits und denen kleiner und mittlerer Gesellschaften andererseits bleiben unausgeglichen. Erscheinen die

68 Unter cash flow wird der Saldo aus liquiditätswirksamen Erträgen und Aufwendungen verstanden. Er ist frei von Einflüssen der Buchhaltung und steht insofern für die Ausschüttung an die Kapitalgeber in Form von Zinsen und Dividenden respektive Kurssteigerungen zur Verfügung. Zu ihn beinhaltenden Zahlungsgrößen vgl. Busse von Colbe 1997, 274-278; Bea 1997, 541. Zum discounted cash flow als Erfolgsmaßstab eines Unternehmens vgl. Copeland, Koller, Murrin 1998, 99-120. Helaba Trust Beratungs- und Management Gesellschaft mbH 2001, 3. 
Substitutionslücken der Börsensegmente mit der allgemein fortschreitenden Vervollkommnung des deutschen Aktienmarktes auch überwindbarer, ist der Wettbewerb um das knappe Finanzkapital zwischen einzelnen Marktsegmenten tatsächlich eingeschränkt. Entgegen dem „law of one price“ koexistieren zwischen ihnen dauerhaft differierende Preise für die Übernahme von Unternehmensrisiken derselben Höhe. Denn es lassen sich die Preisunterschiede auch nicht damit erklären, dass die Aktienkurse kleiner und mittlerer Gesellschaften stärker schwanken als die der Standardwerte. Bislang wurde bei der Gegenüberstellung der $\mathrm{KGV}$ in den einzelnen Marktsegmenten implizit unterstellt, dass die jeweils bewertungsrelevanten Risiken identisch sind. Geringere bewertungsrelevante Risiken drücken sich in geringeren Kursabschlägen aus. Sie schlagen sich demnach in einem entsprechend höheren KGV nieder. Folglich wäre das geringere Bewertungsniveau kleiner und mittlerer Gesellschaften auf höhere Marktrisiken zurückzuführen. Ungleich jenen etwa in den USA sind die Small und Mid Caps am deutschen Aktienmarkt jedoch „,vergleichsweise weniger stark durch Marktschwankungen beeinflußt, haben also ein geringeres Marktrisiko.“70 Bereinigt um diese Risikokomponente müsste das $\mathrm{KGV}$ der Sdax-Titel höhere Werte annehmen als das etwa des Dax 30. Die relative Unterbewertung kleiner und mittlerer Gesellschaften fällt vor diesem Hintergrund sogar noch größer aus.

Nicht nur am Sekundärmarkt ist eine relative Unterbewertung der kleinen und mittleren Gesellschaften zu konstatieren. Auch am Primärmarkt fällt das „underpricing“ für kleine und mittlere Unternehmen höher aus. ${ }^{71}$ Underpricing beschreibt dabei die empirisch gut belegte Tatsache, dass junge Aktien nach der Börseneinführung eines Unternehmens im Durchschnitt einen Kurs erreichen, der höher ist als derjenige, den die Ersterwerber für das Papier bezahlt haben. ${ }^{72}$ Angesichts dieser Geringschätzung kleiner und mittlerer Gesellschaften am deutschen Primär- und Sekundärmarkt verwundert es nicht, dass der seit Banz (1981) und Reinganum (1981) für den US-amerikanischen Aktienmarkt empirisch oft bestätigte negative Zusammenhang zwischen der Marktkapitalisierung eines

70 Oertmann 1994a, 244. Zu bewertungsrelevanten Risiken kleiner und mittlerer Gesellschaften vgl. Abschnitt 3.2.

Vgl. Rock (1986); Schmidt et al. 1988, 1197.

Vgl. Schmidt, Terberger 1997, 436f. Die Phänomene des kurzfristigen Underpricing ... “continue to be a puzzle." Aggarwal 2000, 1075. Vgl. die dort angegebene Literatur. Erklärungsansätze für Kurseffekte bei Aktienemissionen gibt Brakmann 1993, 45-131. 
Unternehmens und seiner risiko- und transaktionskostenbereinigten Aktienrendite (Small Firm Effect) am deutschen Aktienmarkt nur eingeschränkt Gültigkeit hat. ${ }^{73}$ In einer allgemein abwärts gerichteten Kursentwicklung (Down-Market) gewinnen Small und Mid Caps an relativer Bedeutung und verzeichnen schließlich eine höhere Rendite. So stellte der Mdax - getragen wiederum von nur einigen wenigen Titeln etwa im Down-Market des Jahres 2000 eine renditeträchtige Anlagealternative zu den „schwächelnden“ Titeln des Dax 30 und Nemax 50 dar. „Sollten sich Blue Chips und Wachstumswerte im Jahresverlauf jedoch wie erwartet erholen und damit das Anlegerinteresse wieder stärker auf sich ziehen, besteht für Midcaps das Risiko des erneuten Abgleitens ins Mauerblümchen-Dasein. ${ }^{674}$ In Phasen boomender Börsen hingegen ist für den deutschen Aktiensekundärmarkt anstatt eines Kleinfirmeneffektes umgekehrt von einem „Big-Firm-Effekt“ auszugehen. ${ }^{75}$ Die Rendite für das Halten von Einzelrisiken kleiner und mittlerer Gesellschaften fällt demnach am deutschen Aktiemarkt in Up-Markets regelmäßig unterhalb des marktgerechten Niveau aus.

Bislang festzuhalten ist, dass die Präferenz der Anleger für Standardwerte a) absolut wie relativ (zur jeweiligen Kapitalisierung) höhere Umsätze, b) insbesondere in UpMarkets höhere relative Bewertungsniveaus am Sekundär- wie am Primärmarkt und c) höhere Aktienrenditen in Up-Markets hervorbringt. Eine Anlegerpräferenz senkt die Kosten der Eigenkapitalbeschaffung bei den bevorzugten Gesellschaften und erleichtert Folgeemissionen, die wiederum die Marktkapitalisierung der ohnehin großen Unternehmen vergrößern. Eine hohe Kapitalisierung und hohe Umsätze bedingen sich somit wechselseitig. Die Anlegerpräferenz für bestimmte Unternehmenstypen zeitigt eine sich selbst verstärkende Konzentration von Börsenumsatz und Kapitalisierung, wie es die annähernde Kongruenz der Lorenzkurven der Abbildung 2.1 nahe legt. Bei der relativ hohen Bewertung der präferierten Standardwerte handelt es sich also nicht um eine nur vorübergehende spekulative Blase, die - mehr oder weniger auf einzelwirtschaftlicher Rationalität gründend - früher oder später zu platzen droht, da sich das Kursniveau von den Dabei mag für einzelne Segmente phasenweise auch der Small Firm-Effekt zutreffen. Vgl. Deutsche Börse AG, Geschäftsbericht 1995, 23.

74 Helaba Trust Beratungs- und Management Gesellschaft mbH 2001, 5.

75 Vgl. Abschnitt 4.5.
} 
inneren fundamentalen Unternehmenswerten fortbewegen. ${ }^{76}$ Es ist vielmehr - gemäß dem Ansatz der Reflexivität $-{ }^{77}$ davon auszugehen, dass sich tendenziell der innere Wert eines Unternehmens seinem vergleichsweise hoch bewerteten Kursniveau angleicht. So verursachen die für die Standardwerte geringeren Eigenkapitalkosten eine ,self fulfilling prophecy“.

Umgekehrt lassen sich für vernachlässigte kleine und mittlere Gesellschaften mit einer geringeren Bewertung neue Aktien - wenn überhaupt - zu einem nur geringeren Emissionserlös über den Primärmarkt plazieren. Die Kosten der Eigenkapitalbeschaffung sind für sie höher, was ihr Unternehmenswachstum relativ behindert. $^{78}$ Unterbewertete Aktien vergrößern außerdem die Gefahr einer „feindlichen“ Übernahme. ${ }^{79}$ Um Unterbewertungen am Sekundärmarkt zu verhindern, besteht aus diesen Gründen ein Anreiz zum Rückkauf eigener Aktien. ${ }^{80}$ Mit dieser Art der Aktiennachfrage lässt sich zwar das KGV möglicherweise erhöhen. ${ }^{81}$ Der Rückkauf unterliquider Aktien ist allerdings nicht zwangsläufig mit einer Kurssteigerung verbunden. So kann der Rückkauf eigener Aktien das umlaufende Aktienkapital verringern, ohne die damit sinkende Marktkapitalisierung durch einen entsprechenden Kursanstieg auf dem ursprünglichen Niveau zu halten. Neben dem Rückkauf eigener Aktien stellt ein „Delisting“ oder ein „Going Private“ weitere Möglichkeiten dar, ${ }^{82}$,die börsennotierten Unternehmen aus ihrer Falle zu holen. “83 Ein Verschwinden der Unternehmen am unteren Ende des Aktienmarktes

76 Zu Bubble-Theorien vgl. Jarchow 1994, 255-258; Flood, Garber (1980); Blanchard, Watson (1982); Jüttner (1989); Marterbauer 1994, 14f.

77 Vgl. Soros 1998a, 58ff. Die Fundamentaldaten versteht Soros als vom Sekundärmarkt abhängige Variablen. Mit ihrem zeitlichen Voranschreiten gehe die Bevorzugung der Standardwerte von der Finanzsphäre auf die Realökonomie über. Abbild (Kursverlauf) und Abzubildendes (Unternehmenswert) würden im Gleichgewicht nicht nur deshalb übereinstimmen, weil die Abbildfunktion, sprich der Aktienmarktmechanismus, effizient sei. Auch führe das Abbild eine Anpassung des Abzubildenden an das Abbild herbei. Dabei könne die ,virtuelle“ Welt der Aktienmärkte durchaus die dominierende Rolle gegenüber der realen Welt einnehmen: der Aktienmarkt gewissermaßen als „Fake“. Vgl. Geier (1999).

78 Nach einer Studie von Coopers \& Lybrand $(1994,85)$ führen mittelständische Unternehmen in Großbritannien mangelnde Liquidität am Sekundärmarkt als wesentliches Hindernis bei der Durchführung von Kapitalerhöhungen an.

79 Vgl. Fischer 2000, 36.

80 Gesetzlich geregelt dürfen börsennotierte Aktiengesellschaften in Deutschland bis zu 10\% der eigenen Aktien zurückkaufen. Vgl. Maier 1998, 36.

81 Fraglich ist, ob der Rückkauf eigener Aktien bei vernachlässigten Gesellschaften das Kursniveau (Zähler) in einem Maße anhebt, das den ansteigenden Gewinn pro Aktie (Nenner) kompensiert.

82 Unter einem „Delisting“ versteht man den Widerruf der Zulassung von börennotierten Wertpapieren. Der Begriff „Going Private“ beschreibt das weitergehende Ziel einer Gesellschaft, ihre Aktien dem Börsenhandel insgesamt zu entziehen. Vgl. Sohbi (2001).

83 F.A.Z. v. 14.03.01, Nr. 62, 34. 
verringert dabei nur rechnerisch die Konzentration des Sekundärmarktes. ${ }^{84}$ Außerdem sind es vor allem Unternehmen mit gesunder Substanz, das heißt mit vergleichsweise hohen Einzahlungsüberschüssen (Cash Flows), die aufgrund einer ausgeprägten Unterbewertung einerseits Anreize haben, aus dem Aktienmarkt auszuscheiden und denen Private Equity-Geber andererseits das Verlassen des Aktienmarktes ermöglichen. ${ }^{85}$ Denn insbesondere die „guten“ Einzelrisiken haben bei einer grundsätzlichen Vernachlässigung ihrer Börsensegmente einen Anreiz, der „Tränke“ des Aktienmarktes den Rücken zuzukehren. Infolgedessen besteht für die Segmente kleiner und mittlerer Gesellschaften auf lange Sicht die Gefahr, dass eine Notierung der zurückbleibenden Unternehmen für sie mit einem Signal „schlechter“ Qualität verbunden ist. ${ }^{86}$

Die Erwartung, mit insgesamt zunehmendem Anlegerinteresse am deutschen Sekundärmarkt eine gleichmäßigere Verteilung des Kapitalaufkommens auf die einzelnen Segmente zu erhalten, hat sich in den letzten Jahren nicht erfüllt. Der Börsenboom seit Mitte der 90er Jahre konnte der Konzentration offenbar nicht entgegenwirken. Dass aus den allgemein zu erwartenden Liquiditätszuflüssen an den Aktienmarkt künftig hohe Sickereffekte zu Gunsten der Small und Mid Caps in beachtlichem Umfang resultieren, ist angesichts der zunehmenden Institutionalisierung des Sekundärmarktes unwahrscheinlich. Institutionelle Anleger gelten als Protagonisten der beschriebenen Anlegerkonzentration. ${ }^{87}$ Eine stärker kapitalgedeckte Altersversorgung und eine Übertragung von Verpflichtungen der Unternehmen aus Pensionsrückstellungen auf Pensionsfonds wird die Position institutioneller Anleger stärken. ${ }^{88}$ Auch eine anhaltende Konsolidierung der

„Ein nicht unerheblicher Teil der Mdax-Werte wird uns in zwölf Monaten nicht mehr zur Verfügung stehen, Delisting (der Rückzug von der Börse) wird 2001 ein ganz großes Thema. Gemessen am Cdax und Mdax sind fünf bis zehn Prozent Delistings realistisch“, meint Jörss im Handelsblatt v. 01.12.2000. Ein gängiges Muster ist, dass ein Venture-Capital-Fonds ein Unternehmen den Aktionären zu einer attraktiven Prämie abkauft, das Management ersetzt und die Restrukturierung und die anschließende Expansion mit Fremdkapital finanziert.

Vgl. Abschnitt 4.2.3.

Vgl. Eberstadt 1999, 275.

"... the second important change is the rise of investment funds... the new kind of institutional investor, like other financial institutions, concentrate their influence on just a very small group of companies. In 1997, institutional investors for instance owned 48\% of Daimler-Benz, 51\% of MAN, $85 \%$ of Preussag, $65 \%$ of Deutsche Babcock, $85 \%$ of Linde, $71 \%$ of Lufthansa and $50 \%$ of Hoechst (Deutsches Aktieninstitut 1998, table $08.5-1,1997$ ) ... The position of pension funds as institutional investors is becoming stronger and the funds are mainly interested in the DAX 30 firms which are guided by shareholder value principles." Jürgens, Naumann, Rupp 2000, 58+71. Vgl. a. Hauck 1988, 18; F.A.Z. v. 03.03.01, Nr. 53, 25. 
öffentlichen Haushalte - eingefordert etwa durch die Maastricht-Kriterien - sowie anstehende Vermögensübertragungen im Zuge von Erbschaften lassen eine Umschichtung in Aktienpapiere erwarten, von der insbesondere die Institutionellen profitieren dürften. Bei der Zusammenstellung ihrer Portfeuilles agieren sie branchenorientiert. Über die nationale Fragmentierung eines die gesamte westliche Hemisphäre umspannenden Aktienmarktes sind sie in zunehmendem Maße erhaben. ${ }^{89}$ Zusammen mit der Beseitigung von Währungsrisiken durch die europäische Einheitswährung trägt dieses Anlegerverhalten zu einer fortschreitenden De-Segmentierung der nationalen Aktienmärkte an ihren oberen Enden bei. ${ }^{90}$ In die gleiche Richtung wirkt die Beseitigung der steuerlichen Benachteiligung institutioneller Anleger aus dem Ausland durch die Umstellung vom Vollanrechnungs- zum Halbeinkünfteverfahren und der Absenkung des Spitzensteuersatzes der Körperschaftssteuer auf 25\% (für einbehaltene wie ausgeschüttete Gewinne). ${ }^{91}$ Dies dürfte den Anteil deutscher Blue Chips in den Portefeuilles ausländischer Anleger erwartungsgemäß erhöhen. ${ }^{92}$ Mit einer erwarteten Gewichtung von über $28 \%$ im Euro Stoxx 50 werden insbesondere deutsche Standardwerte zu künftigen Nettogewinnern einer Umschichtung in europäische Portefeuilles institutioneller Anleger erwartet. ${ }^{93}$ All diese Entwicklungen sprechen dafür, dass sich die ohnehin hohe Anlegerkonzentration am deutschen Sekundärmarkt abermals erhöhen, zumindest aber nicht abnehmen wird. Der Löwenanteil einer zusätzlichen Geldeinheit zum deutschen Aktienmarkt wird vermutlich auch künftig großenteils in Segmente für Big und weniger in Segmente für Small und Mid Caps fließen.

„Investierten deutsche institutionelle Investoren bis Ende $1998 \mathrm{zu}$ etwa 70\% in deutschen Anlagen und nur zu 30\% in ausländischen Werten, so dürfte sich dieses Verhältnis im EuroAktienmarkt auf $25 \%$ zu $75 \%$ in den nächsten Jahren umkehren.“ Die Aktiengesellschaft 8/1999, R328; vgl. Eberstadt 1999, 274; vgl. a. Helg et al. (1995).

90 Vgl. Kotz 1998, $262 \mathrm{f}$.

91 Eichel 2000, 75f. Es zeigt sich, dass der Anrechnungsvorteil den inländischen Anlegern zwischen 1989 und $1995 \mathrm{zu}$ einer um durchschnittlich mehr als 50\% höheren Dividendenrendite verholfen hat. Während also z.B. aus Sicht des Weltkapitalmarktes deutsche Aktien 1995 eine Dividendenrendite von 2,24\% erwirtschaftet haben, werden durch die Steuergutschrift einem (steuerbefreiten) deutschen Investor 3,2\% offeriert. Dabei ist zudem zu beachten, dass diese Zahlen die Benachteiligung der ausländischen Anleger insofern unterzeichnen, als diese Anleger üblicherweise zusätzlich Kapitalertragssteuer entrichten müssen, während für die Inländer die Kapitalertragssteuer nur eine Vorauszahlung auf die anfallende Einkommenssteuer ist. Vgl. Fuest, Huber 2000, 352. 
$\mathrm{Zu}$ konstatieren ist, dass der allgemeine Trend der Vervollkommnung des deutschen Aktienmarktes und mit ihr die Homogenisierung in den Segmenten am oberen Ende merklich voranschreitet. Vorwiegend im Dax 30 nimmt der Informationsstand der Anleger ebenso zu wie ihre Reaktionsgeschwindigkeit. Sachliche und räumliche Präferenzen verlieren an Bedeutung. Es reduziert sich die Aktie eines Standardwertes im Wesentlichen auf das erwartete Rendite/Risiko-Profil. Der Homogenisierung am oberen Ende des Aktienmarktes steht eine weitgehende Vernachlässigung der Teilmärkte für kleine und mittlere Gesellschaften der Old Economy am unteren Ende des Aktienmarktes gegenüber. Entgegen dem allgemeinen Homogenisierungstrend wird der Kapitalfluss zwischen den Segmenten - scheinbar paradox - durch „weiche“ Faktoren wie die Unternehmensgröße und -branche beeinflusst. Die Aufteilung der Kapitalströme auf die einzelnen Segmente ist geprägt von Anlegerpräferenzen für Blue Chips und für den betrachteten Untersuchungszeitraum auch für Wachstumswerte aus dem Bereich der New Economy. Die Segmente und Teilmärkte der Small und Mid Caps der Old Economy werden von Eigenkapitalgebern hingegen weitgehend vernachlässigt. Bei der Wahl des Anlagesegments rückt das am oberen Ende des Sekundärmarktes immer zentraler werdende Kriterium des Rendite/Risiko-Profils einer Einzelanlage in den Hintergrund. Angesichts einer an Bedeutung zunehmender Institutioneller Anleger ist auch künftig nicht mit einem gleichmäßigeren Kapitalaufkommen zwischen den Segmenten zu rechnen.

Federführend für das Fragmentieren des deutschen Gesamtsekundärmarktes sind letztlich die Träger der Börsen. ${ }^{94}$ Es dokumentiert sich hierin ihr Streben nach einer Ausweitung der Wertpapiergeschäfte. Denn nur wer im Wettbewerb unter den Börsen ein hohes Transaktionsvolumen hervorbringt, kann bei abnehmenden Durchschnittskosten den Preis für einzelne Transaktionen senken, was für Banken und andere Aktienhändler, die ihre Aufträge mal nach Frankurt, mal nach London oder Paris vergeben, von entscheidendem Interesse ist. Ein insgesamt hohes Transaktionsvolumen ist deshalb Voraussetzung dafür, bestimmte fixkostenintensive Computerprogramme gewinnträchtig implementieren und eine Infrastruktur für

94 Die Frankfurter Wertpapierbörse etwa wird getragen von der Deutschen Börse AG, deren Großaktionäre die vier großen deutschen Privatbanken und die Regionalbörsen sind. 
derivative Transaktionen etablieren $\mathrm{zu}$ können: ${ }^{95}$ „Size matters“! Da die Börsenbetreiber in starker Konkurrenz zueinander um die höchsten Umsätze stehen, fällt ihr Hauptaugenmerk auf die umsatzstarken Gesellschaften - die großen eben. Mit der Schaffung von Börsensegmenten für kleine und mittlere Gesellschaften mit je eigenständigen institutionellen Profilen ist darüber hinaus das Ziel der Börsenbetreiber verbunden, die allgemeine Anlagebereitschaft und im Gefolge die Zahl der Unternehmen am Aktienmarkt zu erhöhen. Mag die Segmentierung des Sekundärmarktes auch zur Gewinnmaximierung der Börsenbetreiber beitragen; ob sie auch zum höchstmöglichen Transaktionsvolumen in jedem einzelnen Segment führt, ist ungewiss. Möglicherweise trägt die von den Börsenbetreibern vollzogene Segmentierung zur Konzentration des Finanzkapitals, zu einer mithin geminderten Anlagebereitschaft in einzelnen Teilmärkten und -segmenten bei. Vermessen wäre jedoch die Einschätzung, die Konzentration des Eigenkapitals allein auf die Segmentierung durch die Börsenbetreiber oder den Gesetzgeber zurückzuführen,

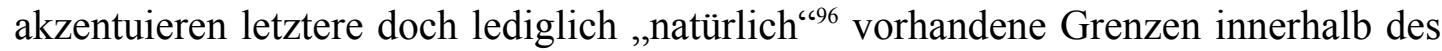
Gesamtmarktes.

Wesentliche Einnahmequellen der Deutsche Börse AG ist das Computerprogramm Xetra und die Derivatenbörse Eurex. Vgl. Rohwetter 2001, 27.

Zur Unterscheidung einer willkürlichen von einer natürlichen Segmentierung vgl. Bauer 1977, $16-23$. 


\subsection{Unterliquidität am deutschen Aktiensekundärmarkt}

Der letzte Abschnitt verdeutlichte die Präferenz der Anleger am deutschen Aktiensekundärmarkt für die Segmente großer Gesellschaften. In ihnen werden die Anteilsscheine von einer Vielzahl von Akteuren gehandelt. In beliebiger Menge wechseln die Aktien ohne Zeitverluste und Kurseingeständnisse von nennenswertem Umfang zu geringen Transaktionskosten den Eigner. Besteht zu jedem Zeitpunkt eine ausreichende Anzahl von miteinander kompatiblen Kauf- und Verkaufsplänen um den markträumenden Aktienkurs (Markttiefe), und werden auch großvolumige Aufträge von der jeweiligen Marktgegenseite absorbiert (Marktbreite), ${ }^{97}$ unterliegt der Aktienkurs c.p. keinen erratischen Schwankungen. Sofern darüber hinaus rationale Anlegererwartungen vorliegen und der Preismechanismus uneingeschränkt flexibel ist, bewegt sich dieser Aktienkurs - nach Abschlag für das bewertungsrelevante Risiko - entlang dem inneren Wert eines Unternehmens. ${ }^{98} \mathrm{Zu}$ diesem den fundamentalen Unternehmenswert (Fair Value) widerspiegelnden Aktienkurs, herrscht Markträumung zwischen den Aktienanbietern und nachfragern. ${ }^{99}$ Alle Kauf- und Verkaufspläne werden zum fundamental richtig bewerteten Kurs ausgeführt. Ein solcher Sekundärmarkt ist informations- ${ }^{100}$ bewertungs- ${ }^{101}$ und transaktionseffizient. Ein jederzeitiger Transaktionswunsch ist in beliebiger Menge zu eben diesem fundamentalen Kurs ohne Transaktionskosten

97 Vgl. Upper 2000, 6. Der Begriff der „Marktbreite“ wird gelegentlich auch als täglich fortgeschriebener Saldo der gestiegenen und gefallenen Titel eines Aktienmarktes verstanden. Der innere Wert eines Unternehmens wird über seinen Substanzwert oder den Ertragswert, das heißt die in Zukunft $\mathrm{zu}$ erwartenden diskontierten Einzahlungsüberschüsse aus den Unternehmensinvestitionen ermittelt. Danach bemisst sich der fundamentale Kurs einer Aktie. Vgl. Abschnitt 3.1.

$\mathrm{Zu}$ einem fundamentalen Bewertungsmodell für den deutschen Aktienmarkt vgl. Jandura, Rehkugler 1999, 25-31. Informationseffizienz, dass der Marktwert einer Aktie nicht dauerhaft von ihrem Ertragswert eines Unternehmens abweichen kann. „A market in which prices always 'fully reflected' available information is called 'efficient'." Fama 1970, 383. Nach dem Ausmaß der Reflexion von Informationen in Aktienkursen wird zwischen einer weak, semi-strong und einer strong Form der Informationseffizienz unterschieden. Vgl. ebenda, 383ff. „A weaker and economically more sensible version of the efficiency hypothesis says that prices reflect information to the point where the marginal benefits of acting on information ... do not exceed the marginal costs ..." Fama 1991, 1575. Vgl. a. Franke, Hax 1990, 315-320. Zu weiteren Definitionen vgl. Sapusek 1998, 31-34. Zu einer kritischen Auseinandersetzung mit der „efficient market hypothesis“vgl. Findlay, Williams (2000-2001). Je nach der Güte der Abbildung, sprich dem Grad der Bewertungseffizienz, erwächst für einzelne Anleger die Möglichkeit, Kursveränderungen $\mathrm{zu}$ antizipieren. Sind bereits alle bewertungsrelevanten Informationen in den Kursen enthalten, gibt es keinen Informationsvorsprung mehr. Preise in informationseffizienten Märkten sind „faire“ Preise und somit gute Indikatoren für den Wert der ihnen zugrunde liegenden Güter. Sie entsprechen dem inneren Wert dieser Assets und im Fall von Aktienmärkten dem Barwert der erwarteten Einzahlungsüberschüsse der unternehmerischen Investitionen. 
durchführbar. Ungleichgewichtspreise existieren hier nur vorübergehend. Entsprechende Gegenaufträge führen etwaig abweichende Kurse mit (unendlich) hoher Reaktionsgeschwindigkeit zum fundamental richtig bewerteten Aktienkurs zurück („Marktelastizität“). Überhänge der einen oder der anderen Marktseite sind zum fundamentalen Aktienkurs somit dauerhaft nicht möglich.

Aktien, die an einem solch effizienten Sekundärmarkt gehandelt werden, sind - wie man sagt - vollkommen liquide. ${ }^{102}$ Der hier verwendete Liquiditätsbegriff ist ein objektbezogener. ${ }^{103}$ Er stellt weder auf die Zahlungsfähigkeit eines Wirtschaftssubjektes ab, noch wird er als Synonym gebraucht für finanzielle Objekte wie Zahlungsmittel, Devisenreserven oder Bankguthaben von Nichtbanken. ${ }^{104} \mathrm{Er}$ beschreibt vielmehr die reibungslose Transformierbarkeit von Vermögensobjekten ${ }^{105}$ Liquidität im Sinne von Liquidier- oder Monetisierbarkeit von Vermögensteilen also. ${ }^{106}$ Bei vollkommener Liquidität trifft jedes geplante Aktienangebot zum fundamental richtig bewerteten Kurs jederzeit auf eine entsprechende Aktiennachfrage. Aktienangebot und -nachfrage stimmen zum Fair Value in zeitlicher und mengenmäßiger Hinsicht überein. Für eine vollständig liquide Aktie gilt die jederzeitige Verfügbarkeit ihres vollen monetären Gegenwertes ohne Inkaufnahme im- oder expliziter Transaktionskosten; ${ }^{107}$ die Liquidität einer Aktie misst somit ihre „Nähe zum Geld“. ${ }^{108}$ Da bei vollkommener Liquidität jederzeit alle ausgeschütteten Aktien eines Unternehmens zum fundamentalen Kurs geplantermaßen einen Eigner haben, besteht eine Identität von nachgefragtem Eigenkapital der Unternehmen in Höhe des emittierten und fundamental richtig bewerteten Aktienkapitals und dem Eigenkapitalangebot seitens der Investoren.

\footnotetext{
102 Vgl. Fuhrmann 1994, 8ff.

103 Vgl. Cramp (1987).

104 Zu verschiedenen Liquiditätsbegriffen vgl. Vormbaum 1995, 112-116.

105 Vgl. Stützel 1959, 622; Jarchow 1966, 8-10.

106 Vgl. Gramp 1970, 14-16; Nicklisch 1932, 456; Fischer 1964, 324f. Dieser Liquiditätsbegriff steht in enger Beziehung zu dem von Le Coutre (1926, 284f.) geprägten Begriff der ,absoluten Liquidität"،.

107 Vgl. Bernstein 1987, 224; Schwartz 1992, 666; Gerke, Rasch 1993, 312; Oesterhelweg, Schiereck 1993, 390. Zu weiteren Definitionen vgl. Thadden 1999, $992 \mathrm{f}$.

108 Die Liquidität des Vermögensgegenstandes Geld ist deshalb vollkommen, sein Liquiditätsgrad beträgt folglich 1 .
} 
Werden zum fundamentalen Kurs weniger Aktien zu halten geplant als ausgeschüttet sind, liegt ein Überhang der Aktienangebotsseite vor. Es herrscht Unterliquidität. ${ }^{109}$ Bei Unterliquidität wird zum fundamentalen Aktienkurs folglich ex ante weniger Eigenkapital angeboten, als von den Unternehmen an Aktien emittiert wurde. Dieser Nachfrageüberschuss an Eigenkapital entspricht der Differenz aus geplantem Aktienangebot und niedrigerer geplanter Aktiennachfrage. Aktienanbieter, die zum geplanten Transaktionszeitpunkt auf eine geringere Nachfrage stoßen, müssen ihr geplantes Transaktionsvolumen einschränken, auf einen Kaufwunsch in entsprechender Höhe warten, (explizite) Gebühren einer Vermittlung zahlen oder (implizit) Kursabschläge in Kauf nehmen; anders ist die sich stets einstellende ex post-Identität von Aktienangebot und -nachfrage nicht $\mathrm{zu}$ bewerkstelligen. Unterliquidität geht deshalb stets mit Transaktionskosten für die Eigner der vernachlässigten Gesellschaften einher. Die Zeit-, Mengen- und Kurskomponente der Unterliquiditätskosten bedingen sich dabei gegenseitig: Ein Disagio etwa beschleunigt die Ausführung und erhöht die Anzahl der umsetzbaren Aktien; die Inkaufnahme einer längeren Wartezeit verringert den notwendigen Kursabschlag und ermöglicht ebenfalls ein höheres Transaktionsvolumen; und ein eingeschränktes Transaktionsvolumen verringert die Wartezeit sowie die so genannten MarketImpact-Kosten. Je schneller und je mehr die Eigner unterliquide Aktien planen zu verkaufen, desto eher sind sie zur Inkaufnahme von Kursabschlägen bereit. Das Ausmaß der Kurszugeständnisse bestimmt das Ausmaß der Unterbewertung einer Aktie. Dabei gilt: Je kurselastischer das Aktienangebot reagiert, desto weniger sinkt das Kursniveau anlässlich eines (exogenen) Rückgangs der Aktiennachfrage unter den fundamentalen Aktienkurs - vorausgesetzt die Aktiennachfrage ist nicht vollkommen kurselastisch. ${ }^{110}$ Je mehr die Eigner einer Gesellschaft also „abzuwarten“ bereit sind anstatt Kurseingeständnisse hinzunehmen, desto geringer fällt die Unterbewertung aus. Umgekehrt fällt das Bewertungsniveau umso tiefer

\footnotetext{
109 Dementsprechend ist eine Aktie überliquide bei einem Nachfrageüberhang nach Aktien wie es etwa in der Anfangsphase des Neuen Marktes der Fall war.

110 Liquiditätsorientierte Anleger gewichten die Inkaufnahme von Preiszugeständnissen höher als die Kosten des Wartens, weshalb ihr Aktienangebot vergleichsweise kurselastisch reagiert. Liquiditätsorientierte Anleger schränken ihr Aktienangebot anlässlich einer Unterbewertung deutlicher ein als Arbitrageure und informationsorientierte Anleger. Für sie besteht aufgrund besserer Anlagemöglichkeiten ein drängendes Transaktionsmotiv, weshalb Preiseingeständnisse eher in Kauf genommen werden. Auf eine marginale Unterbewertung reagiert ihr Aktienangebot deshalb weniger kurselastisch. Vgl. Rasch 1996, 63f.
} 
unter den fundamentalen Kurs, je weniger kurselastisch Aktienangebot, aber auch die Aktiennachfrage reagieren. ${ }^{111}$

Die Operationalisier- und Quantifizierbarkeit des Unterliquiditätsgrades einer Aktie wird durch die Dreidimensionalität der Unterliquiditätskosten - Zeitverlust, Mengenrestriktionen, ex- und implizite Kurseingeständnisse - erschwert. Eine Aktie nach seinem Liquiditätsgrad zu beurteilen, verlangt die Berücksichtigung aller drei Aspekte. Aber auch der spezielle Kontext der Marktteilnehmer ist für die Einschätzung des Liquiditätsgrades, wie folgendes Beispiel zu verdeutlichen sucht:

Angenommen, von zwei gleich großen Aktienbeständen besteht der erste Aktienbestand zur einen Hälfte aus leicht und zur anderen Hälfte aus schwer veräußerbaren Aktien und der zweite Aktienbestand aus durchgehend mäßig leicht veräußerbaren Aktien. Wünscht der Vermögensbesitzer kleinere Mengen zu veräußern, erachtet er ersteren Aktienbestand für liquider. Wünscht er hingegen größere Mengen zu veräußern, bewertet er den zweiten Bestand als den liquideren. Eine ähnlich asymmetrische Einschätzung der Liquidität beider Aktienbestände gilt bei unterschiedlichen Umwandlungsfristen. Mit zunehmender geplanter Umwandlungsfrist wird der Vermögensbesitzer im betrachteten Beispiel zunächst den ersten Vermögensbestand, später den zweiten Vermögensbestand für liquider erachten.

Bereits eine eindeutige ordinale Liquiditätseinteilung von Vermögenswerten ist also nur möglich, ist zugleich definiert, in Bezug auf welchen persönlich gewünschten Umwandlungsbetrag und auf welche persönlich gewünschte Umwandlungsfrist gemessen werden soll. Vor diesem Hintergund nimmt es nicht Wunder, dass es das Kriterium zur Messung der Unterliquidität einer Aktie nicht gibt. Um sich dem Liquiditätsgrad einer einzelnen Aktie oder eines ganzen Marktsegmentes - bei gegebener Marktorganisation $-{ }^{112}$ rechnerisch dennoch zu nähern, wird seit Demsetz $(1968)^{113}$ eine Vielzahl von Maßen bemüht, die allesamt nur einzelne Aspekte des Unterliquiditätsphänomens ausleuchten: ${ }^{114}$ Es werden - um nur einige zu nennen Umsatzzahlen errechnet, Kurs-Gewinn-Verhältnisse und Marktkapitalisierungen

$111 \quad$ Dies kann zum Verschwinden eines Marktes führen, wie der Fall infungibler spezifischer Güter zeigt. Spezifische Güter stehen in einem losen substitutiven Verhältnis zueinander. Um die Marktgegenseite zum Tausch zu bewegen, müssen extreme Preiszugeständnisse erfolgen, die als Quasi-Rente ausgenutzt zu werden drohen. Deshalb werden spezifische Güter regelmäßig dem Marktmechanismus entzogen und in einer kooperativen oder hierarchischen Form hergestellt, bezogen und vertrieben.

112 Den Zusammenhang von Marktorganisation und Liquidität beleuchtet Theissen 1999, 238ff.

113 Demsetz (1968) liefert das klassische Referenzmodell eines liquiden Marktes. Zu weiterführender Literatur vgl. Grossman, Miller (1988). 
verglichen, die Zahl der Aktionäre und der Konzentrationsgrad des Aktienbesitzes ermittelt, die Anzahl der Transaktionsvermittler (Market-Maker) angegeben, Mehrfachnotierungen an in- und ausländischen Börsenplätzen einbezogen, Umschlaghäufigkeiten des Streubesitzes, Mengenelastizitäten der Kurse, Transaktionen pro Zeitintervall berechnet, wobei auch all diese Meßmethoden noch Raum für weitergehende anlegerbezogene Differenzierungen lassen. ${ }^{115}$ Andere Indikatoren sind etwa die Höhe des beanspruchten „Greenshoe“, ${ }^{116}$ die zeitliche Verschiebung und Reduzierung der geplanten Kapitalerhöhung, die Anzahl der Tage ohne Börsenumsätze, Mehrfachlistings, der Zeitpunkt des Erstgangs an die Börse, die Höhe des Underpricings, die Aktienkurszusätze nach Handelsschluss, das Preisniveau einer Aktie und so genannte Liquiditätsraten ${ }^{117}$. Zur Berechnung der Liquiditätsgrade ganzer Börsen und nicht nur einzelner Aktien findet häufig der Turnover (Börsenumsatz gemessen an der Börsenkapitalisierung des Gesamtsekundärmarktes) und der Value Traded (Börsenumsatz im Verhältnis zum BSP) Anwendung.

Durch die vorherrschende Anlegerpräferenz für bestimmte Segmente und Teilmärkte in einem insgesamt fragmentierten Gesamtmarkt ist es möglich, dass rationalen Anlegern identisch erscheinende Rendite/Risiko-Profile in verschiedenen Segmenten unterschiedliche Liquiditätsgrade aufweisen. Enge Teilmärkte mit jeweils wenigen Transaktionspartnern, geringen Transaktionsvolumina und einer fehlenden Erneuerungskraft können markttiefen und -breiten Teilmärkten mit einer ausgeprägten „,resiliency“ und „,immediacy“ (hin zu den fundamentalen Kursen) gegenüberstehen. Wie in keinem anderen Segment des deutschen Sekundärmarktes sind die Aktien des Dax 30 zu einem hohen Grad liquide. Der Handel mit Aktien kleiner und mittlerer Gesellschaften ist hingegen nur eingeschränkt möglich. Ihre Aktien sind notorisch unterliquide. $\mathrm{Zu}$ bestimmten Zeitpunkten sind etwaige Transaktionswünsche in bestimmter Menge nicht ausführbar. Es fehlt schlicht die entsprechende Marktgegenseite. Dies sei an den jeweiligen Handelsfrequenzen

$114 \quad$ Vgl. Krainer, LeRoy 1997, 7; Schwartz 1992, 666; Upper 2000, 8f.

115 Vgl. Rudolph 1992, 338.

116 Im Vorfeld einer Emission ist ungewiss, wie groß die Aktiennachfrage letztlich sein wird. Das in petto gehaltene, bei einer unerwartet hohen Nachfrage nachschießbare Aktienkapital wird als „Greenshoe“ bezeichnet.

117 Hierbei handelt es sich um die Quote aus durchschnittlichem Handelsvolumen zu durchschnittlichen Kursveränderungen in einer Periode. Vgl. z.B. Cooper, Groth, Avera 
(infrequent trading) illustriert, und zwar anhand der differierenden Anzahl an Preisfeststellungen der Cdax-Werte an einem als repräsentativ zu bezeichnenden Börsentag im Xetra-Handel. ${ }^{118}$ Am 10.03.00 bildete der Cdax 688 inländische Unternehmen aus dem Amtlichen Handel an der Frankfurter Wertpapierbörse ab. Neben den Dax 100-Werten notierten 185 Unternehmen im Nemax all shares und 137 im Smax all shares. Die übrigen noch kleineren 266 Cdax-Werte gehörten keinem weiteren Index an, entstammten aber auch nicht dem noch weitaus weniger liquiden Freiverkehr. Innerhalb der Börsenzeit (8'30') verzeichnete jeder Dax 30Wert im Durschnitt 980 (variable) Preisfeststellungen, das sind fast zwei Fixings pro Minute. ${ }^{119}$ Die Kurse der Mdax-Werte wurden durchschnittlich 36 mal festgestellt mit bereits 21 Unternehmen, die 5 Preisfixings und weniger aufwiesen. ${ }^{120}$ Die Nemax-Werte verzeichneten 117 und die des Smax 10 Fixings. ${ }^{121}$ Die übrigen 266 Aktien erfuhren im Durchschnitt nur knapp 5 Preisfixings. Dies entspricht durchschnittlich einer Preisfeststellung in 13/4 Stunden. 114 dieser Unternehmen, immerhin also 43\%, verzeichneten überhaupt keine Preisfeststellungen, mithin auch keinen Umsatz.

Das am häufigsten verwendete Liquiditätsmaß ist die quotierte oder effektive GeldBrief-Spanne (Bid-Ask-Spread), die Differenz also zwischen dem An- und Verkaufskurs. ${ }^{122}$ Je höher die Liquidität einer Aktie ist, desto geringer fallen die Kursauf- und -abschläge für Käufer wie Verkäufer bezogen auf einen Zeitpunkt aus. Abbildung 2.4 verdeutlicht, dass die Höhe der Geld-Brief-Spanne mit der Anlegerpräferenz für große und medienwirksame Unternehmen abnimmt. So beträgt etwa der Bid-Ask-Spread für besonders umsatzschwache Werte des Geregelten

(1985). Zu den Grenzen der Aussagekraft dieses Liquiditätsmaßes vgl. Grossman, Miller 1988, 630f.

118 Ein ähnliches, wenn auch nicht ganz so deutliches Bild zeichnet der Parketthandel.

119 Im Gegensatz zum kontinuierlichen Preisfeststellungsverfahren werden bei den periodischen Verfahren, den „Gesamtpreis- oder Einheitskursverfahren“, an der Börse eintreffende Orders zunächst gesammelt und zu jeweils festgelegten Zeitpunkten, ein- oder mehrmals täglich, in einer zweiseitigen Auktion, bei der ein für alle Anleger einheitlicher Kurs nach dem Meistausführungsprinzip ermittelt wird, zur Ausführung gebracht.

120 An nicht wenigen Börsentagen bleiben ca. 5-10 Mdax-Werte auch gänzlich ungehandelt.

121 Sieht man von den beiden weit überdurchschnittlich gehandelten Werten der Ifco Systems N.V. und Knorr Capital Partner AG ab, so gelangt man bei den Smax-Werten nur auf durchschnittlich 5 Preisfixings.

Die Geld-Brief-Spanne hängt nicht nur vom Liquiditätsgrad ab. Auch bestimmt die Struktur des Marktes für Market-Maker, das Preisfindungsverfahren und die Diversifikation der Market-Maker-Portfolios ihre Höhe. Zur Kritik am Bid-Ask-Spread als Liquiditätsmaß vgl. Grossman; Miller 1988, 628-630; Glosten, Harris 1988, $123 \mathrm{f}$. 
Marktes und des Amtlichen Handels mehr als das Zehnfache von dem der Dax 30Werte (Abbildung 4).

Abbildung 2.4 Bid-Ask-Spreads nach Segmenten / Segmentausschnitten ${ }^{123}$

\begin{tabular}{|c|c|}
\hline Marktsegmente bzw. Sekundärmarktausschnitte & Geld-Brief-Spanne \\
\hline $\operatorname{Dax}(n=30)$ & $0,43 \%$ \\
\hline $\operatorname{Mdax}(\mathrm{n}=70)$ & $1,85 \%$ \\
\hline umsatzschwächste Mdax-Titel $(\mathrm{n}=30)$ & $2,88 \%^{\mathrm{a}}$ \\
\hline umsatzstärkste Neue Markt - Titel $(\mathrm{n}=30)$ & $1,13 \%$ \\
\hline umsatzschwächste Neue Markt - Titel $(\mathrm{n}=30)$ & $1,69 \%$ \\
\hline umsatzstärkste Sdax-Titel $(\mathrm{n}=30)$ & $2,02 \%$ \\
\hline umsatzschwächste Sdax-Titel $(\mathrm{n}=30)$ & $2,73 \%$ \\
\hline $\begin{array}{l}\text { besonders umsatzschwache Werte des Geregelten } \\
\text { Marktes und Amtlichen Handels }(n=30)\end{array}$ & $4,48 \%{ }^{\mathrm{b}}$ \\
\hline
\end{tabular}

Die Geld-Brief-Spanne stellen Kosten dar, die im Durchschnitt für die Akteure bei jeder einzelnen Transaktion anfallen. Die am Bid-Ask-Spread gemessenen langfristigen Kosten der Unterliquidität übersteigen jedoch jene bei einmaliger

123 Es handelt sich „nur“ um die explizite Geld-Brief-Spanne. Der implizite spread für Werte mit geringem Umsatzvolumen ist höher als für Aktien mit hohen und häufigen Umsätzen. Vgl. Beiker 1993, 135; Haller, Stoll 1989, $702 f$.

124 Die bid-ask spreads in Xetra sind nicht generell geringer als im Parketthandel. Dennoch ist der elektronische Handel weniger attraktiv für unterliquide Werte. Dies hängt damit zusammen, dass die spreads im elektronischen Handelssystem empfindlicher auf Veränderungen der Renditevolatilität reagieren. Vgl. Theissen (2002). 
Inkaufnahme um ein Vielfaches. Sie machen einen nicht unwesentlichen Teil des Aktienwertes aus, wie folgendes Beispiel veranschaulicht: ${ }^{125}$

Wenngleich eine exakte intersubjektive Quantifizierbarkeit der Unterliquiditätskosten einer Aktie - wie oben erläutert - nicht möglich ist, sei der Einfachheit halber angenommen, dass sich die Unterliquiditätskosten allein im Spread widerspiegeln. In Anlehnung an die Werte der Abbildung 2.4 beträgt die durchschnittliche Geld-Brief-Spanne eines liquiden Dax 30-Wertes im Durchschnitt 0,43\% vom Mittelkurs, die eines unterliquiden Wertes aus dem Geregelten Markt oder Amtlichen Handel durchschnittlich 4,48\%. Der Streubesitz des ersteren wird 11/4 mal, der des letzteren 2/5 mal per annum umgeschlagen (vgl. Abbildung 2.2). Annahmegemäß betrage der risikobereinigte Zinsfuß 8\% p.a. Wir erhalten somit einen Barwert aller künftig anfallenden Spreads in Höhe von

$0,412\left(0,0448 \times \sum_{t=0}^{\infty} \frac{1}{1,08^{t}}\right)=24,9$

für besonders umsatzschwache Aktien des Geregelten Marktes und des Amtlichen Handels und einen Barwert aller künftig anfallenden Spreads in Höhe von ,nur“"

$$
1,257\left(0,0043 \times \sum_{t=0}^{\infty} \frac{1}{1,08^{t}}\right)=7,3 \%
$$

für einen durchschnittlichen Dax 30-Wert. Mit einer Differenz von ergo 17,6\%-Punkten machen die Unterliquiditätskosten in Form differierender GeldBrief-Spannen für erstere einen empfindlich höheren Anteil am Kurs aus.

Um die Kosten aufzuwiegen, die für Händler weniger liquider Aktien auf lange Sicht mehr anfallen, müssen die betreffenden Unternehmen eine entsprechend höhere risikobereinigte Rendite real erwirtschaften. ${ }^{126}$ Umgekehrt reduziert ein hoher Grad an Marktliquidität die Renditeforderungen der Investoren. Liquidität mindert damit die laufenden Eigenkapitalkosten für ein Unternehmen. Obendrein ermöglicht Liquidität am Sekundärmarkt einen höheren Emissionspreis bei Folgeplazierungen am Primärmarkt.

\footnotetext{
125 Vgl. Amihud, Mendelson (1986).

126 Zum Zusammenhang zwischen dem Liquiditätsgrad einer Aktie und den Kapitalkosten der emittierenden Gesellschaft vgl. Cooper, Groth, Avera (1985); Amihud, Mendelson 1991b, 236; Foerster, Karolyi (1996).
} 
Unterschiedliche Unterliquiditätskosten bewirken eine Selektion der Anlegerschaft je nach Planungshorizont in den einzelnen Segmenten. Nach dem so genannten Klienteleffekt ${ }^{127}$ agieren Eigenkapitalgeber, die Informationsvorsprünge auszunutzen beabsichtigen, vorzugsweise in liquiden Segmenten. Hier sind die Unterliquiditätskosten einer schnellen Reaktion vergleichsweise gering. Anleger mit kürzerem Planungshorizont meiden hingegen Segmente kleiner und mittlerer Aktien, sind hier doch hohe Umschlaghäufigkeiten nicht immer realisierbar. Die Wahrscheinlichkeit, zum geplanten Zeitpunkt das gewünschte Transaktionsvolumen ohne Abschlag veräußern zu können, ist in unterliquiden Segmenten kleiner und mittlerer Gesellschaften nämlich geringer. Es verbleiben in unterliquiden Segmenten folglich Anleger mit einer eher längeren geplanten Haltedauer. Aufgrund dieses Klienteleffekts sind die Segmente der kleinen und mittleren Gesellschaften weniger anfällig für Unterliquiditätsschocks, das heißt für einen plötzlichen Rückgang des Eigenkapitalangebots. ${ }^{128}$ Die Werte des Dax 30 hingegen verfügen dank einer Anlegerpräferenz für sie zwar über ein grundsätzlich höheres Eigenkapitalangebot. Eine Beschleunigung der durchschnittlichen Umschlaghäufigkeit der Anleger beispielsweise kann diesen „Liquiditätspuffer“ jedoch schnell aufbrauchen. Selbst grundsätzlich hochliquide Marktsegmente sind letztlich nicht vor einem unterschießenden Eigenkapitalangebot gefeit. Während das Phänomen der Unterliquidität für Standardwerte - wie die regelmäßige Erholung etwa nach Börsencrashs zeigt - nur vorübergehender Natur ist, stellt sie für Small und Mid Caps eine dauerhafte Erscheinung dar. Offenbar liegen verschiedene Arten von Unterliquidität vor.

\footnotetext{
127 Vgl. Amihud, Mendelson 1991a, 59.

128 Vgl. Amihud, Mendelson (1990).
} 


\subsection{Arten der Unterliquidität}

Unterliquidität stellt sich als ein im Verhältnis zum ausgeschütteten und damit nachgefragten Aktienkapital zurückbleibendes Eigenkapitalangebot dar; zum fundamentalen Kurs werden mehr Aktien angeboten als nachgefragt. Ein Angebotsüberhang an Aktien kann sich auf einzelne Zeitpunkte beziehen oder sich über einen (begrenzten) Zeitraum erstrecken. Demnach handelt es sich um eine punktuelle Unterliquidität oder um eine persistente Unterliquidität. Bezogen auf einen unterschiedlichen Zeithorizont weichen in beiden Fällen die geplante Verkaufsmenge und die geplante Kaufmenge voneinander ab. $\mathrm{Zu}$ einzelnen Zeitpunkten oder zeitpunkübergreifend lassen Profildiskrepanzen zwischen Aktienangebot und Aktiennachfrage den Aktienmarkt zum fundamentalen Kurs hierbei ungeräumt. Es herrscht eine Art „Mismatch“129. Bei punktueller Unterliquidität liegt zum jeweiligen fundamentalen Aktienkurs ein „unterschießendes“ Eigenkapitalangebot bezogen auf einen einzelnen Zeitpunkt einer geplanten Transaktion, bei persistenter Unterliquidität bezogen auf einen (begrenzten) Zeitraum vor. Besteht für einen betrachteten Zeitraum lediglich punktuelle Unterliquidität, liegen Überhänge mal der einen, mal der anderen Marktseite vor. Über die einzelnen Zeitpunkte hinweg betrachtet, gleichen sich Aktienangebot und -nachfrage jedoch aus. Das geplante Aktienangebot und die geplante Aktiennachfrage stimmen im Betrachtungszeitraum in diesem Fall überein; es herrscht somit bei persistenter Liquidität punktuell Unterliquidität. Beide Arten der Mismatch-Unterliquidität können aber auch gemeinsam in Erscheinung treten. So können Profildiskrepanzen zwischen beiden Marktseiten zu einzelnen Zeitpunkten auftreten, wenn sich über den Zeitraum die Überhänge der betrachteten Zeitpunkte in der Summe nicht ausgleichen, wenn also eine persistente Unter- oder auch Überliquidität besteht. Eine punktuelle Unterliquidität ist sogar dann möglich, wenn zum fundamentalen Aktienkurs phasenweise eine überschießende Aktiennachfrage, das heißt persistente Überliquidität herrscht. Begreift man etwa die Konzentration des Anlegerinteresses auf Standardwerte als persistente Überliquidität, besteht in ihren Segmenten zu bestimmten Zeitpunkten möglicherweise ein geplantes Angebotsüberhang an Aktien, das heißt punktuelle Unterliquidität. In bestimmten

129 Der Begriff des „Mismatch“ bezieht sich in der arbeitsmarktökonomischen Literatur auf regionale oder qualifikatorische Diskrepanzen zwischen dem Arbeitsangebot und der Arbeitsnachfrage. Vgl. etwa Heise 1996, 71f. 
Phasen wie etwa zu Börsencrashs tritt aber auch in Segmenten der Standardwerte persistente Unterliquidität auf, nämlich dann, wenn die Aktiennachfrage - zumindest über einen begrenzten Zeitraum - einbricht.

Das Ausmaß an Mismatch-Unterliquidität hängt davon $a b$, inwiefern sich Aktienangebot und Aktiennachfrage auf die einzelnen Zeitpunkte ungleich verteilen. Die Profildiskrepanzen zwischen beiden Marktseiten nehmen zu, wenn sich das Transaktionsvolumen eines Zeitraums c.p. auf mehrere Zeitpunkte ausdifferenziert etwa durch eine Verlängerung der Börsenhandelszeit oder durch einen Anstieg der geplanten Handelsfrequenz (Vergleich der Szenarien 1 und 2 in Abbildung 2.5). Profildiskrepanzen zwischen Aktienangebot und -nachfrage treten außerdem umso deutlicher zu Tage, je geringer das für den Zeitraum geplante Transaktionsvolumen beider Marktseiten ist (Vergleich der Szenarien 2 und 4). An einem Markt mit grundsätzlich hohem Transaktionsvolumen treten die zeitpunktbezogenen Überhänge einer Marktseite weniger in Erscheinung. Der Anteil des realisierten Transaktionsvolumens am geplanten Transaktionsvolumen nimmt überproportional $\mathrm{ab}$, wenn das geplante Transaktionsniveau - in absoluten Zahlen $-{ }^{130}$ auf alle Zeitpunkte und auf beiden Marktseiten gleichmäßig verteilt zurückgeht. Ein solcher Effekt ist etwa vorstellbar bei der Einführung einer zusätzlichen Handelsplattform (neben dem konventionellen Parkett- der elektronische Xetra-Handel) oder einer Aufteilung des unveränderten Handelsvolumens auf weitere Regionalbörsen. Neben dem Ausmaß an zeitpunkt- und zeitraumbezogenen Profildiskrepanzen zwischen beiden Marktseiten beeinflusst damit auch das auf beiden Seiten geplante Transaktionsvolumen den Liquiditätsgrad einer Aktie, eines Segments oder des Gesamtmarktes. Aus analytischen Gründen lässt sich neben der (strukturellen) Mismatch-Unterliquidität von punktueller und persistenter zeitlicher Reichweite auch eine in Hinsicht auf das geplante Transaktionsvolumen niveaubedingte Art von Unterliquidität ausmachen. Beide „strukturellen“ Arten der Unterliquidität sind mit der niveaubedingten Unterliquidität eng verwoben. ${ }^{131}$ Wie bei einer persistenten Form der Unterliquidität etwa zeichnet sich auch die niveaubedingte Unterliquidität

130 Anders verhält es sich bei einem relativ gleichen Rückgang des geplanten Transaktionsvolumens. Geben etwa beide Marktseiten um x \% nach, erhöht sich am Ausmaß der Unterliquidität selbstverständlich nichts.

131 Erhöht sich das Transaktionsvolumen gegen unendlich, verschwinden limitational auch die Profildiskrepanzen punktueller oder persistenter Art. Außerdem verschwindet niveaubedingte Unterliquidität automatisch bei nicht vorhandenen Profildiskrepanzen. 
für einen betrachteten Zeitraum dadurch aus, dass mehr Aktien angeboten als nachgefragt werden, dass sich das Eigenkapitalangebot phasenweise also zurückzieht. Fraglich ist deshalb, inwiefern sich beide Arten von Unterliquidität voneinander unterscheiden. Bei persistenter Unterliquidität besteht über den betrachteten Zeitraum hinweg eine langfristige Tendenz zum erneuten Anstieg der Aktiennachfrage. Nach einer Phase abnehmender Aktiennachfrage, erholt sie sich wieder (Szenario $3 \rightarrow$ Szenario 2). Persistente Unterliquidität stellt insofern keine permanente, sondern eine nach einem Zeitraum vorübergehende „Unterschuss,,Situation der Aktiennachfrage dar. Demgegenüber ist für niveaubedingte Unterliquidität kennzeichnend, dass es langfristig diese Tendenz zu einer wieder ansteigenden Aktiennachfrage nicht gibt. Der Nachfrageunterschuss an Aktien ist von dauerhafter Natur und kann nur dadurch reduziert werden, dass emittierte Aktien dem Umlauf entzogen werden und sich das Aktienangebot auf diese Weise langfristig der dauerhaft gesunkenen Aktiennachfrage anpasst. Dadurch sinkt beidseitig das geplante Transaktionsvolumen (Szenario $3 \rightarrow$ Szenario 4).

\section{Abbildung 2.5 Arten der Unterliquidität in zeitlicher Abfolge}
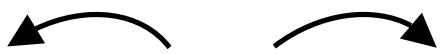

Szenario1 $\rightarrow$ Szenario $2 \rightarrow$ Szenario $3 \quad$ Szenario 4

\begin{tabular}{|c|c|c|c|c|c|c|c|c|}
\hline & $\mathrm{A}^{\mathrm{gepl}}$ & $\mathrm{N}^{\text {gepl }}$ & $\mathrm{A}^{\text {gepl }}$ & $\mathrm{N}^{\text {gepl }}$ & $\mathrm{A}^{\text {gepl }}$ & $\mathrm{N}^{\mathrm{gepl}}$ & $\mathrm{A}^{\text {gepl }}$ & $\mathrm{N}^{\mathrm{gepl}}$ \\
\hline $\mathrm{T}_{1}$ & 40 & 40 & 50 & 30 & 50 & 20 & 40 & 20 \\
\hline $\mathrm{T}_{2}$ & 0 & 0 & 0 & 30 & 0 & 20 & 0 & 20 \\
\hline $\mathrm{T}_{3}$ & 10 & 20 & 0 & 10 & 0 & 0 & 0 & 0 \\
\hline $\mathrm{T}_{4}$ & 20 & 60 & 10 & 70 & 10 & 60 & 0 & 60 \\
\hline $\mathrm{T}_{5}$ & 0 & 0 & 20 & 0 & 20 & 0 & 10 & 0 \\
\hline $\mathrm{T}_{6}$ & 50 & 10 & 40 & 0 & 40 & 0 & 30 & 0 \\
\hline $\mathrm{T}_{7}$ & 30 & 20 & 30 & 10 & 30 & 0 & 20 & 0 \\
\hline$\sum \mathrm{TK}^{\text {gepl }}$ & 150 & 150 & 150 & 150 & 150 & 100 & 100 & 100 \\
\hline $\mathrm{A}^{\text {real }}$ & \multicolumn{2}{|c|}{$\frac{1}{100}$} & \multicolumn{2}{|c|}{$\frac{1}{50}$} & \multicolumn{2}{|c|}{30} & \multicolumn{2}{|c|}{20} \\
\hline$\frac{A^{\text {real }}}{A^{\text {gepl }}}$ & \multicolumn{2}{|c|}{$2 / 3$} & \multicolumn{2}{|c|}{$1 / 3$} & \multicolumn{2}{|c|}{$1 / 5$} & \multicolumn{2}{|c|}{$1 / 5$} \\
\hline
\end{tabular}

$\mathrm{A}=$ Aktienangebot; $\mathrm{N}=$ Aktiennachfrage; $\mathrm{TK}=$ Transaktionsvolumen; $\mathrm{T}=$ Zeitpunkt 
Die Liquidität einer Aktie wird im Beispiel der Abbildung 2.5 durch das Verhältnis von realisiertem zu geplantem Aktienangebot in vier verschiedenen Szenarien über einen betrachteten Zeitraum von jeweils 7 Zeitpunkten zum jeweiligen fundamentalen Kurs wiedergegeben. ${ }^{132}$ Unterliquidität drückt sich dabei in einem Wert kleiner als Eins aus. Das gewählte Liquiditätsmaß umfasst die punktuelle und persistente genauso wie die niveaubedingte Form der Unterliquidität. Es verringert sich die Liquidität einer Aktie einerseits mit einer zunehmenden Profildiskrepanz zwischen den Marktseiten; sei sie punktueller Art (Szenario $1 \rightarrow$ Szenario 2) oder wie der Vergleich von Szenario 2 und 3 verdeutlicht - persistenter Art. Die Liquidität der betrachteten Aktie nimmt aber auch andererseits mit sinkendem geplanten Transaktionsvolumen infolge eines überproportionalen Rückgangs des realisierten Angebots ab (Szenario $2 \rightarrow$ Szenario 4). Das gewählte Liquiditätsmaß berücksichtigt damit sowohl das geplante Transaktionsvolumen als auch die Profildiskrepanzen inkompatibler Transaktionspläne. Stimmen Kauf- und Verkaufspläne, wenn auch auf niedrigem Transaktionsniveau, in allen Zeitpunkten überein, wäre die Aktie nach dem gewählten Liquiditätsmaß jedoch vollkommen liquide. Jedes geplante Aktienangebot träfe auf eine entsprechende Nachfrage und würde auch realisiert werden. Das Liquiditätsmaß beträgt in diesem (unrealistischen) Grenzfall Eins. ${ }^{133}$ An diesem Fall wird deutlich, dass niveaubedingte Unterliquidität für das gewählte Liquiditätsmaß eine nur katalysierende Funktion übernimmt, hingegen keine eigenständige. Dem gewählten Liquiditätsmaß folgend, kann es ohne Mismatch-Unterliquidität also keine niveaubedingte Unterliquidität geben. Darin liegt ein Schwachpunkt des gewählten Liquiditätsmaßes. Denn zum einen erfasst das gewählte Liquiditätsmaß in dem Fall nicht das etwaige Entziehen von Aktien aus dem Umlauf etwa durch Aktienrückkäufe. Zum anderen geht dabei unter, dass ein hohes geplantes Transaktionsvolumen auf beiden Marktseiten, mithin ein hohes Maß an niveaubedingter Liquidität erst die Voraussetzung dafür schafft, dass der Verlauf eines Aktienkurses die fundamentalen Veränderungen eines Unternehmenswertes angemessen und zeitnah wiederzugeben in der Lage ist. Die Übereinstimmung von

132 Das Liquiditätsmaß lässt offen, ob sich die Überhangsituationen ex-post über eine Anpassung der Preise oder der Transaktionsmengen ausgleichen.

133 Das gewählte Liquiditätsmaß sieht keine Überliquidität vor. Zwar ist in einzelnen Teilperioden ein Nachfrageüberhang möglich, doch können logisch zwingend insgesamt nicht mehr Aktienangebote realisiert als geplant werden. 
geplantem Aktienangebot und geplanter Aktiennachfrage ist nur eine notwendige, aber keine hinreichende Bedingung für einen informations- und bewertungseffizienten Sekundärmarkt. Dass der Aktienkurs den sich ständig verändernden inneren Wert eines Unternehmens widerspiegeln kann, setzt in einzelnen Teilperioden deshalb ein entsprechendes Transaktionsvolumen voraus, das heißt ein bestimmtes Niveau an Liquidität. Insofern erhöht sich durch ein ansteigendes Transaktionsniveau nicht nur die Liquidität eines Marktes, sondern es verbessert sich auch grundsätzlich seine Informations- und Bewertungseffizienz. Vollkommene Liquidität im Sinne eines bewertungs- und informationseffizienten Sekundärmarktes verlangt neben einer $\mathrm{zu}$ jedem Zeitpunkt vollständigen Kompatibilität beider Marktseiten somit ein hinreichendes Transaktionsvolumen, das fundamentale Veränderungen des Unternehmens in den Aktienkursen angemessen und zeitnah abzubilden vermag. Entgegen dem gewählten Liquiditätsmaß kann niveaubedingte Unterliquidität im Sinne einer Bewertungs- und Informationseffizienz insofern auch dann existieren, wenn eine Aktie punktuell und persistent vollkommen liquide ist.

In den vier Szenarien der Abbildung 2.5 führt der fundamental richtig bewertete Aktienkurs auf dem betrachteten Markt zu einzelnen Zeitpunkten keine Markträumung herbei. Über die gesamte Periode betrachtet, entsprechen sich Aktienangebot und -nachfrage in den Szenarien S1, S2 und S4. In diesen Szenarien ist die betrachtete Aktie persistent liquide. In S1 ist der Anteil des realisierten am geplanten Aktienangebot vergleichsweise am höchsten. Der Liquiditätsgrad halbiert sich in S2, nehmen doch die Profildiskrepanzen zwischen beiden Marktseiten in punktueller Hinsicht zu. Mit sieben anstatt nur fünf Teilperioden differenziert sich der Handel in S2 im Vergleich zu S1 mit $\mathrm{T}_{2}$ und $\mathrm{T}_{5}$ zeitlich aus. Zusätzlich verlieren Angebot und Nachfrage in den Teilperioden $\mathrm{T}_{1}, \mathrm{~T}_{4}$ und $\mathrm{T}_{7}$ an Kompatibilität. S2 zeichnet somit gewissermaßen das Bild eines weniger tiefen und weniger effizienten Marktes als S1. Die Summe des beiderseitig geplanten Transaktionsvolumens bleibt im betrachteten Zeitraum $\left(\mathrm{T}_{1}-\mathrm{T}_{7}\right)$ allerdings unverändert. Reagieren die Kurse der betrachteten Aktie infolge punktueller Unterliquidität auch auf die jeweiligen Überhänge zu den einzelnen Zeitpunkten entsprechend, schwanken sie in S2 mehr als in S1 um den fundamentalen Aktienkurs. Denn in S2 nimmt im Vergleich zu S1 die punktuelle Unterliquidität $\mathrm{zu}$, während in beiden Szenarien eine 
zeitraumbezogene, das heißt persistente Liquidität herrscht. Dadurch, dass das Transaktionsvolumen in beiden Szenarien konstant bleibt, ändert sich auch nichts am Ausmaß niveaubedingter Unterliquidität. In Szenario S3 liegt neben punktueller auch eine persistente Mismatch-Unterliquidität vor. Bezogen auf den betrachteten Zeitraum wird hier weniger Eigenkapital bereitzustellen geplant, als die Unternehmen in Höhe der emittierten Aktien Eigenkapital „nachfragen“. Deshalb ist im Normalfall zu erwarten, dass das Kursniveau in S3 unter das fundamentale Bewertungsniveau absinkt. In S4 sinkt die Liquidität des Marktes niveaubedingt, da hier das geplante Transaktionsvolumen gleichmäßig auf beide Marktseiten und auf alle geplanten Transaktionszeitpunkte verteilt abnimmt.

Begreift man die vier Szenarien als ein nacheinander ablaufendes Schema, geht dem niveaubedingten Anstieg der Unterliquidität in S4 ein zeitraumbezogener Rückgang der Aktiennachfrage in S3 voraus. Diese einseitige Abnahme der Aktiennachfrage in S3 lässt sich etwa mit der Halbierung des Liquiditätsgrades in S2 im Übergang von S1 erklären. Die im Vergleich zu S1 in S2 angestiegene punktuelle Unterliquidität erhöht den Spread, das heißt die Transaktionskosten, und verringert dadurch die Aktiennachfrage in S3. Der Finanzkapitalabfluss führt in anderen Bereichen (wie im Rentenbereich, auf den Märkten ausländischer Aktien und alternativer Anlagen) zu steigenden Kursen, was wiederum den Abfluss beschleunigt. Es ergibt sich aus einer anfänglich gestiegenen punktuellen Unterliquidität eine persistente Unterliquidität. Dieser Rückgang kann im Extrem sogar in einen unterliquditätsbedingten Crash münden; ${ }^{134}$ eine solche ,asset deflation“ ergibt sich - wohlgemerkt! unterliquiditätsbedingt und nicht aus spekulativen, massenpsychologischen oder aus Gründen, die den fundamentalen Werten der Unternehmen erwachsen. Infolge eines punktuellen Unterliquiditätsschocks in S2 - im Verhältnis zu S1 - schränken also die Eigenkapitalgeber ihre geplante Aktiennachfrage in S3 ein. Ein persistentes Abweichen der geplanten Aktiennachfrage vom geplanten Aktienangebot (S3) mag periodenübergreifend, das heißt für $\mathrm{T} \rightarrow \infty$, jedoch nicht Bestand haben. So kann sich die in S3 zurückgegangene persistente Mismatch-Liquidität auf höherem nachfrageseitigen Transaktionsniveau wieder erholen, wie in S2 der Fall. Nach einem Rückgang der Aktiennachfrage in S3 ist in Segmenten niveaubedingt liquider

134 “...we found that on October $19^{\text {th }}[1987]$ the dollar bid-ask spread increased by more than $63 \%$ compared to its pre-Crash level [on a sample of NYSE stocks included in the S\&P 500 list]..." Amihud, Mendelson 1991b, 238; vgl. Grossman, Miller 1988, 631. 
Aktien früher oder später mit einer Erholung des Eigenkapitalangebots auf ehemals hohem Transaktionsvolumen zu rechnen $(\mathrm{S} 3 \rightarrow \mathrm{S} 2)$. Die geplante Aktiennachfrage passt sich hier dem nach wie vor hohen Aktienangebot jenseits der Periode von S3 wieder an. Denn in niveaubedingt liquiden Segmenten ist der Kursrückgang in S3 mit einem entsprechenden Anstieg der erwarteten Rendite verbunden. Auf kurz oder lang veranlasst der Anstieg der Renditeerwartung die Eigenkapitalgeber, ihre Aktiennachfrage so lange auszuweiten, bis das ursprüngliche fundamentale Kursniveau wieder erreicht ist. Bei einem konstanten Aktienangebot erreicht die geplante Aktiennachfrage dabei ebenfalls das ursprüngliche Volumen (S2). Insbesondere in Segmenten der Standardwerte ist $\mathrm{zu}$ beobachten, dass sich ihr Liquiditätsgrad nach einem (strukturellen) Unterliquiditätsschock erneut auf höherem Transaktionsniveau wie in S2 stabilisiert, dass die persistente Unterliquidität langfristig betrachtet vorübergeht und sich nicht $\mathrm{zu}$ einer niveaubedingten Unterliquidität entwickelt. ${ }^{135}$

Während eine kurselastische Nachfrage nach zeitweise unterbewerteten Standardwerten das Transaktionsvolumen erneut anhebt und somit das Kursniveau zum fundamentalen Bewertungsniveau führt, ist die Aktiennachfrage nach kleinen und mittleren Gesellschaften weitaus weniger kurselastisch. Die Anleger reagieren mit dauerhafter Unterbewertung und einer dauerhaft eingeschränkten Aktiennachfrage, das heißt mit niveaubedingter Unterliquidität (S4). Eine Unterbewertung niveaubedingt unterliquider Aktien geht nach dem Rückgang der Aktiennachfrage in S3 offenbar nicht mit einer steigenden, sondern mit einer allenfalls konstanten Renditeerwartung einher. Bei einer kursunelastischen Renditeerwartung bleiben das geplante Aktienangebot und die geplante Aktiennachfrage zunächst unverändert, da beide eine Funktion der (nicht ansteigenden) Renditeerwartung darstellen. ${ }^{136}$ Zum fundamentalen Aktienkurs bleibt ein Angebotsüberhang an Aktien bestehen. S3 stellt in diesem Fall deshalb nicht nur ein periodenbezogenes, sondern auch ein periodenübergreifendes, langfristiges

135 Gleichwohl zeigt die Geschichte, dass ein Börsencrash sich auch im Standardwertebereich zu einer dauerhaft unterschießenden Aktiennachfrage und somit zu niveaubedingter Unterliquidität ausweiten kann.

136 Ist ein sinkendes Kursniveau mit einer sogar abnehmenden Renditeerwartung verbunden, verlaufen Aktienangebot und Aktiennachfrage anomal. Sinkende Kurse bringen dann ein steigendes Aktienangebot und eine sinkende Nachfrage hervor. Das „Unterschuss“-Angebot an Eigenkapital mithin das Ausmaß an Unterliquidität nimmt mit der Unterbewertung zu anstatt ab wie bei einem normalen Verlauf der Aktienangebots- und -nachfragekurven. 
Gleichgewicht dar. Die Aktien erfahren eine dauerhafte Unterbewertung, da von der kursunelastischen Renditeerwartung keine veränderten Aktienkauf- oder Verkaufspläne ausgehen. Das geplante Eigenkapitalangebot unterschreitet somit zur fundamental richtigen Bewertung periodenübergreifend das umlaufende Aktienkapital. Nicht nur zum fundamentalen, sondern auch zu jedem anderen darunter liegenden Kurs herrscht Unterliquidität, das heißt ein Angebotsüberhang an Aktien. ${ }^{137}$ Es verfestigt sich die persistente Unterliquidität des Szenarios S3 zu einer niveaubedingten Unterliquidität in S4.

S4 veranschaulicht den Fall eines langfristig ebenfalls sinkenden Aktienangebots bei dauerhafter Unterbewertung. In Anlehnung an das Gresham'sche Gesetz entziehen Anteilseigner ihre unterbewerteten Titel langfristig dem Umlauf. Der Anstieg des Festbesitzanteils, das heißt der Rückgang des Streubesitzanteils, an einem Unternehmen ist somit das Ergebnis einer dauerhaften Unterbewertung seiner Aktien. ${ }^{138}$ Dabei passen sich die Pläne der Aktienanbieter in S4 über die betrachtete Periode hinweg ihrer Realisierbarkeit durch die in S3 gesunkene Aktiennachfrage auf niedrigem Transaktionsniveau an. ${ }^{139}$ Durch eine Senkung des Streubesitzes gleichen sich Aktienangebot und Aktiennachfrage an; besser gesagt: die niedrige Nachfrage nach niveaubedingt unterliquiden Aktien bestimmt langfristig das Aktienangebot. ${ }^{140}$ Insofern ist die Aktiennachfrage für die Ausweitung des realisierten Transaktionsvolumens, mithin die Erhöhung der niveaubedingten Liquidität von großer Bedeutung. Während sich das Aktienangebot langfristig an die Aktiennachfrage bei niveaubedingter Unterliquidität anpasst, gilt Umgekehrtes nur eingeschränkt. Wie die Erfahrungen am deutschen Aktiensekundärmarkt zeigen, lässt sich mit einer Erhöhung des Streubesitzanteils kleiner und mittlerer Gesellschaften nur geringfügig seine Aktiennachfrage stimulieren und somit die niveaubedingte Unterliquidität senken. Neben der Erhöhung des Festbesitzes besteht eine andere Variante der Angleichung des Aktienangebots an die Aktiennachfrage darin, eigene

137 Dieser Mechanismus eines zunehmend unterschießenden Eigenkapitalangebots besteht realistischerweise nur innerhalb einer Bandbreite der Aktienkurse. Bei einem Kurs nahe Null ist zu erwarten, dass die Aktiennachfrage erneut ansteigt. Lassen sich Aktien jedoch auch mit sehr großen Preiszugeständnissen nicht veräußern, herrscht nicht nur Unter-, sondern sogar Illiquidität.

138 Vgl. Kreps 1994, 563; Meyer (1957).

139 Dies entspricht der Tatsache, dass kleine und mittlere Gesellschaften, die dauerhaft unterbewertet sind, einen geringeren Streubesitz aufweisen als die niveaubedingt liquiden Standardwerte. Vgl. Spalte 4 in Abbildung 2.2. 
Aktien zurückzukaufen. Eigene, niveaubedingt unterliquide und unterbewertete Aktien zu erwerben, erhöht zwar kurzfristig die Aktiennachfrage. Mittel- und langfristig impliziert der Rückkauf eigener Aktien jedoch ebenfalls eine Reduzierung des umlaufenden Aktienangebotes. ${ }^{141}$ Damit wird regelmäßig bezweckt, die Unterbewertung der nicht ausreichend nachgefragten Aktien zu beseitigen. Gelingt dies nicht, bestehen für eine Gesellschaft weitere Möglichkeiten darin, sich vom Aktienmarkt in Form eines Delistings oder eines Going Privates gänzlich zurückzuziehen, oder zumindest das Volumen geplanter Folgeemissionen zu kürzen. Auch hierbei handelt es sich letztlich um Maßnahmen, das Aktienangebot an die niedrigere Aktiennachfrage anzupassen. Auf niedrigem geplanten Transaktionsvolumen passen sich durch diese Maßnahmen zwar Aktienangebot an Aktiennachfrage an - allerdings auf Kosten der niveaubedingten Form von Unterliquidität. Im Übergang von S3 zu S4 heben sich im gewählten Beispiel beide Effekte gegenseitig auf: Der Rückgang der persistenten Unterliquidität entspricht dem Anstieg der niveaubedingten Unterliquidität. Das Liquiditätsmaß weist in beiden Szenarien einen Wert von 0,2 auf.

Mit dem Ergebnis eines höheren Liquiditätsgrades und damit auch geringerer Transaktionskosten einer Aktie agieren an manchen Märkten so genannte Market Makers. Sie übernehmen die Funktion, Profildiskrepanzen zwischen Aktienangebot und -nachfrage punktueller und persistenter Art einzuebnen. Findet der Markt zu bestimmten Zeitpunkten und in bestimmten Teilperioden aus sich heraus nicht zur Räumung, stärken Market Makers den Part der jeweils kürzeren Marktseite. Sie ermöglichen vor allem in niveaubedingt liquiden Segmenten einen jederzeitigen Handel von Aktien, indem sie ,immediacy“, das heißt eine sofortige Handelbarkeit anbieten. Zu fortlaufenden Geld- und Briefkursen („Quotes“) kaufen und verkaufen Market Makers als Handelsvermittler entsprechend der jeweiligen Überhangsituation vorübergehend bestimmte Aktienmengen. ${ }^{142}$ Eine auf diese Weise einem Markt

140 Auf einem Zirkulationsmarkt gilt, dass jeder Aktienanbieter logisch zwingend vormals Aktiennachfrager gewesen sein muss, aber nicht umgekehrt. Vgl. Castan (1997).

Im Gegensatz zu Market Maker-Verfahren werden bei Auktionsverfahren Kauf- und Verkaufsaufträge der Anleger an ein Limitorderbuch geleitet, in dem sie, geordnet nach Preis und Eingabezeit, einander gegenübergestellt werden. Auf Grundlage der vorliegenden Orders werden dann markträumende Gleichgewichtskurse ermittelt. Kennzeichen des Auktionsverfahrens ist, dass die Orders der Anleger direkt miteinander konkurrieren und gegeneinander ausgeführt werden. $\mathrm{Zu}$ hybriden Verfahren (Specialist- oder Order DisplaySystemen) vgl. Rasch 1996, 118 f. 
verliehene Liquidität mindert zwar den „Mismatch“ zwischen beiden Marktseiten. Doch kommen Market Makers an einem niveaubedingt unterliquiden Markt als Endnachfrager nicht in Frage. Einem Markt niveaubedingte Liquidität direkt zu verleihen, ist Market Makers nicht möglich, gleichen sie doch lediglich die Profildiskrepanzen zwischen den einzelnen Zeitpunkten und Teilperioden aus. Folglich sind Market Makers an niveaubedingt unterliquiden Segmenten nicht oder nur eingeschränkt tätig. ${ }^{143}$ Einzelnen Gesellschaften, die in Vergessenheit zu geraten drohen, werden deswegen in manchen Segmenten so genannte Designated Sponsors, das heißt Betreuer anbeigestellt. ${ }^{144}$ Ihre Aufgabe ist es, auf Anfrage von Marktteilnehmern oder auf eigene Initiative verbindliche Geld-Brief-Kurse in begrenzter Bandbreite für maximale Transaktionsvolumina innerhalb bestimmter Fristen ins Orderbuch zu stellen. ${ }^{145}$ Wie Market Makers reduzieren sie damit sowohl punktuelle als auch persistente Unterliquidität. Darüber hinaus übernehmen Designated Sponsors die kürzere Marktseite nicht nur vorübergehend, sondern längerfristig. Im Gegensatz zu Market Makers sind die Positionen der Designated Sponsors beim Handel einzelner Aktien nämlich langfristig nicht unbedingt glattgestellt. Designated Sponsors sind damit betraut, insbesondere solche Aktienwerte dauerhaft mit niveaubedingter Liquidität $\mathrm{zu}$ versorgen, welche Market Makers meiden - die der Small und Mid Caps eben. ${ }^{146}$ Mit dem Angebot niveaubedingter Liquidität ist die Hoffnung verbunden, das Gesamtinteresse der Anleger an niveaubedingt unterliquiden Aktien $\mathrm{zu}$ wecken. Aber auch diese liquiditätssteigernde Maßnahme zu Gunsten der Small und Mid Caps war von nur eingeschränkter Effektivität. ${ }^{147}$ Beck (1999) stellt nüchtern fest, „[e]in Betreuer löse das Liquiditätsproblem nicht; im Neuen Markt gebe es für jeden Titel zwei Betreuer,

143 „In the long run the number of market makers will adjust until, in equilibrium, the returns to each from assuming the risk of waiting to trade the ultimate buyers just balance the costs of maintaining a continous presence in the market. This adjustment determins the equilibrium amount of immediacy provided..." Grossman, Miller 1988, 617. Einer Studie von Gerke zufolge beträgt der Umsatzanteil der Designated Sponsors im Mdax ca. $7 \%$, im Neuen Markt ca. 14\%. Zu Mindestanforderungen von Designated Sponsors vgl. www.xetra.de unter der Rubrik „Der Handel/ Designated Sponsor / Betreuer Guide“".

146 Bei Erfüllung der Mindestanforderungen werden die Transaktionskosten der Designated Sponsors von der Deutsche Börse AG rückvergütet. Zwischen Emittenten und Designated Sponsor kann ein Vertrag eine Entlohnung beinhalten. Ihre Höhe variiert sehr stark und ist von seinen Leistungen (nur Handel oder auch Research, Investor Relations) abhängig. Mit 479 (Stand 13.04.00) ist nur die Hälfte aller börsennotierten inländischen Unternehmen betreut: 55 Mdax, 246 Neuer Markt inkl. Dual-Listing, 178 Smax und sonstige. Ausländische betreute Werte: 85. Im Rahmen von Xetra Release 3 (Start 12.10.98), einer komplementären Stufe zur Erweiterung des Funktionsumfangs von Xetra, war geplant, alle damals ca. 2.000 an 
und dennoch gebe es dort Liquiditätsprobleme.“148 Die Institutionen der Market Makers und Designated Sponsors vermögen zwar das Auseinanderklaffen von Angebot und Nachfrage zeitlich zu überbrücken, mithin (strukturelle) MismatchUnterliquidität $\mathrm{zu}$ reduzieren. Aufgrund ihrer Professionalität, Diversifizierungsmöglichkeiten und Größenvorteile reduzieren sie damit auch volkswirtschaftliche Kosten; ${ }^{149}$ dabei spielt die wettbewerbliche Ausgestaltung des Marktes für „,immediacy“ eine wesentliche Rolle. ${ }^{150}$ Liquiditätshändler können aber nur einen begrenzten Beitrag für die niveaubedingte Liquidität der Small und Mid Caps leisten. ${ }^{151}$ Niveaubedingte Liquidität hängt eben nicht von den genannten „,institutionellen Arrangements, sondern auch von stabilen Angebots- und Nachfrageverhältnissen ab... "Technische" Maßnahmen zur Verbesserung der Marktliquidität können daher kein Ersatz für ein langfristiges, an fundamentalen Größen orientiertes Handeln der Kapitalmarktakteure sein. “152

Festzuhalten ist: Innerhalb eines Zeitraums ist eine punktuelle von einer persistenten Mismatch-Unterliquidität zu unterscheiden. Liegt für einen (begrenzten) Zeitraum ein Angebotsüberhang an Aktien vor (persistente Unterliquidität), tendieren beide Marktseiten auf (sehr) lange Sicht dahin, sich erneut auszugleichen; entweder auf wieder gestiegenem Transaktionsniveau oder auf dauerhaft geringerem Transaktionsniveau. In letzterem Fall liegt niveaubedingte Unterliquidität vor, die symptomatisch für die Segmente kleiner und mittlerer Gesellschaften ist. Im folgenden Abschnitt ist der Frage nachzugehen, ob sich niveaubedingte Unterliquidität breiter Börsensegmente auf die gesamtwirtschaftliche Entwicklung negativ auswirkt, mithin wirtschaftspolitische Gegenmaßnahmen zu ergreifen sind. Mit einer Beschränkung auf die Form niveaubedingter Unterliquidität ist keineswegs die volkswirtschaftliche Bedeutung der Mismatch-Unterliquidität für gering zu erachten; insbesondere wenn man dabei an die damit einhergehenden volatilen

der FWB notierten in- und ausländischen Aktiengesellschaften mit mindestens einem Designated Sponsor zu versehen. Vgl. Die Aktiengesellschaft 12/1998 R502. Beck 1999, 25.

"These gains arise essentially from diversification - the spreading of the transferred risks over the entire group of market makers. The larger that group, the lower, c.p., the risk and expected return per unit traded by each and hence also the lower the effective cost of immediacy to the customers." Grossman, Miller 1988, 618f.

Kontraintuitiv stellt Glosten $(1989,211)$ fest: "While competing market makers will expect a zero profit on every trade, the monopolist will average his profits across trades. This implies a more liquid market when there is extensive trading on private information."

Vgl. Gerke 1995, 22.

Deutsche Bundesbank 1998, 67. 
Kursschwankungen denkt. ${ }^{153}$ Doch gebietet sich für den betrachteten Unternehmenskreis kleiner und mittlerer Gesellschaften am deutschen Aktiensekundärmarkt eine Schwerpunktsetzung auf die niveaubedingte Form von Unterliquidität. Germany were found to be insignificant.” Arestis, Demetriades, Luintel 2001, 37. 


\subsection{Niveaubedingte Unterliquidität und wirtschaftliche Entwicklung}

In Deutschland hat sich die Produktions-Produktivitäts-Schere seit Beginn der 60er Jahre trotz sinkender Zuwachsraten der gesamtwirtschaftlichen Produktivität gespreizt. ${ }^{154}$ Der Produktionszuwachs ist $\mathrm{zu}$ gering, um ein vollbeschäftigungskonformes Arbeitsplatzangebot $\mathrm{zu}$ schaffen. $\mathrm{Um}$ die Wachstumslücke zu schließen, kommt dem technischen Fortschritt besondere Bedeutung zu. Insbesondere - auf der Anwendung von Schlüsseltechnologien basierenden $-{ }^{155}$ radikalen Produktinnovationen ${ }^{156}$ wird eine große Bedeutung für eine nachhaltige gesamtwirtschaftliche Nachfrageexpansion beigemessen. ${ }^{157}$ Nicht zuletzt kommt dem Investitionsverhalten kleiner und mittlerer Unternehmen eine besondere Rolle zu, verfügt die deutsche Wirtschaft doch über ein ausgeprägt mittelständisches Rückgrat. ${ }^{158}$ Kleine und mittlere Unternehmen beschäftigen - laut Angaben des Bonner Instituts für Mittelstandsforschung - 70\% aller Arbeitnehmer, führen 46\% aller Bruttoinvestitionen durch und tragen 57\% zur Bruttowertschöpfung aller Unternehmen bei. ${ }^{159}$ Ihre Innovationen und Gründungen bilden - wider die NeoSchumpeter-Hypothesen $-{ }^{160}$ den „Humus“ für gesamtwirtschaftliches Wachstum. ${ }^{161}$

154 Vgl. Pätzold 1993, 273-278.

155 Die Langen Aufschwünge der wirtschaftlichen Entwicklung wurden seit 200 Jahren stets von so genannten Basisinnovationen getragen. Vgl. Kromphardt 1987, 199f.

156 „Produktinnovationen sind neuartige, bisher auf dem Markt noch nicht angebotene Produkte oder wesentlich verbesserte bereits vorhandene Produkte. Sie zeichnen sich durch einen bestimmten Neuheitsgrad bezüglich der Gebrauchseigenschaften und einen höheren Kundennutzen gegenüber vorausgegangenen bzw. vergleichbaren Produkten aus.“ Sabisch 1991, 64.

157 Um in einem Lange-Wellen-Abschwung langfristiges Wachstum zu induzieren, ist nachfrageseitig das Überwinden des 1. Gossen'schen Gesetzes durch die Schaffung neuer Produkte erforderlich. Vgl. Nefiodow 1996, 63-89; Kühne 1991, 7-61.

158 Die deutsche Wirtschaft besteht, was die Anzahl der Unternehmen, deren Beitrag zur Wertschöpfung, die Innovationskraft und den Beschäftigungseffekt angeht, überwiegend aus mittelständischen Unternehmen. Von den über 3 Mio. Unternehmen gehören 99,8\% der Gruppe kleiner und mittlerer Unternehmen an - nach der Mittelstandsdefinition des Bonner Instituts für Mittelstandsforschung: Unternehmen mit bis zu 500 Arbeitnehmern und weniger als 100 Mio. DM Jahresumsatz bzw. weniger als 25 Mio. DM Jahresumsatz in den Branchen Handelsvermittlung, Einzelhandel, Verkehr und Nachrichtenübermittlung, Banken, Versicherungen und Dienstleistungen. Vgl. Baums 1997, 1943; Scholz 2000, 519; Leopold, Frommann 1998, 1-3.

159 Vgl. http://www.ifm-bonn.de/dienste/daten.htm.

160 Schumpeter 1980, 174f. „Wir müssen vielmehr anerkennen, daß die Großunternehmung zum kräftigsten Motor dieses Fortschritts und insbesondere der langfristigen Ausdehnung der Gesamtproduktion geworden ist ... " $\mathrm{Zu}$ den Neo-Schumpeter-Hypothesen vgl. Schmidt 1996, 99-104; Ahrns, Feser 1991, 64-66. Die Größe eines Unternehmens erleichtert u.a. dadurch die Innovationstätigkeit, als mit ihr die entsprechende Verfügbarkeit finanzieller Mittel einhergeht. Vgl. Galbraith 1952, 92.

$161 \mathrm{Ob}$ große Unternehmen innovativer sind als kleine, ist in der Literatur umstritten. Vgl. Perlitz, Löbler 1989, 9-22. Nach der jüngeren Literatur zeichnet sich im Innovationsbereich eine Art Arbeitsteilung zwischen großen und kleinen Unternehmen ab. Vgl. Herdzina 1999, 43f.; 
Der für die gesamtwirtschaftlichen Belange notwendige technische Fortschritt ist in hohem Maße kapitalgebunden. ${ }^{162}$ Für langfristige Investitionen von erheblicher finanzieller Belastung sind die Möglichkeiten kleiner und mittlerer Unternehmen zur Innenfinanzierung oft begrenzt. Ihre Möglichkeiten der Akkumulation durch Einbehalten von Gewinnen, Zurückhalten erwirtschafteter Abschreibungen und Umschichten des Vermögens innerhalb des Unternehmens sind jedoch begrenzt. ${ }^{163}$ Auch ist - so wird häufig beklagt - ihre Eigenkapitalquote $\mathrm{zu}$ gering. ${ }^{164} \mathrm{Zur}$ Finanzierung von Unternehmensinvestitionen ist deshalb die Bereitstellung externen Fremd- oder Eigenkapitals erforderlich. Dass sich Unternehmen oft durch fehlende Eigen- oder Fremdmittel in ihrer Innovationstätigkeit beschränkt sehen, ${ }^{165}$ deutet auf ein finanzierungsbedingtes Hindernis des Unternehmenswachstums hin. Geeignete Finanzinstitutionen sind unerlässlich, jene Investitionen zu ermöglichen, die einen vollbeschäftigungskonformen Anstieg des gesamtwirtschaftlichen Wachstums induzieren. ${ }^{166}$ Es verwundert deshalb nicht, dass in der jüngeren Literatur die „,coevolution of the real and financial sectors of an economy" 167 als Kennzeichen wirtschaftlicher Entwicklung wieder verstärkt betont wird; ${ }^{168}$ empirische Untersuchungen neueren Datums bekräftigen den Gleichlauf von Real- und

Weigand 1996, 45f.; Liu, Yang (2000). Für kmU spricht ein relativ hohes Maß an Kreativität und Flexibilität. Small is beautiful! Getreu diesem Motto betreiben immer mehr Unternehmen down sizing, outsourcing, contracting out, Fokussierung auf Kernkompetenzen und Entflechtungen. Diese Entwicklungen scheinen jenen theoretischen Strang zu bestätigen, der die Neo-Schumpeter-Hypothesen verneint und die X-Ineffizienz in Unternehmen betont. Nach Perlitz, Löbler $(1989,38)$ ist für das Zustandekommen von Innovationen neben technischem Wissen und dem „Entrepreneur“ insbesondere investititionsbereites Kapital nötig. Die Vorstellung, dass kapitalgebundenes Investivkapital einen entscheidenden Beitrag zur wirtschaftlichen Entwicklung leistet, hat eine weit zurückreichende Tradition. Vgl. BöhmBawerk (1891), Hicks (1969). In: Bencivenga, Smith, Starr 2000, 770. „In Skandinavien, Frankreich und den Vereinigten Staaten macht man uns seit Jahren vor, wie man mit einem Steuerrecht, das einbehaltene Gewinne präferiert, hohe Wachstumsraten erzielen kann.“ Eichel 2000, 77.

Die Eigenkapitalausstattung ostdeutscher Unternehmen war 1998 mit 23,4\% fast ebenso hoch wie die westdeutscher Unternehmen. Vgl. KfW-Beiträge zur Mittelstands- und Strukturpolitik, Bd. 19, 18. In den USA und Großbritannien sind Eigenkapitalanteile an den Bilanzsummen von 40 bis $50 \%$ die Regel. Zu Schwierigkeiten des Ländervergleichs von Eigenkapitalquoten (Einstellung stiller Reserven, Bewertung von Bilanzpositionen) vgl. Deutsche Bundesbank, Monatsbericht (1994); DIW-Wochenbericht 48/01. Vgl. Winker 1996, 24.

Für die vorliegende Diskussion ist unerheblich, ob mit einer höheren Aktienliquidität das Niveau wirtschaftlichen Wachstums einmalig ansteigt oder ob es zu einem Anstieg der Wachstumsrate kommt. Nach neueren Ansätzen, die das Wachstum pfadabhängig verstehen, mag einem einmalig höheren Ausbringungsniveau sogar ein dauerhaft höherer Wachstumspfad folgen. Smith 1998, 451; vgl. a. Boyd, Smith $(1996,1998)$. 
Finanzsektor. ${ }^{169}$ In breitangelegten Zeitreihen- und Querschnittsanalysen ergeben sich darin anhand ausgewählter Maße signifikante Korrelationen der Entwicklung etwa des Bankensystems mit gesamtwirtschaftlichem Wachstum.

Für die wirtschaftliche Entwicklung kommt dem Finanzsektor in der jüngeren Literatur neben einer begleitenden auch eine katalysierende Bedeutung zu. Auf der Grundlage neuerer theoretischer Erkenntnisse findet der lange Zeit vernachlässigte Zusammenhang zwischen Finanzinstitutionen und wirtschaftlicher Entwicklung in den letzten Jahren zunehmende Beachtung. ${ }^{170}$ Man widmet sich der Frage, ob eine effiziente Ausgestaltung der Finanzinstitutionen nicht nur parallel zu wirtschaftlichem Wachstum erfolgt, sondern ob die jeweilige Verfasstheit der Finanzinstitutionen reales Wachstum sogar $\mathrm{zu}$ induzieren vermag: Where finance leads, enterprise follows? Diese Frage wird von jüngeren Untersuchungen überwiegend bejaht. ${ }^{171}$ Effiziente Finanzinstitutionen,$\ldots$ are the underlying determinant of the long-run performance of economies.“172 „Kapitalmärkte seien der Schlüssel zu mehr Wohlstand und Beschäftigung. “" ${ }^{\text {173 }}$ Ineffiziente Finanzmärkte hingegen würden reales Wachstum mit entsprechenden Auswirkungen auf die Beschäftigungsentwicklung beeinträchtigen. Eine nicht hinreichende Herausbildung effizienter Finanzinstitutionen schaffe Pfadabhängigkeiten und schränke Wachstum ein. ${ }^{174}$

Diese Auffassung ist bemerkenswert, spielte der Finanzsektor in der Wachstumstheorie doch lange Zeit eine untergeordnete Rolle. „Most of macroeconomic theory presumes that the financial system functions smoothly - and smoothly enough to justify abstracting from financial considerations." ${ }^{175}$ Die lange Zeit

168 Die These von der parallelen Entwicklung von Finanzinstitutionen und der Realökonomie reicht weit in die Vergangenheit zurück. Vgl. Bagehot 1874, 6; Schumpeter 1926, 144+205; Cameron (1967); Goldsmith (1969); McKinnon (1973). Vgl. etwa Levine, Zervos (1998); Neusser, Kugler (1998); Rousseau, Wachtel (1998); De Gregorio, Guidotti (1995); King, Levine (1993); Ghani (1992); Gelb (1989).

170 "Betts and Bhattacharya ... establish that the potential for development traps and for oscillation - damped or otherwise - depends on the severity of the CSV problem in capital markets." Smith 1998, 455. Einen Überblick gibt etwa Gertler (1988).

171 Vgl. Lehr, Wang (2000); Levine, Loayza, Beck (2000); Grennwood, Jovanovic (1999).

$172 \quad$ North 1990, 107

173 F.A.Z. v. 19.03.99, Nr. 66, 25: „Der Neue Markt ist keine Seifenblase“. Vgl. etwa Deutsche Börse AG, Geschäftsbericht 1995, 24f. zu den erwarteten Auswirkungen des Neuen Marktes auf den deutschen Arbeitsmarkt.

174 Vgl. Frenkel, Hemmer 1999, 310-318.

175 Gertler 1988, 559. 
vorherrschende Geringschätzung einer für gesamtwirtschaftliches Wachstum adäquaten Finanzinfrastruktur war der Auffassung geschuldet, die gesellschaftlichen Koordinationsmechanismen würden quasi automatisch für die Existenz und Effizienz jeweils geeigneter Finanzinstitutionen sorgen. ${ }^{176}$ Vertreter eines evolutionären Rationalismus räumen zwar mit Robinson (1979) ein, ,that there are many excellent ideas which cannot be implemented because those who have conceived them are unable to back them with finance. “177 Doch gelte letztlich: „,when a strong impulse to invest is fettered by lack of finance devices are invented to release it ... and habits and institutions are developed accordingly. ${ }^{\text {“178 }}$ Dieser Auffassung zufolge verfügen Finanzmarktmechanismen über endogene Kräfte der Selbstregulierung. Finanzinstitutionen seien demnach langfristig kein Engpassfaktor in der gesamtwirtschaftlichen Entwicklung. ${ }^{179}$ Die Bereitstellung externen Finanzkapitals sei grundsätzlich in ausreichendem Umfang verfügbar. Von daher erfahre die Volkswirtschaft keine wachstumsrelevante Restriktion von Seiten der Unternehmensfinanzierung, um auf ihrer Produktionsmöglichkeitenkurve wirtschaften zu können. Die Rolle der Finanzmärkte in der volkswirtschaftlichen Diskussion sei, so wird folglich vielfach behauptet, ${ }^{180}$ aufgebauscht und - mit Lucas - „badly over-stressed“"181 “[W]here enterprise leads finance follows”182.

"The growing importance of stock markets around the world has recently opened a new avenue of research into the relationship between financial development and economic growth, which focuses on the effects of stock market development." ${ }^{183}$ Eine höhere Eigenkapitalquote deutscher Unternehmen - so wird vielfach betont erleichtert die Möglichkeiten zur Finanzierung von Unternehmenswachstum und Innovationen, vergrößert ihre Unabhängigkeit, da Eigenkapital keiner konkreten Verwendungsvorschrift unterliegt, erhöht ihre Kreditwürdigkeit und Stabilität in Krisensituationen, schützt vor Konkurs und geht bei einer allgemeinen

\footnotetext{
176 White (1999, Kap.1) zeigt den (endogenen) Evolutionsprozess monetärer Institutionen auf.

177 Robinson 1979, 20.

178 Robinson 1979, 20. Vgl. a. Gurley, Shaw 1955, 520; Schlick 1994, 26.

179 Vgl. Frenkel, Hemmer 1999, 307.

180 "In a recent survey of development economics, Stern (1989) does not mention the role of the financial sytsem in economic growth... Similarily, a recent collection of essays by the pioneers of development economics, including three Nobel price-winners, does not describe the role of financial system in economic growth." Levine, Zervos 1996, 326. 
Konditionenspreizung voraussichtlich in Zukunft mit geringeren Kapitalkosten einher. ${ }^{184}$ Eine hohe Eigenkapitalquote deutscher Unternehmen korreliert signifikant positiv mit ihren Gewinnvariablen und erweitert damit die Möglichkeiten zur Innenfinanzierung. ${ }^{185} \mathrm{Im}$ Rahmen der allgemein positiven Börsenentwicklungen fiel in Deutschland in den letzten Jahren insbesondere dem Neuen Markt eine große volkswirtschaftliche Bedeutung - auch als Exitmöglichkeit für die expandierende

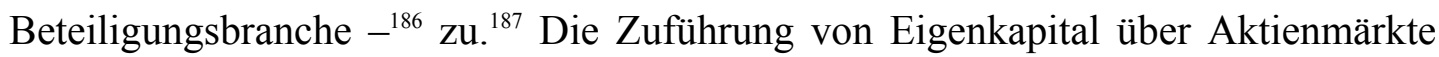
für kleine und mittlere Unternehmen der Old Economy stand in der wissenschaftlichen Diskussion bislang im Hintergrund. Umso fraglicher ist, ob das Phänomen niveaubedingter Unterliquidität des „Rückgrats“ der deutschen Wirtschaft am Sekundärmarkt ein Hindernis für Wachstum und Beschäftigung darstellt. ${ }^{188}$ Diese Frage zerfällt mit der gesamtwirtschaftlichen Bedeutung des Aktiensekundärmarktes im Allgemeinen und der gesamtwirtschaftlichen Bedeutung niveaubedingter Unterliquidität im Speziellen in zwei Teilbereiche.

Die gesamtwirtschaftliche Bedeutung von Sekundärmärkten erschließt sich einem nicht sofort, alloziieren sie knappes Finanzkapital doch nicht direkt in seine volkswirtschaftlich beste Verwendung; lenken sie Ersparnisse doch nicht unmittelbar in die Bildung von Sach- und Humankapital. ${ }^{189}$ Schließlich wechseln die Anteilsscheine an einem Unternehmen durch eine Transaktion am

183 Arestis, Demetriades, Luintel 2001, 16. Die weltweite Aktienmarktkapitalisierung wuchs von \$2 Billionen in 1982 auf \$15,2 Billionen in 1996, was einer durchschnittlichen Wachstumsrate von $15 \%$ entspricht.

184 Zu jüngeren Entwicklungen an den Finanzmärkten sowie zu „Basel II“ vgl. Kapitel 6.

185 Vgl. Deutsche Bundesbank 1999b, 46. Eine Bestandsaufnahme der Verschuldung deutscher Unternehmen im europäischen Vergleich liefern Ramb 2000, 2; Hansen 1996a, 36-39. Zu einer kritischen Beurteilung der Eigenkapitallücke aus (neo-)institutionenökonomischer Sicht vgl. Schmidt,Terberger 1997, 452-460. „Und vielleicht sind die sinkenden Eigenkapitalquoten ein Ausdruck davon, daß sich die Möglichkeiten, solche [Eigner-Manager-] Probleme einzudämmen, im Laufe der Zeit zugunsten der Fremdfinanzierung verschoben haben." Feess 1997, 459. $\mathrm{Zu}$ einer strukturierten Diskussion des Irrelevanztheorems aus institutionenökonomischr Sicht vgl. Harris, Raviv (1991). "Improving liquidity is also important from the viewpoint of ensuring exit possibilities for initial risk investors in the company.” Jürgens, Naumann, Rupp 2000, 70.

187 „Wenn auch der Neue Markt ... Alternativen für die Unternehmensfinanzierung aufzeigen, so darf nicht übersehen werden, dass sich für ein erfolgreiches IPO bisher erst wenige hundert Unternehmen qualifiziert haben. "Scholz 2000, 520. Vgl. Horn 1994, 3.

189 Im Rahmen dieser Arbeit stehen allokative Wirkungen des Aktienmarktes, die sich aus der Konzentration des Sekundärhandels ergeben, im Vordergrund. Selbstverständlich hat die Anlegerpräferenz auch Auswirkungen auf das gesellschaftliche Freiheitsziel im Sinne einer verzerrten Chancengleichheit und auf Verteilungsfragen. Robinson, Eatwell 1974, 317: „Die ökonomische Hauptrolle einer Börse besteht mehr in der Erhaltung und Ausdehnung der Rentiervermögen, als in der Bereitstellung finanzieller Mittel für Industrie und Regierung.“ 
Aktiensekundärmarkt „nur“ die Eigentümer. Des ungeachtet sind Sekundärmärkte keine „side-show“ im gesamtwirtschaftlichen Kontext. Sie tragen zu einer besseren Allokationseffizienz bei, wenn auch nur indirekt. ${ }^{190}$ Am Aktienmarkt wird die parzellierte Ersparnis der Anleger mit kurzer Anlagefrist in hohe Summen langfristig haftendes Investivkapitals überführt wird. ${ }^{191}$ Außerdem werden unternehmerische Investitionsrisiken auf zahlreiche Kapitalgeber verteilt. ${ }^{192}$ Ohne die Transferierbarkeit fungibler Anteilsscheine am Sekundärmarkt sind diese Transformationsleistungen des Aktienmarktes, die bei geringen Transaktionskosten ${ }^{193}$ zu sinkenden Eigenkapitalkosten für die Unternehmen führen, ${ }^{194}$ eingeschränkt. Dank fortgeschrittener Technisierung und Liberalisierung, ${ }^{195}$ dank standardisierter Kontraktformen, festgelegter Konventionen und einer weitgehenden Vertragssicherheit - auf globalen Kapitalmärkten - ${ }^{196}$ können Anleger ihre Anteilseignerschaft zu geringen Such-, Verhandlungs- und Prüfkosten über den Sekundärhandel aufkündigen. Durch die Diversifizierung ihrer Portefeuilles reduzieren sie unsystematische Unternehmensrisiken. Dadurch abnehmende Renditeforderungen an die Unternehmen bewirken abermals sinkende Eigenkapitalkosten und ermöglichen desweiteren Investitionen mit vergleichsweise geringen internen Verzinsungen. Sekundärmärkte tragen damit nicht nur dazu bei, dass die unternehmerischen Invesitionsströme breiter, sondern dass sie auch länger werden. Abnehmende Kapitalkosten werten nämlich solche langfristigen Investitionen auf, die erst $\mathrm{zu}$ späteren Zeitpunkten $\mathrm{zu}$ Einzahlungen führen. Sekundärmärkte senken damit den Grad der gesamtwirtschaftlichen Myopie. Kurzum: Aktiensekundärmärkte wirken sich positiv auf das Niveau von gesamtwirtschaftlicher Investition und Ersparnis aus. ${ }^{197}$ Darüber hinaus schaffen sie für die Eigenkapitalgeber einen Anreiz, bewertungsrelevante Informationen über einzelne Unternehmen in einem hoch spezialisierten wirtschaftlichen Produktionsprozess einzuholen (Informationseffizienz) und tragen auf diese Weise

190 Eine ausführliche Funktionenbestimmung des Aktienmarktes liefert Lüthje 1970, 57-61.

191 Damit löst der Sekundärmarkt das Problem der so genannten konstitutionellen Schwäche am langen Ende des Marktes. Vgl. Hicks 1939, 146.

192 Vgl. Rasch 1996, 60f.

193 Die Kosten der Bereitstellung bzw. Beschaffung von Eigenkapital treiben einen Keil zwischen die Finanzierungskosten eines Kapitalnehmers und der Rendite, die den Kapitalgeber nach Abzug aller Gebühren, Steuern und sonstigen Kosten zufließt. Je höher diese Marge ist, desto geringer sind gesamtwirtschaftliche Ersparnis, Investitionen und damit auch das Wirtschaftswachstum.

194 Zur Berechnung von Kapitalkosten vgl. etwa Schulze (1994).

195 Zur Finanzmarktliberalisierung vgl. Mooslechner (1995).

196 Zur Integration der globalen Kapitalmärkte vgl. Obstfeld 1998, 9-13. 
zur Informationsgenerierung und Wissensdiffusion (knowledge spillovers) ${ }^{198}$ und zur Bewertungseffizienz bei - wenngleich sich im unrealistischen Grenzfall vollkommener Informations- und Bewertungseffizienz das Problem des so genannten Informationsparadoxons stellt: ${ }^{199}$ Der künftige Kursverlauf einer Aktie ist hier nur mehr Gegenstand von Zufalls- oder Chaostheorien - der informations- und bewertungseffiziente Sekundärmarkt als ,,pure gambling market ${ }^{\star 200} .{ }^{201}$

Conditio sine qua non für die Transaktionskosten-, Bewertungs- und Informationseffizienz eines Sekundärmarktes ist die Liquidität des an ihm gehandelten Gutes. Nur bei ausreichender Liquidität kann das Geschehen am Finanzmarkt jenes im Realen zu geringen Transaktionskosten „richtig“ bewertet abbilden und Risiken beseitigen. Liquidität ist darüber hinaus Voraussetzung für den Einsatz derivativer Instrumente zur Absicherung imponderabler Kursverläufe, ermöglicht damit erst die Vollständigkeit des Sekundärmarktes. ${ }^{202}$ Jeder Anleger kann sich über den bereits heute bekannten Tausch bedingter Ansprüche auch gegen solche Risiken absichern, die durch Diversifizierung seines Portefeuilles nicht beseitigt werden können. ${ }^{203}$ Durch niveaubedingte Unterliquidität werden die genannten Arten der Effizienz eines Sekundärmarktes beeinträchtigt. Die mit niveaubedingter Unterliquidität einhergehende Unterbewertung erschwert das

197 Vgl. Greenwood, Smith (1997).

198 Vgl. Bhattacharya, Ritter (1983); Bhattacharya (1993); Greenwood, Jovanovic (1990); Williamson (1986). Durch einen höheren Informationsstand verbessert sich das Corporate Governance. Vgl. dazu Holmström, Tirole (1993).

Mit zunehmender Informations- und Bewertungseffizienz eines Marktes sinkt der Anreiz für Anleger zur Informationsproduktion. Jede individuell erhaltene Information ist dann bereits in den Kursen enthalten. Die Beschaffung von Informationen wird folglich für das Individuum irrational. Wie also können sich Informationen in Aktienkurse einpreisen, wenn sie erst gar nicht beschafft werden?! Vgl. Grossman, Stiglitz (1980); Sapusek 1998, 113-140. Stiglitz 1982, 123.

201 Liegt ein informationseffizienter Markt vom Grade 1 vor, sind Kursänderungen im Zeitverlauf voneinander gänzlich unabhängig, d.h. für einzelne Anleger nur durch einen Zufallsprozess beschreibbar. „Die beste Prognose für den Preis der nächsten Periode ist der heute den Marktteilnehmern bekannte Preis, was dem Martingale-Modell bzw. dem Random-WalkModell entspricht.“ Vgl. Sapusek 1998, 1. Wird nicht von einer konstanten, sondern von einer positiven erwarteten Gleichgewichtsrendite ausgegangen, wird ein so genanntes Submartingale-Modell zu Grunde gelegt.

Das Vorliegen von Pareto-Effizienz setzt voraus, dass für alle unsicheren, an bestimmte künftige Umweltzustände gebundenen Konsumansprüche der Wirtschaftssubjekte Märkte existieren, an denen sich jeder Akteur über den bereits heute verabredeten Tausch dieser bedingten Ansprüche (Arrow-Debreu-Verträge) gegen ökonomische Risiken absichern kann. Die Marktstruktur nennt man in diesem Fall vollständig. Diese Absicherungswirkung lässt sich an Aktienmärkten durch Optionsgeschäfte erreichen. Sofern alle möglichen Umweltzustände durch entsprechende Kombinationen ausreichend vieler, in ihren Merkmalsausprägungen unabhängiger Wertpapiere abgedeckt werden können, spricht man dementsprechend von einem vollständigen Aktienmarkt. 
Indizieren fundamentaler Veränderungen in den jeweiligen Kursen, sie nimmt damit den Anreiz für die Anleger, bewertungsrelevante Informationen zu beschaffen, und sie verhindert die Etablierung versicherungstechnischer Instrumente im marktwirtschaftlichen Prozess. Für die betroffenen Unternehmen erschwert niveaubedingte Unterliquidität die Beschaffung von Eigenkapital. Falls Folgeemissionen überhaupt sowie fristgerecht möglich sind, senkt sie ihren Ausgabekurs (Underpricing) und erhöht auf diese Weise die Kosten der Eigenkapitalbeschaffung. Niveaubedingte Unterliquidität verhindert damit, dass Finanzkapital stets in seine volkswirtschaftlich besten Verwendungen gelenkt wird, in jene Unternehmen also, die - unter Berücksichtung des bewertungsrelevanten Risikos - den höchsten Ertrag abzuwerfen erwarten lassen. ${ }^{204}$ Gesamtwirtschaftliche Effizienzverluste entstehen also dadurch, dass am segmentierten deutschen Sekundärmarkt Eigenkapital zum einen nur eingeschränkt, das heißt nicht in Höhe des ausgeschütteten fundamental richtig bewerteten Aktienvolumens, und zum anderen nicht entsprechend ihrer relativen Knappheit eingesetzt wird. ${ }^{205}$ Diese Effizienzverluste übertragen sich vom Sekundärmarkt auf den Primärmarkt. Insbesondere in Phasen eines hohen Eigenkapitalbedarfs der Unternehmen wirkt sich dies hemmend auf das gesamtwirtschaftliche Wachstum - wie im Vorfeld eines Langen Aufschwungs. ${ }^{206}$ Niveaubedingte Unterliquidität wirkt sich außerdem einschränkend auf die Beschaffung von Fremdkapital aus. So können unterbewertete, weil unterliquide Aktien nur eingeschränkt als Sicherheiten einer Kreditvergabe dienen. Niveaubedingte Unterliquidität erhöht folglich nicht nur die Eigenkapitalkosten, sondern verteuert auch die Aufnahme von Fremdkapital. Mit unterliquiden Anteilsscheinen steigen für die betroffenen Unternehmen aber nicht nur die Kreditkonditionen. Darüber hinaus nimmt auch die Wahrscheinlichkeit zu, am Kreditmarkt rationiert zu werden.

Niveaubedingte Unterliquidität mindert damit das Volumen des bereit zu stellenden Fremd- und Eigenkapitals und verzerrt seine Allokation. Sie verzerrt außerdem auch die Allokation des bereits emittierten Kapitals - nämlich dann, wenn sich die

\footnotetext{
$203 \quad$ Vgl. Deutsche Bundesbank 1998, 64.

204 Vgl. Weber 1999, 304.

205 Allgemein gesprochen muss das Verhältnis der Grenzprodukte zweier exemplarischer Gesellschaften im gesamtwirtschaftlichen Optimum ihrem Faktorpreisverhältnis, das heißt der geforderten wie erwarteten Rendite entsprechen. Vgl. Tschach 2000, 46. Vgl. Gerke 1995, 17. 
Investitionsentscheidungen der Unternehmen an den erwarteten Kursveränderungen orientieren, die mangels Liquidität aber nicht die fundamentalen Veränderungen widerspiegeln. Durch die Aussicht, sich künftig Kapital (möglichst günstig) beschaffen zu können, sind Manager motiviert, ihre Investitionssentscheidungen am Chart ihres Unternehmens zu orientieren. Das durch niveaubedingte Unterliqudität verzerrte Geschehen an den Sekundärmärkten übernimmt damit eine Lenkungsfunktion für die Auswahl vorzunehmender Realinvestitionen. Auch als Indikator für die Leistungsfähigkeit des Managements wird die fortwährende Berücksichtigung der Aktienkursentwicklung verstanden. Für Manager niveaubedingt unterliquider Unternehmen schränkt sich jedoch die Möglichkeit ein, ihre Leistungen am Aktienkurs messen sowie ihre Entlohnung an den Aktienkurs koppeln zu können, was regelmäßig mit Einkommenseinbußen verbunden ist. Damit einhergehende Wettbewerbsverzerrungen am Arbeitsmarkt der Agenten führen zu einem „brain drain“ zu Lasten der unterliquiden Gesellschaften - und zwar nicht nur im Bereich des Top-Managements. Ferner schlagen sich unterbewertete, weil unterliquide Aktien negativ auf die Produktnachfrage nieder, wenn die Aktien nämlich in einer vermögensabhängigen Konsumfunktion Teile des Gesamtvermögensbestandes sind. ${ }^{207}$ Die Nachfrage nach Produkten unterliquider Gesellschaften wird möglicherweise durch einen wettbewerbsverzerrenden Imageschaden zusätzlich beeinträchtigt. Denn unterbewertete Aktienkurse schaden der Reputation eines Unternehmens insgesamt.

Nach diesen Überlegungen ist die Frage danach, ob niveaubedingt unterliquide Aktien des „Rückgrats“ der deutschen Wirtschaft ein Hindernis für das gesamtwirtschaftliche Wachstum und Beschäftigung darstellt, der Richtung nach zu bejahen. Die durch niveaubedingte Unterliquidität entstehenden Effizienzverluste ließen sich kompensieren, würde man die Finanzierung über Aktienmärkte adäquat ersetzen können. Nach heutigem Wissensstand lässt sich diese Frage nicht erschöpfend zu beantworten. Es sind jedoch an der Substituierbarkeit Zweifel angebracht: Eine alternative Eigenkapitalfinanzierung über den Beteiligungsmarkt stellt mangels Masse keine wirkliche Alternative für den traditionellen Mittelstand

207 In welchem Ausmaß sich der Grad der Aktienliquidität auf die gesamtwirtschaftliche Nachfrage auswirkt, hängt nicht zuletzt vom Gewicht der Aktien in der volkswirtschaftlichen Konsumfunktion und der marginalen Konsumquote der Anteilseigner ab. 
dar. ${ }^{208}$ Einerseits kaprizieren sich nicht-börsliche Risikokapitalgeber in Deutschland zu einem wesentlichen Anteil auf die ,early-stage“-Finanzierung der New Economy, andererseits geht eine (direkte) Beteiligungsfinanzierung mit dem Einräumen von Mitspracherechten für die Beteiligungsgeber einher, die von vielen Mittelständlern nicht toleriert wird. Außerdem sind Private Equity-Geber in einer fortgeschrittenen Reifephase der finanzierten Unternehmen oft auf Aktienmärkte als Exitmöglichkeit ihrer Investitionen angewiesen. (Nicht-börsliche) Beteiligungsfinanzierungen und die Existenz von Aktienmärkten zur Beschaffung von Eigenkapital sind deshalb eher als Komplementäre, denn als Substitute zu begreifen. Auch an der Substituierbarkeit einer Aktienfinanzierung durch die Bereitstellung langfristigen Fremd- oder Mezzaninekapitals sind insbesondere für kleine und mittlere Gesellschaften der Old Economy mit eingeschränkter Innenfinanzierungskraft Zweifel angebracht. Denn zum einen sind die verschiedenen Finanzierungsformen für die jeweiligen Kapitalgeber und -nehmer - entgegen dem Irrelevanztheorem Modiglianis und Millers (1958) - ${ }^{209}$ mit unterschiedlichen Anreizstrukturen verbunden. ${ }^{210}$ Zum anderen ist zu beobachten, dass auch andere Märkte wie etwa der Kreditmarkt nicht frei von Kapitalrationierung $\mathrm{zu}$ Lasten des Mittelstandes sind. ${ }^{211}$ Die stark angestiegene Nachfrage nach Aktienfinanzierungen in den letzten Jahren deutet ebenfalls auf eine nur eingeschränkte Substituierbarkeit dieser Art von Finanzierung

208 Trotz starker Neuinvestitionen in den letzten Jahren (über 6 Mrd. DM in 1999) nimmt sich die Bereitstellung von Eigenkapital an nicht börsennotierte deutsche Unternehmen (Private Equity) mit nicht einmal 0,2\% vom BSP selbst im europäischen Vergleich bescheiden aus. Vgl. Fischer 2000, 36; Die Aktiengesellschaft 6/1999, R215-R218. Dabei werden europaweit zwei Drittel des BSP von nicht börsennotierten Unternehmen erwirtschaftet. Vgl. F.A.Z. v. 14.02.01, Nr. 38, 35. Insbesondere das Modell der Unternehmensbeteiligungsgesellschaften (im Sinne des Gesetzes über Unternehmensbeteiligungsgesellschaften v. 17.12. 1986 - UBBG) hat sich nicht durchsetzen können. Nach einem Höchststand im Jahr 1992 mit 16 Gesellschaften hat deren Zahl kontinuierlich abgenommen, so dass Anfang 1997 nur noch 9 Unternehmensbeteiligungsgesellschaften existierten. Ein völliger Fehlschlag war die Einführung so genannter Beteiligungs-Sondervermögen nach dem Gesetz über Kapitalanlagegesellschaften ( $\$ \S 25 \mathrm{a}$ ff KAGG). Bisher wurde nach den veröffentlichten Informationen hierzu kein einziges Beteiligungs-Sondervermögen mit Beteiligungen an nichtbörsennotierten Unternehmen aufgelegt. Vgl. Baums 1997, 1943. Zu aktuellen Tendenzen des deutschen Beteiligungskapitalmarktes vgl. KfW 2001b, 17-23. Eine Einschätzung zum Venture-Capital Marktpotenzial geben Leopold, Frommann 1998, 106-113. Zu Arten des außerbörslichen Eigenkapitalmarktes vgl. Leopold (1993).

Der rigorosen Version des Modigliani/Miller-Theorems zufolge ist für zwei Unternehmen derselben Risikoklasse die Kapitalstruktur und auch die Art der Bereitstellung von externem Kapital belanglos. Das Irrelevanztheorem basiert jedoch auf unrealistischen Annahmen wie die Risikoneutralität der Marktteilnehmer, homogene Erwartungen, vollkommene Informationen, ein neutrales Steuersystem und gleiche Marktkonditionen für alle Beteiligten. Vgl. a. Miller (1988); Schmidt, Terberger 1997, 456-460; Myers (1984). Vgl. Feess 1997, 533f.

211 Vgl. Brost 2000, 23f. Zu den Entwicklungen an den Finanzmärkten und dem künftig zu erwartenden Bankenverhalten vgl. Kapitel 6. 
hin. Es spricht mehr für ein komplementäres Verhältnis von Unternehmensfinanzierungen einzelner Kapitalmärkte zur Finanzierung des deutschen Mittelstandes. Effizienzverluste am Aktiensekundärmarkt verengen die Spielräume, sich anderweitig finanzieren zu können. Gelänge hingegen eine weitgehend friktionslose Finanzierung der kmU über den Aktienmarkt, würden sie günstige Konditionen auch am Kreditmarkt erwirken und der Kreditrationierung entkommen können. ${ }^{212}$ Den Unternehmen stünden dadurch außerdem ein größeres Beteiligungsangebot und auch ein höheres Potenzial an Selbstfinanzierung zur Verfügung. Zwar ist es richtig, "that even large stock markets are unimportant sources for corporate finance" ${ }^{213}$. Aufgrund seiner komplementären Beziehung zu anderen Finanzierungsquellen ist die ,wirkliche“ Bedeutung von Aktienmärkten für die Unternehmensfinanzierung jedoch um ein Vielfaches höher einzuschätzen, als allein der numerische Anteil der Aktien- an der Gesamtfinanzierung erwarten lässt.

Mag die „wirkliche“ Bedeutung der Aktienmärkte auch erheblich sein, ist das Auftreten niveaubedingter Unterliquidität nicht zwangsläufig beklagenswert. Ist man nämlich der Überzeugung, dass ein kapitalmarktbasiertes Finanzsystem für die Finanzierung gesamtwirtschaftlich notwendiger Produktinnovationen grundsätzlich nicht geeignet sei, geriert das Phänomen niveaubedingter Unterliquidität zu einem Garanten dafür, dass sich Aktienmärkte in ungewünschtem Ausmaß nicht auszubreiten in der Lage sind. ${ }^{214}$ Der komparative Vorteil eines mehr bankorientierten Finanzierungssystems wird mancherseits in der Finanzierbarkeit besonders riskanter Investitionen gesehen. In Übereinstimmung mit der „,delegated monitor"-These ${ }^{215}$ verursacht die Kontrolle von Unternehmen hohe Fixkosten. Die Unternehmenskontrolle sei deshalb besser aus der Hand weniger (delegierter) Finanzintermediäre wie Banken zu gewährleisten anstatt durch eine Vielzahl von Aktionären, für die sie ein öffentliches Gut darstelle. ${ }^{216}$ Gegen diesen komparativen Vorteil eines bankbasierten Finanzierungssystems lässt sich der Einwand erheben, die Informationsproduktion durch Kreditinstitute falle im Verhältnis zu einer

\footnotetext{
212 Unterstellt man, dass alle Unternehmen mit einem Jahresumsatz von mehr als 10 Mio. DM potenziell börsenfähig und -willig sind (2\% aller Unternehmen in Deutschland), so ergibt sich ein Potenzial für Aktienfinanzierungen von etwa 65.000 Unternehmen.

213 Levine, Zervos 1996, 324; Mayer (1988).

214 Zu Vor- und Nachteilen von bank- versus kapitalmarktbasierten Finanzsystemen vgl. Rajan, Zingales (1995, 1998); Edwards, Fischer (1994); Corbett, Jenkinson (1994). 
marktbasierten Finanzierung insgeamt geringer aus. ${ }^{217}$ Die Konkurrenz in der Informationsproduktion durch eine Vielzahl von Aktionären führe zu einer höheren Transparenz über die Unternehmen. Im Übrigen sei die Anteilseignerstruktur insbesondere in Deutschland nicht derart parzelliert, dass für alle Eigner die Unternehmenskontrolle ein öffentliches Gut darstelle. Die im Rahmen der Theorie der Finanzintermediation ${ }^{218}$ geführte Debatte über die relative Effizienz der unterschiedlichen Finanzierungssysteme ist theoretisch weiterhin ergebnisoffen. ${ }^{219}$ Vermutlich wird auch die künftige Entwicklung zu keiner eindeutigen Klärung darüber führen, ob ein markt- einem bankbasierten Finanzierungssystem - vor dem Hintergrund der deutschen ,design-connectedness ${ }^{\text {‘220 }}$ - letztlich überlegen ist. Auch ein Blick in die Vergangenheit gibt keinen Aufschluss darüber. ${ }^{221}$ Wider eine breite und wissenschaftlich fundierte Kritik ist das kapitalmarktorientierte Finanzsystem seit einigen Jahren für Viele faktisch „das Leitbild, das mit der Begeisterung des gerade Konvertierten vertreten wird.“222 Stellt niveaubedingte Unterliquidität für die Befürworter dieser Entwicklung ein Wachstumshindernis dar, ist sie für die Gegner einer zunehmenden Marktorientierung langfristig ein Garant für wirtschaftliche Entwicklung.

„In order that people should be willing ... to sink large amounts of capital, ... it is the availability of liquid funds which is crucial. This condition was satisfied in England ... by the the first half of the eighteenth century ... The liquid asset was there, as it

216 Ein Plädoyer für die letztendliche Überlegenheit eines bankbasierten deutschen Finanzsystems gibt Kotz (1998, 1999, 2000).

217 Eine Vielzahl von Anteilseignern bietet eine größere Meinungsvielfalt und sorgt für die ständige Kontrolle des unternehmerischen Gebarens, was von besonderer Bedeutung ist bei unsicheren Produktionsfunktionen im technologischen Wandel, wie es Investitionen in die Erstellung von Produktinnovationen darstellen. Dagegen würde bankbasierte Systeme Vorteile bei der Finanzierung traditioneller Industrie- oder Handwerkszweige besitzen. Vgl. Allen 1993, 92-95; Allen, Gale 1994, 41f. Grundlegende Arbeiten sind Gurley, Shaw (1960); Brainard, Tobin (1961). Eine zusammenfassende Literaturübersicht geben Hellwig (1990) und Allen 1993, 86-90.

219 Vgl. Kotz 1999, 322.

220 Der Begriff stellt darauf ab, dass es kein absolut, sondern nur relativ zum jeweiligen Wirtschafts- und Gesellschaftssystem bestes Finanzsystem gibt. Vgl. Milgrom, Roberts 1992, 33; vgl. a. Kotz 2000, 638.

Vgl. etwa Rasch 1996, 44f.; Michie 1987, 50-57 + 110; Gurley, Shaw 1967, 259; Fritsch 1981, 7; Gömmel 1992, 153. Chandler 1990, 415ff.

Kotz 1999, 322. "This paper has painted two possible scenarios for the development of financial markets and corporate systems in Europe. The first is a steady process of convergence towards market-based systems with greater accountability, disclosure and transparency. The second is a persistence and intensification of systemic differences with companies shopping around for appropriate financial and legal arrangements. The most likely outcome is a mixture of the two." Mayer 1999, 54f. 
would not have been even a few years earlier.“223 Diese Auffassung einer wachstumsförderlichen Wirkung liquider Anlagen gilt neueren Untersuchungen zufolge als empirisch erhärtet. Levine (1991) sowie Bencivenga, Smith und Starr (1995) greifen die These Hicks' auf, nach der die Liquidierbarkeit fungibler Anteilsscheine an Unternehmen eine notwendige Bedingung für das Eingehen hochrentierlicher langfristiger idiosynkratischer Großinvestitionen ist. Die Bestätigung der Hicks'schen These in diesen Untersuchungen läuft konform mit weiteren empirischen Analysen von Atje, Jovanovic (1992). Sie erkennen für 40 Länder eine signifikante Korrelation wirtschaftlichen Wachstums in der Periode 1980 bis 1988 mit der Liquidität der untersuchten Aktienmärkte - gemessen am Value Traded (Börsenumsatz im Verhältnis zum BSP). Levine und Zervos (1998) erhöhen abermals die Anzahl der Länder um fast 20\% und verdoppeln nahezu den Untersuchungszeitraum. Sie fügen überdies mit dem Turnover (Börsenumsatz zur Marktgröße) einen weiteren Indikator für die Liquidität der Kapitalmärkte hinzu. ${ }^{224}$ Nach ihren Ergebnissen "the liquidity measures are positively significantly correlated with Output Growth, Capital Stock Growth, and Productivity Growth..."225 Danach folgt die Entwicklung des Finanzsektors nicht einfach dem wirtschaftlichen Wachstum. Vielmehr induziert Marktliquidität anders herum ein höheres wirtschaftliches Wachstum. Um die Sensibilität der Marktliquidität auf das Wirtschaftswachstum klarer herauszuarbeiten, berücksichtigen Levine, Zervos (1998) wirtschaftliche und politische Faktoren. Ein hohes Maß an Aktienmarktliquidität sei demnach selbst dann ein robuster Indikator für die Wachstumsraten des realen Pro/Kopf-Einkommens, der Kapitalakkumulation und der Produktivität, wenn abstrahiert wird von der anfänglichen Höhe des Volkseinkommens, der Investitionen in das Ausbildungswesen, der Stabilität der politischen Verhältnisse sowie der Art der Fiskalpolitik. ${ }^{226}$ Neben Querschnitts- legen

223 Hicks 1969, 143-145.

224 So kann ein Aktiensegment gemessen am Value Traded zwar unterliquide, gemessen am Turnover jedoch äußerst liquide sein. Der Turnover hat gegenüber dem Value Traded den Vorteil, dass der inflationäre Effekt eines nicht fundamentaltheoretisch $\mathrm{zu}$ begründenden Kursanstiegs automatisch verschwindet, da er sowohl im Zähler wie auch im Nenner vorkommt. Bereingt um diesen Preiseffekt kann die positive Auswirkung der Sekundärmarktliquidität auf das Wachstum auch für den Value Traded eindeutig bestätigt werden.

225 Levine, Zervos 1998, 543. Zu qualitativ gleichen Ergebnissen gelangen Levine (1991) Holmström, Tirole (1993) sowie Bencivenga, Smith und Starr (1995, 1996). Auch sie konstatieren eine signifikant positive Korrelation zwischen der Liquidität am Aktienmarkt und dem Wachstum, der Kapitalakkumulation und den Produktivitätsfortschritten.

226 Vgl. Atje, Jovanovic (1992, 1993); Demirgüç-Kunt, Levine (1993); Levine, Zervos (1994, 1998); Gurley, Shaw (1955, 1960, 1967). 
auch Zeitreihenanalysen positive Auswirkungen der Aktienmarktliquidität auf das reale (Pro Kopf-)Einkommen eines Landes nahe. ${ }^{227}$ Nach Obstfeld (1994) tragen die größeren Möglichkeiten einer Risikodiversifizierung auf liquiden international integrierten Aktienmärkten zu einer verbesserten Ressourcenallokation und einer Beschleunigung der Wachstumsrate bei. Rajan, Zingales (1998) zeigen, dass Branchen, die sich extern finanzieren, eher in Ländern mit entwickelten Finanzmärkten prosperieren. Demirgüç-Kunt, Maksimovic (1996) stellen fest, dass Unternehmen, mikroökonomisch untersucht, in Ländern mit gut funktionierenden Kredit- und Eigenkapitalmärkten schneller wachsen als allein auf der Grundlage von Firmeneigenschaften vorhersagbar wäre. Die Marktliquidität eines Aktienwertes als Indikator für die Finanzierbarkeit eines Unternehmens über die Kapitalmärkte sei außedem auch ,positively associated with corporate debt-equity ratios in developing countries. “228

Die Wachstumswirkung liquider Aktienmärkte ist den genannten empirischen Untersuchungen zufolge eindeutig positiv. Diese Ergebnisse sind umso bemerkenswerter, als ein Liquiditätsanstieg in der Literatur als durchaus auch mit negativen Wachstumseffekten behaftet diskutiert wird. "Of all maxims of orthodox finance, none, surely, is more anti-social than the fetish of liquidity, the doctrine that it is a positive virtue on the part of investment institutions to concentrate their resources upon the holding of "liquid" securities."229 Liquiditätsbedingt negative Wachstumseffekte ergeben sich grundsätzlich aus Folgendem:230

- Mit steigender Liquidität sinken die Transaktionskosten. Eine damit ansteigende Rendite erhöht den Anreiz, zu Lasten des Primärmarktes mehr am Sekundärmarkt zu investieren. Dies kann einen beschränkenden Effekt auf die gesamtwirtschaftliche Investition hervorrufen. ${ }^{231}$

- Hochliquide Aktienmärkte lösen sich mit zunehmender Technisierung und Liberalisierung des Aktienmarktes derart vom realwirtschaftlichen Arestis, Demetriades, Luintel (2001) und die dort angegebenen Literaturhinweise. Demirgüç-Kunt, Maksimovic (1996).

Keynes 1936, 155.

Vgl. a. Levine (1997); Demirgü - Kunt, Levine (1996a,b).

Vgl. Shleifer; Summers (1988) Moreover, Devereux, Smith (1994) emphasize that greater risk sharing through internationally integrated stock markets can actually reduce saving rates and slow economic growth. In jüngeren empirischen Untersuchungen wird dieser Aspekt bezweifelt. Vgl. Bencivenga, Smith, Starr 1995, 156; dies. (2000). 
Geschehen, dass die Geschwindigkeit ihrer Preis- und Mengenbewegungen im Verhältnis zur Geschwindigkeit im realwirtschaftlichen Bereich zunimmt. Als Ergebnis einer solchen Entwicklung reagieren die Anleger weniger auf realwirtschaftliche Veränderungen als vielmehr auf Veränderungen des Aktienmarktes selbst. Ein um sich selbst „kreisender“ Aktienmarkt erzeugt eine Panoplie an derivativen Instrumenten von allerdings geringer realwirtschaftlicher Grenzproduktivität. ${ }^{232}$ In der damit einhergehenden Abwanderung von Finanzkapital in volkswirtschaftlich weniger produktive Finanzmarktbereiche dokumentiert sich ein relativer „Bedeutungsverlust der Real- gegenüber der Finanzinvestition. “233

- Mit dem Abzug von Finanzkapital aus realwirtschaftlich produktiven Bereichen in den Sekundärmarkt samt seiner derivativen Märkte geht ein „brain drain“ einher. Aufgrund hoher Verdienstmöglichkeiten ziehen Sekundärmärkte mit einem hohen Maß an Liquidität knappes qualifiziertes Humankapital aus dem realwirtschaftlichen Bereich ab. „This measure of success attained by Wall Street, regarded as an institution of which the proper social purpose is to direct new investment into the most profitable channels in terms of future yield, cannot be claimed as one of the outstanding triumphs of laissez-faire capitalism - which is not surprising, if I am right in thinking that the best brains of Wall Street have been in fact directed toward a different object.". ${ }^{234}$

- Mit zunehmender Liquidität und Umschlaghäufigkeit einer Aktie werden Aktionäre nicht immer bereit sein, die fixen Kosten der Informationsproduktion $\mathrm{zu}$ übernehmen. ${ }^{235}$ Auch ihrer Kontrollaufgabe des Managements werden sie immer weniger gerecht. ${ }^{236}$ Die Anteilseigner vernachlässigen ihre Funktion als Korrektiv für mitunter myopisch handelnde

232 Fraglich ist, ob es sich bei jedem neuen Finanzinstrument um eine Innovation im Schumpeter'schen Sinne handelt. Oft werden für heterogenisierte Märkte neue Produkte geschaffen. Dies ist durchaus mit dem Erzielen von Renten für die Entwickler verbunden, aber nicht notwendigerweise mit einer Vervollständigung der Finanzmärkte. Denn dadurch werden nicht mehrere unsichere Zustände in der Zukunft handel- und versicherbar. Es erweist sich jedoch im Einzelnen als schwierig zu zeigen, dass etwa die hunderttausendste EUR investiert in eine spezielle Option mit der Daimler Chrysler AG als Underlying für die Volkswirtschaft mehr Nutzen stiftet als in die Liquidität eines niveaubedingt unterliquiden Wertes. Heise 1996, 141.

Keynes 1936, 159.

"Stiglitz $(1985,1994)$ says that stock market liquidity will not enhance incentives for acquiring information about firms or exerting corporate governance.” Levine, Zervos 1996, 324.

Zum trade-off zwischen einem hohen Grad an Liquidität auf der einen und einem hohen Grad an Kontrolle durch die Aktionäre vgl. Shleifer, Vishny (1986); Bolton, Thadden (1998). 
und ineffektiv arbeitende Manager. Corporate Governance avanciert für die Eigner zu einem öffentlichen Gut. Eine geringe Informationsproduktion und diffusion sowie eine fehlende Unternehmenskontrolle durch die Anteilseigner verhindert eine effiziente Ressourcenallokation und beeinträchtigt gesamtwirtschaftliches Wachstum. ${ }^{237}$ „Intertemporal risk sharing will no longer be possible once widely-used and liquid markets are established." ${ }^{238}$

- Anstelle der direkten Kontrolle der Manager durch die Aktionäre bricht sich zwar eine takeover-Mentalität Bahn. ${ }^{239}$ Jedoch erweist sich die damit einhergehende Kontrolle des Managements ebenfalls oft als kontraproduktiv. ${ }^{240}$ Ein „outgesourcte“ Markt für Unternehmenskontrolle kann die Interessen der Aktionäre an den langfristig rentabelsten Investitionsobjekten nicht vertreten. ${ }^{241}$ So genannte „Raider“ würden vielmehr den Unternehmen ihre kurzfristigen Interessen diktieren.

- Die Loslösung der Kursentwicklung an hochliquiden Aktienmärkten von den fundamentalen Faktoren in der Realwirtschaft ${ }^{242}$ (excess volatility) erleichtert die Bildung destabilisierender spekulativer Blasen. Beim Zerplatzen können ganze Sozial-, Wirtschafts- und Gesellschaftssysteme in Mitleidenschaft gezogen werden. ${ }^{243}$ „As the organisation of investment markets improves, the risk of the predominance of speculation does, however, increase ... Speculators may do no harm as bubbles on a steady stream of enterprise. But the position is serious when enterprise becomes the bubble on a whirlpool of speculation. When the capital development of a country becomes a by-product of the activities of a casino, the job is likely to be ill-done. “244

- Das Bilden und Zerplatzen von spekulativen Blasen wird eine ausgeprägte Kurzsichtigkeit der Anleger gefördert, die insbesondere an hochliquiden Aktienmärkten vorherrschend ist (Klienteleffekt). Der Aktienmarkt geriert sich als Quelle gesamtwirtschaftlicher Unsicherheit. Er wird zum „Zockermarkt“, eine Art Spielkasino, dessen Treiben in einem nahezu interventionsfreien Raum ein nur bedingt realwirtschaftliches Korrektiv

Vgl. Shleifer, Vishny (1986); Shleifer, Summers (1988); Bhide (1993).

Vgl. Allen, Gale 1994, 44.

Vgl. Delingat (1996).

Vgl. Morck, Shleifer, Vishny (1989); Shleifer and Summers (1988)

Vgl. Shleifer, Vishny (1986); Bhide (1993).

Vgl. Shiller (1981, 1989).

Vgl. Scherz (1994).

Keynes 1936, 158f. 
erfährt, dafür aber über bedeutende Transmissionskanäle auf realwirtschaftliche Größen einwirkt.

Diese allokativen wie stabilitätspolitischen Gegenargumente eines hohen Liquiditätsgrades von Aktien mögen zwar für ,überliquide“ Marktsegmente zutreffen. Denn die genannten Aspekte setzen Handelsvolumina voraus, die mit fundamentalen Veränderungen zu erklären, nur mehr schwerlich möglich sind. Doch diese Argumente ins Feld $\mathrm{zu}$ führen gegen wirtschaftspolitische Versuche, den Liquiditätsgrad in den Segmenten für Small und Mid Caps anzuheben, entbehrt jeder Grundlage. Dass von ihnen die genannten wachstumsschädlichen Effekte ausgehen, ist nicht zu erwarten. Die oben erwähnten empirischen Untersuchungen, die in großer Überstimmung insgesamt positive Wachstumswirkungen von liquiden Aktienmärkten nachgewiesen haben, umfassen zwangsläufig auch diese die wirtschaftliche Entwicklung konterkarrierenden Effekte eines „zu“ hohen Grades an Marktliquidität. Die Analysen differenzieren nämlich nicht nach den wachstumsförderlichen Auswirkungen bestimmter Teilsegmente eines Gesamtmarktes. Da die negativen Wachstumswirkungen nicht von Segmenten kleiner und mittlerer Gesellschaften ausgehen, ist zu erwarten, dass sich eine höhere Liquidität ihrer Aktienwerte sogar noch stärker positiv auf die gesamtwirtschaftliche Entwicklung auswirkt, als nach den Untersuchungen ohnehin zu vermuten ist. "Keynes was right that liquidity is a problem, but he was wrong in failing to recognize that liquidity is also the solution." ${ }^{245}$ Es besteht offenbar ein wirtschaftspolitisches Handlungsgebot dahingehend, den Grad niveaubedingter Liquidität in Segmenten kleiner und mittlerer Gesellschaften in marktkonformer Weise zu erhöhen. Um für ein entsprechendes staatliches Handeln eine theoretische Grundlage zu schaffen, sind die Entstehungsursachen für niveaubedingte Unterliquidität im Rahmen des Sekundärmarktmechanismus zu klären. Dazu ist im Folgenden zunächst der Funktionsmechanismus des Sekundärmarktes zu beleuchten. 
3 Niveaubedingte Unterliquidität

am

Capital Asset Pricing Model 


\subsection{Der Funktionsmechanismus des Sekundärmarktes ${ }^{246}$}

Niveaubedingte Unterliquidität stellt sich als ein dauerhaft überschießendes Aktienangebot zum fundamentalen Aktienkurs dar. An einem informations-, bewertungs- und transaktionseffizienten Sekundärmarkt bemisst sich der fundamentale Kurs einer Aktie an der Identität des Kapitalwertes der Unternehmensinvestitionen und dem Marktwert aller Unternehmensanteile. Beträgt etwa der Barwert künftiger sicherer Einzahlungsüberschüsse aus den Investitionen eines Unternehmens, das heißt sein discounted cash flow (dcf), 100 Mio. EUR, beläuft sich die Marktkapitalisierung im Gleichgewicht eines effizienten Sekundärmarktes aus Arbitragegründen auf denselben Betrag. ${ }^{247}$ Bei einer ausgegebenen Anzahl von 1 Mio. Aktien liegt der fundamentale Aktienkurs folglich bei 100 EUR pro Aktie. Anders als im Beispiel handelt es sich beim Erwirtschaften des def jedoch meist um einen unsicheren Zahlungsstrom. Deshalb ist die Übernahme des bewertungsrelevanten Aktienrisikos neben dem Konsumverzicht mit einem Kursabschlag verbunden. Der fundamentale Aktienkurs liegt im Beispiel bei unsicherem Zahlungsstrom deshalb unter 100 EUR pro Aktie. Fraglich ist, wie sich der Kursabschlag, aus der die geforderte und erwartete Rendite erwächst, ${ }^{248}$ für die Übernahme des Aktienrisikos berechnet. ${ }^{249}$

Im Mittelpunkt der Kapitalmarkttheorie steht die Frage nach der Höhe des bewertungsrelevanten Risikos, das bei einem discounted cash flow eines Unternehmens unter Unsicherheit den fundamentalen Aktienkurs sowie daraus abgeleitet die geforderte und die erwartete Aktienrendite im Gleichgewicht bestimmt. ${ }^{250}$ „Harter Kern“(251 der vorwiegend neoklassisch geprägten

246 Der Funktionsmechanismus des Sekundärmarktes wird im Rahmen des CAPM dargestellt. Im Kern reproduziert dieser Abschnitt Lehrbuchstoff. Für eine ausführliche Darstellung vgl. etwa Schmidt, Terberger 1997, Kap. 9; Haugen 1987, chap. 6; Kosfeld 1996, Kap. 2.

247 Liegt die Marktkapitalisierung unterhalb des discounted cash flow, liegt ein Anreiz vor, das am Sekundärmarkt unterbewertete Unternehmen aufzukaufen, et vice versa.

248 Macht das durch den Markt bewertete Risiko samt Konsumverzicht $10 \%$ des discoutd cash flows pro Aktie aus, liegt der fundamentale Aktienkurs bei 90 EUR und die geforderte wie erwartete Rendite bei ca. 10,1\%.

Die erwartete Rendite setzt sich aus den ausgeschütteten Cash Flows in Form von Dividenden oder aus ihrer Thesaurierung zusammen. Das Einbehalten von Cash Flows ruft an effizienten Sekundärmärkten eine entsprechende Kurssteigerung hervor. Kurssteigerungen in Form einbehaltener Cash Flows können deshalb auch als künftige Dividenden verstanden werden, die auf den gegenwärtigen Zeitpunkt abdiskontiert werden. 
Kapitalmarkttheorie stellt das in Erwartungsgrößen formulierte positive Capital Asset Pricing Model (CAPM) dar. ${ }^{252}$ Das CAPM bewertet risikobehaftete Anlagen unter Unsicherheit im statischen Gleichgewicht des Kapitalmarktes. ${ }^{253}$ Aufbauend auf der normativen Portefeuille-Theorie erfolgt die Bestimmung des Rendite/RisikoProfils einer Einzelaktie in Bezug auf das von allen rationalen Anlegern gehaltene effiziente Portefeuille, dessen Teil die $\mathrm{zu}$ bepreisende Einzelaktie ist. $^{254}$ Bei Vorhandensein einer risikolosen Anlagemöglichkeit ${ }^{25}$ erfolgt die Zusammenstellung unsicherer Einzelanlagen zum effizienten Portefeuille gemäß der Tobin-Separation ${ }^{256}$ unabhängig von der Risikoneigung der Anleger. Ungeachtet ihrer Risikoeinstellung halten alle Anleger im Gleichgewicht ebendieses effiziente Portefeuille. ${ }^{257}$ Die zentralen Entscheidungsparameter für Investments sind die zu erwartende Rendite und das damit verbundene Risiko. Letzteres wird durch die statistischen Verteilungsparameter Varianz und Standardabweichung gemessen. Nach der so genannten Bernoullirationalität werden auf der Grundlage des $(\mu-\sigma)-$ Kriteriums einzelne Aktien in das effiziente Portefeuille so lange aufgenommen, so lange sie mit dem höchsten Erwartungswert des Nutzens verbunden sind. ${ }^{258}$ Das Portefeuille

im Kursverlauf einer Aktie sichtbar. Aus der Projektion vergangener Chartformationen in die Zukunft werden Aussagen für die künftige Kursentwicklung getroffen. Vgl. Welcker 1982, 1923. Lakatos 1974, 130. Aufgrund methodologischer Grundentscheidungen seiner Protagonisten ist der „harte Kern“ eines Forschungsprogramms unwiderlegbar. Anomalien dürfen nur im „Schutzgürtel“" von Hilfs- und Beobachtungshypothesen und Anfangsbedingungen zu Veränderungen führen. Vgl. Chalmers 1994, Kap. 7+9. Einen Überblick zu Anomalien am Aktienmarkt gibt Sapusek 1998, 141-186.

Das CAPM geht auf Sharpe (1964), Lintner (1965a,b), Treynor (1965) und Mossin (1966) zurück. Für seine Entwicklung erhielt Sharpe 1990 den Nobelpreis für Wirtschaftswissenschaften.

Der Kapitalmarkt ist die Gesamtheit der Möglichkeiten, frühere gegen spätere Zahlungen zu tauschen, wobei eine Zahlung heute den Preis für die zugehörige Gegenleistung in Form zukünftiger Zahlungen angibt. Gehandelt werden am Kapitalmarkt Rechte an physischen Gütern, nicht aber Geld. Geld ist im Klassisch-Neoklassischen Sinne kein Kapital. So leisten etwa Aktionäre im Akt des Erwerbs einen Verzicht auf den Verbrauch physischer Produktion. Gegen den Erhalt einer verbrieften Forderung in Form einer Aktie übertragen Aktionäre den Verzicht auf den Verbrauch physischer Produktion im Austausch an Unternehmen. Diese verwenden das erhaltene Kapital fortwährend für Unternehmensinvestitionen. Zur Portefeuille-Theorie vgl. Jarchow 1998, 40-69. Die Portefeuille-Theorie geht zurück auf Markowitz (1952) und Tobin (1957/58). Zur Ableitung des CAPM aus der State Preference Theory vgl. Franke, Hax 1990, 306-311; Unser 1999, $125 f$. Dies könnte z.B. eine zehnjährige Bundesanleihe sein.

Vgl. Tobin 1957/58, 82ff.

Das Entscheidungsproblem besteht in der Aufteilung eines gegebenen Investitionsbetrages auf mehrere riskante Alternativen mit der Zielsetzung der Maximierung des Erwartungswertes des Nutzens der riskanten zukünftigen Zahlungsströme. Ein Portefeuille ist dann effizient zusammengestellt, wenn es - unter Berücksichtigung einer risikolosen Anlage - den größten Erwartungswert pro Varianzrisiko abwirft.

Um eine Entscheidung anhand des Mittelwertes $(\mu)$ und der Varianz $\left(\sigma^{2}\right)$ als vernünftig im Sinne des Bernoulli-Kriteriums zu bezeichnen, muss die Nutzenfunktion des Entscheiders quadratisch sein, oder es muss eine Normalverteilung der Renditen vorliegen. 
umfasst dann logisch zwingend alle am Markt umlaufenden Aktien im Verhältnis ihrer jeweiligen fundamental richtig bewerteten Marktkapitalisierung. Das ungeachtet seiner Risikoneigung von einem rationalen Anleger gehaltene Portefeuille, das geplantermaßen alle am Sekundärmarkt umlaufenden Aktien gemäß ihrer richtig bewerteten Marktkapitalisierung beinhaltet, wird in der Literatur als Marktportefeuille bezeichnet. Je nach Risikoeinstellung des Anlegers nimmt das Marktportefeuille in seinem Vermögensbestand einen mehr oder weniger großen Anteil ein. ${ }^{259}$

Die Anleger sind im CAPM annahmegemäß mehr oder weniger risikoscheu, sie verfügen über einen Planungshorizont von einer Periode. Ihre Erwartungen sind homogen $^{260}$ und rational $^{261}$. An einem annahmegemäß vollkommenen ${ }^{262}$ und effizienten Finanzmarkt entspricht die erwartete Rendite eines Einzelanlegers deshalb dem Erwartungswert, welcher sich aus der für die Renditeerklärung relevanten Informationsmenge bestimmen lässt. „,[E]xpectations, ..., are the same as predictions of the relevant economic theory." ${ }^{263}$ Die Erwartungen der Anleger sind deshalb weder pessimistisch noch optimistisch. Frei von Präferenzen lehnen die Anleger ihre Entscheidungen über Kauf, Verkauf oder Halten unsicherer Anlagen

Dies trifft auch für einen negativen Vermögensbestand, das heißt bei einer Kreditaufnahme zu. Homogene Erwartungen ergeben sich aus symmetrisch verteilten und vollständigen Informationen über die Renditeverteilung. Vgl. Hörnstein 1990, 115. Die Homogenitätsannahme ist grundlegende Voraussetzung für die Aggregation individueller Verhaltensweisen im Gleichgewicht. Sie ist aber nicht sehr realistisch. Vgl. Bagwell (1991); Shleifer (1986); Frankfurter, Philipps (1996). Lintner (1969) hat als erster gezeigt, dass wesentliche Ergebnisse des CAPM bei Einführung heterogener Erwartungen bestehen bleiben. An die Stelle der homogenen Renditeerwartungen und (Ko-)Varianzen treten die gewogenen Mittelwerte der Erwartungsparameter aller Anleger. Die Idee der Aktienbewertung anhand der erwarteten zukünftigen Dividenden ist eine wichtige Konkretisierung des allgemeinen Konzeptes „rationaler Erwartungen“. Vgl. Muth (1961). Muth folgend, nennt man Erwartungen rational, erfüllen sie sich im Durchschnitt.

An einem vollkommenen Finanzmarkt gibt es keine Transaktionskosten, Steuern, Ein- und Austrittsbarrieren. Die Anlegerstruktur ist polypolistisch. Einzelne Anleger haben keinen Einfluss auf das Unternehmen oder den Kurs. Sie verfügen über vollkommene Informationen und sind frei von Präferenzen. Die Anteilsscheine eines Unternehmens sind beliebig teilbar. Das von Brennan (1970) entwickelte CAPM berücksichtigt die unterschiedliche Behandlung von Kursgewinnen und Dividenden. Die erwartete Rendite einer Anlage hängt dann nicht mehr nur vom bewertungsrelevanten Risiko, sondern auch von der Dividendenrendite der Anlage ab. Da Anleger mit unterschiedlichen Steuersätzen verschiedene strukturierte Portefeuilles realisieren, muss das Marktportefeuille wie bei der Einführung heterogener Erwartungen nicht mehr effizient sein. Zur Einführung weiterer Marktunvollkommenheiten vgl. Perridon, Steiner 1999, 271. 
objektiven Wahrscheinlichkeiten an. ${ }^{264}$ Spekulationsgewinne sind deshalb allenfalls temporär im Rahmen eines „fair game“" erzielbar. ${ }^{265}$

Da sich die Handlungen der Anleger an den objektiven Wahrscheinlichkeiten anlehnen, kommen genau die Kurse zustande, die die Anleger in Einsicht der objektiven Informationslage subjektiv erwarten. Somit stimmen die erwartete und die tatsächliche Rendite überein. Rationale Anleger haben deswegen keinen Anlass, ihre Erwartungsbildung und damit einhergehende Entscheidungsregeln zu verändern. Im Gleichgewicht entspricht die erwartete nicht nur der tatsächlichen, sondern auch der geforderten Rendite. Denn eine über der erwarteten markträumenden Gleichgewichtsrendite des CAPM liegende Rendite $\mathrm{zu}$ fordern, ist an einem annahmegemäß polypolistischen Aktienmarkt einzelnen Anlegern als Preisnehmer verwehrt. Eine darunter liegende Rendite zu fordern, ist unter den neoklassischen Annahmen irrational. Deshalb fordert der rationale Anleger im beschriebenen Modell genau die Rendite, die er gemäß Einsichtnahme in die objektive Informationslage auch erwartet. ${ }^{266}$ Wie im eingangs geschilderten Beispiel dargestellt, muss die von den Anlegern geforderte Rendite darüber hinaus real erwirtschaftet werden. Je nach Zusammensetzung der Kapitalstruktur bedarf es einer Internen Verzinsung der Unternehmensinvestitionen in entsprechender Höhe. In einer vollständig eigenfinanzierten Aktiengesellschaft entspricht die Interne Verzinsung des eingesetzten Eigenkapitals der von den Anlegern geforderten Rendite. ${ }^{267}$ Zur Ermittlung der Kapitalwerte möglicher Investitionen wird in diesem Fall die geforderte Aktienrendite als Zinsfuß $\mathrm{r}_{\mathrm{i}}^{\text {int }} \mathrm{zu}$ Grunde gelegt. ${ }^{268} \mathrm{Im}$ Gleichgewicht

264 Machlup (1983) kritisiert den Gebrauch des Terminus ,rationale Erwartung“ dahingehend, dass „Rationalität“ in der Ökonomik stets die Übereinstimmung von Handlung und Meinung bedeuten würde, nicht aber eine Orientierung der Handlung an der „Objektiven Wirklichkeit“. Die „Fair-Game“-Bedingung impliziert, dass sich im Durchschnitt Gewinne und Verluste ausgleichen. Spekulationen sind langfristig auf informationseffizienten Märkten ausgeschlossen.

Als Preisnehmer hat der einzelne Eigenkapital gebende Anleger weder Einfluss auf die Interne Verzinsung als für die Eigentümer angestrebte Aktienrendite. Noch kann er mit seinen verschwindend geringen Umsätzen den Aktienkurs wesentlich und dauerhaft verändern. Ihm bleibt nur die Möglichkeit des, ,voting by feet“.

267 Bei abnehmender Grenzproduktivität werden vollständig aktienfinanzierte Unternehmensinvestitionen solange ausgeweitet, bis die Eigenkapitalkosten in Höhe der Renditeforderung dem Gegenwartswert der unternehmerischen Investitionsreihe entsprechen. Mit zunehmendem bewertungsrelevanten Risiko einer Anlage erhöhen sich die Eigenkapitalkosten des Unternehmens. Seinen Niederschlag findet dies in der Diskontierung der Zahlungsreihen von Unternehmensinvestitionen. Letztere erhalten einen entsprechenden Risikoabschlag beziehungsweise der Diskontfaktor einen Risikoaufschlag. Rolfes (1992) ist der Auffassung, dass die im Rahmen einer Anwendung der Kapitalwertmethode typischerweise gesetzte Prämisse eines einheitlichen Kalkulationszinsfußes für alle Betrachtungsperioden 
stimmen die geforderte Rendite $\mu_{\mathrm{i}}{ }^{\text {gef }}$, die erwartete Rendite $\mu_{\mathrm{i}}^{\text {erw }}$ und die tatsächlich erzielte Rendite $r_{i}^{\text {tat }}$ mit der Internen Verzinsung $r_{i}^{\text {int }}$ der einzelnen Aktien überein. Für jede einzelne Aktie i gilt im Gleichgewicht: ${ }^{269}$

$r_{i}^{\text {int }}=\mu_{i}^{\text {gef }}=\mu_{i}^{\text {erw }}=r_{i}^{\text {tat }}$

Im Übereinklang dieser vier Größen aller $\mathrm{n}$ unsicheren Anlagen des Marktportefeuilles dokumentiert sich das (statische) CAPM-Gleichgewicht. Die Forderungen der Anleger erfüllen sich und es gibt keinen Anlass, diese Pläne zu ändern. Die geforderte und erwartete Rendite des Marktportefeuilles, dessen Teil die Einzelaktie i ist, beläuft sich auf das - nach ihren jeweiligen Portefeuilleanteilen gewogenen Mitteln aller Einzelrenditen n. Das CAPM-Gleichgewicht ist insofern markträumend, als für den rationalen Anleger kein Transaktionsbedarf am Sekundärmarkt besteht. Die von den Unternehmen emittierten Aktien finden im Gleichgewicht geplantermaßen eine Aktiennachfrage in entsprechender Höhe. Die Aktien sind im CAPM-Gleichgewicht liquide. Die von der Renditeforderung seitens der Anleger abhängige Eigenkapitalnachfrage der Unternehmen und das von der Renditeerwartung abhängige Eigenkapitalangebot seitens der Anleger stimmen im CAPM-Gleichgewicht überein. Da die erwartete und die geforderte Rendite identisch sind und in ihrer Flexibilität keinen Restriktionen oder Rigiditäten unterliegen, führen sie das CAPM-Gleichgewicht in eine I-S-Identität als Ausdruck gesamtwirtschaftlichen Kapitalmarktgleichgewichts. Die von den Renditeforderungen abhängigen Unternehmensinvestitionen entsprechen der Ersparnis, die wiederum eine Funktion der Renditeerwartung durch die Anleger ist. Der Mechanismus frei fluktuierender Kurse respektive Renditen gewährleistet letztlich die Übereinstimmung von Investition und Ersparnis.

Bestimmungsgröße der geforderten und erwarteten Rendite einer Anlage i ist ihr bewertungsrelevantes Risiko. Für die Bestimmung des bewertungsrelevanten Risikos einer Aktie i ist zu berücksichtigen, dass die Aktie i Teil des vom rationalen Anleger

durch den differenzierteren Ansatz von je nach Laufzeit unterschiedlichen Kalkulationszinsfüßen $\mathrm{zu}$ ersetzen ist. Weil diese nach einzelnen Perioden differierenden Zinsfüße aus der „tatsächlich“ herrschenden Zinsstruktur herzuleiten sind, bezeichnet er sein Konzept als „marktzinsorientierte“ Investitionsrechnung. gemeint, wird dies mit der Verwendung des Buchstabens ,r“ deutlich gemacht. 
gehaltenen Marktportefeuilles ist. Im Marktportefeuille werden durch Mischung einzelne Risiken diversifiziert, das heißt durch Streuung beseitigt. ${ }^{270}$ Dadurch reduziert sich das Marktportefeuillerisiko regelmäßig auf einen Wert, der unter dem gewogenen Durchschnitt des Gesamtrisikos seiner Einzelaktien $\left(\operatorname{var}\left(\mathrm{r}_{\mathrm{n}}\right)=\sigma_{\mathrm{n}}^{2}\right)$ liegt. Das bewertungsrelevante Risiko einer einzelnen Aktie i bemisst sich folglich nur nach ihrem Beitrag zum Risiko des Marktportefeuilles. Dieses entspricht seiner Sensitivität mit den Schwankungen der Portefeuillerendite. Bewegt sich etwa die erwartete Rendite einer Einzelaktie stets in die gleiche Richtung wie die des Marktportefeuilles mit allerdings größeren Schwankungen, liegt das bewertungsrelevante Risiko der einzelnen Aktie über dem (Markt-) Portefeuillerisiko. Ist die Schwankung einer Einzelrendite geringer als die der gleichgerichteten Marktrendite, trägt die Einzelaktie unterduchschnittlich zum Portefeuillerisiko bei. Formal drückt sich die Schwankungsintensität der Rendite einer Einzelaktie i mit der des Marktportefeuilles in der Kovarianz der Einzelrendite mit der Portefeuillerendite $\operatorname{cov}\left(\mathrm{r}_{\mathrm{i}}, \mathrm{r}_{\mathrm{M}}\right)$ aus. ${ }^{271}$ Bewegt sich der Kurs einer Aktie, mithin ihre Rendite exakt mit dem des Marktportefeuilles, beträgt das Risiko einer Aktie somit dem des Marktportefeuilles. Denn für das Risiko einer Aktie i gilt bei $r_{i}=r_{M}$ : $\operatorname{cov}\left(\mathrm{r}_{\mathrm{i}}, \mathrm{r}_{\mathrm{M}}\right)=\operatorname{cov}\left(\mathrm{r}_{\mathrm{M}}, \mathrm{r}_{\mathrm{M}}\right)=\operatorname{var}\left(\mathrm{r}_{\mathrm{M}}\right)=\sigma_{\mathrm{M}}^{2}$. Schwankt die Rendite der Einzelaktie weniger (mehr) als die des Marktportefeuilles, wird für die einzelne Aktie ein geringeres (höheres) Risiko vom Markt entgolten als für die Übernahme des Marktportefeuilles. Das Gesamtrisiko einer Einzelaktie $\left(\operatorname{var}\left(\mu_{\mathrm{i}}\right)=\sigma_{\mathrm{i}}^{2}\right)$ beziehungsweise das Risiko des ihr zugrundeliegenden discounted cash flows der Unternehmensinvestitionen mag um ein Vielfaches darüber liegen. Das diversifizierbare, so genannte unsystematische Risiko einer Aktie verschwindet beim Halten des effizienten Marktportefeuilles. Der Markt entlohnt im Gleichgewicht des CAPM nur das durch Portefeuillebildung nicht diversifizierbare, das so genannte systematische Risiko einer Aktie. Rationale Anleger, die das optimal diversifizierte Marktportefeuille halten, tragen demnach

270 Korrelieren alle Aktien vollkommen positiv miteinander, ist keine Risikodiversifizierung zwischen den Einzelaktien möglich. Bei vollständig positiver Korrelation $(+1)$ kann durch Portfeuille-Bildung lediglich eine einfache Durchschnittsbildung des Risikos erreicht werden (Risikozerfällung). Bei einem Korrelationskoeffizienten kleiner als +1 und größer als -1 ist durch Portefeuille-Bildung eine Reduzierung des Risikos unter die Durchschnittsbildung möglich (Risikodiversifikation). Bei einem Korrelationskoeffizienten von -1 ist durch Portefeuille-Bildung sogar eine völlige Beseitigung des Risikos möglich (Risikokompensation). $\operatorname{cov}\left(\mathrm{r}_{\mathrm{i}}, \mathrm{r}_{\mathrm{M}}\right)=$ Standardabweichung der Einzelaktie $\mathrm{i} \times$ Standardabweichung des Marktportefeuilles $\mathrm{M} \times$ Korrelationskoeffizient zwischen i und $\mathrm{M}$. 
lediglich systematische Risiken. ${ }^{272}$ Unsystematisches, aktienindividuelles Risiko zu übernehmen, ist für Anleger irrational, hieße es, auf die Möglichkeiten der Diversifizierung durch das Halten des effizienten Marktportefeuilles zu verzichten. ${ }^{273}$ Systematische Risiken betreffen alle Einzelanlagen, wenn auch in unterschiedlichem Ausmaß wie etwa konjunkturelle oder inflationäre Einflüsse oder jene in Verbindung mit Wechselkursveränderungen. ${ }^{274}$ Das systematische Risiko beinhaltet somit nur markt- hingegen keine diversifizierbaren unternehmensspezifischen Faktoren. Etwa die an seiner Marktkapitalisierung gemessene Größe eines Unternehmens stellt eine diversifizierbare Risikokomponente dar, die im Gleichgewicht des Kapitalmarktes nicht entgolten wird. Denn das Marktportefeuille umfasst Unternehmen unterschiedlicher Größe, so dass sich durch das Halten des effizienten Marktportefeuilles etwaige aus einer Unternehmensgröße erwachsende Risiken diversifizieren lassen.

Es ist zunächst festzuhalten: Anders als die Portefeuillerendite setzt sich das Portefeuillerisiko nur aus einem Teil der Einzelrisiken zusammen, nämlich den gewogenen Kovarianzwerten der Einzelrenditen mit der Rendite des Marktportefeuilles. Darin drückt sich der nicht-diversifizierbare systematische Teil des Einzelrisikos als durch den Markt einzig bewertungsrelevant aus. Für das Bereitstellen von Eigenkapital mit bewertungsrelevanten Risiken fordern die Anleger im Gegenzug eine Prämie $\mu_{\mathrm{i}}$. Diese Forderung beinhaltet neben der Übernahme systematischen (Kovarianz-)Risikos einen Ausgleich für den damit einhergehenden Konsumverzicht in Höhe des risikolosen Zinssatzes $r_{f}$. Aufgrund von Arbitragefreiheit muss das Entgelt für das Bereitstellen einer Einheit risikobehafteten

272 Zwar lassen sich einzelwirtschaftlich auch systematische Risiken beseitigen durch einen entsprechenden entgeltlichen Einsatz derivativer Instrumente. Gesamtwirtschaftlich gelingt dies jedoch nicht. So ,taucht das - auch im Aggregat nicht zum Verschwinden zu bringende systematische Risiko am Ende vor allem als Kreditrisiko in den Banken auf.“ Kotz 2000, 642. Vgl. a. Kimball (2000).

273 In der Literatur wird behauptet, dass unsystematische Risiken bei etwa 15-20 Aktien pro Portefeuille nahezu vollkommen diversifizierbar sind. Vgl. Brealy, Myers 1984, 124ff.; Sharpe, Gordon 1990, 218; Van Horne 1989, 70 f.

274 Die Beziehungen zwischen den Einzelrenditen wird durch eine Vielzahl - im CAPM nicht spezifizierter - ökonomischer Faktoren bestimmt. Mit dem Multi-Beta-CAPM existiert ein Mehrfaktorenmodell, das auf den gleichgewichtstheoretischen Annahmen des CAPM beruht. Durch eine Aufspaltung bewertungsrelevanter Risiken in mehrere Teilportefeuilles und die Interpretation der Teilportefeuilles als Risikofaktoren kann das Multi-Beta-CAPM abgeleitet werden. Dabei korrelieren die einzelnen Teilportefeuilles perfekt mit dem zu simulierenden Faktor und weisen mit den übrigen Faktoren eine Korrelation von 0 auf. Das systematische Risiko wird dabei so auf $\mathrm{n}$ Teilportefeuilles aufgespalten, dass die Aggregation der Teilportefeuilles zum Marktportefeuille führt. Vgl. Sharpe (1977). 
Eigenkapitals im CAPM-Gleichgewicht für jede Aktie identisch sein. ${ }^{275}$ Die geforderte Rendite pro Risikoeinheit beträgt für zwei beliebige Aktien i und j im Gleichgewicht demnach:

$$
\frac{\mu_{i}-r_{f}}{\operatorname{cov}\left(r_{i}, r_{M}\right)}=\frac{\mu_{j}-r_{f}}{\operatorname{cov}\left(r_{j}, r_{M}\right)}
$$

Forderten Anleger für die Übernahme einer bewertungsrelevanten Risikoeinheit durch die Aktie i eine höhere Prämie als für die Übernahme einer Risikoeinheit durch die Aktie j, wäre dies gleichbedeutend mit einer Unterbewertung der Aktie i gegenüber der Aktie j. Erstere würde zusätzliche Nachfrage auf sich ziehen. Letztere würde verstärkt angeboten werden, so dass sich letztlich die Gleichgewichtsbedingung (3.2) erneut einstellt. ${ }^{276}$ Was für einzelne Aktien gilt, gilt der Proportionalität wegen auch für das Marktportefeuille:

$$
\frac{\mu_{i}-r_{f}}{\operatorname{cov}\left(r_{i}, r_{M}\right)}=\frac{\mu_{M}-r_{f}}{\operatorname{var}\left(r_{M}\right)} \Leftrightarrow \mu_{i}=r_{f}+\frac{\left(\mu_{M}-r_{f}\right)}{\operatorname{var}\left(r_{M}\right)} \operatorname{cov}\left(r_{i}, r_{M}\right)
$$

Durch einfache Umformungen ergibt sich die geforderte Rendite einer Einzelaktie in Abhängigkeit vom risikolosen Zinssatz $r_{f}$ und der mit dem systematischen (Kovarianz-)Risiko einer einzelnen Aktie gewichteten Prämie für die Übernahme einer Portefeuillerisikoeinheit. Bewegt sich die Rendite der Aktie i im Gleichschritt mit der des Marktportefeuilles $\left(\operatorname{cov}\left(\mathrm{r}_{\mathrm{i}}, \mathrm{r}_{\mathrm{M}}\right)=\operatorname{var}\left(\mathrm{r}_{\mathrm{M}}\right)\right)$, entspricht die Rendite der einzelnen Aktie der des Marktportfeuilles: $\mu_{\mathrm{i}}=\mu_{\mathrm{M}}$. Ein Kovarianzwert, der das Portefeuille(varianz-)risiko übersteigt, wird mit einer Rendite oberhalb der Portefeuillerendite entlohnt, et vice versa. Als Volatilitätsmaß für die Rendite einer einzelnen Anlage relativ zum Gesamtmarkt operationalisiert und quantifiziert $\beta_{\mathrm{i}}$ das systematische Risiko einer Aktie. ${ }^{277}$ Es gilt für die n umlaufenden Aktien:

$$
\Leftrightarrow \mu_{n}=r_{f}+\left(\mu_{M}-r_{f}\right) \beta_{n} \quad \text { mit } \beta_{n}=\frac{\operatorname{cov}\left(r_{n}, r_{M}\right)}{\operatorname{var}\left(r_{M}\right)}
$$
Buchstabe $\beta$ meist dann verwendet, wenn der theoretisch abgeleitete Wert gemeint ist. Wird dagegen die Schreibweise „Beta“ gebraucht, ist der empirisch ermittelte Wert gemeint. 
Die Division der aktienindividuellen Risikomaße $\operatorname{cov}\left(r_{n}, r_{M}\right)$ mit der Varianz der Renditeverteilung des Marktportefeuilles standardisiert das aktienindividuelle Risikomaß $\beta_{\mathrm{n}}$ zu einer dimensionslosen Größe. $\beta_{\mathrm{i}}$ drückt aus, um wieviel Prozent sich der Wert einer Einzelanlage mit dem Marktportefeuille bewegt. ${ }^{278}$ Beträgt $\beta_{\mathrm{i}}$ Eins beziehungsweise 100\%, entspricht die betrachtete Anlage dem Risiko-RenditeProfil des Marktportefeuilles. Sind bei $\beta_{\mathrm{i}}<1$ die Schwankungen einer Einzelanlage weniger erratisch als die des Marktportefeuilles, ist die betrachtete Anlage weniger riskant, also auch mit einer geringeren Renditeforderung und -erwartung verbunden. Bei einem bewertungsrelevanten Risiko $\beta_{\mathrm{i}}>1$ übersteigt die Riskoprämie einer einzelnen Aktie die des Marktportefeuilles. Ein $\beta_{\mathrm{n}}$-Wert von etwa $-0,9$ bedeutet, dass sich die Einzelaktie entgegen dem Trend des Marktportefeuilles bewegt, allerdings mit einer etwas geringeren Schwankungsbreite als das Marktportefeuille.

\section{Abbildung 3.1 $\quad$ Wertpapiermarktlinie im Up-Market}

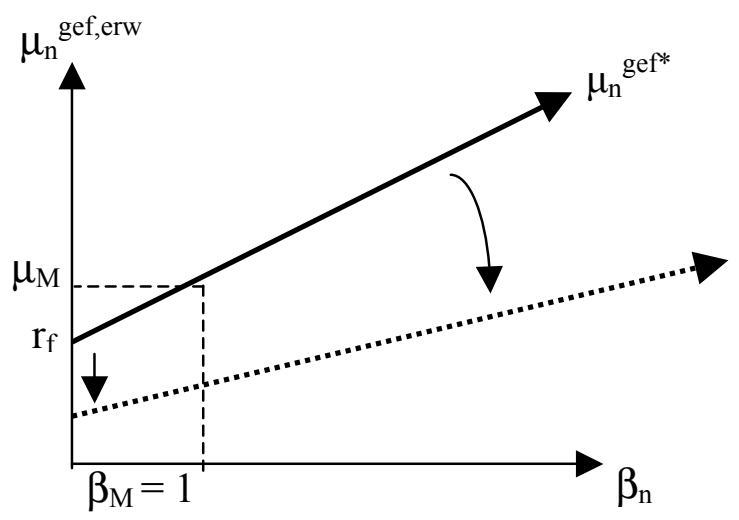

Da im Gleichgewicht die Übernahme einer marginalen Risikoeinheit $\beta_{\mathrm{n}}$ aufgrund der Arbitragefreiheit für alle Aktien und Aktienportefeuilles gleich sein muss, ist die von ihrem bewertungsrelevanten Risiko einer Einzelaktie $\beta_{\mathrm{i}}$ abhängige Funktion der geforderten und erwarteten Aktienrendite $\mu_{\mathrm{i}}$ am Sekundärmarkt linear (Abbildung 3.1). Es besteht ein festes Austauschverhältnis zwischen der Renditeforderung und erwartung der n Aktien und ihren $\beta_{\mathrm{n}}$-Risiken, und zwar uneingeschränkt für alle $\beta_{\mathrm{n}} \rightarrow$ $\infty .{ }^{279}$ Die Wertpapiermarktlinie verläuft ins Unendliche, da alle Aktien, auch solche für $\beta_{\mathrm{n}} \rightarrow \infty$ Teile des effizienten Marktportefeuilles sind. Eine systematische 
Missachtung irgendeiner Risikogruppe findet seitens der Anleger im CAPM nicht statt. Jedes Einzelrisiko ist im Gleichgewicht des CAPM dank vollkommener Informationen genau auf der Linearen $\mathrm{zu}$ verorten. Der unbegrenzt lineare Zusammenhang von geforderter, erwarteter und tatsächlicher Rendite und Interner Verzinsung einerseits sowie dem in $\beta$ gemessenen bewertungsrelevanten Risiko einer Anlage andererseits wird in der Literatur als Wertpapiermarktlinie (,security market line ${ }^{(280}$ ) bezeichnet. Der Ordinatenabschnitt der Wertpapiermarktlinie entspricht dem Ausgleich für den Konsumverzicht einer Bereitstellung von Eigenkapital $r_{\mathrm{f} .}{ }^{281}$ Die Steigung der Wertpapiermarktlinie beläuft sich auf die (Risiko-)Prämie, die der Markt für die Übernahme einer Einheit des Portefeuillerisikos hergibt.

Die Höhe der Risikoprämie für die Übernahme einer Risikoeinheit, mithin die Steigung der Wertpapiermarktlinie hängt von verschiedenen Faktoren ab. Mit einer beispielsweise steigenden Risikoaversion des Anlegers kommt es in seinem Gesamtvermögensbestand c.p. zu einer Umschichtung der Kapitalbereitstellung. Bei zunächst unveränderter Wertpapiermarktlinie nimmt der geplante Anteil des alle unsicheren Anlagen umfassenden Marktportefeuilles ab, der Anteil sicherer Anlagen im Gesamtvermögen nimmt zu. Für das Übernehmen einer Risikoeinheit am Aktienmarkt reicht dem zunehmend risikoaversen Anleger nämlich die bisher erwartete Rendite nicht mehr aus. Der sich daraus ergebende Rückgang des Kursniveaus erhöht uno actu die Renditeforderung und -erwartung. Bei zunehmender Risikoaversion wird somit die Wertpapiermarktlinie steiler. Mit dem Zufluss des Kapitals im sicheren Anlagenbereich sinkt gleichzeitig die risikolose Rendite $r_{f}$. Deshalb sind Phasen geringer Risikoneigungen der Anleger durch eine vergleichsweise steile Wertpapiermarktlinie gekennzeichnet, beginnend auf niedrigem Niveau, das heißt bei einem geringen Ordinatenabschnitt. Umgekehrt fließt mit abnehmender Risikoaversion mehr Kapital aus dem sicheren Anlagebereich in riskante Anlagen, so dass sich die Wertpapiermarktlinie nicht nur verflacht, sondern sich auch insgesamt nach oben verlagert. 
Ein zweiter Einflussfaktor auf die Steigung der Wertpapiermarktlinie geht von der realwirtschaftlichen Sphäre aus. Erhöht sich etwa im Rahmen eines Konjunkturverlaufs die Interne Verzinsung der Unternehmensinvestitionen im Laufe der betrachteten Periode, hat dies ebenfalls Auswirkungen auf die geforderte und erwartete Rendite seines eingesetzten Eigenkapitals. Der rationale Anleger passt seine Renditeforderung und -erwartung an den steigenden Unternehmenswert nach oben an. Bei unveränderter Risikoneigung werden dadurch zwar risikobehaftete und risikolose Anlagen im Gesamtvermögensbestand der Anleger nicht miteinander umgeschichtet, so dass der Ordinatenabschnitt der Wertpapiermarktlinie konstant bleibt. Jedoch erhöht sich beim (überraschenden) Erwirtschaften einer allgemein höheren Internen Verzinsung durch die Unternehmen auch die Renditeforderung und -erwartung für die Übernahme einer bewertungsrelevanten Risikoeinheit. Die Wertpapiermarktlinie wird entsprechend steiler. Erwirtschaften hingegen die Aktiengesellschaften eine überraschend geringere Interne Verzinsung des eingesetzten Eigenkapitals wie etwa bei einem konjunkturellen Abschwung, fordern Anleger c.p. geringere Renditen ein. Die Wertpapiermarktlinie verflacht sich. Eine sinkende Interne Verzinsung führt also wie eine abnehmende Risikoaversion zu einer flacheren, eine vergleichsweise hohe Interne Verzinsung führt wie eine zunehmende Risikoaversion $\mathrm{zu}$ einer steileren Wertpapiermarktlinie. Die Steigung der Wertpapiermarktlinie nimmt also in solchen Phasen zu, in denen die diskontierten Einzahlungsüberschüsse aus den Unternehmensinvestitionen ansteigen und die Anleger risikoaverser werden, et vice versa.

Weitere Einflüsse auf die Steigung der Wertpapiermarktlinie gehen etwa von der Geld- und Fiskalpolitik aus. Im Zuge einer expansiven Geld- und Fiskalpolitik erhöht sich möglicherweise die Interne Verzinsung unternehmerischer Investitionen mit der soeben beschriebenen Folge einer höheren Renditeforderung und -erwartung. Allerdings resultiert aus einer expansiven Geldpolitik auch ein eher sinkendes und aus einer expansiven Fiskalpolitik ein eher steigendes Zinsniveau. ${ }^{282}$ Damit bewirkt erstere wirtschaftspolitische Maßnahme einen Kapitalzufluss zum Aktienmarkt, letztere einen entsprechenden Abfluss. Folglich geht eine expansive Geldpolitik mit einem (konterkarrierenden) Effekt der Verflachung, eine expansive Fiskalpolitik mit einem (verstärkenden) Effekt des Anstiegs der Wertpapiermarktlinie einher. Ferner

$282 \quad$ Vgl. Abschnitt 4.4 
wirkt auch ein exogen verursachter Kapitalabfluss vom Sekundärmarkt weg (losgelöst etwa durch ein sinkendes Interesse in- und ausländischer Eigenkapitalgeber an Aktien im Euroraum), hin in andere Märkte positiv auf die Steigung der Wertpapiermarktlinie, et vice versa.

Phasen gesamtwirtschaftlichen Abschwungs sind regelmäßig durch eine zunehmende Risikoaversion bei abnehmenden Investitionserträgen der Unternehmen gekennzeichnet. ${ }^{283}$ Wie sich beide Effekte auf die Steigung der Wertpapiermarktlinie auswirken, hängt im Einzelfall von ihrer jeweiligen Stärke wie auch von ihrer Antizipation durch die Anleger zu Beginn der betrachteten Periode ab; auch ist für die Steigung der Wertpapiermarktlinie von Bedeutung, wie sich etwaige geld- und fiskalpolitische Maßnahmen auf die Interne Verzinsung sowie die Flüsse zwischen den Kapitalmärkten auswirken. Einerseits können Anleger in Abschwungphasen für die Übernahme einer systematischen Risikoeinheit bei einem sinkenden Unternehmenswert eine nur abnehmende Kompensation fordern. Andererseits erfordert die Übernahme eines bestimmten Risikos bei abnehmender Risikoneigung eine größere Ausgleichszahlung pro übernommener bewertungsrelevanter Risikoeinheit. Diese beiden entgegengesetzten Wirkungen auf das Steigungsmaß der Wertpapiermarktlinie lassen sich chronologisch insofern miteinander vereinbaren, als in der Frühphase eines Abschwungs im Gegensatz zu seiner Spätphase die Risikoaversion relativ hoch ist. So ist zu erwarten, dass die Wertpapiermarktlinie erst in einer realwirtschaftlichen Talsohle die geringste Steigung annimmt. Umgekehrt ist zu erwarten, dass die Wertpapiermarktlinie ihre größte Steigung im Boom eines konjunkturellen Zyklus annimmt. Hier wirken sich (noch) hohe Interne Verzinsungen sowie eine bereits sinkende Risikoneigung der Anleger auf die Steigung der Wertpapiermarktlinie positiv aus. Außerdem ist in einer Phase gesamtwirtschaftlicher Überhitzung mit einem Anstieg des (risikolosen) Zinsniveau $\mathrm{zu}$ rechnen, so dass es zusätzlich $\mathrm{zu}$ einer Parallelverschiebung der Wertpapiermarktlinie nach oben kommt, et vice versa. Sinkt im Zuge einer Rezession das Angebot an sicheren Anlagemöglichkeiten, das heißt, nimmt die Unternehmensnachfrage nach festverzinslichem Kapital in sicheren Anlagenbereich ab, stößt der Kapitalzufluss aus dem Sekundärmarkt auf immer weniger Anlagemöglichkeiten im sicheren Bereich. Folglich sinkt der risikolose Zinssatz $\mathrm{r}_{\mathrm{f}}$ 
umso deutlicher. Steigt jedoch im weiteren Verlauf einer Rezession das Angebot an sicheren Anlagemöglichkeiten für die Kapitalgeber, kann das vom Aktienmarkt zufließende Kapital auf ein möglicherweise noch größer gewordenes Anlagespektum im sicheren Bereich stoßen. Die Wertpapiermarktlinie verlagert sich in diesem Fall mit einer steigenden Rendite im sicheren Renditebereich $r_{\mathrm{f}}$ in einer Rezession sogar nach oben.

Abbildung 3.2 Die Wertpapiermarktlinie als Konjunkturindikator

\begin{tabular}{l|c|c}
\multicolumn{2}{c|}{ Rering } & Hoch \\
\hline In Klammern: (Ordinatenabschnitt; Steigung) & Aufschwung & Boom \\
\hline
\end{tabular}

Vor dem Hintergrund dieser Überlegungen kann die Lage der Wertpapiermarktlinie die Funktion eines Konjunkturindikators übernehmen (Abbildung 3.2). Dabei verlaufen Risikoneigung und Interne Verzinsung im konjunkturellen Verlauf entgegengesetzt. Die in Abbildung 3.1 skizzierte Drehung und Verlagerung der Wertpapiermarktlinie nach unten, kann demnach als eine Veränderung von einer Phase des Booms mit (noch) ansteigenden Werten der Cash Flows und einer bereits hohen Risikoaversion hin zur Talsohle mit (noch) sinkenden Internen Verzinsungen, aber einer bereits hohen Risikoneigungen der Anleger interpretiert werden. Im Gegensatz zum Boom ist dann die Talsohle eines realwirtschaftlichen Zyklus durch eine eher flache Wertpapiermarktlinie gekennzeichnet. Zeichnet sich beim Durchschreiten der Talsohle ein Aufschwung ab, führt eine zunehmende Interne Verzinsung $\mathrm{zu}$ einem Anstieg der Wertpapiermarktlinie in Form einer Drehung sowie einer Verlagerung nach oben. Eine zunehmende Risikoaversion verleiht dann in der Spätphase eines Aufschwungs der Steigung der Wertpapiermarktlinie einen zusätzlichen Schub. Der einsetzende Beginn einer Rezession ist aufgrund einer sinkenden Internen Verzinsung mit einer Verflachung der Wertpapiermarktlinie sowie ihrer Verlagerung nach unten verbunden. Erst in der Spätphase einer 
Rezession, das heißt bei einer nach wie vor abnehmenden Internen Verzinsung des eingesetzten Eigenkapitals, aber einer zunehmenden Risikoneigung der Anleger ist mit der geringsten - möglicherweise auch einer negativen - Steigung der Wertpapiermarktlinie zu rechnen. Eine Wertpapiermarktlinie von „mittlerer“ Steigung lässt sich alternativ als eine Situation mit steigenden Internen Verzinsungen bei hoher Risikoneigung (Aufschwung) oder umgekehrt als eine Situation mit sinkenden Internen Verzinsungen bei hoher Risikoaversion (Abschwung) interpretieren.

Elegant in seiner Erscheinung stößt bereits die von den genannten Einflussfaktoren unabhängige Darstellung des CAPM auf mitunter fundamentale Kritik. ${ }^{284}$ Eingewandt wird insbesondere, das Marktportefeuille sei angesichts der unüberschaubaren Zahl von unsicheren Anlagemöglichkeiten praktisch unbestimmbar. Eine Einzelanlage könne deshalb lediglich im Verhältnis zu einem beliebig ausgewählten Portefeuille wie etwa dem Cdax stehen, nicht aber zu dem real nicht existenten Marktportefeuille (so genanntes Index- beziehungsweise Surrogatproblem). ${ }^{285}$ Die Bewertung einer Einzelanlage anhand des CAPM werde somit unmöglich; dass zahllose empirische Tests des CAPM nicht zu einheitlichen Resultaten geführt haben, verwundert insofern kaum. ${ }^{286}$ Wie jede andere Theorie auch ist das CAPM tautologisch. Die Linearität seiner Wertpapiermarktlinie ist logisch zwingend der Annahme geschuldet, dass sich die Anleger nur am erwarteten Ertragswert und an der Varianz der Rendite orientieren. ${ }^{287}$ Zwar lässt sich die Aussage eines linearen Zusammenhangs zwischen Rendite und Risiko mit einem beliebigen Portefeuille verifizieren, an Hand des real nicht existenten Marktportefeuilles allerdings nicht falsifizieren. Das CAPM sei deshalb nicht überprüfbar. Es verliere somit im Popper'schen Sinne den Anspruch, Theorie zu

284 Zu einer Zusammenfassung der Kritik am CAPM vgl. Haugen 1987, 152-159; Oertmann 1997, 21f.; Perridon, Steiner 1999, 273; Kruschwitz, Löffler 1997, 645.

285 Nach dem CAPM besteht das Marktportefeuille aus sämtlichen risikobehafteten Vermögenstiteln, unabhängig davon, ob diese handelbar sind oder nicht. Roll (1977) bemängelt daher die Unmöglichkeit einer praktischen Ermittlung einzelner Renditen. Ein empirischer Test über eine signifikant positive Rendite/Risiko-Beziehung könne daher nur einer Überprüfung des jeweiligen Indexes gleichkommen, nicht aber den im CAPM postulierten Rendite/Risiko-Zusammenhang bestätigen oder verwerfen. Vgl. Roll 1977, 130f. + 147ff.; Hamerle, Rösch (1995). erhebliche methodische Schwierigkeiten; die bisher vorliegenden empirischen Ergebnisse für den deutschen Kapitalmarkt lassen keine eindeutigen Aussagen über die empirische Gültigkeit des CAPM zu, schließen sie allerdings auch nicht aus (Möller 1988). Franke, Hax 1990, 285. Vgl. Unser 1999, 113; Oertmann 1997, 21. 
sein. Weitere Kritik wird geäußert hinsichtlich einer angenommenen Normalverteilung der Renditen, des $\beta$-Risikos als alleinigem bewertungsrelevanten Risiko, ${ }^{288}$ der Bestimmbarkeit der risikolosen Anlage ${ }^{289}$, des beschränkten einperiodigen Planungshorizontes ${ }^{290}$ und anderen Annahmen des dargestellten Grundmodells. ${ }^{291}$

Unbestritten liegen dem skizzierten Grundmodell des CAPM realitätsferne Annahmen zugrunde. Doch erzeugen entsprechende Modifikationen der Annahmen des Grundmodells keine Veränderungen im Wesentlichen. Einzig die Komplexität der Darstellung nähme zu. ${ }^{292}$ Allen Anfechtungen zum Trotz ist das CAPM für Theorie und Praxis das maßgebende Bewertungsmodell für unsichere Kapitalmarktanlagen. ${ }^{293}$ Es ist "alive and well. ${ }^{{ }^{294}}$ Das CAPM ist von der Konzeption her klar. Wenn auch behaftet mit den genannten empirischen, methodischen und wissenschaftstheoretischen Problemen, ist es ein in sich schlüssiges gedankliches Konstrukt. Bereits der Mangel an einer ebenbürtigen theoretischen Alternative „Zwingt“ zu einer weiteren theoretischen Beschäftigung mit ihm. ${ }^{295}$ „Es scheint keinen anderen Weg zu geben, als sich mit den Schwächen des CAPM erneut

Vgl. Abschnitt 3.2.

Sind alle Anlagen risikobehaftet, stellen sich Anleger je nach Risikoneigung unterschiedliche Portefeuilles zusammen. Black (1972) verzichtet im Zero-Beta-CAPM dennoch auf die Existenz einer risikolosen Anlage. An deren Stelle tritt ein mit dem Marktportefeuille nicht korreliertes varianzminimales Portefeuille. Vgl. Haugen 1987, 148f.

Investitionen erbringen meist Einzahlungsüberschüsse in mehreren Perioden. Zur Mehrperiodigkeit vgl. Singleton (1990); Fama (1977); Merton (1973a,b); Breeden (1979); Lee (1976); Gilster (1983). Ob und wie sich das CAPM auf die Bewertung mehrperiodischer Vorteilsströme anwenden lässt, ist immer noch umstritten. Vgl. Stapelton, Subrahmanyam (1978); Haley, Schall (1979). Schrittweise rückwärts rechnend und das CAPM jeweils auf die Summe aus dem Einzahlungsüberschuss anwendend, gelangt man im Prinzip zu dem Wert im Entscheidungszeitpunkt $\mathrm{t}_{0}$.

Für einen Überblick über die Weiterentwicklungen des CAPM und andere Beta-Preis-Modelle vgl. Oertmann 1997, Kap. 2+3; Perridon, Steiner 1999, 269-272.

Vgl. Haugen 1987, 138.

Vgl. Oertmann 1994a, 231; Copeland, Weston 1988, $212 \mathrm{ff}$.

Levy 1997, 143. Vgl. a. Amihud, Christensen, Mendelson (1992); Jagannathan, Wang (1993).

Etwa die mit dem CAPM konkurrierende Arbitrage Pricing Theory (APT) liefert keine absolute Bewertung der Finanztitel, sondern eine die Relation von Wertpapierpreisen beschreibende Gleichung bei Abwesenheit von Arbitragemöglichkeiten (hierzu ist die Annahme über die Risikoeinstellung zwingend erforderlich). Die APT stellt zwar eine deskriptive Theorie realer Renditegenerierungsprozesse dar, erlaubt aber nicht die Lösung des Grundproblems der optimalen Zusammenstellung eines Portefeuilles. Auch die APT weist aus methodologischer Sicht die Schwäche auf, dass sie für eine Falsifizierung zu unbestimmt ist. Weder Anzahl noch Inhalt der renditebestimmenden Faktoren werden angegeben. Es lässt sich sogar zeigen, dass beliebige Faktoren die Preisgleichung der APT ergeben, sofern nur ein varianzeffizientes Portefeuille aus einer Linearkombination dieser Faktoren gebildet werden kann. $\mathrm{Zu}$ einer kritischen Auseinandersetzung mit der auf Ross (1976) zurückgehenden APT vgl. Kruschwitz, Löffler 1997, 649f;; Unser 1999, 122-125; Bray (1994); Gilles, LeRoy (1991). 
auseinanderzusetzen... ${ }^{\text {(296 }}$ Das CAPM steht damit nach wie vor Pate als Referenzmodell, an dem es sich ,abzuarbeiten“ gilt. Dazu wird im nun folgenden Abschnitt zu verdeutlichen sein, dass niveaubedingte Unterliquidität als das Ergebnis eines dauerhaft existierenden Angebotsüberhangs im Rahmen des CAPM eine Anomalie darstellt.

296 Kruschwitz, Löffler 1997, 650. Sie stellen der APT ein etwa "Todesurteil” aus, vgl. ebd.; Hamerle, Rösch 1996, 2. 


\subsection{Niveaubedingte Unterliquidität und CAPM}

Das CAPM beschreibt bei Liquidität aller unsicheren Anlagen den Zustand eines markträumenden Kapitalmarktgleichgewichts. Die Aktien sind in seinem statischen Gleichgewicht fundamental „richtig“ bewertet. Die Renditeforderungen der Anleger werden realisiert. Im CAPM-Gleichgewicht besteht somit kein Anlass zum Handel von Aktien. Angesichts beträchtlicher Kapitalströme (vor allem in Segmenten liquider Aktien) befindet sich der deutsche Aktiensekundärmarkt offenbar nicht in einem derartigen CAPM-Gleichgewicht. Fraglich ist, ob die festzustellenden Transaktionsvolumina eine Tendenz zum markträumenden Gleichgewicht des CAPM beschreiben, ob die Wertpapiermarktlinie des CAPM dynamisch betrachtet ein stabiles Gleichgewicht darstellt. Als statisches Gleichgewicht konzipiert, enthält das CAPM selber keine Aussage darüber, ob und gegebenenfalls wie Anpassungsprozesse ablaufen. Weil Abweichungen von der Wertpapiermarktlinie annahmegemäß ausgeschlossen sind, ${ }^{297}$ ist dem CAPM ein etwaiger Rückführungsprozess zum beschriebenen Gleichgewicht nicht immanent. Das CAPM lässt indes vielmehr offen, ob es überhaupt einen Anpassungsmechanismus hin zum markträumenden Gleichgewicht gibt. Ein in dynamischer Hinsicht markträumendes Gleichgewicht ist mit der statischen Welt des CAPM genauso (wenig) vereinbar wie ein nicht-markträumendes.

In der finanzwirtschaftlichen Literatur ist für das CAPM in dynamischer Hinsicht jedoch der Konvergenzgedanke vorherrschend. ${ }^{298}$ Die Unterbewertung einer unterliquiden Aktie drückt sich in einer Renditeerwartung aus, die oberhalb der geforderten Rendite in Gestalt der Wertpapiermarktlinie liegt. Durch die Erzielbarkeit einer erwarteten Überrendite bauen entsprechende Kaufaufträge die

\footnotetext{
297 Das der Wertpapiermarktlinie zu Grunde liegende Marktportefeuille umfasst alle Anlagen, also auch die von der Wertpapiermarktlinie abweichende Anlage. Um die Bewegung einer Einzelanlage im Verhältnis zum Markportefeuille zu beschreiben, bedarf es einer gedanklichen Herausnahme der zu vergleichenden Anlage aus dem Marktportefeuille. Dabei ergeben sich jedoch möglicherweise Rückwirkungen auf die Wahrscheinlichkeitsverteilung der Rendite des Marktportefeuilles. Eine Gegenüberstellung von Einzelanlage und Marktportefeuille stößt insofern auf konzeptionelle Probleme. Dieser Einwand eines feed-back ist jedoch praktisch vernachlässigenswert. Eine Einzelanlage ist im Vergleich zum Marktportefeuille für gewöhnlich als $\mathrm{zu}$ klein $\mathrm{zu}$ bezeichnen, um nachhaltigen Einfluss auf das gesamte Aktienbündel ausüben zu können. Vgl. Saelzle (1976). setzt die überwiegend neoklassisch geprägte Literatur implizit Konvergenz voraus.
} 
Unterbewertung - je nach Grad der Informationseffizienz und der Rationalität - ${ }^{299}$ früher oder später ab und gleichen die Renditeerwartung der Renditeforderung uno actu an. Auch überbewertete Aktien können vor dem Hintergrund dieses Konvergenzgedankens nur vorübergehend mit einer erwarteten Rendite/RisikoSituation unterhalb der Wertpapiermarktlinie beschrieben werden. Vom Fundamentalwert abweichende Aktien werden dank eines flexiblen Mechanismus erwarteter Renditen durch stabilisierende Spekulationen zur gleichgewichtigen Wertpapiermarktlinie zurückgeführt. Ob unter- oder überbewertet, die erwartete Rendite konvergiert mit der geforderten Rendite in Gestalt der CAPMWertpapiermarktlinie. ${ }^{300}$ Nach einem temporären Abweichen der Renditeerwartung von der Wertpapiermarktlinie stellt sich somit auf lange Sicht die Identität von anlegerseitigem Eigenkapitalangebot und unternehmerischer Eigenkapitalnachfrage (I-S-Identität des Aktienmarktes) wieder ein. Diesem Konvergenzgedanken folgend, ist das CAPM-Gleichgewicht auch in dynamischer Hinsicht als stabil zu bezeichnen. ${ }^{301}$ Die Situation eines Angebotsüberhangs an Aktien in Plangrößen, sprich Unterliquidität, kann folglich nur temporärer Natur sein. Denn die Renditeerwartung ist im Konvergenzfall kurselastisch und entwickelt sich entgegen dem Kursverlauf. Flexible Renditeerwartungen führen dazu, dass alle Aktien in vollem Umfang und im Verhältnis ihrer fundamental richtig bewerteten Kapitalisierungen langfristig geplante Bestandteile des Marktportefeuilles sind. ${ }^{302}$

Im Unterschied zur unterstellten Konvergenz des CAPM manifestiert sich in niveaubedingter Unterliquidität, einem Zustand dauerhaft eingeschränkten Eigenkapitalangebots, die Inflexibilität der erwarteten Rendite nach oben. ${ }^{303}$ Die

Im Fall semirationaler Erwartungen kennen Wirtschaftssubjekte zwar die langfristig geforderte und erwartete Gleichgewichtsrendite gemäß der Wertpapiermarktlinie, jedoch nicht ihren Anpassungspfad. Sie erwarten jedoch, dass sich die laufende erwartete Rendite auf die langfristige zubewegt. Dabei ist die erwartete Änderung der Kurse von der Periode t-1 zur Periode t proportional zur Abweichung der langfristig erwarteten Rendite von der laufenden erwarteten Rendite. Genauer lautet die Erwartungshypothese: $\mu_{t}-\mu_{t-1}=\theta\left(\mu_{\text {langfristig }}-\mu_{t-1}\right)$, wobei für den die Anpassungsgeschwindigkeit ausdrückenden Koeffizienten $\theta$ gilt: $0<\theta<1$. $\theta$ stellt in zweierlei Hinsicht ein stabilisierendes Element dar. Zum einen werden mit zunehmendem $\theta$ aus divergierenden tendenziell konvergierende Entwicklungen. Zum anderen können steigende Werte von $\theta$ innerhalb einer konvergierenden Anpassung zyklische Entwicklungen unterdrücken. Vgl. Jarchow 2000, 10-14.

Vgl. Felderer Homburg 1987, 72-74.

Vgl. Rubinstein 1973, 172.

Eine systematische Vernachlässigung bestimmter Aktienwerte steht in dezidiertem Gegensatz zu Aussagen der Portefeuille-Theorie, nach der rationale Anleger ihr Portefeuille breit streuen, um unsystematische Risiken zu diversifizieren. Vgl. Abschnitt 2.3. 
Renditeerwartung der Anleger unterbewerteter, weil unterliquider Gesellschaften befindet sich offenbar nicht ober-, sondern unterhalb der markträumenden Wertpapiermarktlinie. „Die Argumentation „Geringe Liquidität, daher (ceteris paribus) hohe erwartete Rendite“ scheint zumindest in Deutschland nicht zu gelten.“604 Die relative Unterbewertung unterliquider Aktien kleiner und mittlerer deutscher Gesellschaften ist in Up-Markets besonders hoch. Von einer Konvergenz der erwarteten an die geforderte Rendite kann deshalb insbesondere in solchen Marktphasen des deutschen Sekundärmarktes nicht die Rede sein. Vor dem Hintergrund niveaubedingter Unterliquidität ist der Mechanismus einer nach oben frei fluktuierenden Renditeerwartung offenbar nur eingeschränkt funktionstüchtig. Er vermag ein dauerhaft überschießendes Aktienangebot nicht ins markträumende Gleichgewicht des CAPM zu führen. Die Unterbewertung einer niveaubedingt unterliquiden Aktie geht mit einer Renditeerwartung unterhalb der Wertpapiermarktlinie einher. Niveaubedingt unterliquide Aktien finden deshalb auch auf lange Sicht nicht in vollem Umfang Eingang in die von allen Anlegern gehaltenen Portefeuilles.

Abbildung 3.3 veranschaulicht eine niveaubedingt unterliquide Aktie c im Rahmen des CAPM (rechtes Diagramm). Ihr bewertungsrelevantes Risiko beträgt $\beta_{\mathrm{C}}$. Für einen Anleger ist eine nach der Wertpapiermarktlinie markträumende Renditeforderung in Höhe von $\mu_{\mathrm{C}}{ }^{\text {gef* }}$ jedoch nicht zu erwarten. Die Renditeerwartung beläuft sich trotz Unterbewertung auf nur mehr $\mu_{\mathrm{C}}{ }_{\mathrm{C}}^{\text {erw }}$. Es besteht folglich eine Lücke zwischen der geforderten und der erwarteten Rendite. Da rationale Anleger nach ihren Erwartungen handeln, stellt sich auch nur eine Rendite unterhalb der Wertpapiermarktlinie ein. Für eine niveaubedingt unterliquide Aktien $\mathrm{c}$ ist somit im nicht-markträumenden Gleichgewicht folgende Ungleichung kennzeichnend:

$r_{c}^{\text {int }}=\mu_{c}{ }^{\text {gef }}>\mu_{c}^{\text {erw }}=r_{c}^{\text {tat }} 305$

304 Sattler 1994, 250. Es kann somit die so genannte Illiquiditätshypothese von Amihud, Mendelson (1991b), nach der unterliquide Wertpapiere im Durchschnitt vor Transaktionskosten eine höhere Rendite erwirtschaften als liquide Titel, für den deutschen Aktienmarkt nicht bestätigt werden. „In equilibrium, the return on an asset is an increasing function of its transaction cost. This is because, as one might expect, investors require a compensation for bearing these costs." Amihud, Mendelson 1991b, 236.

Genau genommen umfasst die hier verwendete Renditeerwartung $\mu_{\mathrm{c}}^{\text {erw }}$ ein bewertungsrelevantes, aber vom Markt nicht entgoltenes Risiko, das mit einer markträumenden Renditeforderung in Höhe von $\mu_{\mathrm{c}}^{\text {gef }}$ einhergeht. Es handelt sich insofern um eine Art „Netto“-Renditeerwartung. Vgl. Abschnitt 4.3.2. 
Abbildung 3.3 Eine niveaubedingt unterliquide Aktie am CAPM

Eigenkapitalangebot und ,-nachfrage“ am Sekundärmarkt

Up-Market mit erwarteter Renditeobergrenze

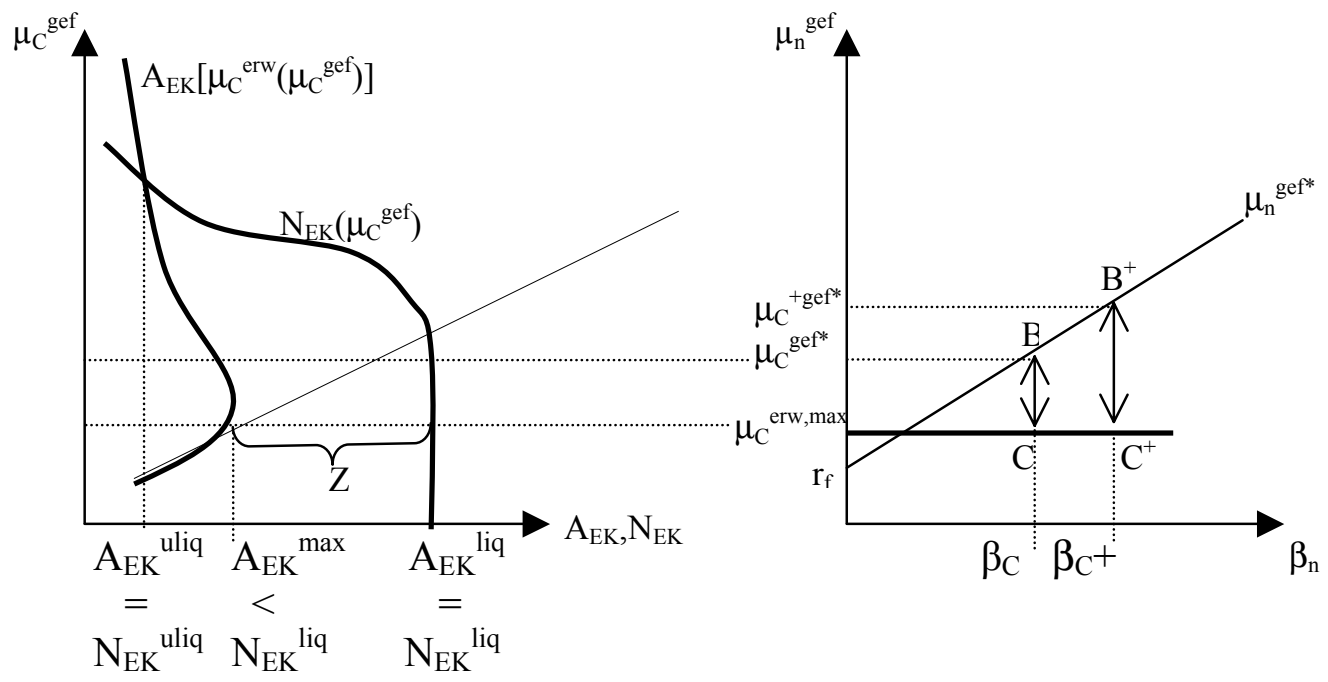

Zum Eigenkapitalangebot: Die Renditeerwartung $\mu_{\mathrm{C}}^{\text {erw }}$ sei nach oben derart inflexibel, dass sie zugleich die maximal erzielbare Renditeerwartung für das Halten der Aktie c ist. Kursabschläge vom fundamental richtig bewerteten Niveau in jedweder Höhe führen zu keiner höheren Renditeerwartung. Eine über $\mu_{\mathrm{C}}^{\text {erw,max }}$ liegende Rendite $\mathrm{zu}$ fordern, schlägt sich nicht in einer steigenden, sondern sogar sinkenden Renditeerwartung nieder. Deshalb verläuft die Eigenkapitalangebotskurve bis $\mu_{C}{ }^{\text {erw,max }}$ mit positiver, darüber hinaus rückwärts geneigt mit negativer Steigung (linkes Diagramm). Das geplante Eigenkapitalangebot ist nämlich eine positive Funktion der erwarteten Rendite, die wiederum ab einem bestimmten Niveau negativ von der Renditeforderung abhängt: $A_{E K}\left[\mu_{C}{ }^{\text {erw }}\left(\mu_{C}{ }^{\text {gef }}\right)\right]$. Bis zum Erreichen des Erwartungsmaximums ist die geforderte mit der erwarteten Rendite positiv korreliert. Über das Erwartungsmaximum hinaus ist eine höhere Forderung mit einer sinkenden Renditeerwartung verbunden. ${ }^{306}$ Daraus resultiert die negative Steigung des oberen

306 Niveaubedingte Unterliquidität beinhaltet aber nicht nur den betrachteten Fall einer rückwärtsgeneigten Eigenkapitalangebotskurve, sondern auch alle übrigen Situationen, in denen das Eigenkapitalangebot zur markträumenden Renditeforderung $\mu_{\mathrm{C}}{ }^{\text {gef* }}$ geringer ist als das ausgeschüttete Aktienkapital: Betrüge c.p. die Elastizität der Renditeerwartung in Bezug auf die Renditeforderung etwa Null, verliefe die Kurve des Eigenkapitalangebotes vollkommen unelastisch und nicht rückwärts geneigt. Würde sich mit zunehmender Höhe die 
Teils der Eigenkapitalangebotskurve. Je elastischer die Renditeerwartung auf die Renditeforderung in diesem Bereich und je elatischer das Eigenkapitalangebot auf Änderungen der Renditeerwartung reagiert, desto flacher verläuft der rückwärtsgeneigte Teil der Eigenkapitalangebotskurve und desto größer der Nachfrageüberhang an Eigenkapital.

Zur Eigenkapitalnachfrage: Auf der Eigenkapitalnachfrageseite buhlt das betrachtete Unternehmen c dauerhaft um die Gunst der Aktionäre. Entscheidungen über Unternehmensinvestitionen mit dem bereitgestellten Eigenkapital werden deshalb mit der Maßgabe getroffen, als frage es jederzeit das bereits erhaltene Eigenkapital nach. ${ }^{307}$ Dabei hängt die „Quasi“-Nachfrage $\mathrm{N}_{\mathrm{EK}}$ des Unternehmens c nach Eigenkapital am Sekundärmarkt unmittelbar von der geforderten Rendite $\mu_{C}{ }^{\text {gef }} a b$. Dass das Unternehmen c das emittierte Aktienkapital bis zur Renditeforderung $\mu_{\mathrm{C}}{ }^{\text {gef* }}$ jederzeit aufs Neue am Sekundärmarkt „nachfragt“, indem es die Interne Verzinsung der Unternehmensinvestitionen auf die Renditeforderungen entsprechend ausrichtet, verdeutlicht sich am unelastischen Verlauf der Nachfragekurve bis zur marktgerechten Renditeforderung. Für eine Renditeforderung oberhalb der marktgerechten Renditeforderung $\mu_{\mathrm{C}}{ }^{\text {gef* }}$ nimmt die Nachfrage je nach den Möglichkeiten des Unternehmens c ab, auf andere Weise Eigenkapital oder ein entsprechendes Substitut aufnehmen zu können. Deshalb ist die Eigenkapitalnachfrage oberhalb von $\mu_{\mathrm{C}}{ }^{\text {gef* }}$ negativ geneigt. ${ }^{308} \mathrm{Im}$ diesem elastischen Teil der Eigenkapitalnachfragekurve kommt zum Ausdruck, dass das Unternehmen $\mathrm{C}$ nun weniger unternehmerische Investitionsprojekte mit steigender

Renditeforderung immer schwächer, allerdings noch positiv auf die Renditeerwartung auswirken, wäre der Verlauf der Eigenkapitalangebotskurve konvex. Mit steigender Renditeforderung würde das Eigenkapitalangebot weniger steigen als es mit abnehmender Renditeforderung sinken würde. Auch diese Fälle stellen niveaubedingte Unterliquidität dar. Umgekehrt drückt sich Überliquidität einer Aktie in einem konkaven Verlauf der Eigenkapitalangebotsfunktion aus. Mit zunehmender Renditeforderung steigt c.p. die Renditeerwartung überproportional. Das Eigenkapitalangebot steigt in diesem Fall mehr, als es sinkt, wenn die geforderte Rendite abnimmt. In diesem Fall läg zur markträumenden Renditeforderung $\mu_{\mathrm{C}}{ }^{\text {gef* }}$ ein Angebotsüberhang an Eigenkapital vor.

Eine von Null verschiedene „Eigenkapitalnachfrage” eines Unternehmens am Sekundärmarkt setzt voraus, dass es sich bei der Emission von Aktien nicht um eine einmalige SpotTransaktion zwischen dem Unternehmen und seinen Eigenkapitalgebern, sondern es sich vielmehr um eine relationale Verbindung handelt. $\mathrm{Zu}$ den Gründen für das Interesse eines Unternehmens an der Liquidität seiner Aktien am Sekundärmarkt vgl. Abschnitt 4.2.1. 
Renditeforderung ausführen. Bis zur markträumenden Renditeforderung $\mu_{\mathrm{C}^{\text {gef* }}}$ ist die Eigenkapitalnachfrage allerdings vollkommen unelastisch.

Planen die Anleger bei maximaler Renditeerwartung, dem betrachteten Unternehmen dauerhaft weniger Eigenkapital bereitzustellen, als zum Emissionszeitpunkt ausgegeben und von den Unternehmen nachgefragt wird, herrscht niveaubedingte Unterliquidität. Es liegt einer dauerhafter Nachfrageüberhang nach Eigenkapital in Höhe von $\mathrm{Z}$ vor. Seitens der Unternehmen wird in Höhe der ausgeschütteten Aktien zur Renditeforderung $\mu_{\mathrm{C}}{ }^{\text {gef* }}$ mehr Eigenkapital nachgefragt, als seitens der Anleger angeboten wird. Die geplante Ersparnis der Eigenkapitalgeber unterschreitet die geplanten Investitionen der Unternehmen: $\left(\mathrm{S}^{\text {gepl }}<\mathrm{I}^{\text {gepl }}\right)$. Je nach Kurvenverlauf können Eigenkapitalangebot und -nachfrage dennoch zu einem Übereinklang beider Größen kommen, dann allerdings bei einer oberhalb der Wertpapiermarktlinie liegenden Renditeforderung und auf einem niedrigeren geplanten I-S-Niveau des Aktienmarktes. ${ }^{309}$ Für niveaubedingte Unterliquidität ist allerdings kennzeichnend, dass sich ein Eigenkapitalangebot unterhalb von $A_{E K}{ }^{l i q}=N_{E K}{ }^{l i q}$ einstellt.

Ein eingeschränktes Eigenkapitalangebot für die c-Aktie bewirkt, dass sich Informationen über fundamentale Unternehmensveränderungen in den Aktienkursen - wenn überhaupt - dann nur in verzögerter Geschwindigkeit widerspiegeln. Ein längerer Anpassungspfad der Kurse an fundamentale Veränderungen kann dazu führen, dass sich Informationen mit entgegengesetzter Wirkung auf den Kurs teils überlagern und somit aufheben. Diese Einschränkungen in der Informations- und Bewertungseffizienz des Sekundärmarktes haben zur Folge, dass der Kurs der niveaubedingt unterliquiden Aktie c weniger mit dem Marktportefeuille schwankt. Unterliquide Aktien kleiner Gesellschaften reagieren auf Veränderungen des Marktportfeuilles unter Umständen erst nach einigen Tagen - und dies sehr unstetig. Deshalb spiegeln sich die systematischen Risiken erst nach einiger Zeit in den Kursen dieser Aktien wider. ${ }^{310}$ Niveaubedingte Unterliquidität verursacht deswegen geringere bewertungsrelevante Beta-Risiken bei den betroffenen Gesellschaften („Risk-Mismeasurement-Hypothese“ ${ }^{\text {(311 }) . ~}{ }^{312}$ Risikomessungen etwa auf täglicher Vgl. Szenario 4 in Abschnitt 2.3.

Vgl. Hotz 1989, 33.

Vgl. Sapusek 1998, 148.

Zur Unterschätzung der Beta-Faktoren kleiner Unternehmen vgl. Roll (1981); Scholes, Williams (1977) sowie Keim (1983). 
Ertragsbasis unterschätzen somit das Risiko von Aktien mit schwachen Umsätzen regelmäßig. ${ }^{313}$ Vor diesem Hintergrund verwundert es nicht, dass Aktien kleiner Gesellschaften in Deutschland - im Unterschied zu denen in den USA und Japan tatsächlich ein besonders niedriges systematisches Risiko aufweisen. ${ }^{314}$ Während in den USA für kleinere Unternehmen Beta-Werte zwischen 1,0 und 2,0 ausgewiesen werden, liegen sie für deutsche Kleinunternehmen mit knapp 0,6 deutlich unter dem des Marktportefeuilles $(1,0)$ sowie denen der Standardwerte. ${ }^{315}$ Mit einem unterliquiditätsbedingten Beta-Abschlag verringert sich für die Anleger zwar auf kurze Sicht die Differenz zwischen geforderter und erwarteter Rendite. Eine geringere Volatilität auf kurze Sicht mag die Schwankungen der Aktienrendite im Verhältnis zur Portefeuillerendite auf lange Sicht allerdings unbeeinträchtigt lassen. Da für Anleger unterliquider Aktien infolge des Klienteleffekts eine längere Haltedauer zutreffend ist, trägt eine unterliquiditätsbedingte Beta-Reduzierung faktisch nicht $\mathrm{zu}$ einem geringeren Anlegerrisiko im Sinne einer langfristigen, am Marktporteuille gemessenen Renditeentwicklung bei. Insofern ist die Aussagekraft des Beta-Faktors auf kurz- und mittelfristiger Basis als Risikomaß einer unterliquiden Anlage von eingeschränkter Aussagekraft. Das tatsächlich bewertungsrelevante Risiko einer unterliquiden Aktie fällt möglicherweise höher aus, als es die relativ geringen Beta-Werte der deutschen Small und Mid Caps ausweisen. Im Beispiel der Abbildung 3.3 wirkt sich dieser Fall in einer entsprechend größeren Differenz zwischen der geforderten und der erwarteten Rendite $\left(\mathrm{B}^{+}-\mathrm{C}^{+}\right.$anstatt $\left.\mathrm{B}-\mathrm{C}\right)$ aus.

Neben der niveaubedingten Unterliquidität besteht auch aus anderen Gründen Anlass zur Vermutung, dass $\beta_{\mathrm{C}}$ das Risiko einer unterliquiden Aktie c zu gering ausweist, mithin diversifizierbares Varianzrisiko für bewertungsrelevant $\mathrm{zu}$ erachten ist. ${ }^{316}$

313 Dimson, Marsh (1983) haben Methoden entwickelt, die dieses "Thiness“-Problem reduzieren oder sogar beseitigen.

Vgl. Sattler $1994,247$.

Vgl. Beiker 1993, 87; Dhawan (2001). Eigene Berechnungen haben ergeben, dass das 250Tage Beta eines durchschnittlichen Dax 30-Wertes zum 29.8.00 bezogen auf den DAX 30 als simuliertes Marktportefeuille 0,69842 bei einer Varianz von 0,336 beträgt. Der entsprechende Wert für Mdax-Titel lag mit 0,747 bei einer Varianz von 0,347 bezogen auf den Mdax als simuliertes Marktportefeuille allerdings in etwa gleich auf.

316 Beim Risikofaktor Alpha erklärt den „,abnormen“ Ertrag, welcher aufgrund von Über- oder Unterbewertungen von Aktien erzielt (ex post) oder erwartet (ex ante) werden kann. Vgl. Hotz 1989, 19f. „When one stock has a higher or lower rate of return than another stock with the same beta, when it does better or worse against the market than its beta would have predicted, this is said to be due to its alpha factor, or the various residual nonmarket influences unique to each stock. If you can select enough stocks with positive alphas, your portfolio will perform 
Denn in vielen Fällen befinden sich unterliquide Aktien in nicht breit diversifizierten Anlegerportefeuilles. Neben dem systematischen $\beta$-Risiko ist in Portefeuilles von geringer Streuung somit auch unsystematisches, so genanntes $\alpha$-Risiko insbesondere für die Bewertung kleiner und mittlerer Gesellschaften relevant. Die Erweiterung des Katalogs bewertungsrelevanter Risiken hat entsprechende Auswirkungen auf die Interne Verzinsung unternehmerischer Investitionsobjekte kleiner und mittlerer Gesellschaften. "Casual observation suggests that the use of risk to refer to own variance rather than correlation with the market, affects the behavior of firms." ${ }^{317}$ Neben dem Varianzrisiko spielen auch andere Faktoren für die Bestimmung bewertungsrelevanter Risiken eine bedeutende Rolle wie etwa eine mangelnde Unabhängigkeit und Stationarität der Renditen, die Schiefe und Wölbung von Renditeverteilungen. ${ }^{318}$ Auch ist das totale Ausfallrisiko ${ }^{319}$ sowie die Wahrscheinlichkeit des Erreichens einer befriedigenden Portefeuillerendite relevant. $^{320}$ Bei einer Berücksichtigung dieser Faktoren zur Bestimmung bewertungsrelevanter Risiken handelt es sich genau genommen um Verstöße gegen die Grundannahmen der Portefeuilletheorie. In der weiteren Untersuchung wird das $\beta$-Risiko aus Gründen einer größeren Wirklichkeitsnähe dennoch durch das $\tau$-Risiko ersetzt. Ohne es genau zu spezifizieren, umfasst das $\tau$-Risiko neben dem $\beta$-Faktor möglicherweise weitere bewertungsrelevante Risiko-Komponenten. ${ }^{321}$

better than its beta would have indicated for a given market movement." Cohen, Zinbarg, Zeikel 1975, 53.

$317 \quad$ Stiglitz 1981, 321.

318 Die Annahme einer Normalverteilung für die Dichte der Ausfallwahrscheinlichkeit erleichtert zwar die Rechenarbeit. Es genügen dann Mittelwert und Streuung zur Kennzeichnung der Verteilung, und man muss sich nicht um deren Schiefe oder Wölbung kümmern. Es ist aber zu berücksichtigen, dass die Renditeverteilung insbesondere unterliquider Aktien von der Normalverteilung abweichen kann. Empirische Untersuchungen haben ergeben, dass auch die Varianz, die Wahrscheinlichkeit bzw. das Ausmaß eines Verlustes, die Schiefe und Wölbung der Wahrscheinlichkeitsverteilung und Unternehmensinformationen als Risikomaß eine Rolle spielen. Vgl. Unser 1999, $121 \mathrm{f}$.

Vgl. Beiker 1993, 85. Abweichend von der Normalverteilung weisen Ausfallrisiken sowohl eine positive Schiefe auf (sie sind linkssteil) als auch ein „fat-tailed downside risk“ auf. Beides führt $\mathrm{zu}$ einer systematischen Unterschätzung des unerwarteten (und oft besonders hohen) Verlustes. Vgl. Kotz 2000, 640.

Obwohl die Notwendigkeit neuer Risikodefinitionen als Basis finanzierungstheoretischer Modelle in der Literatur bekräftigt wird, ist die Zahl der auf der Basis verhaltensgestützter Risikomaße entwickelten Preisbildungsmodelle allerdings noch sehr spärlich. Vgl. De Bondt, Thaler 1989, 199.

Vgl. Levy 1978, 643ff.; Beiker 1993, $91+106$; Mayers 1972, 224. Es gibt eine wachsende Zahl von Hinweisen, denen zufolge das Einfaktorenmodell des CAPM nicht die Renditestruktur unsicherer Anlagen erklären kann. Beginnend mit Banz (1981) und Reinganum (1981) wird die Bewertungsrelevanz von Unternehmensgrößen aufgezeigt. Fama, French (1992) verweisen auf die Bewertungsrelevanz des KGV, Buchwert/Marktwert-Relationen, der Unternehmensgröße, die vergangene Kursentwicklung sowie makroökonomische Variablen. "We find a significant relation between required rates of return and these measures after adjust- 
Die Sonderstellung, die den kleinen und mittleren Gesellschaften in Portefeuillehaltung und Risikobewertung beigemessen wird, hat dazu geführt, den Aktiengesamtmarkt auch in der Theorie segmentiert $\mathrm{zu}$ betrachten. Transaktionskostenunterschiede, ${ }^{322}$ ein ungleicher Informationsfluss ${ }^{323}$ und Mengenbeschränkungen am Kreditmarkt ${ }^{324}$,are the main motivation for the development of GCAPM." ${ }^{25}$ Dem Generalized CAPM nach gibt es nicht nur die eine Wertpapiermarktlinie des Gesamtmarktes mit unsicheren Anlagen, die alle vom rationalen Anleger gehalten werden. ${ }^{326}$ Vielmehr konzentrieren sich die unterschiedlichen Anleger jeweils auf bestimmte Aktien. Der Gesamtmarkt für unsichere Anlagen zerfällt mit der Parzellierung des Marktportefeuilles analog in einzelne Teilmärkte mit einer jeweils unterschiedlichen Bewertung des Rendite/Risiko-Profils von Einzelaktien. Es gibt somit nicht nur eine einzige CAPMWertpapiermarktlinie, sondern eine Vielzahl segmentspezifischer Wertpapiermarktlinien. $^{327}$ Kleinfirmenportefeuilles können durch die Marktsegmentierung im Verhältnis zum Marktportefeuille eine unabhängige Eigenbewegung erfahren. ${ }^{328}$ So können sich zum einen die bewertungsrelevanten Risiken $\tau$ je nach Marktsegment unterscheiden. Zum anderen können auch die Steigungen der einzelnen Wertpapiermarktlinien zwischen den Marktsegmenten differieren. Anlegerpräferenzen für bestimmte Segmente hindern dabei die Marktkräfte daran, die sich unterscheidenden Prämien pro übernommene Risikoeinheit zwischen den Segmenten einander anzugleichen. Prämieren Teilmärkte die Übernahme von Risiken in unterschiedlicher Höhe, muss dies bei einem segmentierten Gesamtmarkt nicht zwangsläufig Arbitrageure anlocken. Zwischen

ing for the Fama and French risk factors, and also after accounting for the effects of the stock price level." Brennan, Subrahmanyam 1996, 441. ,as a result of transaction costs, indivisibility of investment, or even the cost of keeping track of the new financial development of all securities, the $k_{\text {th }}$ investor decides to invest only in $n_{k}$ securities." Levy 1978, 644. ,... if all investors'information sets were complete, then the model would reduce to the standard Sharpe-Lintner-Mossin Capital Asset Pricing Model. Therefore, it is assumed that investors generally know only about a subset of the available securities and that these subsets differ across investors." Merton 1987, 488. „The Tobin-Sharpe-Lintner assumption of unlimited borrowing opportunity at the riskless rate ... are highly unrealistic.“ Markowitz 1990, 224.

Levy 1997, 137.

Da das CAPM von der Ausnahme friktionsloser Kapitalmärkte ausgeht, wird es als Spezialfall des generell von Transaktionskosten und Marktsegmentierung ausgehenden GCAPM betrachtet. 
den Teilsegmenten können Risikoprämien im GCAPM dauerhaft differieren. Aktien in vernachlässigten Segmenten können somit gleichermaßen unterbewertet wie auch mit einer vergleichsweise geringen Renditeerwartung verbunden sein.

Mit Hilfe des GCAPM lassen sich zwar die Folgen der Anlegerpräferenz für Standardwerte einerseits, die Venachlässigung kleiner und mittlerer Gesellschaften andererseits abbilden. Die Ursachen einer Segmentierung des Gesamtmarktes in beund übervorteilte Gesellschaften liefert allerdings auch das GCAPM nicht. Im Einzelnen bleiben folgende Fragen offen:

- Im Verlaufe eines Börsencrashs sind es insbesondere Standardwerte, die aufgrund des Klienteleffektes an Liquidität einbüßen. Doch warum erholt sich der Liquiditätsgrad der Blue Chips im Gefolge einer alle Marktsegmente betreffenden „asset deflation“, während für Small und Mid Caps die Unterliquidität ein dauerhaftes Phänomen darstellt? ? $^{329}$

- Institutionelle Anleger kommen dem Ideal des rationalen Anlegers am nächsten. Wieso meiden sie unterbewertete Small und Mid Caps? Diese Frage drängt sich um so mehr auf, als institutionelle Anleger in zunehmendem Maße zu einer längerfristigen Strategie des „Relationship Investing“6330 übergehen. ${ }^{331}$ Das Problem einer zeitlich erschwerten Veräußerbarkeit unterliquider Aktien müsste mit einer längeren geplanten Haltedauer von abnehmender Bedeutung sein; zumal Umschichtungen im Portefeuille auch nur schrittweise vollzogen werden.

- Um aus der Masse der am Sekundärmarkt Vernachlässigten herauszustechen, könnte eine Gesellschaft die Interne Verzinsung der unternehmerischen Investitionsobjekte einseitig erhöhen. Wäre infolgedessen nicht auch mit einer höheren Renditeerwartung für die Anleger zu rechnen? Zwar erhöht dies die Finanzierungskosten der vernachlässigten Gesellschaften, doch müssten nicht

328 Vgl. Beiker 1993, 94. Mit Hilfe eines differenzierten Blicks durch das GCAPM lässt sich theoretisch die Anomalie des Firmengrößeneffektes auflösen. Vgl. Levy 1997, 119.

Vgl. den Übergang von Szenario 3 zu Szenario 2 beziehungsweise 4 in Abschnitt 2.3.

Darunter ist eine langfristige Beteiligung in Form großer Aktienpakete samt Übernahme von Direktorenmandaten zu verstehen. Vgl. Schäfer 1997, 126.

Bushee (1998) deckt für den US-Markt eine grundsätzliche Langfristorientierung institutioneller Anleger auf. So argumentiert er, „relative to individual investors, the large stockholdings and sophistication of institutional investors allows them to monitor and discipline managers, ensuring that managers choose R\&D levels to maximize long-run value rather than to meet short-term earnings goals." Bushee 1998, 330. 
zumindest solche Unternehmen mit einem überdurchschnittlichen Cash FlowPotenzial auf diese Weise auf sich aufmerksam machen können?

- Die Differenz zwischen geforderter und erwarteter Rendite geht möglicherweise auf einen zu geringen Informationsstand der Anleger über den Fundamentalwert des unterliquiden Unternehmens zurück. Fraglich ist, warum ein von niveaubedingter Unterliquidität betroffenes Unternehmen die Renditeerwartung seiner Anleger nicht dadurch erhöht, dass es eine entsprechende Investor Relation-orientierte Informations- und Marketingstrategie betreibt. Warum ziehen sich Unternehmen unterliquider Aktien stattdessen vom Sekundärmarkt zurück und nehmen die negativen Auswirkungen etwa eines Delistings in Kauf?

Das CAPM samt seiner Modifizierungen lässt diese Fragen der Unterliquiditätsforschung unbeantwortet. "The questions we address ... have however been greatly overlooked by the traditional finance literature because this literature has almost exclusively focused on prices and stocks, rather than flows." ${ }^{332}$ Es drängt sich die grundsätzliche Frage auf, ${ }^{333} \mathrm{ob}$ "the paradigm of rational and optimal behavior must be largely discarded if knowledge in finance is to significantly advance. Others ${ }^{[334]}$ believe that most of the important empirical anomalies surrounding the current theory can be resolved within that traditional paradigm. ${ }^{935}$ Das Verlassen des in der Kapitalmarkttheorie vorherrschenden Paradigmas rationaler Wirtschaftssubjekte ist mit dem Einwand verbunden, ein dauerhaftes Phänomen wie niveaubedingte Unterliquidität würde langfristig nur dann Bestand haben können, wenn es rationalen Gesetzmäßigkeiten folgen würde. „Irrationalismen“336 und marktexogenen Faktoren mag dabei zwar eine katalysierende Wirkung zukommen, ${ }^{337}$ jedoch greifen solche Ansätze in einer zunehmend durchrationalisierten und liberalisierten Aktienwelt für die Entstehung niveaubedingter Unterliquidität zu kurz. Erst durch eine Erklärung des Phänomens im Rahmen des vorherrschenden Forschungsprojektes wird ersichtlich, wie es marktimmanent $\mathrm{zu}$ der geschilderten

Martin, Rey 2000, 1348.

Vgl. hierzu die Aufsätze in Hogarth, Reder (1986).

Unter ihnen befindet sich auch der Autor der vorliegenden Arbeit. Merton 1987, 484.

Es ist dabei insbesondere an Ansätze des Herding, des Noise-Trading sowie des Behavioral Finance zu denken. Vgl. Abschnitt 4.5.

337 Vgl. Abschnitt 4.5. 
divergenten Entwicklung der Börsensegmente kommen kann. ${ }^{338}$ Niveaubedingte Unterliquidität als Versagen des Preismechanismus $\mathrm{zu}$ begreifen, macht ferner theoretische Modelle wie das CAPM als Grundlage für wirtschaftspolitisches laissezfaire zu einer „unsicheren Rückzugsbastion“. Denn die Übervorteilung kleiner und mittlerer Gesellschaften ist dann weniger als exogen verursachtes Ungleichgewichts-, sondern vielmehr als marktimmanentes Gleichgewichtsphänomen zu verstehen. Der Auffassung, es seien ausschließlich „die Mängel des Steuerrechtes, des Gesellschaftsrechtes, des Börsenrechtes und die Eigentümlichkeiten des Kreditwesens und nicht irgendwelche systemimmanenten Fehler der Marktwirtschaft, die den Kapitalzutritt der nicht börsennotierten Unternehmen so erschweren“6339, ist damit allenfalls einschränkt beizupflichten.

Das CAPM ist mit seiner friktionslosen und markträumenden Gleichgewichtserklärung von Preisen am Aktienmarkt ein Geisteskind der frühen Nachkriegsjahre. ${ }^{340}$ Überkommen ist der Tenor des CAPM, das Verhalten der Anleger nach dem Risiko der Kapitalanlage als Teil eines Ganzen zu erklären, keinesweg. ${ }^{341}$ Ganz im Gegenteil erfährt das CAPM mit der Expansion der Aktienmärkte und der damit einhergehenden Probleme, die „Spreu vom Weizen zu trennen“, eine Renaissance. ${ }^{342} \mathrm{Um}$ das CAPM auch für diese zunehmende Selektionsproblematik fruchtbar $\mathrm{zu}$ machen, bedarf es allerdings einiger Modifizierungen. So sind im CAPM finanzwirtschaftliche Entscheidungs- und

338 Modellimmanente Faktoren wie etwa das Vorhandensein von Informationsasymmetrien sind mit dem Markt „,natürlicherweise“ verbunden. Sie lassen sich grundsätzlich nicht beseitigen, allenfalls reduzieren.

Gerke 1972, 16f. Ähnlich auch Albach, Lutter 1988, 17: „So hat das deutsche Aktienrecht mit seinen statischen Größenmerkmalen, unterstützt vom deutschen Börsenrecht, die Börse zum „Markt für Sparbücher“ entarten lassen. Aktienrecht und Börse dienen dem Bedürfnis der Sparer nach Mobilität und Sicherheit ihrer Anlage bei großen Aktiengesellschaften, die der Börse für ihre Finanzierungsaufgaben auch weitgehend entsagen könnten.“

„The philosophical roots of mean-variance, CAPM, and Efficient-Market-Hypothesis ... stemmed from the optimism of the early postwar years - the 1950 and 1960s. That was a time when people believed that men and women of good will could remake the awful world of the 1930s into a new world: well-behaved, functioning within clearly recognized standard deviations, and with generally accepted means to which matters would inexorably regress... In such a world, financial markets are always orderly and risk is recognizable, measurable, manageable, and containable. In such a world, in short, you have some sense of where the shocks will be coming from... In the wake of stunning losses of wealth, nominal as well as real, risk control became an explicit goal for the first time in many years - an occasion for which the new theories of portfolio management were made to order." Bernstein 1995, $10 \mathrm{f}$. Vgl. Unser 1999, 134-140.

342 Vgl. Kimball 2000, 3f.; Kapitel 6. „... the risk allocation function [of the financial system, d.A.] is rapidly evolving due to increased financial innovavtion." Mason 1995, 153. 
Informationsprobleme von Wirtschaftssubjekten allzu verkürzt enthalten. ${ }^{343}$ Das CAPM hinterlässt ein institutionelles Vakuum. Für sich genommen dient es zur Beschreibung der Realität deshalb nur eingeschränkt. Es bedarf gewissermaßen seiner Institutionalisierung. ${ }^{344}, \ldots$ I believe that even modest recognition of institutional structures and information costs can go a long way toward explaining financial behavior that is otherwise seen as anomalous to the standard frictionless-market model.“6345 Mit dem unbegrenzten und linearen Gleichlauf der erwarteten und der geforderten Rendite in Gestalt der markträumenden Wertpapiermarktlinie kann das CAPM nur als Ausgangspunkt weiterführender (neo-)institutionenökonomischer ${ }^{346}$ beziehungsweise neukeynesianischer ${ }^{347}$ - Überlegungen dienen. ${ }^{348}$ Von einem institutionenökonomischen Rationierungsansatz ist für die Erklärung niveaubedingter Unterliquidität im nächsten Kapitel Bereicherndes zu erwarten. Mikroökonomisch fundiert und auf dem Boden der neoklassischen Gleichgewichtstheorie verortet, ${ }^{349}$ spürte die (Neo-)institutionenökonomik bereits marktimmanente Rigiditäten in Preismechanismen an anderen Märkten auf: „Wenn schon auf Gegenwartsmärkten keine Gewähr für jederzeitiges Handeln zu markträumenden Preisen besteht, um wie viel weniger kann dies für Zukunftsmärkte angenommen werden. “350

\footnotetext{
$343 \quad$ "In the basic framework of Arrow and Debreu intermediaries or financial market institutions play no real role; there are no frictions for intermediaries to adress." Smith 1998, 452.

344 Vgl. Schmidt, Terberger 1997, 377f.

345 Merton 1987, 486f.

346 Einen Überblick geben Richter, Furubotn (1996); Löchel (1995); Feldmann (1995). Zu einer kritischen Auseinandersetzung mit der (Neo-)Institutionenökonomik und ihrer Abgrenzung vom Amerikanischen Institutionalismus vgl. Reuter (1994a,b).

347 "The New Keynesian Economics begins with Keynes' basic insights. But it recognizes the need for a more radical departure from the neoclassical framework, and for a much deeper study of the consequences of imperfections in capital markets, imperfections which can be explained by the costs of information." Greenwald, Stiglitz 1987a, 123. Zum rationierungstheoretischen Ansatz der Neuen Makroökonomik vgl. Kurz (1981).

348 Kap. 4 versucht sich an einer Synthese aus (neo-)institutionenökonomischen Erkenntnissen und dem neoklassischen Referenzmodell des CAPM. Dabei kann es aus methodischen Gründen streng genommen nur ein fehlerhafter Versuch sein, handelt es sich beim CAPM doch um ein in sich schlüssiges und damit nur unter Inkaufnahme von Ungenauigkeiten modifzierbares Modell.

Dugger bezeichnet die (Neo-)institutionenökonomik deshalb als „,branch of neoclassical orthodoxy" und als ,ideological defense of the status quo masquerading as economic science.“ Dugger 1989, 2. 
4 Niveaubedingte Unterliquidität

als Ergebnis einer

Kapitalrationierung am Sekundärmarkt 


\subsection{Ein Überblick}

Im statischen Gleichgewicht des CAPM halten rationale Anleger in ihrem effizient diversifizierten (Markt-)Portefeuille alle umlaufenden Aktien im Verhältnis ihrer fundamental richtig bewerteten Marktkapitalisierung. Niveaubedingte Unterliquidität spielt in einer solchen Modellwelt keine Rolle. Realiter sind viele Aktien am deutschen Sekundärmarkt hingegen dadurch gekennzeichnet, dass sie zum fundamentalen Kurs dauerhaft nicht in vollem Umfang nachgefragt werden. Diejenigen Anleger, die niveaubedingt unterliquide Aktien (ungeplantermaßen) im Portefeuille halten, finden - wenn überhaupt - einen Abnehmer nur unter Aufwendung mitunter empfindlicher Transaktionskosten. Bei einer fundamental richtigen Bewertung von Aktien ist das Eigenkapitalangebot seitens der Anleger dauerhaft geringer als das ausgeschüttete Aktienkapital. Eine sich damit einstellende Unterbewertung führt nicht automatisch $\mathrm{zu}$ einem steigenden Angebot an Eigenkapital. Grafisch stellte sich niveaubedingte Unterliquidität in einer konvexen oder sogar rückwärts geneigten Eigenkapitalangebotskurve dar. ${ }^{351}$ Es dokumentiert sich darin, dass ein Anstieg der geforderten Rendite nicht oder nur unterproportional zu einem Anstieg der erwarteten Rendite führt.

Um die nach oben inflexible Renditeerwartung zu erklären, wird in der vorliegenden Arbeit eine komparativ-statische Gleichgewichtsuntersuchung angestellt. Das markträumende Eigenkapitalangebot im CAPM-Gleichgewicht wird dem Eigenkapitalangebot in einem nicht-markträumendem Gleichgewicht gegenübergestellt. Es wird $\mathrm{zu}$ zeigen sein, dass die Unterinformiertheit der Eigenkapitalgeber rationalerweise zu einem eingeschränkten Eigenkapitalangebot in Form einer niveaubedingten Unterliquidität am unteren Ende des Aktienmarktes führen kann. ,[T]he difficulty of distinguishing good quality from bad is inherent in the business world; this may indeed explain many economic institutions and may in fact be one of the more important aspects of uncertainty. “" ${ }^{652}$ Der Annahmenkatalog des CAPM wird außerdem dahingehend erweitert, dass zur Informations- auch eine Ertragsasymmetrie zu Lasten der Eigenkapitalgeber tritt. Demnach sind Aktionäre nur unterproportional an den Investitionserträgen eines Unternehmens beteiligt. Nachdem die Eigenkapitalgeber mit einer Rendite auf satisfizierendem Niveau

$351 \quad$ Vgl. Abschnitt 3.2. 
bedient worden sind, fließen zusätzliche Einzahlungsüberschüsse der Investitionserträge in den „Organzational Slack ${ }^{6353}$ eines Unternehmens oder werden für X-Ineffizienzen an verschiedenen Stellen eines Unternehmens ausgegeben. Eine nur unterproportionale Partizipation der Eigenkapitalgeber an den Investitionserträgen ist insbesondere für Stakeholder-Ökonomien wie der deutschen von Relevanz.

Die Informations- und Ertragsasymmetrien (Abschnitt 4.2.2) zwischen den Prinzipalen und den Agenten bestehen im Rahmen einer zwischen ihnen relationalen Beziehung (Abschnitt 4.2.1). Unter diesen institutionellen Voraussetzungen sammeln sich am Sekundärmarkt für die Eigenkapitalgeber grundsätzlich gute wie auch schlechte Einzelrisiken, das heißt „plums“ und „lemons“ (Abschnitt 4.2.3). Dabei bemisst sich die Güte einer Gesellschaft für die Eigenkapitalgeber nach ihrer bewertungsrelevanten Risikohöhe. Es besteht für die rationalen Anleger bei der von Durchschnittswerten geleiteten Bildung seines Portefeuilles (Abschnitt 4.3.1) insofern ein Selektionsproblem. Unter Berücksichtigung adverser Selektionen (Abschnitt 4.3.2) und adverser Anreize (Abschnitt 4.3.3) stößt die zu erwartende Netto-Rendite der Anleger möglicherweise an eine Obergrenze, die sich unterhalb der markträumenden Renditeerwartung gemäß der Wertpapiermarktlinie befindet. In diesem Fall schränkt der renditemaximierende Eigenkapitalgeber sein Angebot dauerhaft ein (Abschnitt 4.3.4). Es entsteht eine Kapitalrationierung am Sekundärmarkt im langfristigen Gleichgewicht des Grundmodells. Die Unterinvestition der Eigenkapitalgeber am Sekundärmarkt wird durch bestimmte Modellerweiterungen teils ver-, teils entschärft (Abschnitte 4.3.5); auch geld- und fiskalpolitische Maßnahmen üben Einfluss auf das Ausmaß der Sekundärmarktrationierung aus (Abschnitt 4.4).

Im Falle einer Sekundärmarktrationierung ist das Einfordern einer markträumenden Rendite gemäß der Wertpapiermarktlinie in Teilen des Anlegerportefeuilles (Pools) mit einer sinkenden Netto-Renditeerwartung verbunden. Eine Rendite oberhalb der markträumenden Wertpapiermarktlinie zu fordern, kann dabei für ihn ebenfalls

„Als Ergebnis der Darstellung deutscher Übersetzungen des Begriffs „Organizational Slack“ kann festgehalten werden, daß keine der aufgeführten Übersetzungen überzeugt. Aus diesem Grund wird auch keine dieser Übersetzungen in dieser Arbeit verwendet." Scharfenkamp 1987, 43. $\mathrm{Zu}$ Definitionen vgl. ebenda, 22-44. 
irrational sein. Das in Aussicht-Stellen einer höheren Internen Verzinsung durch ein vernachlässigtes Unternehmen wird von rationalen Anlegern nämlich als das Signal eines „lemon“ gewertet. Bei einer markträumenden Renditeforderung muss im gewählten institutionenökonomischen Modellrahmen die Netto-Renditeerwartung somit weder zwingend linear noch unbegrenzt ansteigend verlaufen:

\begin{abstract}
"Today, the classical capital ideas are suspected of suffering from kurtosis, skewness, and other less familiar malignancies. They are under attack from non-linear hypothesis, overwhelmed by fears of discontinuities rather than pricing volatilities and factors, and frequently made irrelevant by exotic new financial instruments that come in unfamiliar shapes and hedge unfamiliar risks. As the mathematics that define these risks grows increasingly complex, the dimensions, contours, and limits of risk - are becoming correspondingly obscure. These developments do not mean that the classical concepts of finance were in some sense ,wrong“. The argument runs the other way. The effort to abandon the beautiful and coherent logic of the classical ideas reflects, not on the ideas, but on the changing environment in which we live." ${ }^{354}$
\end{abstract}

Zwar verliert der institutionenökonomische Ansatz „gegenüber der Neoklassik an Eleganz, erkauft sich aber damit eine Realitätsnähe, die neoklassische Modelle oft schmerzlich vermissen lassen.“" ${ }^{\text {355 }}$ So ziehen Anleger bei unvollkommenen Informationen über die Höhe der $\mathrm{zu}$ finanzierenden Risiken aus der Renditeforderung Rückschlüsse für die Bestimmung der Risiken. Der Preis für die Bereitstellung von Eigenkapital übernimmt neben seiner Funktion als Knappheitsindikator damit zusätzlich eine Anzeigefunktion für die Qualität des gehandelten Gutes. ${ }^{356}$ Wie auch an anderen Märkten ,,price serves a function in addition to that usually ascribed to it in economic theory: It conveys information and affects behavior. Quality depends on price. Of course, in standard economic theory, higher quality items will sell at higher prices: Prices depend on quality." 357 Die Höhe des geforderten Preises für das Bereitstellen von Eigenkapital bietet den unterinformierten Eigenkapitalgebern einerseits Hinweise zur Qualität, das heißt zur Höhe des Risikos der zu finanzierenden Gesellschaften. Die Höhe der

\footnotetext{
354 Bernstein 1995, 10. Ähnlich auch Smith 1998, 451: “And, indeed, it is now standard in analyses of unemployment or asset pricing to impose various kinds of nonconvexities or to impose a structure of markets that is, exogenously, incomplete." Dittus 1985, 79.

Rigide Marktpreise können endogen als Ergebnis der Annahme abgeleitet werden, dass Güter nicht homogen sind und Preise damit möglicherweise auch einen Einfluss auf die Qualität des gehandelten Gutes haben können. Vgl. Arrow (1963); Akerlof (1970). Stiglitz 1987, 3.
} 
Renditeforderung bestimmt aber auch andererseits die Interne Verzinsung der Unternehmensinvestitionen und damit auch die Art der unternehmerischen Investitionsobjekte. Auf diese Weise übt die Renditeforderung der Eigenkapitalgeber Einfluss auf die Qualität der $\mathrm{zu}$ finanzierenden Risiken aus. Die mit einer veränderten Renditeforderung einhergehende veränderte Risikosituation der zu finanzierenden Unternehmensinvestitionen findet dann in der NettoRenditeerwartung der Eigenkapitalgeber Berücksichtigung. Die geforderte Rendite reguliert also nicht nur die Quantität von Angebots- und Nachfragemengen. Rationale Anleger nutzen den Preis für die Überlassung von Eigenkapital bei Unterinformiertheit auch als Instrument, die Qualität unsicherer Unternehmensinvestitionen $\mathrm{zu}$,screenen“. Neben der Allokations- und der Informationsfunktion fällt der geforderten Rendite somit eine Anreiz- und Auslesefunktion $\mathrm{zu}^{358}$

Renditeforderungen haben einerseits einen eindeutig positiven, weil die Renditeerwartung steigernden Effekt auf die Höhe des Eigenkapitalangebots. Die Höhe der Renditeforderung beeinflusst aber auch andererseits die Höhe der bewertungsrelevanten Risiken $\tau_{\mathrm{n}}$. Die Renditeforderung bestimmt damit mittelbar wie unmittelbar die Netto-Renditeerwartung der Anleger sowie das Angebot an Eigenkapital: $A_{E K}=\left[\mu_{n}{ }^{\text {erw }}\left(\mu_{n}{ }^{\text {gef }} ; \tau_{n}\left(\mu_{n}{ }^{\text {gef }}\right)\right]\right.$. Eine Veränderung der Renditeforderung kann sich uneindeutigerweise expansiv oder restriktiv auf das Eigenkapitalangebot auswirken. Führt etwa ein Anstieg der Renditeforderung zu einer erwarteten Risikoerhöhung von hinreichender Stärke, resultiert daraus (scheinbar paradox) eine sinkende Netto-Renditeerwartung und - grafisch gesprochen - ein konvexer oder sogar rückwärts geneigter Verlauf der Eigenkapitalangebotskurve. Eine gemäß der CAPM-Wertpapiermarktlinie markträumende Rendite $\mathrm{zu}$ fordern, verschlechtert möglicherweise die Zusammensetzung der zu finanzierenden Einzelrisiken in einem $\mathrm{Maße}$, das eine Renditeforderung unterhalb der markträumenden rational erscheinen lässt. Der daraus resultierende Angebotsüberhang an Aktien stellt - wohlgemerkt! eine theoretische Möglichkeit dar, keine Zwangsläufigkeit. Das Bereitstellen von Verlauf der Kurven von Angebot und Nachfrage haben könnten, erscheint jenen als widersinnig, die im Geiste der klassischen Wirtschaftslehre erzogen wurden. Die Kurven sollen den Preis bestimmen. Wenn diese selbst den Markteinflüssen unterlägen, so würden die Preise aufhören, eindeutig festgelegt werden zu können. Anstelle eines Gleichgewichtes sähen wir 
Eigenkapital am Sekundärmarkt von markträumendem Umfang, wie es das CAPM impliziert, wird zwar theoretisch nicht ausgeschlossen. Aber auch andere nichtmarkträumende Gleichgewichtssituationen sind unter den genannten Bedingungen möglich. Ist die Netto-Renditeerwartung bei einer Renditeforderung unterhalb der markträumenden größer als bei der markträumenden Renditeforderung, schränken die Anleger ihr Eigenkapitalangebot also dauerhaft ein.

Vor dem Hintergrund realitätsnaher Annahmen (Informations- und Ertragsasymmetrien) stellt sich das Phänomen niveaubedingter Unterliquidität nicht als Marktanomalie dar. Darin, dass das Eigenkapitalangebot infolge einer inflexiblen (Netto-)Renditeerwartung möglicherweise dauerhaft hinter der Eigenkapitalnachfrage zurückbleibt, äußert sich vielmehr die Funktionsfähigkeit des Marktmechanismus. "The Law of Supply and Demand is not in fact a law, nor should it be viewed as an assumption needed for competitive analysis. It is rather a result generated by the underlying assumptions..."359 Wenn die erwartete NettoRendite den Sekundärmarkt wie im CAPM räumt, ist dies Ausfluss bestimmter modellspezifischer Annahmen. Markträumung ist jedoch keine generelle Eigenschaft real existierender Märkte. Sie ist Ausfluss der speziellen Annahme, dass Preise keine Auslese-, Anreiz- und Qualitätsanzeigefunktion übernehmen in einer Welt homogener Aktien von notorischer Güte und kostenloser Informationsbeschaffung. Dabei ist zu betonen, dass ein eingeschränktes Eigenkapitalangebot nicht auf eine monopolistische oder oligopolistische Angebotsstruktur zurückzuführen ist. Niveaubedingte Unterliquidität stellt sich vielmehr als das Ergebnis eines Marktversagens innerhalb einer polypolistischen Wettbewerbsstruktur mit einer Vielzahl von Eigenkapital anbietenden Prinzipalen und Eigenkapital in Höhe der ausgeschütteten Aktien „,nachfragenden“ Agenten dar; ohnehin lässt sich bei einer Einschränkung des Eigenkapitalangebots entlang einer Preis-Absatz-Kurve nicht von einer Rationierung der Nachfrageseite sprechen. Trotz der polypolistischen Marktstruktur sind die Eigenkapitalgeber nicht nur Mengenanpasser, die den Preis für die Überlassung von Eigenkapital als Datum nehmen. Bei unvollkommenen Informationen sind die Anleger an einem polypolistischen Sekundärmarkt Preisnehmer und -setzer zugleich. Einerseits halten die Wirtschaftssubjekte die

uns sich ständig verändernden Preisen gegenüber ... Die Performance einer Aktie kann die Performance der Aktiengesellschaft beeinflussen." Vgl. Soros 1998a, 39+41. 
Preise bewusst konstant auf nicht-markträumendem Niveau, andererseits besitzen die Preise in deren Mengenkalkül den Charakter exogen fixierter Daten. ${ }^{360}$ Zwar haben die Anleger keinen monopolistischen Preissetzungsspielraum, doch muss die markträumende nicht gleichzeitig auch die gewinnmaximale Rendite sein. Liegt letztere unterhalb ersterer, ist es als Eigenkapitalgeber einzelwirtschaftlich rational, weniger Rendite $\mathrm{zu}$ fordern und in den Sekundärmarkt dauerhaft weniger Eigenkapital zu investieren, als seitens der Unternehmen in Form der ausgeschütteten Aktien nachgefragt wird.

Dass das Eigenkapitalangebot dauerhaft die Eigenkapitalnachfrage unterschreitet, setzt zum einen eine mit steigender Renditeforderung hinreichende Verschlechterung der zu finanzierenden Risiken voraus. Zum anderen muss die Angebotskurve grafisch gesprochen - links von der Nachfragekurve liegen. ${ }^{361}$ Würde sich das Eigenkapitalangebot nämlich erst auf einem entsprechend höheren Renditeniveau rückwärts neigen, würde sich kein Nachfrageüberhang nach Eigenkapital einstellen. Abgesehen von zufälligen Gegebenheiten setzt die Existenz eines eingeschränkten Eigenkapitalangebots eine (ungefähre) Kenntnis der Eigenkapitalgeber darüber voraus, wie sich die Investitionstätigkeiten der zu finanzierenden Gesellschaften mit steigenden Renditeforderungen verändern. Die Erwartung einer Qualitätseinbuße bei steigender Renditeforderung impliziert ein Antizipieren der Eigenkapitalnachfrage durch die Anleger. Dies erfordert einen theoretischen Ansatz, der die Interdependenz zwischen dem Eigenkapitalangebots- und dem Eigenkapitalnachfrageverhalten berücksichtigt. Würde lediglich auf eine rückwärts geneigte Eigenkapitalangebotskurve abgestellt werden, ohne das Verhalten der nachfragenden Unternehmen zu berücksichtigen, könnte allenfalls eine notwendige, indes keine hinreichende Bedingung für die mögliche Existenz eines nicht markträumenden Angebots gefunden werden. So wird im Folgenden zu berücksichtigen sein, dass bei der Erklärung niveaubedingter Unterliquidität das Eigenkapitalangebot nicht zufällig hinter der „Eigenkapitalnachfrage“ am Sekundärmarkt, sondern als Reaktion auf das

$360 \quad$ Der scheinbare Widerspruch in den unterstellten Verhaltensweisen kann auch als Widerspruch in den unterstellten Marktformen gefasst werden. Einem alten Argument Arrows (1959) zufolge sind vollkommener Wettbewerb und die Fähigkeit der Akteure, durch ihre Entscheidung, die Preise zu verändern, unvereinbar. Im Modell der Neuen Makroökonomik scheint diese Unvereinbarkeit jedoch aufgehoben zu sein: Das individuelle Wirtschaftssubjekt fungiert einerseits als Preissetzer in einer Welt, die durch eine Art monopolistischen Wettbewerb gekennzeichnet ist, andererseits als Preisnehmer und Mengenanpasser in einer Welt vollkommener Konkurrenz. 
Verhalten der Nachfrageseite infolge einer veränderten Renditeforderung zurückbleibt.

Schränken Anleger ihr Angebot an Eigenkapital ein, ist die Art der rationierten Bereitstellung von Eigenkapital auf grundsätzlich zwei Wegen möglich (Abschnitt 4.5). ${ }^{362}$ Das eingeschränkte Eigenkapitalangebot kann sich auf alle Unternehmen gleichmäßig verteilen. Demnach fände eine Teilrationierung der Kapitalmenge pro Unternehmen statt (so genannte Typ I- bzw. proportionale Rationierung). ${ }^{363}$ Im Falle einer solchen Typ-I-Rationierung ließe sich der konvexe oder sogar rückwärts geneigte Verlauf der Eigenkapitalangebotskurve etwa auf eine abnehmende Grenzproduktivität des Eigenkapitals zurückführen. Diese produktionstechnische Erklärung greift jedoch zu kurz, wird nicht das Kapitalvolumen pro Unternehmen, sondern - wie im Fall niveaubedingter Unterliquidität am deutschen Aktiensekundärmarkt - die Anzahl der Unternehmen rationiert, denen Eigenkapital zuteil wird. In diesem Fall konzentriert sich das Eigenkapitalangebot auf die Aktien der einen Unternehmen, während die Aktien der anderen Unternehmen vernachlässigt werden. Man spricht von Voll-, Typ-II-, 0/1- oder von prioritärer Rationierung. ${ }^{364}$ Niveaubedingte Unterliquidität kleiner und mittlerer Gesellschaften stellt sich demnach als eine nash-gleichgewichtige Vollrationierung am Sekundärmarkt dar. ${ }^{365}$ Das nicht-markträumende Gleichgewicht einer dauerhaft niveaubedingten Unterliquidität geht in diesem Fall zu Lasten bestimmter

$361 \quad$ Vgl. Abbildung 3.3 in Abschnitt 3.2.

362 Es ist Vorsicht geboten beim Rationierungsbegriff: Punkte auf der Angebots- und Nachfragekurve implizieren nicht einfach unterschiedliche Kapitalvolumina bei unterschiedlichen Renditeforderungen, sondern sie beinhalten auch unterschiedliche Produktqualitäten. Vgl. Schlick 1994, 89. Es wird in der Literatur deswegen sogar die Meinung vertreten, bei unterschiedlicher Qualität könne von Rationierung gar nicht die Rede sein. Vgl. Milde, Riley 1988, 107. Vgl. Keeton (1979). Vgl. a. Stiglitz (1970, 1972); Freimer, Gordon (1960); Jaffee (1971); Stigler (1967).

364 Die Grundidee der Rationierung vom Typ II geht im Wesentlichen zurück auf Jaffee, Russell (1976), Keeton (1979) und insbesondere Stiglitz, Weiss (1981). In Anlehnung an Definitionen zu Rationierungen am Fremdkapitalmarkt vgl. Jaffee, Stiglitz 1990, 847-849. „Credit rationing is broadly defined as a situation in which there exists an excess demand for loans because quoted loan rates are below the Walrasian market-clearing level. There are a number of different types of credit rationing depending on how excess demand is defined, on whether the excess demand is temporary or continuing, and most importantly on the factors that cause the loan rate to be depressed."

365 Unter einem Nash-Gleichgewicht versteht man ein (spieltheoretisches) Gleichgewichtskonzept, das eine Situation beschreibt, in der kein Akteur seinen Nutzen durch abweichendes Verhalten erhöhen kann, sofern alle anderen Akteure bei ihren Strategien bleiben. Vgl. Feess 1997, 4758. $\mathrm{Da}$ es sich um eine dynamische Entscheidungssituation bei asymmetrischer Informationsverteilung handelt, müsste streng genommen von einem perfekten Bayesianischen Gleichgewicht die Rede sein. Vgl. ebd. 386ff. 
Unternehmensgruppen. ${ }^{366}$ Da für Anleger das Rendite-Risiko-Profil einzelner Aktien bei asymmetrischen Informationen nicht erkennbar ist, bedarf es zur Rationierung bestimmter Aktien anderer Auswahlkriterien. Nach Kriterien wie etwa der Unternehmensgröße, dem Medienstanding, der Branche und dem Börsensegment ausgewählt, werden Aktien bestimmter Unternehmenstypen gar nicht, andere voll rationiert. ${ }^{367}$ Mit einer pauschalen Kategorisierung nach solch „äußeren“ Merkmalen tritt eine Bewertung einzelner Aktien nach ihren spezifisch zu erwartenden RenditeRisiko-Profilen in den Hintergrund. Den Aktionären aufgrund unvollkommener Informationen hinsichtlich des erwarteten Risiko-Rendite-Profils identisch erscheinende Unternehmen können dabei unterschiedlich behandelt werden. Wird den Einen Eigenkapital in Höhe des emittierten und fundamental richtig bewerteten Aktienkapitals zuteil, werden die Anderen vollends vernachlässigt. Das Hinzuziehen von solchen „sekundär“-ökonomischen Selektionskriterien zur Rationierung der Eigennachfrage stellt nicht notwendigerweise ein Abrücken vom gewinnmaximierenden Kalkül der Eigenkapitalgeber dar. Eine Unterscheidung nach diesen sekundär-ökonomischen Kriterien mag auf Durchschnittswerten erfolgen, die sich in einer ex-post Betrachtung als langfristig gewinnmaximierend herausgestellt haben. Wird dabei ein ganzer Pool von Unternehmen, deren erwartetes Rendite/Risiko-Profil sich für die Anleger von Rendite/Risiko-Profilen anderer Unternehmensgruppen unterscheidet, kategorisch rationiert, spricht die Literatur von „redlining“. 368 In einer durchschnittlichen Betrachtung aller Unternehmen einer Renditeklasse (Poolbildung) werden unter den genannten Bedingungen auch solche Aktienwerte vernachlässigt, die bei vollkommenen Informationen den „primären“ ökonomischen Kriterien von Risiko und Ertrag zufolge ein vergleichsweise noch gutes Ergebnis erwarten lassen. Aufgrund ihrer Zugehörigkeit zu einer nach sekundären Kriterien bestimmten Unternehmensgruppe droht jedoch auch diesen

366 In ihrer Erscheinung als Typ-II Rationierung lässt sich niveaubedingte Unterliquidität deshalb nur unbefriedigend auf eine „natürliche“ Rate im Rahmen von Suchtheorien zurückführen. Vgl. Heise 1996, 68. Denn warum sollte in Segmenten für Small und Mid Caps mehr und länger gesucht werden als in den Segmenten der Standardwerte?! Teilsegmente können auch nach räumlichen Kriterien unterschieden werden. So sind von Kapitalrationierungen bereichernde Einblicke internationaler Kapitalflüsse zu erwarten, wie etwa für die fundamental kaum zu erklärende andauernde außenwirtschaftliche Schwäche des EURO; einer jungen Währung mit einer geringen Reputation der ihn stützenden Organisation. Zur anhaltenden Unterliquidität zukunftsträchtiger Finanzplätze vgl. etwa Cambié 1995, 54f. Während in der vorliegenden Arbeit bestimmte Unternehmenstypen in einer Renditeforderungsklasse rationiert werden, versteht man unter ,red-lining“ den Fall einer Rationierung aller Gesellschaften einer solchen Klasse. Vgl. Stiglitz, Weiss 1981, 406f.; Jaffee, Stiglitz 1990, 859f. Dabei müssen nicht die (netto)ertragsstärksten Klassen mit Kapital bedacht werden. Vgl. Wette (1983). 
guten Einzelrisiken, „,in einen Topf geworfen“ und - aus Gründen einzelwirtschaftlicher Rationalität - am Sekundärmarkt diskriminiert, das heißt rationiert zu werden. Eine pauschale Übervorteilung ganzer Unternehmensgruppen, losgelöst von einer fundamentalen Einzelfallbetrachtung, führt dazu, dass auch gute Einzelrisiken in die „Unterliquiditätsfalle“ einer nach oben rigiden NettoRenditeerwartung geraten. Sich daraus ergebende Finanzierungsbeschränkungen können dabei langfristig im Sinne einer „self-fullfilling-prophecy“ zu einer tatsächlichen Verschlechterung ursprünglich guter, aber vernachlässigte Einzelrisiken führen. Umgekehrt mögen sich bei einer Konzentration der Anleger auf Standardwerte unter ihnen auch „schlechte“, das heißt deutlich unterhalb der Wertpapiermarktlinie befindliche Einzelrisiken befinden. Eine diskriminierende Form der Kapitalrationierung mag zwar für die Anleger einzelwirtschaftlich rational, für die Gesamtwirtschaft hingegen nicht optimal sein. Denn infolge eines nach oben rigiden Preismechanismus impliziert Vollrationierung sowohl eine Unterinvestition der Eigenkapitalgeber am Sekundärmarkt insgesamt als auch eine Fehlallokation des Eigenkapitals innerhalb des Aktiengesamtmarktes. Das Gefangenendilemma ${ }^{369}$ einer Vollrationierung am Sekundärmarkt ruft deshalb die Wirtschaftspolitik auf den Plan (Kapitel 5). 
4.2 Institutionenökonomischer Modellrahmen 


\subsubsection{Die Anteilseignerschaft als relationaler Vertrag}

Der Vertrag, der mit dem Erwerb einer Aktie zwischen dem Unternehmen als Aktienemittent und seinen Anteilseignern zustande kommt, erfüllt wesentliche Elemente eines „,idealtypischen“ Vertrages ${ }^{370}$ : Leistung (Zahlung des Aktienpreises an einen Dritten) und Gegenleistung (Gewährung gesetzlicher und satzungsmäßiger Rechte wie etwa die Teilnahme an der Hauptversammlung etc.) sind ex ante weitgehend festgelegt; Aktienkurs und Handelsvolumen sowie Börsenplatz und Zeitpunkt der Transaktion sind eindeutig spezifiziert und gegenüber Dritten verifizierbar; es herrscht weitgehende Annonymität, persönliche Beziehungen zwischen den Kontraktparteien spielen keine Rolle; die Eigenkapitalgeber verfügen über alternative Vertragspartner, eine Abwanderung der Anleger zur nächstbesten Alternative ist $\mathrm{zu}$ relativ geringen Transaktionskosten möglich; auch der Aktienemittent ist nicht abhängig von einzelnen Kleinaktionären. Die Renditeforderungen der Anleger sind zwar nicht Bestandteil eines zwischen Emittenten und Anteilseignern zustande kommenden Vertrages. Doch wird den Unternehmensmanagern die (ungefähre) Höhe der geforderten Rendite direkt über den Kontakt mit seinen Aktionären etwa zu Hauptversammlungen oder indirekt durch das „voting by feet“ der Anleger offenbar. Für die Anleger ist zum Zeitpunkt der Bereitstellung von Eigenkapital hingegen unklar, ob ihren Renditeforderungen in Form einer entsprechenden Internen Verzinsung der unternehmerischen Investitionsobjekte nachgekommen wird. Ein weiterer Unsicherheitsfaktor besteht für die Anleger darin, dass das Risiko der zu finanzierenden Unternehmensprojekte für sie nicht zu erkennen ist. Mangels direkter Eingriffsmöglichkeiten besteht für die Anleger diese Unklarheit über das zu erwartende Rendite/Risiko-Profil über den gesamten Zeitraum der Bereitstellung von Eigenkapital fort. ${ }^{371}$ Rechtlich ist die „Vertragslücke“ hinsichtlich der angestrebten Höhe der zu erwirtschaftenden Rendite

$370 \quad$ Vgl. Eger 1995, 13f.

371 Die vorliegende Arbeit reiht sich ein in die weit zurück reichende Thematik der Trennung von Eigentum am Unternehmen und seiner Kontrolle. „The directors of such a [joint-stock] companies, being the managers rather of other people's money than of their own, it cannot well be expected, that they should watch over it with the same anxious vigilance with which the partners in a private copartnery frequently watch over their own. Like the stewards of a rich man, they are apt to consider attention to small matters as not for their master's honour, and very easily give themselves a dispensation from having it. Negligence and profusion, therefore, must always prevail, more or less, in the management of the affairs of such a company" Adam Smith 1776, 304. Einen Überblick über die Prinzipal-Agenten-Theorie bieten Shleifer, Vishny (1997a). Vgl. a. die bahnbrechenden Aufsätze von Berle, Means (1932); Jensen, Meckling (1976). 
sowie der Güte des zu finanzierenden Risikos zu Lasten der Eigenkapitalgeber geregelt. Zwar gewähren Aktiengesellschaften ihren Anteilseignern diverse Möglichkeiten einer Mitwirkung auf das Unternehmensergebnis über die Hauptversammlung und den Aufsichtsrat; ${ }^{372}$ dabei hängt das Ausmaß der Partizipationsmöglichkeiten auch von der Art der ausgegebenen Aktientitel ab. ${ }^{373}$ Doch ist das Recht auf Gewinnbeteiligung einer jeden Aktie letztlich ein nur residuales. $^{374}$ Nach der Begleichung aller Kontraktforderungen wie etwa Lieferantenforderungen, Zinsen, Löhne, Gehälter, Abgaben und Steuern fällt (Stamm-)Aktionären der etwaige variable Rest des Bilanzgewinns zu. Dieser erfolgt in ausgeschütteter Form durch die Zahlung von Dividenden oder bei Gewinnthesaurierung und Wiederanlage im Unternehmen durch entsprechende Aktienkurssteigerungen, sofern der Sekundärmarkt bewertungs- und informationseffizient ist. ${ }^{375}$ In Anbetracht dieses nur residualen Dividendenrechts sind Unternehmen keineswegs gegenüber ihren Eigenkapitalgebern verpflichtet, die von ihnen geforderte Rendite bei Eingehen eines bestimmten Investitionsrisikos durch eine entsprechende Interne Verzinsung $\mathrm{zu}$ erwirtschaften. Die Anteilseignerschaft an einem Unternehmen verbindet sich zunächst lediglich mit der Hoffnung der Anleger auf eine Gewinnentstehung und -verwendung, die eine gemäß der CAPM-Wertpapierlinie marktgerechte „Verzinsung“ zeitigt. Insofern sind Renditeforderungen der Aktionäre kein Parameter, den der Aktienemittent in sein unternehmerisches Investitionsgebaren einzubeziehen rechtlich verpflichtet ist. Ganz im Gegenteil legt die zeitliche Abgeschlossenheit des oft annonymen

$372 \quad \mathrm{Zu}$ nennen sind das Partizipationsrecht an einer ordentlichen Jahreshauptversammlung, ein (eingeschränktes) Auskunftsrecht, Stimmrechte bei der Bestellung der Mitglieder des Aufsichtsrates, der Verwendung des Bilanzgewinns, der Entlastung des Vorstands und des Aufsichtsrates, bei der Bestellung der Abschlussprüfer, bei Satzungsänderungen, bei der Kapitalbeschaffung und -herabsetzung, der Bestellung von Prüfern zu Vorgängen bei der Gründung, der Geschäftsführung sowie bei der Auflösung der Gesellschaft. Vgl. Grill; Perczynski 1988, 299-303.

Im Vergleich zu den gewöhnlichen gesetzlichen und satzungsgemäßen Rechten einer Stammaktie gewähren Vorzugsaktien Mehrfachstimmrechte, Dividendenvorzüge (kumulative Vorzugsaktie; Prioritätsaktie) oder Vorzüge beim Anteil am Liquidationserlös. Vgl. a. Abschnitt 5.2.

„Die Aktionäre haben Anspruch auf den Bilanzgewinn, soweit er nicht nach Gesetz oder Satzung, durch Hauptversammlungsbeschluß nach Absatz 3 [Bildung offener Rücklagen oder Gewinnvortrag, d.A.] oder als zusätzlicher Aufwand auf Grund des Gewinnverwendungsbeschlusses von der Verteilung unter die Aktionäre ausgeschlossen ist.“ (§58 Abs. 4 AktG)

375 Mit Modigliani, Miller $(1958,1961)$ hat die Gewinnausschüttung keine Auswirkungen auf die Aktienrendite, sofern ein friktionsloser Markt vorliegt und die Investitionstätigkeit der Unternehmen unverändert bleibt. Dass faktisch die Höhe der Dividendenrendite relevant ist für die Aktienrendite, wirft das sogenannte „dividend puzzle“ auf. Vgl. Black (1976). Zu einer neueren Untersuchung vgl. La Porta, Lopez-de-Silvanes, Shleifer, Vishny (2000). 
Kapitaltransfers über den Primärmarkt die Vermutung nahe, dass es sich bei der Beschaffung von Eigenkapital über die Börse um eine allein punktuelle SpotTransaktionen handelt ohne Verpflichtung seitens der Unternehmen für ein bestimmtes künftiges Investitionsgebaren. Zwar bemisst sich der Emissionserlös - an einem vollkommenen Finanzmarkt - nach der Höhe der künftigen Einzahlungsüberschüsse aus den geplanten Unternehmensinvestitionen; ob diese auch realisiert werden, ist zwischen dem Emittenten und den Anteilseignern vertraglich nicht geregelt. Das unbefristet haftende Eigenkapital steht dem Emittenten, das heißt seinen Unternehmensmanagern rechtlich gesehen zur freien Verfügung. Insofern erscheinen Sekundärmärkte für die Emittenten als eine Art „side-show“, die von realwirtschaftlichen Unternehmensentscheidungen dichotomisch getrennt zu sehen ist. Und der Sekundärmarkt ist lediglich ein Ort, an dem die Rechte aus der Anteilseignerschaft an einer Aktiengesellschaft gehandelt werden. So gesehen reduziert sich die Beziehung zwischen dem Emittenten und seinen Eigenkapitalgebern auf eine punktuelle Spot-Transaktion am Primärmarkt.

Wenn sich Aktiengesellschaften dennoch nach den Vorgaben des Sekundärmarktes richten, verweist dies auf ihre Absichten, den Aktienmarkt dauerhaft auch für Folgeemissionen zu nutzen. ${ }^{376}$ Will ein Unternehmen einen hohen Abschlag vom Wert künftiger Emissionen vermeiden (Underpricing), wird nämlich das Erwirtschaften einer marktgerechten „Verzinsung“ des einmal bereitgestellten Eigenkapitals in jeder Periode aufs Neue zu einer notwendigen, wenn auch keiner hinreichenden Bedingung. ${ }^{377}$ Mit der Ausweitung langfristig haftenden Eigenkapitals senkt ein Unternehmen faktisch das am Sekundärmarkt bewertungsrelevante Risiko. Eine dadurch abnehmende Renditeforderung senkt einerseits die Eigenkapitalkosten für das Unternehmen. Andererseits sinkt auch der Risikoaufschlag bei einer Fremdfinanzierung; womit die Wahrscheinlichkeit kreditrationiert zu werden,

376 Selbstverständlich trifft dies nur auf jene Unternehmen zu, welche langfristig expandieren und etwaige Verlustsituationen temporärer Art nicht zu kaschieren gedenken. Aus analytischen Gründen ist dies die Gruppe von Unternehmen, die Gegenstand der Untersuchung ist. Denn schließlich soll gezeigt werden, dass selbst Unternehmen mit einer erfolgreichen Ausrichtung der Investitionstätigkeit auf die Renditeforderungen der Anleger am Sekundärmarkt diskriminiert werden können.

377 Ob sich ein Unternehmen an die Vorgaben des Sekundärmarktes hält, vermögen Aktionäre nur ex post zu beurteilen. Da Eigenkapitalgeber relativ unterinformiert sind und auf die Verwendung ihres zur Verfügung gestellten Kapitals nur eingeschränkten Einfluss haben, wird für sie wichtig, ob Unternehmen die in der Vergangenheit geforderte Rendite verfehlten oder tatsächlich real erwirtschafteten. Vergangenes Verhalten der Unternehmen findet somit Eingang in die begrenzt rationalen Erwartungen der Aktionäre. 
obendrein abnimmt. Weitere Gründe für eine Ausrichtung der unternehmerischen Investitionstätigkeit an den Renditeforderungen der Anleger und einem folglich hohen Kursniveau bestehen für die Manager des Emittenten darin, dass sich ihre eigenen Leistungen am Barometer des Aktienkursverlaufs messen lassen. Der Chart einer Aktie wird dadurch $\mathrm{zu}$ einer kostengünstigen Form einer „costly state verification ${ }^{6378}$ am Arbeitsmarkt der Agenten bei unvollkommenen Informationen. Außerdem wird die Vergütung von Managern oft an den Aktienkurs des Unternehmens gekoppelt, um eine Interessenkonformität zwischen den Prinzipalen und den Agenten herzustellen. Das Geschehen am Sekundärmarkt ist deshalb auch direkt für das Einkommen der Manager von Bedeutung. Mit dem Ziel, die Motivation der Mitarbeiter im Sinne der Anlegerinteressen zu steigern, kann auch ihr Einkommen etwa in Form von Mitarbeiteraktien an den Aktienkurs gekoppelt werden; womit sich bei steigenden Aktienkursen Lohnkosten einsparen lassen. Schließich ist der Aktiensekundärmarkt für Emittenten auch deshalb von Bedeutung, weil das Renommé eines Unternehmens mit seiner Aktienkursentwicklung steigt; was sich auch auf das Konsumentenverhalten nach den vom Unternehmen angebotenen Produkte entsprechend positiv auswirkt. Ferner beugt das Angleichen der Internen Verzinsung von Unternehmensinvestitionen an die Renditevorgaben der Anleger dem Arbitrageprozess einer feindlichen Übernahme samt anschließender Veräußerung des in seine Einzelteile zerlegten Unternehmens vor. Angesichts dieser Vielzahl von Anreizen für das Management eines Aktienemittenten ist das Nachkommen der Aktionärsforderungen im Zuge einer allgemeinen Shareholder Value-Orientierung ${ }^{379}$ von Bedeutung. Diese Anreize führen grundsätzlich zu einer Wahrung der Renditeinteressen der Prinzipale durch die Agenten. Das „voting by

378 Townsend 1979, 265.

379 Vgl. die zu einem „Klassiker“ avancierte Monografie von Rappaport (1986). Der Shareholder Value ist der finanzielle Wert des Eigenkapitals eines Unternehmens für den Eigentümer, das heißt der Ertragswert des Eigenkapitals. Dieser Ertragswert ist nach den Methoden der dynamischen Investitionsrechnung aus den - unter Berücksichtigung der Unsicherheit der Schätzung - in der Zukunft vermutlich erzielten Einzahlungsüberschüsse, die international heute allgemein als Cash-Flow bezeichnet werden, zu ermitteln. Nach der Ausrichtung des Unternehmensgebarens auf den Eigentümerwert, entspricht der „Shareholder Value“ dem mit den Kapitalkosten diskontierten Barwert der Operativen Cash Flows innerhalb eines überschaubaren Zeitraumes plus anschließendem Restwert des Unternehmens abzüglich des Marktwertes des Fremdkapitals. Dabei sind die Eigenkapitalkosten dem CAPM zu entnehmen. Zur Maximierung des Eigentümerwertes respektive Shareholder Value nach der Discounted Cash Flow-Methode vgl. Rappaport 1995, 53-80; Perridon, Steiner 1999, 16; Michel 1996, 7998; Busse von Colbe (1997); Bühner (1994); Hansen 1996a, 48-50; Bea (1997). Mit dem Begriff der Shareholder Value-Orientierung gelangte die Maximierung des Eigentümerwertes eine Renaissance. Neu am Shareholder Value-Ansatz ist die Verwendung des Ertragswertes zur 
feet" der Anleger zu geringen Transaktionskosten birgt für das Management deshalb ein gewisses Drohpotenzial. Der formal nur residuale Gewinnanspruch der Anteilseigner wird durch die Abhängigkeit des Managements von liquiden und fundamental richtig bewerteten Aktien faktisch aufgewertet. „Jeder erfolgreiche Börsengang erfordert den langfristigen Aufbau und die Pflege der Beziehungen zu potenziellen Investoren und externen Analysten. Die Finanzkommunikation vor, während und nach dem Börsengang ist wesentlicher Bestandteil des Gelingens einer Emission. Neben obligatorischen Kommunikationsmaßnahmen wie die Durchführung von Hauptversammlungen, Ad-hoc-Mitteilungen und Veröffentlichungen von Geschäfts- und Zwischenberichten sind freiwillige Maßnahmen wie Pressekonferenzen oder Road-Shows zu nennen. “‘380

Aus diesen Gründen ist das Verhältnis zwischen den Emittenten und seinen Anteilseigner durch eine gegenseitige Abhängigkeit geprägt. Die Anteilseignerschaft an einer Aktiengesellschaft ist folglich weniger mit einem klassischen, idealtypischen Vertrag zu beschreiben. ${ }^{381}$ Bei einer Anteilseignerschaft handelt es sich vielmehr um einen komplexen Langzeitvertrag ${ }^{382}$ mit einer relationalen Beziehung zwischen dem Eigenkapitalnehmer und seinen - sich wohlgemerkt ändernden - Eigenkapitalgebern. ${ }^{383}$ Langzeitverträge lassen sich nicht durch seine Laufzeit von kurz- oder mittelfristigen Verträgen abgrenzen. Bei Unklarheit über das zu finanzierende Rendite/Risiko-Profil einerseits und über die Dauer der Kapitalbereitstellung andererseits handelt es sich vielmehr um einen Vertrag, dessen Leistung (Bereitstellen von Eigenkapital) und Gegenleistung (marktgerechte

strategischen Steuerung und Kontrolle ganzer Unternehmen, einzelner Konzerngesellschaften und Geschäftsbereiche. Vgl. Busse von Colbe 1997, 274. Scholz 2000, 520 .

Auf der Grundlage von Macneil (1974) unterscheiden Goldberg (1976) und Williamson (1976) den relationalen vom klassichen Vertrag für den Bereich der Wirtschaftswissenschaften. Macneil's erkenntnisleitendes Interesse geht dahin, das reale Verhalten beim Abschluss und während der Durchführung von Verträgen mit dem juristischen Konzept des Vertrages in Einklang zu bringen. Das ausschließlich am diskreten Leistungsaustausch orientierte klassische und neoklassische Vertragsrecht versagt für ihn regelmäßig angesichts der sozialökonomischen Entwicklung hin zur postindustriellen Dienstleistungsgesellschaft, in der die Beziehungen zwischen den Vertragspartnern von Belang sind. Vgl. Jickeli 1996, 28f; Kern (1992).

„Der komplexe Langzeitvertrag hat viele Erscheinungsformen und daher existiert, soweit ich sehe, auch noch keine präzise Definition.“ Niklisch 1987, 17. Vgl. Eger 1995, 19-62.

Unter relationale Beziehungen fällt etwa die laufende Geschäftsverbindung, die fortgesetze Lieferung, das Dauerschuldverhältnis, der symbiotische Vertrag, der Organisationsvertrag und das Konzept des Vertragsnetzes. Vgl. Jickeli 1996, 23-27. Der Begriff der Relationalität findet Anwendung in verbal-analytischen Untersuchungen. In formalen Optimierungsmodellen spricht man synnonym von ,unvollständigen Verträgen“. Vgl. Richter, Furubotn 1996, 173175. 
Verzinsung) zeitlich auseinanderfallen. Im Rahmen eines relationalen Vertrages wandelt sich somit der do ut des-Mechanismus von einer zeitpunkt- in eine zeitraumbezogene Austauschbeziehung, deren Existenz stets bedroht ist, da sie auf eine beiderseitige Kooperation angewiesen ist. Um sich gegenüber den Eigenkapitalgebern kooperativ verhalten zu können, müssen die Präferenzen der Anleger definiert werden. Da die Ermitlung aller einzelnen Anlegerpräferenzen, aber auch deren Aggregation, Schwierigkeiten für die Manager bereitet, wird mit der Maximierung des Unternehmenswertes ein abgeleitetes Unternehmensziel herangezogen. ${ }^{384}$ Was die Gewinnmaximierung in statischer ist in dynamischer Hinsicht die Wahl eines unternehmerischen Wachstumspfades, der bei Konstanz des eingesetzten Eigenkapitals den diskontierten Einzahlungsüberschuss von Unternehmensinvestitionen maximiert. ${ }^{385}$ An einem effizienten Sekundärmarkt spiegelt sich diese Unternehmenswertmaximierung dann spiegelbildlich in einem maximalen Eigentümerwert beziehungsweise Shareholder Value wider. ${ }^{386}$

Die Maximierung des Unternehmens- beziehungsweise Eigentümerwertes setzt zweierlei voraus: Zum einen sind solche Investitionsobjekte auszuwählen, deren erwartete Rendite/Risiko-Profile sich auf oder oberhalb der CAPMWertpapiermarklinie befinden. ${ }^{387}$ Zum anderen bedarf es eines vollständigen Partizipierens der Anleger an jeder zusätzlichen Einheit erwirtschafteten discounted cash flows - nach Begleichen der Kontraktforderungen aller anderen Unternehmensbeteiligten (Stakeholder). Das Ausrichten des unternehmerischen Investitionsgebarens auf die Interessen der Eigentümer bedeutet somit, dass alle Stakeholder eines Unternehmens durch vertraglich fixierte Ansprüche ausreichend beachtet sind und dadurch aus dem Prozess der Investitionsauswahl und seiner Ertragsverteilung ausgeschlossen sind. Im Zuge einer Maximierung des Eigentümerwertes finden weder Entscheidungs- noch Verteilungskämpfe zwischen

Vgl. Hermann 1998, 3; Copeland, Koller, Murrin (1998).

Zur Kapitalwertmethode vgl. Schmidt, Terberger 1997, 128-138 sowie 152-168.

Unter Arrow-Debreu-Annahmen (vgl. Feess 1997, 297-322) ist die Maximierung des Unternehmenswertes pareto-effizient. Steinherr (1998) begrüßt die Amerikanisierung der Finanzierung als fortschrittliche Demokratisierung, als Zugewinn an Freiheit für die Individuen gegen den oppressiven Staat. Aus dem Blickwinkel der Anteilseigner ist dem - zunächst! - in einer Arrow-Debreu-Welt nichts entgegenzusetzen.

Die gesamte Investitionstätigkeit eines Unternehmens umfasst mehrere Teilprojekte. Die Ertragsverteilung für das (Gesamt-)Investitionsprojekt ergibt sich aus der gewogenen Ertragsverteilungen der Teilprojekte. In Abhängigkeit von der Kovarianz der Ertragsverteilungen der Teilprojekte kann sich ein risikomindernder Effekt in der unternehmerischen Gesamtinvestition einstellen. 
den Unternehmensbeteiligten zu Lasten der Anteilseigner statt. „The expected return to an equity investor ... would be exactly the same as the expected return to the projects itself..." ${ }^{988}$ Erst eine Ausrichtung der unternehmerischen Investitionstätigkeit in genannter allokativer wie distributiver Hinsicht maximiert den Unternehmenswert im Sinne der Shareholder. Werden die Aktionärsforderungen durch das Unternehmensmanagement auf diese Weise vollständig erfüllt, „equity finance is free from adverse selection and moral hazard effects“. ${ }^{389}$ Außerdem dokumentiert sich in der Umsetzung der Eigenkapitalgeberinteressen durch die Unternehmensmanager, dass ,[e]quity capital can finance those risky, productive borrowers for whom asymmetric information is acute, while banks concentrate their finance on the well-established, safe borrowers.“ De Meza und Webb (1987) sind folglich der Auffassung, die Überschussnachfrage nach Kapital bei Vorliegen von Kapitalrationierung an den Fremdkapitalmärkten könne durch die Bereitstellung von Eigenkapital geschlossen werden: „With equity finance the underinvestment property of the Stiglitz-Weiss model disappears, and social efficiency obtains. “390 Denn "the entrepreneur and his financier unanimously agree on the choice of a project,... Thus the share contract eliminates the conflict of interest which arises under debt finance. Consequently, in equilibrium there can be no rationing in the market for share contracts." ${ }^{391}$ Doch fällt die Auswahl von Unternehmensinvestitionen wirklich in Übereinstimmung zwischen den Prinzipalen und den Agenten?

\footnotetext{
$388 \quad$ Cho $1986,197$.

389 Cho 1986, 197. Vgl. a. Bester, Hellwig 1987a, 155-157.

390 De Meza, Webb 1987, 282.

$391 \quad$ Bester, Hellwig 1987, 23.
} 


\subsubsection{Informations- und Ertragsasymmetrien}

Die Maximierung des Unternehmens- beziehungsweise Eigentümerwertes setzt - wie im vorherigen Abschnitt geschildert - zum einen voraus, dass jene Investitionsobjekte ausgewählt werden, deren Rendite/Risiko-Profile mindestens auf der CAPM-Wertpapiermarktlinie liegen und damit keine negativen Kapitalwerten aufweisen. Zum anderen ist eine uneingeschränkte Partizipation der Eigner an den diskontierten Einzahlungsüberschüssen nach Begleichen aller Kontraktforderungen notwendig. Beide Bedingungen werden in der realen, nicht-walrasianischen Welt verletzt. Denn die Erfüllung beider Bedingungen setzt ein Management voraus, das sich als Erfüllungsgehilfe der Eigentümer versteht. Es ist realistischerweise eher davon auszugehen, dass die Agenten auf der Grundlage eines Mindestnutzens ihre Leistungen nach Maßgabe ihrer eigenen Interessen optimieren. ${ }^{392}$ Hierbei wägen sie in einer marginalen Kalkulation ihren Aufwand etwa in Form von Arbeitsanstrengungen $^{393}$ mit der $\mathrm{zu}$ erwartenden Arbeitsentlohnung ${ }^{394}$ ab. Zur Eigennutzmaximierung der Manager sind Unternehmensinvestitionen notwendig, zu deren Realisierung es des Inputs verschiedener Faktoren wie etwa Eigenkapital und Humankapital der Mitarbeiter bedarf. Die für die Zufuhr dieser Faktoren einhergehenden Kosten sind von den Managern als Nebenbedingungen ihres Kalküls zu berücksichtigen, den Gegenwartswert ihres eigenen monetären wie nichtmonetären Lebenszeiteinkommens zu maximieren.

Die relationale Beziehung zwischen den Prinzipalen und den Agenten weist eine konstitutionelle Schwäche dahingehend auf, ${ }^{395}$ dass die Informationsdefizite zu Lasten ersterer nur unter Aufwendung von Kosten zu beheben sind; ${ }^{396}$ es liegt eine

392 Aus Gründen der Vereinfachung ist im Folgenden nur das Top-management gemeint. Auswirkungen der Unternehmenshierarchie werden ausgeblendet.

393 " $[\mathrm{M}]$ anagers ... will equate their marginal disutility of effort with their share of their marginal product rather than with their total marginal product. Therefore, too little effort will be forthcoming from agents." Stiglitz, Weiss 1981, 407. Dazu gehört auch der Konsum am Arbeitsplatz, die Prestigeausgaben, die Ausgaben zur Ausweitung des Machtbereichs sowie zur Sicherung des Arbeitsplatzes. Schmidt, Terberger 1997, 439f. Vgl. Elsner 1987, 5-7.

396 Der Grad der Informiertheit bleibt unter den Anlegern annahmegemäß gleich. Wang (1994) sieht Informationsasymmetrien zwischen den Anlegern als ursächlich für das Transaktionsvolumen am Sekundärmarkt, mithin den Liquiditätsgrad von Anlagen. „Investors trade among themselves because they are different... I focus on the effect of information asymmetry on the behavior of volume." Wang 1994, 130. Vgl. a. die dort angegeben Literatur. Asymmetrische Informationen zwischen Alt- und Neueigner spielen im Wesentlichen bei nicht-börsennotierten Kapitalgesellschaften eine Rolle. Bei börsennotierten 
„,costly state verification“ vor, weshalb es für die Eigenkapitalgeber nicht rational sein kann, vollkommen informiert $\mathrm{zu}$ sein. Die Informationen sind für die Eigenkapitalgeber nicht nur unvollkommen, sondern darüber hinaus asymmetrisch verteilt, da die Agenten über Informationen hinsichtlich der unternehmerischen Investitionstätigkeit verfügen, die die Prinzipale nicht haben. ${ }^{397}$ Die asymmetrische Informationsverteilung und die fehlenden Kontrollmöglichkeiten der Eigentümer bieten Managern den Raum, die Ansprüche der Eigenkapitalgeber lediglich als eine Nebenbedingung zu behandeln, wenn auch als eine gewichtige. Ein im Interesse der Eigner zu niedriges Anstrengungsniveau der Manager führt etwa zu einer Auswahl von Unternehmensinvestitionen, die für eine Maximierung des Unternehmenswertes möglicherweise suboptimal ist, also auch unterhalb der referenziellen CAPMWertpapiermarktlinie liegen kann. ${ }^{398}$ Denn Manager gewichten die Ertrags- und Aufwandskomponenten von Investitionen anders, als es die Eigner tun. Zusätzlich mögen Manager bei der Kapitalwertberechnung einer unternehmerischen Investitionsreihe einen anderen Myopiegrad respektive Diskontfaktor zu Grunde legen. Neben einer für die Eigenkapitalgeber ineffizienten Auswahl von Unternehmensinvestitionen impliziert eine Entlohnung der Manager durch moral hazard und hold-up ein für die Eigenkapitalgeber suboptimales Verhalten. In diesem Fall sind die Eigenkapitalgeber nach Begleichen aller Kontraktforderungen nur unvollständig an den erwirtschafteten Einzahlungsüberschüssen beteiligt. ${ }^{399}$ Kurzum: Die Agenten nutzen die asymmetrische Informationsverteilung bei der Auswahl der unternehmerischen Investitionen sowie bei der Verteilung der dabei anfallenden Erträge zu Lasten der Eigner opportunistisch aus. ${ }^{400}$ Dabei nimmt die Neigung der Manager zum Konsum nicht-monetärer Vorteile (Perk Consumption, Consumption

Aktiengesellschaften mit einer annonymen Anlegerstruktur ist diese Art der Informationsasymmetrien sekundär.

Denkbar wäre auch eine inverse Informationsasymmetrie, das heißt ein Informationsvorsprung der Eigenkapitalgeber im Verhältnis zu den Managern, insbesondere bei jungen Unternehmen mit wenigen Erfahrungswerten. Doch ist dieser Fall für den betrachteten Unternehmenskreis börsennotierter Unternehmen der „Old Economy“ weniger relevant. Dieses Phänomen wird in der Literatur unter dem Unterinvestitionsbegriff diskutiert. Vgl. Wosnitza 1990, 947; Jensen, Meckling 1998, 100.

Unter moral hazard versteht man das opportunistische Ausnutzen von Informationsasymmetrien. „Hold-up“ steht für das Berauben von Quasi-Renten spezifischer Investitionen. Dabei wird ein Aktienerwerb nachträglich zu einer versunkenen, locked-in Spezifität. Es findet eine „fundamentale Transformation“ der Bedingungen bezüglich der Zahl der Wettbewerber (vgl. Williamson 1985, 12) statt. Damit ist eine nach der Bereitstellung von Eigenkapital auftretende strategische Verwundbarkeit des Aktionärs in einer vorvertraglich noch wettbewerblichen Konstellation gemeint. Zum Unterschied vgl. Alchian, Woodward $1988,67 f$. 
on the Job) sowie $\mathrm{zu}$ einem geringen Anstrengungsniveau (Shirking) mit abnehmendem Eigentumsanteil der Manager zu. ${ }^{401}$ Denn "the more equity held by the entrepreneur the greater the extent to which his return depends upon project success. ${ } 402$

Die Bereitstellung von Eigenkapital geht also mit so genannten Agency-Kosten für die Prinzipale einher. Um diese einzuschränken, wurden - in Übereinstimmung mit der institutionenökonomischen Literatur $-^{403}$ positive wie negative Sanktionen zu Gunsten der Eigenkapitalgeber entwickelt. ${ }^{404}$ Es handelt sich neben den Einflussmöglichkeiten des Aufsichtsrats, den Haftungsvorschriften des Vorstandes und dem eingeschränkten Einfluss des Vorstandes auf Ausschüttungen um erfolgsabhängige Vergütungsvereinbarungen entlang den Eignerinteressen ${ }^{405}$ etwa in Form von Aktienoptionsplänen, ${ }^{406}$ um feindliche Übernahmen als ein Markt für Unternehmenskontrolle, ${ }^{407}$ um erhöhte Transparenzvorschriften, um die Etablierung eines Zwanges zur Ausschüttung von Gewinnen, aber auch um informelle Einflussmöglichkeiten von Großaktionären. Die Fortentwicklung der Aktienmärkte und eine zunehmende Wettbewerbsintensität um knappes weltweit vagabundierendes Eigenkapital, ${ }^{408}$ eine hohe Wettbewerbsintensität auch auf den Produkt- und

400

Unter Opportunismus versteht Williamson (1985) ein eigennutzmaximierendes Verhalten unter Zuhilfenahme von List. Vgl. Williamson 1985, 47.

Vgl. Jensen, Meckling 1976, 309+312-319.

De Meza, Webb 1990, 206.

"As during the 1970s, major US manufacturing corporations struggled with these very real problems of excessive centralization and innovative competition, a group of American financial economists developed an approach to corporate governance known as agency theory... The rate of return on corporate stock was their measure of superior performance, and the maximization of shareholder value became their creed." Lazonick, O'Sullivan 2000, 15f.; vgl. a. die dort angegebene Literatur.

Die Bedeutung des Coase-Theorems als Instrument zur Internalisierung der zu Lasten der Eigner externalisierten Agency-Kosten ist bei Vorliegen asymmetrischer Informationen eingeschränkt.

Vgl. Diamond, Verrecchia (1982); Jensen, Murphy (1990); Heckermann (1975).

Durch das Eingehen etwa einer short call Position erhält der Manager das sonst entgeltliche Recht, eine bestimmte Menge von Aktien zu einem bestimmten Kurs innerhalb einer bestimmten Frist von einem Stillhalter (long Position) kaufen zu können. Damit partizipiert er an Kurssteigerungen. In Deutschland bemißt sich der variable Teil der Manager-Vergütung meist am Jahresüberschuß oder auch an der Dividende statt an der Steigerung des Shareholder Value. „Stock options in Germany are generally only a supplement to normal salaries.“ Jürgens, Naumann, Rupp 2000, 74.

Vgl. Laffont, Tirole (1988); Scharfstein (1988); Stein (1988). In Deutschland ist der „Markt für Unternehmenskontrolle“ im Vergleich $\mathrm{zu}$ den angelsächsischen Ländern von vernachlässigenswerter Bedeutung. An ihre Stelle treten zum einen Kreditinstitute, die durch Auflagen bei der Kreditvergabe oder durch Beteiligung an Unternehmen Einfluss auf das Management ausüben. Zum anderen hat sich in Deutschland durch Kapitalverflechtungen und wechselseitige Wahrnehmung von Aufsichtsratsmandaten ein System der Selbstkontrolle des Managements herausgebildet.

Vgl. Diamond, Verrecchia (1982); Jensen, Murphy (1990). 
Faktormärkten sowie auf dem Markt für Manager und ein um sich greifendes auf den Shareholder Value gerichtetes Denken tragen ebenfalls zu einer Annäherung an die aus Eignersicht - first best-Lösung einer Maximierung des Eigentümerwerts bei. ${ }^{409}$ All diesen Disziplinierungsmechanismen zum Trotz lassen sich die Manager-EignerKonflikte jedoch nicht vollends beseitigen, allenfalls reduzieren. ${ }^{410}$ Maßgeblich dafür sind nicht zuletzt das Free Rider-Verhalten von Anlegern und die Kosten der Informationsbeschaffung und der Etablierung entsprechender Institutionen. Die first best-Lösung einer Maximierung des Eigentümer- respektive Unternehmenswertes ist deshalb nur annäherungsweise realisierbar.

Dass Manager für die unvollkommen informierten Eigenkapitalgeber suboptimale Investitionen auswählen und sie sie bei der Verteilung der diskontierten Einzahlungsüberschüsse nur unvollständig beteiligen, hängt nicht nur mit den managereigenen Interessen zusammen. „[T]he discussion ... on divergence of interests between stockholders and managers represent only a small segment of a seriously neglected problem: the shaping of intraorganizational conflict.",411 Unternehmen bestehen aus einem Nexus vertraglicher Beziehungen zwischen den Angehörigen unterschiedlicher Interessengruppen. Faktisch haben neben den Eignern auch andere Anspruchsgruppen eines Unternehmens (Stakeholder) ${ }^{412}$ sowohl Einfluss auf die Wahl der Unternehmensinvestitionen als auch auf die Verteilung der Investitionserträge jenseits ihrer Kontraktforderungen. ${ }^{413}$ „Gelingt es einem Unternehmen nicht, die finanziellen Ansprüche seiner Anspruchsgruppen zu befriedigen, so wird es aufhören, eine lebensfähige Organisation zu sein. Mitarbeiter,

$\mathrm{Zu}$ einer geschichtlichen Analyse des Shareholder Value-Ansatzes vgl. Lazonick, O'Sullivan (2000).

410 Vgl. Wosnitza 1991, 39-49; Shleifer; Vishny 1997a, 741; Morck, Shleifer, Vishny 1989, 842; Stiglitz 1981, 328f. Der diskretionäre Spielraum der Manager lässt sich auch durch Vergütungsvereinbarungen nicht gänzlich einschränken. Denn Börsenkurse werden nicht nur vom fundamentalen Faktor des Underlyings beeinflusst, sondern z.B. auch von Änderungen der Kapitalmarktzinsen, von der allgemeinen oder der Branchenkonjunktur. Optionen sind mit einer Hebelwirkung ausgestattet, die zu einer über- oder unterproportionalen Beteiligung an Kursveränderungen führen.

$411 \quad$ Nelson, Winter 1982, 56.

412 „A stakeholder in an organization is (by definition) any group or individual who can affect or is affected by the achievement of the organization's objectives." Freeman, R.E. 1984, 46. Uneindeutig in seinen genauen Definitionsbestandteilen geht der Stakeholder-Begriff zurück auf Dill (1958) und Cyert, March (1963). In den 70er Jahren wurde er u.a. von System- und Organisationstheoretikern aufgegriffen und in verschiedene Richtungen weiterentwickelt. Einen Überblick bieten Freeman, R.E. (1984), Meier-Scherling 1996, 102-116. 
Kunden und Lieferanten werden ihm einfach ihre Unterstützung entziehen. ${ }^{6414}$ Auch Stakeholder erheben somit Ansprüche auf den etwaigen Unternehmenserfolg. ${ }^{415}$ Außerdem üben auch sie Einfluss auf die Auswahl von Unternehmensinvestitionstätigkeit aus. Die Vorstellung, dass allein die Ansprüche der Eigenkapitalgeber (und der Manager) die Investitionstätigkeit eines Unternehmens diktieren und sie die Einzahlungsüberschüsse untereinander aufteilen, während alle anderen Unternehmensbeteiligten erfolgsunabhängige Forderungen hegen, geht an der Wirklichkeit vorbei $-^{416}$ insbesondere an der des „kooperativen Kapitalismus ${ }^{\text {“4117 }}$ " "[T] he modern financial environment is too complex and varies too much from country to country to support ready induction of underlying principles." ${ }^{418}$ Insbesondere die so genannte rheinische Variante des Kapitalismus erfordert die Bedienung der Ansprüche auch der Stakeholder durch das Management. ${ }^{419}$ Ein hoher Gläubigerschutz in einem bankdominierten Finanzierungssystem, Mitbestimmungsgesetze, der Aufsichtsrat als Vertretungsorgan der Arbeitnehmer, eine ausgeprägte Sozialgesetzgebung, ökologische Auflagen, kurzum eine verfassungsgemäße Sozialbindung des Eigentums (Art.14, Abs.2 GG) - diese Institutionen binden die unternehmerischen Investitionsentscheidungen in allokativer wie auch in distributiver Hinsicht an die Belange einer Schar von Unternehmensbeteiligten: ${ }^{420}$ Gläubiger, Arbeitnehmer, Anwohner, Gemeinden, Kunden, öffentliche Gebietskörperschaften. ${ }^{421}$ Es verwundert insofern nicht, dass bezüglich einer sich allgemein ausbreitenden Shareholder-Orientierung für Deutschland konstatiert wird: „there are changes but the impact is marginal.”422 Das kooperative deutsche Unternehmensverständnis spiegelt sich in der täglichen

\footnotetext{
$414 \quad$ Rappaport 1995, 13.

$415 \quad$ Vgl. Kotz 1999, 316.

416 „Ist die Suche nach Handlungs- und Gestaltungsprinzipien einer solchen „,erweiterten Stakeholder-Ökonomie“ nicht zwecklos, bestenfalls naiv, da wider alle historischen (Mega-) Trends? ... Da Institutionen sehr beharrend sind, und da die Institutionen des alten ShareholderKapitalismus durch die Globalisierung noch einmal neue Nahrung bekommen, wird diese Entwicklung von vielen nicht gesehen. Sie ist aber, praktisch und theoretisch, in vollem Gange.“ Biesecker 1998, 2.

417 Windolf, Beyer 1999, 1.

$418 \quad$ Myers 2000, 1006.

419 Vgl. Soskice (1996).

420 Zur rechtlichen und politischen Einflußnahme auf die deutsche Publikumsgesellschaft vgl. Meier-Scherling 1996, 272-286.

421 Vgl. Nissen 1999, 2f.

422 Jürgens, Naumann, Rupp 2000, 75 und 66-74.
} 
Rechtspraxis wider, ${ }^{423}$ aber auch findet es in nicht unbeachtlichen Teilen der deutschen Betriebswirtschaftslehre seinen Niederschlag. ${ }^{424}$ Geradezu propagiert wird es von zahlreichen Führungspersönlichkeiten. ${ }^{425}$ Sehen sich Manager auch deklamatorisch - in der Hauptpflicht der Aktionäre, ${ }^{426}$ ist selbst zur Verfolgung des (langfristig ausgerichteten) interessenmonistischen Ziels einer Maximierung des Eigentümerwertes die Berücksichtigung anderer Anspruchsgruppen vonnöten. ${ }^{427} \mathrm{Mag}$ die Sozialbindung des Eigentums in der deutschen Stakeholder-Ökonomie auch im allgemeinen Langfristinteresse der Aktionäre sein,,${ }^{428}$ impliziert sie doch andererseits im Einzelfall eine Einschränkung der Shareholder-Interessen. Denn die „Stabilität“ eines Unternehmens, die mit der Einbeziehung von Stakeholder-Interessen in die unternehmerische Investitionstätigkeit verbunden ist, stellt für Aktionäre ein öffentliches Gut dar. Verweigern Stakeholder einzelner Unternehmen den Input ihrer Faktoren, betrifft dies nur einen kleinen und austauschbaren Teil im Aktienportefeuille der Anleger. Deshalb stellt der Einfluss der Stakeholder auf die Investitionstätigkeit einzelner Unternehmen neben den Managerinteressen eine weitere Abweichung von der Maximierung des Eigentümer- respektive Unternehmenswertes dar.

Stakeholder schließen zwar in der Regel mit einem Unternehmen Festbetragskontrakte als Gegenleistung für den Einsatz ihrer jeweiligen Inputs ab.

423 In Deutschland lässt sich dieser korporatistische Gedanke auf Walter Rathenaus Vorstellung vom „Unternehmen an sich“ zurückführen. Vgl. Rathenau 1917, 38f. Sie hat in der Version des "Unternehmensinteresses" besonders unter Juristen breite Anerkennung gefunden.

424 Wagner spricht sogar von einem fachspezifischen Ziel-Schisma, das in den Bereichen Personal und Organisation sowie Marketing gegenüber der Shareholder-orientierten Investitions- und Finanzierungstheorie besteht. Vgl. Wagner 1997, 474ff.

425 Vgl. Meier-Scherling 1996, 3. Stellvertretend für viele deutsche Führungspersönlichkeiten Herrhausen 1971, 30: „Die gesellschaftspolitische Mission liegt nicht nur in der ökonomischen, sondern auch in der ganz allgemeinen Verpflichtung des Managers gegenüber den Belangen der res publica." Vgl. Wagner 1997, 489; Douglas Daft, CEO der Coca Cola Inc.: "Gutes zu tun, ist gut für das Geschäft. Es bringt Geschäftserfolg und damit auch Shareholder-Value. Ein "Unternehmen als guter Bürger" ist Teil eines lokalen Gemeinwesens und unterstützt dort zum Beispiel Schulen. Oder den Umweltschutz. Der wird auch für unsere Kunden ein immer wichtigeres Thema." In: Die ZEIT v. 17.08.00, Nr. 34, 19. In der Maximierung des Eigentümer- respektive Unternehmenswertes sehen sie meist eine Interessenharmonie aller Unternehmensbeteiligten. Vgl. Rappaport 1995, $12 \mathrm{f}$.

Cornell, Shapiro (1987) weisen auf die negativen finanziellen Auswirkungen (auch für die Eigenkapitalgeber) hin, die aus einer Nichtbeachtung dieser Wünsche resultieren wie z.B. höhere Löhne aufgrund eines schlechten Betriebsklimas oder unsicherer Arbeitsverhältnisse, höhere Zinszahlungen wegen mangelnder Beachtung der Wünsche der Kreditgeber.

"The business entreprise should make its major decisions for long-term shareowner benefit. In doing so, it needs to consider which other stakeholder considerations represent the "enlightened self-interest" of the shareowners - those interests that support the long-term gains for such shareowners." Mahoney 1997, 327. 
Neben den expliziten vertraglichen Regelungen der Koalitionspartner sind jedoch auch implizite Vertragselemente von Bedeutung. Die Erfüllung impliziter Stakeholder-Ansprüche ist gesetzlich nicht bindend, wird aber von den Stakeholdern dennoch erwartet wie etwa Aufstiegsmöglichkeiten für Mitarbeiter, Sicherheit des Arbeitsplatzes, die Langfristigkeit von Lieferbeziehungen, Wahrung ökologischer und sozialer Verantwortlichkeiten. Von der Erfüllung solcher impliziten Ansprüche machen die Stakeholder eines Unternehmens den Einsatz ihrer Faktoren oft abhängig. Da ihr Input für das Unternehmensmanagement zu einem bestimmten Grad weder beobachtbar noch kontrollierbar ist, liegen asymmetrische Informationen zu Lasten des Managements vor. Die Festbetragskontrakte mit Stakeholdern sind unvollständig und weisen ebenfalls relationale Elemente auf. So ist etwa der Arbeitsmarktmechanismus nur eingeschränkt in der Lage, vollständige Verträge über Umfang und Qualität der Leistungen der Mitarbeiter zu bewerkstelligen. "[W]hen investments in highly specialized human capital are important to the way that a firm creates wealth, employees, as well as shareholders, are likely to be residual claimants and, therefore, residual risk-bearers. ${ }^{" 429} \mathrm{Da}$ das Management zur Maximierung ihres Eigennutzes quantitativ wie qualitativ auf den Input der „Festbetrags“-Kontrahenten angewiesen ist, gibt es auch ihren impliziten Ansprüchen teilweise statt. Diese können entgeltlicher, aber auch nicht-entgeltlicher Natur sein. Beispielsweise wird versucht, die Macht der über firmenspezifisches Wissen verfügenden Arbeitnehmer, einen geringen sowie qualitativ schlechten Arbeitseinsatz einzubringen, durch die Zahlung so genannter Effizienzlöhne zu regulieren. ${ }^{430}$ Hierbei handelt es sich um eine Vergütung oberhalb der den Arbeitsmarkt räumenden Marktlöhne. Diese wiederum speisen sich aus den Einzahlungsüberschüssen der Unternehmensinvestitionen. Eine andere am deutschen Kapitalmarkt an Bedeutung zunehmende Gruppe der Bondholder orientieren sich weniger am Ziel der Maximierung des Marktwertes eines Unternehmens. Der Bondholder-Value orientiert sich vielmehr an Stabilitätszielen wie etwa der Zahlungsfähigkeit eines Unternehmens. Um bei eingeschränkten Eigenkapitalbeschaffungsmöglichkeiten Fremdkapitalgeber zu aquirieren, bedarf es einer Berücksichtigung auch ihrer Ansprüche in der unternehmerischen Investitionstätigkeit. ${ }^{431}$ Obgleich sowohl Arbeitnehmer als auch Bondholder grundsätzlich nur Festbetragsansprüche an das Unternehmen binden,

\footnotetext{
$429 \quad$ Blair 1995, 238.

430 Vgl. Heise 1996, 78-84.

431 Vgl. Papon 2001, 15.
} 
sind vor diesem Hintergrund neben anderen auch sie vom Management wie „Restbetragsbeteiligte“ mit Einfluss auf Art und Verteilung der unternehmerischen Investitionstätigkeit $\mathrm{zu}$ berücksichtigen. ${ }^{432}$ Es enstehen Kosten, die über die Festkontraktbeträge hinaus erwirtschaftet werden müssen.

Die Unvollständigkeit der Verträge beziehungsweise die Ineffizienz des Marktmechanismus ruft das Management als eine Instanz auf den Plan, die die verschiedenen Ansprüche der Unternehmensbeteiligten reguliert. Die Stabilisierung des unternehmerischen Vertragsgeflechts durch das Management ist umso bedeutender, als auch zwischen den einzelnen Beteiligten eines Unternehmens asymmetrische Informationen, Externalitäten und auch unterschiedliche Ein- und austrittsbarrieren bestehen. ${ }^{433}$ Außerdem führt der gesamtwirtschaftliche Wandel zu permanenten Verschiebungen im Machtgefüge eines Unternehmens. Die Stabilisierungsfunktion des Managements besteht darin, den Input der Stakeholder wie der Eigenkapitalgeber in Dauer, Umfang und Qualität zu gewährleisten. ${ }^{434}$ Den Zustand einer „quasi-resolution of conflict ${ }^{6435}$ zwischen den mitunter koalierenden Stakeholdern herzustellen, ist wesentliche Aufgabe des Managements als ausbalancierende Instanz zwischen Anspruchsgruppen, die über mehr oder weniger Einflussmöglichkeiten verfügen. Dabei ist die Erhaltung des unternehmerischen Produktionsprozesses in seinem reibungslosen Ablauf umso gefährdeter, je weniger substituierbar die einzelnen Inputfaktoren sind. Für die Maximierung des eigenen Nutzens stehen die Agenten eines Unternehmens also mehr oder weniger vor der Aufgabe, die Ansprüche der Stakeholder bei den unternehmerischen Investitionsentscheidungen in allokativer wie in distributiver Hinsicht $\mathrm{zu}$ berücksichtigen und miteinander auszutarieren. ${ }^{436}$

Da die Interessen der einzelnen Koalitionspartner teils divergieren, ist die Entscheidungsfindung anhand eines originären (aggregierten) Unternehmensziels nahezu unmöglich. Eine Minimalforderung aller Koalitionspartner als „kleinster gemeinsamer Nenner“ postuliert die Erhaltung des Unternehmens als

\footnotetext{
432 Cornell, Shapiro (1987).

433 Das Coase-Theorem kommt innerhalb von Unternehmen infolge hoher Transaktionskosten und unausgewogenen Machtkonstellationen schwerlich zum Tragen. Nissen 1999, 9.

Nelson, Winter 1982, 56.

Keynes $(1933,565)$ spricht von „Selbstsozialisierung von Großunternehmen“.
} 
Einkommensquelle. ${ }^{437}$ Der dazu notwendige konstante Zufluss an Inputs aller Unternehmensbeteiligten erfordert eine im Vergleich zur Eigentümerwertmaximierung möglicherweise abweichende Auswahl von Unternehmensinvestitionen. Dies trifft vor allem dann $\mathrm{zu}$, wenn aus den Unternehmensinvestitionen für die Stakeholder höhere Risiken als für die Eigenkapitalgeber erwachsen. Davon ist angesichts geringerer Diversifizierungsmöglichkeiten der Stakeholder regelmäßig auszugehen. Ein Unternehmen, das von den Stakeholdern nicht-diversifizierbare Risiken in der Auswahl seiner Investitionstätigkeit berücksichtigt, muss eine höhere Interne Verzinsung für die Auswahl effizienter Unternehmensinvestitionen zu Grunde legen. Durch die Berücksichtigung von Unternehmensrisiken, die für Stakeholder bewertungsrelevant sind, wird somit insgesamt weniger investiert; es wird in Projekte mit früher einsetzenden Nettoeinzahlungen und in weniger riskante Projekte investiert. ${ }^{438}$ Es , „äßt sich feststellen, daß die Bereitschaft in risikobehaftete Projekte zu investieren bei einem Stakeholder-orientierten Ansatz geringer ausfällt. Dies ergibt sich zum einen dadurch, daß der Wert einer Investition aus der Shareholder Value-Sicht höher ist. Zum anderen schmälert bei der Stakeholder-orientierten Sichtweise das Vorhandensein von unsystematischen Risiken die optimale Investitionsintensität. “4439

In distributiver Hinsicht drückt sich die Berücksichtigung der Stakeholder-Interessen in der unternehmerischen Investitionstätigkeit darin aus, dass ihre Ansprüche auch jenseits der Festbetragskontrakte zu befriedigen sind. Das Ausmaß ihrer Partizipation an den Einzahlungsüberschüssen der unternehmerischen Investitionstätigkeit hängt von den Bedeutung ihrer Inputs sowie ihrer Möglichkeiten ab, ihren Beitrag innerhalb des Festbetragskontraktes, etwa durch Bummeln, zu variieren. Es bedarf gewisser Anreizzahlungen an die Stakeholder, um die Zufuhr ihrer Beiträge zur Wertschöpfung des Gesamtunternehmens in notwendigem Maße aufrecht zu erhalten. Die Höhe der Anreizzahlungen hängt außerdem von den erwirtschafteten Einzahlungsüberschüssen $\mathrm{ab}$. In Zeiten, in denen hohe Einzahlungsüberschüsse erwirtschaftet werden, übersteigen die Ressourcenzuweisungen an die Stakeholder ihre Festbetragsansprüche. Umgekehrt beläuft sich in weniger erfolgreichen Phasen

$437 \quad$ Vgl. Hermann 1998, 48.

438 Vgl. Hermann 1998, 183-196. 
der Anspruch der Stakeholder lediglich in Höhe ihrer Kontraktforderungen. Mit der Kontraktforderung als Mindestanspruch entsteht eine Anspruchsasymmetrie der Stakeholder gegenüber dem Management. $\mathrm{Da}$ sich in Phasen geringer Einzahlungsüberschüsse ihre Ansprüche nicht oder nur mit einer gewissen zeitlichen Verzögerung nach unten anpassen, muss das Management in solchen Phasen vorsorgen, in denen das Unternehmen einen hohen Cash Flow erwirtschaftet. Über das Anreiz-Beitrags-Gleichgewicht aller Unternehmensbeteiligter hinaus muss das Management in erfolgreichen Phasen Organizational Slack deshalb zurückhalten, um den Input der Unternehmensbeteiligten auch in Phasen geringer Cash Flows gewährleisten $\mathrm{zu}$ können. ${ }^{440}$ Organizational Slack befindet sich in den stillen Reserven eines Unternehmens, in Überkreuzkompensationen, offenen Rücklagen und Rückstellungen; nach deutschen Rechnungslegungsvorschriften sind bestimmte Ausschüttungen an die Eigenkapitalgeber sogar rechtlich eingeschränkt. ${ }^{441}$ In realwirtschaftlich weniger erfolgreichen Perioden löst das Management den gebildeten Slack dann wieder auf. Es ist „auch für eine schlanke Unternehmung entscheidend, genügend notwendigen Slack zu besitzen, sofern Ziele wie Flexibilität, Innovationsfähigkeit und Qualitätsverbesserungen verfolgt werden. “442 Im Gegensatz zur X-Ineffizienz ${ }^{443}$ übernimmt Organizational Slack somit eine stabilisierende Pufferfunktion im Unternehmen. ${ }^{444}$ Das Auf- und Abbauen von Organizational Slack ist umso erforderlicher, wenn sich die Ansprüche der Unternehmensbeteiligten den erratisch fluktuierenden Einzahlungsüberschüssen nur langfristig anpassen. Neben anderen Ursachen bedarf es also wegen der kurzfristigen Nicht-Anpassung beharrlicher Stakeholder-Ansprüche der Bildung eines finanziellen Puffers -

439 Hermann 1998, 196. Zur Unterlassung von Investitionen mit positivem Kapitalwert (Unterinvestition) in jungen innovativen Unternehmen vgl. Heitzer 2000, 149f.

440 Vgl. Cyert, March 1963, 36-38. „Organizational Slack“ beschreibt in statischer Hinsicht eine Redundanz oder eine überschießende Ressourcenbindung über das organisatorisch unbedingt erforderliche Maß hinaus. Er entsteht in Form von Zeit, Geld, Mitarbeitern, Anreizen oder einer überdimensionierten Organisationsstruktur. Zu Entstehung und Arten von Slack vgl. Backmann 1999, 9-16. Es gibt zahlreiche Versuche, ,slack“ ins Deutsche zu übertragen. Da jeder Versuch jeweils nur einen Teilaspekt beleuchtet, wird in der vorliegenden Arbeit der engliche Begriff übernommen.

$441 \quad$ Vgl. Wagner 1997, 480.

442 Fallgatter 1995, 220. Auch fördert Slack die Innovationsfähigkeit eines Unternehmens, da wegen reichlich vorhandener Ressourcen auch riskantere Entwicklungsprojekte durchgeführt werden können. Vgl. Cyert, March 1992, $189 f$.

443 Vgl. Leibenstein (1966).

444 Organizational Slack und X-Ineffizienzen kennzeichnen eine im Vergleich zur Kostenminimierung der traditionellen Theorie ineffiziente Allokation von Faktoren innerhalb der Unternehmung. X-Ineffizienzen werden begründet mit Unkenntnis der Produktions- und Kostensituation sowie mit Nicht-Ausnutzung der vollen Arbeitskapazität; es ist rational, sie in 
Organizational Slack eben. Dieser dient wohlgemerkt nicht nur zur Befriedigung von Begehrlichkeiten des Managements. In erster Linie ist der Aufbau von Organizational Slack zur (außerplanmäßigen) Bedienung der Stakeholder-Ansprüche vonnöten. Allerdings kann das Unternehmensmanagement erst mit der entsprechenden Kompetenz ausgestattet auf diese Weise die in einer StakeholderÖkonomie divergierenden Interessengruppen miteinander in Übereinklang bringen. ${ }^{445}$ Zur Bildung und Auflösung Organizational Slacks muss das Management über Gestaltungsmöglichkeiten hinsichtlich des Bilanzgewinns verfügen. Was mit letzterem geschieht, liegt weitgehend im Ermessensspielraum der Manager. Zwar obliegt der Hauptversammlung einer Aktiengesellschaft das Recht, über die Gewinnverwendung zu entscheiden. ${ }^{446}$ Dieses Recht ist in seiner Bedeutung jedoch dadurch eingeschränkt, dass die Hauptversammlung sich an den bereits vom Management festgestellten Jahresabschluss halten muss. ${ }^{447}$ Außerdem wird der Einfluss der Stakeholder auf den Entstehungs- und Verteilungsprozess der Unternehmensinvestitionen durch entrichtete ,side payments“ reduziert.

Das Einbehalten von Organizational Slack aus dem Cash Flow setzt ein bestimmtes Maximierungsverhalten der Eigenkapitalgeber voraus. In Anlehnung an Simon (1955) ist in einer Umwelt, über die nur unvollständige Informationen bestehen, ${ }^{448}$ von einem nur satisfizierenden Verhalten eingeschränkt rationaler Eigenkapitalgeber auszugehen. ${ }^{449}$ Satisfizierung ist mit Simon (1961) jedoch nicht Ausfluss einer eingeschränkten Rationalität, “[which, d.A.] refers to rational principles underlying non-optimizing adaptive behavior of real people." ${ }^{450}$ Eingeschränkte Rationalität impliziert kein teilweise irrationales Verhalten. Vielmehr stellt sich die

dem Grad zu beseitigen, als die mit der Beseitigung verbundenen Kosten des Anreizes und der Kontrolle nicht die Kosteneinsparungen übertreffen. Vgl. Leibenstein 1966, 397-413.

Vgl. §119 Abs.1 Ziff.2 AktG.

Vgl. §174 Abs.1 S.2 AktG. Zum Auskunftsverweigerungsrecht des Managements hinsichtlich der Wertansätze in der Jahresbilanz sowie der Bewertungs- und Abschreibungsmethoden vgl. $\$ 131$ Abs.3 AktG. Zu einer jüngeren negativ ausfallenden Bewertung der Informationspolitik deutscher Aktiengesellschaften sowie der deutschen Rechnungslegung vgl. FAZ v. 28.7.00, Nr. 173, 25+27. Die meisten deutschen Aktiengesellschaften benutzen noch immer nicht die anlegerfreundlichen Rechnungslegungssysteme IAS oder US-GAAP. Zum Thema HGB versus IAS/US-GAAP vgl. Zeitschrift für das gesamte Kreditwesen 4/1998, 148-173.

Alternative Maximierungsansätze geben Schumann, Meyer, Ströbele 1999, 465-467.

Da ihre Erkenntnis- und Informationsverarbeitungskapazitäten beschränkt sind, können die Akteure sich nur eingeschränkt rational verhalten. Zur „,bounded rationality“ vgl. Williamson 1985, 46f. Dieser beruft sich auf Simon (1955), der maximierendes durch satisfizierendes Verhalten ersetzt.

Selten 1999, 3. 
Entscheidungsfindung eines beschränkt rationalen Wirtschaftssubjektes als ein Suchprozess dar, der geleitet wird von ,aspiration levels ${ }^{\text {(451 }}$, von mittel- und langfristig variierenden Anspruchsniveaus also. Das Verhalten der Eigenkapitalgeber ist somit zwar ,intentionally rational, but limitedly so“. ${ }^{452}$ In diesem Sinne ist es seitens der Eigenkapitalgeber rational, eine „nur“ befriedigende Rendite des eingesetzten Eigenkapitals $\mathrm{zu}$ fordern. ${ }^{453}$ Informationsunvollkommenheiten heben damit die Annahme rationalen Verhaltens nicht auf. ${ }^{454}$ Sie modifizieren die unterstellte Rationalität lediglich dahingehend, dass sich die (satisfizierenden) Forderungen unvollkommen informierter Eigenkapitalgeber stufenweise, das heißt in „nur“ mittel- bis langfristig variablen Anspruchsniveaus der maximal möglichen Forderungen annähern. "[O]wners can assess the performance of their top management only by a relatively general comparison with other similar firms and with the stock market as a whole. The ignorance of stockholders drastically reduces the amount of marginal switching they do from one stock to another that appears to be enjoying better performance... The force of competition among different stocks, which would in theory be expected to put pressure on top management to maximize the rate of growth of its stock price, is in fact severely weakened by ignorance. As a result, owners tend to act as "satisficers" instead of "maximizers".,"455

Wie die Stakeholder verfolgen damit auch die Eigenkapitalgeber auf lange Sicht ein variables, auf kurze Sicht jedoch ein konstantes, das heißt satisfizierendes Anspruchsniveau. Ein solches Beharrungsvermögen der Aktionäre drückt sich etwa in der Kontinuität der Dividendenzahlungen aus, die nachweislich zahlreiche deutsche Aktiengesellschaften praktizieren. ${ }^{456}$ Ein auf kurze Sicht satisfizierendes

\footnotetext{
$451 \quad$ "An aspiration level is a value of a goal variable which must be reached or surpassed by a satisfactory decision alternative. .. Simon coined the word "satisficing" for this process." Selten 1999, 1; vgl. Simon 1959, 262-264. Zur Aspiration adaption theory vgl. Selten 1999, 7-16.

452 Simon 1961, 24.

453 Simon erklärt die Satisfizierung von Individuen psychologisch. Vgl. Simon 1959, 262f. Roy (1952), Telser (1955), Kataoka (1963) entwickelten Varianten eines ,safety-first“-Prinzips als Optimalitätskriterium für Investitionsentscheidungen der Anleger unter Risiko. Nach diesen Ansätzen fokussieren Anleger ausschießlich auf die Wahrscheinlichkeit, eine bestimmte Rendite beziehungnsweise ein bestimmtes Ergebnis zu verfehlen. Diese zentrale Annahme ist allerdings mit dem Bernoulliprinzip nicht mehr vereinbar.

$455 \quad$ Monsen, Downs 1965, 225.

456 Zur Dividendenausschüttung im Lichte des Signaling vgl. John, Williams (1985). Als empirisch gesichert kann die Politik der Dividendenkontinuität betrachtet werden; schon 1956 veröffentlichte Lintner eine Studie, in der er seine Hypothese bestätigte, die Gewinnausschüttungsentscheidung in der Aktiengesellschaft liege primär in der Frage, ob der in der Vergangenheit ausgeschüttete Dividendensatz geändert werden solle oder nicht. Dieses Ergebnis wurde seitdem in mehreren Vergleichsstudien auch für die Bundesrepublik
} 
Anspruchsniveau der Eigenkapitalgeber impliziert eine nur degressive Ertragsbeteiligung der Eigner am erwirtschafteten Cash Flow. Mit zunehmenden Einzahlungsüberschüssen ist der Grenzanteil der Aktionäre daran folglich abnehmend (Abbildung 4.1). Ähnlich wie das (1. Gossen'sche) Gesetz abnehmenden Grenznutzens $^{457}$ impliziert Satisfizierung für Eigenkapitalgeber eine mit steigendem diskontierten Cash Flow (dcf) abnehmende Grenzforderung. ${ }^{458}$ Mit einem ansteigenden erwirtschafteten Barwert künftiger Einzahlungsüberschüsse, steigt der Anteil der Eigenkapitalgeber daran nur unterproportional. "Contributors of capital ... must be assured a rate of return sufficient to induce them to contribute their capital to the enterprise." ${ }^{459}$

\section{Abbildung 4.1 Asymmetrische Beteiligung an den unternehmerischen Investitionserträgen}

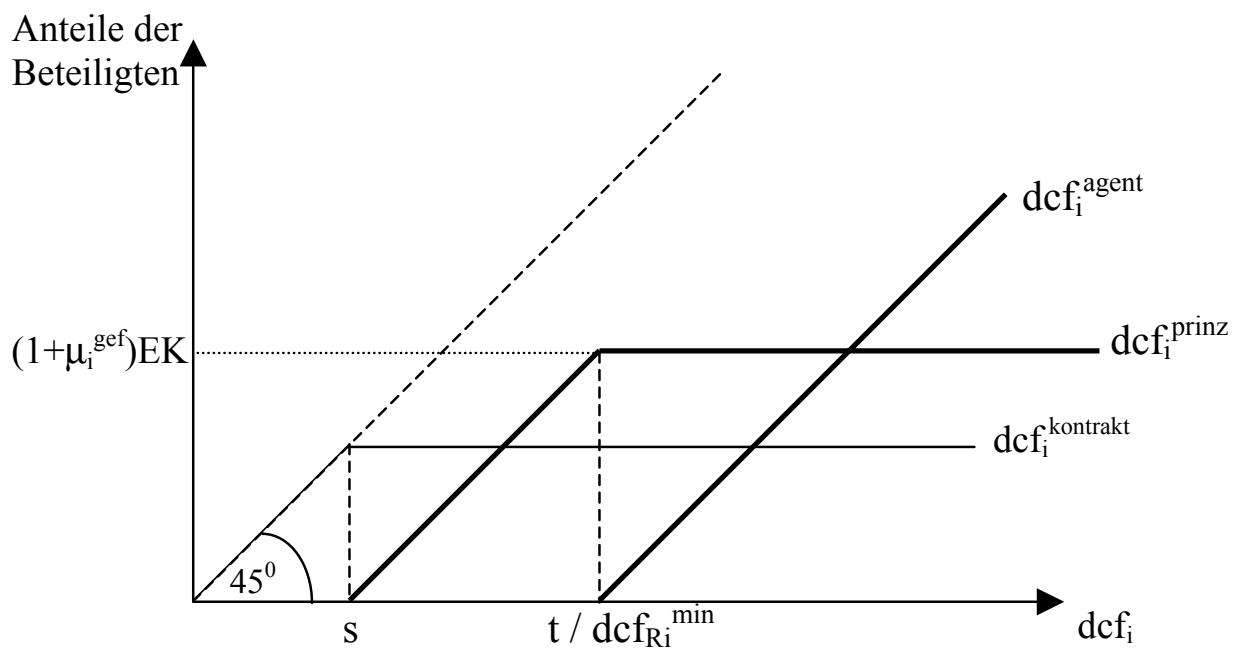

Der diskontierte Einzahlungsüberschuss dcf $_{\mathrm{i}}$ eines Unternehmens i entfällt auf die Kontraktforderungen der Stakeholder $\mathrm{dcf}_{\mathrm{i}}^{\text {kontrakt }}$, auf das satisfizierende Anspruchsniveau seiner Eigenkapitalgeber $\mathrm{dcf}_{\mathrm{i}}^{\text {prinz }}$ sowie auf den Anteil der Agenten

Deutschland bestätigt. Vgl. Wosnitza 1990, 949; Hansen 1996a, 42. “Outside equity depends on an intertemporal constraint: the dividend paid today must ensure outside investors' participation for at least one more period." Myers 2000, 1007 Vgl. a. Zwiebel (1996) und Warther (1997), die eine bestimmte Dividende als minimale Auszahlung modellieren, welche nötig ist, damit Anteilseigner nicht in den Prozess der Gewinnverwendung intervernieren.

Während die Theorie des Unternehmens aus der Konzeption satisfizierenden Verhaltens wesentliche Anstöße erhielt, steht die Ausgestaltung einer entsprechenden Haushaltstheorie noch aus. Vgl. Schumann, Meyer, Ströbele 1999, 99. 
$\operatorname{dcf}_{\mathrm{i}}^{\text {agent }}$, aus dem die Manager Organizational Slack bilden und X-Ineffizienzen finanzieren. Nach Erfüllung der Kontraktforderungen durch die Stakeholder werden bis zur Erfüllung einer bestimmten satisfizierenden Renditeforderung zunächst die Interessen der Eigenkapitalgeber in den Mittelpunkt gestellt $\left(\mathrm{s}<\mathrm{dcf}_{\mathrm{i}}<\mathrm{t}\right)$. Jede erwirtschaftete Einheit an $\operatorname{dcf}_{\mathrm{i}}$ geht bis zum Punkt $\mathrm{s}$ in vollem Umfang an die Festbetragsbeteiligten und von s bis $t$ in ausgeschütteter oder - bei Vorliegen eines bewertungseffizienten Marktes - in thesaurierter Form vollständig an die Eigenkapitalgeber. ${ }^{460}$ Die Höhe des satisfizierenden Anspruchsniveaus der Eigner leitet sich dabei aus dem CAPM ab $\left(\mu_{\mathrm{i}}{ }^{\text {gef }}\right)$. Der Ertrag der Eigner ab einem erwirtschafteten Cash Flow größer als t verläuft, wenn auch nicht - wie aus Vereinfachunsgründen im Beispiel gewählt - Null, so doch degressiv; der „Ertrag“ der Manager dcf $_{\mathrm{i}}^{\text {agent }}$ hingegen progressiv. Da eine zusätzlich anfallende Einheit an Cash Flow dcf $\mathrm{f}_{\mathrm{i}}$ (von s) bis Punkt $\mathrm{t}$ den Prinzipalen und ab $\mathrm{t}$ den Agenten zufällt, liegt eine asymmetrische vermögensmäßige Betroffenheit zwischen den Eigenkapitalgebern und -nehmern vor (Ertragsasymmetrie). Aus dem mit dcf $f_{i}^{\text {agent }}>0$ gebildeten Organizational Slack speisen sich die für den Erhalt eines Unternehmens stabilisierenden Zahlungen an die Stakeholder, die Manager und die Shareholder. Im Vergleich zur First-Best-Lösung eines Manager-Eigners, der in einer Arrow-DebreuWelt nach Begleichen aller Kontraktforderungen ( $\mathrm{ab}$ dem Punkt s) vollständig an einem zusätzlichen Einzahlungsüberschuss dcf $_{\mathrm{i}}$ partizipiert, unterliegen die Eigner hier gewissermaßen einer progressiven Steuer; ihre nur unterproportionale Beteiligung an den Einzahlungsüberschüssen kann als ein nicht-staatliches Instrument der Umverteilung innerhalb eines Unternehmens interpretiert werden. Der Handlungs- und Verteilungsspielraum der Agenten steigt mit zunehmendem $\operatorname{dcf}_{\mathrm{i}}^{\text {agent }}$. Doch erst nach Begleichen aller Kontrakt- wie Marktforderungen in Punkt $t$ beginnt ihre Dispositionsfreiheit. Um zumindest die Kontraktforderungen der Stakeholder und die Marktforderungen der Eigner (bei einem Organizational Slack =0) zu erwirtschaften, muss ein Unternehmen Investitionen mit einem $\mathrm{dcf}_{\mathrm{i}}$ in Höhe von mindestens $\mathrm{t}$ auswählen. Ein über $\mathrm{t}$ hinaus entstehender $\mathrm{dcf}_{\mathrm{i}}$, steht dann dem Management für eigene Zwecke, das heißt für X-Ineffizienzen sowie vor allem für

460 Hierbei handelt es sich selbstverständlich um eine vereinfachte Darstellung. Ist den Eignern ein stets gleichbleibender Ertrag etwa im Rahmen der Dividendenkontinuität zuzukommen, verläuft die Ertragskurve der Eigner nicht erst ab t, sondern bereits vom Ursprung parallel zur Abszisse. Möglicherweise ist hier Organizational Slack aufzulösen. Worauf es trotz aller anderen möglichen Verläufe in Abbildung 4.1 ankommt, ist ein letztlich degressiver Ertragsverlauf der Prinzipale sowie spiegelbildlich eine progressive Beteiligung der Agenten. 
die Bildung von Organizational Slack zur Verfügung. Diese für die Agenten anfallende Finanzmasse wird deshalb als „free discounted cash flow“ bezeichnet, ${ }^{461}$ womit man die dem Unternehmen nach Abzug aller notwendigen Auszahlungen zur Verfügung stehenden Mittel meint. Manager haben somit einen Anreiz, Unternehmensinvestitionen mit oberhalb von t liegenden Cash Flows zu realisieren, was jedoch gegen die Interessen der Eigner verstoßen kann (Gestaltungsasymmetrie). Denn eine Erhöhung des $\operatorname{dcf}_{\mathrm{i}}$ im Zuge einer progressiven Ertragsbeteiligung der Manager kann Auswirkungen auf das Risiko der zu finanzierenden Investitionen haben. Einerseits sind die Eigenkapitalgeber dann aufgrund der Ertragsasymmetrie an einem steigenden dcf $_{i}$ auf kurze Sicht gar nicht und auf mittelere bis lange Sicht nur eventuell und unterproportional beteiligt. Andererseits „partizipieren“ sie jedoch vollständig an den nicht-diversifizierbaren Risiken, die mit einem höheren $\operatorname{dcf}_{\mathrm{i}}$ einhergehen. Für Aktionäre „the punishment for grievous error is ... greater than the reward for outstanding success. This asymmetry between failure and success tends to make the managers of a diffused-ownership firm behave differently from the managers of the type of owner-managed firm envisioned by traditional theory." 462 Die Ertragsasymmetrie zwischen den Prinipalen und den Agenten hat Auswirkungen auf die Art der Gesellschaften, die Eigenkapitalgeber am Sekundärmarkt antreffen. Dies zu untersuchen, ist Gegenstand des nun folgenden Abschnitts.

461 „Free cash flow is cash flow in excess of that required to fund all projects that have positive net present values when discounted at the relevant cost of capital. Conflicts of interest between shareholders and managers over payout policies are especially severe when the organization generates substantial free cash flow. The problem is how to motivate managers to disgorge the cash rather than investing it at below the cost of capital or wasting it on organization inefficiencies." Jensen 1986, 323. 


\subsubsection{Selektion des Sekundärmarktes?}

In der Literatur weit verbreitet, ist die Auffassung, dass eine Aktienemission und somit eine Notierung am Sekundärmarkt ein schlechtes Signal für die Qualität eines Unternehmens darstellt. Diese Vorstellung ist Ausfluss einer - im Unterschied zur vorliegenden Arbeit - als linear unterstellten Beteiligung der Eigenkapitalgeber am erwirtschafteten Cash Flow dcfi. Teilen sich nach Bedienung der StakeholderAnsprüche die Investitionserträge für alle dcf $f_{i}$ proportional auf die Prinzipale und die Agenten (samt Organizational Slack) auf, besteht seitens der Eigenkapitalgeber wie des Managements ein gleichgerichtetes Interesse an Unternehmensinvestitionen mit möglichst hohen Einzahlungsüberschüssen. Aufgrund einer dann unbegrenzten Beteiligung der Eigenkapitalgeber am Cash Flow besteht für die Manager guter Unternehmen ein Anreiz, Investitionen weitgehend fremd- statt eigenzufinanzieren. Denn durch eine möglichst geringe Aktienfinanzierung ließe sich der steigende Anteil der Eigenkapitalgeber an hohen Cash Flows begrenzen. Die Bevorzugung von Fremdkapital, mithin ein hoher Verschuldungsgrad ist demzufolge Ausfluss eines Gewinnkalküls der Manager von Unternehmen mit hohen Cash FlowInvestitionen. ${ }^{463}$ Der Tendenz nach notieren am Aktienmarkt c.p. folglich Unternehmen mit vergleichsweise geringen dcf-Werten. ${ }^{464}$ "Entrepreneurs with higher expected returns prefer debt over equity. Intuitively, „good“ entrepreneurs are more reluctant to have the investors share in their success." ${ }^{465} \mathrm{Da}$ eine Aktienemission bei einer annahmegemäß proportionalen Beteiligung der Eigner am Cash Flow ein somit schlechtes „Label“ darstellt, sind die Kosten der Eigenkapitalbeschaffung für die börsennotierten Aktiengesellschaften - prohibitiv hoch. Dies entspricht der so genannten Hackordnungs- beziehungsweise "pecking order"-Hypothese. ${ }^{466}$ " [R]aising funds by issuing new equity may be a highly costly,

463 Die Verschuldung eines Unternehmens zeitigt einen Leverage-Effekt, das heißt eine Hebelwirkung dahingehend, dass gegenüber dem Fall reiner Eigenfinanzierung neben einem renditesteigernden Effekt auch das Risiko des Eigenkapitals steigt. Vgl. Schmidt, Terberger 1997, 244f.

464 Vgl. Ross (1977). Myers, Majluf (1984) erklären den adversen Selektionseffekt einer Eigenkapitalbeschaffung dadurch, dass Manager im Spannungsverhältnis zwischen Alt- und Neueignern Partei ergreifen, und zwar für die Insider (Altaktionäre) und gegen die Outsider (Neuaktionäre). Alteigner eines, guten“ Unternehmens sind demnach weniger an der Aufnahme neuer Aktionäre interessiert.

465 Hellmann, Stiglitz 1995, 2.

466 Unternehmen bevorzugen danach aus Kostengründen innenfinanzierte Mittel und ziehen, wenn notwendig, die Emission von Obligation der von Aktien vor. Vgl. Fazzari, Hubbard, Petersen 1988, 156. Zur theoretischen Fundierung der "pecking-order"-Hypothese vgl. Myers, Majluf (1984); vgl. a. Schulze 1994, 82-86. 
if not prohibitively difficult undertaking." ${ }^{467}$ Da eine Börsennotierung bei Zugrundelegung einer proportionalen Beteiligung der Eigenkapitalgeber an den Einzahlungsüberschüssen der unternehmerischen Investitionstätigkeit nicht nur ein schlechtes Signal darstellt, sondern zudem kostenintensiv ist, ,firms are reluctant to issue new equity." ${ }^{468}$

Die Überlegung, ausgerechnet börsennotierte Unternehmen seien „schlechte“ Unternehmen, erscheint in Anbetracht der letztjährigen Renaissance des Aktienmarktes sowie der Klagen über zu geringe Eigenkapitalquoten geradezu absurd. Fraglich ist, wieso es realiter überhaupt und in zunehmendem Maße eine Finanzierung über Aktienmärkte gibt, wenn der teure Rückgriff auf aktienfinanziertes Eigenkapital als Zeichen schlechter Qualität, das heißt eines vergleichsweise geringen Cash Flows gedeutet wird. ${ }^{469}$ Entgegen der theoretischen Auffassung, bei einer Aktienausgabe handele es sich um ein per se schlechtes, kann es sich offenbar tatsächlich auch um ein positives Signal handeln, wenn ein Unternehmen die teuerste Form der Außenfinanzierung wählt und dabei gleichzeitig das Leverage-Risiko reduziert. ${ }^{470}$ Demnach würden sich am Aktienmarkt vielmehr Unternehmen unterschiedlicher Qualität sammeln. In Übereinklang mit der Realität steht vielmehr, dass sich am Sekundärmarkt „gute“ wie auch „schlechte“ Unternehmen gleichermaßen befinden; dass sich mit einer Börsennotierung also nicht automatisch die „Spreu vom Weizen“ trennt. Vor dem Hintergrund der Diskrepanz zwischen der theoretischen Auffassung (bei Zugrundelegung einer proportionalen Beteiligung der Eigner am Cash Flow) und der realen Wahrnehmung wird in der Literatur gelegentlich eine differenzierte Auffassung vertreten: "Whereas

Greenwald, Stiglitz 1990, 2.

Asquith, Mullins 1986, 65.

"The chief difficulty with this analysis of the debt-equity choice is that we end up leaving no room at all for stock issues... But it is possible to rationalize equity in models based on information asymmetries alone... Suppose there is a large information asymmetry about the future variance rate. If investors underestimate the variance rate, the firm will be tempted to issue debt, but if they overestimate the variance rate the firm will be tempted to issue equity. Thus equity issues are not completely ruled out in equilibrium. Giammarino and Neave (1982) set up a model in which the managers and investors share the same information about everything except risk. In this case, equity dominate debt, because the only time managers want to issue debt is when they know the firm is riskier than investors think. Investors realizing this, refuse to buy. Only equity, or perhaps a convertible security, is issued in equilibrium... Firms actually seem to favor debt over equity issues, not the reverse. We believe asymmetric information about firm value is a stronger determinant of financing behavior than asymmetric information about risk ... although future empirical research could of course prove us wrong." Myers, Majluf 1984, 209.

Vgl. Bernanke, Gertler, Gilchrist 1996, 5; Asquith, Mullins 1986, 64. 
moral hazard concerning the entrepreneur's effort choice affect outside equity finance more than outside debt finance, the reverse is true of moral hazard concerning the riskiness of the entrepreneur's strategy choice." ${ }^{\text {471 }}$ Eine Aktienemission signalisiere folglich nicht per se eine schlechte Qualität, so doch die Art der $\mathrm{zu}$ finanzierenden Unternehmensinvestitionen. Es seien zwar - wie oben behauptet - Unternehmen mit geringerem Cash Flow, die tendenziell eine Eigenkapitalfinanzierung bevorzugen. Doch seien ihre Unternehmensinvestitionen auch weniger riskant. "Equity markets by contrast attract entrepreneurs with lower expected returns, but also with lower risk." ${ }^{972}$ Die hohe Eigenkapitalquote eines Unternehmens deute folglich auf ein vergleichsweise geringes, wenn auch marktgerechtes Rendite/Risiko-Profil der Unternehmensinvestitionen hin. Auch dieses Signal einer Eigenkapitalfinanzierung hinsichtlich der Art der zu finanzierenden Risiken am Aktienmarkt erscheint insofern unrealistisch, als Eigenkapital gerade dazu dient, riskante Unternehmensinvestitionen zu vollziehen.

Dass die Wahl der Finanzierungsform eine bestimmte Güte oder Art der zu finanzierenden Unternehmensinvestitionen signalisiert, erübrigt sich bei einer annahmegemäß degressiven Ertragsbeteiligung der Eigner am erwirtschafteten Cash Flow nach Begleichen der Kontraktforderungen. Mit der Annahme einer „quasifestverzinslichen“ Entlohnung für die Bereitstellung von Eigenkapital entfällt der oben geschilderte adverse Selektionsmechanismus des Sekundärmarktes. Legt man nämlich eine nur unterproportionale Partizipation der Eigner an den Einzahlungsüberschüssen zu Grunde, befinden sich am Sekundärmarkt Unternehmen mit hohem wie mit geringem Cash Flow. Es gibt keinen besonderen Anreiz für Unternehmen mit hohem dcf, die Fremd- der Eigenkapitalfinanzierung vorzuziehen. ${ }^{473}$ Nach Bedienung der satisfizierenden Anspruchsniveaus von Fremdwie Eigenkapitalgebern stehen die darüber hinaus gehenden Erträge nämlich dem Management - ungeachtet der Finanzierungsform - als „free discounted cash flow“ für Organizational Slack oder X-Ineffizienzen zur Verfügung. Insofern lassen aktienfinanzierte Unternehmensinvestitionen nicht per se auf einen im Vergleich zu fremdkapitalfinanzierten Unternehmensinvestitionen geringeren Cash Flow eines Unternehmens schließen; auch ist bei Zugrundelegung einer unterproportionalen

\footnotetext{
$471 \quad$ Hellwig 1989, 278.

472 Hellmann, Stiglitz 1995, 2.
} 
Beteiligung der Eigner an den unternehmerischen Investitionserträgen nicht mit einem durchschnittlich geringeren Rendite/Risiko-Profil börsennotierter Unternehmen im Verhältnis zu nicht-börsennotierten Unternehmen zu rechnen. Da eine Aktiennotierung im Fall eines degressiv angenommenen Ertragsverlaufs der Eigner nicht generell mit einer adversen Selektion behaftet ist, sammeln sich demzufolge am Aktienmarkt Unternehmen unterschiedlicher Qualität: Unternehmen mit geringen und hohen Cash Flows sowie mit über- und unterdurchschnittlichen Rendite/Risiko-Profilen ihrer Investitionsprojekte.

Ob die Beschaffung von Eigenkapital mit einem bestimmten Signal für die Güte und die Art der zu finanzierenden Unternehmensinvestitionen einhergeht, ist somit theoretisch offen. ${ }^{474}$ Diese Frage scheint auch eher akademischer Natur zu sein. In der realen Welt stehen Anleger angesichts der Vielzahl von Anlagemöglichkeiten vor der Auswahl mehr oder weniger guter Einzelrisiken - unabhängig davon, ob sie im Vergleich zu fremdkapitalfinanzierten Unternehmen der Art oder der Güte nach unterschiedlich beschaffen sind. Die (relativ) guten von den (relativ) schlechten Einzelrisiken zu trennen, ist für unvollkommen informierte Anleger praktisch nur eingeschränkt möglich. Das nur in Grenzen kontrollierbare Auftreten unterschiedlicher Unternehmenstypen am Sekundärmarkt wirft ein Selektionsproblem für die Eigenkapitalgeber auf. Folglich lässt sich die Frage, ob niveaubedingte Unterliquidität ein Selektionsproblem des Sekundärmarktes ist, verneinen. Niveaubedingte Unterliquidität ist vor dem Hintergrund der Ertragsasymmetrie vielmehr als ein Selektionsproblem am Sekundärmarkt zu begreifen. Denn es befinden sich dort sowohl gute als auch für Eigenkapitalgeber schlechte Einzelrisiken. Die Ursache für Sekundärmarktrationierung ist weniger in einer fragwürdigen Selektion des Sekundärmarktes, sondern vielmehr im Selektionsproblem der Eigenkapitalgeber zwischen „guten“ und „schlechten“ Einzelrisiken am Sekundärmarkt zu suchen.

Dass der Sekundärmarkt nicht per se zwischen guten und schlechten Unternehmen selektiert, ist letztlich der Überlegung geschuldet, dass die Bereitstellung von Eigenkapital eine quasi-festverzinsliche Vereinbarung darstellt. Bis zu einem höheren Eigenkapitalkosten einzusparen.

474 Vgl. Asquith, Mullins 1986, 61-66.
} 
satisfizierenden Niveau sind die Eigenkapitalgeber an den Investitionserträgen vollständig, zumindest jedoch proportional, darüber hinaus nur unterpropotional beteiligt - wie die Fremdkapitalgeber. Sonstigen Unterschieden zum Trotz ${ }^{475}$ ist beiden Formen der Außenfinanzierung (Eigenkapital, Fremdkapital) das Zahlungsversprechen des Kapital nehmenden Unternehmens gemeinsam, das unternehmerische Investitionsgebaren derart $\mathrm{zu}$ gestalten, dass die jeweiligen Kapitalgeber - hier etwa das Kreditinstitut, dort die Aktionäre - ihr eingesetztes Kapital „verzinst“ zurückerhalten. ${ }^{476}$ Bei einer Bereitstellung von Fremdkapital sind mit der Rückzahlungshöhe und der Rückzahlungsfrist wesentliche Bestandteile des Zahlungsversprechens vertraglich festgelegt. Bei einer Bereitstellung von Eigenkapital über den Aktienmarkt hingegen erwächst die Höhe der Renditeforderung und die Dauer der Anteilseignerschaft unmittelbar dem Marktgeschehen. Nicht ein expliziter Vertrag, sondern - modelltheoretisch gesprochen - die Wertpapiermarktlinie des CAPM diktiert den Preis zur Überlassung von Eigenkapital als Pendant zum vertraglich festgelegten Kreditzins. Die Dauer der Eigenkapitalbereitstellung ergibt sich aus dem Vergleich mit einer alternativ zu erwartenden Rendite. Steht zu erwarten, dass die geforderte marktgerechte Rendite verfehlt wird, droht die sofortige Aufkündigung der Kapitalüberlassung am Sekundärmarkt - falls möglich - durch das Abtreten der Anteilseignerschaft an einen Dritten. Ob Kredite oder Aktien - beide Formen der Außenfinanzierung implizieren das Erwirtschaften einer marktgerechten „Verzinsung“ innerhalb einer bestimmten Frist. Was in dem einen Fall vertraglich fixiert ist, erwächst im anderen Fall unmittelbar dem Marktgeschehen. "Debt and equity are sometimes hard to distinguish..." ${ }^{477} \mathrm{Zu}$ unterscheiden sind Fremd- und Eigenkapitalfinanzierung im Grad der vertraglichen Festlegung des Zahlungsversprechens an die Kapitalgeber. Mithin lassen sich die Stammaktie und der Standardkreditvertrag als Endpunkte eines Kontinuums möglicher Formen der Kapitalüberlassung verstehen. Während der Standardkreditvertrag das Zahlungsversprechen weitestgehend vertraglich regelt, ist

\footnotetext{
$475 \quad$ Vgl. Grill, Perczynski 1988, 304.

476 Lüthje (1970, 15) und Ehrlicher (1968) zählen Aktien deshalb zum Handelsgegenstand des Kreditmarktes. Vgl. Lüthje 1970, 45. Ehrlicher (1968) unterscheidet den Kapitalmarkt in einen Markt im engeren Sinne, auf dem langfristige Wertpapiere (Aktien und Obligationen) gehandelt werden, und in einen Markt im weiteren Sinne mit sämtlichen langfristigen Ausleihungen und Anlagen. Die Aktienbörse ist Teil des Kapitalmarktes im engeren Sinne und wird zusammen mit den Märkten für andere langfristige Wertpapiere in Wertpapierbörsen organisiert. Auch Schumpeter $(1961,698)$ betont die Einbettung der Wertpapierbörse in andere Kreditmärkte und deren Interdependenz. Myers 2000, 1006.
} 
das Zahlungsversprechen einer Stammaktie Gegenstand einer unmittelbar marktmäßigen, relationalen Beziehung zwischen Eigenkapitalgebern und -nehmern. Zwischen beiden Polen einer institutionellen Ausgestaltung von Kapitalbereitstellung und Zahlungsversprechen sind etwa Floating Rate Notes $^{478}$ einzuordnen, die durch einen höheren Grad an Relationalität als Standardkreditverträge gekennzeichnet sind, aber einen geringeren als Vorzugsaktien und Genußscheine. ${ }^{479}$ Entsprechend ist auch Mezzaninekapital $^{480}$ etwa in Form von Gesellschafterdarlehen, stillen Beteiligungen und nachrangigen Darlehen als hybride Finanzierungsform zwischen einer vertraglichen und einer relational marktmäßigen Bereitstellung von Kapital einzuordnen. Trotz dieser Unterschiede im Detail weisen alle Finanzierungsformen eine asymmetrische Beteiligung von Kapitalgebern und -nehmern an den Investitionserträgen eines Unternehmens auf. Dies eröffnet der Unterliquiditätsforschung Raum für eine Übertragung von Ansätzen der Kapitalrationierung vom Fremdkapitalmarkt auf den Aktiensekundärmarkt. ${ }^{481}$ „Although these results are presented in the context of credit markets, we show ... that they are applicable to a wide class of principal-agent problems..." ${ }^{482}$

478 Hierbei handelt es sich um Kreditverträge mit einer variablen Verzinsung, die sich etwa am Libor, Fibor oder Euribor bemisst.

479 Vgl. Siebel (1997).

$480 \quad$ Zum Mezzaninebegriff vgl. Leopold, Frommann 1998, 319.

481 Die nachfolgende Darstellung erfolgt in Anlehnung an Stiglitz, Weiss (1981). Vgl. a. Stiglitz (1982, 1984, 1987, 1988); Stiglitz, Weiss (1983, 1986, 1987a,b); Greenwald, Stiglitz (1987a,b, 1988a,b,c); Greenwald, Stiglitz, Weiss (1984). Einen Überblick über die Kapitalrationierungsansätze vor Stiglitz, Weiss (1981) bietet Baltensperger (1978). Zu Kreditrationierung im Konjunkturverlauf vgl. Azariadis, Smith (1998). 
4.3 Sekundärmarktrationierung 


\subsubsection{Poolbildung im Anlegerportefeuille}

Unter den Annahmen des CAPM hält der rationale Anleger in seinem (Markt-) Portefeuille gleichgewichtig alle sich am Markt befindlichen fundamental richtig bewerteten unsicheren Anlagen im Verhältnis ihrer Marktwerte. ${ }^{483}$ Der rationale Anleger ist vollständig informiert über das bewertungsrelevante Risiko einzelner Aktienwerte und vermag deshalb jede einzelne Aktie auf, unter oder über der gleichgewichtigen Wertpapiermarktlinie des CAPM einzuordnen. Ist sein Informationsstand über das bewertungsrelevante Einzelrisiko hingegen unvollkommen, kann der Anleger unsichere Anlagen im Verhältnis zur Wertpapiermarktlinie nicht genau spezifizieren. Er geht von einer Einzelfallbetrachtung zu einer Durchschnittsbetrachtung ihm mehr oder weniger ähnlich erscheinender Einzelrisiken über. Wie im CAPM befinden sich dabei (aus analytischen Gründen zunächst noch) alle umlaufenden Aktienwerte im Portefeuille des rationalen Anlegers. Allerdings ist dieses Portefeuille nach der Höhe der Renditeforderungen in verschiedene Risikoklassen, in so genannte Pools unterteilt. Aktien ähnlich erscheinenden bewertungsrelevanten Risikos werden in einem Pool zusammengefasst - unabhängig von der Größe der Aktiengesellschaften, ihrem Medienstanding und anderen Attributen, die für eine Vollrationierung am Sekundärmarkt entscheidend sind. Von allen Aktien eines Pools wird dieselbe Rendite gefordert, welche sich auf einem für die Eigenkapitalgeber satisfizierenden Anspruchsniveau befindet. Innerhalb eines Risikopools befinden sich über- wie unterdurchschnittliche Einzelrisiken. Die unterdurchschnittlichen Risiken stellen die für die Eigenkapitalgeber guten Einzelrisiken (,plums“), die überdurchschnittlichen Einzelrisiken die für die Eigenkapitalgeber schlechten Einzelrisiken (,lemons“) dar. ${ }^{484}$ Dabei „subventionieren“ die guten die schlechten Unternehmen eines Pools insofern, als erstere im Sinne der Eigenkapitalgeber den Pooldurchschnitt erhöhen, den hingegen letzere senken. Doch vermag der unvollkommen informierte Eigenkapitalgeber zwischen guten und schlechten Einzelrisiken nicht zu unterscheiden. Es wird deshalb von den Unternehmen mit den schlechten wie den guten Einzelrisiken dieselbe Rendite gefordert. Die geforderte Rendite werde von den guten, aber auch von den schlechten Einzelrisiken eines Pools mit einer

483 Betragen beispielsweise die fundamental richtig bewerteten Marktkapitalisierungen der einzigen beiden unsicheren Anlagen $\mathrm{A}=600.000$ EUR und $\mathrm{B}=400.000$ EUR, besteht das Marktportefeuille zu $60 \%$ aus A- und zu $40 \%$ aus B-Aktien. 
entsprechenden Internen Verzinsung ihrer Unternehmensinvestitionen real erwirtschaftet. ${ }^{485}$ Sowohl die guten als auch die schlechten Gesellschaften eines Pools kommen somit ungeachtet ihres tatsächlich bewertungsrelevanten Einzelrisikos der durchschnittlich geforderten Rendite der Anleger nach.

Je mehr Informationen der Anleger über die Einzelrisiken verfügt, desto differenzierter kann er alle Aktienwerte seines Portefeuilles nach durchschnittlichen Risiken in Pools gruppieren. Damit nimmt die Anzahl der Pools zu und die Anzahl der Unternehmen, die in einer Risikoklasse gepoolt werden, ab. Im Grenzfall vollkommener Informationen besteht eine einzelne Risikoklasse wie im CAPM aus nur einem Aktienwert. Je unvollkommener hingegen die Informationen hinsichtlich des bewertungsrelevanten Risikos von Einzelrisiken sind, desto undifferenzierter werden Einzelrisiken in einer Gruppe gepoolt. Die Anzahl der Pools ist dann geringer und die Anzahl der Aktienwerte innerhalb eines Risikopools größer. Bei einer vergleichsweise geringen Informiertheit der Anleger sind die guten und schlechten Einzelrisiken innerhalb eines Pools besonders breit gestreut. Die bewertungsrelevante Risikohöhe der Einzelwerte vermag der Anleger nicht näher zu spezifizieren. Er betrachtet die Aktien eines Pools derselben Renditeforderung deshalb als gleichwertig, so dass ein Einzelrisiko scheinbar dem durchschnittlichen Risiko entspricht. Den Durchschnitt des Risikopools sowie die Wahrscheinlichkeitsverteilung und -dichte aller Einzelrisiken eines Pools ist dem Eigenkapitalgeber dabei etwa durch Branchenstudien und -prognosen bekannt. ${ }^{486} \mathrm{Um}$ die Qualität eines einzelnen Unternehmens zu erkennen, fehlen den Aktionären jedoch nicht nur hinreichende Informationen, sondern auch adäquate ScreeningInstrumente, mit Hilfe derer ihr Informationsstand vervollkommnet werden könnte. Aktionäre behandeln einzelne Unternehmen einer Risikoklasse deshalb nicht für sich genommen, sondern qua Kenntnis der Verteilungs- und der Dichtefunktion aller Unternehmensinvestitionen eines Pools im Rahmen einer Durchschnittsbetrachtung.

$484 \quad$ Vgl. Emons (2001).

485 Auf solche Einzelrisiken, die den Renditeforderungen der Anleger nicht oder nicht in ausreichendem Umfang nachkommen, wird in der Untersuchung verzichtet. Denn schließlich soll gezeigt werden, dass auch solche Unternehmen, die sich den Renditevorgaben des Sekundärmarktes unterwerfen, durch niveaubedingte Unterliquidität übervorteilt werden. Bei Berücksichtigung auch jener Gesellschaften, die die Renditevorgaben des Sekundärmarktes nicht umsetzen, vergrößert sich das Selektionsproblem der Eigenkapitalgeber abermals.

Diese Annahme ist notwendig für die Existenz einer Gleichgewichtslinie, die bewertungsrelevante Risiken mit einer Renditeforderung positiv verbindet. Lägen auch keine 
Die Informationsasymmetrie der rationalen Anleger besteht also darin, dass sie nur das durchschnittlich bewertungsrelevante Risiko der einzelnen Aktienpools ihrer Gesamtportefeuilles, jedoch nicht die Höhe der Einzelrisiken innerhalb der Pools kennen.

Sind die Anleger nur mehr unvollkommen informiert über die Bewertungsrelevanz von Einzelrisiken, allerdings sehr wohl informiert über Durchschnittsrisiken von (Teil-)Gruppen innerhalb des Gesamtportefeuilles, ist eine Bewertung von Einzelaktien in einem Vergleich zum (Markt-)Portefeuille nicht mehr möglich. Die Bewertung einer Einzelaktie erfolgt im Rahmen einer vergleichenden Betrachtung zwischen dem Durchschnittsrisiko der Teilgruppe, in die das Einzelrisiko gepoolt ist, und dem (Markt-)Portefeuille. ${ }^{47}$ Die referenzielle Wertpapiermarktlinie des auf einer Betrachtung von Einzelaktien beruhenden CAPM wird in der Welt unvollkommener Informationen der Anleger deshalb ergänzt durch eine Beziehung zwischen der Renditeforderung und -erwartung sowie dem bewertungsrelevanten Risiko der einzelnen Risikopools bestehend aus jeweils ähnlich erscheinenden Einzelrisiken. Diese Beziehung ist ebenso linear. Werden nämlich mindestens drei Risikoklassen vom rationalen Anleger gebildet, ist es aus Arbitragegründen für die Eigenkapitalgeber rational, die Unternehmen so zu poolen, dass jeder Pool pro zusätzlich übernommene durchschnittlich bewertungsrelevante Risikoeinheit marginal dieselbe Rendite abzuwerfen erwarten lässt. Kombinationen aus der Renditeforderung an die Gesellschaften eines Pools und seinem (bekannten) durchschnittlichen Poolrisiko müssen sich deswegen - ähnlich der CAPMWertpapiermarktlinie - im Gleichgewicht auf einer linearen, aber diesmal auf Aktienpools basierenden Kurve befinden, der im Folgenden genannten Poollinie. Wäre dem nicht so, könnte der Anleger allein durch Umgruppierungen einzelner Gesellschaften zwischen den Pools eine positive Rendite erzielen. Die Begradigung einer vorübergehend nicht-linearen Poollinie wird durch den Wettbewerbsdruck beschleunigt, dem insbesondere institutionelle Anleger ausgesetzt sind. Die (lineare) Poollinie ist also der geografische Gleichgewichtsort von Renditeforderung und

Durchschnittswerte hinsichtlich einer Teilgruppe von Einzelrisiken vor, erfolgte die Renditeforderung beliebig. zu Schwankungen des (Markt-)Portefeuilles. Der einzige Unterschied besteht darin, dass nicht die Wahrscheinlichkeitsverteilung der Einzel-, sondern der Gesamtrenditen zugrunde gelegt werden. Vgl. Abschnitt 3.1. 
bewertungsrelevantem Risiko aller Pooldurchschnitte. Die Einzelrisiken eines Pools befinden sich je nachdem, ob es sich um ein „lemon“ oder um ein „plum“ handelt, rechts oder links der Poollinie. Die die Durchschnittsrisiken abbildende Poollinie dient den Eigenkapitalgebern als Vergleichsmaßstab dafür, ob Veränderungen wie etwa durch die Forderung einer höheren Rendite $\mathrm{zu}$ vollziehen rational ist. Renditeforderungen an die Gesellschaften eines Pools, die dazu führen, dass ein durchschnittliches Poolrisiko durch adverse Selektionen unter die Poollinie rutscht, werden unterlassen; solche, die zu einer Verortung eines durchschnittlichen Poolrisikos oberhalb der Poollinie führen, werden dagegen unternommen.

Was für die unvollkommen informierten Eigenkapitalgeber die Poollinie ist, ist für die vollständig informierten Unternehmensmanager als „Eigenkapitalnachfrager“ die CAPM-Wertpapiermarktlinie. ${ }^{488}$ Eigenkapitalanbieter und -nachfrager haben also grundsätzlich unterschiedliche Referenzkurven. Bei Vorliegen symmetrischer Informationen sind Wertpapiermarkt- und Poollinie kongruent. Vor dem Hintergrund asymmetrischer Informationen befindet sich jedoch die Poollinie regelmäßig rechts der CAPM-Wertpapiermarktlinie, im Bereich eines unterschießenden Eigenkapitalangebots also. Denn es ist für die Prinzipale zu erwarten, dass die Agenten ihren Informationsvorsprung dahingehend ausnutzen, im Durchschnitt riskantere Unternehmensinvestitionen vorzunehmen, indem sie zu einer bestimmten Renditeforderung der Anleger insgeheim riskantere Unternehmensprojekte auswählen. Durch die Auswahl von Unternehmensinvestitionen mit geringeren Projektwahrscheinlichkeiten fallen erwartungsgemäß höhere Einzahlungsüberschüsse an. Diese stehen den Agenten zur Bildung von Organizational Slack, aber auch für X-Ineffizienzen zur Verfügung $\left(=\mathrm{dcf}_{\mathrm{i}}{ }^{\text {agent }}\right){ }^{489}$ So ist zu erwarten, dass auch die jeweils besten, das heißt geringsten Einzelrisiken der Pools allenfalls bewertungsrelevante Risiken in Höhe der Wertpapiermarktlinie, aber nicht links davon aufweisen. Da mit zunehmender Informationsasymmetrie die Anzahl der Einzelrisiken in einem Pool und damit die Spannbreite zwischen überund unterdurchschnittlichen Aktienwerten zunimmt, ist die Poollinie umso weiter (rechts) von der Wertpapiermarktlinie entfernt, je unvollkommener die Anleger informiert sind. Umgekehrt verkleinert sich mit abnehmender Informationsasymmetrie die Streuung der Einzelrisiken im Pool, und es nähert sich

488 Zu unvollkommenen Informationen auch der Agenten vgl. Abschnitt 5.4. 
die Poollinie (der Eigenkapitalgeber) der Wertpapiermarktlinie (der Eigenkapitalnehmer) an. Dasjenige Risiko, das sich aus der positiven Differenz zwischen der Wertpapiermarktlinie und der Poollinie ergibt, ist auf die fehlende Spezifizierbarkeit der Einzelrisiken in einem Pool bei asymmetrischen Informationen zurückzuführen. Die fehlende Spezifizierbarkeit der Einzelrisiken eines Pools betrifft je nach Ausmaß der Informationsvollkommenheiten mehr oder weniger alle Aktien. Das Risiko einer Falschspezifizierung von Einzelaktien ist durch eine breite Streuung im Anlegerportefeuille deshalb nicht vollständig diversifizierbar. Es handelt sich insofern um ein firmenunspezifisches und folglich am Kapitalmarkt bewertungsrelevantes Risiko. Ob der Markt die Übernahme dieses bewertungsrelevanten Risikos im Rahmen einer entsprechend höheren Renditeforderung auch tatsächlich kompensiert, hängt - wie zu zeigen sein wird nicht zuletzt von der Steigung der Poollinie ab. Die Steigungen der Pool- wie der Wertpapiermarktlinie können identisch sein, müssen es aber nicht. Die Steigung der Poollinie hängt nämlich neben den in Abschnitt 3.1 genannten Steigungsfaktoren (Risikoneigung der Anleger, Höhe der Internen Verzinsung, Kapitalzufluss, Geldund Fiskalpolitik) auch vom Ausmaß der Informiertheit über die einzelnen Risikoklassen ab. Haben die Anleger etwa besonders gute Kenntnisse über die Aktien im Hochrisikobereich, nähert sich die Poollinie in diesem Bereich der Wertpapiermarktlinie stärker an. Die Steigung der Poollinie verliefe folglich steiler als die der Wertpapiermarktlinie.

Niveaubedingte Unterliquidität als das Ergebnis einer Vollrationierung am Aktiensekundärmarkt stellt sich als ein in geplanten Größen, nicht nur vorübergehender, sondern dauerhafter Überhang der Eigenkapitalnachfrage dar. Zu zeigen ist deshalb, dass sich die Poollinie qua höherer Renditeforderungen der Wertpapiermarktlinie langfristig nicht anzupassen vermag. Die Poollinie verharrt vielmehr dauerhaft rechts von der Wertpapiermarktlinie, im Bereich eines unterhalb des markträumenden Niveaus liegenden Eigenkapitalangebots. Dabei werden allerdings bestimmte Einzelrisiken innerhalb der Pools nachgefragt, andere identisch erscheinende werden vernachlässigt. Es liegt eine Ungleichbehandlung von Einzelrisiken eines Pools durch rationale Eigenkapitalgeber vor. ${ }^{490}$ Kriterium dafür,

\footnotetext{
$489 \quad$ Vgl. Abschnitt 4.2.2.

490 Wird ein ganzer Pool von Aktienwerten eines bestimmten Durchschnittsrisikos rationiert, liegt der Fall des ,redlining“" vor. Vgl. Abschnitt 4.1.
} 
welche Einzelaktien eines Pools be- und welche übervorteilt werden, kann aufgrund ihrer fehlenden Spezifizierbarkeit unmöglich die Höhe der bewertungsrelevanten Einzelrisiken sein. Vielmehr sind es so genannte sekundäre Kriterien, die über Beoder Missachtung seitens der Anleger entscheiden. Ein Erklärungsansatz für die ungleiche Behandlung von identisch erscheinenden Einzelrisiken bedarf insofern einer gewissermaßen horizontalen Analyse, als Gesellschaften eines einzelnen Pools zu betrachten sind. Nur auf diese Weise wird deutlich, dass eine in ökonomischer Hinsicht rationale Diskriminierung gleich erscheinender Aktienwerte zu Lasten bestimmter Unternehmenstypen stattfindet, mithin ein Versagen des Aktiensekundärmarktmechanismus vorliegt. Für die Analyse niveaubedingter Unterliquidität als Vollrationierung fällt deshalb in einem ersten Schritt das Augenmerk exemplarisch auf einen einzelnen Pool. In ihrer Aggregation mit den rationierten Aktienwerten anderer Pools ergibt sich dann in einem zweiten Schritt das Bild eines segmentierten Gesamtaktienmarktes. Eine vor dem Hintergund unvollkommener Informationen eingeschränkte Eigenkapitalangebotsmenge in Verbindung mit einer Konzentration des Eigenkapitalangebots auf nur wenige Aktienwerte (sprich: Vollrationierung) zeichnet sich folglich verantwortlich dafür, dass der deutsche Aktiensekundärmarkt in beschriebener Art segmentiert ist.

Die nun folgende Untersuchung verengt die Betrachtung auf den ersten Schritt einer „horizontalen“ Analyse eines exemplarischen Risikopools i. Es wird der mögliche Fall aufzuzeigen sein, dass innerhalb eines Pools i im langfristigen Gleichgewicht a) das Eigenkapitalangebot hinter der Eigenkapitalnachfrage dauerhaft zurückbleibt und das eingeschränkte Eigenkapitalangebot b) $\mathrm{zu}$ Lasten bestimmter Unternehemenstypen geht. Eine bei unvollkommenen Informationen durchzuführende Anhebung der Renditeforderung (mit einer beabsichtigten Annäherung der Pool- an die markträumende Wertpapiermarktlinie beziehungsweise des Eigenkapitalangebots an die Eigenkapitalnachfrage) geht möglicherweise mit einer adversen Veränderung der Zusammensetzung des Pools und einem infolgedessen steigenden Durchschnittsrisiko einher. Das sich mit höheren Renditeforderungen verschlechternde Durchschnittsrisiko eines Pools kompensiert die zusätzlich geforderte Rendite in diesem Fall derart über, dass die höhere Renditeforderung sogar $\mathrm{zu}$ einem Rückgang der - die Risikoverschlechterung umfassenden - Netto-Renditeerwartung führt. Der im CAPM zu einer Markträumung 
von Eigenkapitalangebot und -nachfrage tendierende Preis- beziehungsweise Renditemechanismus wird auf diese Weise außer Kraft gesetzt. Denn eine markträumende (Netto-) Rendite ist nur dann zu erwarten, wenn sich das Poolrisiko anlässlich einer Renditeforderung in Höhe der Wertpapiermarktlinie durch eine sich advers veränderte Poolzusammensetzung nicht überproportional erhöht. Eine NettoRenditeerwartung, die infolge adverser Selektionen, aber auch adverser Anreize dauerhaft unterhalb der markträumenden CAPM-Wertpapiermarktlinie verharrt, geht dann mit einer im Gleichgewicht gesamtwirtschaftlichen Unterinvestition am Aktiensekundärmarkt einher, das heißt dauerhaft mit einem Tobin'schen q kleiner als Eins. $^{491}$ 


\subsubsection{Adverse Selektion}

In einer Welt unvollkommener Informationen, in der Preise Einfluss auf die Qualität von Gütern haben, geht eine Anhebung der Renditeforderung für die Einzelrisiken eines Pools mit einer veränderten Zusammensetzung des Pools einher. Verschlechtert sich etwa das durchschnittliche Poolrisiko in einem Ausmaß, dass die gestiegene Renditeforderung überkompensiert, ist es für die Eigenkapitalgeber rational, den Anstieg der Renditeforderung zu unterlassen. Die Netto-Renditeerwartung wird in diesem Fall infolge einer ansteigenden Renditeforderung negativ. Das Eigenkapitalangebot verharrt infolgessen dauerhaft unterhalb seines markträumenden Niveaus. Betrifft dies mehrere Pools im Anlegerportefeuille, verbleibt die Poollinie im langfristigen (nicht-markträumenden) Gleichgewicht rechts von der Wertpapiermarktlinie. Vor dem Hintergrund eines zunächst einperiodigen Planungshorizontes ist im nun Folgenden zu zeigen, ${ }^{492}$ dass es für Eigenkapitalgeber bei Vorliegen asymmetrisch verteilter Informationen sowie asymmetrisch verteilter unternehmerischer Investitionserträge im Rahmen einer relationalen PrinzipalAgenten-Beziehung rational sein kann, dauerhaft eine nicht-markträumende Rendite, das heißt eine Rendite unterhalb der Wertpapiermarktlinie zu fordern. Dazu wird in einem ersten Schritt erläutert, dass die Anleger grundsätzlich einen Anreiz haben, weniger Eigenkapital anzubieten als im markträumenden CAPM. In einem zweiten Schritt wird der Frage nachzugehen sein, ob und unter welchen Bedingungen es sich hierbei um einen dauerhaften Überhang der Eigenkapitalnachfrage handelt.

In Unkenntnis der genauen Risikohöhe einzelner Aktien zerfällt das Gesamtportefeuille des rationalen Anlegers in einzelne Risikoklassen. Einer dieser Pools besteht aus einzelnen Aktien, die dem unterinformierten Anleger von ähnlicher Risikohöhe erscheinen. Von den sieben Gesellschaften eines exemplarisch betrachteten Pools i (vergleiche linkes Diagramm der Abbildung 4.2) wird bei einem durchschnittlichen bewertungsrelevanten Poolrisiko $\tau_{\mathrm{i}}^{\varnothing}$ gemäß der Poollinie $\mu_{\mathrm{n}}^{\text {gefø }}$ pauschal eine „Verzinsung“ des eingesetzten Eigenkapitals von $\mu_{\mathrm{i}}^{\text {gef }}$ gefordert. Für den Anleger erkennbar ist die Verteilung der Einzelrisiken im Pool i, nicht aber die Spezifizierung seiner Einzelrisiken. Die beiden überdurchschnittlich riskanten Einzelrisiken, von denen der unterinformierte Anleger nicht weiß, welche der sieben

492 Zur Mehrperiodigkeit vgl. Abschnitt 4.3.5.3. 
Aktienwerte sich dahinter verbergen, liegen vom Pooldurchschnitt $\tau_{\mathrm{i}}^{\varnothing}$ vergleichsweise weit entfernt. Die breite Streuung der Einzelrisiken lässt deshalb auf einen eher geringen Informationsstand des rationalen Anlegers schließen.

Abbildung 4.2 Adverse Selektion im Up-Market ${ }^{493}$

$\underline{\text { Informationsasymmetrie }}$

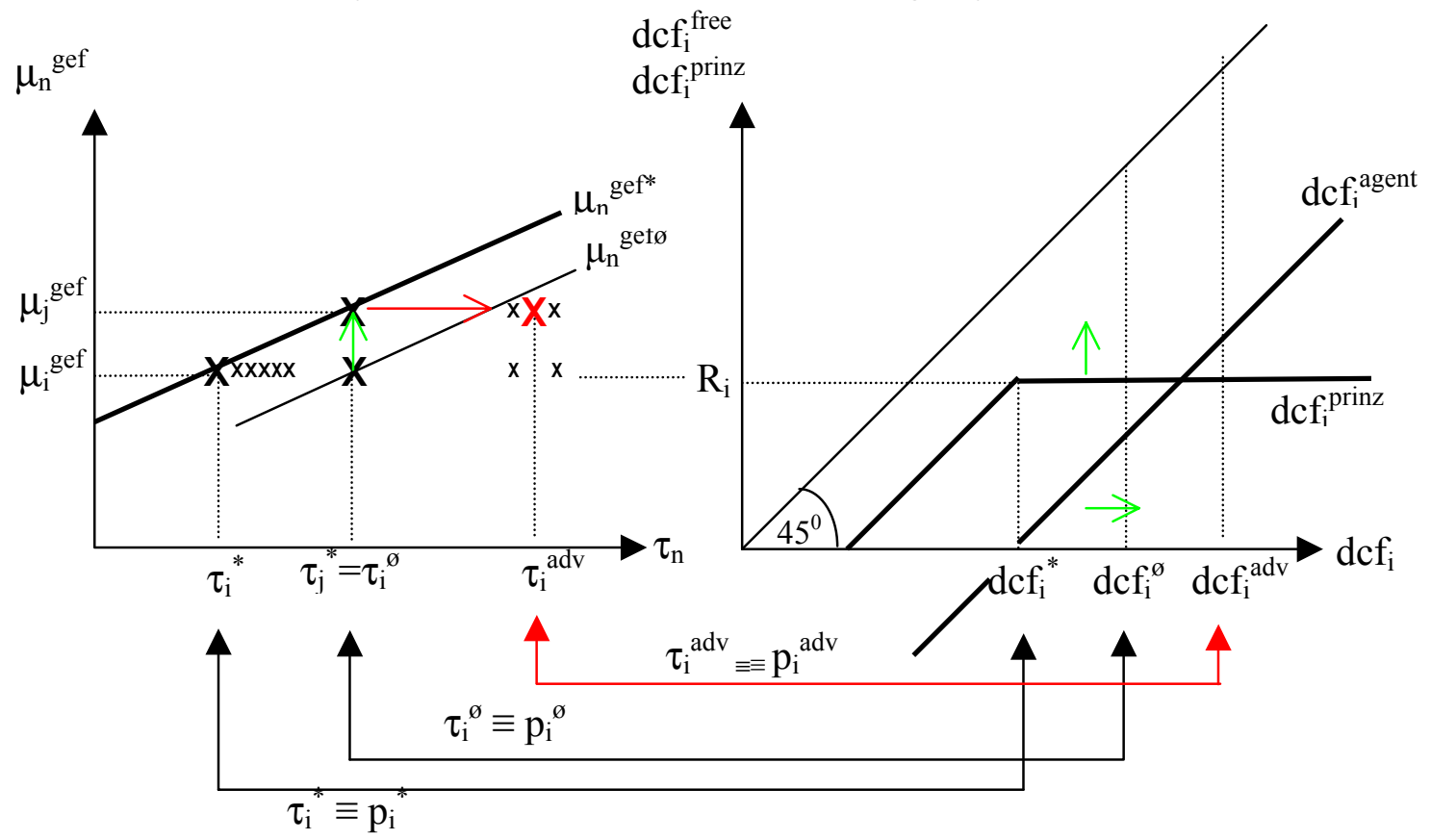

Nach Begleichen der Kontraktforderungen werden die erwirtschafteten Erträge aus der unternehmerischen Investitionstätigkeit $\operatorname{dcf}_{\mathrm{i}}$ des Durchschnittsunternehmens im Pool $\mathrm{i}$ auf die Eigenkapitalgeber in Höhe von $\mathrm{dcf}_{\mathrm{i}}^{\text {prinz }}$ sowie auf die Manager zur Bildung von Organizational Slack oder für X-Ineffizienzen $\left(\mathrm{dcf}_{\mathrm{i}}^{\text {agent }}\right)$ asymmetrisch aufgeteilt (vergleiche rechtes Diagramm). In Anbetracht der Relationalität ihrer Beziehung $\mathrm{zu}$ den Prinzipalen sorgen die Agenten der Poolunternehmen für das Erwirtschaften der geforderten Rendite $\mu_{\mathrm{i}}{ }^{\text {gef }}$. Dazu ist ein unternehmerischer Investitionsertrag für die Prinzipale am zu erwirtschaftenden Cash Flow $\left(\mathrm{dcf}_{\mathrm{i}}\right)$ in Rendite/Risiko-Kombination eine Überrendite mit einem entsprechenden Angebotsüberhang an Eigenkapital. Sofern keine Rigiditäten des Marktmechanismus nach unten bestehen, tendiert der Aktienmarkt von einer solchen Situation ausgehend zum markträumenden Gleichgewicht der Wertpapiermarktlinie. 
Höhe von $\mathrm{R}_{\mathrm{i}}=\left(1+\mu_{\mathrm{i}}{ }^{\text {gef }}\right)$ Ek vonnöten. ${ }^{494}$ Nach Begleichen der Kontraktforderungen fallen die Einzahlungsüberschüsse bis dcf $_{\mathrm{i}}{ }^{*}$ vollständig den Eigenkapitalgebern, aufgrund der Ertragsasymmetrie darüber hinaus den Managern zu.

Der als konstant angenommene Investitionsertrag $\mathrm{R}_{\mathrm{i}}$ lässt sich durch verschiedene unternehmerische Investitionsobjekte realisieren. ${ }^{495} \mathrm{Um}$ für die Prinzipale den Investitionsertrag $R_{i} z u$ erwirtschaften, können die Agenten zwischen unsicheren Investitionsprojekten mit einem vergleichsweise hohen erwarteten Cash Flow dcf bei einer geringen Erfolgswahrscheinlichkeit $p_{i}$ oder etwa (im Extremfall) dem Projekt mit dem geringsten Projektertrag $\mathrm{dcf}_{\mathrm{i}}{ }^{*}$ bei hoher Erfolgswahrscheinlichkeit $\mathrm{p}_{\mathrm{i}}{ }^{*}$ wählen; letzteres Investitionsprojekt, bei dem eine finanzielle Masse weder für Organizational Slack noch für X-Ineffizienzen übrig bleibt $\left(\operatorname{dcf}_{i}^{\text {agent }}=0\right)$, wird bei vollkommenen Investitionen gemäß der CAPM-Wertpapiermarktlinie unternommen. Entsprechend dem bewertungsrelevanten Risiko $\tau_{i}^{ø}$ liegt die Projektwahrscheinlichkeit der Unternehmensinvestitionen des Pools i bei durchschnittlich $\mathrm{p}_{\mathrm{i}}^{\varnothing}$, $496 \mathrm{ihr}$ erwarteter Einzahlungsüberschuss bei $\operatorname{dcf}_{\mathrm{i}}^{\varnothing}$. Die Zufallsvariable der erwarteten Einzahlungsüberschüsse $\mathrm{dcf}_{\mathrm{i}}$ ist dabei derart (normal)verteilt, dass ihm im Sinne eines „mean preserving spread“4497 bei konstantem erwarteten Investitionsertrag $R_{i}$ jeweils gegenläufige Projektwahrscheinlichkeiten $p_{i}$ zugeordnet sind. Das Produkt aus Projektwahrscheinlichkeit $p_{i}$ und dem erwartetem Cash Flow $\left(\mathrm{dcf}_{\mathrm{i}}\right)$ ergibt demnach stets den von den - annahmegemäß risikoneutralen ${ }^{498}$ Anlegern geforderten Investitionsertrag $\mathrm{R}_{\mathrm{i}}$. Zum Erwirtschaften dieses von den

494 Die Höhe des konstant eingesetzten Eigenkapitals EK betrage vereinfachungshalber 1. Somit gilt $\mathrm{R}_{\mathrm{i}}=1+\mu_{\mathrm{i}}{ }^{\text {gef }}$.

495 In Abschnitt 4.3.5.4 wird die angenommene Konstanz des Investitionsertrages $\mathrm{R}_{\mathrm{i}}$ aufgehoben.

496 Implizit wird unterstellt, dass geringere Projektwahrscheinlichkeiten $\mathrm{p}_{\mathrm{i}}$ nicht vollends diversifiziert werden können.

497 Als Maß für das Risiko eines Investitionsertrags $R_{i}$ fungiert die Erfolgswahrscheinlichkeit der ihm zugrundeliegenden Projekte. Das Risiko des Investitionsertrags wird im Sinne eines ,mean preserving spread“ definiert. Vgl. Rothschild, Stiglitz (1970). Bei gleichem Erwartungswert des Investitionsertrags $\mathrm{R}_{\mathrm{i}}$ wird ein Projekt mit seiner Varianz risikoreicher. Bei einem mean preserving spread weist die Dichtefunktion von $\mathrm{R}_{\mathrm{i}}$ einen konstanten Erwartungswert durch die beiden Zufallsvariablen $\mathrm{dcf}_{\mathrm{i}}$ und $\mathrm{p}_{\mathrm{i}}$, jedoch unterschiedliche Projektwahrscheinlichkeiten auf. Durch einen so genannten mean preserving spread verlagern sich die Wahrscheinlichkeitsgewichte der Dichtefunktion von $\mathrm{R}_{\mathrm{i}}$ vom Zentrum in seine Flügel. Dadurch wird bei riskanten Projekten das Integral der Verteilungsfunktion größer. Vgl. ebenda, 227231.

498 Risikoneutralität innerhalb eines Pools hinsichtlich $\mathrm{R}_{\mathrm{i}}$ stellt eine Inkonsistenz zur angenommenen Risikoaversion der Anleger zwischen den Pools hinsichtlich der Zusammenstellung der Aktien zum Portefeuille dar. Doch verbirgt sich hinter diesem scheinbaren Widerspruch nur eine Vereinfachung, die im Folgenden aufgehoben wird. Dieser „Kunstgriff“ ändert qualitativ nichts am möglichen Auftreten einer adversen Selektion. Ob der Projektertrag $\operatorname{dcf}_{\mathrm{i}}$ bei sinkendem $\mathrm{p}_{\mathrm{i}}$ proportional - wie bei Risikoneutralität - oder 
Anlegern geforderten Investitionsertrages $R_{i}$ bedarf es Unternehmensinvestitionen mit einem bewertungsrelevanten Mindestrisiko in Höhe von $\tau_{i}^{*}$. Andernfalls müsste - falls vorhanden - Organizational Slack aufgelöst werden. Das Mindestrisiko zu unterschreiten, wäre für Manager irrational, da sie mit einer Offenlegung aller Informationen, das heißt bei einer symmetrischen Informationsverteilung einen $\mathrm{dcf}_{\mathrm{i}}^{\text {agent }}$ in derselben Höhe (von Null) einstreichen würden. Um einen Anteil am Cash Flow $\mathrm{dcf}_{\mathrm{i}}^{\text {agent }}<0 \mathrm{zu}$ verhindern, steht $\mathrm{zu}$ erwarten, dass die Agenten riskantere Kombinationen als $\mathrm{dcf}_{\mathrm{i}}{ }^{*} / \mathrm{p}_{\mathrm{i}}{ }^{*}$ anstreben. Es gilt also für alle $\mathrm{dcf}_{\mathrm{i}} \geq \mathrm{dcf}_{\mathrm{i}}{ }^{*}$ :

$$
\mathrm{p}_{\mathrm{i}} \times \mathrm{dcf}_{\mathrm{i}}=\mathrm{R}_{\mathrm{i}}=\text { const. } \Leftrightarrow \mathrm{dcf}_{\mathrm{i}}=\mathrm{R}_{\mathrm{i}} / \mathrm{p}_{\mathrm{i}}
$$

Durch einfache Differenzierung ergibt sich:

$$
\Rightarrow \quad \operatorname{ddcf}_{\mathrm{i}} / \mathrm{dp}_{\mathrm{i}}=-\mathrm{R}_{\mathrm{i}} / \mathrm{p}_{\mathrm{i}}^{2}=-\mathrm{dcf}_{\mathrm{i}} / \mathrm{p}_{\mathrm{i}}<0 \text {. }
$$

Für das Erreichen des von den Eigenkapitalgebern geforderten Investitionsertrages $\mathrm{R}_{\mathrm{i}}$ stehen die Wahrscheinlichkeiten möglicher Projekte $\mathrm{p}_{\mathrm{i}}$ und seine erwarteten Einzahlungsüberschüsse $\mathrm{dcf}_{\mathrm{i}}$ in einem inversen Verhältnis. Bei der Grenzkombination $\left(\mathrm{dcf}_{\mathrm{i}}{ }^{*} / \mathrm{p}_{\mathrm{i}}{ }^{*}\right)$ der unternehmerischen Investitionstätigkeit kommen die Manager der Renditeforderung $\mu_{\mathrm{i}}{ }^{\text {gef }}$ respektive dem geforderten Investitionsertrag $\mathrm{R}_{\mathrm{i}}$ nach und lassen dabei ihren Informationsvorsprung gegenüber den Eigenkapitalgebern verstreichen. Nach Begleichen der Kontraktforderungen fallen sämtliche Einzahlungsüberschüsse $\left(\mathrm{dcf}_{\mathrm{i}}^{*}\right)$ in diesem Fall den Eigenkapitalgebern zu. Im exemplarisch betrachteten Pool i wählen die Manager der guten, das heißt der unterdurchschnittlichen Einzelrisiken annäherungsweise derart beschaffene Projekte mit relativ hohen Projektwahrscheinlichkeiten und relativ geringen erwarteten Einzahlungsüberschüssen $\mathrm{dcf}_{\mathrm{i}}$ aus. Dass sich die Manager aller Unternehmen eines Pools für anlegerfreundliche $\left(\mathrm{dcf}_{\mathrm{i}} ; \mathrm{p}_{\mathrm{i}}\right)$-Kombination entscheiden, ist jedoch unwahrscheinlich. So wirft die anlegerfreundlichste Kombination $\left(\operatorname{dcf}_{i}^{*} / \mathrm{p}_{\mathrm{i}}^{*}\right)$ für die Manager nur den Mindest-Cash Flow $\operatorname{dcf}_{\mathrm{i}}^{\text {agent }}=0 \mathrm{ab}$. Indem Manager riskantere Investitionsprojekte auswählen, können sie die dabei erwartungsgemäß anfallende Finanzmasse für Organizational Slack und für X-Ineffizienzen nutzen. Da das

überproportional - wie bei Risikoaversion - ansteigt, um einen konstanten erwarteten Investitionsertrag $R_{i}$ zu erzielen, ist für das grundsätzliche Phänomen adverser Selektion unerheblich. 
Management des durchschnittlichen Unternehmens im betrachteten Pool i der Renditeforderung $\mu_{\mathrm{i}}^{\text {gef }}$ nachkommt, indem es durchschnittlich Investitionsobjekte mit einer geringeren Erfolgswahrscheinlichkeit von $\mathrm{p}_{\mathrm{i}}^{\varnothing}<\mathrm{p}_{\mathrm{i}}{ }^{*}$ respektive einem bewertungsrelevanten Risiko $\tau_{\mathrm{i}}^{\varnothing}>\tau_{\mathrm{i}}{ }^{*}$ unternimmt, erwirtschaftet es einen erwartungsgemäß positiven freien Cash Flow $\operatorname{dcf}_{i}^{\text {free }}\left(=p_{i} \times \operatorname{dcf}_{i}^{\text {agent }}\right)$. Die Agenten haben also einen Anreiz, den geforderten Investitionsertrag $\mathrm{R}_{\mathrm{i}}$ mit Hilfe von vergleichsweise riskanten Investitionsobjekten anzusteuern, die vorzugsweise einen hohen $\mathrm{dcf}_{\mathrm{i}}$-Wert mit einer geringen Projektwahrscheinlichkeit $\mathrm{p}_{\mathrm{i}}$ kombinieren. Unter Vernachlässigung der Kontraktforderungen steigt nämlich der für die Agenten frei verwendbare Cash Flow dcf ${ }_{i}^{\text {free }}$ mit riskanteren Unternehmensprojekten an:

$$
\left.\mathrm{dcf}_{\mathrm{i}}^{\text {free }}=\mathrm{p}_{\mathrm{i}}\left[\mathrm{dcf}_{\mathrm{i}}-\left(1+\mu_{\mathrm{i}}^{\mathrm{gef}}\right) \mathrm{EK}\right)\right] .
$$

Mit Hilfe von Gleichung (4.2) folgt als Differenzialgleichung:

$$
\Rightarrow \mathrm{ddcf}_{\mathrm{i}}^{\text {free }} / \mathrm{dp}_{\mathrm{i}}=\mathrm{dcf}_{\mathrm{i}}+\mathrm{p}_{\mathrm{i}}\left(\mathrm{ddcf}_{\mathrm{i}} / \mathrm{dp}_{\mathrm{i}}\right)-\left(1+\mu_{\mathrm{i}}{ }^{\text {gef }}\right) \mathrm{EK}=-\left(1+\mu_{\mathrm{i}}{ }^{\mathrm{gef}}\right) \mathrm{EK}<0 .
$$

Mit steigender Wahrscheinlichkeit der Unternehmensinvestitionen $p_{i}$ sinkt der für die Agenten frei verwendbare Cash Flow $\mathrm{dcf}_{\mathrm{i}}^{\text {free }}$, et vice versa. Manager werden somit für die Inkaufnahme höherer Anstrengungen, die gegebenenfalls mit riskanteren Unternehmensinvestitionen höherer $\mathrm{dcf}_{\mathrm{i}}$-Werte verbunden sind, durch einen höheren Cash Flow $\mathrm{dcf}_{\mathrm{i}}^{\text {free }}$ entschädigt. Sie neigen insofern $\mathrm{zu}$ einer Externalisierung der Projektrisiken $\mathrm{zu}$ Lasten der Eigenkapitalgeber. Diese ehalten trotz höherer tatsächlich eingegangener Risiken „nur“ den geforderten Investitionsertrag $\mathrm{R}_{\mathrm{i}}$. Es besteht für Manager deshalb ein Anreiz, $\left(\mathrm{dcf}_{\mathrm{i}} / \mathrm{p}_{\mathrm{i}}\right)$-Kombination $\mathrm{zu}$ wählen, die an einem Up-Market einer Rendite/Risiko-Situation rechts unterhalb der Wertpapiermarktlinie gleichkommen. Aus der Informations- und Ertragsasymmetrie entwickelt sich somit eine Gestaltungsasymmetrie dergestalt, dass Manager riskantere Unternehmensinvestitionen vornehmen als im CAPM-Gleichgewicht eines markträumenden Eigenkapitalangebots gemäß der Wertpapiermarktlinie der Fall ist. Welche $\left(\mathrm{dcf}_{\mathrm{i}} / \mathrm{p}_{\mathrm{i}}\right)$-Kombination die Agenten des Pools $\mathrm{i}$ wählen, hängt von der einzelwirtschaftlichen Notwendigkeit, Organizational Slack bilden zu müssen, von den Konsumbedürfnissen der Manager am Arbeitsplatz und nicht zuletzt von ihrer Risikoneigung ab. Diese Aspekte bestimmen, in welchem Umfang die Eigner ihren 
Informationsvorsprung ausnutzen, das heißt grafisch gesprochen, wie weit die Poolvon der Wertpapiermarktlinie entfernt liegt.

Bislang wurde auf formalem Wege lediglich gezeigt, dass das von den Managern durchschnittlich angesteuerte Rendite/Risiko-Profil des Pools i $\left(\mu_{\mathrm{i}}{ }^{\mathrm{gef}} / \tau_{\mathrm{i}}{ }^{\varnothing}\right)$ sich gemäß der Poollinie unterhalb der markträumenden Wertpapiermarktlinie befindet. Bei einem konstanten Investitionsertrag $\mathrm{R}_{\mathrm{i}}$ partizipieren die Eigenkapitalgeber hierbei schlechterdings am höheren bewertungsrelevanten Risiko $\tau_{i}^{\varnothing}\left(>\tau_{i}^{*}\right)$. Da sie an den höheren bewertungsrelevanten Risiken voll, an den höheren Einzahlungsüberschüssen hingegen nicht beteiligt sind, schränken sie bei Rendite/Risiko-Profilen unterhalb der Wertpapiermarktlinie ihr Eigenkapitalangebot ein. Denn die Netto-Renditeerwartung wird im Verhältnis zur CAPM-Kombination $\left(\mu_{\mathrm{i}}^{\mathrm{gef}} / \tau_{\mathrm{i}}^{*}\right)$ durch die Auswahl riskanterer Investitionsprojekte beeinträchtigt. Die Anleger bieten deshalb bei unvollkommenen Informationen weniger Eigenkapital an, als an fundamental richtig bewerteten Aktien umlaufen. Fraglich ist, ob das eingeschränkte Eigenkapitalangebot dauerhaft ist oder in eine zusätzliche Renditeforderung mündet und damit einen Anstieg des Eigenkapitalangebots bis hin zur Markträumung herbeiführt. Im betrachteten Beispiel der Abbildung 4.2 hätte eine Anhebung des Anspruchsniveaus von $\mu_{\mathrm{i}}^{\text {gef }}$ auf $\mu_{\mathrm{j}}^{\text {gef }}$ (grüner Pfeil des linken Diagramms) c.p. zur Folge, dass sich der Überhang der Eigenkapitalnachfrage beseitigen würde. Neben einem gewinnsteigernden Effekt ergibt sich durch eine Renditeforderung in Höhe von nun $\mu_{\mathrm{j}}^{\text {gef }}$ jedoch auch eine Veränderung in der Zusammensetzung des Pools (roter Pfeil). ${ }^{499}$ Die Verschlechterung der Poolzusammensetzung ist verhältnismäßig stark ausgeprägt, da die fünf (unbekannten) guten der insgesamt sieben Einzelrisiken aus dem Pool i ausscheiden. Für die fünf unterdurchschnittlichen Einzelrisiken des Pools fällt die erhöhte Renditeforderung zu hoch aus, um nicht Organizational Slack auflösen zu müssen $\left(\mathrm{dcf}_{\mathrm{i}}^{\text {free }}<0\right)$. Es verbleiben lediglich die beiden schlechten Einzelrisiken im Pool um welche Gesellschaften es sich dabei im Einzelnen auch immer handeln mag. Sie realisieren zwar die erhöhte Renditeforderung. Jedoch ,[a]s the price of equity rises, the relative proportion of honest entrepreneurs decreases, and the relative proportion

499 Eine höhere Rendite $\mathrm{j}$ von den Unternehmen eines Pools i zu fordern, führt streng genommen zu einer Verschmelzung der Pools i und j. Aus Vereinfachungsgründen wird der Effekt der Verschmelzung von ursprünglich getrennt behandelten Risikoklassen hier vernachlässigt. Es wird lediglich ein Vergleich der Risikosituation von Pool j mit Pool i nach Erhöhung der Renditeforderung angestellt. 
of looters increases. In fact, sufficiently high prices of equity only looters will be left, and the investors' returns always turn negative." ${ }^{" 500}$ Es ist $\mathrm{zu}$ untersuchen, unter welchen Bedingungen der für die Anleger positive Gewinneffekt einer höheren Renditeforderung, welche durch die im Pool verbleibenden Gesellschaften auch zu erwarten ist, stärker wiegt als der negative Gewinneffekt, der dadurch entsteht, dass sich die Zusammensetzung des Pools verschlechtert. Beide entgegengesetzten Effekte spiegeln sich in der Veränderung des Ertrages $E\left(\equiv p_{i} \times \operatorname{dcf}_{i}^{\text {prinz }}\right)$ des rationalen Anlegers wider:

$$
\begin{gathered}
E=\frac{\left(1+\mu_{i}^{\text {gef }}\right) E K}{\tau_{i}\left(p_{i}\right)} \equiv p_{i}\left(1+\mu_{i}^{\text {gef }}\right) E K \\
\text { mit } \tau_{\mathrm{i}} \equiv 1 / \mathrm{p}_{\mathrm{i}} \text { und } \mathrm{dcf}_{\mathrm{i}}^{\text {prinz }}=\mathrm{R}_{\mathrm{i}}=\left(1+\mu_{\mathrm{i}}^{\text {gef }}\right) \mathrm{EK}
\end{gathered}
$$

Da den Aktionären die Dichtefunktion der Wahrscheinlichkeitsverteilung $g\left(p_{i}\right)$ unternehmerischer Investitionsobjekte bekannt ist, lässt sich der erwartete Ertrag E der Aktionäre umformen in:

$$
\mathrm{E}=\int_{0}^{p_{i}^{*}}\left(1+\mu_{i}^{g e f}\right) E K g\left(p_{i}\right) d p_{i}=\left(1+\mu_{i}^{g e f}\right) E K \int_{0}^{p_{i}^{*}} g\left(p_{i}\right) d p_{i}
$$

mit der maximalen Wahrscheinlichkeit $\mathrm{p}_{\mathrm{i}}{ }^{*}\left(\equiv \tau_{\mathrm{i}}{ }^{*}\right)$, oberhalb derer die Agenten nicht als Eigenkapitalnachfrager am Sekundärmarkt auftreten. Dabei stellt $\int_{0}^{p_{i}^{*}} g\left(p_{i}\right) d p_{i}$ die Zahl der Einzelrisiken im betrachteten Pool und $\mathrm{g}\left(\mathrm{p}_{\mathrm{i}}\right)$ die den Eigenkapitalgebern bekannte Dichtefunktion der Verteilung von unternehmerischen Projektwahrscheinlichkeiten dar. Bezieht man den erwarteten Ertrag E auf das eingesetzte Eigenkapital EK, erhält man als Quotienten $\rho_{i}^{\varnothing}$ die erwartete NettoRendite des für den Pool zur Verfügung gestellten Eigenkapitals; die NettoRenditeerwartung $\rho_{\mathrm{i}}^{\varnothing}$ unterscheidet sich von der bisher verwendeten (Brutto)Renditeerwartung $\mu_{\mathrm{i}}$ - wie bereits mehrfach angeklungen - darin, dass letztere im Gegensatz zu ersterer nicht die veränderte Risikozusammensetzung nach Anhebung

$500 \quad$ Vgl. Hellmann, Stiglitz 1995, 30. 
der Renditeforderung berücksichtigt. ${ }^{501}$ Dabei gilt für alle Rendite/Risiko-Profile auf der Poollinie, dass ihre Netto-Renditeerwartungen stets identisch sind. Durch eine Variation der Rendite/Risiko-Kombinationen entlang der Poollinie lässt sich die Netto-Renditeerwartung somit nicht erhöhen. In einer Bewegung auf der Poollinie etwa nach oben wird jede zusätzliche Rendite gerade durch das ansteigende Risiko aufgezehrt, so dass netto keine höhere Rendite $\rho_{\mathrm{i}}^{ø} \mathrm{zu}$ erwarten ist:

$$
\rho_{i}^{ø}=\frac{E}{E K}=\frac{\left(1+\mu_{i}^{g e f}\right) E K \int_{0}^{p_{i}^{*}} g\left(p_{i}\right) d p_{i}}{E K \int_{0}^{p_{i}^{*}} g\left(p_{i}\right) d p_{i}}
$$

Ob eine höhere Rendite zu fordern rational ist, verdeutlicht sich an der Veränderung der erwarteten Netto-Rendite $\rho_{i}^{ø}$ des für den Pool i zur Verfügung gestellten Eigenkapitals. Dabei hängt die Veränderung von $\rho_{\mathrm{i}}^{ø}$ vom Ausmaß diverser Effekte $\mathrm{ab}$, die in der folgenden Differenzialgleichung zu unterscheiden sind: ${ }^{502}$

$$
\begin{aligned}
& \frac{d \rho_{i}^{ø}}{d \mu_{n}^{g e f}}=\int_{0}^{p_{i}^{*}} g\left(p_{i}\right) d p_{i}[\int_{0}^{p_{i}^{*}} \overbrace{\left.p_{i} g\left(p_{i}\right) d p_{i}+\left(1+\mu_{i}^{g e f}\right) p_{i}^{*} g\left(p_{i}^{*}\right)\left(\frac{d p_{i}^{*}}{d \mu_{n}^{g e f}}\right)\right]}^{\mathrm{a}(+)} \\
& \underbrace{\mathrm{b}(+)}_{\mathrm{d}(+)} \overbrace{}^{\left(\frac{d p_{i}^{*}}{d \mu_{n}^{g e f}}\right) g\left(p_{i}^{*}\right)\left[\left(1+\mu_{i}^{g e f}\right) \int_{0}^{p_{i}^{*}} p_{i} g\left(p_{i}\right) d p_{i}\right]}
\end{aligned}
$$

Nimmt Gleichung 4.8 einen positiven Wert an, steigt die erwartete Netto-Rendite mit einer marginalen Anhebung der Renditeforderung. Es lohnt sich folglich für den Eigenkapitalgeber, die Renditeforderung um eine Einheit anzuheben. Entsprechend läge das Rendite/Risiko-Profil des Pools zu einer diskret angehobenen Renditeforderung $\left(\mu_{\mathrm{j}}^{\mathrm{gef}} / \tau_{\mathrm{i}}^{\mathrm{adv}}\right)$ im Fall eines positiven Wertes von Gleichung 4.8 links oberhalb der Poollinie. Es würde damit ein geringeres Risiko aufweisen als die

\footnotetext{
$501 \quad$ Ungeachtet seiner Zusammensetzung erwirtschaften alle Unternehmen des Pools die geforderte Rendite vor wie auch nach ihrer Anhebung durch eine entsprechende die Interne Verzinsung der Unternehmensinvestitionen. Die geforderte Rendite der sich im Pool befindlichen Unternehmen entspricht daher auch der erwarteten (Brutto-)Rendite.

502 Vgl. Hillier, Imbrahimo 1993, 280f.
} 
vergleichbare Pool-Kombination $\left(\mu_{\mathrm{j}}{ }^{\mathrm{gef}} / \tau_{\mathrm{j}}{ }^{\sigma}\right)$. Die adverse Selektion der Poolzusammensetzung infolge einer höheren Renditeforderung fiele unterproportional aus. Umgekehrt ist eine Anhebung der Renditeforderung zu unterlassen, fällt Gleichung (4.8) negativ aus; im betrachteten Beispiel der Abbildung 4.2 fällt die Rendite/Risiko-Kombination $\left(\mu_{\mathrm{j}}{ }^{\mathrm{gef}} / \tau_{\mathrm{i}}{ }^{\text {adv }}\right)$ nach einer diskreten Erhöhung der Renditeforderung auf das markträumende Niveau unter die Poollinie.

Entscheidend für Richtung und Ausmaß der veränderten Netto-Renditeerwartung sind die einzelnen Terme (a) bis (d) in Gleichung 4.8. Die Terme (a) bis (c) drücken dabei die Veränderung des Ertrags $\mathrm{E}$ aus, die sich durch einen Anstieg der Renditeforderung ergeben, das heißt die Veränderung des Zählers von Gleichung (4.7). Der Term (d) spiegelt die Veränderung des eingesetzten Eigenkapitals wider, das heißt den veränderten Nenner von Gleichung (4.7). Mit steigender Renditeforderung geht die im Pool verbleibende Anzahl von Unternehmen zurück und mit der Verkleinerung des Pools das eingesetzte Eigenkapital. Unter Berücksichtigung des Substraktionszeichens ist Term (d) somit positv, erhöht also bei steigender Renditeforderung die erwartete Netto-Rendite. Term (a) entspricht der ursprünglichen Anzahl der sich im Pool befindlichen Unternehmen und ist ebenfalls positiv. Term (b) entspricht der Zunahme des Ertrags dadurch, dass die im Pool verbleibenden Unternehmen der gestiegenen Renditeforderung nachkommen. Ein Anstieg der geforderten Rendite senkt aber auch gleichzeitig den den frei verfügbaren Cash Flow $\mathrm{dcf}_{\mathrm{i}}^{\text {free }}$, und zwar den der guten Einzelrisiken unter das Mindest-Niveau von Null. Infolgedessen scheiden die für die Eigenkapitalgeber guten, marginalen Unternehmen aus dem betrachteten Pool der Eigenkapital nachfragenden Unternehmen aus. Bei einer nicht nur marginalen, sondern diskreten Erhöhung der Renditeforderung etwa auf das Anspruchsniveau $\mu_{\mathrm{j}}{ }^{\text {gef }}$ verlassen alle fünf Gesellschaften links der Poollinie mit einem unterdurchschnittlich bewertungsrelevanten Risiko $\tau_{\mathrm{i}}$ (mit $\tau_{\mathrm{i}}^{*} \leq \tau_{\mathrm{i}}<\tau_{\mathrm{i}}^{\varnothing}$ ) den Pool i. Es ist für die Eigenkapitalgeber zu erwarten, dass die fünf guten Risiken die erhöhte Renditeforderung durch das Realisieren ihrer Investitionsobjekte nicht erwirtschaften, mithin in einer einperiodigen Betrachtung ausscheiden. ${ }^{503} \mathrm{Um} \mathrm{im}$ Pool derer zu verbleiben, die der höheren Renditeforderung nachkommen, bedarf es

503 Denkbar in einem mehrperiodigen Ansatz ist, dass die guten Unternehmen in einem solchen Fall der Renditeforderung ,zumindest“ gemäß der Wertpapiermarktlinie nachkommen und darüber hinaus Organizational Slack auflösen. Vgl. Abschnitt 4.3.5.3. 
eines kritischen Mindestrisikos der unternehmerischen Investitionsobjekte. Ein Anstieg der geforderten Rendite von $\mu_{\mathrm{i}}^{\text {gef }}$ auf $\mu_{\mathrm{j}}{ }^{\text {gef }}$ beziehungsweise des geforderten Investitionsertrages von $R_{i}$ auf $R_{j}$ erhöht das für die Manager kritische unternehmerische Mindestrisiko. Die guten Grenzrisiken scheiden aus dem Pool aus, da sie zur Erwirtschaftung des gestiegenen Renditeanspruchs $R_{j}$ nur mehr einen Organizational Slack kleiner Null erzielen, was weniger ist, als würden sie ihren Informationsvorsprung unausgenutzt lassen. Nun mehr links von der Wertpapiermarktlinie anzuordnen, würden sich die guten Unternehmen somit besser stehen, sorgten sie für symmetrisch verteilte Informationen; daraus lässt sich über den bislang unterstellten einperiodigen Betrachtungszeitraum ein Anreiz für die guten Einzelrisiken dergestalt ableiten, dass sie ihren Informationsvorsprung gegenüber den Eigenkapitalgebern mittel- bis langfristig abbauen. ${ }^{504}$ In einer einperiodigen Betrachtung kommen die ausscheidenden, guten Einzelrisiken den höheren Renditeforderungen durch eine entsprechende Interne Verzinsung jedoch gar nicht oder nur eingeschränkt nach. Dabei wird das Ausscheiden guter Einzelrisiken zum einen durch die Risikoneigung der Agenten beeinflusst. So erachten risikoaverse Manager ihre marginalen Investitionsobjekte für unprofitabler als risikofreudige Manager. ${ }^{505}$ Der Austritt der guten Unternehmen wird zum anderen durch das Ausmaß der Unterbewertung verstärkt, von dem im Zuge der Poolbildung die guten Einzelrisiken besonders betroffen sind. Denn unterbewertete Aktien erhöhen die Eigenkapitalkosten, bergen die Gefahr einer feindlichen Übernahme, mindern die Liquidationserlöse realer Vermögensobjekte sowie das Ansehen des Unternehmens, gehen mit Einschränkungen auf dem Arbeitsmarkt für Agenten einher und erhöhen letztlich auch die Kosten für die Zertifizierung der tatsächlichen Unternehmensqualität. Kurzum: unterbewertete Kurse veranlassen gute, vom Ausscheiden bedrohte Unternehmen eher zum Austritt aus dem Pool.

Nach Anhebung der Renditeforderung auf $\mu_{\mathrm{j}}{ }^{\text {gef }}$ verbleiben im betrachteten Beispiel der Abbildung 4.2 die beiden überdurchschnittlich riskanten Einzelrisiken des Beispiels im Pool. Sie sind die einzigen Unternehmen, die bei der gestiegenen Renditeforderung keinen Organizational Slack auflösen müssen, um mit ihren

504 Damit kann die Renditeanhebung zu einer Screening-Methode: Der rationale Anleger könnte sich in einer mehrperiodigen Betrachtung auf die ausscheidenden, weil erwartungsgemäß guten Unternehmen kaprizieren. Dabei entsteht allerdings der Anreiz einer Imitation für die schlechten Einzelrisiken. Vgl. hierzu Abschnitt 4.3.5.3. Vgl. Smith 1998, 457f. 
geplanten Investitionsobjekten den Forderungen der Anleger nachkommen zu können. Wenngleich auch für sie ein höheres Anspruchsniveau der Aktionäre c.p. mit einem Rückgang der frei verfügbaren Cash Flows verbunden ist, gibt es jedoch keine Notwendigkeit für sie, den Pool verlassen zu müssen. Denn im Unterschied zu den guten, können die schlechten Einzelrisiken des Pools i nach wie vor Organizational Slack aufbauen. Aus Sicht der Eigenkapitalgeber findet folglich eine Negativauslese statt, die im betrachteten Beispiel ein Ausmaß annimmt, das die erwartete Netto-Rendite sinken lässt. Eine adverse Selektion senkt die durchschnittliche Projektwahrscheinlichkeit von $\mathrm{p}_{i}{ }^{\varnothing}$ auf $\mathrm{p}_{\mathrm{i}}^{\text {adv }}$, womit das bewertungsrelevante Risiko von $\tau_{\mathrm{i}}^{ø}$ auf $\tau_{\mathrm{i}}^{\text {adv }}$ ansteigt. Dieser Effekt drückt sich in einem negativen Term (c) der Gleichung (4.8) aus. Das steigende Poolrisiko in Gestalt des Terms (c) kompensiert die übrigen auf die Renditeerwartung positiv wirkenden Effekte (a), (b) und (d) einer gestiegenen Renditeforderung im Beispiel der Abbildung 4.2 über. Ob eine adverse Veränderung der Poolzusammensetzung hinreichend groß ist, um die erwartete Netto-Rendite zu senken, bemisst sich grafisch betrachtet an der Steigung der Poollinie. Mit zunehmender Steigung der Poolline nimmt das akzeptable Ausmaß adversen Selektionsrisikos ab. Im Unterschied zu einem Up-market mit steiler Poollinie kompensiert der adverse Selektions- den favorablen Gewinneffekt bei einer hinreichend flachen Poollinie nicht über. Verliefe also die Steigung der Poollinie in Abbildung 4.2 deutlich flacher als eingezeichnet, wäre der adverse Selektionseffekt möglicherweise weniger dominant. Die Wahrscheinlichkeit, eine höhere Rendite rationalerweise nicht $\mathrm{zu}$ fordern und ein folglich nicht-markträumendes Eigenkapitalvolumen anzubieten, steigt also mit zunehmender Steilheit der Poollinie. ${ }^{506}$

Das Ausmaß der Verschlechterung eines Pools infolge einer höheren Renditeforderung hängt von der Anzahl und der Art der ausscheidenden Gesellschaften ab. Dies wird wiederum von der ursprünglichen Verteilung der Einzelrisiken im Pool bestimmt. So vergrößert sich etwa das Ausmaß der adversen Selektion umso mehr, je weiter die Einzelrisiken um das Durchschnittsrisiko des Pools gestreut sind (Abbildung 4.3). Denn durch das Ausscheiden der guten Einzelrisiken verbleiben bei einer ziemlich breiten Streuung vergleichsweise hohe Einzelrisiken im Pool. Mit einer heterogenen Zusammensetzung des Pools und einer

506 Dieser Aspekt wird in Abschnitt 4.3.4 vertieft. 
infolgedessen ausgeprägten adversen Selektion sinkt somit das Eigenkapitalangebot am Sekundärmarkt. Die Heterogenität ist wiederum Ausfluss der asymmetrischen Informationsverteilung. Ein rationaler Anleger mit nahezu vollkommenen Informationen wird insbesondere Aktienwerte ähnlichen Risikos poolen wie im Beispiel den Pool r. Für schlecht informierte Eigenkapitalgeber sind hingegen eher die Aktienpools $\mathrm{s}$ und $\mathrm{t}$ repräsentativ mit einem deutlichen Anstieg des durchschnittlichen Poolrisikos von $\tau_{\mathrm{s}, \mathrm{t}}{ }^{ø}$ auf $\tau_{\mathrm{s}, \mathrm{t}}^{\mathrm{adv}}$ nach Auscheiden der unterdurchschnittlichen Einzelrisiken. Die Wahrscheinlichkeit, dass eine adverse Selektion die erwartete Netto-Rendite infolge einer höheren Renditeforderung reduziert, nimmt also grundsätzlich mit dem Grad der Uninformiertheit der Anleger zu. Ein Vergleich der beiden Pools s und t zeigt darüber hinaus, dass es beim Anstieg des durchschnittlichen Poolrisikos nicht nur auf die Breite der Streuung ankommt. Auch die Art, wie die Einzelrisiken im Pool verteilt sind, ist entscheidend für die Höhe der adversen Risikoveränderung.

Im Beispiel der Abbildung 4.3 wird (aus analytischen Gründen) davon ausgegangen, dass das durchschnittliche Poolrisiko $\tau_{\mathrm{n}}{ }^{ø}$ in der Ausgangssituation, das heißt vor Anhebung der Renditeforderung in allen drei Pools ungeachtet des Grades ihrer Heterogenität gleich hoch und damit auch unabhängig vom Grad der Informationsunvollkommenheiten ist. Doch vergrößert sich der Tendenz nach mit abnehmenden Informationen nicht nur die Streuung der Einzelrisiken um ein durchschnittliches Poolrisiko, sondern auch das durchschnittliche Poolrisiko vom markträumenden Risiko, das heißt die Entfernung der Pool- von der Wertpapiermarktlinie. Denn eine bessere Spezifizierbarkeit von Einzelrisiken ermöglicht den Anlegern eine weniger abweichende Einschätzung des Pooldurchschnitts von der Wertpapiermarktlinie.

Abbildung 4.3 Adverse Selektion in Abhängigkeit vom Informationsgrad
und der Verteilung der Einzelrisiken

\begin{tabular}{|c|l|l|l|l|l|l|l|l|l|l|l|l|l|}
\hline$\tau_{\mathrm{n}}$ & 1,0 & 1,1 & 1,2 & 1,3 & 1,4 & $\mathbf{1 , 5}=\tau_{\mathbf{n}}^{\mathbf{0}}$ & 1,6 & 1,7 & 1,8 & 1,9 & 2,0 & 2,1 & $\tau_{\mathbf{n}}^{\left.\mathbf{a d v}_{1}\right)}$ \\
\hline
\end{tabular}




\begin{tabular}{|c|c|c|c|c|c|c|c|c|c|c|c|c|}
\hline Pool r & & & & $\mathrm{X}_{\text {gut }}$ & $\mathrm{X}_{\text {gut }}$ & $\tau_{r}^{\varnothing}$ & $\mathrm{X}_{\mathrm{schl}}$ & $\mathrm{X}_{\mathrm{sch} 1}$ & & & & 1,65 \\
\hline Pool s & & & $\mathrm{X}_{\text {gut }}$ & $\mathrm{X}_{\text {gut }}$ & $\mathrm{X}_{\text {gut }}$ & $\tau_{\mathrm{s}}{ }^{\varnothing}$ & & & & & $\mathrm{X}_{\mathrm{schl}}$ & 2,1 \\
\hline Pool t & $X_{\text {gut }}$ & $X_{\text {gut }}$ & & & & $\tau_{t}^{\varnothing}$ & & & $\mathrm{X}_{\mathrm{schl}}$ & $\mathrm{X}_{\mathrm{schl}}$ & & 1,95 \\
\hline
\end{tabular}

1) Dieser durchschnittliche Risikowert ergibt sich nach Anhebung der Renditeforderung dadurch, dass die unterdurchschnittlichen Einzelrisiken aus dem Pool scheiden.

Im Fall adverser Selektion stehen einzelne Gesellschaften der Risikopools angesichts höherer Renditeforderungen vor den beiden Möglichkeiten „Bleiben oder Gehen“. Denkbar ist darüber hinaus, dass einzelnen Unternehmen verschiedene Investitionsprojekte von unterschiedlichem Risiko zur Auswahl stehen. Anlässlich einer höheren Renditeforderung können die Agenten in diesem Fall die unternehmerische Investitionstätigkeit derart modifizieren, dass sie riskanter wird. Die Eigenkapitalgeber kennen in diesem Fall zwar wieder die Verteilungen der möglichen Projekte, können die konkrete Auswahl der Investitionsprojekte eines Unternehmens aber weder beobachten noch kontrollieren. Wie der folgende Abschnitt zeigt, besteht für die Manager der Unternehmen eines Pools ein adverser Anreiz darin, durch eine gestiegene Renditeforderung die für die Anleger schlechten, weil riskanten Investitionen auszuwählen. Damit eröffnet sich für die guten Einzelrisiken des Pools eine Alternative zum Ausscheiden: ... selber ein schlechtes Poolrisiko zu werden! 


\subsubsection{Adverse Anreize}

Der adverse Selektionsmechanismus beruht auf dem Ausscheiden der für die Eigenkapitalgeber guten Einzelrisiken eines Pools und dem Verbleib seiner schlechten Einzelrisiken. Dass die guten Unternehmen zum Ausscheiden veranlasst werden, hat maßgeblich damit zu tun, dass sie ihre Investitionstätigkeit nach einer höheren Renditeforderung nicht zu verändern in der Lage sind. Können die Manager der guten Unternehmen stattdessen ihre Investitionen modifizieren oder sogar anders zusammenstellen, besteht für sie eine Alternative zum Austritt. Mit der Auswahl nun mehr riskanter Projekte mit höheren erwarteten Einzahlungsüberschüssen können sie den frei verfügbaren Cash Flow auf einem Niveau halten, dass sie zumindest nicht zu einer Auflösung Organizational Slacks gezwungen sind, um den Renditeforderungen der Anleger Genüge leisten zu können. Mit der Auswahl dieser neuen Investitionsprojekte verschlechtern sich jedoch die für die Eigenkapitalgeber vormals guten Einzelrisiken. Der adverse Anreiz für die Manager eines Unternehmens, risikoreichere Investitionen zu realisieren, ist für Einzelrisiken in der Nähe der Wertpapiermarktlinie besonders hoch. Denn sie betrifft das Ausscheiden durch einen Anstieg der Renditeforderung umso mehr. Aber auch die schlechten Einzelrisiken eines Pools sind versucht, ihren Managerertrag auf ursprünglichem Niveau durch zunehmend risikoreiche Unternehmensinvestitionen aufrecht $\mathrm{zu}$ erhalten. Durch adverse Anreize verschlechtert sich also die Güte der ausgewählten Investitionen bei gleichbleibender Unternehmenszahl im Pool. Während die Manager anlässlich einer höheren Renditeforderung die weniger riskanten Investitionen aussortieren, forcieren sie die für die Anleger schlechten Unternehmensprojekte. Denn erstere sind für das Management nicht mehr rentabel, doch letztere schon. Formal ergibt sich der adverse Anreizeffekt aus dem Differenzieren des Managerertrags $\operatorname{dcf}_{i}^{\text {free }}$ nach der geforderten Rendite: ${ }^{507}$

$$
\begin{array}{r}
\quad \operatorname{dcf}_{\mathrm{i}}^{\text {free }}=p_{\mathrm{i}}\left[\mathrm{dcf}_{\mathrm{i}}-\left(1+\mu_{\mathrm{i}}^{\mathrm{gef}}\right) \mathrm{EK}\right] \\
\Rightarrow \quad \mathrm{ddcf} f_{\mathrm{i}}^{\text {free }} / \mathrm{d} \mu_{\mathrm{n}}{ }^{\text {gef }}=-\mathrm{p}_{\mathrm{i}} \mathrm{EK}
\end{array}
$$

Der Managerertrag dcf free ${ }_{\mathrm{i}}^{\text {free }}$ st im Zuge steigender Renditeforderungen umso mehr, je höher die Erfolgswahrscheinlichkeit der Unternehmensinvestitionen $p_{i}$ ist, das heißt je weniger riskant die Investitionen sind. Ist zur gegebenen Renditeforderung

507 Kontraktforderungen bleiben der Einfachheit halber vernachlässigt. 
$\mu_{\mathrm{i}}{ }^{\text {gef }}$ eine (risikoneutrale) Geschäftsführung indifferent zwischen der Ausführung eines riskanten Investitionsprojektes $\mathrm{n}$ und eines weniger riskanten Investitionsprojektes $\mathrm{m}\left(\mathrm{p}_{\mathrm{n}}<\mathrm{p}_{\mathrm{m}}\right)$, ist der frei verfügbare Cash Flow $\mathrm{dcf}_{\mathrm{i}}^{\text {free }}$ in beiden Fällen identisch (Abbildung 4.4). Ein marginaler Anstieg der Renditeforderung etwa auf $\mu_{\mathrm{j}}^{\text {gef }}$ geht für die Manager beim weniger riskanten Projekt $\mathrm{m}$ mit einen größeren Ertragseinbruch als beim riskanteren Investitionsprojekt $\mathrm{n}$ verbunden. Dies resultiert daraus, dass - formal ausgedrückt - sich die Steigungen beider Kurven $-\mathrm{p}_{\mathrm{i}}$ EK mit den Projektwahrscheinlichkeiten $\mathrm{p}_{\mathrm{m}}$ und $\mathrm{p}_{\mathrm{n}}$ unterscheiden.

Abbildung 4.4 Adverser Anreizmechanismus

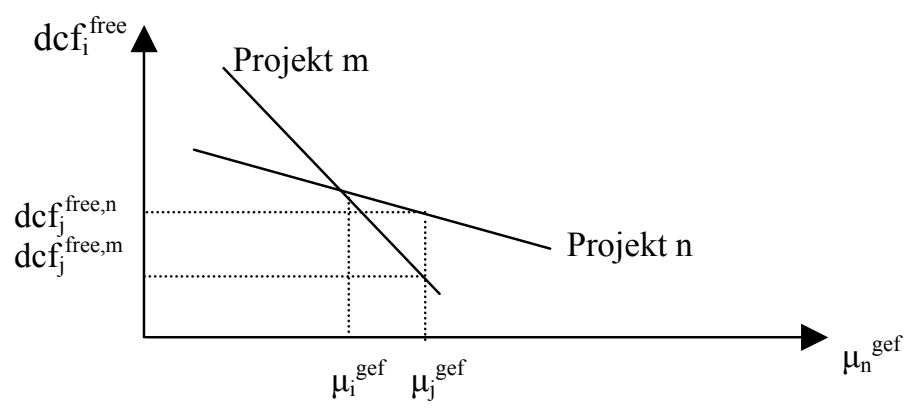

Bei einer Renditeforderung unterhalb von $\mu_{\mathrm{i}}{ }^{\text {gef }}$ wählen die Manager das weniger riskante Projekt $\mathrm{m}$. Bei einer darüber liegenden Renditeforderung bevorzugen die Manager das riskante Projekt n. Der Grund für den größeren Rückgang des Managerertrags bei weniger riskanten Investitionsobjekten infolge eines Anstiegs der geforderten Rendite besteht darin, dass das weniger riskante Projekt m den Anstieg der Renditeforderung mit größerer Wahrscheinlichkeit erzielen muss. Um die höhere Zahlungsverpflichtung bei Übernahme des weniger riskanten Projektes $\mathrm{m} \mathrm{zu}$ umgehen, wählen die Manager rationalerweise eher die für die Aktionäre schlechten Investitionsobjekte aus. Auf diese Weise verursacht ein Anstieg der Renditeforderung neben einer adversen Selektion für die Eigenkapitalgeber eine Verschlechterung der zu finanzierenden Investitionen dadurch, dass die Agenten vor allem der guten, aber auch der schlechten Unternehmen einen Anreiz haben, risikoreichere Investitionsprojekte durchzuführen. 


\subsubsection{Unterinvestition am Sekundärmarkt}

Das Verlassen des Pools durch die guten Einzelrisiken (adverse Selektion) sowie das Unterlassen der guten Projekte bei konstanter Poolzusammensetzung (adverse Anreize) - beides führt ausgehend von einer Situation eingeschränkten Eigenkapitalangebots dazu, dass es möglicherweise irrational für Aktionäre ist, eine höhere, den Markt räumende Rendite $\mathrm{zu}$ fordern. Die Anforderungen an die Unternehmen eines bestimmten Pools i von $\mu_{\mathrm{i}}{ }^{\text {gef }}$ auf $\mu_{\mathrm{j}}{ }^{\text {gef }}$ auszuweiten, ist - wie in Abbildung 4.5 dargestellt - bei einer hinreichend steilen Poollinie mit einem überproportionalen Anstieg des bewertungsrelevanten Risikos von $\tau_{i}^{\varnothing}$ auf $\tau_{i}^{\text {adv }}$ verbunden (Quadrant V). Die Verschlechterung des Poolrisikos kompensiert im Fall der fett gedruckten schwarzen steileren Poollinie $\mu_{\mathrm{n}}^{\text {gefø }}$ den Gewinneffekt einer höheren Renditeforderung über. Die Rendite/Risiko-Kombination befindet sich nach erhöhter Renditeforderung $\mu_{\mathrm{j}}{ }^{\mathrm{gef}} / \tau_{\mathrm{i}}{ }^{\text {adv }}$ unterhalb der Poollinie. ${ }^{508}$ Dennoch eine höhere Rendite zu fordern, hieße, eine geringere erwartete Netto-Rendite in Kauf zu nehmen (Quadrant II). Entspricht die geforderte Rendite $\mu_{\mathrm{i}}{ }^{\text {gef }}$ in der Ausgangssituation bereits der maximal $\mathrm{zu}$ erwartenden Netto-Rendite $\rho_{\mathrm{i}}^{\varnothing, \max }$, ist eine Erhöhung der Renditeforderung über dieses Maß hinaus mit Einbußen für die Aktionäre verbunden. Die Obergrenze der maximal zu fordernden Rendite $\mu_{\mathrm{i}}{ }^{\text {gef }}$ insgesamt zu überschreiten, ist deshalb für Eigenkapitalgeber irrational. ${ }^{509}$ Gleichen sich Eigenkapitalangebot und -nachfrage bei $\mu_{\mathrm{i}}{ }^{\text {gef }}$ nicht bereits aus, gibt es keine kompetitiven Kräfte, die eine Markträumung herbeiführen könnten. Eine über $\mu_{\mathrm{i}}^{\text {gef }}$ liegende Rendite oder gar die gemäß Wertpapiermarktlinie markträumende Rendite $\mu_{\mathrm{j}}{ }^{\text {gef }} \mathrm{zu}$ fordern, ist mit einer Ertragseinbuße verbunden. So verharrt die erwartete Rendite dauerhaft unterhalb der Wertpapiermarktlinie bei unvollkommenen Informationen - im Bereich eines Überhangs der Eigenkapitalnachfrage.

\footnotetext{
508 Es sei unterstellt, dass die Poollinie eine Parallele zur Wertpapiermarktlinie bildet. Bei zunehmenden Informationsunvollkommenheiten etwa im Hochrisikobereich verläuft die Poolflacher als die Wertpapiermarktlinie. $\mu_{\mathrm{i}}{ }^{\text {gef }}$ ausgehend mit einer monoton sinkenden Renditeerwartung verbunden ist. Diese vereinfachende Annahme wird in Abschnitt 4..3.5.1 aufgehoben. Es kommt dort zu einem bimodalen Verlauf der Netto-Renditeerwartung $\rho_{\mathrm{i}}^{\varnothing}$ in Abhängigkeit von der Renditeforderung $\mu_{\mathrm{n}}^{\text {gef }}$.
} 
Abbildung 4.5 Unterinvestition am Sekundärmarkt ${ }^{510}$

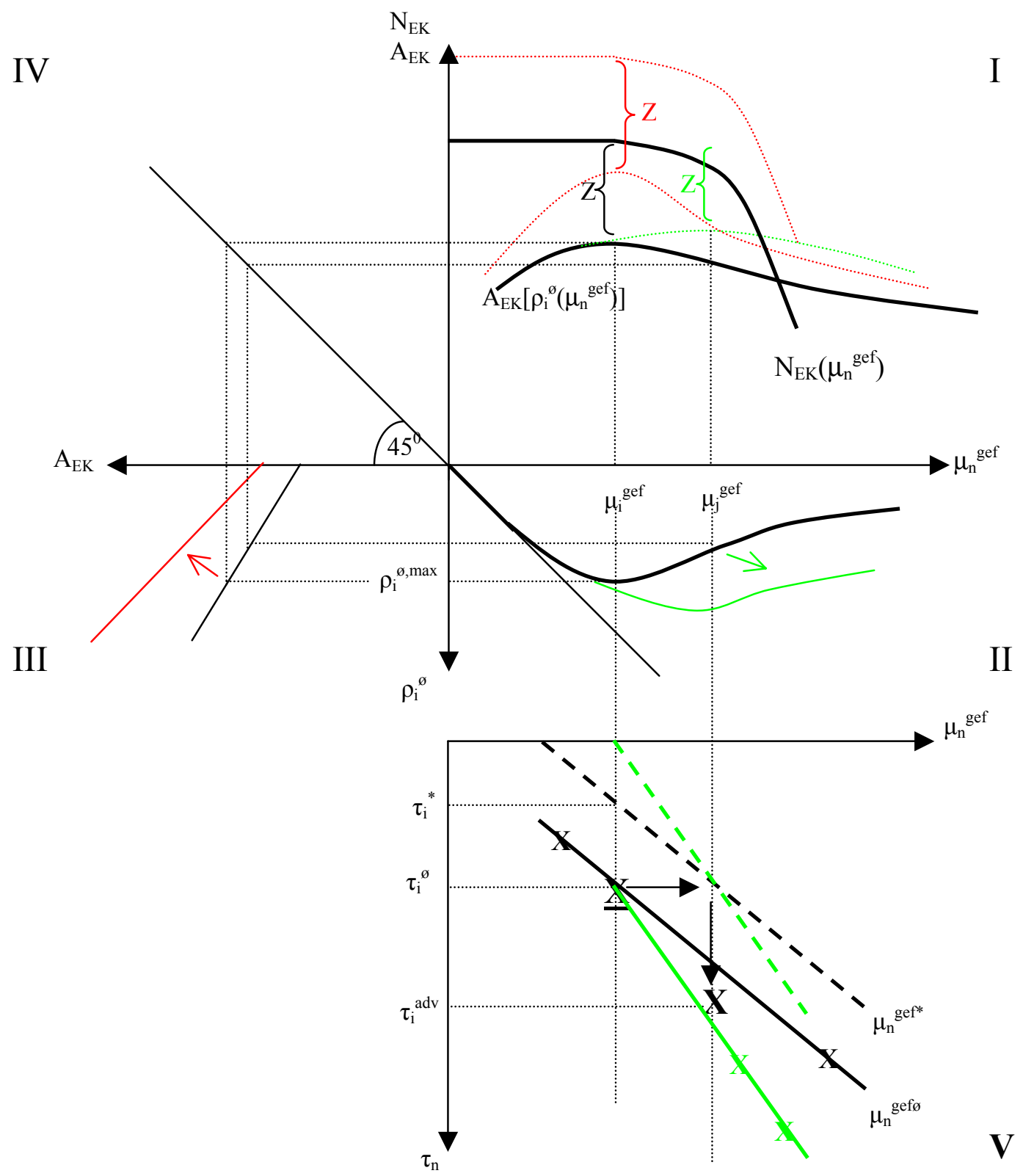

Ein nicht-monoton ansteigender Verlauf der erwarteten Netto-Rendite $\rho_{\mathrm{i}}^{\varnothing}$ in Bezug auf die Renditeforderung schlägt sich in einer letztlich von der Renditeforderung abhängigen rückwärts geneigten Kurve des Eigenkapitalangebots für die Unternehmen des Pools nieder (Quadrant I). Der Verlauf des Eigenkapitalangebots ist das Spiegelbild der nicht-monotonen Beziehung zwischen der erwarteten Netto-

$510 \quad$ Quadrant I und V entsprechen der Abbildung 3.3. Dabei entspricht $\mathrm{A}_{\mathrm{EK}}\left[\rho_{\mathrm{i}}^{\varnothing}\left(\mu_{\mathrm{n}}{ }^{\text {gef }}\right)\right]$ der dortigen Angebotsfunktion $\mathrm{A}_{\mathrm{EK}}\left[\mu_{\mathrm{C}}{ }^{\text {erw }}\left(\mu_{\mathrm{C}}{ }^{\text {gef }}\right)\right]$. Vgl. Abschnitt 3.2. 
Rendite und der geforderten Rendite. Das Eigenkapitalangebot verläuft bis $\mu_{\mathrm{i}}{ }^{\text {gef }}$ beziehungsweise $\rho_{\mathrm{i}}^{\varnothing, \max }$ zunächst positiv, oberhalb davon negativ geneigt. Die Eigenkapitalnachfrage verläuft bis zum markträumenden Renditeniveau (bei vollkommenen Informationen) $\mu_{\mathrm{i}}{ }^{\text {gef }}$ zunächst vollkommen unelastisch. ${ }^{511}$ Darüber hinaus reagiert die Eigenkapitalnachfrage negativ auf die geforderte Rendite $\mu_{\mathrm{n}}{ }^{\text {gef }}$. Durch das Ausscheiden der weniger riskanten Unternehmen im Pool lässt die Nachfrage nach Eigenkapital nach; dieser nachfragesenkende Effekt ist besonders ausgeprägt bei einer starken Selektion. Mit dem Ausscheiden von Unternehmen aus dem Pool nimmt zwar der Überhang der Nachfrage nach Eigenkapital, ${ }^{512}$ doch ist dieser Rückgang lediglich auf die adverse Selektion, nicht aber auf einen Anstieg des Angebots zurückzuführen. Da bei vollkommenen Informationen Eigenkapitalangebot und -nachfrage sich gemäß der Wertpapiermarktlinie entsprechen, klafft zum Anspruchsniveau $\mu_{\mathrm{i}}{ }^{\text {gef }}$, das maximal zu fordern rational ist, ein dauerhafter Überhang der Eigenkapitalnachfrage in Höhe von Z. Die Eigenkapitalnachfrage wird in dieser Höhe rationiert. Unter bestimmten Konstellationen von Angebot und Nachfrage ist Markträumung $(Z=0)$ auch bei unvollkommenen Informationen am Sekundärmarkt möglich, sie ist aber nicht - wie bei vollkommenen Informationen - als eine Art Naturgesestz zu betrachten. Markträumung ist bei einem nicht-monotonen Verlauf in dem Ausnahmefall dann möglich, wenn die Angebots- die Nachfragekurve zur maximalen Renditeerwartung $\rho_{\mathrm{i}}^{\varnothing, \max }$ bei $\mu_{\mathrm{i}}{ }^{\text {gef }}$ gerade tangiert. Ansonsten impliziert die Übereinstimmung von Eigenkapitalangebot und -nachfrage einen über $\mu_{\mathrm{i}}{ }^{\text {gef }}$ hinaus aufstrebenden Ast der erwarteten Netto-Rendite.

Im Vergleich der schwarzen mit der grünen Poollinie in Quadrant V wird deutlich, dass adverse Selektions- und Anreizrisiken und von daher auch das Ausmaß an Sekundärmarktrationierung vor allem bei einer steilen Poollinie zum Tragen kommen. ${ }^{513}$ Diese theoretisch abgeleitete Erkenntnis stimmt durchaus mit der

511 Darin kommt zum Ausdruck, dass die Manager aller Unternehmen des Pools bis zum markträumenden Renditeniveau $\mu_{\mathrm{i}}^{\text {gef }}$ mit einer entsprechenden Internen Verzinsung der unternehmerischen Investitionsprojekte das zur Verfügung gestellte Eigenkapital zu verzinsen gedenken. Sie verhalten sich, als ob sie zu jedem Zeitpunkt Eigenkapital in Höhe des über den Primärmarkt einmalig erhaltenen Eigenkapitals zu jedem Zeitpunkt erneut nachfragen. Baltensperger, Devinney $(1985,491)$ behaupten, dass der nachfrageseitige Mengeneffekt, der sich bei einer ausgeprägt adversen Selektion im Ausscheiden vergleichsweise vieler Unternehmen im Pool ausdrückt, in der Regel das Ausmaß des unterschießenden Eigenkapitalangebotes allenfalls abzuschwächen, aber nicht zu beseitigen vermag.

Dass die flache, grüne Wertpapiermarktlinie mit einem größeren Ordinatenabschnitt eingezeichnet wird, hat allein analytische Gründe. Wie Abschnitt 3.1 zeigte, geht eine Verflachung der Wertpapiermarktlinie normalerweise mit einer Verlagerung nach unten einher. 
Beobachtung überein, dass die relative Vernachlässigung kleiner und mittlerer Gesellschaften am deutschen Sekundärmarkt in Up-Markets besonders hoch ist. ${ }^{514}$ Dies trifft insbesondere auf die Phase eines konjunkturellen Höhepunktes mit (noch) ansteigenden Internen Verzinsungen, einer im Vorfeld des anstehenden Abschwungs bereits aufkommenden Risikoaversion und einem immer wichtiger werdenden „guten“ Informationsstand bei einer Anlageentscheidung. Umgekehrt fallen die adversen Selektions- und Anreizrisiken bei einer flachen Poollinie weniger ins Gewicht, wie vor allem in einer konjunkturellen Talphase (noch) abnehmender Interner Verzinsungen und einer aufkommenden Risikoneigung der Anleger. Insofern geht von der Sekundärmarktrationierung auf die konjunkturellen Umschwünge eine verstärkende Wirkung aus. ${ }^{515}$ Im Einzelnen hängt das Auftreten und das Ausmaß einer Sekundärmarktrationierung im langfristigen Gleichgewicht ab von:

- ... der anlegerseits noch nicht antizipierten Veränderung der Internen Verzinsung der sich im Pool befindlichen Gesellschaften. Eine ansteigende Interne Verzinsung wie etwa in konjunkturellen Phasen des Aufschwungs bewirkt eine Poollinie mit ausgeprägt positiver Steigung. Mit einer ansteigenden Poollinie erhöht sich die Wahrscheinlichkeit, dass adverse Selektionen und Anreize die Netto-Renditeerwartung senken, mithin eine Rationierung der Eigenkapital nachfragenden Seite am Sekundärmarkt bewirken. Bei einer flachen Poollinie fallen die adversen Anreiz- und Selektionsrisiken deshalb nicht so sehr ins Gewicht, weil die Anleger für eine marginal höhere Rendite einen größeren Anstieg des bewertungsrelevanten Risikos in Kauf nähmen. Eine hinreichend flache Wertpapiermarktlinie im Beispiel der Abbildung 4.5 impliziert einen Anstieg der Netto-Renditeerwartung infolge einer Anhebung der Renditeforderung. Grafisch drückt sich dies in einer Verschiebung der Kurve der erwarteten Netto-Rendite nach unten aus (Quadrant II). Im Zuge einer Verflachung der Poollinie nähert sich die maximal zu erwartende Netto-Rendite $\rho_{\mathrm{i}}^{\emptyset, \max }$ somit der markträumenden Renditeforderung $\mu_{\mathrm{j}}^{\text {gef }}$ bei Poolbildung an. Dementsprechend verringert sich das Ausmaß der Sekundärmarktrationierung (grünes Z).

$514 \quad$ Vgl. Abschnitt 2.1. 
- ... der Risikoneigung der Anleger. Wie erläutert übt sie einerseits einen positiven Einfluss auf die Steigung der Wertpapiermarktlinie aus. Andererseits steigt die Risikoelastizität des Eigenkapitalangebots an. Das Eigenkapitalangebot reagiert mit einer mengenmäßig größeren Einschränkung auf ein marginal höheres Risiko. Eine minimale Abweichung des durchschnittlich bewertungsrelevanten Poolrisikos $\tau_{\mathrm{i}}^{\varnothing}$ vom markträumenden Risiko $\tau_{\mathrm{i}}{ }^{*}$ führt bei abnehmender Risikoneigung zu einer relativ hohen Einschränkung des Eigenkapitalangebots. Im Grenzfall der Risikoneutralität, das heißt bei einer Risikoelastizität des Eigenkapitalangebots von Null bliebe das Eigenkapitalangebot bei durchschnittlichen Poolrisiken unterschiedlicher Bewertungsrelevanz unverändert. Bei einem tatsächlich bewertungsrelevanten Risiko $\tau_{i}^{\varnothing}>\tau_{i}^{*}$ bliebe ein risikoneutrales Eigenkapitalangebot letztlich auf markträumendem Niveau verhaftet. Alle emittierten Aktien würden trotz eines durchschnittlich höheren Poolrisikos geplantermaßen nachgefragt werden. Je höher jedoch die Risikoaversion ist, desto empfindlicher reagiert das Eigenkapitalangebot auf eine marginale Abweichung des durchschnittlichen Poolrisikos von der Wertpapiermarktlinie und desto größer ist das Ausmaß des unterschießenden Eigenkapitalangebots.

- ... dem Grad der Informationsunvollkommenheit. Dieser bestimmt die Streuung der Einzelrisiken im betrachteten Pool. Je breiter c.p. die Einzelrisiken im Pool gestreut sind und je mehr gute Einzelrisiken bei einer Anhebung der Renditeforderung aus dem Pool ausscheiden, desto größer ist das Ausmaß einer adversen Selektion, mithin das des unterschießenden Eigenkapitalangebots. Sind die Anleger relativ gut informiert über die Höhe der einzelnen Risiken, führt ein Anstieg der Renditeforderung $\mathrm{zu}$ einer vergleichsweise geringen Verschlechterung der Poolrisiken, da die Einzelrisiken nahe des Durchschnittsrisikos streuen. Die Obergrenze der erwarteten Netto-Rendite steigt bei guter Informiertheit der Anleger folglich mit einer höheren Forderung an und mit ihr auch grundsätzlich das Eigenkapitalangebot. Infolge eines höheren Grades an Informationsvollkommenheit ist somit ein Rückgang der 
Sekundärmarktrationierung zu erwarten. Das Eigenkapitalangebot nähert sich dem Nachfrageniveau in Höhe des ausgeschütteten und fundamental richtig bewerteten Aktienkapitals (grünes Z). Neben der Streuung der Einzelrisiken im Pool bestimmt der Grad der Informationsunvollkommenheit außerdem die Differenz zwischen dem tatsächlich bewertungsrelevanten Poolrisiko $\tau_{\mathrm{i}}^{\varnothing}$ und dem markträumenden Risiko bei vollkommenen Informationen $\tau_{i}{ }^{*}$, das heißt dem Abstand zwischen der Wertpapiermarkt- und der Poollinie. Ihre Entfernung nimmt mit der Unvollkommenheit der Informationen zu. Sie ist ebenfalls mitentscheidend für das Ausmaß von Z. Bei vollkommenen Informationen würde jeder Pool aus genau einer Aktie bestehen und die Poollinie mit der Wertpapiermarktlinie zusammenfallen. Der Angebotsmangel würde folglich Null betragen. Je höher jedoch das durchschnittliche Poolrisiko $\tau_{\mathrm{i}}^{\varnothing}$ vom bewertungsrelevanten Risiko $\tau_{i}^{*}$ entfernt liegt, desto höher fällt - bei einer Risikoelastizität des Eigenkapitalangebots ungleich Null - das Ausmaß der Sekundärmarktrationierung $\mathrm{Z}$ aus.

Nimmt im Zuge einer Liquiditätshausse am Aktienmarkt das Niveau des Eigenkapitalangebots durch eine exogene Angebotsausweitung allgemein zu (Parallelverschiebung der Angebotskurve in Quadrant III) oder reagiert das Eigenkapitalangebot stärker auf die erwartete Netto-Rendite (Drehung im Achsenabschnitt), ist ebenfalls mit einer Zunahme des Eigenkapitalangebots in Pool i $\mathrm{zu}$ rechnen. ${ }^{516}$ Derartige mengenexpansive Entwicklungen sind in „Rationierungsregimen“ wie ein sich öffnendes Ventil mit liquiditätserhöhenden Auswirkungen, wobei die maximal erzielbare Netto-Rendite $\rho_{\mathrm{i}}^{\varnothing, \max }$ unverändert bleibt. ${ }^{517}$ Einer Reduzierung von $\mathrm{Z}$ durch eine dauerhafte Ausweitung des Eigenkapitalangebots steht jedoch mittel- bis langfristig eine Ausweitung auch der Eigenkapitalnachfrage entgegen. Angesichts des zuströmenden Eigenkapitals sehen sich die Unternehmen im Durchschnitt durch einen allgemeinen Anstieg des Kursniveaus $\mathrm{zu}$ weiteren Emissionen veranlasst. Es verschiebt sich deshalb in Quadrant I sowohl die Angebots- als auch die Nachfragekurve nach oben. ${ }^{518}$ In

\footnotetext{
516 Die am deutschen Aktiensenkundärmarkt zu beobachtende Institutionalisierung deutet auf eine zunehmende Sensibilität der Kapitalströme auf die Netto-Renditeerwartungen hin. Vgl. Blinder 1987, 339.

Die Interdependenz der Beziehung zwischen der Angebots- und der Nachfragekurve erklärt sich letztlich daraus, dass beide Marktseiten von derselben Dichtefunktion $\mathrm{g}\left(\mathrm{p}_{\mathrm{i}}\right)$ der Projektertragsverteilung abhängen. Vgl. Stiglitz 1987, 3; Hillier, Ibrahimo 1993, 286.
} 
welcher Geschwindigkeit sich die Eigenkapitalnachfrage der anhaltenden Liquiditätshausse anpasst, hängt vom Ausmaß ihrer Antizipation ab. Im betrachteten Beispiel der Abbildung 4.5 werden insoweit rationale Erwartungen unterstellt, als das Ausmaß an Sekundärmarktrationierung (rotes Z) innerhalb des Betrachtungszeitraumens (von nach wie vor einer Periode) gleich bleibt. Bei einer auf die Liquiditätshausse überschießenden Reaktion der Aktien emittierenden Gesellschaften wächst das Ausmaß der Sekundärmarktrationierung sogar über ihr ursprüngliches Niveau hinaus. Aufgrund der zumindest auf lange Sicht bestehenden Interdependenz beider Marktseiten ist mit dem Schließen der Eigenkapitalangebotslücke durch einen stärker werdenden Kapitalstrom nicht zu rechnen. ${ }^{519}$

\section{Abbildung 4.6 Maximale Annäherung der Pool- an die Wertpapiermarktlinie}

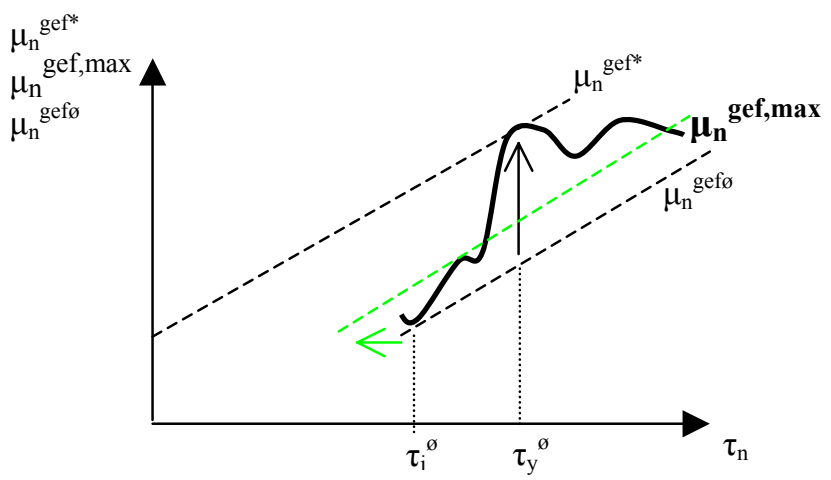

Die bisher genannten Einflussgrößen bezogen sich stets auf das Eigenkapitalangebot für einen einzelnen Risikopool i. Hebt man diese „horizontale“ Betrachtung auf, sieht man sich vor das Problem gestellt, dass eine allgemeine Anhebung der Renditeforderungen für alle n Pools eines Anlegerportefeuilles adverse Selektionen und Anreize von jeweils unterschiedlicher Stärke hervorrufen. Da höhere Renditeanforderungen die erwartete Netto-Rendite einiger Pools erhöht, die anderer Pools - wie etwa im Fall des Pools i - hingegen nicht, fällt der Anstieg der Renditeforderungen von Pool $\mathrm{zu}$ Pool rationalerweise unterschiedlich aus

519 Dies erklärt, warum die hohen Kapitalzuflüsse der letzten Jahre an den deutschen Aktienmarkt nicht mit einer größeren Gleichverteilung des Kapitalaufkommens zwischen den Segmenten verbunden war. Vgl. Abschnitt 2.1. Für die wirtschaftspolitischen Implikationen (Kapitel 5) wird auf Maßnahmen verzichtet, die den generellen Kapitalstrom zu Gunsten des Aktienmarktes erhöht. Dass bedeutet jedoch nicht, dass speziell für vollrationierte Unternehmen angebotsseitige Anreizeffekte $\mathrm{zu}$ einer langfristig sinkenden Sekundärmarktrationierung führen können. Vgl. Abschnitt 5.5. 
(Abbildung 4.6); bei einem in allen Pools gleichmäßigen Anstieg der Renditeforderung verlöre die Poollinie als der geografische Ort der durchschnittlich erwarteten Poolrisiken ihre Linearität, nähme eventuell sogar einen nicht-monotonen Verlauf an. Eine Erhöhung der Renditeforderung ist solange rational, bis eine marginale Erhöhung der Renditeforderung gerade kompensiert wird durch die Verschlechterung der Poolqualität. Ist die Obergrenze der zu erwartenden NettoRendite erreicht, ist die Renditeforderung nach oben in Richtung markträumendes Niveau nicht weiter anzuheben. Bis auf den Pool y im Beispiel der Abbildung 4.6 befinden sich alle Renditeforderungen, die die Netto-Renditeerwartungen der $\mathrm{n}$ Pools jeweils maximieren, unterhalb der Wertpapiermarktlinie. Verbindet man die geometrischen Orte der maximalen Renditeforderungen aller Pools des Portefeuilles $\mathrm{zu}$ einer Kurve $\mu_{\mathrm{n}}{ }^{\text {gef,max }}$ ergeben sich variierende Teilabschnitte, das heißt Bereiche, die mal positive und mal negative Steigungen aufweisen. Während eine Erhöhung der Renditeforderung für den Pool i mit einer sofort abnehmenden NettoRenditeerwartung verbunden ist, fällt der adverse Anreiz- und Selektionsmechanismus für den Pool y bis zur markträumenden Renditeforderung in einem derart schwachen Maße aus, dass sich die erwartete Netto-Rendite nicht verringert. Für Anleger des Pools y ist es deshalb rational, die gemäß der Wertpapiermarktlinie markträumende Rendite zu fordern. Von allen übrigen Pools können mehr oder weniger über der Poollinie $\mu_{\mathrm{n}}^{\text {gefø }}$ liegende Renditen gefordert werden. Mit einer jeweiligen Erhöhung der geforderten Rendite bis zur maximalen Netto-Renditeerwartung eines Pools findet eine Durchmischung zwischen ursprünglichen Einzelrisiken einer Risikoklasse und den neu hinzukommenden statt. Damit einhergehende Veränderungen auf den Verlauf der ursprünglichen Poollinie $\mu_{\mathrm{n}}{ }^{\text {gefø }}$ induzieren Umgruppierungen, die dann eine neue Poolline erneut linearen Verlaufs erzeugen (grün gestrichelte Kurve in Abbildung 4.6). Im Zuge dieser erneuten Glättung der Poollinie nähert sich die Pool- der Wertpapiermarktlinie an. Möglicherweise verändert sich auch das Steigungsmaß der Poollinie. In der Verschiebung und Drehung der Poollinie kommt das Potenzial zum Ausdruck, die Renditeforderungen in Richtung markträumende Wertpapiermarkt erhöhen und damit das Eigenkapitalangebot ausweiten zu können, ohne übermäßige adverse Selektions- und Anreizrisiken in den jeweiligen Pools in Kauf nehmen zu müssen. 


\subsubsection{Modellerweiterungen}




\subsubsection{Bimodale Netto-Renditeerwartung}

Manch kritischer Leser mag sich die Frage stellen, wie Aktien überhaupt emittiert werden können, wenn sie wenig später von den Anlegern vernachlässigt werden. Müsste die Eigenkapitalnachfrage des plazierten Aktienkapitals nicht bereits am Aktienprimärmarkt rationiert werden? Doch müssen Aktien, bevor sie am Sekundärmarkt rationiert werden, zunächst einmal am Primärmarkt nachgefragt werden. Liegt in der unterschiedlichen Beachtung der Eigenkapitalgeber am Primärund am Sekundärmarkt nicht ein Widerspruch? - Rationierte Unternehmen verschwinden in zunehmendem Maße vom Sekundärmarkt, um im Rahmen eines Going Private außerhalb einer Börsennotierung Eigenkapital $\mathrm{zu}$ beziehen. Die Tatsache, dass Unternehmen, die am Sekundärmarkt rationiert werden, bei einem Going Private im Beteiligungsbereich hingegen auf ein größeres Eigenkapitalangebot stoßen, scheint ein ähnliches Paradoxon $\mathrm{zu}$ sein. ${ }^{520}$ In beiden Fällen, hier am Beteiligungsmarkt, dort am Aktienprimärmarkt, wird Unternehmen Eigenkapital zuteil, das ihnen am Sekundärmarkt verwehrt wird.

Ein Grund für diese scheinbar widersprüchlichen Eigenkapitalangebotsmengen an unterschiedlichen Märkten liegt in einem unterschiedlichen Informationsgrad begründet. Am Primärmarkt fällt der Fokus der Medien und des Kapitalmarktresearch regelmäßig auf Neuemissionen. Dies produziert und verbreitet Informationen zu Gunsten der Erstabnehmer, so dass zum Zeitpunkt der Emission für Eigenkapitalgeber vergleichsweise geringe Informationsasymmetrien bestehen. ${ }^{521}$ Auch die Informiertheit der Eigenkapitalgeber steigt bei einer Beteiligungsfinanzierung im Rahmen eines Going Private mit ihrer Nähe zum Unternehmen. ${ }^{522}$ Beide Informationseffekte dürften eine expansive Wirkung auf das Eigenkapitalangebot nicht verfehlen. Neben einer besseren Informationsausstattung der Eigenkapitalgeber als Erklärung für ein unterschiedliches Eigenkapitalangebot zu Gunsten derselben Unternehmen an unterschiedlichen Märkten ist ein weiterer, ein Preiseffekt von Bedeutung. Die Renditeforderung der Eigenkapitalgeber liegt am Primär- wie auch am Beteiligungsmarkt regelmäßig höher als am Sekundärmarkt. Die unterbewertete Ausgabe von Aktien (Underpricing) insbesondere bei kleinen und

$520 \quad \mathrm{Zu}$ Arten der Beteiligung vgl. Pleschak, Kulicke, Stummer 1998, 13-27.

521 Unbestritten gibt es realiter auch manipulierende Informationen, die das Eigenkapitalangebot kurzfristig stimulieren. 
mittleren Gesellschaften ist mit der regelmäßigen Erwartung auf einen kräftigen Kursanstieg nach der Emission verbunden. Es manifestiert sich darin die Forderung der Anleger am Aktienprimärmarkt nach einer vergleichsweise hohen Rendite im Tausch für die Bereitstellung von Eigenkapital. Während es für das emittierende Unternehmen am Primärmarkt um den unmittelbaren Erhalt von Eigenkapital geht, wird am Sekundärmarkt „nur“ die Anteilseignerschaft übertragen. Daraus ergibt sich für die Eigenkapitalgeber am Primärmarkt eine stärkere Position, eine höhere Renditeforderung, sogar über dem markträumenden Niveau durchzusetzen. Ebenso wie für den Primär- ist auch für den Beteiligungsmarkt eine höhere Renditeforderung kennzeichnend. „Die Zielrendite für eine einzelne Beteiligung wird mit etwa 30\% angegeben. ${ }^{6523}$ Angesichts dieser durch Kontroll- und Mitentscheidungsrechte durchsetzbaren Zielrendite ist nachvollziehbar, dass vor allem kleine und mittlere Gesellschaften des traditionellen Mittelstands nicht ohne weiteres für die Bereitstellung von Private Equity Capital prädestiniert sind. Eine Beteiligungsfinanzierung ist für außerbörsliche Eigenkapitalgeber mit geringen Exit-, das heißt Veräußerungsmöglichkeiten ihrer Investitionen verbunden. ${ }^{524}$ Deshalb wird im Gegenzug von den Beteiligungsnehmern regelmäßig eine höhere Rendite gefordert, die durchzusetzen mit Hilfe einer größeren Einsichtnahme in das Unternehmensgeschehen und größeren Einflusskanälen auf die Unternehmensinvestitionen möglich wird. Während ersterer Aspekt eine geringere Informationsasymmetrie impliziert, geht letzterer mit einer abgeschwächten Ertragsasymmetrie einher. Die Reduzierung beider Asymmetrien führt bei einer Erhöhung der Renditeforderung im Rahmen einer außerbörslichen Finanzierung zu einem sinkenden Ausmaß adverser Anreiz- und Selektionsrisiken. Insoweit kann eine im Vergleich zum Sekundärmarkt höhere Rendite am Beteiligungsmarkt nicht nur gefordert, sondern auch netto erwartet werden. ${ }^{525}$

Doch auch ungeachtet unverminderter Informations- und Ertragsasymmetrien kann es selbst bei ausgeprägt adversen Selektionen und Anreizen rational sein, eine markträumende oder sogar noch darüber hinaus liegende Rendite zu fordern. Eine Renditeforderung über ein bestimmtes Niveau hinaus, war in der bisherigen Untersuchung stets mit einer sinkenden Netto-Renditeerwartung verbunden. Befindet

\footnotetext{
522 Vgl. Engel 2001, 2.

$523 \quad$ KfW 1999, 17; vgl. a. Engel 2001, 1.

524 Vgl. Leopold, Frommann 1998, 3.
} 
sich die erwartete Netto-Renditeerwartung unterhalb der markträumenden CAPMWertpapiermarktlinie wurde das Angebot an Eigenkapital eingeschränkt und die Eigenkapitalnachfrage am Sekundärmarkt rationiert. Auch jeder weitere Anstieg der Renditeforderung führte nicht zu einem Anstieg der erwarteten Netto-Rendite. Dass vor diesem Hintergrund eine deutlich höhere Renditeforderung wie am Aktienprimär- oder am Beteiligungsmarkt zu einem höheren Eigenkapitalangebot führen soll, scheint deswegen zunächst fraglich. Doch löst sich dieser scheinbare Widerspruch darin auf, dass die Kurve der erwarteten Netto-Rendite bislang als unimodal, das heißt mit einem einzigen (globalen) Maximum unterstellt wurde. Je nach Streuung der Einzelrisiken innerhalb eines Aktienpools kann die erwartete Netto-Rendite jedoch auch über mindestens ein zusätzliches (lokales) Maximum verfügen, das heißt einen multimodalen Verlauf annehmen. ${ }^{526}$ Bereits bei Vorliegen eines bimodalen Verlaufs der erwarteten Netto-Renditekurve (Abbildung 4.7) erschließt sich einem die zunehmende Bedeutung von Beteiligungsfinanzierern für vormals am Sekundärmarkt rationierte Gesellschaften sowie die weiterer Eigenkapitalgeber über den ,grauen Kapitalmarkt ${ }^{6527}$. Auch wird deutlich, warum Aktien, bevor sie am Sekundärmarkt rationiert werden, am Primärmarkt zunächst emittiert werden können. Infolge eines deutlich höheren Forderungsniveaus in Verbindung mit einer bimodalen Netto-Renditeerwartung ist an diesen Märkten mit einem höheren Eigenkapitalangebot zu rechnen als am Sekundärmarkt.

\section{Abbildung 4.7 Bimodale Netto-Renditeerwartung ${ }^{528}$}

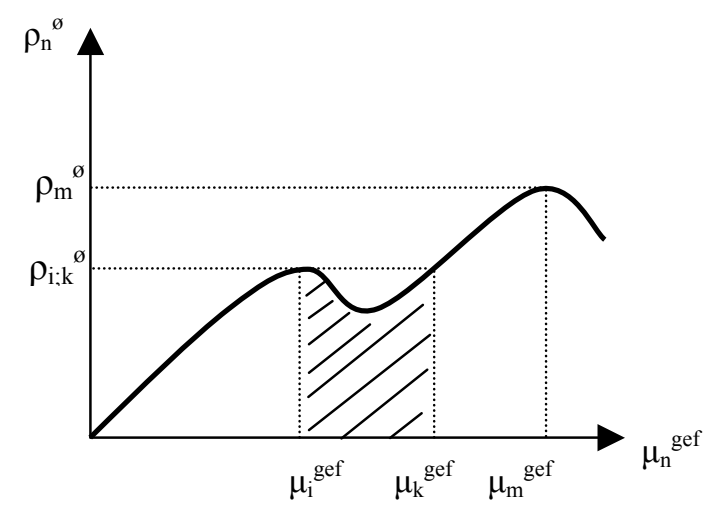

$525 \quad$ Vgl. Leopold, Frommann 1998, 34-37.

526 Der Fall mehrerer lokaler Opima sei aus Vereinfachungsgründen durch den Fall eines nur bimodalen Verlaufs der erwarteten Rendite ersetzt. Bei Vorhandensein mehrerer Optima im Verlauf der Erwartungskurve kann es entsprechend geboten sein, mehrere Renditen zu fordern. Vgl. Leopold 1993, 348-362. 
Eine Erhöhung der Renditeforderung von $\mu_{\mathrm{i}}^{\text {gef }}$ auf das gemäß der Wertpapiermarktlinie markträumende Niveau $\mu_{\mathrm{j}}^{\text {gef }}$ (zwischen $\mu_{\mathrm{i}}{ }^{\text {gef }}$ und $\mu_{\mathrm{k}}{ }^{\text {gef }}$ ) ist in jedem Fall mit einem Rückgang der erwarteten Netto-Rendite verbunden. Eine in diesem Ausmaß höhere Rendite zu fordern, ist deswegen für die Eigenkapitalgeber (am Sekundärmarkt) irrational. Selbst eine diskrete Erhöhung auf das Forderungsniveau in Höhe von $\mu_{\mathrm{k}}{ }^{\text {gef }}$, das sich über der Wertpapiermarktlinie befindet, verspricht keine höhere Netto-Renditeerwartung. Allerdings führt eine darüber hinaus liegende Renditeforderung zu einer erwarteten Netto-Rendite größer als $\rho_{\mathrm{i}, \mathrm{k}}{ }^{ø}$. Lässt sich aus der Position des Anteilseigners am Sekundärmarkt nicht eine in diesem Ausmaß höhere Rendite einfordern, ist es rational, dort das Eigenkapitalangebot entsprechend einzuschränken. Am Primär- oder auch am Beteiligungsmarkt, wo die Eigenkapitalgeber kraft ihrer größeren Bedeutung für die Unternehmen einen empfindlichen Forderungssprung durchzusetzen imstande sind, mag dies anders sein. Hier besteht für die Eigenkapitalgeber die Möglichkeit, in den Bereich einer solch höheren erwarteten Netto-Rendite vorzustoßen. Vor dem Hintergund des bimodalen Verlaufs der Netto-Rendite kann es also für Anleger durchaus rational sein, für denselben Unternehmenspool an Märkten unterschiedlicher Durchsetzungsstärke der Eigenkapitalgeber unterschiedliche Renditen $\mathrm{zu}$ fordern und damit auch unterschiedliche Mengen an Eigenkapital anzubieten. Während dieselben Anleger einen bestimmten Pool am Sekundärmarkt bei $\mu_{\mathrm{i}}{ }^{\text {gef }}$ rationieren, bieten sie $\mathrm{ihm}$ über den Beteiligungsmarkt ein Eigenkapitalvolumen von möglicherweise sogar über markträumendem Niveau an. Die höchste Netto-Renditeerwartung erzielen die Eigenkapitalgeber im Beispiel der Abbildung 4.7, verlegen sie sich auf den - für Venture Capitalists so typischen Hochrisikobereich und fordern $\mu_{\mathrm{m}}$ gef . Einer solchen Forderung nachzukommen, ist allerdings nur für jene Gesellschaften des Pools i mit den höchsten Risiken möglich. Selbst zum Teil überdurchschnittliche Einzelrisiken des Pools stellen ihre Nachfrage nach Eigenkapital ein bei einer Renditeforderung, die sich wie $\mu_{\mathrm{m}}^{\text {gef }}$ deutlich über dem markträumenden Niveau der Wertpapiermarktlinie befindet. Selbst diese Gesellschaften mit sogar über dem Pooldurchschnitt riskanten Unternehmensinvestitionen, die am Sekundärmarkt bei einer Renditeforderung von 
$\mu_{\mathrm{i}}^{\text {gef }}$ rationiert werden, können hier auch am Beteiligungsmarkt nicht mit einem Eigenkapitalangebot rechnen. Sie vermögen auch an einer „2. Runde“ der Eigenkapitalvergabe über den Beteiligungsmarkt nicht zu partizipieren. 


\subsubsection{Ungewissheit}

Je nach Grad der Informationsvollkommenheit vermochte der rationale Anleger bislang sein Gesamtportefeuille in verschiedene Pools mit ihm ähnlich erscheinenden Einzelrisiken zu unterteilen. Wenn dem Eigenkapitalgeber auch nicht die exakte Höhe einzelner Risiken bekannt ist, weiß er doch stets um die Verteilungs- und Dichtefunktion der Risikoverteilung in den einzelnen Pools und damit auch um die Höhe der durchschnittlichen Poolrisiken. Je nach Streuung der Einzelrisiken kann eine höhere Renditeforderung eine inakzeptable Verschlechterung des durchschnittlichen Poolrisikos herbeiführen. Ob eine adverse Veränderung der Zusammensetzung eines Pools für eine Anhebung der Renditeforderung hinnehmbar ist oder nicht, hängt nicht unwesentlich von der Risikoneigung der Anleger ab, das heißt von der Steigung der Poollinie. Risikoaverse Anleger verlangen für die Übernahme einer marginalen Erhöhung adversen Risikos eine höhere Entschädigung als weniger risikoaverse Eigenkapitalgeber. So mag das Ausmaß einer adversen Selektion anlässlich einer bestimmten Anhebung der Renditeforderung für den vergleichsweise wenig risikoaversen Anleger akzeptabel sein, für den stärker risikoaversen hingegen nicht. Dadurch wirkt sich die Risikoneigung der Anleger auf die angebotene Menge an Eigenkapital, mithin das Ausmaß der Unterinvestition aus. ${ }^{529}$

Unabhängig davon, ob der Anleger risikoavers ist oder nicht, bedarf er statistischer Kenntnisse über die Risikoverteilung innerhalb der Pools, denen er für die Ausrichtung seines Handelns vertraut. Ist dem so, besteht zwar Unsicherheit über die Spezifizierung der Einzelrisiken in den Pools, aber keine Ungewissheit über die Verteilung der Einzelrisiken im Pool. Unter Ungewissheit ist der Zustand zu verstehen, in dem nicht alle möglichen Umweltausprägungen künftiger Entwicklungen von den Eigenkapitalgebern spezifiziert werden können. ${ }^{530}$ Können bei einer Investitionsentscheidung für das Eintreten aller relevanten Umweltzustände keine Wahrscheinlichkeiten, also weder objektive noch subjektive, angegeben werden, handelt es sich um eine Entscheidung unter Ungewissheit. ${ }^{531}$ Bei Ungewissheit geht es nicht nur um ein Zuwenig an Informationen für die

529 Im CAPM hat die Risikoneigung der Anleger lediglich Einfluss auf die Höhe der frei fluktuierenden Renditeforderung, die das Eigenkapitalangebot konstant hält. 
Eigenkapitalgeber. Auch ein Zuviel an Informationen, das statistisch-ökonometrisch schwer auszuwerten ist, stellt die Anleger vor das Ungewissheitsproblem einer fehlerhaften Selektion.

\begin{abstract}
„We should not conclude from this that everything depends on waves of irrational psychology. On the contrary, the state of long-term expectation is often steady, and, even when it is not, the other factors exert their compensating effects. We are merely reminding ourselves that human decisions affecting the future, whether personal or political or economic, cannot depend on strict mathematical expectation, since the basis for making such calculations does not exist; and that it is our innate urge to activity which makes the wheels go round, our rational selves choosing between the alternatives as best we are able, calculating where we can, but often falling back for our motive on whim or sentiment or chance." ${ }^{532}$
\end{abstract}

Während man unter Unsicherheit also die Entscheidungssituation versteht, in der es a-priori- oder a-posteriori-Wahrscheinlichkeiten gibt, impliziert Ungewissheit einen fehlenden statistisch-ökonometrischen Zugriff auf die Welt. ${ }^{533}$ Bei Vorliegen von Ungewissheit haben die Eigner eines Unternehmens somit auch zur Wahrscheinlichkeitsverteilung und -dichte der Poolrisiken wenn überhaupt, dann nur eingeschränkt belastbare Informationen. Vergrößert sich der ökonometrisch nicht zugängliche Bereich oder werden die Akteure pessimistischer hinsichtlich der Aussagekraft der vorhandenen Informationen, steigt die Ungewissheit. ${ }^{534}$ Ungewissheit hinsichtlich eines durchschnittlichen Poolrisikos entsteht oder weitet sich etwa aus, wenn die Anzahl der Einflussfaktoren der bewertungsrelevanten Risiken zunimmt und in ihrer Wirkung für die Eigenkapitalgeber undurchschaubarer werden, wenn die Kosten der Informationsbeschaffung teurer werden oder wenn Informationsproduzenten ein geringeres Vertrauen entgegengebracht wird.

Eine solche Entwicklung zunehmender Ungewissheit ist mit Phasen zunehmender, aber auch mit Phasen abnehmender Risikoaversion hinsichtlich des geringer werdenden statistisch verlässlichen Bereichs sehr wohl vereinbar. So ist im Zuge einer allgemeinen Euphorie eines Börsenbooms zwar einerseits risikofreudiges Verhalten $\mathrm{zu}$ konstatieren. Andererseits wird durch eine Vielzahl von

$531 \quad$ Vgl. Perridon, Steiner 1993, $97 \mathrm{f}$.

532 Keynes 1936, $162 \mathrm{f}$.

533 Zum Konzept der Ungewissheit in der postkeynesianischen Tradition vgl. Neal (1996); Kregel (1998); Bernstein (1998); vgl. a. Felderer, Homburg 1999, $100 f$.

534 "The state of long-term expectation, upon which our decisions are based, does not solely depend, therefore, on the most probable forecast we can make. It also depends on the confidence with which we make this forecast - on how highly we rate the likelihood of our best forecast turning out quite wrong." Wolfson 1996, 6. 
Neuemissionen, durch eine abnehmende Verlässlichkeit des Kapitalmarktresearch sowie durch eine abnehmende Verwertbarkeit der von ihnen ermittelten Indikatoren der „Nebel der Ungewissheit“ dichter. In diesem nicht ungewöhnlichen Fall geht eine zunehmende Risikoneigung der Anleger mit zunehmender Ungewissheit einher. ${ }^{535}$ Es ist somit möglich, dass das Eigenkapitalangebot letztlich durch die steigende Ungewissheit sinkt, obwohl mit einer abnehmenden Risikoaversion die Renditeforderungen pro übernommene bewertungsrelevante Risikoeinheit sinken, das heißt, obwohl sich die Poollinie verflacht. Im geschilderten Grundmodell war bislang ein zunehmend eingeschränktes Eigenkapitalangebot stets Ausfluss einer steiler werdenden Poollinie. Das Ausmaß der Sekundärmarktrationierung war insofern positiv abhängig von der Risikoaversion der Anleger. Jede Erhöhung der Renditeforderung in einer stärker risikoaversen Anlegerschaft ging tendenziell mit einem Rückgang des Eigenkapitalangebots und damit einer Zunahme der Sekundärmarktrationierung einher. ${ }^{536}$ Deshalb stellt es eine Erweiterung des Grundmodells dar, dass das Eigenkapitalangebot durch eine zunehmende Ungewissheit auch bei abnehmender Risikoaversion sinken kann. Während eine abnehmende Risikoaversion einen Anstieg der Renditeforderung rationalerweise bewirkt, nimmt davon unabhängig das Ausmaß der Rationierung infolge steigender Ungewissheit zu. In Phasen zunehmender Risikoaversion hingegen geht von einer steigenden Ungewissheit ein verstärkender Effekt auf das Ausmaß der Sekundärmarktrationierung aus.

Den Einfluss von Unsicherheit auf das Ausmaß der Kapitalrationierung am Sekundärmarkt im Rahmen des Grundmodells veranschaulicht Abbildung 4.8. ${ }^{537}$ In der Ausgangssituation eines konjunkturellen Abschwungs mit einer ausgeprägten Risikoaversion (steile, gestrichelte Poollinie in Quadrant V) ist das Ausmaß der Sekundärmarktrationierung mit $Z_{\mathrm{Ab}}$ vergleichsweise groß (Quadrant I). Eine im weiteren Konjunkturverlauf zunehmende Risikoneigung der Anleger wird als eine Verflachung der Nutzenfunktion $U_{\text {Auf }}$ dargestellt, die die Möglichkeitenkurve (MK)

535 "Even though Keynes took a dim view of the impact of a "liquid" stock market on the stability of the economy and the efficiency of ressource allocation, his repeated emphasis on the importance of animal spirits in a world of uncertainty reveals his conviction that economic growth is critically dependent on an environment that encourages risk taking." Bernstein 1998, 17. Vgl. a. Davidson (1991), Crotty (1994). Vgl. Wolfson 1996, 460. 
an einer optimalerweise nun höheren erwarteten Risiko/Rendite-Kombination tangiert (Quadrant VI). ${ }^{538}$

Abbildung 4.8 Sekundärmarktrationierung bei abnehmender Risikoaversion und zunehmender Ungewissheit

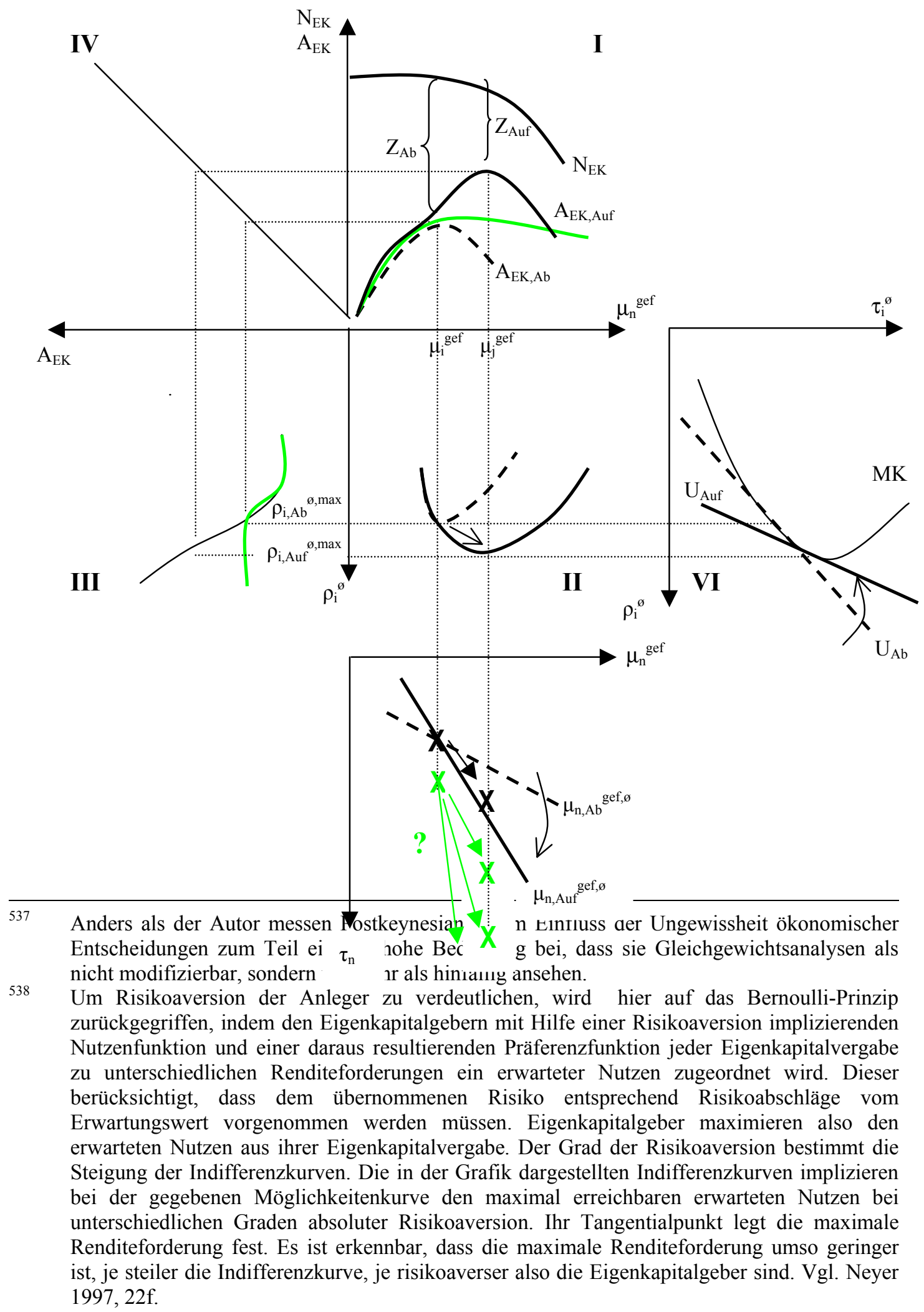


Damit einher geht eine Erhöhung der Renditeforderung und der NettoRenditeerwartung (Quadrant II) sowie eine Verflachung der Poollinie (Quadrant V). ${ }^{539}$ Dieser Effekt führt für sich genommen $\mathrm{zu}$ einer Ausweitung des Eigenkapitalangebots respektive eines Rückgangs der Sekundärmarktrationierung von $Z_{\mathrm{Ab}}$ auf $Z_{\mathrm{Auf}}$ (Quadrant I). Gleichzeitig steige jedoch annahmegemäß die Ungewissheit unter den Eigenkapitalgeber. Verantwortlich für den zunehmenden Grad an Ungewissheit können etwa politische Einflussfaktoren sein, aber auch die Unkenntnis über das Verhalten der internationalen Anlegerschaft, eine unüberschaubar gewordene Zahl börsennotierter Gesellschaften oder das sinkende Vermögen der Eigenkapitalgeber, die entscheidenden renditebeeinflussenden Unternehmensfaktoren $\mathrm{zu}$ isolieren. Das Kapitalmarktresearch wirkt dieser Ungewissheit zwar grundsätzlich entgegen, muss aber nicht zur vollständigen Stabilisierung der Anlegererwartungen führen. Ungewissheit drückt sich in der Abbildung 4.8 darin aus, dass das sicher geglaubte durchschnittliche Poolrisiko vor Anhebung der Renditeforderung möglicherweise ebenso höher zu veranschlagen ist wie die adversen Anreiz- und Selektionsrisiken infolge der höheren Renditeforderungen (grün eingezeichnete Rendite/Risiko-Kombinationen in Quadrant V). Aufgrund dieser Ungewissheit nimmt die Renditeelastizität des Eigenkapitalangebots ab (Quadrant III). Eine dementsprechend veränderte Eigenkapitalangebotskurve (Quadrant I) belässt das Ausmaß der Sekundärmarktrationierung auf dem ursprünglichen Niveau - der abnehmenden Risikoaversion zum Trotz. Im Gesamtergebnis steigt zwar das Maximum der NettoRenditeerwartung, das Ausmaß der Unterinvestition der Anleger bleibt jedoch unverändert. Es ist hier somit der Fall eines bei zunehmender Risikoneigung und Renditeforderung konstanten Ausmaßes an Sekundärmarktrationierung dargestellt. ${ }^{540}$ Die abnehmende Risikoaversion geht im betrachteten Beispiel zwar mit einer

539 Auf den Effekt einer höheren Internen Verzinsung der Unternehmensinvestitionen im konjunkturellen Aufschwung, wird aus Vereinfachungsgründen verzichtet. Ein Aufschwung wäre grafisch betrachtet mit einer Verlagerung der Möglichkeitenkurven nach unten verbunden.

Dass bei zunehmender Ungewissheit weniger Eigenkapital angeboten, verdeutlicht dass der Aktienmarkt nicht als sicherer „Hafen“ angesehen wird. „In an uncertain world whenever liabilities are specified and enforceable only in terms of money, the holding of money is a valuable choice.” Keynes 1936, 236f.; vgl. a. Davidson 1991, 141. 
höheren Renditeforderung einher, nicht aber mit einer sinkenden Sekundärmarktrationierung. 


\subsubsection{Mehrperiodigkeit}

Im Grundmodell erfüllen die Unternehmen eines Pools den geforderten Renditeanspruch aufgrund ihrer relationalen Beziehung zu den Eigenkapitalgebern dadurch, dass sie eine entsprechende Interne Verzinsung real erwirtschaften. Die Unternehmensmanager nutzen ihren Informationsvorsprung aus, indem sie höhere unternehmerische Investitionsrisiken eingehen. Hierbei handelt es sich um eine Form des Defektierens, das von den Eigenkapitalgebern mit einem eingeschränkten Eigenkapitalangebot erwidert wird. Damit wählen sowohl die Agenten als auch die Prinzipale eine Strategie der Nicht-Kooperation. ${ }^{541}$ Das Rationierungsgleichgewicht im Grundmodell entspricht dem beiderseitig unkooperativen Handeln im Rahmen eines Gefangenendilemmas bei einperiodigem Zeithorizont. Der unterstellte Planungszeitraum gibt nicht angemessen die insbesondere deutsche Realität wider. Institutionelle Anleger hegen ein oft intensives und langjähriges Verhältnis zu den beteiligten Unternehmen. Selbst in den USA gehen institutionelle Anleger vermehrt dazu über, sich langfristig und mit großen Aktienpaketen an Unternehmen zu beteiligen und dabei auch Direktorenmandate zu übernehmen, kurzum: Relationship Investing zu betreiben. ${ }^{542}$ Damit bezwecken die institutionellen Eigenkapitalgeber einen höheren Informationsgrad sowie eine größere Beteiligung an den Investitionserträgen, was beides zu einem sinkenden Ausmaß der Kapitalrationierung am Sekundärmarkt führt.

Im Rahmen eines Relationship Investing zielen vertrauensbildende Maßnahmen der Agenten darauf ab, die Notwendigkeit einer Einflussnahme und Überwachung durch die Prinzipale zu reduzieren. Dazu zählen neben der rechtzeitigen Bereitstellung von sachgerechten Informationen verschiedene Anstrengungen zum Aufbau von Reputation. Für die Agenten besteht aus Reputationsgründen ein Anreiz zu kooperativem Verhalten gegenüber den Eigenkapitalgebern, was diese ebenfalls zu kooperativem Handeln motiviert. Ein Aufeinandertreffen von Prinzipalen und Agenten über mehrere Perioden mag deshalb für beide Seiten eine andere Strategie hervorrufen als über einen Zeithorizont von nur einer Periode. Durch ein

\footnotetext{
541 Im so genannten Bayesianischen Gleichgewicht werden spieltheoretische Lösungen für statische Spiele bei asymmetrischen Informationen ermittelt. Dabei maximieren alle Spieler ihren Erwartungsnutzen als Funktion der Wahrscheinlichkeitsverteilung über die Typen der anderen und deren zugehörigen Strategien. Vgl. Porter 1992, 16ff; Bernstein 1998, 23f.
} 
längerfristiges „Miteinander“ kann sich eine so genannte Kollusionslösung entlang der Wertpapiermarktlinie einstellen. ${ }^{543}$ „The one-shot incentive problem of Jensen and Meckling is replaced by an ongoing relation involving strategic behaviour on all sides." ${ }^{544}$ In einem mehrperidigen Kontext ist $\mathrm{zu}$ vermuten, dass Informationsasymmetrien und Ungewissheit sowie der Opportunismus der Agenten zu Gunsten der Eigenkapitalgeber abnehmen. Die Gefahr, durch nicht-kooperatives Verhalten wahrscheinliche zukünftige Gewinne zu verspielen, schafft Anreize zur Kooperation zwischen beiden Akteuren, den Eigenkapitalgebern wie den Managern. ${ }^{545}$ Fraglich ist, ob ein längerfristiges Verhältnis zwischen Eigenkapitalgebern und -nehmern $\mathrm{zu}$ einer Reduzierung oder gar $\mathrm{zu}$ einer Beseitigung der Sekundärmarktrationierung führt, ${ }^{546}$ disziplinieren doch die Sanktionsmöglichkeiten beider Seiten $\mathrm{zu}$ einem jeweils kooperativen Handeln. ${ }^{547}$ Sowohl die Eigenkapitalgeber in Form geringerer Poolrisiken als auch die Unternehmen in Form höherer Eigenkapitalangebote wären langfristig Nutznießer einer längerfristigen Beziehung. Der Aufbau langfristiger Vertrauensbeziehungen zwischen Prinzipalen und Agenten ist zwar auf kurze Sicht ein Einschnitt in den Aktionsraum beider Seiten. So wird ein schneller Kauf und Verkauf von Aktien durch ein eingeschränktes „,voting by feet“ etwa durch Rückspracheverpflichtungen erschwert. Den Anlegern ermöglichen derartige Eingeständnisse im Gegenzug aber eine höhere Transparenz und Interessenvertretung bei der Investitionstätigkeit der Unternehmen. Umgekehrt sind Manager bei Kooperation zu mehr Transparenz und einer geringeren Zurückhaltung und vor allem Verschwendung von Einzahlungsüberschüssen gezwungen. Sie erhalten dadurch den Freiraum, jene die Entscheidungsträger ihren gemeinsamen Nutzen maximieren, indem sie (zumindest vom Ergebnis her) kooperativ handeln.

$544 \quad$ Hellwig 1989, 284.

545 Vgl. Jickeli 1996, 60.

Eine vollständige Beseitigung der Kapitalrationierung bedeutet, dass die Agenten nur Investitionsrisiken gemäß der Wertpapiermarktlinie eingehen und die Anleger Eigenkapital auf ebenfalls markträumendem Niveau bereitstellen.

547 Im Rahmen eines so genannten perfekten Bayesianischen Gleichgewichts wird eine spieltheoretische Lösung für dynamische Spiele bei asymmetrischer Informationsverteilung ermittelt. Die Spielzüge der einzelnen Stufen werden dazu verwendet, um die Wahrscheinlichkeitsverteilung über den Typenvektor der anderen Spieler aufzubessern. Der konzeptionell schwierige Punkt ist, dass jeder Spieler versuchen wird, aus dem beobachteten Verhalten seiner Mitspieler (stochatische) Rückschlüsse auf deren Typen zu ziehen, was diese wiederum bei ihrem Verhalten einkalkulieren werden. Häufig gibt es daher nur Gleichgewichte in gemischten Strategien, was die praktische Bedeutung spieltheoretischer Lösungen für solch komplexe (allerdings auch hochgradig relevante) Entscheidungssituationen einschränkt.
} 
Unternehmensinvestitionen vollziehen zu können, deren Profitabilität sich den Anlegern bei einem einperiodigen Planungshorizont nicht sofort erschlie $3 t{ }^{548}$

Grundsätzlich lässt sich im Rahmen etwa einer so genannten Tit For Tat-Strategie unter bestimmten Bedingungen eine Kooperationslösung herbeiführen. $\mathrm{Zu}$ einer vollständigen Beseitigung der Sekundärmarktrationierung bedarf es dafür bereits mit der Annahme eines unendlichen Zeithorizonts einer unrealistischen notwendigen Bedingung. ${ }^{549}$ So sind mehrperiodige „Spiele“ mit begrenzter Rundenanzahl rationalerweise mit der Gefahr einer so genannten Rückwärtsinduktion des Defektierens von der letzten in die jeweils vorherigen Spielrunden verbunden. ${ }^{550}$ Bei einer endlichen Anzahl von Spielrunden besteht für die Eigenkapitalgeber in der letzten Periode nämlich ein Anreiz zu unkooperativem Verhalten, das durch Antizipation unkooperatives Verhalten auch in allen vorherigen Spielrunden erzeugt. Eine von vornherein zeitlich begrenzte Spielsituation wird jedoch der Beziehung zwischen Prinzipalen und Agenten in der Realität ebenfalls nicht gerecht. ${ }^{551}$ Sowohl die Prinzipale als auch die Agenten wissen nicht, wie lange die jeweilige Gegenseite zur Kooperationsstrategie neigt. Es ist deshalb für die nachfolgende Untersuchung von einem so genannten Superspiel auszugehen, in dem die Akteure nicht den Abbruch eines möglicherweise unbegrenzten Spiels kennen. Die Anzahl der Aufeinandertreffen ist damit endlich, für Prinzipale wie Agenten ist jedoch ex ante das Ende unbekannt. Beide Akteure können deshalb kooperieren (Auswahl wenig riskanter Investitionsobjekte / Nicht-Rationierung) oder defektieren (Auswahl riskanter Investitionsobjekte / Rationierung). ${ }^{552}$ Superspiele sind in der Spieltheorie dadurch gekennzeichnet, dass es unendlich viele teilspielperfekte Gleichgewichte gibt (Folk-Theorem). Es ist deshalb nur im Einzelfall, das heißt unter Berücksichtigung aller Rahmenbedingungen der Akteure, zu eruieren, welche

\footnotetext{
$548 \quad$ Vgl. Schäfer 1997, 126.

Eine weitere Bedingung besteht etwa darin, dass es keine Präferenz der Akteure für gegenwärtige Kooperationserträge gibt, künftige Erträge also nicht abzudiskontieren sind. Vgl. Haubrich 1989, 11.

$550 \quad$ Vgl. Neus 1993, 899-902.

551 Erwecken realiter manche Aktiengesellschaften vor allem kleiner und mittlerer Größe auch den Eindruck einer myopischen Einstellung zur Börse, im Folgenden sei dennoch von einem unbegrenzten Planungshorizont der Unternehmen ausgegangen. Schließlich ist aus analytischen Gründen jener Fall interessanter, in dem der Marktzutritt für solche Unternehmen erschwert ist, die eine erfolgreiche Maximierung des Marktwertes der Kapitalanteile durchführen. Vgl. etwa Feess 1997, 437. 
Strategien zu verfolgen für Prinzipale wie Agenten rational sind. Allgemein lassen sich folgende Tendenzaussagen treffen:

- Um ein beiderseits kooperatives Verhalten zu erzeugen, müssen Manager und Anleger über die verschiedenen Einzelsituationen hinaus ein für beide Seiten bindendes Arrangement herausfinden, dass das Verhalten für die jeweils andere Seite „dechiffrierbar“ macht. Eine solche Übereinkunft über den Durchsetzungsmechanismus einer beiderseitigen Kooperation liegt insofern außerhalb des Spiels, als sie über das reine Handeln hinaus das jeweils eigene Verhalten in Reaktion auf das Verhalten des Gegenüber leitet. Zum Auffinden eines derartigen „Grundverständnisses“ jenseits der einzelnen Situationen bedarf es formeller Abreden oder einer informellen Kommunikation durch Rückgriff etwa auf gesellschaftliche Konventionen, bestimmte Lernverhaltensstrategien (z.B. im Rahmen der besagten Tit For Tat-Strategie) oder so genannter Fokalpunkte. ${ }^{553}$ Wesentlich dabei ist, dass die Akteure symmetrische Zielsetzungen haben und Umweltbedingungen identisch interpretieren. Der Kontext der Situation, der „Background“ der Spieler oder andere unscharfe Faktoren sind von Bedeutung. Eine zunehmende Entfremdung der Anteilseigner der Unternehmen und ein abnehmender Identifikationsgrad der Eigenkapitalgeber mit seinem Unternehmen, eine Internationalisierung der Anlegerstruktur sowie die Internationalität des Unternehmensmanagements ${ }^{554}$ erschweren vor diesem Hintergrund c.p. das Auffinden eines gemeinsamen $\mathrm{zu}$ Kooperation neigenden Verständnisses. In die grundsätzlich andere Richtung wirkt hingegen beispielsweise die Ausgabe von Belegschaftsaktien und ein anlegerfreundliches Investor Relationship.

- Die Wahrscheinlichkeit einer Kollusionslösung nimmt mit abnehmender Anzahl der Akteure auf beiden Seiten zu. Zum einen nimmt für die Unternehmensmanager der Anreiz zum Trittbrettfahren ab, wenn die Zahl der Unternehmen in einem Pool gering ist. Dass das Ausmaß der Rationierung in einem dynamischen Kontext abnimmt, setzt damit eine hinreichende

553 Ein Gleichgewicht, auf das sich die Individuen konzentrieren, wird Fokalpunkt oder gleichgewicht genannt, das Organisationsprinzip (Gleichheit, Eindeutigkeit, Geographie, alphabetische Reihenfolge) als Fokalprinzip.Vgl. Kreps 1994, 372f., 462.

554 In diesem Zusammenhang sei beispielhaft auf die verschiedenen „Kulturen“ bei der Daimler/Chrysler-Fusion verwiesen. 
Spezifizierbarkeit der Einzelrisiken in möglichst viele Pools voraus. Bei einem hohen Informationsstand der Eigenkapitalgeber und einer folglich geringen Anzahl an Einzelrisiken in einem Pool kann sich dann eine kooperative Lösung in einem dynamischen Kontext leichter entfalten. Zum anderen steht zu erwarten, dass mit abnehmender Anzahl an Eigenkapitalgebern eines Unternehmens, die Einfluss auf das Unternehmen ausüben, das heißt mit einer kleineren Anlegerstreuung die Wahrscheinlichkeit für ein anlegerseits kooperatives Verhalten zunimmt. In Publikumsgesellschaften ist eine Kollusionslösung hingegen weniger wahrscheinlich. Denn mit sinkendem Anteil der Eigenkapitalgeber nimmt auch die Fähigkeit und Bereitschaft ab, mit entsprechenden Sanktionen eine langfristige Kooperation anzupeilen. Eine steuerlich begünstigte - künftig fortschreitende Zersplitterung der Aktionärsschaft in Deutschland und die damit einhergehende Aufteilung der Mehrheitsbesitze auf mehrere Köpfe (Stichwort: „Das Ende der Deutschland $\mathrm{AG}^{\text {“) }}$ ) dürfte der Wahrscheinlichkeit einer Kollusion insofern entgegenwirken.

- Entfernt sich die Kursentwicklung der Aktien eines Pools zunehmend von ihren Inneren Werten und unterliegt erratischen Schwankungen, die nicht von Fundamentalfaktoren verursacht werden, üben andere, nicht identifizierbare Faktoren in einem zunehmenden Maße Einfluss auf die Aktienrendite aus. Derartige „Noisy Observables“ erhöhen den Grad der Ungewissheit. Steigt der Einfluss nicht-fundamentaler Faktoren auf Kurs und Rendite an, ist für die Eigenkapitalgeber das tatsächliche Verhalten der Manager hinter den Noisy Observables in immer geringerem Maße erkennbar. Ist der positive Kursverlauf tatsächlich auf eine günstige Zwischenbilanz des Unternehmens oder doch nur auf spekulative Umstände zurückzuführen? Weichen die Unternehmensmanager des Pools umgekehrt von ihrer Strategie der Kooperation ab und suchen tatsächlich riskantere Projekte aus? Erkennen die Prinzipale fälschlicherweise in einer bestimmten Datenlage ein unkooperatives Verhalten seitens der Agenten, bringt eine mit dieser Fehleinschätzung einhergehende Bestrafung in Form einer Kapitalrationierung möglicherweise tatsächlich unkooperatives Verhalten der Agenten und somit ein Abweichen vom optimalen Gleichgewicht hervor. Noise verzerrt die Transparenz über das Verhalten der jeweiligen Gegenseite. ${ }^{555}$ Schwer

${ }^{555} \mathrm{Zu}$ Phänomen des Noise vgl. Abschnitt 4.5. 
erkennbare Zufallskomponenten wirken sich auf die Wahrscheinlichkeit einer Kollusionslösung somit negativ aus. ${ }^{556}$

- Fristigkeit und Intensität der Beziehung zwischen Eigenkapitalgebern und nehmern hängt auch von der Inanspruchnahme sonstiger Leistungen ab. Es dürfte für die Frage einer Kooperation nicht ohne Belang sein, ob die Agenten zu ihren Prinzipalen auch noch über andere Bande als der reinen Eigenkapitalbereitstellung verbunden sind. Durch Bindungen auch am Kreditmarkt, am Aktienprimärmarkt und an unterschiedlichen Produktmärkten lässt sich die Gegenseitigkeit stärken. Ein sich damit ergebender umfassender und impliziter „Groß“-Vertrag kann kooperatives Verhalten im Bereich der Eigenkapialbereitstellung fördern. Nimmt etwa die Bedeutung der deutschen Universalbanken als Großaktionäre, als Kreditgeber, als Emissionsbanken etc. ab, und wird die Wertschöpfungskette auf verschiedene Finanzmarktakteure übertragen, sinkt infolge nachlassender Verbundenheit ihre Bereitschaft zur Kooperation mit den Eigenkapital nachfragenden Gesellschaften. ${ }^{557}$

Langfristige Beziehungen zwischen den Prinzipalen und den Agenten bergen Potenziale zur Reduzierung der Sekundärmarktrationierung. $\mathrm{Ob}$ diese auch tatsächlich abgerufen werden, bleibt angesichts der genannten sich künftig am deutschen Aktienmarkt verstärkenden Entwicklungen allerdings fraglich. Deshalb ist auch unter Einbezug eines mehrperiodigen Kontextes mit adversen Selektionen und Anreizen zu rechnen. Gelingt es nicht, die guten Einzelrisiken vor dem Ausscheiden zu bewahren, ist zu hinterfragen, ob das Ausscheiden von Gesellschaften, das heißt das Nicht-Bedienen einer höheren Renditeforderung von den Eigenkapitalgebern langfristig als Signal eines unterdurchschnittlichen Einzelrisikos zu werten ist. Vermögen Anleger (im Nachhinein) zu erkennen, welche Gesellschaften genau ausgetreten sind, können sie ihre Anlagestrategie auf eben diese guten Gesellschaften ausrichten. Die Renditeerhöhung kann sich in einer mehrperiodigen Betrachtung insofern als ein Screening-Instrument herausstellen, als sich die Ausgeschiedenen qua ihrer Austritte für Anleger als gute Einzelrisiken darstellen. Bei denjenigen Gesellschaften, die trotz höherer Renditeforderung im Pool bleiben, würde es sich folglich um die für die Eigenkapitalgeber schlechten Einzelrisiken handeln. Während

$556 \quad$ Vgl. Feess 1997, 464f. 
sich von den guten Unternehmen eine Rendite in Höhe von nach wie vor $\mu_{\mathrm{i}}{ }^{\text {gef }}$ einfordern ließe, könnte sich der Anspruch an die schlechten Einzelrisiken auf die höhere Rendite $\mu_{\mathrm{j}}{ }^{\text {gef }}$ belaufen. Bei einer Anhebung beider Renditeforderungen in einer „2. Runde“ ließe sich das Screening-Instrument Renditeforderung zu einer weiteren Spezifizierung der Einzelrisiken nutzen etc. Letztlich wären alle Einzelrisiken des Pools wie im CAPM entlang der Wertpapiermarktlinie mit je eigenen Renditeforderungen versehen.

Gegen das Funkionieren eines solchen Screenings lässt sich neben den dabei anfallenden Transaktionskosten einwenden, dass die guten Einzelrisiken unter den Ausgeschiedenen von jenen zu trennen sind, die nur vorgeben, gute Einzelrisiken zu sein, von ihren Imitatoren also. Der Anreiz für schlechte Einzelrisiken, sich als gute $\mathrm{zu}$ präsentieren und die Eigenkapitalnachfrage infolge einer höheren Renditeforderung einzustellen, führt langfristig erneut $\mathrm{zu}$ einem Poolinggleichgewicht. Es verwässert sich durch die Imitation der guten durch die schlechten Einzelrisiken die Renditeforderung als funktionsfähiges ScreeningInstrument. Die Eigenkapitalgeber vermögen zwischen den „wahren“ Guten und den nur vorgetäuscht guten Einzelrisiken nicht $\mathrm{zu}$ unterscheiden. Sich als Eigenkapitalgeber in einem mehrperioden Planungshorizont also auf diejenigen Unternehmen zu kaprizieren, die im Gefolge einer Renditeerhöhung ausscheiden, erscheint angesichts dieses langfristigen Pooling-Mechanismus nicht geeignet, um das Problem adverser Selektionen und Anreize zu überwinden. Ein weiteres Problem bei einer Trennung von über- und unterdurchschnittlichen Einzelrisiken besteht darin, dass sich unter denjenigen Gesellschaften, die trotz höherer Renditeforderungen im Pool verbleiben, auch gute Einzelrisiken befinden mögen, welche Organizational Slack aufzulösen bereit sind. Dieses Gegenargument einer trennscharfen Separierbarkeit guter von schlechten Einzelrisiken durch eine Anhebung der Renditeforderung ist umso zutreffender, als eine Anhebung der Renditeforderung theoretisch nicht zwingend mit einer adversen, sondern auch mit einer favorablen Selektion einhergehen kann. 


\subsubsection{Favorable Selektion}

Kann im Rahmen einer Poolbildung des Anlegerportefeuilles ein unterdurchschnittlich riskantes Unternehmen eine höhere Renditeforderung nicht aus dem Investitionsertrag $R_{i}\left(=p_{i} \times d c f_{i}\right)$ bedienen, scheidet es bei einer einperiodigen Betrachtung aus dem Pool aus; ein mehrperiodiger Zeithorizont der Prinzipale und Agenten mag zu einer Reduzierung, vermutlich nicht aber zu einer Beseitigung der Sekundärmarktrationierung beitragen. Da es sich bei den ausscheidenden Gesellschaften vornehmlich um die für die guten Einzelrisiken handelt, findet eine adverse Selektion im Zuge einer Anhebung der Renditeforderung statt. Das Ausscheiden eines guten Unternehmens setzt voraus, dass die den Agenten zur Auswahl stehenden Investitionsobjekte von der Anhebung der Renditeforderung durch die Eigner unbeeinflusst bleiben. Zwar neigen die Manager auch guter Gesellschaften im Fall adverser Anreize zur Auswahl von schlechteren $\left(\operatorname{dcf}_{\mathrm{i}} / \mathrm{p}_{\mathrm{i}}\right)$ Kombinationen. Aber auch hier bleiben die zur Verfügung stehenden Investitionsprojekte unabhängig von der Höhe der Renditeforderung. Die einzelnen Investitionsobjekte eines Unternehmens i weisen nach wie vor dieselben Kombinationen aus unternehmerischem Projektertrag $\operatorname{dcf}_{\mathrm{i}}$ und Projektwahrscheinlichkeit $p_{i}$ auf. Das Auftreten einer für die Eigenkapitalgeber adversen Selektion gründet somit auf der Annahme von der Renditeforderung unabhängiger und konstanter Investitionserträge $R_{i}$ der vom Ausscheiden bedrohten guten Einzelrisken. Doch ist nicht unrealistisch, dass das Management anlässlich einer steigenden Renditeforderung vorhandene X-Ineffizienzen, die mit den Unternehmensinvestitionen einhergehen mit der Zielsetzung ausmerzt, im Pool verbleiben zu können. Dies kann sich entweder in einem höheren Projektertrag dcf $_{\mathrm{i}}$ oder einem sinkenden Projektrisiko $\mathrm{p}_{\mathrm{i}}$ niederschlagen. Beides führt $\mathrm{zu}$ einem ansteigenden Investitionsertrag $\mathrm{R}_{\mathrm{i}}$. Hebt man die Annahme der bei steigender Renditeforderung unveränderten Investitionserträge auf, indem eine steigende Renditeforderung positiv mit den Projekterträgen $\operatorname{dcf}_{n}$ oder den Projektwahrscheinlichkeiten $\mathrm{p}_{\mathrm{n}}$ korreliert, ${ }^{558}$ kann eine adverse Selektion hinfällig werden. Mit einem höheren erzielbaren Investitionsertrag $R_{i}$ bedienen auch die guten Einzelrisiken die höheren Renditeforderungen der Anleger. Sie können damit ihr

558 Entscheidend ist, dass sich bei einem steigenden Anspruchsniveau der Eigenkapitalgeber der Investitionsertrag $R_{i}$ einiger Projekte erhöhen lässt, unabhängig davon, wie sich die Projekterträge $\operatorname{dcf}_{\mathrm{i}}$ und -wahrscheinlichkeiten $\mathrm{p}_{\mathrm{i}}$ im Einzelnen verändern. 
Ausscheiden aus dem Pool vermeiden. Ganz im Gegenteil: Wenn insbesondere die guten Gesellschaften über die Möglichkeit und vor allem auch über die Bereitschaft verfügen, die Investitionserträge durch eine Optimierung der Unternehmensprojekte zu steigern, kann sich anstelle einer adversen sogar eine favorable Selektion einstellen, ${ }^{559}$ wie folgendes Beispiel verdeutlicht:

Anleger fordern von den beiden folgenden Unternehmen eines Aktienpools i der scheinbar selben Risikoklasse eine Rendite in Höhe von $\mu_{\mathrm{i}}{ }^{\text {gef }}$. Diese Renditeforderung ist durch einen Investitionsertrag von jeweils $R_{i}=100$ Einheiten zu bedienen. Es handelt sich im Pool um folgende Unternehmen:

Gutes Einzelrisiko A: Projektertrag $\mathrm{dcf}_{\mathrm{A}}=100$; Projektrisiko $\mathrm{p}_{\mathrm{A}}=1,0 \Rightarrow$ (geforderter) Investitionsertrag $\mathrm{R}_{\mathrm{A}}=100$; Projektanteil der Anleger im Erfolgsfall $\mathrm{dcf}_{\mathrm{A}}{ }^{\text {prinz }}=100$; erwarteter Netto-Ertrag für die Anleger $\mathrm{E}_{\mathrm{A}}=100\left(=\mathrm{dcf}_{\mathrm{A}}{ }^{\text {prinz }} \times \mathrm{p}_{\mathrm{A}}\right)$; Projektanteil für die Manager im Erfolgsfall $\mathrm{dcf}_{\mathrm{A}}{ }^{\text {agent }}=0$; frei verfügbarer Cash Flow $\operatorname{dcf}_{\mathrm{A}}^{\text {free }}=0\left(=\mathrm{dcf}_{\mathrm{A}}{ }^{\text {agent }} \times \mathrm{p}_{\mathrm{A}}\right)$.

Schlechtes Einzelrisiko B: Projektertrag $\operatorname{dcf}_{\mathrm{B}}=111,1$ Projektrisiko $\mathrm{p}_{\mathrm{B}}=0,9 \Rightarrow$ (geforderter) Investitionsertrag $\mathrm{R}_{\mathrm{B}}=100$; Projektanteil der Anleger im Erfolgsfall $\mathrm{dcf}_{\mathrm{B}}{ }^{\text {prinz }}=100$; erwarteter Netto-Ertrag für die Anleger $\mathrm{E}_{\mathrm{B}}=90\left(=\mathrm{dcf}_{\mathrm{B}}{ }^{\text {prinz }} \times \mathrm{p}_{\mathrm{B}}\right)$; Projektanteil für die Manager im Erfolgsfall $\mathrm{dcf}_{\mathrm{B}}{ }^{\text {agent }}=11,1$; frei verfügbarer Cash Flow $\mathrm{dcf}_{\mathrm{B}}{ }^{\text {free }}=10\left(=\mathrm{dcf}_{\mathrm{B}}{ }^{\text {agent }} \times \mathrm{p}_{\mathrm{B}}\right)$.

Bei Unternehmen A handelt es sich um ein marginales Poolprojekt links von der Poollinie, genauer gesagt auf der markträumenden Wertpapiermarktlinie (mit einem frei verfügbaren Cash Flow von $\mathrm{dcf}_{\mathrm{A}}^{\text {free }}=0$ ). Unternehmen $\mathrm{B}$ stellt ein vergleichsweise schlechtes, weil riskanteres Einzelrisiko dar $\left(\mathrm{dcf}_{\mathrm{B}}{ }^{\text {free }}=10\right)$ rechts der Poollinie dar. Für den Eigenkapitalgeber ergibt sich bei einer paritätischen Zusammensetzung des Pools aus beiden Gesellschaften ein durchschnittlich erwarteter Netto-Ertrag von $95\left[=\left(E_{A}+E_{B}\right): 2\right]$. Das durchschnittliche Poolrisiko erfordert gemäß der Wertpapiermarktlinie annahmegemäß einen Investitionsertrag von 105. Deshalb erwägt der Anleger eine entsprechende Erhöhung der Renditeforderung. Um diese Forderung erfüllen zu können, müsste das gute Unternehmen A (c.p.) im Erfolgsfall Organizational Slack auflösen, weshalb es aus dem Pool ausscheidet. Für die Agenten des Unternehmens B würde zwar der frei verfügbare Cash Flow dcf ${ }_{\mathrm{B}}^{\text {free }}$ von 10 auf 5,5 $(=6,1 \times 0,9)$ sinken. Jedoch muss Unternehmen B nicht auf einen finanziellen Puffer zurückgreifen, weshalb es im Pool verbleibt. Es liegt somit eine adverse Selektion vor. Vom verbleibenden Unternehmen $B$ ist bei der gestiegenen Renditeforderung ein Netto-Ertrag $E_{B}$ von 94,5 (= 105 x 0,9) zu erwarten. Durch die Anhebung der Rendite sinkt der erwartete Netto-Ertrag für Anleger deshalb nach der adversen Selektion um $0,5(=95-94,5)$. Ein Renditeanspruch, der einen Investitionsertrag von 100 übersteigt, stellt sich deshalb als nicht rational dar, zumindest nicht unter der Bedingung konstanter Projekterträge und -risiken des Unternehmens A.

Vermögen die Eigenkapitalgeber nicht nur den Mindest-Investitionsertrag einzufordern, sondern darüber hinaus auch auf die Höhe der Einzahlungsübeschüsse oder die Eintrittswahrscheinlichkeit von A Einfluss auszuüben, kann sich eine mit steigender Renditeforderung favorable Selektion einstellen. Erwirken die

Die Modelle sind nicht "robust" hinsichtlich kleiner Modellveränderungen. “... the implications for theory and policy may differ from model to model. It is, therefore, important to choose between the different models before attempting to draw firm conclusions from the literature." Hillier, Ibrahimo 1993, 300. Vgl. a. Hillier, Worrall (1994). 
Eigenkapitalgeber für Unternehmen A (und zwar nur für Unternehmen A) etwa einen Anstieg der Einzahlungsüberschüsse $\operatorname{dcf}_{\mathrm{A}}$ von 100 auf 112, ist es für die Eigenkapitalgeber rational, eine Rendite zu fordern, die einem Investitionsertrag von $\mathrm{R}_{\mathrm{A}}=112$ entspricht. Die entsprechende Renditeforderung liegt oberhalb der Wertpapiermarktlinie. Das Unternehmen B zieht sich in diesem Fall aus dem Pool zurück. Für B wäre nämlich Organizational Slack aufzulösen, um dieser Renditeforderung Genüge leisten zu können. Von Unternehmen A ist hingegen ein Netto-Ertrag $\mathrm{E}_{\mathrm{A}}$ in Höhe von $112(=112$ x 1,0) zu erwarten. Das Ausscheiden des risikoreicheren Unternehmens B ist im Sinne des Eigenkapitalgebers, steigert es doch den durchschnittlich zu erwartenden Netto-Ertrag von 95 (bei Poolbildung) auf 112 nach favorabler Selektion.

Wie das Beispiel veranschaulicht, geht ein Anstieg der Renditeforderung mit einer höheren erwarteten Netto-Rendite für die Eigenkapitalgeber einher, wenn das

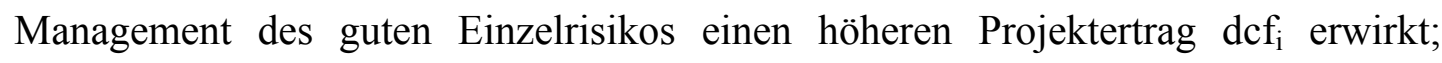
Gleiches würde eine Verringerung des Projektrisikos in entsprechender Höhe bewirken. In beiden Fällen erzwingt eine höhere Renditeforderung einen Anstieg des unternehmerischen Investitionsertrages der guten Einzelrisiken. Ist der Einfluss der Anleger auf die Unternehmensprojekte der guten Einzelrisiken hinreichend groß, kann eine höhere Rendite zu fordern für Eigenkapitalgeber somit rational sein; nicht obwohl, sondern gerade weil mit der dadurch bewirkten Selektion eine Verbesserung des durchschnittlichen Poolrisikos einhergeht. Ist der Investitionsertrag, den gute Gesellschaften nun zu erwirtschaften in der Lage sind, hinreichend groß, tritt an die Stelle einer adversen eine favorable Selektion. ${ }^{560}$ Hierbei ändert sich die Zusammensetzung des Pools dahingehend, dass die schlechtere Einzelrisiken aus dem Pool gedrängt werden.

Damit der Fall favorabler Selektion eintreten kann, müssen allerdings zwei Bedingungen erfüllt sein. Zum einen muss die neuerliche Renditeforderung sich auf einem hinreichend hohen Niveau bewegen, dass schlechte Einzelrisiken zum Ausscheiden bewegt. Dafür muss mindestens eine Rendite gefordert werden, die das durchschnittliche Poolrisiko entlang der Wertpapiermarktlinie abwerfen würde. Erst $\mathrm{ab}$ dieser Forderungshöhe beginnen nämlich die knapp überdurchschnittlichen Einzelrisiken, Organizational Slack aufzehren zu müssen, um den Ansprüchen der Anleger genügen zu können. Aus dieser Tatsache leitet sich eine Tendenzaussage dahingehend ab, dass die Wahrscheinlichkeit einer favorablen im Gegensatz zu einer adversen Selektion mit ansteigender Wertpapiermarktlinie abnimmt. Je flacher die Wertpapiermarktlinie ist, desto geringer ist die geforderte Rendite, mit der ein 
Ausscheiden der schlechten Einzelrisiken erwirkt werden kann. Außerdem steigt die Wahrscheinlichkeit des Auftretens einer favoralen Selektion mit zunehmendem Informationsstand der Anleger. Denn zum einen befinden sich die schlechten Einzelrisiken näher am Pooldurchschnitt und zum anderen ist die Entfernung der Pool- zur Wertpapiermarktlinie geringer. Beides verringert den Mindestanstieg der Renditeforderung, von dem aus die schlechten Einzelrisiken ausscheiden. Eine zweite Voraussetzung für das Auftreten einer favorablen Selektion besteht darin, dass die vom Ausscheiden bedrohten überdurchschnittlichen Poolrisiken anders als die guten Einzelrisiken nicht zu einer Anhebung der Investitionserträge bereit oder in der Lage sind. ${ }^{561}$

Infolge einer favorablen Selektion wird das durchschnittliche Poolrisiko mit steigender Renditeforderung sicherer. Da sich im Zuge einer favorablen Selektion die Qualität der Poolzusammensetzung verbessert, besteht für die Aktionäre ein Anreiz, die Renditeforderungen möglicherweise über das markträumende Niveau zu erhöhen. Eine Rendite oberhalb der Wertpapiermarktlinie $\mathrm{zu}$ fordern, ist auf der Eigenkapitalangebotsseite mit einer Überhangsituation verbunden. In diesem Fall, wird im Verhältnis zur Markträumung nicht $\mathrm{zu}$ wenig, sondern $\mathrm{zu}$ viel an Eigenkapital angeboten. Im Gegensatz zum Fall adverser Selektion, in dem eine unterhalb der Wertpapiermarktlinie verharrende Renditeforderung Unterinvestition bewirkt, liegt bei favorabler Selektion somit eine Überinvestition am Aktiensekundärmarkt vor. ${ }^{562}$ "If it is assumed that projects differ in expected return then rationing no longer remains a possibility and the outcome would be overinvestment rather than underinvestment relative to the first-best." ${ }^{963}$ Die Folge eines Überschussangebots an Eigenkapital am Sekundärmarkt ist „Überliquidität“, was sich in einer ansteigenden Umlaufgeschwindigkeit von Aktien, einer Überbewertung ihres Kursniveaus und folglich verbilligten Kosten der Eigenkapitalbeschaffung aus wirkt.

\footnotetext{
$560 \quad$ Vgl. de Meza, Webb (1987); Bester (1985a); Neyer (2000).

561 Erhöhen im umgekehrten Fall nur die ,schlechten“ Einzelrisiken den Investitionsertrag, wird eine Anhebung der Renditeforderung umso wahrscheinlicher.

Vgl. Bester (1985a). Bernanke, Gertler (1987) und Black, de Meza (1990) zeigen unter Berücksichtigung kleiner Modellmodifikationen, dass auch bei Vorhandensein asymmetrischer Informationen hinsichtlich der variierenden Investitionserträge $\mathrm{R}_{\mathrm{i}}$ Unterinvestition möglich ist. Hillier, Ibrahimo 1993, 289.
} 
Scheinbar stellt das Auftreten einer Positiv- oder einer Negativauslese eine Entweder-Oder-Frage dar, scheiden doch innerhalb eines Pools infolge einer Anhebung der Renditeforderung entweder die guten oder die schlechten Unternehmen aus. Das Auftreten einer favorablen Selektion und damit Überliquidität innerhalb einzelner Segmente widerspricht jedoch nicht einem infolge adverser Anreiz- und Selektionsrisiken vollrationierten Gesamtmarkt. So ist es theoretisch durchaus miteinander vereinbar, dass an einem von adverser Selektion insgesamt betroffenen Gesamtmarkt in denjenigen Segmenten, auf die sich das Eigenkapitalangebot ohnehin konzentriert, ${ }^{564}$ das Phänomen einer favorablen Selektion in Verbindung mit einem Überschussangebot und mit Überliquidität vorherrscht. Denn eine Konzentration des Anlegerinteresses auf bestimmte Teilsegmente erhöht den Einfluss der Eigenkapitalgeber auf die Unternehmensprojekte und damit die Möglichkeit, die Investitionserträge aus den Unternehmensprojekten qua einer höheren Renditeforderung zu steigern. Durch favorable Selektion gekennzeichnete Börsensegmente überliquider Standardwerte würden demnach unterliquiden Nebenwerten gegenüberstehen. Die mögliche Koexistenz beider Selektionsarten impliziert insofern eine Sowohl-Als-AuchErscheinung von Über- und Unterliquidität bezogen auf unterschiedliche Börsensegmente.

564 Zur Konzentration des eingeschränkten Eigenkapitalangebots auf bestimmte Anlagen vgl. Abschnitt 4.5. 


\subsection{Geld- und Fiskalpolitik im "equity channel"}

Das Ausmaß einer Kapitalrationierung am Sekundärmarkt hängt von der Steigung der Poollinie ab. Geld- und fiskalpolitische Maßnahmen üben Einfluss auf die Höhe des Entgelts für die Übernahme einer bewertungsrelevanten Risikoeinheit und damit auf die Steigung der Poollinie aus. ${ }^{565}$ Deshalb ist fraglich, ob von der Sekundärmarktrationierung eine katalysierende Wirkung auf fiskal- und geldpolitsche Maßnahmen ausgeht. Hierzu wird zwischen einer expansiven geldpoltischen Maßnahme in Form einer Geldmengenausweitung und einer expansiven fiskalpolitischen Maßnahme in Form einer Staatsausgabenerhöhung, welche durch die Ausgabe von Staatspapieren finanziert wird, unterschieden. ${ }^{566}$ Dabei ist die Frage zu beleuchten, ob die wirtschaftspolitischen Maßnahmen die Steigung der Poollinie, infolgedessen die adversen Selektions- und Anreizrisiken und damit das Ausmaß an Sekundärmarktrationierung beeinflussen, was dann wiederum auf die ursprünglichen Maßnahmen zurückwirkt.

Geld- und fiskalpolitische Maßnahmen verändern eine vormals als optimal angesehene Vermögenszusammensetzung vermittels des Transmissionsmechanismus relativer Preise. ${ }^{567}$ Sofern sich auf eine geld- und fiskalpolitische Maßnahme die verfügbaren Mittel der Privaten, ihr Vermögen oder die mit den einzelnen Bilanzpositionen verbundenen Erträge oder Aufwendungen verändern, reagieren Wirtschaftseinheiten mit Anpassungsmaßnahmen. Diese betreffen das Finanz- und das Sachvermögen ebenso wie die Verbindlichkeiten. Aber auch die Produktion neuer Güter wird in den Anpassungsmechanismus einbezogen. ${ }^{568}$ Es sind grundsätzlich zwei Wirkungen zu unterscheiden, die von geld- und fiskalpolitischen Maßnahmen ausgehen. Zum einen verändert sich der Vermögensbestand als Ganzes (Vermögenseffekt). Zum anderen geben exogene Schocks Veranlassung zu Umschichtungen in den einzelnen Vermögensteilbereichen (Substitutionseffekt). Es handelt sich bei diesem Transmissionsmechanismus relativer Preise um

\footnotetext{
565 Zum Einfluss monetärer Effekte auf den Aktienmarkt vgl. Jandura, Rehkugler (1999).

566 Die Wirkung von adversem Selektions- und Anreizrisiko auf die entsprechend kontraktiven Maßnahmen sind spiegelbildlich zu betrachten. Unabhängig davon mag sich die Wirksamkeit von expansiven und kontraktiven Maßnahmen grundsätzlich unterscheiden. Vgl. hierzu Kakes (2000).

$567 \quad$ Vgl. Hillmer 1993, Kap. 4.

568 Vgl. Jarchow 1998, 227-233.
} 
portefeuilletheoretische Überlegungen. ${ }^{569}$ Wird der Transmissionsmechanismus der relativen Preise an einer Stelle der Wirkungskette durch Marktfriktionen wie etwa durch einen inflexiblen Preismechanimus am Aktiensekundärmarkt gestört, hat dies eine veränderte Auswirkung der geld- und fiskalpolitischen Maßnahme auf den gesamtwirtschaftlichen Output zur Folge.

Der Einfachheit halber wird zwischen finanziellen Aktiva wie Geld und Wertpapiere einerseits und Anteilsrechten an Unternehmen, das heißt Aktien andererseits unterschieden. Abgesehen vom Extremfall einer vollständigen Komplementarität zwischen den finanziellen Aktiva und den Anteilsrechten wirken sich geld- und fiskalpolitische Maßnahmen über den Transmissionsmechanismus relativer Preise auf die Renditeforderungen der Aktionäre und folglich auch auf die gesamtwirtschaftliche Investitionstätigkeit aus. ${ }^{570}$ Veränderungen etwa des Geldbestandes von Privaten üben dann - da Geld eine Wertaufbewahrungsfunktion erfüllt - Einfluss sowohl auf das Gesamtvermögen als auch auf das relative Gefüge der Teilvermögensbestände untereinander aus. ${ }^{571}$ Von Veränderungen im Geldbestand werden andere finanzielle Aktiva wie Obligationen, aber auch der Bestand an realen Anteilsrechten beziehungsweise Aktien betroffen. Im Zuge einer expansiven geldpolitischen Maßnahme nimmt bei einer Ausweitung der Geldmenge $^{572}$ die Nachfrage nach festverzinslichen Wertpapieren aufgrund ihres substitutionalen Verhältnisses zu. Mit einer folglich sinkenden Rendite für

569 Diese wurden mit der Keynes'schen an Opportunitätskosten orientierten Aufteilung des Geldvermögens auf die Spekulationskasse einerseits und den Wertpapierbestand andererseits vorbereitet und von Friedman auf ein ganzes Spektrum von Anlageentscheidungen übertragen. Die monetaristischen Modelle Friedmans, Brunners und Meltzers unterscheiden sich von jenen des Keynesianismus à la Tobin in Bezug auf das rein analytische Instrumentarium kaum. Mit der Entwicklung der Portefeuilletheorie und deren Übernahme durch den Monetarismus hat eine methodische Konvergenz von Monetarismus und Keynesianismus stattgefunden. Differenzen bestehen in einer unterschiedlichen Einschätzung der Art und Stärke verschiedener Substitutionsbeziehungen zwischen den Vermögensteilbereichen. Vgl. Felderer, Homburg 1999, 245-248; Jarchow 1998, 227.

570 Das aus der Mikroökonomie bekannte Begriffspaar „Substitutionalität-Komplementarität“ bezieht sich auf die Stärke der Reaktion eines Güterverhältnisses (x/y) bei Änderung des relativen Preises. Die Substitutionselastizität besteht aus dem Quotienten einer relativen Veränderung der Gütermischung auf der Grundlage einer relativen Änderung des Preises. Beträgt sie Null, spricht man von vollständiger Komplementarität. Egal, in welchem Verhältnis die Ertragsraten der finanziellen und realen Aktiva stehen, ihre Anteile am Portefeuille bleiben in diesem Fall dieselben. Geht die Substitutionselastizität im anderen Extremfall gegen unendlich liegt vollständige Substitutionalität vor.

$571 \quad$ Es sei von Preiseffekten abstrahiert.

572 Ohne dass sich der Zinssatz bei einer Ausweitung der Geldmenge ändert, verringert sich bei Vorliegen von Kreditrationierung die Lücke zwischen Kreditangebot und -nachfrage. Selbst bei zinsunelastischen Investitionen ist in diesem Fall - in Übereinstimmung mit der so genannten availability doctrine - Geldpolitik wirksam. Vgl. Winker 1994, 6f. 
festverzinsliche Papiere verschiebt sich die Poollinie des Aktiensekunärmarktes parallel nach unten. Der Konsumverzicht, der mit der Bereitstellung von Eigenkapital einhergeht, wird mit einer folglich geringeren festen Verzinsung entgolten. Besteht zwischen festverzinslichen Wertpapieren und realen Aktiva wie Aktien ein zumindest schwach komplementäres Verhältnis, steigt ausgelöst durch eine expansive geldpolitische Maßnahme ebenfalls die Aktiennachfrage. Daraufhin steigende Aktienkurse führen c.p. zu einem Rückgang der Renditeforderung der Anleger pro übernommene Risikoeinheit. Eine expansive geldpolitische Maßnahme führt somit c.p. zu einer Verflachung der Wertpapiermarktlinie und folglich zu einer Abnahme adverser Selektions- und Anreizrisiken. ${ }^{573} \mathrm{Zu}$ dem ohnehin expansiven Effekt sinkender Zinsen im Rahmen einer expansiven Geldpolitik kommt unter den genannten Bedingungen im „equity channel“ der Tendenz nach ein weiterer, auf die Gesamtwirtschaft expansiv wirkender Schub hinzu: eine Abnahme der Sekundärmarktrationierung. ${ }^{574}$ Dieser Sekundärmarktkatalysator wirkt umso stärker, je schwächer die Komplementarität finanzieller und realer Aktiva ausgeprägt ist, das heißt, je mehr sich die Zinssenkung auf die Aktiennachfrage auswirkt. ${ }^{575}$ Dabei hängt der Grad der Komplementarität vom gemeinsamen Korrelationskoeffizienten sowie der Risikoneigung der Wirtschaftssubjekte ab. ${ }^{576} \mathrm{Ob}$ die adversen Selektions- und Anreizrisiken durch eine expansive Geldpolitik auch tatsächlich abnehmen, hängt

573 Mit ihrer Verflachung bewirkt die Poollinie abnehmende Eigenkapitalkosten und damit einen steigenden Fundamentalwert, weshalb die Poollinie tendenziell wieder steiler wird. In die gleiche Richtung wirkt der den Konsum steigernde Pigou-Effekt, der die Interne Verzinsung der Unternehmen steigern dürfte. Andererseits mag der zinssenkende Effekt einer expansiven geldpolitischen Maßnahme die Risikoneigung der Anleger erhöhen, was wiederum zu einer Verflachung der Wertpapiermarktlinie führt. Aus Gründen der Vereinfachung sei deshalb die Wirkung einer geld- oder fiskalpolitischen Maßnahme auf den unternehmerischen Fundamentalwert und die Risikoneigung vernachlässigt. Ohnehin ist die Analyse insofern partialanalytisch, als etwa der Güter- und Arbeitsmarkt nicht berücksichtigt werden. Ob ein expansiver Impuls über die damit einhergehende Zunahme der Güternachfrage das Realeinkommen oder die Preise erhöht, kann ebenfalls nicht gesagt werden.

$574 \mathrm{Zu}$ den in der Literatur bisher erwähnten Transmissionskanäle geldpolitischer Impulse auf die Realwirtschaft über Vermögenspositionen (Zinskanal, Wechselkurskanal, Konsumkanal, Kreditkanal) vgl. Mishkin 1985, 4-9; Hillmer 1993, 211-229; Bernanke, Gertler (1995); Guender, Moersch (1997); Schlick 1994, Kap. 1.2.

575 Während die klassisch-neoklassische Theorie auf der einen, die Keynesianische Geld- und Vermögenstheorie auf der anderen Seite von einer vollständig substitutiven Beziehung zwischen Obligationen und Aktien ausgehen, hält Tobins Portefeuilletheorie eine komplementäre Beziehung für möglich, schließt ein schwach substitutives Verhältnis aber nicht aus. Monetaristen bezweifeln die Komplementarität von finanziellen und realen Aktiva. Vgl. Felderer, Homburg 1999, 220-233.

576 Die Komplementarität zwischen Obligationen und Aktien ist umso stärker, je größer die Risikoaversion und je geringer der Wert (nicht der Betrag) des Korrelationskoeffizienten ist. Bei absoluter Risikoaversion und einer Korrelation kleiner Eins ist die Komplementarität vollständig. 
letztlich von der Verteilung der Einzelrisiken in den Pools zusammen, mithin dem Ausmaß an Informationsunvollkommenheiten am Sekundärmarkt.

Zu dem beschriebenen Substitutionseffekt einer Umschichtung von Geldvermögen in substitutives Wertpapiervermögen sowie bei zumindest schwacher Komplementarität auch in Aktienvermögen tritt ein Vermögenseffekt hinzu. Dieser besteht darin, dass eine (reale) Geldmengenausweitung von den Privaten möglicherweise als Erhöhung des Gesamtvermögens empfunden wird. ${ }^{577}$ Handelt es sich bei Aktien nicht um inferiore Güter, verstärkt der Anstieg des (empfundenen) Gesamtvermögens die Aktiennachfrage erneut mit einer Verflachung der Wertpapiermarktlinie c.p. zur Folge. Denn ein höheres Vermögen lässt die Nachfrage nach Aktien ansteigen, senkt also die Renditeforderungen der Anleger pro übernommene Risikoeinheit. Beide Effekte - der Substitutions- wie der Vermögenseffekt - führen im Equity Channel folglich zur Verstärkung einer geldpolitischen Maßnahme. Im Zuge einer Geldmengenexpansion wird somit der Umfang der Unterliquiditätsfalle der Tendenz nach verringert. Dabei kommt insbesondere den kleinen und mittleren Gesellschaften eine expansive Geldpolitik in Form einer geringeren Kapitalrationierung am Aktiensekundärmarkt zu Gute. ${ }^{578}$ Die Träger der Geldpolitik haben in der Dosierung ihrer Maßnahmen einen etwaig katalysierenden Effekt abnehmender Sekundärmarktrationierung zu berücksichtigen, um ein Überschießen ihrer Maßnahme zu verhindern.

Wie bei einer geld- ist auch bei einer fiskalpolitischen Maßnahme zwischen einem Substitutions- und einem Vermögenseffekt zu unterscheiden. Die Finanzierung von Staatsausgaben durch die Ausgabe von Staatspapieren führt zu einer Ausweitung des Wertpapierangebots und einer folglich steigenden Rendite (mit crowding outs als mögliche Folge). Die Bereitstellung von Eigenkapital geht deswegen mit einem ansteigenden Entgelt für den Konsumverzicht einher, und die Poollinie verschiebt sich zunächst parallel nach oben. Aber auch die Steigung Poollinie erfährt eine Veränderung. Bei einer - erneut unterstellten - zumindest schwach komplementären Beziehung zwischen Obligationen und Aktien nehmen die Aktienkurse infolge des Zinsanstiegs bei nachlassender Aktiennachfrage ab, und die Renditeforderung pro

577 Neuklassiker führen dagegen an, dass Private im Rahmen einer rationalen Erwartungsbildung mit einem steigenden Preisniveau rechnen. Deshalb betrage der Vermögenseffekt einer expansiven Geldpolitik Null. 
übernommene bewertungsrelevante Risikoeinheit infolgedessen zu. Die dadurch steiler werdende Poollinie tendiert zu einem Anstieg des adversen Selektions- und Anreizrisikos, womit die ursprünglich expansive fiskalpolitische Maßnahme durch eine etwaig zunehmende Sekundärmarktrationierung im Equity Channel einen dämpfenden Effekt erhielte. Diesem kontraktiv wirkenden Substitutionseffekt einer expansiven Fiskalpolitik wirkt jedoch ein expansiver Vermögenseffekt entgegen, sofern die Ausgabe von Staatspapieren von den Privaten als Zunahme des gesamten Nettovermögens betrachtet wird. ${ }^{579}$ Vermag der Vermögens- den Substitutionseffekt nicht zu kompensieren, wirkt einer fiskalpolitischen - anders als bei einer geldpolitischen - Maßnahme das retardierende Moment eines eingeschränkten Eigenkapitalangebots am Sekundärmarkt entgegen.

Neben den geschilderten negativen Wachstumswirkungen, ${ }^{580}$ die von einer niveaubedingten Unterliquidität der kleinen und mittleren Gesellschaften ausgehen, gibt es somit auch aus Gründen der Wirksamkeit fiskalpolitischer Maßnahmen ein Interesse an der Ursachenbekämpfung von Sekundärmarktrationierung. Auch die Träger der Geldpolitik haben in der Dosierung und im Timing ihrer Maßnahmen einen multiplikativen Transmissionskanal durch die Unvollkommenheiten des Aktienmarktes $\mathrm{zu}$ berücksichtigen. Eine die Renditeforderung der Anleger beeinflussende politische Maßnahme erfährt durch die Sekundärmarktrationierung entweder eine Verstärkung (wie im Fall der Geldpolitik) oder eine Abschwächung (wie im Fall der Fiskalpolitik) von - je nach Auswirkung auf die adversen Selektions- und Anreizrisiken - möglicherweise empfindlichem Ausmaß. ${ }^{581}$ Daher sind im Equity Channel über- beziehungsweise „unterschießende“ Reaktionen durch das Geschehen am Aktiensekundärmarkt zu erwarten. Die Existenz eines die Geldund Fiskalpolitik katalysierenden Equity Channel spült letztlich „Wasser auf die Mühlen“ der so genannten Credit School, die im Gegensatz zur „monetary school“

Diese Aussage ist übereinstimmend mit Gertler, Gilchrist (1994).

Gegen diese als „Fiskalillusion“ bezeichnete Einschätzung lässt sich einwenden, dass der Staat einerseits Zinsen für die aufgenommene Schuld zu zahlen hat, und er andererseits Tilgungen leisten muss, sofern es sich nicht um Obligationen mit unendlicher Laufzeit handelt, was aber nur aufschiebende Wirkung hat. Zum Bestreiten dieser zusätzlichen Ausgaben ist der Staat früher oder später bei sonst unveränderter Nachfrage zu einer Steuererhöhung gezwungen. Deshalb - so die Neuklassiker - werden die Privaten die Ausgabe von Staatspapieren nicht als Nettovermögenszuwachs ansehen. Dies entspricht dem Ricardianischen Äquivalenztheorem, wonach die Wirkungen steuer- und kreditfinanzierter Staatsausgaben letztlich gleichwertig sind.

Vgl. Abschnitt 2.4.

Vgl. Mankiw (1986). 
davon ausgeht, dass Kapitalmarktmechanismen nicht reibungslos funktionieren und wirtschaftspolitische Maßnahmen für sich genommen somit nur eine eingeschränkte Wirkung zeitigen. ${ }^{582}$

\subsection{Unterliquiditätsfalle}

Im CAPM hält jeder Anleger ungeachtet seiner Risikoneigung das alle unsichere Einzelanlagen umfassende Marktportefeuille. Diese Vorstellung wurde bislang aufrechterhalten. Jeder Anleger hält ebendieses Marktportefeuille, gruppiert die Einzelanlagen in Pools mit ihm ähnlich erscheinenden Einzelrisiken, die die Anleger nicht im Eizelnen exakt, sondern nur pauschal spezifizieren können. Die im Zuge einer Erhöhung der Renditeforderung drohende Negativauslese führt zu einem die Eigenkapitalnachfrage dauerhaft unterschreitenden Eigenkapitalangebot. Offen blieb bislang die Frage, ob alle Einzelanlagen im gleichen Maße weniger an Eigenkapital erhalten (Typ-I- oder Teil-Rationierung) oder ob sich die Eigenkapitalgeber auf einige Anlagen kaprizieren, andere hingegen vernachlässigen (Typ-II- oder VollRationierung). Im Fall einer Teilrationierung sind alle Aktien - wie im CAPM - im Verhältnis ihrer Kapitalisierung Teile des (Markt-)portefeuilles, wenn auch - anders als im CAPM - das ausgeschüttete Aktienkapital nicht in vollem Ausmaß geplantermaßen nachgefragt wird. Im Fall einer Vollrationierung einiger Aktien bei gleichzeitiger Konzentration des Eigenkapitalangebots auf andere Werte finden hingegen einige Einzelanlagen geplantermaßen Eingang ins Portefeuille des rationalen Anlegers, andere jedoch nicht. Bei Vollrationierung wird der Aktiengesamtmarkt in grundsätzlich bevorzugte und grundsätzlich vernachlässigte Teilmärkte segmentiert. Im Gegensatz zur Teil- bedarf es bei einer Vollrationierung Kriterien, nach denen zwischen be- und übervorteilten Gesellschaften unterschieden wird. Diesen Auswahlkritien kommt bei Vorliegen von Vollrationierung entscheidende Bedeutung für das Kapitalaufkommen in den einzelnen Marktsegmenten und Teilmärkten zu.

Eine Vollrationierung ist mit Kosten einer effektiven Kategorisierung zwischen beund übervorteilten Aktienwerten verbunden. Würden einige Aktienwerte des Pools voll-, andere gar nicht rationiert werden, verringert sich die Anzahl der sich im Pool

582 Zur Rolle von Kreditmarkt-Unvollkommenheiten in der Makroökonomik vgl. Gertler (1988); Hubbard (1998); Bernanke (1993); Kashyap, Stein (1994); King, Levine (1993); Jaffee, Stiglitz 1990, 884; Blinder, Stiglitz (1983); Blinder (1987; 1989, Kap. 4). 
befindlichen Aktienwerte, mithin auch die Möglichkeiten des Reduzierens von Portefeuillerisiken. Deshalb verzichtet der Anleger bei einer Vollrationierung auch auf Diversifizierungseffekte, die im Zuge einer breiten Streuung des Portefeuilles entstehen. Aus diesen Gründen erscheint eine gleichmäßige Verteilung des eingeschränkten Eigenkapitalangebots auf alle Aktien zunächst als der überlegene Rationierungstyp. Allerdings geht eine Beachtung aller börsennotierten Gesellschaften ebenfalls mit hohen Transaktionskosten einher. Und eine gewisse Diversifizierung von Risiken lässt sich bereits dadurch erreichen, dass man sich auf Unternehmen mit einer breit gestreuten Investitionstätigkeit verlegt - auf die größeren Unternehmen eben. Offenbar lassen sich in der Realität durch eine Konzentration des eingeschränkten Eigenkapitalangebots auf nur wenige Gesellschaften im Zuge einer Voll- anstelle einer Teilrationierung entscheidende Intensivierungserträge erzielen. Die Überprüfung und Überwachung von Einzelrisiken ist mit hohen fixen Stückkosten verbunden. Eine Konzentration der Researchkapazitäten auf relativ wenige Einzelanlagen bringt bei der Informationsproduktion somit sinkende Durchschnittkosten, aber auch Lerneffekte ein. Darüber hinaus gewinnen Anleger, die ihr Eigenkapital auf nur wenige Unternehmen konzentrieren, an Durchsetzungskraft gegenüber den Agenten, aber auch anderen Anteilseignern. Dies trägt zu einer größeren Beteiligung an den unternehmerischen Investitionserträgen (Ertragssymmetrie) und zum Erhalt von Insider-Informationen (Informationssymmetrie und Gewissheit) bei. ${ }^{583}$ Bei einer Einflussnahme auf die Investitionstätigkeit der Unternehmen kann sogar eine favorable Selektion erwirkt werden. "Liquid assets also provide a safe haven for not committing one's monetary claims on resources ..."584

Vollrationierung bringt einen "unsatisfied fringe"585 von Gesellschaften am Sekundärmarkt hervor, die in die Unterliquiditätsfalle einer nach oben rigiden NettoRenditeerwartung tappen. ${ }^{56}$ In die Unterliquiditätsfalle geraten für die

583 Zum Insider-Handel vgl. Weber (1993).

$584 \quad$ Davidson 1991, 139.

$585 \quad$ Keynes 1930, 212.

586 Der Begriff der Unterliquiditätsfalle lehnt sich an der Keynes'schen Liquiditätsfalle an. Die Keynes'sche Liquiditätsfalle ist einem erwartungsbedingt nach oben starren Kurs von Anleihen geschuldet. Infolge einer unendlich zinselastischen Spekulationskasse, das heißt bei absoluten Liquiditätspräferenz vermag der Zins eine gewisse Untergrenze nicht zu unterschreiten. Einer zinselastischen privaten Investitionstätigkeit ist somit von Seiten des Preises zur Finanzierung eine „natürliche“ Grenze gesetzt. Vgl. Felderer, Homburg 1999, 144-148; Kromphardt 1987, 175f. Beiden Fallen ist gemeinsam, infolge von Preisrigiditäten einen realwirtschaftlich 
Eigenkapitalgeber gute wie schlechte Einzelrisiken. „,[T]here is no presumption that the market equilibrium allocates [capital] to those for whom the expected return on their investments is highest. ${ }^{6587}$ Befindet sich ein Unternehmen erst einmal in der Lage permanenter Vernachlässigung durch die Aktionäre, ist ihm nur schwer möglich, dieser Unterliquiditätsfalle zu entkommen. Denn die guten unter den rationierten Unternehmen können der Unterliquiditätsfalle nicht dadurch entkommen, dass sie potenziellen Eigenkapitalgebern direkt besondere Leistungen wie etwa Mindestdividenden in Aussicht stellen. Denn für Eigenkapitalgeber ist generell davon auszugehen, dass es insbesondere die schlechteren unter den rationierten Einzelrisiken sind, die zum Nachgeben eines höheren Renditeanspruchs bereit sind. Ein unterdurchschnittliches Poolrisiko, das der Vollrationierung anheim gefallen ist, ist zum Erwirtschaften einer höheren Rendite nicht bereit, wäre doch dafür Organizational Slack aufzulösen. Bietet ein rationiertes Unternehmen potenziellen Eigenkapitalgebern eine höhere Interne Verzinsung an, müssen deshalb letztere vermuten, es handele sich vielmehr um ein schlechtes unterdurchschnittliches Poolrisiko.

Fraglich ist, nach welchen Kriterien Unternehmen eines Pools bei Vollrationierung trotz scheinbar identischen Rendite/Risiko-Profils teils be-, teils übervorteilt werden. Firmenspezifische Kriterien, die im CAPM als unsystematische Risiken für nichtbewertungsrelevant erachtet werden, gelangen bei Vorliegen unvollkommener Informationen $\mathrm{zu}$ besonderer Bedeutung. Nach ihnen wird geurteilt, auf welche Aktienwerte sich das eingeschränkte Eigenkapitalangebot erstreckt und welche Unternehmenstypen umgekehrt in niveaubedingte Unterliquidität fallen. Die Unterscheidung ist nicht beliebig zu treffen. Mit Hilfe der Auswahlkriterien versucht der rationale Eigenkapitalgeber, sich den nicht ersichtlichen Einzelrsiken tatsächlich anzunähern. Die Auswahlkriterien dienen insofern als Instrumente des Screening; allerdings wird ihre Funktionstüchtigkeit bereits dadurch unterwandert, dass sie meist nur das Ergebnis historischer Wahrscheinlichkeiten sind. Bei Zugrundelegung von Auswahlkriterien wird die einzelne Gesellschaft eines Pools nicht als Einzelrisiko, sondern im Rahmen einer Durchschnittsbehandlung je nach ihrer Zugehörigkeit zu bestimmten Unternehmenstypen bewertet. Dabei fallen unter die

kontraktiven Effekt verursachen zu können. Zum Begriff der „illiquidity trap“ vgl. a. Epstein 1995, 309. 
rationierten Aktienwerte auch gute Poolrisiken. Sie werden insofern diskriminiert, als möglicherweise höhere Einzelrisiken qua ihrer Zugehörigkeit zu einer bevorzugten Gruppierung stattdessen Eigenkapital angeboten wird. Die Diskriminierung guter Einzelrisiken ist für den rationalen Eigenkapitalgeber hinzunehmen, solange die Durchschnittsbildung nach Auswahlkriterien insgesamt fundamentaltheoretisch begründet ist. Eine Fehlbewertung von Einzelwerten ist unter der Annahme rationaler Gruppenbildungen in diesem Fall billigend in Kauf zu nehmen. Entbehren die im Folgenden aufgeführten Auswahlkriterien jedoch einer fundamentalen Grundlage und werden sie „lediglich“ aus Transaktionskostengesichtspunkten genutzt, um die komplexe Welt irgendwie auf eine überschaubare Größe zu reduzieren, wird die Funktion der Auswahlkriterien als Screening-Instrument zwischen durchschnittlich guten und durchschnittlich schlechten Unternehmenstypen hinfällig. Möglicherweise findet in diesem Fall eine Fehlbewertung nicht nur einzelner Risiken, sondern sogar ganzer Teilgruppen statt. Eine Missklassifizierung nicht nur einzelner Werte, sondern ganzer Teilgruppen wird vom Wettbewerbdruck, der etwa auf instititutionelle Anleger lastet, nicht zwingend beseitigt. Aufgrund von versunkenen Informations- und Kommunikationskosten gibt es ein Beharrungsvermögen, an einer - wenn auch ineffizienten - Unterscheidung zwischen verschiedenen Unternehmenstypen festzuhalten. ${ }^{588}$

- Unternehmensalter und Dauer der Notierung am Aktienmarkt. Zum einen spielt die zeitliche Dimension eine entscheidende Rolle für den Aufbau und den Bestand einer relationalen Beziehung zwischen Eigenkapitalgebern und -nehmern. Zum anderen steht die zeitliche Komponente für Kontinuität eines Unternehmens. Sich als Anleger auf ältere und seit längerer Zeit an der Börse notierte Gesellschaften zu verlegen, impliziert die Ausnutzung eines Selektionseffektes. Denn im Laufe der Zeit verschwinden realwirtschaftlich unerfolgreiche, das heißt riskante Unternehmen eines Pools von der Börse, ${ }^{589}$ die Zeit wirkt gewissermaßen wie ein Screening-Instrument. Durch diesen Ausleseprozess verändert sich die Verteilung der Renditeerwartungen im Laufe der Zeit. Denn für ein junges Unternehmen ist die Gefahr, den Markt schon bald wieder verlassen zu müssen, vergleichsweise groß. Die Austrittwahrscheinlichkeit sinkt mit der Dauer einer Notierung am Aktienmarkt respektive seinem Unternehmensalter, so dass nach einigen Perioden 
die Restgruppe im Durchschnitt einen höheren Anteil an risikoarmen Firmen aufweist als eine neu in den Markt eintretende, ansonsten völlig identisch erscheinende Vergleichsgruppe. Dass die Aktien von Börsenneulingen in den ersten drei bis fünf Jahren nachweislich eine geringere Rendite abwerfen als bereits länger an der Börse notierenden Werte, passt dabei ins beschriebene Bild. ${ }^{590}$ Auch den guten unter den jungen Gesellschaften fehlt oft ein so genannter track record, der Auskunft über ihre vergangene Performance gibt.

- Tiefe der Geschäftsbeziehungen. Das Verhältnis zwischen Eigenkapitalgebern und -nehmern kann durch andere Bande zusätzlich gestärkt werden wie etwa durch Kreditbeziehungen oder die Teilnahme des Eigenkapitalgebers am Emissionskonsortium des Unternehmens oder sonstige Geschäftsbeziehungen. ${ }^{591}$

- Größe und Bekanntheitsgrad des Börsensegments, in dem eine Aktiengesellschaft notiert. ${ }^{592}$ Reputation und Rahmenbedingungen eines Börsensegments gelten als Indikator für die Güte der dort notierenden Gesellschaften.

- Zugehörigkeit zu einer aus Anlegersicht verheißungsvollen Branche wie etwa Software, Telekommunikation, Biotechnologie etc., denen allgemein positive Gewinnaussichten bescheinigt werden.

- Medienbeachtung des Unternehmens sowie sein "degree for investor recognition" 593 insbesondere durch institutionelle Anleger. ${ }^{594}$ Damit eng verbunden ist auch ein gewisser „Modeeffekt“ unter den Anlegern.

Mit Ausnahme der Branchenzugehörigkeit liegt eine hohe Korrelation der genannten Kriterien mit dem Kriterium der Größe eines Unternehmens gemessen am Marktwert des Eigenkapitals, aber auch an der Beschäftigtenzahl oder der Unternehmensbilanz vor. In der Literatur wird deshalb der Unternehmensgröße als so genannte catch-allVariable besondere Bedeutung beigemessen. Die Größe eines Unternehmens gilt spätestens seit Fama und French (1992) als vom Markt im Gleichgewicht zu

Vgl. Winker 1993a, 10-14; Jovanovic (1982).

Zur Long-run Underperformance des deutschen Aktienmarktes vgl. Sapusek 1998, 182-184.

Vgl. Abschnitt 4.3.5.3.

Vgl. Barry, Brown (1982); Kadlec, McConnell (1994).

Merton 1987, 494.

Vgl. Falkenstein (1996); Arbel, Carvell, Strebel (1983); Arbel, Streibel (1982); Arbel (1985).

Cohen, Zinbarg, Zeikel (1975) messen den Grad der Vernachlässigung eines Unternehmens: „Three different measures of generic neglect were calculated, all relating to institutional holdings as reported by Standard \& Poor's: 1. The number of financial institutions holding common stocks of the firm. 2. The percentage of the firm's outstanding shares held by institutions. 3. A weighted measure of institutional attention calculated as the product of (1) multiplied by (2).“ Cohen; Zinbarg; Zeikel 19xx, 191. 
berücksichtigender eigenständiger Risikofaktor. "Size is widely considered a proxy for capital market access, ..." ${ }^{955}$ Junge mittelständische Unternehmen aus einer Sättigungsbranche der Old Economy sind von den Folgen asymmetrischer Informationen besonders betroffen. ${ }^{596}$ Das Halten ihrer Aktien wird mit allgemein höheren Risiken in Verbindung gebracht. Gründe dafür sind:

- höhere Insiderrisiken ${ }^{597}$

- eine ,patriarchalische“ Informationsvermittlung

- geringere Transparenzvorschriften in Segmenten für Small und Mid Caps; ihre Segmente würden außerdem ein oft $\mathrm{zu}$ geringes eigenständiges Profil aufweisen. ${ }^{598}$

- stärkere Abhängigkeit ihrer Unternehmenserträge von Veränderungen gesamtwirtschaftlicher Größen; eine höhere Konjunkturabhängigkeit ${ }^{599}$ etwa „may result in a 'flight to quality'" ${ }^{600}$ (, was die relativ hohe Zinselastizität der Aktiennachfrage nach kleinen und mittleren Aktientiteln in Hochzinsphasen erklären dürfte.)

- ein geringeres politisches Gewicht, das einzusetzen nicht nur in Krisenzeiten den Aktionären zugute kommt; Großunternehmen kommen aufgrund ihrer gesellschaftspolitischen Bedeutung in den Genuss eines staatlich garantierten Bestandsschutz.

- geringere Ausrichtung der Investitionstätigkeit am Shareholder-Value. Außerdem gibt es für Standardwerte aufgrund ihres öffentlichen Interesses und höherer Transparenzverpflichtungen einen größeren Druck, ihre Investitionsobjekte so auszuwählen, dass sich ihre Risiken einander angleichen. Dieser Anpassungsdruck entfällt bei Gesellschaften kleiner und mittlerer Größe, so dass

Perez-Quiros, Timmermann 2000, 1231.

Gertler, Gilchrist (1994) argumentieren, dass Informationsasymmetrien, die die externe Kapitalbeschaffung verteuern, junge Unternehmen, Unternehmen mit idiosynkratischen Investitionen und Unternehmen mit geringen Sicherheiten betreffen. Sie alle sind i.d.R. kleinere Unternehmen.

Vgl. Gerke 1995, 21.

Dass bspw. der Geregelte Markt kein eigenständiges Image als Wachstums- und Innovationsmarkt entwickeln konnte, wird nicht zuletzt mit einer möglichen Einteilung einer Aktie in verschiedene Marktsegmente, an verschiedenen Börsenplätzen bei Wahl unterschiedlicher Handelsverfahren (variabler Handel oder Kassakurs) begründet.

"Using size as a proxy to credit markets, this work has found that small manufacturing firms experience substantially more procyclical variation in sales, inventories, and short-term debt than larger firms, consistent with the flight-to-quality prediction." Bernanke, Gertler, Gilchrist 1996, 2. Vgl. a. Beiker 1993, 106-111.

Perez-Quiros, Timmermann 2000, 1229. 
bei ihnen eine größere Risikostreuung innerhalb eines Pools $\mathrm{zu}$ erwarten ist. Anhebungen der Renditeforderungen verursachen folglich bei Blue Chips einen geringeren adversen Selektionseffekt als bei kleinen und mittleren Aktienwerten.

- geringere Möglichkeiten zur Ausnutzung von Unternehmensdiversifikationen durch Verbundvorteile ${ }^{601}$

- ein höherer Verschuldungsgrad und weniger Möglichkeiten, aus dem Cash Flow Verbindlichkeiten zu tilgen und Eigenkapital zu bilden. ${ }^{602}$

Aus diesen Gründen lassen kleine und mittlere Gesellschaften höhere Risiken erwarten. Dies führt eigentlich zu einer Eingruppierung kleiner und mittlerer Gesellschaften in Pools, an die höhere Renditeansprüche gestellt werden. Es könnten die höheren Risiken kleiner und mittlerer Gesellschaften aber auch Anlass geben zur Vermutung, dass sie innerhalb eines Pools überdurchschnittliche Poolrisiken darstellen. Ein funktionsfähiger, das heißt nicht rationierender Marktmechanismus hätte in diesem Fall durch einen entsprechenden Renditeaufschlag den Risikofaktor „Unternehmensgröße“ zu kompensieren. Tatsächlich ist in der kapitalmarkttheoretischen Literatur die Auffassung weit verbreitet, der Kauf von Aktien kleiner und mittlerer Gesellschaften sei regelmäßig mit der Forderung einer „Überrendite“ verbunden. ${ }^{603}$ Man spricht vom „Kleinfirmeneffekt"“ ${ }^{604}$ Darunter kann man eine Überrendite als (teilweisen) Ausgleich für das höhere Risiko verstehen, das mit dem Halten kleiner und mittlerer Aktientitel grundsätzlich einhergeht. ${ }^{605}$ Insoweit handelte es sich beim Kleinfirmenefekt weniger um eine Marktanomalie als vielmehr um einen Ausgleich für Risikokomponenten, die im CAPM nicht spezifiziert sind. ${ }^{606}$ Höhere Kosten etwa bei der Informationsbeschaffung sind

601 " $[\mathrm{L}]$ arge firms because of their greater diversification and longer track records, and because of economies of scale in collecting and processing information about their situation - have lower agency costs per dollar of external finance than smaller firms." Größ1, Stahlecker, Wohlers $1999,5$. Vgl. Beiker 1993, 163-169; Größ1, Stahlecker (2000); Größ1, Stahlecker, Wohlers (1999). Vgl. Levy (1990).

604 Erstmals untersucht wurde der Firmengrößeneffekt von Banz (1981) und Reinganum (1981). Vgl. a. Reinganum (1992).

605 Die Überrendite des Kleinfirmeneffekts wird auf verschiedene Effekte zurückgeführt: Taxselling-Hypothese, Window-Dressing institutioneller Anleger sowie eine Interdependenz mit anderen Bewertungsanomalien und Saisonalitäten wie etwa den Januar-, Price/Earnings-, Leverage-, Buchwert / Marktwert-, Autokorrelations-, Winner-Loser-Effekt. Vgl. Sattler 1994, 82-149; Schnittke 1989, 31-113; Beiker 1993, 25-29. „Insgesamt scheint der KFE aber ein eigenständiger Effekt zu sein.“ Beiker 1993, 32. pagatorischen und kalkulatorischen Kosten, also auch den Unterliquiditätskosten die Rede sein. Vgl. Domke (1987). 
Selbstversicherungsbeiträge und müssen folglich bei der Renditeberechnung berücksichtigt werden. Zahlreiche empirische Untersuchungen bestätigen die Anomalie einer transaktions- und risikobereinigten Überrendite - für den USamerikanischen Aktienmarkt. ${ }^{607}$ Für den deutschen Aktienmarkt kann hingegen keine stabile signifikante Überrendite kleiner und mittlerer Gesellschaften festgestellt werden. ${ }^{608}$ Oertmann $(1994 a, b)$ zufolge hängt die renditemäßige Abweichung für den deutschen Aktienmarkt vielmehr von der jeweiligen Marktphase ab. Seinen Erhebungen zufolge schwächt sich in Phasen positiver Risikoprämien, das heißt in Up-Markets der Small Firm Effect deutlich ab. Man könne sogar von einem negativen Kleinfirmeneffekt oder einem "Big-firm-Effect" sprechen. In einer differenzierteren Analyse kommt Sattler (1994) zu einem ähnlichen Ergebnis: „Die durchschnittlichen Renditen eines Size-Portefeuilles ... sind in Up-market-Phasen in der Regel signifikant geringer als die Marktrendite.““609 Dass Gesellschaften kleiner und mittlerer Größe am deutschen Aktienmarkt im Up-Market demzufolge keine Über-, sondern vielmehr eine Unterrendite abwerfen, erhärtet empirisch die These der vorliegenden Arbeit, dass Small und Mid Caps am deutschen Aktienmarkt nicht nur vernachlässigt, sondern darüber hinaus sogar rationiert werden. Denn läge „nur“ eine Vernachlässigung vor, würde eine entsprechende Anlage mit einer Überrendite kompensiert werden. Am deutschen Aktienmarkt erfolgt jedoch offenbar keine Kompensation für kleine und mittlere Gesellschaften - in Up-Markets. Ganz im Gegenteil wird die Bereitstellung von Eigenkapital für rationierte Aktientitel mit einer unterhalb vom Marktniveau liegenden Rendite bestraft. „Namely a truly anomalous regularity would be if an inverse relation between size and return was not obeserved.“6610

Die Vernachlässigung der Aktien kleiner und mittlerer Gesellschaften geht nicht zuletzt auf institutionelle Anleger zurück. Sie zeichnet eine ausgeprägte Anlegerpräferenz für ,stocks that are larger, more liquid, and have relatively low returns dur-

607 Einschränkend muss allerdings gesagt werden, dass bereits die Kosten der Unterliquidität - wie Abschnitt 2.2 zeigte - nicht objektiv bestimmbar sind. Folglich lässt sich keine endgültige Aussage über die Existenz einer solchen Überrendite treffen. Vgl. Beiker (1993). Sattler 1987, 220. Der Autor führt fort: „Dies gilt auch nach einer Bereinigung um die WinnerLoser- und die q-Variable.“

610 Berk 1995, 275. Er erkennt in der Unternehmensgröße den entscheidenden „missing factor“ für eine verbesserte Quantifizierung des CAPM. 
ing the previous year.“611 Institutionelle verdrängen zunehmend Kleinanlegerpräferenzen für Small und Mid Caps durch ihr Angebot indirekter Aktienanlagen über Investmentfonds; oft meiden Investmentfonds im Zuge passiven Index-Benchmarkings ${ }^{612}$ Small und Mid Caps weitgehend. ${ }^{613}$ Mit zunehmender Bedeutung institutioneller Anleger verlegt sich der „durchschnittliche“, repräsentative Anleger immer stärker auf die Aktien großer Gesellschaften mit einem hohen Grad an „media coverage“ und einer Notierung in prestigeträchtigen Segmenten. Ein derartiger Wandel in der Struktur der Aktiennachfrage erhöht das Kurs- und das Renditeniveau der Standardwerte relativ zu Lasten desjenigen vernachlässigter Aktienwerte. ${ }^{614}$

"[T]his compositional shift tends to increase demand for the stock of large companies and decrease demand for the stock of small companies. The compositional shift can, by itself, account for a nearly 50 percent increase in the price of large-company stock relative to small-company stock and can explain part of the disappearance of the historical small-company stock premium... From 1926 to 1979 investors in small companies enjoyed a distinct return advantage over investors in large companies. Using standard classifications for these categories, the small stock premium was 4 percent per year over this time. This premium, first pointed out by Banz (1981) and subsequently analyzed in many academic studies, has reversed in the subsequent twenty years, with large stocks earning a significant premium over small stocks since 1980." ${ }^{.615}$

Die „Flucht“ in Standardwerte zeitigt sich selbst verstärkende Effekte. Je höher die niveaubedingte Liquidität innerhalb eines Segmentes ist, desto mehr Eigenkapital wird angezogen. Je liquider ein Markt ist, desto größer das Anlegerinteresse, desto

611 Gompers, Metrick 2001, 229. Zu grundsätzlichem Meiden kleiner und unterliquider Aktienwerte vgl. a. Falkenstein (1996); Del Guercio (1996); Wermers 1999, 582.

612 Passives Index-Benchmarking impliziert die These informationseffizienter Märkte, nach der Kursveränderungen nicht antizipierbar sind, sondern einem Random Walk folgen. Vgl. Soros 1998a, 58.

613 Investmentfonds zeigen eine eindeutige Präferenz für Standardwerte. In traditionellen Investmentfonds besteht ein jederzeitiges Anteilsrückgaberecht der Anleger. Ein erhöhter Mittelrückfluss macht somit die Liquidierung eines Teils der im Sondervermögen befindlichen Aktien notwendig. Verfügen die Anlageobjekte nicht über eine ausreichende Liquidität, könnten Investmentfonds ihrer gesetzlichen Verpflichtung zu einer jederzeitigen Rücknahme der Anteilsscheine nicht nachkommen. Dieses Problem umgehen geschlossene Fonds (closedend-funds). Bei diesem Fondstyp werden die Aktienanteilsscheine nicht an die Kapitalanlagegesellschaft zurückgegeben, sondern über die Börse abgegeben. Vgl. Baums 1997, 1947. Auch restriktive Anlagevorschriften des Versicherungsaufsichtsgesetzes sehen vor, dass entweder in Dax-Werte oder in sonstige, risikoarme Anlageformen investiert wird. In der vorherrschenden Finanzliteratur werden Nachfrageeffekte dieser Art von Arbitrageuren absorbiert, die mit Hilfe von „sophisticated“ Handelsstrategien für den „korrekten“ Preis sorgen. Wie derartige Arbitrageprozesse möglicherweise ausgehebelt werden, zeigen auch De Long, Shleifer, Summers, Waldmann (1990); Shleifer, Vishny (1997b). Weitere Literaturhinweise liefern Gompers, Metrick 2001, 231. 
liquider wiederum der Markt etc. ${ }^{616}$ Hohe niveaubedingte Liquidität zeitigt somit Netzwerkexternalitäten. ${ }^{617}$ Am anderen Ende des Sekundärmarktes ergibt sich umgekehrt eine eigendynamische Vernachlässigung ohnehin rationierter Unternehmen. Denn fixkostenintensive Research-Aktivitäten über ein Unternehmen einzugehen, ist erst ab einer gewissen Umsatzhöhe rentabel. ${ }^{618}$ Eine entsprechende Losgröße zu erreichen, ist in unterliquiden Märkten jedoch nur eingeschränkt möglich. Deshalb geht die Nachfrage nach detaillierten Unternehmensanalysen seitens der Anleger zurück. „So kann die 30. Analyse eines Standardwertes profitabel sein, während bei Spezial- und Nebenwerten eine erste Studie ihre Kosten nicht erwirtschaften kann. ${ }^{\text {“619 }}$ Ein geringerer Informationsstand der Anleger in Bezug auf kleine und mittlere Gesellschaften führt $\mathrm{zu}$ einer größeren Streuung ihrer Aktienwerte in einem Aktienpool. Sich auf kleine und mittlere Gesellschaften zu verlegen, impliziert somit die größere Gefahr einer adversen Selektion, weshalb sich die Konzentration der Anleger auf Standardwerte abermals verstärkt. Auf diese Weise geraten Small und Mid Caps in einen „Teufelskreis“ niveaubedingter Unterliquidität. ${ }^{620}$

Fruchtbare Hinweise für eine Größenpräferenz der Anleger liefert zudem der kapitalmarkttheoretische Zweig der verhaltensorientierten Finanzmarktforschung (Behavioral Finance). ${ }^{621}$ Sie rückt dabei allerdings vom Menschenbild des rationalen

616

Ein niveaubedingt liquides Marktsegment geht einerseits mit geringen Transaktionskosten einher. Andererseits können rationale informationsorientierte Anleger die höhere Kursvolatilität für sich nutzbar machen. Vgl. a. Kriebel (1962).

Der Nutzen eines einzelnen Netzteilnehmers steigt mit der Gesamtzahl der Netzteilnehmer. Netztwerkexternalitäten sind nicht mit externen Effekten zu verwechseln, da erstere eine Beziehung innerhalb und nicht außerhalb eines Marktes bezeichnen. Netzwerkexternalitäten können allgemein dazu führen, dass bisherige Netzteilnehmer auch bei einem leistungsfähigeren Angebot außerhalb des Netzes im alten Netz verharren, weil der Marktanteil der konkurrierenden Anbieter noch nicht die kritische Höhe erreicht hat, bei dem ihr Netz vergleichbare Externalitäten hervorbringen. Vgl. a. Amihud, Mendelson (1991a, 63f.).
Vgl. Franke, Hax 1990, 372

Rasch 1996, 93.

Vgl. Rasch 1996, 109-111. Neben den Anlegern wird auch ein sinkendes Interesse der Emissionsbanken an der Marktpflege von Nebenwerten (geringe Umsätze = geringe Gebühreneinnahme, Pflichten der Prospekthaftung etc.) angeführt.

Vgl. Unser (1999); Kleidon 1986, 488ff. Derartige Ansätze zeigen etwa, dass Anleger die ihnen zugänglichen Informationen selektiv und subjektiv wahrnehmen und teils nicht-rational verarbeiten. Bei ihren Entscheidungen maximieren sie nicht immer den Erwartungsnutzen. Informationen, die sie als erste erreichen, werden gegenüber den späteren tendenziell übergewichtet. Umgekehrt treten ältere Informationen in der Wahrnehmung gegenüber neu hinzukommenden in den Hintergrund. Ferner gewichten beispielsweise Anleger einfache Informationen stärker als komplexe, die sie noch verarbeiten müssen. So schenken Anleger Berichten von Übernahmen, Umsatzmeldungen und Börsengängen mehr Aufmerksamkeit als den Veröffentlichungen von Bilanzkennzahlen. 
Anlegers ab. Wird der bislang unterstellte rationale Anleger lediglich um einen teils irrationalen Anlegertyp, dem so genannten Noise Trader, ${ }^{622}$ erweitert, gelangt man bereits ebenfalls zu Effekten, die die Divergenz des Sekundärmarktes zusätzlich verstärken. ${ }^{623}$ Verfügen unterinformierte Noise Trader über eine teils irrationale Informationsverarbeitung oder über verzerrte Wahrscheinlichkeitsverteilungen, muss es selbst für den gut informierten rationalen Anleger nicht sinnvoll sein, gemäß den unternehmerischen Fundamentalfaktoren zu handeln. Für den rationalen Anleger ist dabei entscheidend, wie die jeweils (kursbestimmende) Masse handelt, deren Akteure sich wiederum dem vermuteten Handeln wiederum anderer anpassen etc. ${ }^{624}$ Gegen den „Strom“ die unterbewerteten rationierten Gesellschaften für eine Aktienanlage auszuwählen, wird damit immer weniger rational. "[W]here individuals differ, there will be some incentives for acquiring information, but if information is costly, market prices reflect the information of the informed individuals only imperfectly." ${ }^{25}$ Informationen über Fundamentalfaktoren werden somit nicht nur subjektiv, sondern auch objektiv zu einer möglicherweise untergeordneten Sache für eine Aktienanlage. Anstelle der Fundamentalfaktoren geriert die Interaktion der Investorengruppen zu einem den Handel entscheidend beeinflussenden Faktor; so kann in Noise-Trader-Modellen nicht nur die Fluktuation der Kursverläufe zunehmen, sondern auch ihre Fortbewegung von den Fundamentalfaktoren. ${ }^{626}$

Reagieren einzelne Anleger auf das Verhalten der (kursbestimmenden) Masse dahingehend, dass sie es lediglich imitieren, liegt Herdenverhalten vor. ${ }^{627}$

622 Der Begriff „Noise“ für einen Handel auf der Basis bewertungsirrelevanter Informationen hat sich nach der Ansprache von Black, F. (1986) auf der Jahrestagung der American Finance Association in der Literatur durchgesetzt. Einen guten Überblick verschafft Heyl (1995); De Long, Shleifer, Summers, Waldmann (1990). Für Noise Trader werden Verhaltensweisen oder Handelsstrategien angenommen, die durch eine Loslösung der Handelsaktivitäten von fundamentalen Änderungen der objektiven Informationslage über den Unternehmenswert geprägt sind: Orientierung an Preisen der Vergangenheit, verzerrte Erwartungsbildung, emotionale Orientierung, undifferenzierte Orientierung an Expertenmeinungen.

Damit wird es für den rationalen Anleger etwa lohnend, „front running“, das heißt die Nutzung irrationalen Verhaltens der Noise Trader oder „stock picking“ statt Diversifizierung zu betreiben. Vgl. Teh, De Bondt (1997). Stiglitz 1982, 120.

626 Noise-Trader-Modelle sind in der Lage, in der Realität beobachtbare Phänomene wie positive Autokorrelationen der Kurse und Überreaktionen auf Informationen zu erklären.

Einen guten Literaturüberblick zu mutual fund herding gibt Wermers (1999). Vgl. a. Teh, de Bondt (1997); Bannerjee (1992); Grinblatt, Titman, Wermers (1995). Herdenverhalten wird als Motiv in Noise-Trading-Modellen aufgegriffen. Vgl. dazu von Heyl 1995, 174-180; zu Keynes' „Beauty Contest“"vgl. Keynes 1936, 214. 
"Mitlaufen" wird zu einem rationalen Motiv, ${ }^{628}$ wenn ein asymmetrischer Ansehensverlust vorliegt, das heißt, das Verfehlen einer befriedigenden Rendite bei einer Strategie des Abweichens von der Herde mit höheren Sanktionen verbunden ist als bei kollektivem Versagen. ${ }^{629}$ Außerdem kann Herding rationalerweise Lerneffekte hervorbringen. ${ }^{630}$ Herding wird durch den Bezug von Informationen aus identischen Quellen $^{631}$ oder die Anpassung an erfolgreichen Akteuren der Vergangenheit ${ }^{632}$ verstärkt. Bezogen auf das Phänomen niveaubedingt unterliquider Aktien zeitigt Herding eine Eigendynamik, die den ohnehin rationierten Einzelrisiken eines Pools $\mathrm{zu}$ einer weiteren Missachtung gereicht. Dabei sind Mitläufereffekte nicht nur zwischen den Standardwerten einerseits und den Neben- und Spezialwerten andererseits $\mathrm{zu}$ konstatieren, „...funds also show significant levels of herding in small stocks, although they seldom trade such stocks." ${ }^{633}$ 
5 Wirtschaftspolitische Implikationen 


\subsection{Risikoüberwachung: Externes Mittelstands-Rating ${ }^{634}$}

Sekundärmarktrationierung und die infolgedessen niveaubedingte Unterliquidität kleiner und mittlerer Gesellschaften sind das Ergebnis einer inflexiblen, nach oben rigiden Netto-Renditeerwartung der Anleger. Auf Informationsunvollkommenheiten basierende und vom Markt nicht entgoltene Selektions- und Anreizrisiken verhindern, eine markträumende Rendite $\mathrm{zu}$ fordern. Ein entscheidender Ansatz wirtschaftspolitischen Eingreifens hat deshalb bei einer möglichen Fehleinschätzung einer Aktienanlage anzusetzen. Ratingagenturen vermögen dazu einen wesentlichen Beitrag zu leisten. Ratingagenturen sind Produzenten von Erwartungssicherheit. Sie

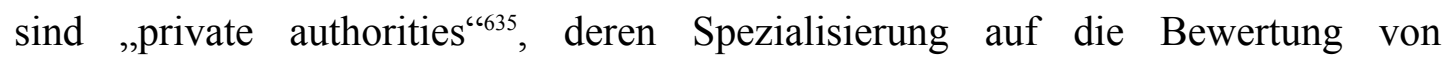
Finanztiteln und Emittenten der Nachfrage nach wirkungsvollen Mechanismen des Umgangs mit einer forcierten Unsicherheit globaler Finanzmärkte nachkommt. ${ }^{636} \mathrm{Die}$ angestrebte Objektivität der Agenturen resultiert daraus, dass ihre Leistungen allein an der Qualität ihrer in Ratings gegossenen Analysen gemessen werden. Im Unterschied zu Banken oder Emissionshäusern wird die Analyse von Ratingagenturen nicht dazu benutzt, um ihren Kunden weitere Beratungs- oder Finanzprodukte zu verkaufen. Die durch Ratings gesteigerte Transparenz erhöht die Effizienz der Allokation von Kapital und Risiko und damit die Funktionsfähigkeit der Märkte. Außerdem reichern die Analysten der Ratingagenturen das Humankapital an den Finanzplätzen mit ihrem Spezialwissen in der Bewertung unternehmerischer Chancen und Risiken an. ${ }^{637}$ Die Analyseberichte der Ratingagenturen liefern eine umfassende, aber fokussierte und entscheidungsorientierte Dokumentation der wesentlichen quantitativen wie auch qualitativen Beurteilungsaspekte und verdichten diese im Rating.

Mit Rating bezeichnet man die durch spezielle Symbole einer Ratingskala (oder einer semantischen Verkettung solcher Symbole) ausgedrückte Meinung einer auf Bonitätsanalysen über die Bestandskraft und Zukunftsfähigkeit eines Emittenten, die

\footnotetext{
634 Das externe Rating meint eine Beurteilung durch externe Agenturen, internes Rating entspricht einer Beurteilung durch Banken. Im Folgenden ist stets von externem Rating die Rede. dynamischen und komplexen Regelungsfeldes nicht mehr in der Lage sind, einen geeigneten regulatorischen Rahmen bereitzustellen, treten private Autoritäten. Strulik 2000, 443.

Vgl. Die Aktiengesellschaft 6/1999, R220.
} 
mit einem bestimmten Finanztitel verbundenen Zahlungsforderungen zu erfüllen. ${ }^{638}$ Ratings stellen mithin auf eine fundamentale Beurteilung von Unternehmen ab. Die Bewertung ist systematisch, öffentlich und zum Teil auch dynamisch. Ratingagenturen können Unternehmen grundsätzlich auf Forderungen der Fremdund auf Forderungen der Eigenkapitalgeber hin überprüfen. Demnach unterscheidet man zwischen Kredit- und Aktien-Ratings. ${ }^{639}$ Je nach den speziellen Anforderungen der Kapitalgeber sind dazu verschiedene Analysemethoden $\mathrm{zu}$ verwenden. ${ }^{640}$ „Der Anspruch ... [von] Rating-Modellen, eine Risikoklassifikation zu erstellen bzw. ein bestimmtes Qualitätskriterium einer Aktie zu bewerten, kann ... sehr informativ sein. Dabei können unterschiedliche Risikopakete und andere kursbeeinflussende Kriterien im Mittelpunkt der Analyse stehen. ${ }^{6641}$ Allerdings sind die Erfahrungen insbesondere der letzten Jahre dahingehend zu berücksichtigen, dass Ratingagenturen zwar auf der einen Seite auf eine Erzeugung von Sicherheit abzielen, auf der anderen Seite jedoch unabdingbar $\mathrm{zu}$ einer fortschreitenden Ausweitung von Unsicherheitspotenzialen beitragen können. So absorbieren Ratingagenturen nicht nur finanzökonomische Unsicherheiten. Sie schaffen auch ein Fundament, von dem aus Risiken eingegangen und entsprechende Gewinne erzielt werden können. ${ }^{642}$

Mit einem auf die Bedürfnisse der Anleger zugeschnittenen effizienten Monitoring lassen sich durch Ratings der Grad der Informiertheit von Eigenkapitalgebern steigern, Unsicherheiten und Ungewissheiten reduzieren. Ratings tragen zur Spezifizierung der sich in einem Aktienpool befindlichen Einzelrisiken bei. Auf diese Weise verringert sich zum einen die Spannbreite der Risiken in einem Pool. Zum anderen nimmt das durchschnittliche Poolrisiko ab, womit sich die Poollinie der Wertpapiermarktlinie annähert. Höhere Risiken werden bei den Verursachern dadurch internalisiert, dass sie in einen Pool mit höheren Renditeforderungen umgruppiert werden. Umgekehrt gelangen niedrigere Einzelrisiken in geringere Forderungsklassen. Unternehmen, von denen vor einem Rating dieselbe Rendite

638 Die Ratingskala, wie sie sich seit der vorletzten Jahrhundertwende in weit mehr als einhundert Ländern der Welt durchgesetzt hat, reicht vom AAA für Top-Adressen bis zur Note D (= „Default“) bei einem insolventen Unternehmen. Vgl. Everling 1999a, 249.

639 Zum Kredit-Rating vgl. etwa Krag, Schmelz, Seekamp (1998).

640 Teegen (1987) unterscheidet Modelle, die Fehlbewertungen von Aktiva aufdecken, von jenen, die Qualitäts- und Risikoaspekte einer Aktie beurteilen. Für die Belange der vorliegenden Arbeit sind letztere von größerer Relevanz.

641 Teegen 1987, 718.

642 Vgl. Varnholt (1995). Zu den Fehleinschätzungen der Ratingagenturen etwa in der Asienkrise vgl. Hillebrand 2001, 162-167. 
gefordert werden, werden deshalb nach einem Rating in teils unterschiedliche Pools eingeteilt. Die Last der unterdurchschnittlichen, guten Einzelrisiken eines Pools, die schlechten $\mathrm{zu}$ subventionieren, wird dadurch reduziert. Eine Anhebung der Renditeforderung auf ein markträumendes Niveau auf der Wertapiermarktlinie bedingt infolgedessen ein geringeres Ausscheiden der unterdurchschnittlichen Einzelrisiken. Die dadurch abnehmenden adversen Selektions- und Anreizrisiken verringern das Ausmaß der Kapitalrationierung am Sekundärmarkt. ${ }^{643}$

„Vorsichtigen Schätzungen zufolge“, so die Financial Times Deutschland vom 19.04.00, ,ist davon auszugehen, dass Moody's und Standard \& Poor's über die von ihnen erteilten Ratings den Fluss von rund 80 Prozent des gesamten Weltkapitals kontrollieren.“ Im Gegensatz zum angelsächsischen Raum ${ }^{644}$ nimmt sich die AktienRatingkultur in Deutschland vor allem im Bereich kleiner und mittlerer Aktiengesellschaften nach wie vor bescheiden aus. Nach Angaben der Europäischen Kommission verfügen zur Zeit nur etwa 170 deutsche Unternehmen über ein externes Rating, ${ }^{645}$ allerdings ist die Tendenz steigend. ${ }^{646}$ Dabei ist die Existenz eines guten Ratings oft Voraussetzung für das Interesse institutioneller Anlegergruppen. ${ }^{647}$ Es gibt zwar eine Reihe von Entwicklungstendenzen, die das Wachstum des Ratingmarktes auch hierzulande beflügeln; ${ }^{648}$ Scholz (2000) beziffert die Anzahl der für ein Rating potenziell in Frage kommenden deutschen Unternehmen etwa auf 200.000. ${ }^{649}$ Doch wird ein flächendeckendes Angebot von MittelstandsRatingagenturen durch verschiedene Aspekte erschwert: Der Aufbau eines effizienten Bewertungs-Systems wirft hohe fixe Kosten auf und ist mit entsprechend

643 Trotz der optimalen Wahl des Niveaus von „monitoring“ und „bonding“ lassen sich nicht die Handlungen durchsetzen, die die reibungslose Kooperation im „first-best“-Optimum kennzeichnen. Es bleibt im „second-best“-Optimum ein „residual loss“ durch mangelnde Kooperation zwischen Prinzipal und Agent in Form so genannter Agency-Kosten. Vgl. Jensen, Meckling 1976, 308; Bernanke (1981).

Aufgrund seiner gesetzlichen Verankerung hat das Rating in den USA große Bedeutung. Vgl. Hohl, Liebig 1999, 504.

Vgl. Deutsche Bank AG 2001, 9.

„1990 waren erst etwa zwei Dutzend deutsche Adressen in Deutschland mit einem Rating von Standard \& Poors oder Moody's Investors Service versehen worden." Scholz 2000, 522.

Vgl. Die Aktiengesellschaft 6/1999, R220. So sind etwa US-amerikanische Pensionsfonds durch Aufsichtsbestimmungen verpflichtet, ihr Vermögen im Wesentlichen in solche Werte anzulegen, die von den Rating-Agenturen mit „,investment grade“ benotet werden. Fallen sie unter diese Note, müssen sie sich von diesen Werten trennen.

Vgl. Everling 1999a, 251f. benennt in diesem Zusammenhang folgende Aspekte: Liberalisierung und Deregulierung, Internationalisierung und Globalisierung, Verbriefung und Finanzinnovationen, Desintermediation und Institutionalisierung, Informations- und Kommunikationstechnologien. Zur Expansion von Ratingagenturen in Europa vgl. Everling (1999b). 
hohen Preisen eines einzelnen Ratings verbunden; ${ }^{650}$ junge Ratingagenturen weisen Größennachteile bei der Informationsgenerierung und -verarbeitung auf; denn um das Risiko von Fehleinschätzungen reduzieren zu können, fehlt es ihnen sowohl an der Größe zu einer breiten Diversifizierung ihres Kundenstamms als auch an einem über viele Jahre herangereiften Expertenwissen. Da man die inzwischen auch hierzulande aktiven Weltmarktführer (Standard \& Poors, Moody's, Fitch) für die Belange des deutschen Mittelstandes nicht geeignet hielt, wurden entsprechende Initiativen in einigen Bundesländern ab Mitte der 90er Jahre wirtschaftspolitisch unterstützt. Anschubfinanzierungen in der Frühphase von Agenturen leisteten vor allem öffentliche Förderbanken, Wirtschaftsverbände und Verlage. ${ }^{651}$ Die EuroRatings AG, die GDUR Mittelstands-Rating AG in Frankfurt gehören zusammen mit der U.R.A. AG und der RS Rating Services in München zu den jüngeren, mit Fokus auf den Mittelstand tätigen Agenturen. Für die EuroRatings AG (größte Kapitaleigner sind die DtA und der Medienkonzern Holtzbrinck) steht erklärtermaßen das Investoren-orientierte Emittenten-Rating, das umsatzstärkere mittelständische Unternehmen den Zugang zum Geld- und Kapitalmarkt öffnen soll, im Mittelpunkt. Mittlerweile sind neben den größeren Mittelstands-Ratingagenturen, die über eigene Analysestäbe und Back-Offices verfügen, zunehmend auch kleinere Anbieter von Unternehmens-Ratings entstanden. Dahinter stehen oft Wirtschaftsprüfer und Unternehmensberater, aber im einzelnen auch oft die Hermes Exportversicherung, die Kreditauskunftei Creditreform, die Technische Universität Ilmenau sowie der Technische Überwachungsverein Rheinland/Berlin-Brandenburg. Bei allen Unterschieden in Qualität und Umfang der jeweiligen Ratingverfahren stehen all diese Anbieter vor dem Problem, dass ihnen als Nachweis der „Treffsicherheit“" ihrer Einstufungen ein historischer Vergleichsmaßstab (track record) fehlt. Um dieses Problem einer „Reputationslücke“ junger Agenturen schneller in den Griff zu bekommen, wurde in Berlin die RatingCert e.V. als Selbstorganisation der Mittelstandsratingagenturen gegründet. Ihre Mitglieder

649

650

Vgl. Scholz (2000), 522

Die Kosten für ein externes Rating liegen zwischen 34.000 EUR bei Standard \& Poors und 5.000 EUR bei U.R.A. Vgl. Deutsche Bank AG 2001, 9. Unternehmen treten üblicherweise mindestens an zwei Agenturen heran. Vgl. Hillebrand 2001, 153; Scholz 2000, 522.

651

Vgl. Landeszentralbank Hessen 2001, 2. Die Aktivitäten der Bayerischen Staatsregierung führten etwa zur Gründung der R@S Rating Services AG mit Sitz in München. Träger ist die GREM Gesellschaft für Rating im Europäischen Mittelstand, die das Ziel verfolgt, eine eigenständige Rating-Kultur in Deutschland zu entwicklen. Gleichzeitig wurde in Frankfurt unter mehrheitlicher Beteiligung der Deutschen Ausgleichsbank (neben der Holtzbrinck- 
verpflichten sich zur Einhaltung von Mindeststandards in Bezug auf Qualität, Objektivität und Transparenz der Bewertungsverfahren. Ziel ist die Etablierung eines qualitätssichernden Gütesiegels für Mittelstands-Ratings. Darüber hinaus will es die Deutsche Vereinigung für Finanzanalyse und Asset Management Finanzteilnehmern und Aufsichtbehörden anhand von Mindestnormen ermöglichen, eine Evaluierung verschiedener externer Ratingverfahren vorzunehmen.

Das Engagement der meisten Ratingagenturen konzentriert sich auf verschiedene Formen des Kreditratings. Wirtschaftspolitisch ist es geboten, die öffentlichen Initiativen in Richtung einer verstärkten Bildung von Aktienrating-Agenturen speziell für die Belange börsennotierter kleiner und mittlerer Gesellschaften auszuweiten. Dabei ist zum einen bei der Existenzgründung von Ratingagenturen im Aktienbereich anzusetzen, wodurch eine auch im internationalen Vergleich klaffende Unternehmenslücke geschlossen werden könnte. Zum anderen gilt es, Wachstum und Alter des Unternehmensbestandes zu fördern. Dadurch kommen die Unternehmen zunehmend in den Genuss oben genannter Lerneffekte, eines track record und von Größenvorteilen (größere Risikodiversifizierung). Die Gefahr, bei einem Engagement der öffentlichen Hand privatwirtschaftliche Initiativen zu verdrängen, ist zum derzeitigen Entwicklungsstand der Angebotsseite als gering einzuschätzen. Von öffentlichen Initiativen etwa in Form von Private-Public-Partnership gehen derzeit für die Anbieterseite vielmehr belebende Elemente aus. Angesichts der langfristig verheißungsvollen Aussichten des Ratingmarktes ist zu erwarten, ${ }^{652}$ dass nach dem „me too“-Prinzip mit rein privatwirtschaftlicher Initiative weitere Agenturen gegründet werden, die sich die erarbeiteten Grundlagen eines Ratingwesens in Deutschland zu Nutze machen. ${ }^{653}$

Die Nachfrage der Unternehmen hängt nicht unwesentlich vom Renommé der anbietenden Agentur ab. ${ }^{654}$ „Auf dem von Wiederholungsgeschäften und Publizität geprägten Markt für Ratings ist Reputation ein kostbares, weil Vertrauen schaffendes

Gruppe, dem Verlag Hoppenstedt, der FGG Finanzierungsgarantie-Gesellschaft) die Euroratings AG gegründet.

Die Änderungen im Bereich der Bankenvorschriften zur Unterlegung von Eigenkapital („Basel II") lassen im Zusammenhang mit den ohnehin stattfindenden Entwicklungen an den Finanzmärkten ein großes Nachfragewachstum auch nach externen Ratings erwarten. Vgl. Kapitel 6. ausgerichtet sind, vgl. Wagner 1991, Kap. III. 
Gut, das mit jedem neuen Urteil auf dem Spiel steht. “655 Sowohl mit einer zunehmenden Anzahl von Ratingagenturen als auch mit zunehmender Anzahl von Ratings pro Unternehmen dürfte die Qualität der Unternehmensbewertungen und damit auch das Renommé der Rating-Agenturen ansteigen. ${ }^{656}$ Vorbehalte an der Wirksamkeit von Ratings seitens der Nachfrager treten durch eine zunehmende Wettbewerbsintensität unter den Agenturen um die besten Ratings im Sinne einer getreuen Abbildung der Unternehmenssituationen in den Hintergrund. Um diese positiven Wettbewerbswirkungen hervorzurufen, bietet sich umso mehr eine öffentliche Mitwirkung und Beteiligung bei der Finanzierung, aber auch bei der Bereitstellung und Verarbeitung von Informationen an. Ein möglicher Rückgriff auf den gleichermaßen aktuellen wie profunden Wissensschatz der Deutschen Bundesbank sowie der bundes- und ländereigenen Förderinstitute über die deutsche Unternehmenslandschaft würde Ratingagenturen ein hohes Maß an Seriösität verleihen. Allerdings ist bei der institutionellen Ausgestaltung eines solchen Informationstransfers der Gefahr Rechnung $\mathrm{zu}$ tragen, dass eine $\mathrm{zu}$ starke Involviertheit vor allem der Bundesbank zu einem auf anderem Felde nicht hinnehmbaren gesamtwirtschaftlichen Vertrauensverlust führen könnte. ${ }^{657}$ Das Engagement von Unternehmensverbänden ist dabei mit gesamtwirtschaftlich weniger Risiken verbunden. Auch sie verfügen über einen großen Informationspool, der für die verstärkte Bildung von Aktien-Ratingagenturen fruchtbar gemacht werden könnte. Auch ihr detailliertes Branchen- und Unternehmenswissen gilt es, für profunde Ratings zu geringen Kosten für die Endnachfrager zu nutzen.

Zusätzlich zur Stärkung der Angebotsseite sind Anreize für eine zunehmende Nachfrage nach Ratings durch die Unternehmen zu setzen. Diese Anreize können finanzieller Art sein. ${ }^{658}$ Aber auch Einsatzmöglichkeiten von Ratings etwa im Rahmen eines Garantie-Systems können - wie der folgende Abschnitt zeigen wird -

$654 \quad$ Vgl. FAZ v. 13.07.99, Nr. 159, 27.

655 Strulik 2000, 452. Vgl. a. Die Aktiengesellschaft 6/1999, R 222.

656 Vielfach wird kritisiert, die Einschätzung von Agenturen folge im Wesentlichen öffentlich verfügbaren Informationen. Ratings würden deshalb einen kaum (statistisch signifikanten) Zusatznutzen stiften. Die Benotungen der Agenturen würden sich überdies veränderten Datenkonstellationen nur langsam anpassen. Mit dieser Inflexibilität ist eine prozyklische Wirkung von Ratings mit folglich destabilisierenden Konsequenzen verbunden. Zur Kritik an der Wirksamkeit von Ratings vgl. Kotz (2000).

657 Die Reputation der Bundesbank könnte Schaden nehmen, wenn sich ihre Bonitätseinstufungen als falsch erweisen sollten. Ein bundesbankeigenes Ratingsystem müsste vom Bundesaufsichtsamt für das Kreditwesen überprüft werden, was möglicherweise ihre Position als unabhängige Behörde beeinträchtigt. Vgl. FAZ v. 17.11.99, Nr. 268, 29f. 
zu einer stärkeren Ratingnachfrage führen. In einem solchen System geht es nicht nur darum, vom Markt unentgoltene adverse Selektions- und Anreizrisiken zu beseitigen, sondern sie auch zu transferieren. Adverse Selektions- und Anreizrisiken, die die Bereitstellung von Eigenkapital beeinträchtigen, sind in Bereiche zu lenken, in denen sie auf Nachfrage stoßen. Um das Risiko aus der Fehleinschätzung einer einzelnen Aktie und um die Gefahr einer Risikoverschlechterung einer einzelnen Aktie kaufen und verkaufen zu können, muss zuvor das Gesamtrisiko einer Aktie identifiziert, operationalisiert und quantifiziert werden. Dazu sind Ratings ein hilfreiches Instrument. 


\subsection{Risikotransfer: Garantieleistungen}

Rating-Agenturen vermögen die Unterinformiertheit der Anleger zu reduzieren und leisten damit einen wichtigen Beitrag zur Selbststeuerung globaler Finanzmärkte. Aber auch sie unterliegen dem Problem der Unterversorgung mit Informationen und sind „nicht in der Lage, die Realkomplexität ihrer Umwelt zu erfassen und gesichertes Wissen zu produzieren." ${ }^{\text {"659 }}$ Deshalb ist auch nicht von ihnen der Zustand symmetrischer Informationsverteilung zu erwarten. Auch mit Hilfe von Ratings kann somit eine exakte Verortung der Einzelrisiken auf der Wertpapiermarktlinie nicht vorgenommen werden. Allenfalls dienen Ratings dazu, die Streuung der Einzelrisiken innerhalb eines Pools $\mathrm{zu}$ verringern und die Pool- der Wertpapiermarktlinie anzunähern. Rating führen lediglich $\mathrm{zu}$ einer weniger ungenauen Risikoeinschätzung der einzelnen Aktien. Beispielsweise muss der Anleger eines nicht gerateten Unternehmenspools davon ausgehen, dass eine bestimmte Aktie seines Pools ein Risiko zwischen den Noten A- und C+ aufweist; nach Durchführung eines Ratings kann er mit einer geringeren Risikospanne etwa zwischen B+ und B- rechnen. Mit der verbesserten Risikospezifizierung nimmt zwar die Gefahr eines adversen Selektions- und Anreizrisikos im Zuge einer markträumenden Renditeforderung grundsätzlich ab. Es entstehen für den Anleger jedoch zwei Fehlerquellen. Zum einen kann eine Fehleinschätzung der RatingAgenturen dergestalt vorliegen, dass sich das Einzelrisiko de facto mir $\mathrm{C}+$ oder noch schlechter außerhalb der angenommenen Bandbreite B+ bis B- befindet. Eine solche Gefahr nimmt mit abnehmender Anzahl von Ratings, denen sich ein Unternehmen unterzieht, zu. Ein weiteres Risiko für die Eigenkapitalgeber besteht darin, dass sich - von den Rating-Agenturen nicht zeitgleich entdeckt - eine Bonitätsverschlechterung der Einzelrisiken über den Grad von B- hinaus einstellt. Diese Gefahr besteht auch dann, wenn sich die Unternehmen einem ständigen Rating aussetzen. Um die Anleger gegen beide mögliche Beeinträchtigungen - die Fehleinschätzung der Agenturen sowie eine unentdeckte Risikoverschlechterung abzusichern, ist es zweckmäßig, dem Anleger ein Bonitätsrisiko in Höhe von mindestens B- zu garantieren. Stellt sich im Laufe des Wettbewerbs der Agenturen um das beste Rating eines Unternehmens heraus, dass das betrachtete Unternehmen

659 Strulik 2000, 447. Franz (2001, 223) sieht methodische Probleme hinsichtlich der Anzahl und der Auswahl der Indikatoren, der Transformation qualitativ erfasster in quantitative 
mit mindestens B- zu hoch geratet wurde, das Unternehmen faktisch also ein größeres Risiko darstellt, kommt es automatisch zu Ausgleichszahlungen seitens der Garantiegeber an den Anleger. Das Vorliegen eines Garantiefalls kann auch daran geknüpft werden, dass sich bei richtiger Einschätzung durch die Rating-Agenturen das Einzelrisiko unter das garantierte Mindestrating von B- verschlechtert. Die im Garantiefall fließenden Leistungen sind als Kompensation für einen unerwarteten Risikoanstieg zu verstehen.

Fraglich ist, in welcher Form Garantien geleistet werden können. Um die Kosten für die Etablierung einer Garantie-Infrastruktur zu minimieren, bietet es sich an, auf bestehende Instrumente zurückzugreifen. Aus diesem Grund kommt das Rechtsinstitut der Vorzugsaktie als Garantieinstrument in Frage. ${ }^{660}$ Vorzugsaktien beinhalten neben den üblichen gesetzlichen und satzungsgemäßen Rechten und Verpflichtungen, die einer Stammaktie erwachsen, besondere Vorrechte. Eine Vorzugsaktie könnte etwa mit dem Recht ausgestattet werden, bei Überschreiten einer bestimmten von den Ratingagenturen festgestellten Risikohöhe in den Genuss genau spezifizierter Vorrechte $\mathrm{zu}$ gelangen. Hierbei sind höhere Dividenden, Zahlungen von Seiten Dritter, der Erhalt von (Mehrfach-)Stimmrechten oder etwa Bevorrechtigungen am Liquidationserlös denkbar. ${ }^{661}$ Gegen einen verbreiteten Einsatz von Vorzugsaktien als Instrument einer Risikogarantie spricht allerdings zum einen ihr Anachronismus, kaprizieren sich doch insbesondere Großanleger bei der Nachbildung von Indizes auf Stammaktien. ${ }^{662}$ Zum anderen würden Vorzugsaktien aufgrund ihrer Heterogenität den Handel ohnehin unterliquider Aktientitel um ein Weiteres zerteilen. ${ }^{63} \mathrm{Um}$ die Liquidität von Aktien $\mathrm{zu}$ verbessern, sind Vorzugsaktien als Garantieinstrument deshalb konterproduktiv.

Indikatoren, dem Verhältnis von zusammengeführten Teildimensionen und der Gewichtung einzelner Indikatoren und Teildimensionen im Verhältnis zueinander.

„Bei den deutschen Aktiengesellschaften sind Vorzugstitel beliebt. Von den 100 größten börsennotierten Unternehmen hat immerhin jedes Fünfte sowohl Stamm- als auch Vorzugsaktien - ..." Slodczyk 2000, 30. Die erste Emission einer stimmrechtslosen Vorzugsaktie hat es 1979 gegeben. Der Anteil der Vorzugsaktien an den Neuemissionen in den Jahren 1983 bis 1987 fiel mit 41\% auffallend hoch aus. Vor allem kleine und mittlerer Gesellschaften bedienten sich des Rechtsinstituts stimmrechtsloser Vorzugsaktien. Vgl. Allmandinger, Güttler 1989, 460.

Grill, Perczynski (1988, 302f.) unterscheiden folgende Arten von Vorzugsaktien: Mehrstimmrechte; kumulative Vorzugsaktien; kumulative stimmrechtslose Vorzugsaktien; Prioritätsaktien; Dividendengarantien dürfen nicht von den emittierenden Aktiengesellschaften selber, sondern nur von Dritten, z.B. dem Staat, der Muttergesellschaft oder Verbänden gegeben werden. 
Im Unterschied zu Vorzugsaktien greifen Finanzderivate wie Optionen, Forwards und Futures nicht in den Handel ihrer ,underlyings“", das heißt der ihnen zu Grunde liegenden Aktien ein. ${ }^{664}$ Derivate initiieren einen bedingten Zahlungsstrom bei Einsetzen eines vertraglich vorher festgelegten Ereignisses gegen Zahlung einer Prämie an den Garantiegeber. Derivate ermöglichen eine Trennung und den separaten Handel einzelner Marktrisikobestandteile. Mit Hilfe derartiger Derivate kann man sich gegen ungewollte Kursveränderungen der ihnen zugrunde liegenden Aktien absichern. Die üblichen Derivate, die Marktpreisschwankungen etwa infolge einer bekannt gewordenen Risikoveränderung absichern, sind für unterliquide Aktien jedoch nicht geeignet. Denn unterliquide Aktien sind dadurch gekennzeichnet, dass ihre Kurse gar nicht, nicht adäquat oder nicht zeitnah auf fundamentale Veränderungen des Unternehmensrisikos reagieren. Herkömmliche Derivate, die Marktpreisrisiken absichern, sind als Garantieinstrument für unvorhergesehene Risikoveränderungen jedoch nur wirksam, wenn sich die Risikoveränderungen in entsprechenden Kursveränderungen niederschlagen. Risikoverschlechterungen, die sich hingegen nicht in den Kursen eines unterliquiden „underlyings“ niederschlagen, werden vom Verkäufer herkömmlicher Derivate nicht übernommen. Verändert sich der Kurs einer unterliquiden Aktie infolge einer Risikoverschlechterung nicht oder nicht ausreichend, bleibt eine Garantiezahlung folglich aus. Die Gefahr einer Risikoverschlechterung verbliebe beim Halter einer unterliquiden Aktie. Da der Garantiefall bei herkömmlichen Derivaten indirekt erfolgt, das heißt an Kursveränderungen der Aktie gekoppelt ist, kommen auch sie als Garantieinstrument gegen eine Fehleinschätzung von Einzelrisiken durch Rating-Agenturen oder eine von ihnen unbeobachtete Risikoverschlechterung nicht in Frage. Dies ist insofern bedauernswert, als die genannten Derivate besonders im Standardwertebereich

664 Als Derivate werden Finanzinstrumente bezeichnet, deren eigener Wert sich aus dem Marktpreis bzw. dem Index eines (oder mehrerer) originärer Basisinstrumente ableitet. Finanzderivate weisen ein auf die Zukunft gerichtetes Vertragselement auf, das als Kauf- oder Verkaufsverpflichtung wie beim Forward- und Futures-Typ oder als Kauf- oder VerkaufsOption ausgestaltet ist. Komplexe derivative Instrumente lassen sich grundsätzlich auf diese beiden Grundtypen zurückführen. Bei Instrumenten vom Forward- oder Futures-Typ gehen beide Vertragspartner mit Abschluss des Kontrakts ein spiegelbildliches Gewinn- bzw. Verlustrisiko ein. Optionen bewirken hingegen eine asymmetrische Risikoübertragung. Der Options-Käufer sichert sich einseitig gegen das Risiko einer für ihn ungünstigen Preisentwicklung ab. Der Options-Verkäufer (,Stillhalter“) trägt zwar dieses Risiko, kann aber mit der Option - anders als bei Forward- und Futures-Kontrakten - von einer für ihn vorteilhaften Preisentwicklung nicht profitieren. Das einseitig übernommene Preisänderungsrisiko wird mit der vom Optionskäufer an den Stillhalter zu entrichtenden Optionsprämie bewertet und abgegolten. 
gängige Instrumente darstellen, mithin eine Ausweitung der Infrastruktur auf die Börsensegmente kleiner und mittlerer Aktiengesellschaften $\mathrm{zu}$ relativ geringen Transaktionskosten machbar erscheint.

Das Vorliegen eines Garantiefalls bedarf einer direkten Koppelung an veränderte Risikosituationen - unabhängig von ihrer (Nicht-)Auswirkung auf Marktpreisveränderungen. Entpuppt sich etwa die Risikoeinschätzung von Ratingagenturen als $\mathrm{zu}$ optimistisch, muss eine effektive Garantieleistung sofort greifen. So genannte Kreditderivate erfüllen eine solche Funktion. ${ }^{665}$ Kreditderivate sind Finanzkontrakte, deren Wert sich vom genau definierten Risiko eines Referenzaktivums (Underlying, Basiswert) ableitet. Kreditderivate sind eine relativ neue Art von Finanzderivaten. Sie unterscheiden sich von den herkömmlichen derivativen Finanzinstrumenten darin, dass sie nicht Marktpreisrisiken, sondern etwa das Risiko einer Herabstufung im Rating, das heißt eine Bonitätsverschlechterung absichern. Der Wert von Kreditderivaten bemisst sich in diesem Fall auf die Risikoeinschätzung eines Emittenten durch eine Ratingagentur. Kreditderivate ermöglichen auf diese Weise die Abspaltung und separate Handelbarkeit des gerateten Unternehmensrisikos vom originären Geschäft. Es lassen sich vier Grundformen von Kreditderivaten identifizieren, von denen im hiesigen Zusammenhang die so genannte Credit Default Option (CDO $)^{666}$ von besonderer Relevanz ist. ${ }^{667}$ Hierbei zahlt der Risikoverkäufer eine einmalige oder bei längerer Haltedauer eine periodische Prämie an den Risikokäufer. ${ }^{668}$ Ersterer erhält dafür von letzterem bei Eintritt einer bei Vertragsabschluss festgelegten Störung wie etwa eines „downgrade“ eine Ausgleichszahlung. ${ }^{669}$ Der Risikokäufer trägt somit das „spreadwidening risk“ einer Aktie, ohne diese tatsächlich erwerben zu müssen. Abbildung

665 Wie bereits der Name verdeutlicht, werden Kreditderivate insbesondere an Fremdkapitalmärkten eingesetzt. Die Grundidee lässt sich dennoch auf den Eigenkapitalmarkt mit dem Ziel einer höheren Aktienliquidität übertragen. Das BAKred ordnet dieses Instrument aufgrund seiner asymmerischen Zahlungs- und Risikostruktur eher als Option, denn als Swap ein.

667 Als weitere Grundformen der Kreditderivate gelten der Total Return Swap, die Credit Spread Option sowie die Credit Linked Note. Letztere wird nicht als eigenständiges Produkt gestaltet, sondern mit Anleihen zu strukturierten Wertpapieren kombiniert. Vgl. Hohl, Liebig 1999, 505509. genommene Versicherungs- respektive Garantieleistung aufkommt. In Abschnitt 5.5 wird die Überlegung anzustellen sein, ob es angebracht ist, die Prämie für Anleger bestimmter Unternehmen staatlicherseits zu übernehmen. 
5.1 skizziert den möglichen Transfer eines Downgrade-Risikos vom ursprünglichen Anteilseigner zu einem risikobewussten Investor.

Abbildung 5.1 Handel eines Downgrade-Risikos per $\mathrm{CDO}^{670}$

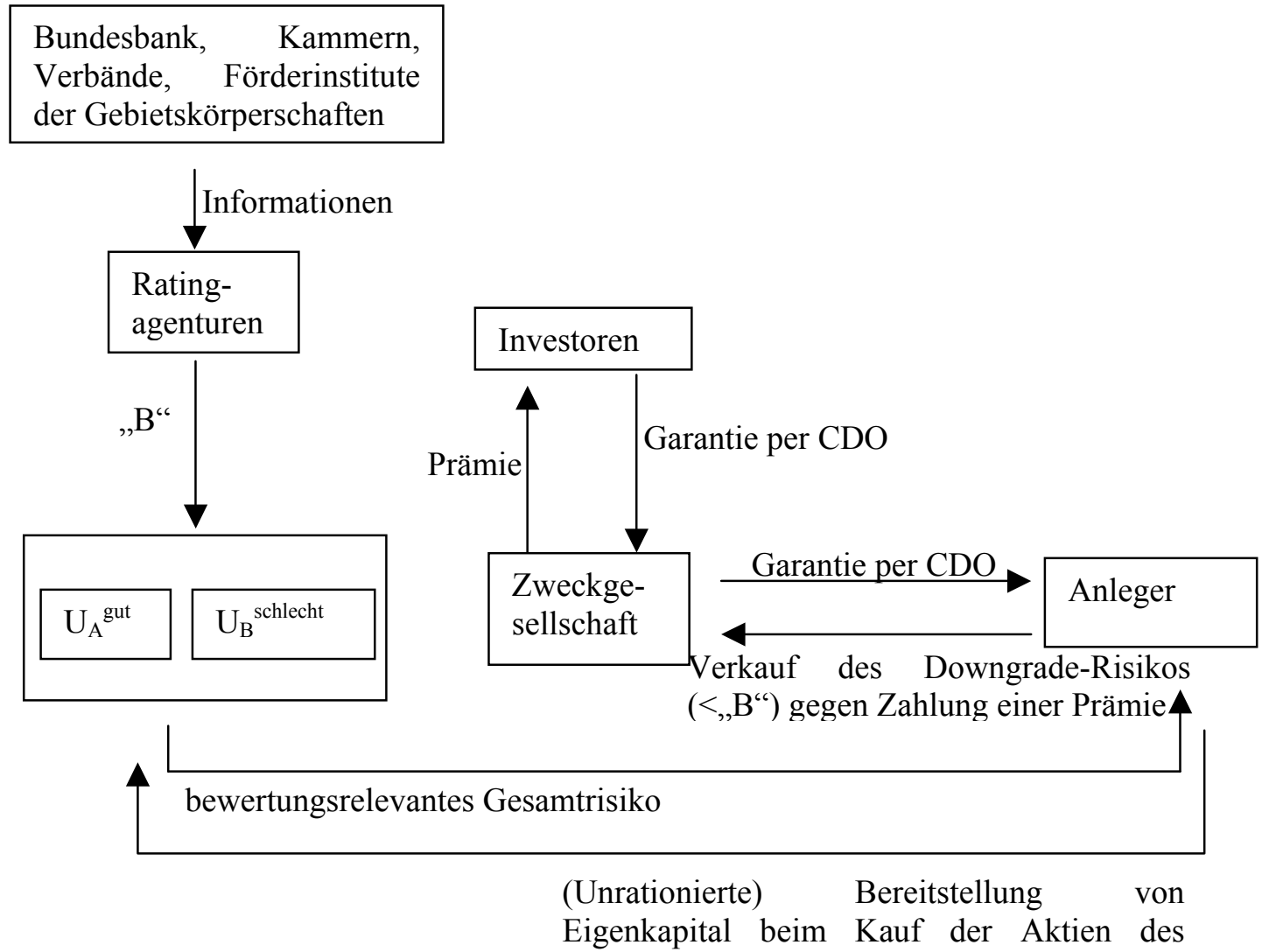

Unter Zuhilfenahme der Informationsbereitstellung übergeordneter Organisationen und Körperschaften des öffentlichen Rechts (Bundesbank, Kammern, Verbände, öffentliche Förderinstitute) raten die Agenturen im gewählten Beispiel zwei exemplarisch betrachtete Unternehmen mit der Note „B“. Durch die Informationsproduktion des Ratings nimmt die Gefahr eines adversen Selektionsund Anreizrisikos zwar ab. Dennoch fordert der rationale Anleger annahmegemäß nicht die gemäß der Wertpapiermarktlinie markträumende Rendite. Ohne die Möglichkeit des Verkaufs eines Downgrade-Risikos würde der Anleger beide Unternehmen vollrationieren. Denn sie gehören zu einem Unternehmenstyp, von dem der Anleger eigentlich ein überdurchschnittliches Poolrisiko erwartet. Erst 
dadurch, dass sich der Eigenkapitalgeber mit Hilfe einer Credit Default Option (CDO) mit der Zahlung einer Prämie an eine Zweckgesellschaft gegen das Downgrade-Risiko unter die Ratingnote B versichert, fragt er die Aktien beider Unternehmen nach und stellt somit in unrationiertem Umfang dem Pool der Risikoklasse „B“ Liquidität zur Verfügung. Garantiegeber ist eine Zweckgesellschaft, die dem Eigenkapitalgeber eine Garantiezahlung zukommen lässt, wenn die Risikosituation einer Aktie - wie im Falle des schlechten Unternehmens $\mathrm{U}_{\mathrm{B}}{ }^{\text {schlecht }}$ - anfangs $\mathrm{zu}$ positiv eingeschätzt wurde oder sie sich im Laufe der Garantiezeit verschlechtert. Neben ihrer Bewertungsfunktion bieten Ratings damit in Verbindung mit CDO's den Anlegern unterliquider Titel die Möglichkeit, denjenigen Teil des Unternehmensrisikos $\mathrm{zu}$ veräußern, der Bonitätsverschlechterungen oder Fehleinschätzungen erwächst. Damit werden adverse Selektions- und Anreizrisiken vom Eigenkapitalgeber auf die Zweckgesellschaft transferiert. Diese wiederum kann übernommene Risiken weiterverkaufen. Vor ihrem Weiterverkauf stellt die Zweckgesellschaft die übernommenen Garantieverpflichtungen jedoch im Zuge eines „financial engineering“ $\mathrm{zu}$ einem neuen Risikoprodukt zusammen und bietet dieses bedarfsgerecht risikobewussten Investoren an. ${ }^{671}$ Wenn die Zweckgesellschaft bereits vor der Übernahme des Downgrade-Risikos einen Investor findet, bleibt sie als Risikovermittlerin sogar von vornherein unbeschadet. Als Ergebnis dieser Transaktionen werden die adversen Selektions- und Anreizrisiken, die zu einer Kapitalrationierung am Sekundärmarkt führen können, in einen Bereich weitergeleitet, in dem sie auf Nachfrage treffen. Durch den Risikotransfer lässt sich der Grad der Aktienliquidität erhöhen und die Effizienz des Finanzmarktes steigern. ${ }^{672}$

Der Markt für Kreditderivate wie CDO's ist in Deutschland noch unterentwickelt, so dass die volkswirtschaftlichen Kosten zur Schaffung einer geeigneten Infrastruktur

670 Die von der Zweckgesellschaft aufgekauften Downgrade-Risiken können an Investoren auch in Form von Credit Linked Notes transferiert werden. Vgl. KfW 2001a, 26.

671 Die im Rahmen des Financial Engineering isolierten Einzelbestandteile werden häufig aggregiert und in einer liquideren Form mit geringeren Transaktionskosten den Kapitalmärkten zur Verfügung gestellt. Vgl. Hohl, Liebig 1999, 510-512. Das Financial Engineering trägt maßgeblich zu einem tiefen Markt für die Übernahme von Downgrade-Risiken seitens risikobewusster Investoren bei. 
zunächst hoch sind. ${ }^{673}$ Auch fehlt noch eine vom Markt akzeptierte Bewertungstheorie für bonitätssensitive Finanzierungstitel. Aufgrund ihrer vielfältigen Einsatzmöglichkeiten im Bereich des Risikomanagements ist jedoch künftig mit einem stetig steigenden Handel mit Kreditderivaten in immer weiteren Bereichen zu rechnen. Durch eine kontinuierliche Durchführung derartig fördernder Transaktionen nimmt die Bedeutung der anfänglichen hohen Fixkosten durch die Nutzung von Skalenerträgen jedoch ab. Öffentliche Förderinstitute wie die KfW übernehmen bereits heute einen aktiven Part im Akquirieren und Bündeln von (Kredit-)Forderungen, die anschließend mittels Verbriefung am Kapitalmarkt refinanziert werden. Damit soll gewährleistet werden, dass sich Kreditinstitute auch im traditionell ertragsarmen Firmenkundengeschäft kleiner und mittlerer Unternehmen künftig weiterhin engagieren. Öffentlichen Förderinstituten könnte auch hinsichtlich der Bildung einer Zweckgesellschaft für die Übernahme, Bündelung und Weitergabe von adversen Selektions- und Anreizrisiken bei Eigenkapitalforderungen besondere Bedeutung zukommen.

will migrate quickly from one market to another, or from one country to another. That is, while hedging acts to reduce independent risk, it can enhance systemic risk." Kimball 2000, 12. 


\subsection{Risikosignale: Eigeninvestitionen ins Downgrade-Risiko}

Die Handelbarkeit adverser Selektions- und Anreizrisiken bietet risikobewussten Investoren Anlagemöglichkeiten im Mittelstandsbereich, ohne das Gesamtrisiko einer unterliquiden Aktie tragen $\mathrm{zu}$ müssen. Es ermöglicht auch den von niveaubedingter Unterliquidität betroffenen Unternehmen, ins eigene Risiko zu investieren. Investieren die beiden Unternehmen des gewählten Beipiels A und B in ihr eigenes „downgrade“-Risiko, geben sie per CDO ihren Eigenkapitalgebern selber eine Garantie für ihre eigene durch ein Rating ausgewiesene Bonität. Es ergäbe sich dabei für beide Unternehmen ein unterschiedlicher Investitionsertrag. Das gute Unternehmen A, das sich zum einen „richtig“ bewertet sieht und überdies keine Bonitätsverschlechterung intendiert, erhält vom Risikoverkäufer eine Prämie für die Übernahme des eigenen Downgrade-Risikos. Das schlechte Unternehmen B, das aufgund einer Fehlbewertung der Ratingagenturen insgeheim mit einem Downgrading rechnen muss und zusätzlich noch riskantere Unternehmensprojekte beabsichtigt, erhält zwar zunächst auch die Optionsprämie für die Übernahme des Risikos eigener Bonitätsverschlechterungen. Doch hat das Unternehmen B im Gegensatz zu Unternehmen A eine Inanspruchnahme der Garantieleistung durch die Eigenkapitalgeber einzukalkulieren. Es gibt insofern einen Fehlanreiz für schlechte Einzelrisiken, in die eigene Bonität zu investieren. Für gute Einzelrisiken hingegen handelt es sich um eine lohnenswerte Investition. Aus diesen Anreizen wird das Ausmaß der Investition einer Gesellschaft ins eigene Risiko zu einem SignalingInstrument. ${ }^{64}$ Denn wegen der negativen Korrelation zwischen der Wahrscheinlichkeit einer Bonitätsverschlechterung und den Signalisierungskosten in Form von eigener Garantieverpflichtungen lässt sich als Anleger an Art und Umfang der Investition eines Unternehmens in die eigene Bonität die „Spreu vom Weizen“ trennen (vgl. Abbildung 5.2). An einer Separation der zuvor gepoolten Unternehmen nach ihrem Bonitätsverschlechterungsrisiko sind Anleger trotz Vorhandensein von Garantieleistungen (durch dritte) zusätzlich interessiert, weil sie ihre Versicherungskosten damit zusätzlich senken können.

$674 \quad$ Im Gegensatz zum Screening geht man beim Signaling davon aus, dass in einer Welt asymmetrisch verteilter Informationen der „erste Schritt“ vom besser Informierten ausgeht. 
Abbildung 5.2 Separationsgleichgewicht durch eigene Garantieverpflichtungen

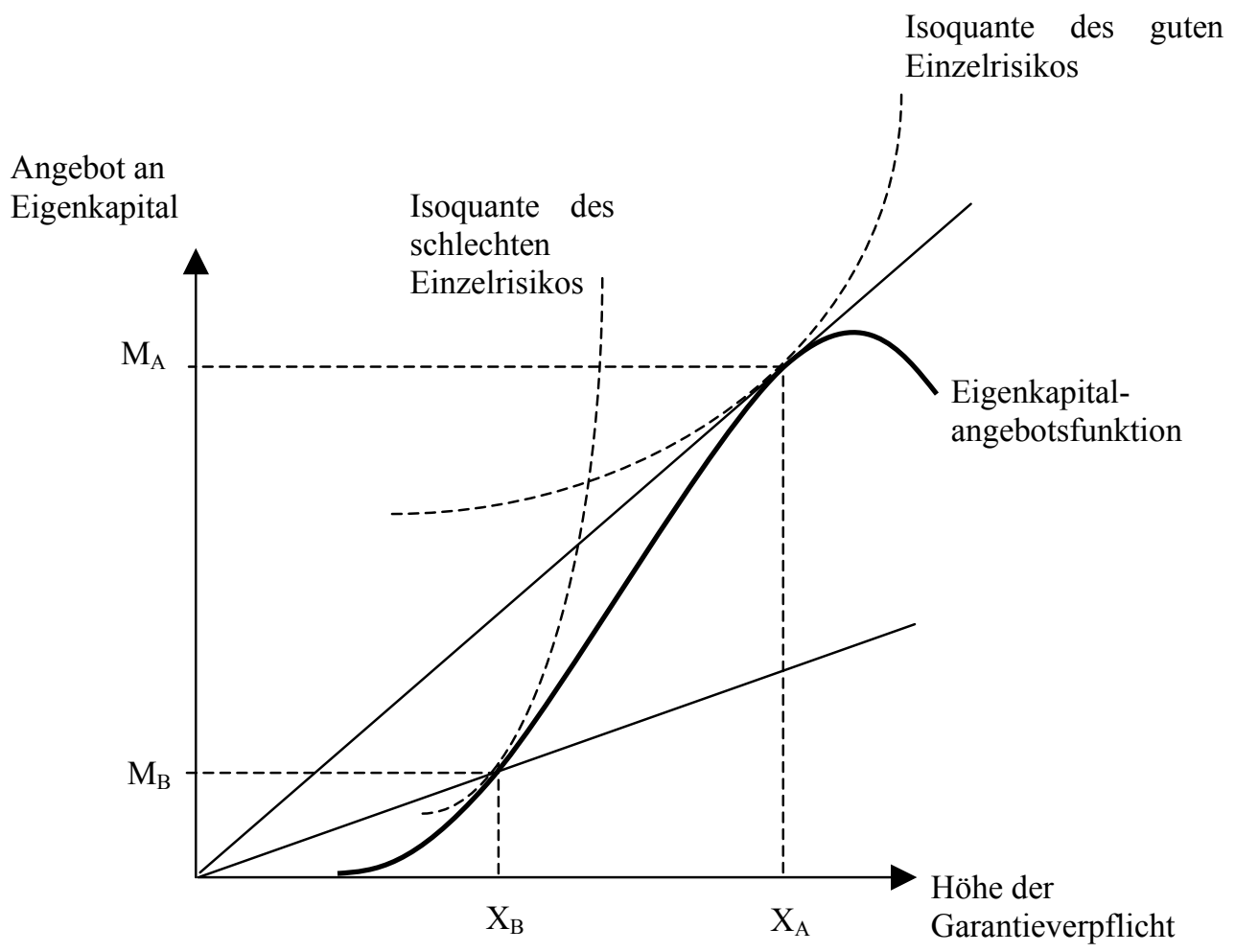

Beide Unternehmen A und B sehen sich in Abbildung 5.2 einer Eigenkapitalangebotskurve seitens der Anleger gegenüber, die von der Art und Höhe der eigens gegebenen Garantieverpflichtungen abhängt. Beide Unternehmen erreichen die Höhe des gewünschten Eigenkapitalangebotes durch Antizipieren dieser Eigenkapitalangebotsfunktion. ${ }^{675}$ Ihre Kurve nimmt ab einem bestimmten Garantieniveau einen s-förmig ansteigenden Verlauf an. Da ein „übertrieben“ hohes Garantieniveau den Anlegern unglaubwürdig erscheinen muss, gehen die Unternehmen von einem maximal erreichbaren Eigenkapitalangebot durch die Bereitstellung einer Garantie aus. Die von der Garantieleistung abhängige Eigenkapitalangebotsfunktion erfährt von dort an eine negative Steigung. An die Eigenkapitalangebotskurve schmiegen sich die maximal möglichen Isoquanten der Unternehmen mit den für die Anleger guten wie schlechten Einzelrisiken an. Dabei verläuft die Isoquante eines schlechten Unternehmens steiler als die eines guten Unternehmens. Die Grenzrate der Substitution vom Eigenkapitalangebot durch

675 Der Einfachheit halber gehen wir davon aus, dass im Gleichgewicht die Unternehmen die Abhängigkeit des Eigenkapitalangebots von der jeweiligen Garantieleistung richtig antizipieren, so dass die antizipierte und die tatsächliche Eigenkapitalangebotskurve zusammenfallen. 
Garantieverpflichtungen ist bei Unternehmen des schlechten Typs durchgängig höher, das heißt, bei einer marginalen Erhöhung der Garantieverpflichtung muss das Eigenkapitalangebot für das schlechte Unternehmen B stets größer sein, um nich die Isoquante $\mathrm{zu}$ verlassen. ${ }^{676}$ Dies hängt damit zusammen, dass die Garantiebeziehungsweise Bondingkosten für schlechte Unternehmen höher als für gute Unternehmen sind. Es ergibt sich daraus ein Trenngleichgewicht mit einer hohen Garantieleistung $\mathrm{X}_{\mathrm{A}}$ der guten Gesellschaft und einem vergleichsweise hohen Eigenkapitalangebot durch die Anleger in Höhe von $\mathrm{M}_{\mathrm{A}}$. Das schlechte Unternehmen hingegen geht eine geringere Garantieleistung $X_{B}$ ein, erhält somit auch nur ein geringeres Eigenkapitalangebot in Höhe von $\mathrm{M}_{\mathrm{B}}$. Dabei ist $\mathrm{zu}$ erwarten, dass das Einbringen hoher eigener Garantieleistungen, wie im Fall des Unternehmens A, einen zusätzlich positiven Einfluss ausübt auf die Güte der unternehmerischen Realinvestitionen. ${ }^{677}$ So ist etwa von der Abgabe eigener hoher Garantien eine disziplinierende Wirkung auf die unternehmerische Investitionstätigkeit zu erwarten. Die Existenz eines Trenngleichgewichts beruht letztlich darauf, dass der bessere Unternehmenstyp die geringeren (marginalen) Garantiekosten aufweist. Die Anreizverträglichkeitsbedingung muss deshalb für das gute Unternehmen derart gestaltet werden, dass der erwartete Vorteil aus einem Mehr an Liquidität der eigenen Aktie höher sein muss als die erwarteten Kosten der Garantieleistung, damit sich eine Abweichung vom Trenngleichgewicht für das gute Einzelrisiko nicht lohnt. Gleiches gilt für das schlechte Unternehmen. Erst dann ist ein dauerhaftes Aufbrechen des Pooling- hin zu einem Separationsgleichgewicht möglich. Im Falle eines vollständigen Sortierens der Einzelrisiken in einem Pool ist die Kapitalrationierung am Sekundärmarkt als (Pooling-)Gleichgewicht auszuschließen. ${ }^{678}$ Wenn das Überschreiten einer bestimmten Garantieschwelle jedoch für die Eigenkapitalgeber von besonderer Bedeutung ist, weist die obige Eigenkapitalangebotsfunktion einen Knick auf, der möglicherweise von der Isoquante der schlechten wie der guten Gesellschaft tangiert wird. ${ }^{679}$ In diesem Fall ist es für beide Unternehmenstypen rational, Garantien in derselben Höhe einzugehen. Trotz ihrer Investitionen ins eigene Downgrade-Risiko lässt sich das

\footnotetext{
676 Dabei sei unterstellt, dass die Grenzproduktivität aus einer Einheit zusätzlich angebotenen Eigenkapitals am Sekundärmarkt für beide Unternehmenstypen identisch ist.

677 Vgl. analog zum Arbeitsmarktfall von Spence (1973,); Feess 1997, 609-614; Spence (1974).

678 Vgl. Bester (1985b, 1987).

679 Vgl. Kreps 1994, 573f. 
Poolinggleichgewicht hier nicht aufbrechen mit einer fortbestehenden Sekundärmarktrationierung als Folge.

Die Wahrscheinlichkeit einer Imitation des guten durch den schlechten Unternehmenstyp nimmt mit der Anzahl der Vertreter pro Unternehmenstyp zu. ${ }^{680}$ Bei einer Vertreterzahl pro Unternehmenstyp von Eins (wie im gewählten Beispiel) führt die Imitation des einzigen schlechten Unternehmens im Pool sofort zu einem Poolinggleichgewicht mit einem „nur“ mittleren Eigenkapitalangebot für beide Unternehmen. Die Anzahl der sich im Pool befindlichen Unternehmen spricht für einen vergleichsweise hohen Informationsstand der Anleger. Nimmt jedoch die Zahl der Vertreter pro Unternehmenstyp mit abnehmenden Informationen zu, ist die Imitation eines einzelnen schlechten Unternehmens mit einem für ihn höheren Eigenkapitalangebot verbunden, da die Imitation durch ein einzelnes Unternehmen sich nur geringfügig auf das Trenngleichgewicht auswirkt. Abweichungen des einzelnen schlechten Unternehmens vom Trenngleichgewicht lohnen sich im Fall vieler Akteure deshalb eher, weil einem schlechten Unternehmen dadurch anstatt eines nur mittleren vielmehr ein hohes Eigenkapitalangebot zuteil wird. Der Anreiz zur Imitation steigt somit bei abnehmender Informiertheit der Eigenkapitalgeber und einer folglich ansteigenden Anzahl von Einzelrisiken in einem Pool. Angesichts dieses höheren Anreizes für schlechte Unternehmen zur Imitation ist mit zunehmender Anzahl von Unternehmen im Pool ein Aufbrechen des gewünschten Trenngleichgewichts, das heißt ein erneutes Poolinggleichgewicht zu erwarten. Um einer Imitation vorzubeugen, muss das gute Unternehmen noch höhere Garantieverpflichtungen eingehen. Die dabei anfallenden Kosten zur Bewahrung eines Separationsgleichgewichts könnten vermieden werden, würden Ratingagenturen im Zuge ihrer Informationsvermittelnden Unternehmensbewertung für eine geringe Vertreterzahl von Unternehmenstypen innerhalb eines Pools sorgen. Ratingagenturen kommt darüber hinaus insbesondere dann eine zusätzliche Bedeutung zu, je ,agressiver“ der schlechte Unternehmenstyp eine Pooling- und der gute Unternehmenstyp eine Separationsstrategie verfolgt. Angesichts der Vielzahl möglicher Einflussfaktoren für das Entstehen eines Pooling- oder eines Separationsgleichgewichtes stellt sich die Frage: "Explaining everything, explaining

$680 \quad$ Vgl. Feess 1997, 607-614. 
nothing?" ${ }^{681}$ Hinsichtlich eines auf Trennung ausgerichteten Signaling bleibt letztlich festzuhalten: ,[T]he conclusions which have been reached are very sensitive to the details of the game-theoretic specification that one looks at. Seemingly minor modifications of the formal model have a major impact on the predicted outcome of "competition" in a market with adverse selection." 682 


\subsection{Risikogemeinschaften: Co-opetition ${ }^{683}$}

Investieren Unternehmen in das Risiko eines eigenen Downgrades durch RatingAgenturen, besteht, wie im letzten Abschnitt dargestellt, die Möglichkeit, nicht die Zwangsläufigkeit einer Separation zwischen guten und schlechten Gesellschaften. Durch Art und Umfang selber eingegangener Garantieverpflichtungen offenbart sich Anlegern die Güte der zu finanzierenden Unternehmensrisiken mit dem Ergebnis eines im Extrem markträumenden Eigenkapitalangebots. Dieses SignalingInstrument setzt allerdings Qualität der Ratings sowie Vertrauen in die Kompetenz der Rating-Agenturen voraus. ${ }^{684}$ Das Engagement einer Gesellschaft in ihr eigenes Downgrade-Risiko impliziert zudem Kenntnisse über das Bewertungssystem der Ratingagenturen. Insbesondere in der Aufbauphase einer Rating-Infrastruktur sind diese Voraussetzungen oft nicht gegeben. Unternehmen haben (noch) kein Vertrauen $\mathrm{zu}$ den Ratingagenturen. Es fehlt den Agenturen am entsprechenden Reputationskapital. Überdies kennen die Unternehmen nicht das Bewertungssystem sowie die Reaktionsfunktion der Ratingagenturen auf eigene Fehleinschätzungen. Ungewissheit verstärkt sich zusätzlich dadurch, dass der Garantiefall möglicherweise einzig einer Fehleinschätzung durch die Ratingagenturen geschuldet sein mag. Außerdem besteht - selbst für gute Unternehmen - oft Ungewissheit und Uninformiertheit über die eigene unternehmerische Investitionsstätigkeit sowie damit einhergehende mögliche Risikoveränderungen. ${ }^{65}$ Diese Aspekte machen das Einbringen eigener Garantieleistungen auch für gute Unternehmen zu einem möglicherweise kostenintensiven Unterfangen, weshalb sie drohen auszubleiben. Unter diesen Voraussetzungen sind die für die Eigenkapitalgeber guten Einzelrisiken weitaus weniger bereit, Garantien abzugeben. $\mathrm{Zu}$ ungewiss ist die erwartete Inanspruchnahme der Garantie und $\mathrm{zu}$ hoch sind die $\mathrm{zu}$ entrichtenden Schadensleistungen im Garantiefall.

683 In Anlehnung an Nalebuff, Brandenburger (1998) handelt es sich hier um gemeinsame „Aktionen“ zur Attrahierung von knappem Eigenkapital - durchgeführt von Aktiengesellschaften, die für sich genommen in einem wettbewerblichen Verhältnis um das Eigenkapitalangebot stehen.

684 Zu Anforderungen und Problemen von Ratingverfahren vgl. KfW 2001b, 27f.

685 In der eingeschränkten Informiertheit der Agenten liegt im Übrigen kein Widerspruch zu der Annahme asymmetrischer Informationen $\mathrm{zu}$ Lasten der Prinzipale. Das Vorliegen asymmetrischer Informationen impliziert lediglich einen geringeren Informationsstand der Prinzipale im Verhältnis zu den Agenten. 
Die mit Eigengarantien einhergehenden Unsicherheiten und Ungewissheiten legen es nahe, Unternehmenskooperationen $\mathrm{zu}$ bilden. Kooperative Organisationsformen zeitigen Synergie- und Lerneffekte im Umgang mit sich wandelnden Umfeld- und Wettbewerbsbedingungen. Sie sind deshalb offen für das Erschließen neuer Tätigkeitsfelder. Vor diesem Hintergrund können Unternehmensnetzwerke anstelle der Einzelunternehmen entweder die Abgabe von Garantien gemeinschaftlich organisieren. Oder sie können einzelnen Unternehmen bei der Vergabe von eigenen Garantien die Möglichkeit einer Rückversicherung bieten. ${ }^{686}$ Damit würde das Eingehen eigener Garantieverpflichtungen für die Unternehmen $\mathrm{zu}$ einer kalkulierbaren Größe werden. Gute Unternehmen, die aus Gründen fehlender Informationen oder Gewissheit über Risikostand und -entwicklung der eigenen Investitionstätigkeit sowie im Umgang mit Ratingagenturen auf eigene Garantieleistungen verzichten, werden bei Vorhandensein einer Rückversicherung oder der Möglichkeit einer gemeinschaftlichen Garantieabgabe zu Investitionen ins eigene Risiko motiviert.

Beide Instrumente ermöglichen den schlechten Einzelrisiken, sich unter die guten zu mischen, wodurch die Wirksamkeit des Signaling durch unternehmerische Investitionen ins eigene Risiko drohen unterwandert zu werden. Dadurch käme es erneut $\mathrm{zu}$ einem Pooling-Gleichgewicht mit einer Kapitalrationierung der Eigenkapitalgeber als Folge. Unabhängig davon, ob eine Unternehmenskooperation Garantien selber leistet oder Einzelunternehmen bloß die Möglichkeit einer Rückversicherung bietet, verfolgt sie jedoch andererseits das Ziel, möglichst gute Einzelrisiken ins Netzwerk aufzunehmen. Mit der Gestaltung unterschiedlicher Arten von Beitragszahlungen kann die Unternehmenskooperation Screening hinsichtlich der Güte der kooperierenden Unternehmen betreiben. ${ }^{677}$ So werden gute Unternehmen - bei einem für alle Unternehmenstypen identischen Grad an

686 Es liegt ein Analogon zum Fremdkapitalmarkt nahe: So genannte Kreditgarantiegemeinschaften für den Mittelstand ersetzen bei kleinen und mittleren Unternehmen im Bedarfsfall fehlende bankgeeignete Kreditsicherheiten durch eine Kreditgarantie. Vgl. Leopold, Frommann 1998, 49.

Screening setzt voraus, dass Typen mit unterschiedlichen Anreizverträglichkeitsbedingungen verschiedene Verträge annehmen, so dass sie für die schlecht informierten Vertragsteller unterscheidbar werden (Trenngleichgewicht). Im Gegensatz zum Signaling geht die Trennung von „Spreu und Weizen“ beim Screening vom schlecht Informierten aus. Den Nutzen des Informierten bei Ablehnung des Vertrages nennt man Reservationsnutzen, so dass die Teilnahmebedingung darin besteht, einen Vertrag anzubieten, der mindestens den Reservationsnutzen stiftet. Vgl. Feess 1997, 616-625. Zur Möglichkeit eines dauerhaften Trenngleichgewichtes vgl. Kreps 1994, 566ff. 
Ungewissheit, Uninformiertheit und Misstrauen sich und den Ratingagenturen gegenüber - dazu neigen, einen Vertrag mit hoher Selbstbeteiligung im Garantiefall und geringem Beitragssatz zu wählen. Stattdessen werden schlechte Unternehmen zu einer geringen Selbstbeteiligung bei einem hohen Versicherungsbeitrag tendieren. Durch eine hohe Selbstbeteiligung im Garantiefall ließe sich der Anteil der schlechten Kooperationsunternehmen bereits bei der Aufnahme ins Unternehmensnetzwerk reduzieren. Insofern stellt das Engagement eines Unternehmens in einer Risikogemeinschaft bereits ein positives Signal dar.

Damit ein Netzwerk derart komplexe Versicherungsaufgaben mit hohen „overhead“Kosten zu übernehmen in der Lage ist, muss es sich bereits in einer fortgeschrittenen Entwicklungsstufe befinden. In einer zunächst losen partnerschaftlichen Gemeinschaft können rationierte, um knappes Anlegerkapital konkurrierende Unternehmen (co-opetition) jedoch mit einer zunächst anderen Zielsetzung zueinander finden. Ähnlich der Initiative der BWSC $^{688}$ können lose Interessengemeinschaften durch die Bildung von realen und virtuellen Plattformen zur transaktionskostenminimalen Steigerung des Bekanntheitsgrades jedes einzelnen Unternehmens führen. ${ }^{69}$ Für den Erfolg von Unternehmensnetzwerken sind der Bekanntheitsgrad der Organisatoren und die Qualität der angebotenen Informationsund Vermittlungsleistungen dienlich. Das Erschließen höherwertiger Kooperationsfelder wie etwa oben skizzierte Versicherungsleistungen ist jedoch mit hohen Kosten verbunden. Moderne Informations- und Kommunikationsmittel tragen zwar zu sinkenden Kooperationskosten bei. Dennoch fallen den geeigneten Trägern der Wirtschaftspolitik wie den Kammern und Verbänden, Ministerien und Förderanstalten des Bundes und der Länder bei der Finanzierung, Organisation und Durchführung der Kooperationen besondere Bedeutung zu. Sie können für die Schaffung angemessener Rahmenbedingungen sorgen sowie als Informationslieferant für die Unternehmen etwa bei der Einführung und

688 Hinter dieser Plattform von Baden-Württembergischen Small Caps (BWSC) verbirgt sich eine regionale Interessengemeinschaft von 13 Unternehmen des Smax mit einer Zielsetzung im Bereich des Marketing. Die Unternehmen planen am Kapitalmarkt gemeinsame Veranstaltungen und wollen durch Bündelung verschiedener Aktivitäten einen stärkeren Bekanntheitsgrad erreichen. Die in der BWSC organisierten Unternehmen repräsentieren über 7,5 Mrd. DM Umsatz und beschäftigen weltweit 18.300 Mitarbeiter. und reale Plattformen für die Bereitstellung von Beteiligungskapital errichtet. Der Zweck dieser Plattformen besteht darin, potenzielle Beteiligungsgeber und -nehmer so zu einer 
Fortentwicklung kooperativer Maßnahmen, als Beratungsdienst für die Netzwerkteilnehmer, als Organisatoren von Kapitalmarktmessen, ${ }^{690}$ als Repräsentant gegenüber politischen Gremien, Börsenorganisationen, Ratingagenturen und Investoren fungieren und nicht zuletzt eine Garantie-Infrastruktur aufbeziehungsweise ausbauen.

Kooperation zu vernetzen, dass ihnen alle benötigten Informationen und Leistungen gebündelt und damit schneller und kostengünstiger vermittelt werden können. Auf Kapitalmarktmessen - wie in Leipzig, Düsseldorf und Hannover - können sich börsennotierte Gesellschaften und andere Unterternehmen, Investment- und Emissionshäuser, Kreditinstitute und Versicherungen, Risikokapitalanlagegesellschaften sowie Immobilienunternehmen präsentieren. 


\subsection{Finanzielle Anreize staatlicher Förderung}

Niveaubedingte Unterliquidität ist einer Netto-Renditeerwartung der Anleger unterhalb des markträumenden Niveaus geschuldet. Das erwartete Rendite/RisikoProfil der $\mathrm{zu}$ finanzierenden Poolrisiken fällt infolge adverser Selektions- und Anreizrisiken insgesamt zu gering aus. Die bisherigen Vorschläge zur Erhöhung des Eigenkapitalangebots im Bereich kleiner und mittlerer Aktiengesellschaften setzten am Risiko an. Sie zielten darauf ab, die bei unvollkommenen Informationen entstehenden adversen Selektions- und Anreizrisiken für (potenzielle) Eigenkapitalgeber zu reduzieren. Ein grundsätzlich anderer Ansatz besteht darin, die erwartete Rendite bei unveränderten Risiken anzuheben. Die Idee dazu ist alt: "The introduction of a substantial Government transfer tax on all transactions might prove the most serviceable reform available, with a view to mitigating the predominance of speculation over enterprise in the United States. “691 Diesen Keynes'schen Gedanken aufgreifend, schlug Tobin (1974) ${ }^{692}$ vor, ,to throw some sand in the wheels of our excessively efficient international money markets ${ }^{\text {(693. }}$. Eine minimale Transaktionssteuer würde den spekulativen, kurzfristigen Devisenhandel entschleunigen, ohne die Kapitalmobilität auf mittlere und längere Sicht wesentlich $\mathrm{zu}$ beeinträchtigen. In der vorliegenden Arbeit gilt es umgekehrt, die Transaktionsgeschwindigkeit in unterliquiden Aktiensegmenten zu erhöhen. Um „Sand aus dem Getriebe“ am unteren Ende des Aktienmarktes zu blasen, bietet sich folglich eine negative, indirekte „Tobinsteuer“ an ${ }^{694}$ die den Handel mit unterliquiden Werten im Verhältnis zu dem liquider Werte subventioniert. ${ }^{695}$ Die staatliche Übernahme eines nicht unwesentlichen Teils der Unterliquiditätskosten erhöht die Renditeerwartung der Anleger kleiner und mittlerer Gesellschaften. Bei unveränderter Risikosituation werden die subventionierten Aktienwerte damit in einen Pool höherer Renditeforderung umgruppiert. Da Pools höherer Renditeforderungen auch ein höheres durchschnittliches Poolrisiko aufweisen, nimmt die Wahrscheinlichkeit einer Rationierung der subventionierten Einzelrisiken

\footnotetext{
$691 \quad$ Keynes $1936,160$.

692 Vgl. Tobin 1974, 88ff.; Huffschmidt (1995); Palley (1999).

693 Tobin 1978 b, 154.

694 Zur Unterscheidung von direkten und indirekten Steuern vgl. Musgrave, Musgrave, Kullmer 1985, 8.

695 Eine steuerliche Begünstigung auch der liquiden Standardwerte reduziert nicht die Ungleichverteilung des Kapitalangebots zwischen den Segmenten. Sie verstärkt außerdem die beschriebenen negativen Wirkungen überliquider Segmente. Vgl. Abschnitt 2.4.
} 
in einer Risikoklasse höheren Forderungsniveaus ab. Auf diese Weise induziert eine negative Tobinsteuer ein höheres Eigenkapitalangebot im Bereich der subventionierten unterliquiden Segmente. ${ }^{696}$ Um Mitnahmeeffekte seitens der Anleger zu vermeiden, sollte die Subventionierung des Handels allerdings die GeldBrief-Spanne einer Transaktion nicht übersteigen. Auch sollte für die Geld-BriefSpanne eine Höchstgrenze festgelegt werden, da andernfalls die Subvention von Maklern abgeschöpft zu werden droht.

Die Wirksamkeit einer indirekten negativen Steuer steigt mit der Umschlaghäufigkeit einer Aktie, entfaltet sie ihre Anreize doch erst beim Handel. Deshalb ist es überlegenswert, in förderungswürdigen Segmenten eine Subventionierung des Handels mit einer negativen direkten Steuer wie etwa einer Reduzierung oder

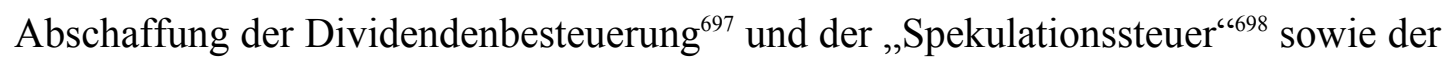
Kapitalertragsteuer ${ }^{699}$ zu flankieren. Auch eine erneute (körperschafts-)steuerliche Begünstigung ausgeschütteter Gewinne käme den Anlegern unterliquider Aktien einerseits $\mathrm{zu}$ Gute. ${ }^{700}$ Denn thesaurierte Gewinne schlagen sich bei unterliquiden Unternehmen nicht unbedingt in einem entsprechend höheren Kurs nieder. Andererseits wird dadurch jedoch für die Unternehmen ein Anreiz geschaffen, Gewinne auszuschütten und nicht unbedingt im Unternehmen zur Finanzierung von

696 Wie sich eine negative Besteuerung einzelner Gesellschaften eines Pools auf die Steigung der gesamten Poollinie und damit das Ausmaß der Kapitalrationierung insgesamt auswirkt, hängt von der Verteilung der subventionierten Gesellschaften in den jeweiligen Pools ab. Konzentrieren sie sich etwa im Niedrigrisikobereich der Poollinie, stellt sich durch die Subventionierung eine Verflachung der Poollinie und damit ein zusätzliches Kapitalangebot ein. Denn für die Übernahme eines marginal höheren Risikos fordern die Anleger nur noch eine geringere Rendite vom Markt. Umgekehrt wird durch eine Subventionierung des Hochrisikobereichs für die Übernahme einer zusätzlichen Risikoeinheit eine höhere Rendite gefordert. Deshalb nehmen in diesem Fall die adversen Selektions- und Anreizrisiken zu, so dass eine Subventionierung sogar insgesamt das Eigenkapitalangebot einschränken kann.

Am 1.1.2001 wurde vom Vollanrechnungs- zum Halbeinkünfteverfahren umgestellt: Ausschüttungen einer Kapitalgesellschaft sind nur noch zur Hälfte mit dem persönlichen Einkommensteuersatz zu versteuern. Jedoch entfällt die Gutschrift der vom Unternehmen bereits gezahlten Körperschaftssteuer auf die Einkommensteuer.

Die „Spekulationssteuer“ ist Teil der Einkommensteuer. Seit 1.1.2001 sind Kursgewinne innerhalb der Spekulationsfrist von einem Jahr zur Hälfte zu versteuern, unter Berücksichtigung einer jährlichen Freigrenze von DM 1.000,-. Mit Beginn des Veranlagungszeitraums 2002 müssen private Anteilseigner ab einer Beteiligung von 1\% (bislang 10\%) an einer Kapitalgesellschaft die Hälfte der Erlöse versteuern. Kapitalgesellschaften müssen die Gewinne aus dem Verkauf von Beteiligungen an anderen Kapitalgesellschaften dagegen nicht versteuern, sofern die Beteiligung länger als ein Jahr dauerte.

$\mathrm{Ab}$ 1.1.2001 wurde die Kapitalertragsteuer für Dividenden und Ausschüttungen von 25\% auf $20 \%$ gesenkt.

$\mathrm{Ab}$ 1.1.2001: Senkung der Körperschaftssteuer auf $25 \%$ sowohl für einbehaltene als für ausgeschüttete Gewinne (bislang $40 \%$ bzw. 30\%). 
Investitionen $\mathrm{zu}$ belassen, was angesichts geringer Eigenkapitalquoten konterproduktiv wäre. Weitere Möglichkeiten der Subventionierung der Renditeerwartung unterliquider Aktien sind: eine steuerliche Bezuschussung größerer Mengen unterliquider Aktien für Kleinanleger, besondere Sparprämien für Anlagen in kleine und mittlere Aktiengesellschaften sowie eine Dividendenprämie beim Ersterwerb einer unterliquiden Aktie (,front end loading ${ }^{6701}$ ).

Eine Subventionierung der Renditeerwartung unterliquider Aktien geht mit der Gefahr einher, dass es den Managern der betroffenen Unternehmen Handlungsspielräume eröffnet, die mitverantwortlich für ein geringes Anlegerinteresse sind: X-Ineffizienzen, Bildung von Organizational Slack. Es bestünde außerdem die Gefahr, dass die Subventionierung die Risiken einzelner Unternehmen und damit die Streuung der Einzelrisiken im Pool vergrößern. Damit wäre für die Anleger letztlich sogar eine Erhöhung adverser Selektions- und Anreizrisiken zu befürchten. Diesen Gefahren kann dadurch begegnet werden, dass man die Zahlung öffentlicher Subventionsgelder an bestimmte Aktivitäten der Gesellschaften knüpft, die ihre Risiken anzeigen wie etwa die Vornahme eines Aktienratings, die Übernahme eigener Garantieleistungen oder die Partizipation an einer Unternehmenskooperation, welche auf eine Trennung von „Spreu und Weizen“ angelegt ist. Der Anreiz eines Unternehmens, an staatliche Subventionen für seine Eigentümer $\mathrm{zu}$ gelangen, wäre insofern mit informationsschaffenden und risikosenkenden Maßnahmen verquickt. Eine auf diese Weise selektiv eingesetzte Subventionierung übernimmt damit auch eine Screening-Funktion. ${ }^{702}$

Die Bindung staatlicher Förderung an informationsschaffende und risikosenkende Maßnahmen ist auch deshalb geboten, weil finanzielle Anreize in Zeiten chronisch leerer Haushaltskassen als eine gesamtwirtschaftliche Investition in die Zukunft politisch leichter durchsetzbar sind. Um die öffentlichen Kassen zu schonen, böte es sich zusätzlich an, die Finanzierung der Subventionen im unterliquiden Bereich entweder den hochliquiden Aktiensegmenten durch eine (positive) Tobinsteuer aufzuerlegen oder die staatliche Bezuschussung der Anleger mit einer Beteiligung durch die unterliquiden Unternehmen $\mathrm{zu}$ verbinden. Eine Beteiligung der Unternehmen käme jedoch einer freiwilligen Anhebung der Aktienrendite gleich.

$701 \quad$ Vgl. Allen 1987, 42f. 
Wie bereits gezeigt, ${ }^{703}$ signalisiert eine Erhöhung der zu erwirtschaftenden Rendite durch ein Unternehmen selber den Anlegern das Vorliegen eines eher schlechten Einzelrisikos. Dies konterkarriert die Screening-Wirkung staatlicher Förderung. Eine fiskalisch gebotene Anbindung einer öffentlichen Bezuschussung an eine Beteiligung der Unternehmen ist deshalb aus informationsökonomischen Gründen abzulehnen. Eine Besteuerung überliquider Standardwerte hingegen ist jedoch nicht nur zur Entlastung öffentlicher Haushalte ratsam, sondern erscheint auch aus allokativen Gründen geboten. Die Begradigung einer etwaigen Überinvestition in hochliquiden Segmenten könnte aufkommensneutral mit der einer Unterinvestition in unterliquiden Segmenten verbunden werden. ${ }^{704}$ Auf diese Weise ließe sich der „Sand“ von dem einen in den anderen Bereich transferieren. Die Einführung einer (positiven) Tobinsteuer am oberen Ende des Aktiensekundärmarktes könnte die destabilisierenden Elemente überliquider Segmente $^{705}$ mit einer Belebung unterliquider Segmente in Übereinklang bringen. Die Einführung einer (positiven) Tobinsteuer war in der Vergangenheit jedoch stets mit großen Schwierigkeiten der Implementierbarkeit verbunden. ${ }^{706}$ Der Tobinsteuer wird zwar regelmäßig konzediert, „the scheme is in the best tradition of the Chicago School." ${ }^{\text {"707 }}$ Allerdings konnte sie sich bislang nicht durchsetzen, stößt sie doch nicht zuletzt auf interessenpolitische Gegenargumente. $^{708}$ So wäre zunächst $\mathrm{zu}$ klären, ob der deutsche Aktiensekundärmarkt in Teilen über ein ökonomisch und auch politisch gewolltes $\mathrm{Maß}$ hinaus als überliquide zu bezeichnen ist. ${ }^{709}$

\footnotetext{
$702 \quad$ Vgl. Abschnitt 5.4.

703 Die adverse Selektion ergibt sich ja gerade dadurch, dass die guten Einzelrisiken bei einer Erhöhung der Renditeforderung aus dem Pool ausscheiden.

704 Vgl. De Meza, Webb (1988, 1990).

$705 \quad$ Vgl. Abschnitt 2.4.

706 Zum aktuellen Stand der Diskussion vgl. Piper 2001, 24.

707 Dornbusch (1993) befürwortet die Spekulationssteuer als marktkonformes Mittel zur Stabilisierung der internationalen Währungsordnung bei flexiblen Wechselkursen. Vgl. Dornbusch 1993,77.

Gründe gegen die Einführung einer Tobinsteuer liefert Kulessa 1996, 99-104. Vgl. a. Tobin $1978 \mathrm{a}, 154$.

Auch die Deutsche Börse AG verdient mit jedem Index Geld. Will zum Beispiel ein Fondsmanager einen Fonds mit Dax-Werten auflegen und dabei mit dem geschützten Namen Dax werben, muss er Lizenzgebühren nach Frankfurt überweisen - pro Jahr ca. DM 20.000. Vgl. Brost 1999, 24.
} 


\section{Zusammenfassung und Ausblick}

Der in Börsenumsatz und Marktkapitalisierung stark fragmentierte deutsche Aktiensekundärmarkt ist im Bereich kleiner und mittlerer Gesellschaften durch niveaubedingte Unterliquidität von einzel- wie gesamtwirtschaftlicher Bedeutung gekennzeichnet. Die Ursachen für dieses Wachstumshemmnis sind vornehmlich darin $\mathrm{zu}$ sehen, dass rationale Anleger einer Stakeholder-Ökonomie in ihrer Unterinformiertheit vom Markt nicht entgoltene adverse Selektions- und Anreizrisiken antizipieren und dabei ihre Angebotsmenge einschränken; dass also letztlich der Preismechanismus inflexibel ist. Das im Rahmen eines „trading at a false price ${ }^{\text {“710 }}$ insgesamt „unterschießende“ Eigenkapitalangebot wird nach Kriterien wie der Unternehmensgröße auf nur wenige Gesellschaften konzentriert. Demgegenüber werden die Aktien kleiner und mittlerer Gesellschaften systematisch vernachlässigt, das heißt rationiert. Adäquate wirtschaftspolitische Maßnahmen zur Ausweitung des Eigenkapitalangebots setzen grundsätzlich an einer verbesserten Ausstattung der Anleger mit Informationen, an der Reduzierung und Transformation adverser Selektions- und Anreizrisiken durch die Bildung eines Garantiesystems, an der Etablierung von Unternehmenskooperationen mit Screening- oder Rückversicherungsfunktionen sowie an einer subventionierten Renditeerwartung für unterbewertete, weil niveaubedingt unterliquide Aktientitel an. Im Einzelnen: Mittelstand-Ratings reduzieren Informationsasymmetrien, mithin die adversen Selektions- und Anreizrisiken, die bei einer markträumenden Renditeforderung entstehen. Neben ihrer Bewertungsfunktion bieten Ratings in Verbindung mit so genannten Credit Default Swaps potenziellen Anlegern unterliquider Titel die Möglichkeit, denjenigen Teil des Unternehmensrisikos $\mathrm{zu}$ veräußern, der unvorhergesehenen Bonitätsverschlechterungen erwächst. Die Handelbarkeit von unternehmerischen Teilrisiken ermöglicht risikobewussten Investoren umgekehrt attraktive Anlagemöglichkeiten. Dadurch erhalten auch die Gesellschaften selber die Möglichkeit, in eigene Risiken zu investieren und damit die Güte ihrer Investitionen zu signalisieren. Durch Kooperationen von rationierten, um knappes Anlegerkapital konkurrierende Unternehmen (co-opetition) lässt sich etwa ihr Bekanntheitsgrad transaktionskostenminimal erhöhen und ihre Risikohöhe signalisieren. Es kann zusätzlich eine Positivauslese dadurch induziert werden, wenn das 
Unternehmensnetzwerk neben reinen Marketing- auch gemeinschaftliche Garantieund Rückversicherungsfunktionen beim Aufkauf unternehmerischer Teilrisiken übernimmt. Finanzielle Anreize staatlicherseits können die Rating-Kultur stimulieren, den Einsatz moderner Finanzinstrumente verbreiten, co-opetition unter den betroffenen Gesellschaften fördern und nicht zuletzt das breite Anlegerinteresse für niveaubedingt unterliquide Aktientitel wecken. Die Implementierung entsprechender Institutionen ist mit einem hohen Einsatz wirtschaftspolitischer Träger verbunden.

$\mathrm{Ob}$ dieser Aufwand in Zeiten knapper öffentlicher Kassen durchsetzbar ist, hängt nicht zuletzt davon ab, ob die sich an den Finanzmärkten derzeit abzeichnenden Veränderungen erwartungsgemäß zu einer Ver- oder $\mathrm{zu}$ einer automatischen Entschärfung der Sekundärmarktrationierung führen werden. Um sich der Frage nach der künftigen Förderungswürdigkeit anzunähern, muss man sich sowohl die Ursachen als auch die Wirkungen der Umbrüche an den Finanzmärkten zunächst vergegenwärtigen: Das durch Relationship Lending geprägte deutsche Finanzierungssystem befindet sich bereits seit einigen Jahren in einem tiefgreifenden und noch lange nicht abgeschlossenen Wandel. Mancher Beobachter spricht sogar von einem „clash of cultures“ der kontinentaleuropäischen Bankenorientierung mit der angelsächsisch geprägten transaktionsorientierten Unternehmensfinanzierung. ${ }^{711}$ Die Kultur der Fremdfinanzierung durch Universalbanken, die als Mittler zwischen Finanzkapitalgebern und -nehmern fungieren, erhält zunehmend Risse. Traditionelle Hausbankbeziehungen werden lockerer; immer mehr Unternehmen wagen den Börsengang und es ist eine dynamische Entwicklung auch des deutschen Beteiligungsmarktes zu erkennen; mit einer zunehmend modularen Zerlegbarkeit der finanziellen Wertschöpfungskette hat ein Trend zur Disintermediation und Securitization (Verbriefung) der Fremdkapitalforderungen eingesetzt; ihre Rolle als Finanzintermediäre ergänzen Banken zunehmend durch Beratung und Begleitung von Unternehmen auf dem Weg zum Kapitalmarkt; immer mehr Spezialbanken treten auf den Plan und auch Universalbanken beginnen einzelne Geschäftsfelder tiefgreifend zu zergliedern, zum Teil formaljuristisch wie etwa die Deutsche Bank 24, zum Teil nur organisatorisch innerhalb der gleichen juristischen Einheit.

711 Vgl. Kotz (1999). 
Die treibenden Kräfte hinter diesen tiefgreifenden Umbrüchen, die sich wechselseitig bedingen und verstärken, sind nicht zuletzt die Deregulierung, die zunehmende Integration der Kapitalmärkte und ihre Harmonisierung etwa durch die europäische Einheitswährung sowie der verstärkte Einsatz moderner Informations- und Kommunikationstechnologien. Moderne Infomations- und Kommunikationstechnologien gehen mit hohen fixen und mit sinkenden Durchschnittskosten einher und veranlassen Banken, in betriebsoptimale Mindestgrößen hineinzuwachsen. ${ }^{712}$ Die Deregulierung und Globalisierung ermöglicht eine Ausweitung der Produktpalette auf erweiterten Märkten und erhöht damit die Anzahl der Konkurrenten auf angestammten Märkten. Alle drei Kräfte zusammengenommen erhöhen die Wettbewerbsintensität im Geschäftsbankensektor. Insbesondere für deutsche Banken wächst der Kostendruck, hinkt im internationalen Vergleich doch ihre Eigenkapitalrendite mit geschätzten 7\% dem doppelt so hohen Wert etwa für US-amerikanische Banken deutlich hinterher. Nicht profitable Bereiche geraten auf den Prüfstand. Niedrige Margen im Firmenkundengeschäft sind deshalb kaum mehr durchzuhalten. Es ist vor allem der im Durchschnitt ertragsarme Firmenkredit, der zunehmend unter Druck gerät. Angesichts der technischen Machbarkeit bepreisen Geschäftsbanken deshalb in sehr viel stärkerem Maß einzelne Risiken. Die Quersubventionierung ertragschwacher durch ertragsstarke Kreditnehmer, die Universalbanken im Rahmen ihrer Strategie zur räumlichen Ausweitung ihrer Absatzmärkte traditionell angewandt haben, wird durch eine stärkere Überprüfung einzelner Kreditnehmer ersetzt. Aufsichtsrechtlich durch „Basel II“713 verstärkt, ist deshalb künftig eine deutliche Konditionenspreizung durch

712 Informationen werden dezentralisiert; sie sind ständig und überall kostenlos verfügbar. Das Monitoring lässt sich hingegen zentralisieren, der Automationsgrad routinemäßiger Arbeiten, vom Geldautomaten bis hin zu einer standardisierten Risikoeinschätzung, steigt. Die Fortschritte in Hard- und Software machen komplizierte Risikomodelle, die große Informationsmengen verarbeiten, möglich. Damit werden auch neue Märkte beherrschbar wie beispielsweise der Handel mit Risiken.

713 Da Kreditausfälle die Sicherheit von Einlagen und die Existenz eines Kreditinstitutes gefährden können, muss bei Kreditvergaben ein bestimmter Betrag an Eigenkapital unterlegt werden. Vgl. $\S 10$ Grundsatz I des KWG. Die Ermittlung der erforderlichen Eigenmittelhinterlegung erfolgt bislang pauschal in wenige Klassen (im Wesentlichen: öffentliche Kreditnehmer, Kreditinstitute, alle übrigen Kunden). Mit „Basel II“ wird das BIZ (als „Zentralbank der Zentralbanken“) den bisherigen Baseler Akkord von 1988 („Basel I“) voraussichtlich ab 2005 dahingehend modifizieren, dass die Eigenkapitalunterlegung einer Kreditvergabe dem einzelnen Kreditrisiko angepasst werden soll. Angesichts der Befürchtung verschlechterter Finanzierungskosten und -möglichkeiten für den Mittelstand hat der Deutsche Bundestag am 08.06.00 eine Entschließung verabschiedet: „Die Bundesregierung wird gebeten, ... eine einseitige Benachteiligung für die mittelständische Wirtschaft zu vermeiden.“ Vgl. F.A.Z. v. 29.11.00, Nr. 278, 49-64; Rode, Moser (1999); Reden 2000, 533. Zusammen mit der Kreditwirtschaft hat das BMWi die „Gemeinsame Erklärung zur Mittelstandsfinanzierung“ 
die Geschäftsbanken zu erwarten. ${ }^{714}$ Sofern höhere Zinsforderungen aus Gründen adverser Selektionen nicht rational erscheinen, wird das Kreditangebot für wahrhaftig oder vermeintlich - hohe Einzelrisiken künftig sogar eingeschränkt. Es stünde damit eine Ausweitung der Kapitalrationierung am Kreditmarkt zu befürchten - insbesondere wenn dieser bereits bei den Großbanken zu beobachtende Trend auf Sparkassen und Genossenschaftsbanken übergreifen sollte. Die höheren Kosten, die etwa den öffentlichen Instituten durch die Einschränkungen in der Gewährträgerhaftung und Anstaltslast entstehen werden, könnten auf dem Wege eines höheren Konditionenniveaus in Verbindung mit einer stärkeren Konditionenspreizung und einer zunehmenden Kreditrationierung aufgefangen werden. ${ }^{715}$

Der zu konstatierende „,decline of traditional banking“6716 verengt den Spielraum von Kreditbeziehungen, die auf langjährigen Hausbankbeziehungen basieren. Hoher fixer Prüfungskosten wegen bringen insbesondere kleine Unternehmen und kleine Kreditvolumina eine für Kreditinstitute ungünstige Aufwands-/Ertragsrelation. Eine Neuausrichtung der Banken insbesondere gegenüber diesen Unternehmen ist daher zu erwarten und zum Teil auch schon zu beobachten. Höhere Einzelrisiken, wie sie etwa die für einen Lange-Wellen-Aufschwung bedeutsamen Produktinnovatoren darstellen, erwartet höhere Fremdfinanzierungskosten oder auch verschärfte Finanzierungsbeschränkungen. Umso größer wird der Anreiz für sie, Eigenkapital, welches gleichzeitig als Sicherheit für die Beschaffung von Fremdkapital dient, möglicherweise auch über den Aktienmarkt aufzunehmen. ${ }^{717}$ Mit einer zunehmend auf den Aktienmarkt ausgerichteten Finanzierung des deutschen Mittelstandes steht deshalb zu befürchten, dass die Kapitalrationierung am Sekundärmarkt als

ausgearbeitet, in der sich die Banken und Sparkassen verpflichten, ausreichend Kredite für den Mittelstand bereitzustellen.

714 Kreditforderungen, für die eine höhere Eigenkapitalunterlegungsvorschrift besteht, als einzelwirtschaftlich sinnvoll erscheint, können über Asset Backed Securities (ABS), die Ursprungsform der Verbriefung, oder darauf aufbauend über synthetische Verbriefungen verkauft werden. Vgl. KfW 2001a, 24-28.

Im Rahmen der Anstaltslast müssen Länder und Gemeinden ihre Geldinstitute mit dem nötigen Kapital ausstatten. Die Gewährträgerhaftung verpflichtet Länder und Kommunen, die Schulden ihrer Kreditinstitute zu bezahlen. Durch diese Staatsgarantien sind die öffentlichen Institute kreditwürdiger als etwa die Großbanken und können sich Geld zu niedrigeren Konditionen leihen. Am 17.07.01 hat die Bundesregierung mit der EU eine Vereinbarung über das Auslaufen der Gewährträgerhaftung für die Landesbanken bis zum Jahr 2005 erzielt. Die Anstaltslast soll dann ebenfalls modifiziert werden. Edwards, Mishkin 1995, 27.

717 Vgl. Größl, Stahlecker (2000); Größl, Stahlecker, Wohlers (1999); Reimer 1999, 200-204; Stenzel (1995). 
gesamtwirtschaftliches Wachstumshemmnis künftig an Bedeutung gewinnen wird; dass sich - um einen Begriff aus dem Amerikanischen Institutionalismus zu bemühen - die „Technik-Institutionen-Dichotomie“"718 weiterhin ausprägt. Dabei besteht ein noch weites Forschungsfeld darin zu untersuchen, auf welche Weise sich die Sekundärmarktrationierung auf andere Märkte überträgt und sie damit die gesamtwirtschaftliche Entwicklung beeinträchtigt. Insbesondere ist die Wechselwirkung zwischen Sekundärmarkt- und Kreditrationierung noch nicht hinreichend beleuchtet, betonte die Literatur doch "the analogy between "equity rationing" and credit rationing more generally." ${ }^{\prime 719}$ Mit dem theoretischen Ansatz einer Vollrationierung kleiner und mittlerer Gesellschaften am Aktiensekundärmarkt wird in der (neo-)institutionenökonomischen Literatur eine Lücke zu schließen sein, die dadurch klaffte, dass ,... a necessary complement to the theory of credit rationing is a theory of informational imperfections in equity markets. “720

Eine in zunehmendem Maße marktorientierte deutsche Finanzierungskultur könnte allerdings auf der anderen Seite auch mit einer Abnahme von Informations- und Ertragsasymmetrien zu Gunsten der Eigenkapitalgeber einhergehen. Diese Asymmetrien erwiesen sich in der vorliegenden Arbeit als conditiones sine qua non für das Auftreten von Sekundärmarktrationierung. Eine mehr auf die ShareholderInteressen zugeschnittene deutsche Finanzierungskultur ließe zum einen erwarten, dass die Bereitschaft und Verpflichtung der Unternehmen zu mehr Transparenz wächst. Zum anderen ist zu erwarten, dass eine verstärkte Shareholder-Ausrichtung die Kultur der Bildung Organizational Slacks zurückdrängt. Dies ginge zwar zu Lasten einer auf Konsens zwischen den Stakeholdern ausgelegten Unternehmensstabilität. Allerdings wäre eine weniger unterproportionale Beteiligung an den Unternehmenserträgen für die Aktionäre von (zumindest kurzfristigem) Interesse. Der Abbau von Informations- und Ertragsasymmetrien würde das Angebot an Eigenkapital auf die beschriebene Weise ausweiten. Nicht trotz, sondern vielmehr wegen einer zunehmenden Kapitalmarktorientierung würde in diesem Fall die

\footnotetext{
718 Theoretische Ansätze zur volkswirtschaftlichen Nicht-Entwicklung weisen durchgängig die Auseinandersetzung mit dem - meist gegensätzlich gedachten - Begriffspaar „Technologie / Institutionen“ auf. Dabei wird Technologie im Wesentlichen als dynamisch, problemlösungsund wohlfahrtsorientiert, Institutionen als statisch und status-quo-fixiert verstanden. Vgl. Ayres 1944, 125; Reuter 1994a, 235-244. 
Sekundärmarktrationierung als gesamtwirtschaftliches Wachstumshemmnis an Bedeutung verlieren.

$\mathrm{Ob}$ eine zunehmend marktorientierte deutsche Finanzierungskultur die gesamtwirtschaftlich hemmende Wirkung der Sekundärmarktrationierung ver- oder entschärft, hängt deshalb von der Stärke der entgegengesetzten Effekte ab. Auf zumindest kurze und mittlere Sicht ist jedoch zu erwarten, dass das deutsche Finanzierungssystem zwar auf eine zunehmende Marktorientierung hinsteuert, es sich aber mit einer in Informations- und Beteiligungsangelegenheiten fortbestehenden vergleichsweise eignerunfreundlichen Unternehmenskultur konfrontiert sieht. Denn die auf Konsens ausgelegte deutsche Unternehmenskultur lässt sich allenfalls sehr langsam verändern. Die Quantifizierung der Kapitalrationierung am deutschen Sekundärmarkt und ihr Vergleich mit angelsächsischen, aber auch mit anderen „Übergangs“-Ökonomien in Querschnittsund Zeitreihenanalysen könnte für diese Frage bereichernde Erkenntnisse liefern.

Die Förderungswürdigkeit des deutschen Aktiensekundärmarktes erscheint vor dem Hintergrund der Veränderungen an den Finanzmärkten deswegen zumindest für die absehbare Zukunft mehr geboten denn je. Damit die „Eule der Minerva“ bereits zur Morgen-Dämmerung der deutschen Aktienmarktentwicklung ihren Flug entfaltet, bedarf es neben der Förderungswürdigkeit aber auch der Förderungsbereitschaft durch die Unternehmenverbände, die Bundesbank sowie vor allem den Bund und die Länder samt ihrer den Mittelstand fördernden Institute. Andernfalls droht vom Aktienmarkt ausgehend eine Art ,zeremonielle Einkapselung“6721. Die Lobby der am Sekundärmarkt vergleichsweise wenigen rationierten Gesellschaften ist in einer traditionell ebenfalls bankorientierten Förderlandschaft allerdings nicht sehr stark ausgeprägt. Die staatliche Unterstützung der allgemein für wichtig erachteten Bereitstellung von Eigenkapital wird deshalb (zumindst zunächst) mehr im

720 Greenwald, Stiglitz, Weiss 1984, 195. Zur theoriegeschichtlichen Entwicklung der Kreditrationierung vgl. Baltensperger, Devinney (1985); Gertler (1988); Allen (1987); Bester, Hellwig (1987); Jaffee, Stiglitz (1990).

721 Sie stellt sich ein, wenn die Gesellschaft auf technologische Innovationen mit einem verstärkten Festhalten am status quo reagiert und nur solche Innovationen sich durchzusetzen imstande sind, welche sich nahtlos in das bestehende Institutionensystem integrieren. Es handelt sich hierbei um den abgeschwächten „past-binding-type“ einer zeremoniellen Einkapselung. Bush benennt zudem den „future-binding-“ und den „Lysenko-type“. Vgl. Bush 1986, 33; Bush 1987, 321; zum Konzept der “ceremonial encapsulation” vgl. a. Reuter 1994a, 262-269. 
außerbörslichen Bereich zu erwarten sein. Es wird die Hebelwirkung insbesondere der Beteiligungsfinanzierung betont, die den Spielraum paralleler und nachfolgender Finanzierungsentscheidungen erweitert. ${ }^{722}$ Eine vergünstigte (Fremdkapital)Refinanzierung für Beteiligungsgeber, eine (partielle) Freistellung ihrer Risiken bei Investitionen in allen Unternehmensphasen bis hin zum Börsengang (seed, start-up, MBO, MBI, Bridge) oder das öffentliche Koinvestment eines privaten Beteiligungsgebers (Leadinvestor) sind hier bereits in der Vergangenheit Ansatzpunkte gewesen. Die Bereitstellung von Eigenkapital über den deutschen Beteiligungsmarkt erfolgte allerdings in jüngerer Vergangenheit im Wesentlichen zur Finanzierung von im Durchschnitt hochrentierlichen Unternehmensinvestitionen der New Economy. Der Bereich der mittelständischen Old Economy ist hingegen nicht mit einer dafür ausreichenden Renditeerwartung verbunden gewesen und blieb weitgehend ausgespart. Wesentlich zur Missachtung des „klassischen“ Mittelstands auch am Beteiligungsmarkt trug bei, dass die in Deutschland noch recht junge Branche der Private Equity-Geber weder über langjähriges Expertenwissen noch über die Erfahrung zur Trennung von „Spreu und (dem für eine Beteiligungsfinanzierung sehr wenigen) Weizen“ in diesem Marktsegment verfügt. Auch erscheint das Gesamtportefeuille des (im Median) durchschnittlichen deutschen Beteiligungsgebers nicht groß genug, um das Portefeuillerisiko entgegen hohen fixen Prüfungskosten breit diversifizieren und damit reduzieren zu können. Durch unvermindert fortzuführende Haftungsfreistellungen, (fremdfinanzierte) Refinanzierungen am unteren Ende des Konditionenspektrums und Koinvestments könnte die öffentliche Hand der „infant industry“ Beteiligungsbranche helfen, künftig Lernkurveneffekte zu erzielen und Größenvorteile zu nutzen. Damit würde ein Weg beschritten werden, den Beteiligungsgebern marktgerechte Renditen im Bereich des klassischen breiten Mittelstandes zu ermöglichen. Doch gibt es im Rahmen der Beteiligungsfinanzierung weitere Förderansätze, die eine verbesserte Bereitstellung von Eigenkapital für mittelständische Unternehmen der Old Economy vorsehen. Bei unterschiedlicher Zusammensetzung der dabei jeweils mitwirkenden Akteure zielen letztlich alle Konstruktionen in unterschiedlicher Weise auf eine im

722 Vgl. Pleschak, Kulicke, Stummer 1998, 26. 
weiten Sinne „Vernichtung der Zeitlichkeit der Zukunft“723 beziehungsweise auf eine „Defuturisierung ${ }^{6724} \mathrm{ab}$. 


\section{Literaturverzeichnis}

Aggarwal, Reena 2000: Stabilization Activities by Underwriters after Initial Public Offerings. In: Journal of Finance, 55 (3), 1075-1103.

Ahrns, Hans-Jürgen; Feser, Hans-Dieter 1991: Wirtschaftspolitik. Problemorientierte Einführung. 5.A. München, Wien.

Akerlof, G.A. 1982: Labor Contracts as Partial Gift Exchange. In: Quarterly Journal of Economics, 97, 543-569.

Akerlof, G.A. 1970: The Market for "Lemons": Quality Uncertainty and the Market Mechanisms. In: Quarterly Journal of Economics, 84, 488-500.

Die Aktiengesellschaft; Zeitschrift für das gesamte Aktienwesen. Köln.

Albach, Horst; Lutter, Marcus 1988: Deregulierung des Aktienrechtes: Das DreiStufen-Modell: ein Entwurf zur Modifizierung des Aktienrechtes im Hinblick auf personalistische Gesellschaftsstrukturen und einen erleichterten Börsenzugang - gefördert von der Bertelsmann Stiftung. Gütersloh.

Alchian, A.A.; Woodward, S. 1988: The Firm is Dead; Long Live the Firm. A Review of Oliver E. Williamson's The Economic Institutions of Capitalism. In: Journal of Economic Literature, 26, 65-79.

Allen, Franklin 1993: Stock markets and resource allocation. In: Mayer, Colin; Vives, Xavier (Hrsg.) 1993: Capital markets and financial intermediation, 81116. Cambridge.

Allen, Linda 1987: The Credit Rationing Phenomenon: A Survey of the Literature. Occasional Paper, 7, NY.

Allen, William T. 1992: Our Schizophrenic Conception of the Business Corporation. In: Cardozo Law Review, 14 (2), 261-281.

Allen, Franklin; Gale, Douglas 1994: A Welfare Comparison of the German and US Financial Systems. LSE Financial Markets Group Discussion Paper, 191.

Allmandinger, Hans; Güttler, Cuno 1989: Die Vorzugsaktie als Instrument am Aktienmarkt. In: Zeitschrift für das gesamte Kreditwesen, 42 (10), 458-462.

Amihud, Yakov; Christensen, B.J.; Mendelson, Haim 1992: Further Evidence on the Risk-Return Relationship. Working Paper. Stanford. 1992.

Amihud, Yakov; Mendelson, Haim 1991a: Liquidity, Asset Prices, and Financial Policy. In: Financial Analysts Journal, Nov.-Dec., 56-66.

Amihud, Y.; Mendelson, H. 1991b: Liquidity and Asset Prices. In: Finanzmarkt und Portfolio Management. 5 (3), 235-240.

Amihud, Y.; Mendelson, H. 1986: Asset Pricing and the Bid-Ask-Spread. In: Journal of Financial Economics, 17, 223-249.

Amihud, Y.; Mendelson, H.; Wood, R.A. 1990: Liquidity and the 1987 Stock Market Crash. In: Journal of Portfolio Management, Spring, 65-69.

Arbel, Avner 1985: Generic Stocks. An Old Product in a New Package. In: Journal of Portfolio Management, 11 (4), 4-13.

Arbel, Avner; Carvell, Steven; Strebel, Paul 1983: Giraffes, Institutions, and Neglected Firms. In: Financial Analysts Journal, May/June, 2-8.

Arbel, Avner; Strebel, Paul 1982: The Neglected and Small Firm Effects. In: Financial Review, 17 (4), 201-218.

Arestis, Philip; Demetriades, Panicos O.; Luintel, Kul B. 2001: Financial Development and Economic Growth: The Role of Stock Markets. In: Journal of Money, Credit, and Banking, 33 (1), 16-41.

Arrows, K.J. 1959: Toward a Theory of Price Adjustment. In: Abramovitz, M. (Hrsg.) 1959: The Allocation of Economic Resources, 41-51. Stanford. 
Arrow, Kenneth Joseph 1963: Social choice and individual values, 2.ed. NY.

Asquith, Paul; Mullins, David W. 1986: Equity Issues and Offering Dilution. In: Journal of Financial Economics, 15, 61-89.

Atje, Raymond; Jovanovic, Boyan 1993: Stock Markets and Development. In: European Economic Review, 37, 632-640.

Atje, R.; Jovanovic, B. 1992: Stock markets and development. NY.

Ayres, Clarence E. 1944: The Theory of Economic Progress. NY.

Azariadis, Costas; Smith, Bruce 1998: Financial Intermediation and Regime Switching in Business Cycles. In: American Economic Review, 88 (3), 516-536.

Backmann, Christian 1999: Grundüberlegungen zur Mobilisierung: OrganizationalSlack, Ressourcen, Effizienz und Effektivität. Diskussionsbeitrag der Wirtschaftswissenschaftlichen Fakultät Ingolstadt der Katholischen Universität Eichstätt, 118.

Bagehot, Walter 1874: Lombardstreet. Der Weltmarkt des Geldes in den Londoner Bankhäusern. Übersetzt von H. Beta. Leipzig.

Bagwell, Laurie Simon 1991: Shareholder Heterogeneity: evidence and implications. In: American Economic Review, 81 (2), 218-221.

Baltensperger, Ernst 1978: Credit Rationing. Issues and Questions. In: Journal of Money, Credit, and Banking, 10 (2), 170-183.

Baltensperger, Ernst; Devinney, Timothy M. 1985: Credit Rationing Theory: A Survey and Synthesis. In: $\mathrm{ZgS} / \mathrm{JITE}, 141,475-502$.

Bannerjee, A.V. 1992: A simple model of herd behavior. In: Quarterly Journal of Economics, 107, 797-818.

Banz, Rolf W. 1981: The Relationship between Return and Market Value of Common Stocks. In: Journal of Financial Economics, 9, 3-18.

Bauer, Erich 1977: Markt-Segmentierung. Stuttgart.

Baums, Theodor 1997: Aktienmarkt und Finanzierung kleiner Unternehmen. In: ZIPDokumentation, 44, 1942-1948.

Bea, Franz Xaver 1997: Sharholder Value. In: WiSt, 26 (10), 541-544.

Beck, Hanno 1999: Immer auf die Kleinen. In: F.A.Z. v. 30.04.99, Nr. 100, 25.

Beiker, Hartmut 1993: Überrenditen und Risiken kleiner Aktiengesellschaften - Eine theoretische und empirische Analyse des deutschen Kapitalmarktes von 1966 bis 1989. Köln.

Benassy, J.-P. 1977: On Quantity Signals and the Foundations of Effective Demand Theory. In: Scandinavian Journal of Economics, 79, 147-168.

Bencivenga, Valerie R.; Smith, Bruce D.; Starr, Ross M. 2000: Secondary Capital Markets, Long-Run Growth, and the Term Structure of Asset Yields. In: International Economic Review, 41 (3), 769-800.

Bencivenga, V. R.; Smith, B. D.; Starr, R. M. 1996: Equity Markets, Transactions Costs, and Capital Accumulation. In: World Bank Economic Review, 10 (2), 241-265.

Bencivenga, V. R.; Smith, B. D.; Starr, R. M. 1995: Transactions Costs, Technological Choice, and Endogenous Growth. In: Journal of Economic Theory, 67, 153177.

Berk, Jonathan B. 1995: A Critique of Size-Related Anomalies. In: Review of Financial Studies, 8 (2), 275-286.

Berle, Adolf; Means, Gardiner 1932: The Modern Corporation and Private Property. NY.

Bernanke, Ben 1993: Credit in macroeconomy. In: Quarterly Review, Federal Reserve Bank of New York, 18, 435-439. 
Bernanke, Ben S. 1981: Bankruptcy, Liquidity and Recession. In: American Economic Review, papers and proceedings, 71, 155-159.

Bernanke, Ben S.; Gertler, Mark 1995: Inside the Black Box: The Credit Channel of Monetary Policy Transmission. In: Journal of Economic Perspectives, 9 (4), 27-48.

Bernanke, B.; Gertler, M. 1989: Agency costs, net worth, and business fluctuations. In: American Economic Review, 79 (1), 14-31.

Bernanke, B.; Gertler, M. 1987: Financial Fragility and Economic Performance, Working Paper 2318, NBER, Massachusetts (USA).

Bernanke, Ben; Gertler, Mark; Gilchrist, Simon 1996: The Financial Accelerator and the Flight to Quality. In: The Review of Economics and Statistics, 78 (1), 1-15.

Bernstein, Peter L. 1995: Risk as a History of Ideas. In: Financial Analysts Journal. Jan.-Febr., 7-11.

Bernstein, P.L. 1998: Stock market risk in a Post Keynesian world. In: Journal of Post Keynessian Economics, 21 (1), 15-24.

Bernstein, P.L. 1987: Liquidity, Stock Markets, and Market Makers. In: Financial Management, 16, 54-62.

Bester, Helmut 1987: The Role of Collateral in Credit Markets with Imperfect Information. In: European Economic Review, 31, 887-899.

Bester, H. 1985a: The level of Investment in Credit Markets with Imperfect Information. In: ZgS / JITE, 141, 503-515.

Bester, H. 1985b: Screening vs. Rationig in Credit Markets with Imperfect Information. In: American Economic Review, 75, 850-855.

Bester, Helmut; Hellwig, Martin 1987: Moral Hazard and Equilibrium Credit Rationing: An Overview of the Issues. Discussion Paper No. A-125, July 1987, Sonderforschungsbereich 303 „Information und die Koordination wirtschaftlicher Aktivitäten" der Universität Bonn.

Bhattacharya, Sudipto 1993: Discussion. In: Mayer, Colin; Vives, Xavier (Hrsg.) 1993: Capital Markets and Financial Intermediation, 108-113. Cambridge.

Bhattacharya, Sudipto; Ritter, Jay R. 1983: Innovation and Communication: Signaling with Partial Disclosure. In: Review of Economic Studies, 50, 331-346.

Bhide, Amar 1993: The hidden costs of stock market liquidity. In: Journal of Finanancial Economics, 34, 1993, 31-51.

Biesecker, Adelheid 1998: Shareholder, Stakeholder and Beyond - Auf dem Weg zu einer Vorsorgenden Wirtschaftsweise. Bremer Diskussionspapiere zur Institutionellen Ökonomie und Sozialökonomie.

Bikhchandani, Sushil; Hirshleifer, David; Welch, Ivo 1992: A theory of fads, fashion, custom, and cultural change as informational cascades. In: Journal of Political Economy, 100, 992-1026.

Black, Fischer 1986: Noise. In: Journal of Finance, 41, 529-543.

Black, F. 1976: The dividend puzzle. In: Journal of Portfolio Management, 2, 5-8.

Black, J.; de Meza, D. 1990: On Giving Credit where it is Due, mimeo. University of Exeters.

Black, F.; Scholes, M. 1972: The valuation of option contracts and a test of market efficiency. In: Journal of Finance, 27, 399-417.

Blair, Margaret 1995: Ownership and Control: Rethinking Corporate Governance for the Twenty-first Century. Washington D.C.

Blanchard, Oliver J.; Watson, Mark W. 1982: Bubbles, Rational Expectations, and Financial Markets. In: Wachtel, Paul (Hrsg.) 1982: Crises in the Economic and Financial Structure. Lexington (Mass.) 295-315.

Blinder, A.S. 1989: Macroeconomics Under Debate, Kap. 4. London. 
Blinder, A.S. 1987: Credit Rationing and Effective Supply Failures. In: Economic Journal, 97, 327-352.

Blinder, A.S.; Stiglitz, J.E. 1983: Money, Credit Constraints, and Economic Activity. In: American Economic Review, papers and proceedings, 73, 297-302.

Blume, L.; Easley, D. 1992: Evolution and Market Behavior. In: Journal of Economic Theory, 58, 9-40.

Bolton, Patrick; Scharfstein, David S. 1998: Corporate Finance, the Theory of the Firm, and Organizations. In: Journal of Economic Perspectives, 12 (4), 95-114.

Bolton, Patrick; Thadden, Ernst-Ludwig 1998: Liquidity and Control: A Dynamic Theory of Corporate Ownership Structure. In: JITE, 154, 177-223.

Bordo, Michael D.; Eichengreen, Barry; Irwin, Douglas A. 2000: Is Globalization Today Really Different Than Globalization a Hundred Years Ago? In: Wirtschaftspolitische Blätter, 2, 121-129.

Boyd, John H.; Smith, Bruce D. 1998: The evolution of debt and equity markets in economic development. In: Economic Theory, 12, 519-560.

Boyd, J.H.; Smith, B.D. 1996: The Coevolution of the Real and Financial Sectors in the Growth Process. In: World Bank Economic Review, 10 (2), 371-396.

Brainard, William; Tobin, James 1961: Financial Intermediaries and the Effectiveness of Monetary Controls. In: Hester, D.; Tobin, James (eds.) 1961: Financial Markets and Economic Activity, Cowles Foundation Monograph, 21. Yale.

Brakmann, Heinrich 1993: Aktienemissionen und Kurseffekte. Deutsche Bezugsrechtsemissionen für die Jahre 1978 bis 1988. Wiesbaden.

Bray, M. 1994: The Arbitrage Pricing Theory is not Robust 1: Variance Matrices and Portfolio Theory in Pictures, Financial Markets Group Discussion Paper Series 178, LSE.

Brealy, Richard; Myers, Stewart 1984: Principles of Corporate Finance, 3. A. NY.

Breeden, D.T. 1979: An intertemporal asset price model with stochatsic consumption and investment opportunities. In: Journal of Financial Economics, 7, 265-296.

Brennan, Michael J. 1970: Taxes, Market Valuation and Corporate Financial Policy. In: National Tax Journal, 23, 417-427.

Brennan, Michael J.; Subrahmanyam, Avanidhar 1996: Market microstructure and asset pricing: On the composition for illiquidity in stock returns. In: Journal of Financial Economics, 41, 441-464.

Brost, Marc 2000: Blank ohne Bank. In: Die Zeit v. 31.05.00, Nr. 23, $23 \mathrm{f}$.

Brost, M. 1999: Kompass für das Kapital. In: Die Zeit v. 26.08.99, Nr. 35, 24.

Bühl, Walter L. 1985: Eine Zukunft für Deutschland. Grundlinien der technologischen, gesellschaftlichen und politischen Entwicklung. München.

Bühner, Rolf (Hrsg.) 1994: Der Shareholder-Value-Report: Erfahrungen, Ergebnisse, Entwicklungen. Landsberg/Lech.

Bühner, Rolf; Tuschke, Anja 1997: Zur Kritik am Shareholder Value - eine ökonomische Analyse. In: BFuP 5, 499-516.

Bush, Paul D. 1987: The Theory of Institutional Change. In: Journal of Economic Issues, 21 (3), 1075-1116.

Bush, P.D. 1986: On the Concept of Ceremonial Encapsulation. In: Review of Institutional Thought, 3, Dez., 25-45.

Bush, P.D. 1979: The Ceremonial Encapsulation of Capital Formation in American Economy. Vortrag während des jährlichen Treffens der Western Social Science Association im April 1979, zitiert nach: Waller, W.T. (Jr.): Ceremonial Encapsulation and Corporate Cultural Hegemony. In: JEI, 21 (1), 321.

Bushee, Brian 1998: The Influence of Institutional Investors on Myopic R\&D Investment Behavior. In: Accounting Review, 73 (3), 305-333. 
Busse von Colbe, Walther 1997: Was ist und was bedeutet Shareholder Value aus betriebswirtschaftlicher Sicht? In: Zeitschrift für Unternehmens- und Gesellschaftsrecht, 2, 271-290.

Cambiè, Silvia M. 1995: Prager Börse vor neuer Belastung. In: Die Bank, 1, $54 \mathrm{f}$.

Cameron, Rondo; Crisp, Olga; Patrick, Hugh T.; Tilly, Richard 1967: Banking in the Early Stages of Industialization. NY.

Castan, Edgar 1997: Rückkauf eigener Aktien. In: WiSt, 9, 465-467.

Chalmers, A.F. 1994: Wege der Wissenschaft. Berlin u.a.

Chandler, Alfred 1990: Scale and Scope. The Dynamics of Industrial Capitalism. Cambridge (Mass.).

Cho, Yoon Je 1986: Inefficiencies from Financial Liberalization in the Absence of Well-Functioning Equity Markets. In: Journal of Money, Credit, and Banking, 18 (2), 191-199.

Coase, R.H. 1937: The Nature of the Firm. In: Economica, 4, 386-405.

Cohen, J.B.; Zinbarg, E.D.; Zeikel, A. 1975: Investment Analysis and Portfolio Management. 4.A. Homewood (Ill.).

Cooper, K.; Groth, J.C.; Avera, W.E. 1985: Liquidity, Exchange Listing, and Common Stock Performance. Working Paper, Texas A\&M University, August 1983.

Coopers \& Lybrand (Hrsg.) 1994: Made in the UK - Middle Market Survey. London.

Copeland, Tom; Koller, Tim; Murrin, Jack 1998: Unternehmenswert. Methoden und Strategien für eine wertorientierte Unternehmensführung. 2.A. Frankfurt / Main.

Copeland, T.E.; Weston, J.F. 1988: Financial Theory and Corporate Policy. Reading (Mass.).

Corbett, Jenny; Jenkinson, Tim 1994: The Financing of Industry, 1970-1989: An International Comparison. Discussion paper, 948. London.

Cornell, B.; Shapiro, A. 1987: Corporate Stakeholders and Corporate Finance. In: Financial Management, 16, 5-14.

Cramp, A.B. 1987: Liquidity. In: Eatwell, John; Milgate, Murray; Newman, Peter (eds.) 1987: The New Palgrave. A Dictionary of Economics, 3, K to P, 211213.

Creditreform $A G$ 2000: Mittelstand am Scheideweg. Jahresbericht. In: www.creditreform.de.

Crotty, James 1994: Are Keynesian Uncertainty and Macrotheory Compatible? Conventional Decision Making, Institutional Structures, and Conditional Stability in Keynesian Macromodels. In: Dymski, G.; Pollin, R. (eds.) 1994: New Perspectives in Monetary Macroeconomics, Michigan.

Cullom, Peter 2001: Der Venture Capital-Markt in Deutschland. In: Venture Capital 2001. Jahrbuch für Beteiligungsfinanzierung, 15-20.

Cyert, R.M.; March, J.G. 1992: A Behavioral Theory of the Firm. 2.A. Cambridge, Oxford.

Cyert, Richard M.; March, James G. 1963: A Behavioral Theory of the Firm. Englewood Cliffs.

Davidson, Paul 1991: Is Probability Theory Relevant for Uncertainty? A Post Keynesian Perspective. In: Journal of Economic Perspectives, 5 (1), 129-143.

De Bondt, Werner F.M.; Teh, Lillyn L. 1997: Herding Behavior and Stock Returns: An Exploratory Investigation. In: Swiss Journal of Economics and Statistics, $133(2 / 2), 293-324$. 
De Bondt, Werner F.M.; Thaler, Richard H. 1989: A Mean Reverting Walk Down Wall Street. In: Journal of Economic Perspectives, 3, 189-202.

De Gregorio, Jose; Giudotti, Pablo E. 1995: Financial Development and Economic Growth. In: World Development, 23 (3), 433-448.

De Long, J. Bradford; Shleifer, Andrei; Summers, Lawrence H.; Waldmann, Robert J. 1990: Noise Trader Risk in Financial Markets. In: Journal of Political Economy, 98 (4), 703-738.

De Meza, D.; Webb, D.C. 1990: Risk, Adverse Selection and Capital Market Failure. In: Economic Journal, 100, 206-214.

De Meza, D.; Webb, D.C. 1988: Credit Market Efficiency and Tax Policy in the Presence of Screening Costs. In: Journal of Public Economics, 36, 1-22.

De Meza, D.; Webb, D.C. 1987: Too Much Investment: A Problem of Asymmetric Information. In: Quarterly Journal of Economics, 102, 281-292.

Del Guercio, Diane 1996: The Distorting Effect of the Prudent-Man Laws on Institutional Equity Investments. In: Journal of Financial Economics, 40, 31-62.

Delingat, Andreas 1996: Unternehmensübernahmen und Agency-Theorie Konflikte zwischen Management, Aktionären und Fremdkapitalgebern um Verfügungsrechte über Ressourcen. Köln.

Demirgüç-Kunt, Asli; Levine, Ross 1996a: Stock Markets, Corporate Finance, and Economic Growth: An Overview. In: World Bank Economic Review, 10 (2), 223-239.

Demirgüç-Kunt, A.; Levine, R. 1996b: Stock Market Development and Financial Intermediaries: Stylized Facts. In: World Bank Economic Review, 10 (2), 291321.

Demirgüç-Kunt, A.; Levine, R. 1993: Stock market development and financial intermediary growth: A research agenda. The World Bank.

Demirgüç-Kunt, Asli; Maksimovic, Vojislav 1996: Stock Market Development and Financing Choices of Firms. In: World Bank Economic Review, 10 (2), 341369.

Demsetz, Harold 1968: The Cost of Transacting. In: Quarterly Journal of Economics, 82, 33-53.

Deutsche Bank AG 2001: Ratingreport Deutschland. Was der Mittelstand über Basel II denkt.

Deutsche Bundesbank 2001: Realzinsen: Entwicklung und Determinanten. In: Monatsbericht, Juli, 33-50.

Deutsche Bundesbank 1999a: Zur Entwicklung der privaten Vermögenssituation seit Beginn der neunziger Jahre. In: Monatsbericht, Jan., 33-50.

Deutsche Bundesbank 1999b: Zur Unternehmensfinanzierung in Deutschland und Frankreich: Eine vergleichende Analyse. In: Monatsbericht, Okt., 29-46.

Deutsche Bundesbank 1998: Strukturveränderungen am deutschen Kapitalmarkt im Vorfeld der Europäischen Währungsunion. In: Monatsbericht, April, 55-70.

Deutsche Bundesbank 1997: Die Akie als Finanzierungs- und Anlageinstrument. In: Monatsbericht, Jan., 27-41.

Deutsche Bundesbank 1994: Eigenmittelausstattung der Unternehmen ausgewählter EG-Länder im Vergleich. In: Monatsbericht, Okt. 1994, 73ff.

Deutsche Bundesbank 1991: Zur Bedeutung der Aktie als Finanzierungsinstrument. In: Monatsbericht, Okt., 22-29.

Devenow, Andrea; Welch, Ivo 1996: Rational Herding in Financial Economics. In: European Economic Review, 40, 603-615.

Devereux, Michael B.; Smith, Gregor W. 1994: International Risk Sharing and Economic Growth. In: International Economic Review, 35 (4), 535-550. 
Dhawan, Rejeev 2001: Firm Size and productivity differentiel: theory and evidence from a panel of US firms. In: Journal of Economic Behavior \& Organization, 44, 269-293.

Diamond, Douglas W. 1984: Financial Intermediation and Delegated Monitoring. In: Review of Economic Studies, 51, 393-414.

Diamond, Douglas W.; Verrecchia, Robert E. 1982: Optimal Managerial Contracts and Equilibrium Security Prices. In: Journal of Finance, 37, 275-287.

Dill, W.R. 1958: Environment as an Influence on Managerial Autonomy. In: Administrative Science Quarterly, 2, 409-433.

Dimson, E.; Marsh, P.R. 1983: The Stability of the UK Risk Measures and the Problem of Thin Trading. In: Journal of Finance, Juni, 753-783.

Dittus, Peter 1985: Neue Institutionenökonomik. Abschied vom Ideal. In: Wirtschaftswoche, Nr. 19, 03.05.85.

Domke, Hans-Martin 1987: Rendite und Risiko von Aktien kleiner Börsengesellschaften: eine empirische Untersuchung der Performance deutscher Nebenwerte in den Jahren 1971 bis 1980. Frankfurt / Main.

Dornbusch, Rüdiger 1993: Eurpean International Economic Policy Issues. In: European Parliament, Directorate General for Research, working papers, Economic Interdependence - New Policy Challenges. Proceedings of the Public. Hearing by the Committee on External Economic Relations. Brussels 28. September.

Dugger, W.M. 1989: Radical Institutionalism. Basic Concepts. In: Ders. (Hrsg.) 1989: Radical Institutionalism. Contemporary Voices, 1-20. NY, London.

Eberstadt, Gerhard 1999: Der Euro und der Kapitalmarkt. In: Zeitschrift für das gesamte Kreditwesen, 6, 272-276.

Edwards, Franklin R.; Mishkin, Frederic S. 1995: The Decline of Traditional Banking: Implications for Financial Stability and Regulatory Policy. In: FRBNY Economic Policy Review, 1(2), 27-45.

Edwards, Jeremy; Fischer, Klaus 1994: Banks, Finance, and Investment in Germany. Cambridge.

Eger, Thomas 1995: Eine ökonomische Theorie von Langzeitverträgen. Marburg.

Ehrlicher, Werner 1968: Geldtheorie. In: Ders.; Esebwein-Rothe, I.; Jürgensen, H.; Rose, K. (Hrsg.) 1968: Kompendium der Volkswirtschaftslehre, Bd. 2., $1 \mathrm{ff}$. Göttingen.

Eichel, Hans 2000: Steuerreform 2000. Mehr Beschäftigung durch Investitionen. In: Wirtschaftsdienst 2000/II, 75-78.

Engel, Dirk 2001: Höheres Beschäftigungswachstum durch Venture Capital? Discussion Paper No. 01-34, Zentrum für Europäische Wirtschaftsforschung $\mathrm{GmbH}(\mathrm{ZEW})$.

Elsner, Wolram 1987: Institutionen und ökonomische Institutionentheorie. Begriffe, Fragestellung, theoriegeschichtliche Ansätze. In: WiSt, 1, 5-14.

Emons, Winand 2001: Information, Märkte, Zitronen und Signale. Zum Nobelpreis an George Akerlof, Michael Spence und Joseph Stiglitz. In: Wirtschaftsdienst 11, 664-668.

Epstein, Gerald 1995: The Illiquidity Trap. In: Eastern Economic Journal, 21 (3), 309-318.

Everling, Oliver 1999a: Credit Rating in Europa. In: WiSt, 5, 249-252.

Everling, Oliver 1999b: Ratingagenturen expandieren in Europa. In: Die Bank, 12, 808f. 
EZB 2000: New Economy - Begriffsklärung und Hauptkriterien. In: Monatsbericht Oktober 2000, $48 f$.

Falkenstein, Eric G. 1996: Preferences for Stock Characteristics As Revealed by Mutual Fund Portfolio Holdings. In: Journal of Finance, 51 (1), 111-135.

Fallgatter, Michael 1995: Grenzen der Schlankheit: Lean Management braucht Organizational Slack. In: zfo, 4, 215-220.

Fama, Eugene F. 1991: Efficient Capital Markets: II. In: Journal of Finance, 46 (5), 1575-1617.

Fama, E.F. 1977: Foundations of Finance. Portfolio decisions and security prices. Oxford.

Fama, E.F. 1970: Efficient Capital Markets: A Review of Theory and Empirical Work. In: Journal of Finance, 25, 383-417.

Fama, Eugene F.; French, Kenneth R. 1992: The Cross-Section of Expected Stock Returns. In: The Journal of Finance, 47 (2), 427-465.

Fazzari, Steven M.; Hubbard, R. Glenn; Petersen, Bruce C. 1988: Financing Constraints and Corporate Investment. Brookings Papers on Economic Activity, 1, 141-195.

Feess, Eberhard 1997: Mikroökonomie. Eine spieltheoretisch- und anwendungsorientierte Einführung. Marburg.

Felderer, Bernhard; Homburg, Stefan 1999: Makroökonomik und neue Makroökonomik. 7.A. Berlin, Heidelberg, NY.

Feldmann, Horst 1995: Eine institutionalistische Revolution? Zur dogmenhistorischen Bedeutung der modernen Institutionenökonomik. Berlin.

Fischer, G. 1964: Allgemeine Betriebswirtschaftslehre, 10.A. Heidelberg.

Fischer, Leonhard 2000: Private Equity in Deutschland - von der Nische zum Wachstumsmotor. In: F.A.Z. v. 31.07.00, Nr. 175, 36.

Flach, Uwe E.; Schwarz, Michaela 1999: Europäische Wahlverwandschaften - der Euro und die Wachstumsbörsen. In: Zeitschrift für das gesamte Kreditwesen 6, 277-278.

Flood, Robert P.; Garber, Peter M. 1980: Market Fundamentals versus Prive-Level Bubbles: The First Test. In: Journal of Political Economy, 88, 745-770.

Foerster, Stephen R.; Karolyi, G. Andrew 1996: The Effects of Market Segmentation and Illiquidity on Asset Prices: Evidence from Foreign Stocks Listing in the US. Working Paper. University of Chicago.

Franke, Günter; Hax, Herbert 1990: Finanzwirtschaft des Unternehmens und Kapitalmarkt. 2.A. Heidelberg, Berlin.

Frankfurter, G.M.; Phillips, H.E. 1996: Normative Implications of equilibrium models:Homogenous expectations and other artifialities. In: Journal of Economic Behavior \& Organization, 31 (1), 67-84.

Franz, Peter 2001: Ranking aller Orte(n) - ein Kommentar. In: Wirtschaft im Wandel 10/2001, 223.

Fried, J.; Howitt, P. 1980: Credit Rationing and Implicit Contract Theory. In: Journal of Money, Credit, and Banking, 12, 471-489.

Freeman, Christopher 1984: Long Waves in the World Economy. London.

Freeman, R.E. 1984: Strategic Management: A Stakeholder Approach, Boston et al., 31-47.

Freimer, Marshall; Gordon, Myron J. 1960: Why Bankers Ration Credit. In: Quarterly Journal of Economics, 74, 397-416.

Frenkel, Michael; Hemmer, Hans-Rimbert 1999: Grundlagen der Wachstumstheorie. München. 
Frick, I. 1994: Große Hoffnungen. In: Industrie, 44, 10-13.

Fritsch, Ulrich 1981: Die Eigenkapitallücke in der Bundesrepublik. Köln.

Froot, Kenneth A.; Scharfstein, David S.; Stein, Jeremy C. 1992: Herd on the Street: informational inefficiencies in a market with short-term speculation. In: Journal of Finance, 47 (4), 1461-1484.

Fuest, Clemens; Huber, Bernd 2000: Das Vollanrechnungsverfahren ist nicht mehr zeitgemäß. In: Wirtschaftsdienst, 2000/VI, 351-355.

Fuhrmann, Wilfried 1994: Geld und Kredit. Prinzipien monetärer Makroökonomik. 2.A. München.

Funk, Lothar 2000: Ein New Economy-Effekt für Deutschland? In: Wirtschaftsdienst, V/2000, 271-276.

Galbraith, J.K. 1952: American Capitalism. Boston.

Geier, Manfred 1999: Fake. Leben in künstlichen Welten. Mythos-LiteraturWissenschaft. Hamburg.

Gelb, Alan H. 1989: Financial Policies, Growth, and Efficiency. wps 202. World Bank, Country Economics Development. Washington, D.C.

Gerke, Wolfgang 1999: Der Neue Markt. In: WiSt, 4, 204-206.

Gerke, W. 1995: „Der Finanzplatz Deutschland braucht Innovation statt Reaktion“. In: Zeitschrift für das gesamte Kreditwesen, 1, 16-26.

Gerke, W. 1972: Kapitalbeteiligungsgesellschaften. Frankfurt / Main.

Gerke, Wolfgang; Rapp, Heinz-Werner 1993: Eigenkapitalbeschaffung durch Erstemission von Aktien. In: Gebhard, Günther; Gerke, Wolfgang; Steiner, Manfred (Hrsg.) 1993: Handbuch des Finanzmanagements. Instrumente und Märkte der Unternehmensfinanzierung, 287-312. München.

Gerke, Wolfgang; Rasch, Sebastian 1993: Europas Wertpapierbörsen im Umbruch Eine Übersicht über den Strukturwandel im europäischen Börsenwesen. In: ZEW-Wirtschaftsanalysen, 1 (3), 306-336.

Gertler, Mark 1988: Financial Structure and Aggregate Economic Activity: An Overview. In: Journal of Money, Credit, and Banking, 20 (3), part 2, 559-588.

Gertler, M.; Gilchrist, S. 1994: Monetary policy, business cycles, and the behavior of small manufacturing firms. In: Quarterly Journal of Economics 109, 309340 .

Ghani, Ejaz 1992: How Financial markets Affect Long-Run Growth: A Cross Country Study. wps 843. World Bank, Operations Evaluation Department. Washington, D.C.

Giammarino, R.M.; Neave, E.H. 1982: The failure of financial contracts and the relevance of financial policy, Working Paper, 82-3, Queens University, Kingston (Ont.).

Gilles, C.; LeRoy, S. F. 1991: On the Arbitrage Pricing Theory, In: Economic Theory, 1, 213-229.

Gilster, J.E. 1983: Capital Market Equilibrium with Divergent Investment Horizon Length Assumptions. In: Journal of Financial and Quantitative Analysis, 18, 257-268.

Glosten, Lawrence R. 1989: Insider Trading, Liquidity, and the Role of the Monopolist Specialist. In: Journal of Business, 62 (2), 211-235.

Glosten, Lawrence R.; Harris, Lawrence E. 1988: Estimating the Components of the Bid/Ask Spread. In: Journal of Financial Economics, 21, 123-142.

Goldberg, V. 1976: Regulation and Administered Contracts. In: Bell Journal, 7, 426448. 
Goldsmith, Raymond W. 1969: Financial Structure and Development. New Haven (Conn.).

Gompers, Paul A.; Metrick, Andrew 2001: Institutional Investors and Equity Prices. In: Quarterly Journal of Economics, 116(1), 229-259.

Gramp, Horst 1970: Liquidität als Determinante der Aktienkurse. Freiburg i.B.

Greenwald, Bruce; Stiglitz, Joseph E. 1993: Financial market imperfections and business cycles. In: Quarterly Journal of Economics, 108, 77-114.

Greenwald, B.; Stiglitz, J.E. 1990: Macroeconomic Models with Equity and Credit Rationing. Working Paper, 3533, NBER.

Greenwald, B.; Stiglitz, J.E. 1988a: Information, Finance Constraints and Business Fluctuations. In: Kohn, Meir; Tsiang, Sho-Chieh (Hrsg.) 1988: Finance Constraints, Expectations, and Macroeconomics, 103-140. Oxford.

Greenwald, B.; Stiglitz, J.E. 1988b: Financial Market Imperfections and Business Cycles. Working Paper 2494, NBER, USA.

Greenwald, B.; Stiglitz, J.E. 1988c: Examining Alternative Macroeconomic Theories. In: Brookings Papers on Economic Activity, 1, 207-270.

Greenwald, B.; Stiglitz, J.E. 1987a: Imperfect Information, Credit Markets and Unemployment. In: European Economic Review, 31, 444-456.

Greenwald, B.; Stiglitz, J.E. 1987b: Keynesian, New Keynesian and New Classical Economics. In: Oxford Economic Papers, 39, 119-133.

Greenwald, B.; Stiglitz, J.E.; Weiss, Andrew 1984: Information and Macroeconomics. Informational Imperfections in the Capital Market and Macroeconomic Fluctuations. In: American Economic Review, papers and proceedings, 74 (2), 194-199.

Greenwood, Jeremy; Jovanovic, Boyan 1999: The Information-Technology Revolution and The Stock Market. In: American Economic Review, papers and proceedings, 89 (2), 116-128.

Greenwood, J.; Jovanovic, B. 1990: Financial Development, Growth, and the Distribution of Income. In: Journal of Political Economy, 98, 1076-1107.

Greenwood, Jeremy; Smith, Bruce 1997: Financial Markets in Development and the Development of Financial Markets. In: Journal of Economic Dynamics and Control, 21, 145-182.

Grill, Wolfgang; Perczynski, Hans 1988: Wirtschaftslehre des Kreditwesens. Landesausgabe Nordrhein-Westfalen. Bad Homburg v.d.H.

Grinblatt, Mark; Titman, Sheridan; Wermers, Russ 1995: Momentum in investment strategies portfolio performance, and herding: A study of mutual fund behavior. In American Economic Review, 85, 1088-1105.

Gömmel, Rainer 1992: Entstehungsgrund und Entwicklung der Effektenbörse im 19. Jahrhundert bis 1914. In: Pohl, Hans (Hrsg.) 1992: Deutsche Börsengeschichte. Frankfurt / Main.

Größl, Ingrid; Stahlecker, Peter 2000: Finanzierungsstrukturen kleiner und mittlerer Unternehmen und Geldpolitik. Diskussionspapier aus dem Institut für Statistik und Ökonometrie der Universität Hamburg, 51.

Größl, Ingrid; Stahlecker, Peter; Wohlers, Eckhardt 1999: Finanzierungsstruktur und Risiken im Unternehmenssektor der Bundesrepublik Deutschland - Eine empirische Bestandsaufnahme. HWWA Discussion Paper, 83.

Grossman, Sanford J.; Miller, Merton H. 1988: Liquidity and Market Structure. In: Journal of Finance, 43 (3), 617-637.

Grossman, Sanford J.; Stiglitz, Joseph E. 1980: On the Impossibility of Informationally Efficient Markets. In: American Economic Review, 70, 393-408. 
Guender, Alfred; Moesch, Mathias 1997: On the Existencs of a Credit Channel of Monetary Policy in Germany. In: Kredit und Kapital, 12 (2), 173-185.

Gurley, J.G.; Shaw, E.S. 1967: Financial Structure and Economic Development. In: Economic Development and Cultural Change, 15 (3), 257-268.

Gurley, John G.; Shaw, E.S. 1960: Money in a theory of finance. Washington, D.C.

Gurley, J.G.; Shaw, E.S. 1955: Financial aspects of economic development. In American Economic Review, 45, 515-538.

Haley, Charles W.; Schall, Lawrence D. 1979: The Theory of Financial Decisions, 2.ed. NY.

Haller, Andreas; Stoll, Hans R. 1989: Market Structure and Transaction Costs - Implied Spreads in the German Stock Market. In: Journal of Banking and Finance, 13, 697-708.

Hamerle, Alfred; Rösch, Daniel 1996: Arbitrage Pricing Theory und empirische Bestimmung von fundamentalen und makroökonomischen Risikofaktoren an Kapitalmärkten. Regensburger Diskussionsbeiträge zur Wirtschaftswissenschaft, Nr. 276.

Hamerle, A.; Rösch, D. 1995: Das Surrogatproblem bei CAPM-Tests. Konsequenzen der Nichtbeobachtbarkeit des Marktportefeuilles bei der empirischen Validierung des CAPM. Regensburger Diskussionsbeiträge zur Wirtschaftswissenschaft, Nr. 274.

Hansen, H. 1996a: Der deutsche Aktienmarkt. Entwicklungen, Veränderungen, Strukturen. In: Die Aktiengesellschaft, Sonderheft Oktober 1996. Köln.

Hansen, Herbert 1996b: Die Beteiligungsverhältnisse am deutschen Aktienmarkt. In: Die Aktiengesellschaft - Report, 3, R87-R90.

Harris, Milton; Raviv, Artur 1991: The Theory of Capital Structure. In: Journal of Finance, 46 (1), 297-355.

Haubrich, J.G. 1989: Financial Intermediation: Delegated Monitoring and LongTerm Relationships. In: Journal of Banking and Finance, 13, 9-20.

Hauck, Michael 1988: Die Bedeutung der Versicherungswirtschaft für den Kapitalmarkt. In: Müller, Wolfgang; Stöhr, Jochen (Hrsg.) 1988: Frankfurter Vorträge zum Versicherungswesen, 16, 5-21. Karlsruhe.

Haugen, Robert A. 1987: Introductory Investment Theory. NJ.

Häuser, Karl 1985: Aktienrendite und Renditenparadoxie: 1964-1983 in der Bundesrepublik Deutschland. Frankfurt / Main.

Hayek, F.A. von 1973: Law, Legislation, and Liberty, Band 1. Chicago. / (deutsch) 1980: Recht, Gesetzgebung und Freiheit, Bd. 1. Landsberg/Lech.

Heckerman, Donald G. 1975: Motivating Managers to make Investment Decisions. In: Journal of Financial Economics, 2, 273-292.

Heise, Arne 1996: Arbeit für Alle - Vision oder Illusion? Marburg.

Heitzer, Bernd 2000: Finanzierung junger innovativer Unternehmen durch Venture Capital-Gesellschaften. Köln.

Helaba Trust Beratungs- und Management Gesellschaft mbH 2001: Asset-Monitor, 4. Kalenderwoche v. 10.01.01. In: http://www.helaba-trust.de.

Helg, Rodolfo; Manasso, Paolo; Monacelli, Tommaso; Rovelli, Riccardo 1995: How much (a)symmetry in Europe? Evidence from Industrial Sectors. In: European Economic Review, 39, 1017-1041.

Hellmann, Thomas; Stiglitz, Joseph 2000: Credit and equity rationing in markets with adverse selection. In: European Economic Review, 44, 281-304. 
Hellmann, Thomas; Stiglitz, Joseph 1995: A Unifying Theory of Credit and Equity Rationing in Markets with Adverse Selection. Research Paper, 1356, Graduate School of Business, Stanford University.

Hellwig, Martin 1990: Banking, Financial Intermediation and Corporate Finance. In: Giovanni, Alberto; Mayer, Colin (Hrsg.) 1990: European Financial Integration, 35-63. Cambridge.

Hellwig, Martin 1989: Asymmetric Information, Financial Markets, and Financial Institutions. Where are we currently going? In: European Economic Review, 33, 277-285.

Hellwig, M. 1987: Some Recent Developments in the Theory of Competition in Markets with Adverse Selection. In: European Economic Review, 31, 319-325.

Herdzina, Klaus 1999: Wettbewerbspolitik. 5.A. Stuttgart.

Hermann, Ralf Martin 1998: Bestimmung von Ausschüttungsstrategien mittels eines Stakeholder-orientierten Ansatzes. Ulm.

Herrhausen, Alfred 1971: Risiken und Chancen der Demokratie. In: Weidemann, Kurt (Hrsg.) 1990: Alfred Herrhausen. Denken-Ordnen-Gestalten, 19-31. Berlin.

Heyl, Daniel C. Freiherr von 1995: Noise als finanzwirtschaftliches Phänomen. Eine theoretische Untersuchung der Bedeutung von Noise am Aktienmarkt. Frankfurt / Main.

Hicks, J.R. 1969: A Theory of Economic History. Oxford.

Hicks, J.R. 1939: Value and capital. An inquiry into some fundamental principles of economic theory. Oxford.

Hillebrand, Ernst 2001: Schlüsselstellung im globalisierten Kapitalismus. Der Einfluss privater Rating-Agenturen auf Finanzmärkte und Politik. In: Die Privatisierung der Weltpolitik, 150-171.

Hillier, Brian; Ibrahimo, M.V. 1993: Asymmetric Information and Models of Credit Rationing. In: Bulletin of Economic Research, 45 (4), 271-304.

Hillier, Brian; Worrall, Tim 1994: The Welfare Implications of Costly Monitoring in the Credit Market. In: Economic Journal, 104, 350-362.

Hillmer, Matthias 1993: Kausalanalyse makroökonomischer Zusammenhänge mit latenten Variablen. Mit einer empirischen Untersuchung des Transmissionsmechanismus monetärer Impulse. Heidelberg.

Hogarth, Robin M.; Reder, Melvin W. 1986: The Behavioral Foundation of Economic Theory. In: Journal of Business, 59 (4), part 2.

Hohl, Stefan; Liebig, Thilo 1999: Kreditderivate - ein Überblick. In: Eller, Roland; Gruber, Walter; Reif, Markus 1999: Handbuch Kreditrisikomodelle und Kreditderivate. Quantifizierung und Management von Kreditrisiken, Strategien mit Kreditderivaten, bankaufsichtliche Anforderungen. Stuttgart. 499-525.

Holmström, Bengt; Tirole, Jean 1993: Market Liquidity and Performance Monitoring. In: Journal of Political Economy, 101 (4), 678-709.

Horn, Ernst-Jürgen 1994: Neuere Entwicklungen auf dem deutschen Kapitalmarkt. Institutionen, Marktstrukturen und Marktergebnisse. Tübingen.

Hörnstein, Elke 1990: Arbitrage- und Gleichgewichtsmodelle in der Kapitalmarkttheorie. Eine vergleichende Analyse der CAPM- und APTAnsätze unter Berücksichtigung ihrer empirischen Überprüfbarkeit. Frankfurt / Main u.a.

Hotz, Pirmin 1989: Das Capital Asset Pricing Model und die Markteffizienzhypothese unter besonderer Berücksichtigung der empirisch beobachteten „Anomalien“ in den amerikanischen und anderen internationalen Aktienmärkten. St. Gallen. 
Hubbard, Glenn R. 1998: Capital-Market Imperfections and Investment. In: Journal of Economic Literature, 36, 193-225.

Huffschmidt, J. 1995: Steuern gegen die Spekulation? Der Tobin-Vorschlag und seine Erweiterung. In: Memo-Forum, Zirkular der „Arbeitsgruppe Alternative Wirtschaftspolitik“, 23, 53-65. Bremen.

Hüttemann, Petra 1997: Kreditderivate im europäischen Kapitalmarkt. Wiesbaden.

Jaffee, Dwight M. 1971: Credit Rationing and the Commercial Loan Market. An Econometric Study of the Structure of the Commercial Loan Market. NY.

Jaffee, Dwight M.; Russell, T. 1976: Imperfect Information and Credit Rationing. In: Quarterly Journal of Economics, 90, 651-666.

Jaffee, Dwight M.; Stiglitz, Joseph 1990: Credit Rationing. In: Friedman, Benjamin M.; Hahn, Frank H. (Hrsg.) 1990: Handbook of Monetary Economics, vol. II, chap. 16, 837-888.

Jagannathan, R.; Wang, Z. 1993: The CAPM is Alive and Well, Federal Reserve Bank of Minneapolis, Staff Report 165.

Jandura, Dirk; Rehkugler, Heinz 1999: Monetäre Effekte auf die Bewertung des deutschen Aktienmarktes. In: Kredit und Kapital, 1, 24-59.

Janßen, Birgit; Rudolph, Bernd 1992: Der Deutsche Aktienindex DAX. Konstruktion und Anwendungsmöglichkeiten. Frankfurt / Main.

Jarchow, Hans-Joachim 2000: Geldpolitik bei extrapolativen, semirationalen und rationalen Inflationserwartungen. Diskussionsbeiträge aus dem Volkswirtschaftlichen Seminar der Universität Göttingen, 102.

Jarchow, H.-J. 1998: Theorie und Politik des Geldes I. Geldtheorie. 10.A. Göttingen.

Jarchow, H.-J. 1994: Monetäre Außenwirtschaft I. Monetäre Außenwirtschaftstheorie. 4.A. Göttingen.

Jarchow, H.-J. 1966: Theoretische Studien zum Liquiditätsproblem. In: Kieler Studien, Forschungsberichte des Instituts für Weltwirtschaft an der Universität Kiel, 75, Tübingen.

Jensen, Michael C. 1986: Agency Costs of Free cash Flow, Corporate Finance and Takeovers. In: American Economic Review, papers and proceedings, 76 (2), 323-329.

Jensen, Michael C.; Meckling, William H. 1998: Unterinvestition. In: Bolton, Patrick; Scharfstein, David S. 1998: Corporate Finance, the Theory of the Firm, and Organizations. Ort.

Jensen, M.C.; Meckling, W.H. 1976: Theory of the Firm: Managerial Behavior, Agency Costs and Ownership Structure. In: Journal of Financial Economics, 3, 305-360.

Jensen, Michael C.; Murphy, Kevin J. 1990: Performance Pay and Top Management Incentives. In: Journal of Political Economy, 98, 225-264.

Jickeli, Joachim 1996: Der langfristige Vertrag. Eine rechtswissenschaftliche Untersuchung auf institutionen-ökonomischer Grundlage. Baden-Baden.

John, Kose; Williams, Joseph 1985: Dividends, Dilution, and Taxes: A Signalling Equilibrium. In: Journal of Finance, 40 (4), 1053-1069.

Jovanovic, B. 1982: Selection and the Evolution of Industry. In: Econometrica, 50 649-670.

Jürgens, Ulrich; Naumann, Katrin; Rupp, Joachim 2000: Shareholder value in an adverse environment: the German case. In: Economy and Society, 29 (1), 54-79.

Jüttner, Johannes 1989: Fundamentals, Bubbles, Trading Strategies: Are they the Causes of Black Monday? In: Kredit und Kapital, 4, 474. 
Kadlec, Gregory B.; McConell, John J. 1994: The Effect of Market Segmentation and Illiquidity on Asset Prices: Evidence from Exchange Listings. In: Journal of Finance, 49 (2), 611-636.

Kakes, Jan 2000: Monetary Policy and Business Cycle Asymmetry in Germany. In: Kredit und Kapital, 2, 182-197.

Kashyap, Anil; Stein, Jeremy 1994: Monetary Policy and Bank Lending. In: Mankiw, Gregory (ed.): Monetary Policy. NBER. Chicago.

Kataoka, Shinji 1963: A Stochatic Programming Model. In: Econometrica, 31, 181196.

Keeton, W.R. 1979: Equilibrium Credit Rationing. New York.

Keim, Donald 1983: Size-Related Anomalies and Stock Return Seasonality - Further Empirical Evidence. In: Journal of Financial Economics, 12, 13-32.

Kern, Hans-Günther 1992: Ökonomische Theorie der Langzeitverträge. In: JuS, 13.

Keynes, John Maynard 1936: The General Theory of Employment Interest and Money. NY.

Keynes, J.M. 1933: Nationale Genügsamkeit. In: Schmollers Jahrbuch, 561-570.

Keynes, J. M. 1930: A Treatise on Money, vol. 1. NY.

KfW 2001a: Die Verbriefung von Mittelstandskrediten durch die KfW. In: Mittelstands- und Strukturpolitik, Bd. 21, 24-30. Frankfurt / Main.

KfW 2001b: Beiträge zur Mittelstands- und Strukturpolitik, Bd. 26. Frankfurt / Main.

KfW 1999: Beiträge zur Mittelstands- und Strukturpolitik, 11/99, Bd. 12. Frankfurt / Main.

Kimball, Ralph C. 2000: Failures in Risk Management. In: New England Economic Review, Jan./Febr., 3-12.

King, Robert G.; Levine, Ross 1993: Finance and Growth: Schumpeter might be right. In: Quarterly Journal of Economics, 717-737.

Kiyotaki, N.; Moore, J. 1997: Credit Cycles. In: Journal of Political Economy, 105, 211-248.

Kleidon, Allan W. 1986: Anomalies in Financial Economics: Blueprint for Change? In: Journal of Business, 59 (4), 469-499.

Knight, Frank 1921: Risk, Uncertainty, and Profit. Boston.

Köhler, Peter 2000: Zweite Reihe - einsame Spitze. In: Handelsblatt v. 01.12.00.

Kosfeld, Reinhold 1996: Kapitalmarktmodelle und Aktienbewertung. Eine statistisch-ökonometrische Analyse. Wiesbaden.

Kotz, Hans Helmut 2000: Basel II - neue Anforderungen an die Bankenaufsicht. In: Zeitschrift für das gesamte Kreditwesen, 12, 638-642.

Kotz, H.H. 1999: "Clash of Cultures"? - Transaktionsorientierung versus Bankenintermediation. In: Nölling, Wilhelm; Schachtschneider, Karl Albrecht; Starbatty, Joachim (Hrsg.) 1999: Sonderdruck aus: Währungsunion und Weltwirtschaft. Festschrift für Wilhelm Hankel, 311-323. Stuttgart.

Kotz, H.H. 1998: Europäisierung der Finanzmärkte: Vom Verschwinden von Substitutionslücken und Schleiern. In: Caesar, Rolf; Scharrer, Hans-Eckart (Hrsg.) 1998: Die Europäische Wirtschafts- und Währungsunion: regionale und globale Herausforderungen, 252-268. Europäische Schriften des Instituts für Europäische Politik, Nr. 76. Bonn.

Krag, Joachim; Schmelz, Michael; Seekamp, Volker 1998: Bonitätsanalyse von Rating-Agenturen. Marburg.

Krainer, John; LeRoy, Stephen F. 1997: Returns on Illiquid Assets: Are They Fair Games? Working Paper. Chicago.

Kregel, J.A. 1998: Aspects of a Post Keynesian theory of finance. In: Journal of Post Keynesian Economics, 21 (1), 111-133. 
Kreps, David M. 1994: Mikroökonomische Theorie. Aus dem Englischen übersetzt von Schnittko, Ulrich K. Landsberg/Lech.

Kriebel, Horst 1962: Börsenumsatzveröffentlichung - ja oder nein? Ein Beitrag zur Kontroverse um die Frage der Umsatzpublizität an den deutschen Wertpapierbörsen. In: Die Aktiengesellschaft, 3, 71-73.

Kromphardt, Jürgen 1993: Wachstum und Konjunktur. Grundlagen der Erklärung und Steuerung des Wachstumsprozesses, 3.A. Göttingen.

Kromphardt, J. 1987: Konzeptionen und Analysen des Kapitalismus - von seiner Entstehung bis zur Gegenwart. Göttingen.

Kruschwitz, Lutz; Löffler, Andreas 1997: Ross' APT ist gescheitert. Was nun? In: Schmalenbachs Zeitschrift für betriebswirtschaftliche Forschung, 49 (7/8), 644-651.

Kuhn, Thomas S. 1979: Die Struktur wissenschaftlicher Revolutionen. 2.A. Frankfurt / Main.

Kühne, Gabriel 1991: Lange Wellen der wirtschaftlichen Entwicklung: theoretische Erklärungsansätze und Verbindungslinien zur Geschichte der Wirtschaftstheorie und Wirtschaftspolitik. Göttingen.

Kulessa, Margareta E. 1996: Die Tobinsteuer zwischen Lenkungs- und Finanzierungsfunktion. In: Wirtschaftsdienst 1996/II, 95-104.

Kurz, Heinz D. 1981: Zum rationierungstheoretischen Ansatz der Neuen Makroökonomik. In: Hagemann, Harald; Kurz, Heinz D.; Schäfer, Wolf (Hrsg.) 1981: Die neue Makroökonomik. Marktungleichgewicht, Rationierung und Beschäftigung. 107-143. Frankfurt / Main, NY.

La Porta, Rafael; Lopez-de-Silvanes, F.; Shleifer, Andrei; Vishny, Robert W. 2000: Agency Problems and Dividend Policies around the World. In: The Journal of Finance, 55 (1), 1-33.

Laffont, Jean-Jacques; Tirole, Jean 1988: Repeated Auctions of Incentive Contracts, Investment, and Bidding Parity with an Application to Takeovers. In: Rand Journal of Economics, 19, 516-537.

Lakatos, Imre 1974: Falsifikation und die Methodologie wissenschaftlicher Forschungsprogramme. In: Ders.; Musgrave, Alan (Hrsg.) 1974: Kritik und Erkenntnisfortschritt, 89-189. Braunschweig.

Landeszentralbank Hessen 2001: Rating am Finanzplatz Frankfurt. In: Frankfurter Finanzmarkt-Bericht, Sept. 2001, Nr. 40.

Landry, Sophie; Radeke, Oliver 1999: Kreditderivate in der Praxis. In: Eller, Roland; Gruber, Walter; Reif, Markus 1999: Handbuch Kreditrisikomodelle und Kreditderivate. Quantifizierung und Management von Kreditrisiken, Strategien mit Kreditderivaten, bankaufsichtliche Anforderungen, 527-574. Stuttgart.

Lazonick, William; O'Sullivan, Mary 2000: Maximizing shareholder value: a new ideology for corporate governance. In: Economy and Society, 29 (1), 13-35.

Le Coutre, W. 1926: Praxis der Bilanzkritik, Bd. II. Berlin, Wien.

Lee, C.F.: 1976: Investment Horizon and the Functional Form of the Capital Asset Pricing Model. In: Review of Economics and Statistics, 58, 356-363.

Lehr, Carol Scotese; Wang, Ping 2000: Dynamic Effects of Financial Intermediation over the Business Cycle. In: Economic Inquiry, 38 (1), 34-57.

Leibenstein, Harvey 1966: Allocative Efficiency vs. "X-Efficiency". In: American Economic Review, 56, 392-415.

Leijonhufvud, Axel 1989: Recent Developments in Macroeconomics and the New Classical View on Money. In: Forschungsgruppe „Postkeynesianische 
Ökonomie“, Diskussionsbeiträge zur gesamtwirtschaftlichen Theorie und Politik, Neue Folge, 13. Bremen.

Leijonhufvud, A. 1981: Effective Demand Failures. In: Ders. (Hrsg.) 1981: Information and Coordination. Essays in Macroeconomic Theory, 103-129. NY, Oxford.

Leopold, Günter 1993: Gewinnung von externem Eigenkapital für nicht börsennotierte Unternehmen. In: Gebhardt, Günther; Gerke, Wolfgang; Steiner, Manfred (Hrsg.) 1993: Handbuch des Finanzmanagements. Instrumente und Märkte der Unternehmensfinanzierung, 345-364. München.

Leopold, Günter; Frommann, Holger 1998: Eigenkapital für den Mittelstand. Venture Capital im In- und Ausland. München.

Levine, Ross 1997: Financial Development and Economic Growth: Views and Agenda. In: Journal of Economic Literature, 35, 688-726.

Levine, R. 1991: Stock Markets, Growth, and Tax Policy. In: Journal of Finance, 46 (4), 1445-1465.

Levine, Ross; Loayza, Norman; Beck, Thorsten 2000: Financial intermediation and growth: Causality and causes. In: Journal of Monetary Economics, 46, 31-77.

Levine, Ross; Zervos, Sara 1998: Stock Markets, Banks, and Economic Growth. In: American Economic Review, 88 (3), 537-558.

Levine, R.; Zervos, S. 1996: Stock Market Development and Long-Run Growth. In: World Bank Economic Review, 10 (2), 323-339.

Levine, R.; Zervos, S. 1994: Stock markets and banks: Revving the engines of growth. The World Bank.

Levy, Haim 1997: Risk and Return: An Experimental Analysis. In: International Economic Review, 38 (1), 119-149.

Levy, H. 1990: Small Firm Effect: Are there Abnormal Returns in the Market? In: Journal of Accounting, Auditing and Finance, 5, 235-276.

Levy, H. 1978: Equilibrium in an Imperfect Market: A Constraint on the Number of Securities in the Portfolio. In: American Economic Review, 68 (4), 643-658.

Lintner, John 1969: The Aggregation of Investors' Diverse Judgment and Preferences in Purely Competitive Securities Markets. In: Journal of Financial and Quantitative Analysis, 4, 347-400.

Lintner, J. 1965a: The Valuation of Risk Assets and the Selection of Risky Investments in Stock Portfolios and Capital Budgets. In: Review of Economics and Statistics, 47, Febr., 13-37.

Lintner, J. 1965b: Security Prices, Risk, and Maximal Gains from Diversification. In: Journal of Finance, 20, Dec., 587-615.

Liu, Pak-Wai; Yang, Xiaokai 2000: The theory of the size of the firm. In: Journal of Economic Behavior \& Organization, 42, 145-165.

Löchel, Horst 1995: Institutionen, Transaktionskosten und wirtschaftliche Entwicklung. Ein Beitrag zur Neuen Institutionenökonomik und zur Theorie von Douglass C. North. Berlin.

Lucas, Robert E., Jr. 1988: On the mechanics of economic development. In: Journal of Monetary Economics, 22, 3-42.

Luce, R.D.; Raiffa, H.1957: Games and Decisions. Introduction and Critical Survey. NY.

Luhmann, Niklas 1990: Die Zukunft kann nicht beginnen: Temporalstrukturen der modernen Gesellschaft. In: Sloterdijk, Peter (Hrsg.) 1990: Vor der Jahrtausendwende: Berichte zur Lage der Zukunft, 119-150. Frankfurt / Main.

Lüthje, Bernd 1970: Die Funktionsfähigkeit der deutschen Aktienbörse. Göttingen. 
Machlup, Fritz 1983: The Rationality of 'Rational Expectations'. In: Kredit und Kapital, 16, 174.

Macneil, J.R. 1974: The Many Futures of Contract. In Southern California Law Review, 47, 691-816.

Mahoney, Richard J. 1997: Shareowners, Stakeholders, and Enlightened SelfInterest. In: Business \& the Contemporary World, 9 (2), 325-336.

Maier, Gerhard 1998: Der große Bluff. Aktienrückkäufe werden auch in Deutschland populär - der Nutzen für die Anleger ist fraglich. In: Die Zeit v. 07.05.98, Nr. $20,36$.

Mankiw, N. Gregory 1986: The Allocation of Credit and Financial Collapse. In: Quarterly Journal of Economics, 101, 455-470.

Marek 2000: Systematisches und unsystematisches Risiko. In: WISU, 2, 2000, 185.

Markowitz, Harry 1990: Risk Adjustment. In: Journal of Accounting, Auditing and Finance, 5, 213-225.

Markowitz, H. 1952: Portfolio Selection. In: Journal of Finance, 7, 77-91.

Marsh, David 2001: Private Stärke. Ein Börsengang bringt Unternehmen auch Nachteile. In: Die Zeit v. 08.02.01, Nr. 7, 25.

Marterbauer, Markus 1994: Bubbles on a Whirlpool of Speculation. Finanzmärkte, Realwirtschaft und Regulierungsnotwendigkeiten. In: Memo-Forum, Zirkular der „Arbeitsgruppe Alternative Wirtschaftspolitik“, 21, Mai, 13-19. Bremen.

Martin, Philippe; Rey, H. 2000: Financial integration and asset returns. In: European Economic Review, 44, 1327-1350

Marx, Karl 1859: Zur Kritik der Politischen Ökonomie. MEW, 13. Berlin.

Mason, Scott P. 1995: The Allocation of Risk. In: Crane, Dwight B. et.al. (eds.) 1995: The Global Financial System. A Functional Perspective, 153-195. Boston (Mass.).

Mayer, Colin 1999: European capital markets: Competition between systems. In: Cahiers BEI / European Investment Bank, 4 (1), 45-57.

Mayer, C. 1988: New Issues in Corporate Finance. In: European Economic Review, 32 (5), 1167-1188.

Mayers, David 1972: Nonmarketable Assets and the Capital Market Equilibrium under Uncertainty. In: Jensen, M.C. (Hrsg.) 1972: Studies in the Theory of Capital Markets, 223-248. NY.

McKinnon, Ronald I.: 1973: Money and Capital in Economic Development. Washington, D.C.

Meier-Scherling von Deutschland, Philipp 1996: Shareholder Value Analyse vs. Stakeholder Management: Unternehmenspolitische Grundkonzeptionen als Ansätze zur Erweiterung der Theorie der Unternehmung. Darmstadt.

Menger, Carl 1883: Untersuchungen über die Methode der Socialwissenschaften und der Politischen Oekonomie insbesondere. Leipzig (Neuauflage Tübingen 1969).

Mensch, Gerhard 1975: Das technologische Patt. Innovationen überwinden die Depression. Frankfurt / Main.

Merton, R.C. 1987: Presidental adress: A Simple Model of Capital Market Equilibrium with Incomplete information. In: Journal of Finance, 42 (3), 483-510.

Merton, R.C. 1973a: Theory of rational option pricing. In: Bell Journal of Economics and Management Science, 4, 141-183.

Merton, R.C. 1973b: An intertemporal capital asset pricing model. In: Econometrica, 41, 867-887.

Meyer, Ingeborg 1957: Geldwertbewußtsein und Münzpolitik: das sogenannte Gresham'sche Gesetz im Lichte der Verhaltensforschung. Köln, Opladen. 
Michel, Uwe 1996: Shareholder Value Management - Neue Aufgaben für das globale strategische Controlling. In: Horvath, Peter (Hrsg.) 1996: Controlling des Strukturwandels. Standortflexibilität und Kundenzufriedenheit schaffen, 79-107. Stuttgart.

Michie, R.C. 1987: The London and New York Stock Exchanges, 1850-1914. London.

Milde, H.; Riley, J.G. 1988: Signalling in Credit Markets. In: Quarterly Journal of Economics, 183, 101-129.

Milgrom, Paul; Roberts, John 1992: Economics, Organization \& Management. NJ.

Miller, M.H. 1988: The Modigliani-Miller propositions after thirty years. In: Journal of Economic Perspectives, 2, 99-120.

Mishkin, Frederic S. 1985: Symposium on the Monetary Transmission Mechanism. In: Journal of Economic Perspectives, 9 (4), 3-10.

Modigliani, F.; Miller, M. 1961: Dividend Policy, Growth, and the Valuation of Shares. In: Journal of Business, 34, 411-433.

Modigliani, F.; Miller, M. 1958: The Cost of Capital, Corporation Finance, and the Theory of Investment. In: American Economic Review, 48, 261-297.

Möller, Hans Peter 1988: Die Bewertung risikobehafteter Anlagen an deutschen Wertpapiermarktbörsen. In: Zeitschrift für betriebswirtschaftliche Forschung, 40, 779-797.

Monsen, R.J.; Downs, A. 1965: A theory of large managerial firms. In: Journal of Political Economy 73, 221-236.

Mooslechner, Peter 1995: Finanzmarktliberalisierung und Wirtschaftspolitik. In: Thomasberger, Claus (Hrsg.) 1995: Europäische Geldpolitik zwischen Marktzwängen und neuen institutionellen Regelungen. Zur politischen Ökonomie der europäischen Währungsintegration, 97-123. Marburg.

Morck, Randall; Shleifer, Andrei; Vishny, Robert W. 1989: Alternative Mechanisms for Corporate Control. In: American Economic Review, 79 (4), 842-852.

Mossin, Jan 1966: Equilibrium in a Capital Asset Market. In: Econometrica, 34 (4), 768-783.

Musgrave, Richard A.; Musgrave, Peggy B.; Kullmer, Lore 1985: Die öffentlichen Finanzen in Theorie und Praxis, 2, 3.A. Tübingen.

Muth, J.F. 1961: Rational Expectations and the Theory of Price Movements. In: Econometrica, 29, 315-335.

Myers, Stewart C. 1984: The Capital Structure Puzzle. In: Journal of Finance, 39 (3), 575-592.

Myers, Stewart C. 2000: Outside Equity. In: Journal of Finance, 55 (3), 1005-1037.

Myers, Stewart C.; Majluf, Nicholas S. 1984: Corporate Financing and Investment Decisions when Firms have Information that Investors do not have. In: Journal of Financial Economics, 13, 187-221.

Nalebuff, Barry J.; Brandenburger, Adam M. 1998: Co-opetition: 1. A revolutionary mindset that combines competition and co-operation; 2 . The game theory strategy that's changing the game business. NY.

Neal, Penny 1996: Keynesian uncertainty in credit markets. In: Journal of Post Keynesian Economics, 18 (3), 397-418.

Nefiodow, Leo A. 1996: Der sechste Kondratieff: Wege zur Produktivität und Vollbeschäftigung im Zeitalter der Information. Sankt Augustin.

Nelson, Richard R.; Winter, Sidney G. 1982: An Evolutionary Theory of Economic Change. Cambridge (Massachusetts), London. 
Neus, Werner 1993: Emissionskredit und Reputationseffekte - Zur Rolle der Banken bei Aktienemissionen. In: Zeitschrift für Betriebswirtschaft, 63, 897-915.

Neusser, K; Kugler, M. 1998: Manufacturing growth and financial development: evidence from OECD countries. In: Review of Economics and Statistics, 80, 638-646.

Neyer, Ulrike 2000: Fehlallokation von Kapital als Folge asymmetrisch verteilter Informationen auf den Kreditmärkten. Implikationen für den Aufbauprozeß der ostdeutschen Wirtschaft. In: Pohl, Rüdiger (2000): Schriften des Instituts für Wirtschaftsforschung Halle, Bd.7. Baden-Baden.

Neyer, Ulrike 1997: Unterinvestition als Folge asymmetrisch verteilter Informationen unter Berücksichtigung risikoaversen Verhaltens der Kreditinstitute Implikationen für Ostdeutschland. Martin-Luther-Universität HalleWittenberg, Wirtschaftswissenschaftliche Fakultät, Volkswirtschaftliche Diskussionsbeiträge, 9.

Niklisch, Fritz 1987: Vorteile für eine Dogmatik für komplexe Langzeitverträge. In: Ders. (Hrsg.) 1987: Der komplexe Langzeitvertrag. Strukturen und Internationale Schiedsgerichtsbarkeit. Heidelberg.

Niklisch, H. 1932: Die Betriebswirtschaft, 7.A. Stuttgart.

Nissen, Dirk 1999: Zur Relevanz des Stakeholder-Ansatzes im Bereich der Biotechnologie. Universität Lüneburg, Fachbereich Wirtschafts- und Sozialwissenschaften. Arbeitsbericht Nr. 213, Juni.

North, Douglas 1990: Institutions, Institutional Change and Economic Performance. Cambridge.

Obstfeld, Maurice 1998: The Global Capital Market: Benefactor or Menace? In: Journal Economic Perspectives, 12 (4), 9-30.

Obstfeld, M. 1994: Risk-Taking, Global Diversification, and Growth. In: American Economic Review, 85, 1310-1329.

Oertmann, Peter 1997: Global Risk Premia on International Investments. Wiesbaden.

Oertmann, Peter 1994a: Size Effect und Performance von deutschen Aktien. In: Finanzmarkt und Portfoliomanagement, 8 (2), 197-211.

Oertmann, Peter 1994b: Firm-Size-Effekt am deutschen Kapitalmarkt. Eine empirische Untersuchung. In: Zeitschrift für betriebswirtschaftliche Forschung, 46 (3), 229-259.

Oesterhelweg, Olaf; Schiereck, Dirk 1993: Meßkonzepte für die Liquidität von Finanzmärkten. In: Die Bank, 7, 390-397.

Pagano, Marco; Röell, Ailsa 1990: Trading Systems in European Stock Exchanges: Current Performance and Policy Options. In: Economic Policy, 10, 63-115.

Palley, Thomas I. 1999: Speculation and Tobin Taxes: Why Sand in the Wheels Can Increase Economic Efficiency. In: Journal of Economics, 69 (2), 113-126.

Papon, Kerstin 2001: Der neue Charme des Fremdkapitals. In: F.A.Z. v. 07.05.01, Nr. 105, 15.

Pätzold, Jürgen 1993: Stabilisierungspolitik. Grundlagen der nachfrage- und angebotsorientierten Wirtschaftspolitik. 5.A. Bern, Stuttgart, Wien.

Perez-Quiros; Timmermann, Allan 2000: Firm Size and Cyclical Variations in Stock Returns. In: The Journal of Finance, 55 (3), 1229-1262.

Perlitz, Manfred; Löbler, Helge 1989: Das Innovationsverhalten in der mittelständischen Industrie. Das Risk/Return Paradoxon. Stuttgart.

Perridon, Louis; Steiner, Manfred 1999: Finanzwirtschaft der Unternehmung. 10.A. München. 
Perridon, Louis; Steiner, Manfred 1993: Finanzwirtschaft der Unternehmung. 7.A. München.

Piper, Nikolaus 2001: James Tobin und das große Missverständnis. Globalisierungsgegner kämpfen für die Tobin-Steuer, die mit der Idee des Erfinders nicht mehr viel zu tun hat. In: Süddeutsche Zeitung v. 22.09.01, 24.

Pleschak, Franz; Kulicke, Marianne; Stummer, Frank 1998: Beteiligungsfinanzierung in Technologieunternehmen der Neuen Bundesländer. Studie für die Technologie-Beteiligungs-Gesellschaft (tbg) der Deutschen Ausgleichsbank (DtA). Bonn.

Porter, M.E. 1992: Capital Choices: Changing the Way America Invests in Industry. Research Report. Council of Competitiveness / Harvard Business School. Washington.

Rajan, Raghuram G.; Zingales, Luigi 1998: Financial Dependence and Growth. In: American Economic Review, 88 (3), 559-586.

Rajan, R.G.; Zingales, L. 1995: What do we know about capital structure? Some Evidence from International Data. In: Journal of Finance 50, 1421-1460.

Ramb, Fred 2000: Verschuldungsstrukturen im Vergleich. Eine Analyse europäischer Unternehmen. In: Kredit und Kapital, 1, 1-38.

Rappaport, A. 1995: Shareholder Value. Wertsteigerung als Maßstab für die Unternehmensführung. Übersetzt von Wolfgang Klien. Stuttgart.

Rappaport, A. 1986: Creating Shareholder value: The New Standard for Business Performance. NY.

Rasch, Sebastian 1996: Der Aktienmarkt für kleine und mittelgroße Unternehmen. Mannheim.

Rasch, S. 1994a: Börsensegmente für Nebenwerte an Europas Börsen. In: ZEW, Discussion Paper, 94-05.

Rasch, S. 1994b: Börsensegmente für Nebenwerte an Europas Börsen. In: Die Bank, 9, $512-517$.

Rathenau, Walter 1917: Vom Aktienwesen. Berlin.

Rau-Bredow, Hans 1992: Zur theoretischen Fundierung der Institutionenökonomie. München.

Reden, Eckart von 2000: Innovative Finanzierungsformen für den Mittelstand. In: Zeitschrift für das gesamte Kreditwesen, 10, 533f.

Reimer, Hauke 1999: Äpfel am Baum lassen. In: Wirtschaftswoche v. 09.12.99, Nr. 50, 200-204.

Reinganum, Marc R. 1992: A Revival of the Small-Firm Effect. In: The Journal of Portfolio Management, spring, 55-62.

Reinganum, M.R. 1981: Misspecification of Capital Asset Pricing. Empirical Anomalies Based on Earnings' Yields and Market Values. In: Journal of Financial Economics, 9, 19-46.

Reuter, Norbert 1994a: Der Institutionalismus. Geschichte und Theorie der evolutionären Ökonomie. Marburg.

Reuter, N. 1994b: Institutionalismus, Neo-Instituionalismus, Neue Instiutionelle Ökonomie und andere „Institutionalismen“. Eine Differenzierung konträrer Konzepte. In: Zeitschrift für Wirtschafts- und Sozialwissenschaften, 114, 5-23.

Richard, Hermann-Josef 1992: Aktienindizes. Grundlagen ihrer Konstruktion und Verwendungsmöglichkeiten unter besonderer Berücksichtigung des Deutschen Aktienindex - DAX. Bergisch Gladbach. 
Richter, Rudolf; Furubotn, Eirik 1996: Neue Institutionenökonomik. Eine Einführung und kritische Würdigung. Übersetzt von Monika Streissler. Tübingen.

Ritthaler, Mona 1994: Kreditrationierung. Weiden, Regensburg.

Robinson, Joan 1979: The Generalization of the General Theory. And Other Essays. 2.A. London u.a.

Robinson, J. 1969: The Economics of Imperfect Competition. 2.A. London, NY.

Robinson, Joan; Eatwell, J. 1974: Einführung in die Volkswirtschaftslehre. München.

Rock, K. 1986: Why new issues are underpriced. In: Journal of Financial Economics, $15,187-212$.

Rode, Michael; Moser, Christian 1999: Die neuen Basler Eigenkapitalanforderungen. In: Zeitschrift für das gesamte Kreditwesen, 14, 720-733.

Rohwetter, Marcus: Wenn der Markt sich selbst zu Markte trägt. In: Die Zeit, Nr. 5, 25.01.01, 27.

Rolfes, Bernd 1992: Moderne Investitionsrechnung. München.

Roll, Richard 1981: A Possible Explanation of the Small Firm Effect. In: Journal of Finance, 36, 879-888.

Roll, R.: 1977: A Critique of the Asset Pricing Theory's Test. Part I. In: Journal of Financial Economics, 4, 129-176.

Ross, Stephen A.1977: The Determinantion of Financial Structure: The Incentive Signalling Approach. In: Bell Journal of Economics, 8, 23-40.

Ross, S. A. 1976: The Arbitrage Theory of Capital Asset Pricing. In: Journal of Economic Theory, 13, 341-360.

Rothschild, M.; Stiglitz, J.E. 1970: Increasing Risk: I. A Definition. In: Journal of Economic Theory, 2, 225-243.

Rousseau, P.L.; Wachtel, P. 1998: Financial Intermediation and economic performance: historical evidence from five industrial countries. In: Journal of Money, Credit, and Banking, 30, 657-678.

Roy, A.D. 1952: Safety First and the Holding of Assets. In: Econometrica, 20, 431449.

Rubinstein, Mark E. 1973: A Mean-Variance Synthesis of Corporate Financial Theory. In: Journal of Finance, 28, 167-181.

Rudolph, Bernd 1992: Effekten- und Wertpapierbörsen, Finanztermin- und Devisenbörsen seit 1945. In: Pohl, Hans (Hrsg.) 1992: Deutsche Börsengeschichte, 293-440. Frankfurt.

Sabisch, Helmut 1991: Produktinnovationen. Stuttgart.

Saelzle, Rainer 1976: Kapitalmarktreaktionen bei Investitionsentscheidungen. In: Die Unternehmung, 30, 319-331.

Sapusek, Annemarie 1998: Informationseffizienz auf Kapitalmärkten. Konzepte und empirische Ergebnisse. Wiesbaden.

Sattler, Ralf Robert 1994: Renditeanomalien am deutschen Aktienmarkt. Aachen.

Schäfer, Annette 1997: Die These der kurzfristorietierten Aktienbewertung. Theoretische und empirische Analyse. Köln.

Scharfenkamp, Norbert 1987: Organisatorische Gestaltung und wirtschaftlicher Erfolg. Organizational Slack als Ergebnis und Einflußfaktor der formalen Organisationsstruktur. Berlin, NY.

Scharfstein, David 1988: The Disciplinary Role of Takeovers. In: Review of Economic Studies, 55, 185-199.

Scharfstein, David; Stein, Jeremy 1990: Herd Behavior and Investment. In: American Eonomic Review, 80, 465-479. 
Scherz, Viktor 1994: Anmerkungen zum vorläufigen Ende der Finanz-Postmoderne. In: Memo-Forum, Nr. 21, 8-12.

Schlick, Oliver 1994: Kreditrationierung und unvollkommene Finanzmärkte. Mikroökonomische Betrachtung und makroökonomische Effekte. Frankfurt / Main u.a.

Schmidt, Ingo 1996: Wettbewerbspolitik und Kartellrecht. Eine Einführung. 5.A. Stuttgart.

Schmidt, Reinhard H. 1986: Grundzüge der Investitions- und Finanzierungstheorie. 2.A. Wiesbaden.

Schmidt, Reinhard H.; Dietz, Friedhelm; Fellermann, Stefan; Hellmann, Norbert; Schommer, Klaus-Peter, Tyrell, Marcel; Wilwerding, Gerhard 1988: Underpricing bei deutschen Erstemissionen 1984/85. In: Zeitschrift für Betriebswirtschaft, 58 (11), 1193-1203.

Schmidt, R.H.; Terberger, Eva 1997: Grundzüge der Investitions- und Finanzierungstheorie. 4.A. Wiesbaden.

Schneider, Dieter 1992: Investition, Finanzierung und Besteuerung. 7.A.

Schnittke, Jürgen 1989: Überrenditeeffekte am deutschen Aktienmarkt. Eine theoretische und empirische Analyse. Münster.

Scholes, Myron; Williams, Joseph 1977: Estimating Betas from Nonsynchronous Data. In: Journal of Financial Economics, 5, 309-327.

Scholz, Manfred 2000: Rating und Mittelstand - eine Bestandsaufnahme für Deutschland. In: Zeitschrift für das gesamte Kreditwesen, 10, 519-522.

Schrader, Torsten 1993: Geregelter Markt und geregelter Freiverkehr. Auswirkungen gesetzgeberischer Eingriffe. Wiesbaden.

Schulze, Stefan 1994: Berechnung von Kapitalkosten. Ein Konzept für nationale und internationale Unternehmen. Wiesbaden.

Schumann, Jochen; Meyer, Ulrich; Ströbele, Wolfgang 1999: Grundzüge der mikroökonomischen Theorie. 7.A. Berlin u.a.

Schumpeter, Joseph A. 1980: Kapitalismus, Sozialismus und Demokratie. München.

Schumpeter, J.A. 1961: Konjunkturzyklen. Eine theoretische, historische und statistische Analyse des kapitalistischen Prozesses; Grundriß der Sozialwissenschaft. Göttingen.

Schumpeter, J.A. 1926: Theorie der wirtschaftlichen Entwicklung. Eine Untersuchung über Unternehmensgewinn, Kapital, Kredit, Zins und den Konjunkturzyklus. 2.A. München, Leipzig.

Schwartz, Robert A. 1992: Market Liquidity. In: Newman, Peter; Milgate, Murray; Eatwell, John (Hrsg.) 1992: The New Palgrave Dictionary, 2, $666 f$.

Selten, Reinhard 1999: What is Bounded Rationality? Paper prepared for the Dahlem Conference 1999. Sonderforschungsbereich 303 an der Universität Bonn ,Information und die Koordination wirtschaftlicher Aktivitäten, Projektbereich B, Discussion Paper, B-454.

Sharpe, William F. 1977: The CAPM: A "Multi-Beta" Interpretation. In: Levy, H.; Sarnat, M. (Hrsg.) 1977: Financial Decision Making under Uncertainty, 127135. NY.

Sharpe, W. F. 1970: Portfolio Theory and Capital Markets. NY.

Sharpe, W.F. 1964: Capital Asset Prices: A Theory of Market Equilibrium under Conditions of Risk. In: Journal of Finance, 19, 425-442.

Sharpe, William F.; Gordon, Alexander 1990: Investments, 4.A. NJ.

Shiller, Robert J. 1989: Market Volatility. Cambridge (Mass.).

Shiller, R.J. 1981: Do Stock Prices move too much to be justified by subsequent Changes in Dividends? In: American Economic Review, 71, 421-436. 
Shleifer, Andrei; Summers, Lawrence 1988: Breach of Trust in Hostile Takeovers. In: Auerbach, A. (ed.) 1988: Corporate Takeovers: Causes and Consequences, 33-56. Chicago.

Shleifer, Andrei; Vishny, Robert W. 1997a: A Survey of Corporate Governance. In: Journal of Finance, 52 (2), 737-783.

Shleifer, A.; Vishny, R.W. 1997b: The Limits of Arbitrage. In: Journal of Finance, 52, 35-55.

Shleifer, A.; Vishny, R.W. 1986: Large Shareholders and Corporate Control. In: Journal of Political Economy, 96 (3), 461-488.

Siebel, Ulf R. 1997: Vorzugsaktien als „Hybride“ Finanzierungsform und ihre Grenzen. In: Zeitschrift für das gesamte Handelsrecht und Wirtschaftsrecht, 161, 628-664.

Simon, Herbert A. 1961: Administrative Behavior. NY.

Simon, H.A. 1959: Theories of Decision Making in Economics and Behavioural Science. In: American Economic Review 49, 253-283.

Simon, H.A. 1957: Models of Man. NY.

Simon, H.A. 1955: A behavioral model of rational choice. In: Quarterly Journal of Economics 69, 99-118.

Singleton, Kenneth J. 1990: Specification and Estimation of Intertemporal Asset Pricing Models. In: Friedman, Benjamin M.; Hahn, Frank H. (Hrsg.) 1990: Handbook of Monetary Economics, vol I, 583-626. Amsterdam et.al.

Slodczyk, Katharina 2000: Scheine zweiter Klasse. Vorzugsaktien sind Papiere ohne Rechte - aber mit Rendite. In: Die Zeit v. 08.06.00, Nr. 24, 30.

Smith, Adam 1776: The Wealth of Nations, Cannan Edition (Modern Library, NY, 1937).

Smith, Bruce D. 1998: Informational asymmetries in macroeconomics and finance: An introduction. In: Economic Theory, 12, 451-460.

Sohbi, Hassan 2001: Delisting und Going Private. Chancen, Techniken und Risiken des Börsenausstiegs. In: Venture Capital 2001. Jahrbuch für Beteiligungsfinanzierung, 103-106.

Soros, George 1998a: Die Alchemie der Finanzen. Wie man die Gedanken des Marktes liest. 2.A. NY. Deutsche Übersetzung von Axel Retz.

Soros, G. 1998b: Die Krise des Globalen Kapitalismus; Offene Gesellschaft in Gefahr. Aus dem Amerikanischen übersetzt von Klaus Binder et al. NY.

Soskice, David 1996: Stakeholding Yes; the German Model No. In: Kelly, Gavin; Kelly, Dominic; Gamble, Andrew 1997: Stakeholder Capitalism. 219-225. Ipswich, Suffolk.

Spence, A.M. 1973: Job Market Signaling. In: Journal of Economics, 87, 355-374.

Spence, A.M. 1974: Market Signaling: Information Transfer in Hiring and Related Screening Processes. Cambridge, (Mass.).

Spremann, Klaus 1996: Wirtschaft, Investition und Finanzierung, 5.A. München, Wien.

Stackelberg, Heinrich von 1934: Marktform und Gleichgewicht. Wien, Berlin.

Stapelton, R.C.; Subrahmanyam, M.G 1978: A multiperiod e asset pricing model. In: Econometrica, 46, 1077-1096.

Stein, Jeremy C.: 1988: Takeover Threates and Managerial Myopia. In: Journal of Political Economy, 96, 61-80.

Steinherr, Alfred 1998: Derivatives. The Wild Beast of Finance. Chichester.

Stenzel, Stefan 1995: Ausserbörslicher Aktienhandel. Berlin.

Stigler, G. 1967: Imperfections in the Capital Market. In: Journal of Political Economy, 85, 287-292. 
Stiglitz, Joseph E. 1988: Money, Credit, and Business Fluctuations. In: Economic Record, 64, 307-322.

Stiglitz, J.E 1987: The Causes and Consequences of the Dependence of Quality on Price. In: Journal of Economic Literature, 25, 1-48.

Stiglitz, J.E. 1984: Price Rigidities and Market Structure. In: American Economic Review, 74, 350-356.

Stiglitz, J.E. 1982: Information and Capital Markets. In: Sharpe, W.F.; Cootner, C. (Hrsg.): Financial Economics: Essays in Honor of Paul Cootner, 118-158. NJ.

Stiglitz, J.E. 1981: Ownership, Control, and Efficient Markets: Some Paradoxies in the Theory of Capital Markets. In: Boyer, Kenneth D.; Shepherd, William G. (Hrsg.) 1981: Economic Regulation: Essays in Honor of James R. Nelson, 311341. Michigan.

Stiglitz, J.E. 1979: Equilibrium in Product Markets with Imperfect Information. In: American Economic Review, 69, papers and proceedings, 339-345.

Stiglitz, J.E. 1972: Some Aspects of the Pure Theory of Corporate Finance: Bankruptcies and Take-Overs. In: Bell Journal of Economics, 3, 458-482.

Stiglitz, J.E. 1970: Perfect and Imperfect Capital Markets. Paper presented to the New Orleans meeting of the Econometric Society.

Stiglitz, Joseph E.; Weiss, Andrew 1991: Sorting Out the Differences Between Screening and Signaling Models. In: Bachrach, M. 1991: Oxford Essays in Mathematical Economics, Oxford.

Stiglitz, J.E.; Weiss, A. 1987a: Macro-Economic Equilibrium and Credit Rationing. Working Paper, 2164, NBER.

Stiglitz, J.E.; Weiss, A. 1987b: Credit Rationing with many Borrowers. In: American Economic Review, 77, 228-231.

Stiglitz, J.E.; Weiss, A. 1986: Credit Rationing and Collateral. In: Edward, J.; Franks, J.; Mayer, C.; Shaefer, S. (Hrsg.) 1986: Recent Developments in Corporate Finance, 101-135. NY.

Stiglitz, J.E.; Weiss, A. 1983: Incentive Effects of Terminations: Applications to the Credit and Labour Markets. In: American Economic Review, 73, 912-927.

Stiglitz, Joseph E.; Weiss, A. 1981: Credit Rationing in Markets with Imperfect Information. In: American Economic Review, 71, 393-410.

Strulik, Torsten 2000: Funktionen und Folgen privater Rating-Agenturen im Kontext der Regulierung globaler Finanzmärkte. In: Soziale Welt: Zeitschrift für sozialwissenschaftliche Forschung und Praxis, 51 (4), 443-462.

Stützel, W. 1959: Liquidität. In: Handwörterbuch der Sozialwissenschaften, zugleich auch Neuauflage des Handwörterbuch der Staatswissenschaften, 6, 622. Tübingen, Göttingen.

Sutton, J. 1990: Explaining Everything, Explaining Nothing? Game Theoretic Models in Industrial Economics. In: European Economic Review, 34, 505-512.

Teh, Lillyn L.; De Bondt, Werner F.M. 1997: Herding Behavior and Stock Returns: An Exploratory Investigation. In: Schweizerische Zeitschrift für Volkswirtschaft und Statistik, 133 (2), 293-323.

Teegen, Kirsten 1987: Aktien-Rating-Modelle in der Anlageentscheidung. In: Österreichisches Bank Archiv, 10, 710-721.

Telser, Lester G. 1955: Safety First and Hedging. In: The Review of Economic Studies, 23, 1-16.

Thadden, Ernst Ludwig von 1999: Liquidity creation through banks and markets: Multiple insurance and limited market access. In: European Economic Review, 43, 991-1006. 
Theissen, Erik 2002: Floor versus Screen Trading: Evidence from the German Stock Market. In: JITE, 158, 32-54.

Theissen, Erik 1999: Liquiditätsmessung auf experimentellen Aktienmärkten. In: Kredit und Kapital, 2, 225-264.

Thieme, Heiko 1999: Brief aus Wall. Die Macht der schweren Werte. In: F.A.Z. v. 23.08.99, Nr. 194, 27.

Tholen, Michael 2000: Basel II: Was erwartet den Mittelstand? In: Zeitschrift für das gesamte Kreditwesen 10, 2000, 547-549.

Tobin, J. 1978a: A Proposal for International Monetary Reform. In: The Eastern Economic Journal, 4 (3-4), 153-159.

Tobin, J. 1978b: Grundsätze der Geld- und Staatsschuldenpolitik. Aus dem Amerikanischen übertragen von Ketterer, Karl-Heinz. Schriften zur monetären Ökonomie, Bd. 6, Baden-Baden.

Tobin, J. 1974: The New Economics One Decade Older. The Eliot Janeway Lectures in Honor of Joseph Schumpeter. Princeton, NJ.

Tobin, J. 1957/58: Liquidity Preference as Behavior Towards Risk. In: Review of Economic Studies, 25, 65-86.

Townsend, Robert M. 1979: Optimal Contracts and Competitive Markets with Costly State Verification. In: Journal of Economic Theory, 21, 265-293.

Treynor, J. 1965: How to rate management of investment funds. In: Harvard Business Review, 43, 63-75.

Tschach, Ingo 2000: Theorie der Entwicklungsfinanzierung. Mit Kleinkreditprogrammen Kredit- und Arbeitsmarktsegementierung überwinden. Göttingen.

Unser, Matthias 1999: Behavioral Finance am Aktienmarkt. Empirische Analysen zum Risikoverhalten individueller Anleger. Bad Soden/Ts.

Upper, Christian 2000: How safe Was the "Safe Haven"? Financial Market Liquidity during the 1998 Turbulences. Discussion paper, 1, Economic Research Group of the Deutsche Bundesbank.

Van Horne, James C. 1989: Financial Management and Policy, 8.A. NJ.

Varnholt, Burkhard 1995: Systemrisiken auf Finanzmärkten unter besonderer Berücksichtigung der Märkte für Derivate. Bern.

Vollmer, Lothar 1993: Börsenhandel mit $\mathrm{GmbH}-$ und $\mathrm{KG}-$ Anteilen sowie Veränderungen des Aktienrechts (,Kleine $A G^{\star}$ ). In: Gebhardt, Günther; Gerke, Wolfgang; Steiner, Manfred 1993: Handbuch des Finanzmanagements. Instrumente und Märkte der Unternehmensfinanzierung, 365-381. München.

Vormbaum, Herbert 1995: Finanzierung der Betriebe. 9.A. Wiesbaden.

Wagner, Franz 1997: Shareholder Value: Eine neue Runde im Konflikt zwischen Kapitalmarkt und Unternehmensinteresse. In: (BFuP), 5, 473-498.

Wagner, Wolf-Christof 1991: Rating mittelständischer Unternehmen. Fundierung und Konzeption einer standardisierten Unternehmensbeurteilung durch Rating. Frankfurt / Main u.a.

Walras, Léon 1874: Eléments d'économie politique pure ou théorie de la richesse social, édition definitive 1926. Paris.

Wang, Jiang 1994: A Model of Competitive Stock Trading Volume. In: Journal of Political Economy, 102 (1), 127-168.

Warther, Vincent A. 1997: Dividend Smoothing: A sleeping dogs explanation. Working paper, University of Michigan Business School. 
Weber, René 1999: Liberalisierung des Kapitalverkehrs. Grundsatzfragen zur Marktöffnung im Finanzbereich. In: WiSt, 6, 304-306.

Weber, Wolfgang 1993: Insider-Handel, Informationsproduktion und Kapitalmarkt: eine institutionenökonomische Analyse. Wiesbaden.

Weigand, Jürgen 1996: Innovationen, Wettbewerb und Konjunktur: eine theoretische und empirische Untersuchung von Innovationsdeterminanten unter Berücksichtigung des Konjunkturverlaufs. Erlangen-Nürnberg.

Welcker, Johannes 1982: Technische Aktienanalyse. Die Methoden der technischen Analyse mit Chart-Übungen. Wien.

Wermers, Russ 1999: Mutual Fund Herding and the Impact on Stock Prices. In: Journal of Finance, 54 (2), 581-622.

Wette, H. 1983: Collateral in Credit Rationing in Markets with Imperfect Information: Note. In: American Economic Review, 73, 442-445.

White, Lawrence H. 1999: The Theory of Monetary Institutions. Oxford.

Williamson, O.E 1985: The Economic Institutions of Capitalism. Firms, Markets, Relational Contracting. NY, London.

Williamson, O.E. 1976: Franchise Bidding for Natural Monopolies - In General and With Respect to CATV. In: Bell Journal of Economics, 22, 233-261.

Williamson, Stephen D. 1986: Costly Monitoring, Financial Intermediation, and Equilibrium Credit Rationing. In: Journal of Monetary Economics, 18, 159179.

Windolf, P.; Beyer J. 1999: Kooperativer Kapitalismus. In: Kölner Zeitschrift für Soziologie und Sozialpsychologie, 47 (1), 1-36.

Winker, Peter 1996: Rationierung auf dem Markt für Unternehmenskredite in der BRD. Tübingen.

Winker, P. 1994: Eine makroökonomische Analyse von Kreditmarkt und Kreditrationierung. Bankkredite in der Bundesrepublik Deutschland 19741989. Diskussionsbeitrag des Sonderforschungsbereichs 178 „Internationalisierung der Wirtschaft“, Serie II - Nr. 220, 04/1994. Konstanz.

Winker, P. 1993a: Firmenalter und Kreditrationierung. Eine mikroökonomische Analyse mit IFO-Umfragen. Diskussionsbeitrag, Sonderforschungsbereich 178 „Internationalisierung der Wirtschaft“, Serie II - Nr. 206, 05/1993. Konstanz.

Winker, P. 1993b: Die Trägheit von Zinssätzen und Kreditrationierung in der Bundesrpublik Deutschland. In: Diskussionsbeiträge des Sonderforschungsbereichs 178 „Internationalisierung der Wirtschaft“, Serie II - Nr. 208, 07/1993. Konstanz.

Wolfson, Martin 1996: A post Keynesian theory of credit rationing. In: Journal of Post Keynesian Economics, 18 (3), 443-470.

Wosnitza, Michael 1991: Das Agency-theoretische Unterinvestitionsproblem in der Publikumsgesellschaft. Heidelberg.

Wosnitza, M. 1990: Das Unterinvestitionsproblem in der Publikumsgesellschaft. In: Zeitschrift für betriebswirtschaftliche Forschung, 42 (11), 947-962.

Zwiebel, Jeffrey 1996: Dynamic capital structure under managerial entrenchment. In: American Economic Review, 86, 1197-1215. 